\title{
Experimental flow characterization and computational model development of aqueous film-forming foam (ARFF) firefighting jets
}

Christopher P. Menchini

Follow this and additional works at: https://researchrepository.wvu.edu/etd

\section{Recommended Citation}

Menchini, Christopher P., "Experimental flow characterization and computational model development of aqueous film-forming foam (ARFF) firefighting jets" (2013). Graduate Theses, Dissertations, and Problem Reports. 7331.

https://researchrepository.wvu.edu/etd/7331

This Dissertation is protected by copyright and/or related rights. It has been brought to you by the The Research Repository @ WVU with permission from the rights-holder(s). You are free to use this Dissertation in any way that is permitted by the copyright and related rights legislation that applies to your use. For other uses you must obtain permission from the rights-holder(s) directly, unless additional rights are indicated by a Creative Commons license in the record and/ or on the work itself. This Dissertation has been accepted for inclusion in WVU Graduate Theses, Dissertations, and Problem Reports collection by an authorized administrator of The Research Repository @ WVU.

For more information, please contact researchrepository@mail.wvu.edu. 


\title{
EXPERIMENTAL FLOW CHARACTERIZATION AND COMPUTATIONAL MODEL DEVELOPMENT OF AQUEOUS FILM-FORMING FOAM (AFFF) FIREFIGHTING JETS
}

by

Christopher P. Menchini

\author{
Dissertation submitted to the \\ Benjamin M. Statler College of Engineering and Mineral Resources \\ at West Virginia University \\ in partial fulfillment of the requirements \\ for the degree of \\ Doctor of Philosophy \\ in \\ Mechanical Engineering \\ Gary J. Morris, Ph.D., Committee Chairperson \\ Douglas S. Dierdorf, Ph.D. \\ Wade W. Huebsch, Ph.D. \\ John M. Kuhlman, Ph.D. \\ David C. Lewellen, Ph.D. \\ Gregory J. Thompson, Ph.D.
}

Department of Mechanical and Aerospace Engineering

Morgantown, West Virginia

2013

Keywords: Aqueous Film-Forming Foam, Aircraft Rescue Firefighting, Turbulent Liquid Jets, Phase Doppler Particle Analysis, Computational Fluid Dynamics, Discrete Phase Modeling, ANSYS Fluent 
UMI Number: 3605852

All rights reserved

INFORMATION TO ALL USERS

The quality of this reproduction is dependent upon the quality of the copy submitted.

In the unlikely event that the author did not send a complete manuscript and there are missing pages, these will be noted. Also, if material had to be removed, a note will indicate the deletion.

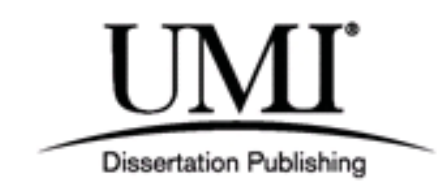

UMI 3605852

Published by ProQuest LLC (2013). Copyright in the Dissertation held by the Author.

Microform Edition (C) ProQuest LLC.

All rights reserved. This work is protected against unauthorized copying under Title 17, United States Code

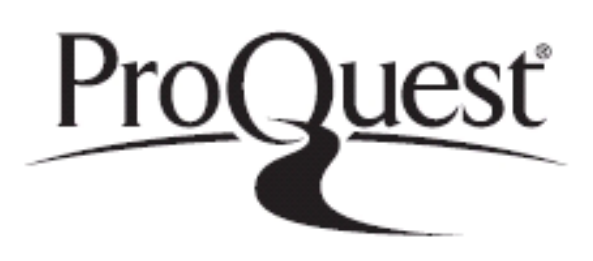

ProQuest LLC.

789 East Eisenhower Parkway

P.O. Box 1346

Ann Arbor, MI 48106 - 1346 


\section{ABSTRACT \\ EXPERIMENTAL FLOW CHARACTERIZATION AND COMPUTATIONAL MODEL DEVELOPMENT OF AQUEOUS FILM-FORMING FOAM (AFFF) FIREFIGHTING JETS}

\section{Christopher P. Menchini}

Over the past few decades, aircraft rescue firefighting (ARFF) research has made technical strides on multiple fronts. Continuing efforts have helped develop computer-aided engineering tools to quantify risk assessment for a variety of ARFF aspects like aircraft pool fire combustion and dynamic crash-related events. To continue this work, a study was conducted to characterize firefighting agent application behavior and to quantify the flow characteristics that differentiate water and AFFF jets. Progress will lead to further simulation capability including a combined aircraft crash-fire-suppression application risk assessment model. An aqueous firefighting agent application laboratory was specially constructed to carry out experiments on firefighting jets ranging from 1 to $11 \mathrm{MPa}$ and 4 to $251 \cdot \mathrm{min}^{-1}$ at AFFF concentration levels ranging from 0 (pure water) to 12 percent by volume. Experimental flow characterization consisted of flow visualization, agent ground pattern distribution analysis, and 2-D phase Doppler particle analysis (PDPA). Flow visualization results depicted minimal differences in terms of overall jet structure between AFFF versus water jets. However, PDPA results showed AFFF enhances jet break-up generating droplet sizes 25 to 100 percent less compared to water jets with AFFF jets lagging water jet velocities by as much as 10 percent in certain instances. Agent ground pattern results confirmed flow performance factors such as ground coverage area, reach, and maximum span all benefit from an increase in nozzle pressure flow rate. An Euler-Lagrange, large eddy simulation computational fluid dynamic (CFD) strategy accounting for droplet collision and break-up was employed to predict firefighting jet flow dynamics with and without the addition of AFFF. AFFF influence was handled computationally via material property variation from pure water in terms of density, viscosity, and surface tension effects. CFD model results were agreeable with flow visualization and phase Doppler data reproducing global trends in both droplet velocity and size data, particularly with respect to the influence of AFFF. However, oversimplified nozzle injection conditions led to greater differences than expected. CFD model result errors were difficult to quantify entirely due to PDPA upper particle size range limitations and complexities associated with direct comparisons to data. High fidelity, near field characterization of AFFFsurface interactions is needed to better understand agent accumulation fluid mechanics with eventual application to fire suppression of aircraft bodies engulfed in fire. 


\section{ACKNOWLEDGEMENTS}

I wish to express my deepest appreciation to all of those who have helped make this work a reality, especially my parents Debbie and Mickey Menchini for a lifetime of support, Drs. Gary Morris, Wade Huebsch, and Doug Dierdorf for their longstanding friendship, technical insight, and guidance in helping to shape overall project direction, Drs. John Kuhlman, Greg Thompson, and David Lewellen for their interest in and review of the work, and to the United States Air Force and Federal Aviation Administration for sponsoring the effort. I would also like to acknowledge Mr. Nick Hillen for his help in constructing the laboratory and in acquiring a portion of the experimental data. I also wish to recognize my co-workers located at Tyndall Air Force Base, FL, especially Mr. Steven Wells, Dr. Mark Enlow, Mr. John Hawk, Mr. Kris Cozart, Mr. Bill Fischer, Mr. Parren Burnette, Ms. Kathy Latza, Mr. John Patnode, and Mr. Mike

McDonald. Without their support, this endeavor would not have been possible. In addition, I would also like to acknowledge the rest of my family, close friends, and co-workers who provided encouragement along the way. 


\section{TABLE OF CONTENTS}

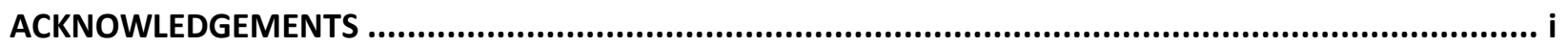

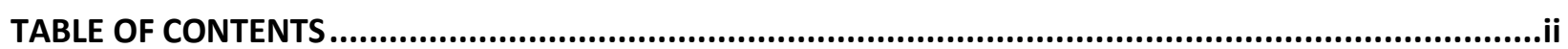

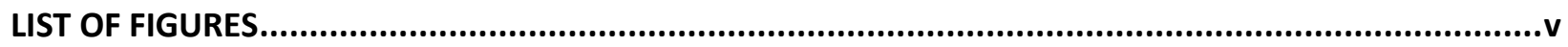

LIST OF TABLES

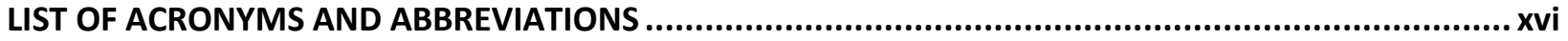

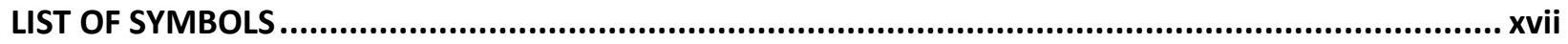

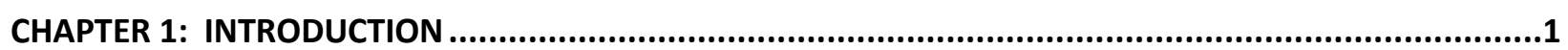

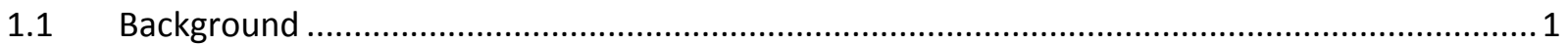

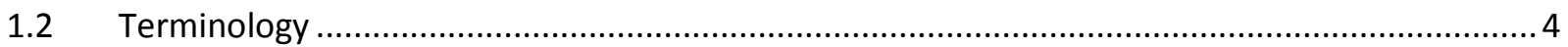

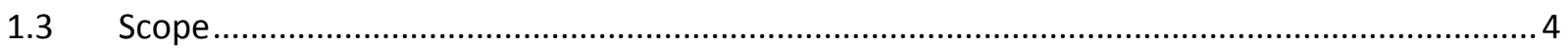

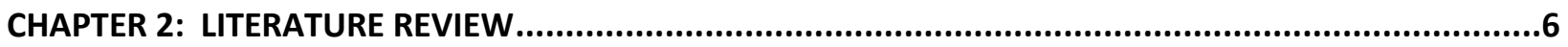

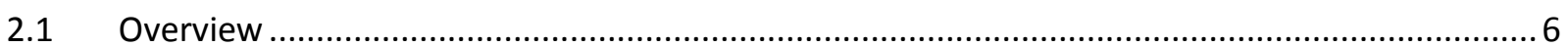

2.2 Physical Characteristics of Aqueous Film-Forming Foam (AFFF) ........................................... 6

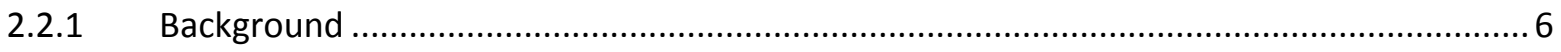

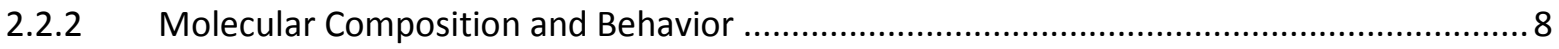

2.2.3 Liquid Continuum Characteristics .............................................................................. 12

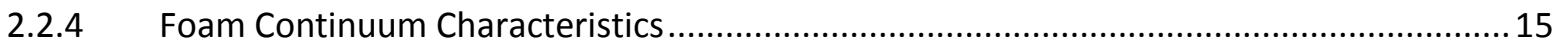

2.3 Turbulent Round Liquid Jets Entering Still Air .................................................................. 17

2.3.1 Early Work on Liquid Jet Instability and Break-up Entering Still Air .................................17

2.3.2 Modern Methods of Classifying Liquid Jet Break-up ........................................................ 20

2.3.3 Secondary Break-up of Liquid Droplets in Still Air ........................................................26

2.3.4 Experimental Flow Characterization with Extension to Firefighting Jets ..........................29

2.3.5 Computational Modeling Methods with Extension to Firefighting Jets ............................34

CHAPTER 3: AQUEOUS FIREFIGHTING AGENT APPLICATION LABORATORY ....................................39

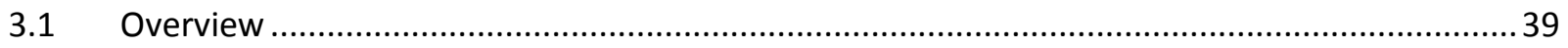

3.2 Firefighting Agent Delivery System ................................................................................ 41

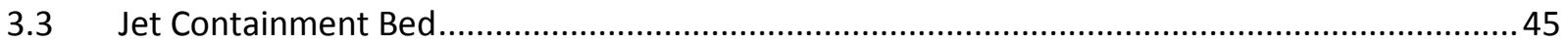

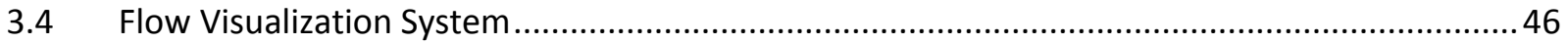

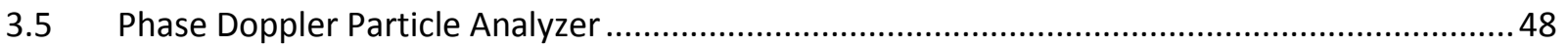

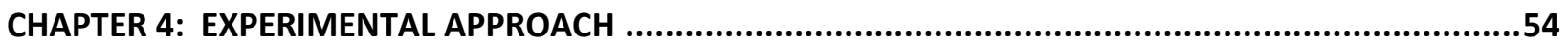

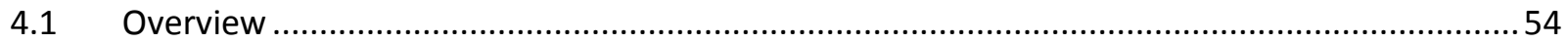

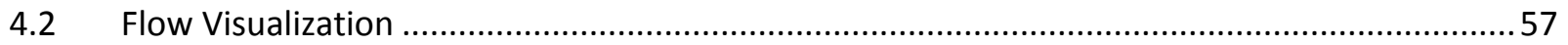

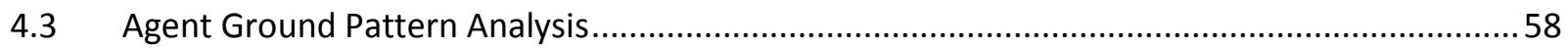




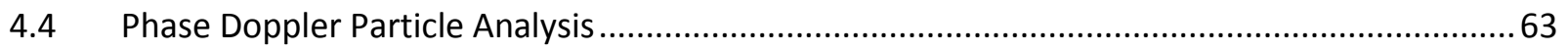

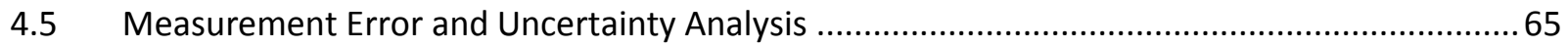

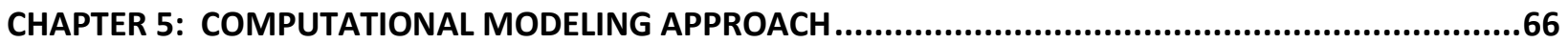

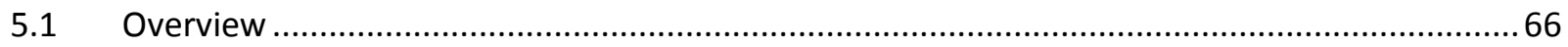

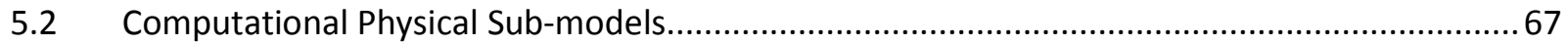

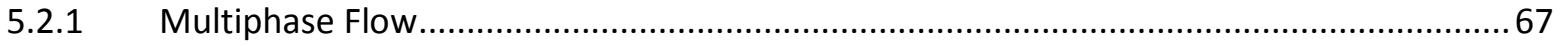

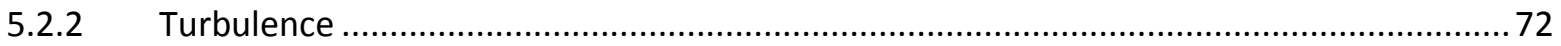

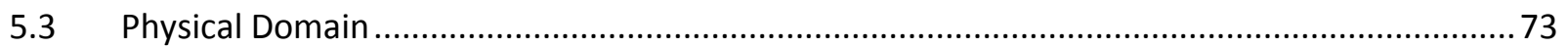

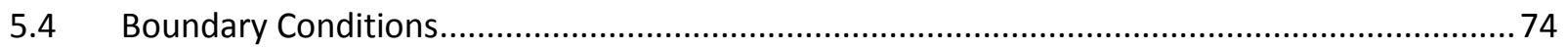

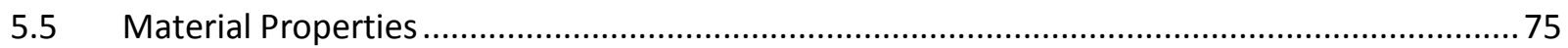

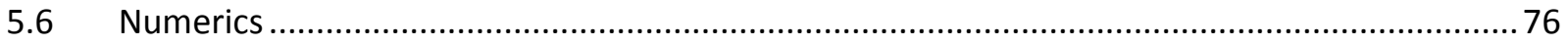

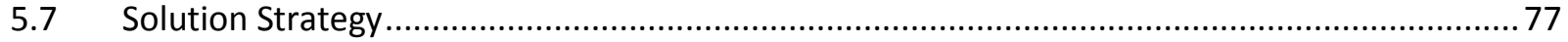

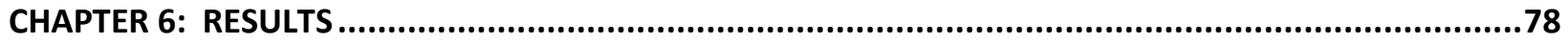

$6.1 \quad$ Experimental Firefighting Jet Flow Visualization Results.................................................... 78

6.1.1 Far Field Flow Visualization Results ............................................................................. 78

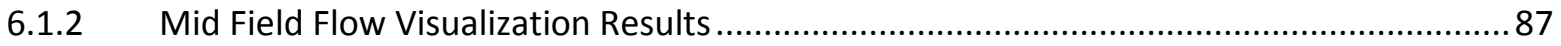

6.1.3 Near Field Flow Visualization Results........................................................................... 91

6.2 Experimental Firefighting Jet Ground Pattern Results .....................................................93

6.2.1 2-D Water Jet Containment Zone Sensitivity Ground Pattern Results ..............................94

6.2.2 2-D Water Jet Ground Pattern Results............................................................................. 95

6.2.3 2-D AFFF Jet Ground Pattern Results ................................................................................ 99

6.2.4 2-D Firefighting Jet Ground Pattern Results Summary ............................................... 102

6.2.5 1-D Firefighting Jet Ground Pattern AFFF Concentration Sensitivity Results .................. 105

6.2.6 Full-Scale 1-D Firefighting Jet Ground Pattern Results ............................................... 105

6.3 Experimental Firefighting Jet Phase Doppler Results ........................................................ 106

6.3.1 Firefighting Jet Phase Doppler Droplet Size Distribution Results ................................... 107

6.3.2 Select Firefighting Jet Phase Doppler Results ............................................................. 110

6.3.3 Firefighting Jet Phase Doppler Results Summary .......................................................... 114

6.3.4 Firefighting Jet Phase Doppler AFFF Concentration Sensitivity Results .........................132

6.4 Computational Firefighting Jet Flow Model Results ............................................................ 137

6.4.1 Computational Firefighting Jet Flow Model Parameter Dependence Results.................. 138

6.4.2 Select Computational Firefighting Jet Flow Model Results Compared to Experiments ... 142

6.4.3 Computational Firefighting Jet Flow Model Results Summary......................................154 


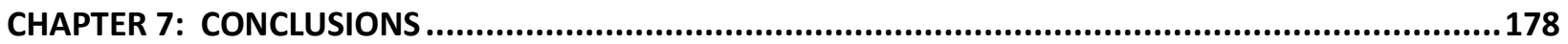

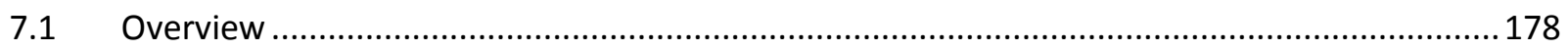

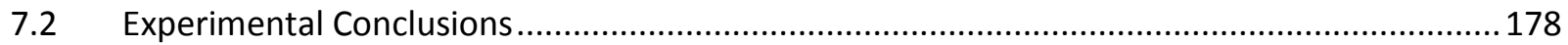

7.3 Computational Firefighting Jet Flow Modeling Conclusions.................................................. 181

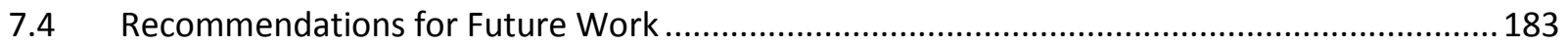

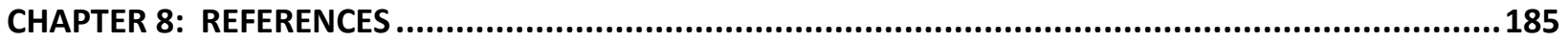

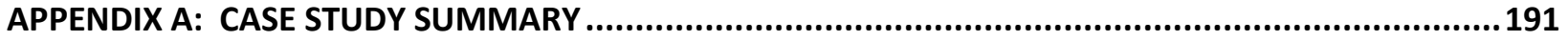

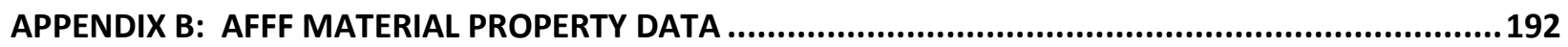

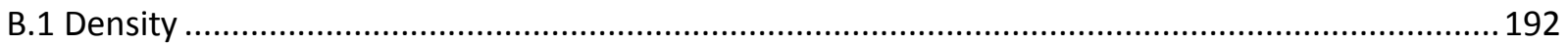

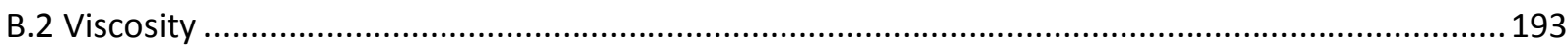

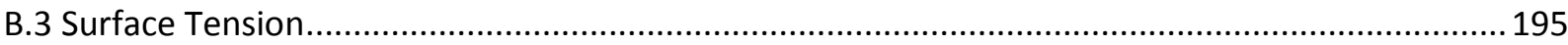

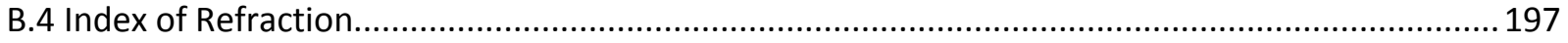

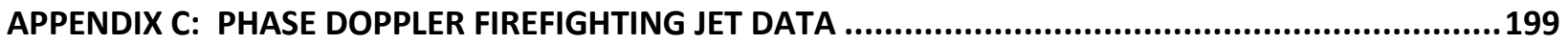

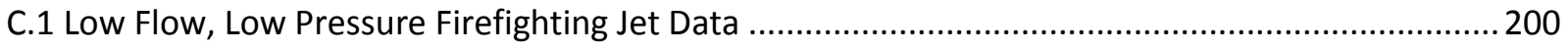

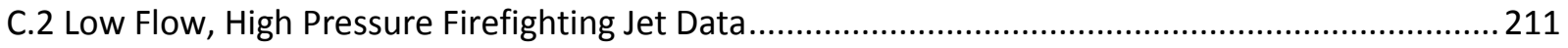

C.3 Medium Flow, Medium Pressure Firefighting Jet Data .............................................................. 222

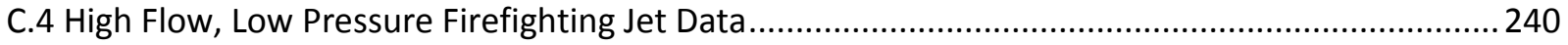

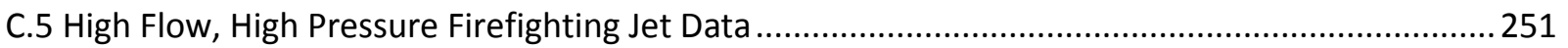

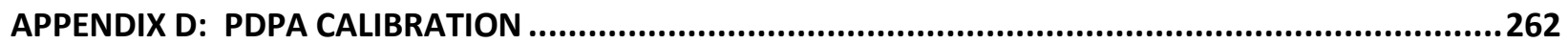




\section{LIST OF FIGURES}

Figure 1.1: (a) A Large-frame Aircraft Mock-up Engulfed by a JP-8 Pool Fire (b) Soot Release Flow Characterization of Various Synthetic Jet Fuel Blends

Figure 1.2: (a) A Fire Suppression Demonstration using Ultra High Pressure (UHP) Firefighting Technology (b) An ARFF Vehicle Undergoing a Mobility Performance Trial ...................................... 2

Figure 1.3: (a) Firefighting Agent Evaluation of a 3-D Running Fuel Fire from an Aircraft Engine Mock-up (b) Firefighting Agent Evaluation on a 2-D pool fire ..................................................... 2

Figure 1.4: The Computational Aircraft-crash-fire-suppression Methodology.......................................... 3

Figure 2.1: (a) AFFF Being Applied to a Medium-frame Aircraft JP-8 Pool Fire (b) AFFF Being Applied to a Large-frame Aircraft JP-8 Pool Fire ........................................................................... 7

Figure 2.2: Examples of Fluorocarbon Surfactants Identified in AFFF [18] ............................................ 10

Figure 2.3: The Conceptual Alignment of Surfactants in an Air-water-fuel Environment $[2,13] \ldots \ldots \ldots . . . .10$

Figure 2.4: An Illustration Depicting the Tendency of Surfactant Molecules to Self-align within an Airborne AFFF Droplet as Surface Age Increases [2] . 11

Figure 2.5: (a) Illustration of a Standard Micelle (b) Interfacial Tension vs. Surfactant Concentration [17]

Figure 2.6: Dynamic Surface Tension vs. Surface Age for (a) Various MIL-SPEC AFFF Formulations and (b) at Different AFFF Concentration Levels [22]

Figure 2.7: (a) Dynamic Surface Tension vs. Surface Age for Various Salinities and (b) Dynamic Surface Tension vs. Surface Age for Various Temperatures [22] 15

Figure 2.8: (a) Shadowgraph Illustrations of Ligaments Peeling Away from an Intact Liquid Jet Core [36] (b) Illustration of the Nozzle Exit Region Depicting Primary and Secondary Breakup [37]

Figure 2.9: The Four Regimes of Liquid Jet Break-up [41] 21

Figure 2.10: Liquid Jet Break-up Regime Classification in Quiescent Air with Respect to Reynolds No. and Ohnesorge No. [35]

Figure 2.11: Liquid Jet Break-up Regimes Classification in Still Air with Respect to Weber No., Reynolds No., and the Gas to Liquid Density Ratio [36].... 24

Figure 2.12: The Jet Stability Curve for Liquid Jets Entering a Gaseous Atmosphere [36] ......................24

Figure 2.13: The Five Droplet Break-up Modes [46] .......................................................................2

Figure 2.14: The Indoor Firefighting Jet Test Facility Layout [49]

Figure 2.15: (a) A Sample Firefighting Jet Concentration Plot $27 \mathrm{~m}$ from a $30^{\circ}$ Inclined Nozzle (b) The Sampler Tube Apparatus Used to Record the Firefighting Jet Concentration Plot [49] 31

Figure 2.16: A Schematic of Theobald's Test Configuration for Measuring Downstream Nozzle Delivery Efficiency [51] 
Figure 2.17: Photographs of Theobald's Test Configuration for Measuring Downstream Nozzle Delivery Efficiency (a) Axial View (b) Horizontal View [51]

Figure 2.18: Theobald's “Continuous Bucket” Results Depicting Dispersion Variation with Nozzle Pressure [51].....

Figure 2.19: (a) A Schematic of the Firefighting Jet Continuity Measurement Apparatus. (b) A Plot Illustrating the Dependence of Firefighting Jet Stability on Nozzle Design [52]... 34

Figure 2.20: Numerical Solutions of Large-scale Firefighting Jet Trajectories [47]. .35

Figure 2.21: A 3-D Model of Liquid Jet Surface and Break-up Near the Nozzle using VOF

Techniques [57]..... 36

Figure 2.22: Time-Evolved CFD Results from an Euler-Lagrange Liquid Jet Flow Model [58] .37

Figure 3.1: Key Components of the Aqueous Firefighting Agent Application Laboratory. 39

Figure 3.2: Key Components of the Aqueous Firefighting Agent Application Laboratory. 40

Figure 3.3: The Aqueous Firefighting Agent Application Laboratory Identifying Key Dimensions .40

Figure 3.4: Firefighting Agent Delivery System Electric Motor Performance [65] .41

Figure 3.5: The Firefighting Agent Delivery System 43

Figure 3.6: The Firefighting Agent Delivery Flow Control Schematic .43

Figure 3.7: The Nozzle Stand and Accompanying Accessories .44

Figure 3.8: Various Views of the Firefighting Jet Containment Bed 46

Figure 3.9: The High Speed Video Camera in Operation .46

Figure 3.10: The XY Galvanometer Scanner in Operation 47

Figure 3.11: Relevant Vector Relations for Determining Droplet Velocity [67]..... .49

Figure 3.12: Light Intensity Scattered by a Particle Passing through the Probe Volume [66] 49

Figure 3.13: (a) Methods of Light Scatter off of a Particle Based on Mie Theory (b) The

Orientation of Each Optical Parameter Involved in Measuring Phase Shift $[66,68]$ 50

Figure 3.14: A Schematic of PDPA Components in Forward Scatter Mode [70].....................................52

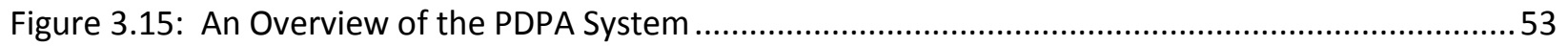

Figure 4.1: The Stoneage Waterblast Tools AP4 Nozzle .54

Figure 4.2: The Firefighting Agent Delivery System Performance Envelope and Associated Firefighting Jet Experiments .55

Figure 4.3: The Main Focal Planes Used for Firefighting Jet Flow Visualization .58

Figure 4.4: (a) The Agent Ground Pattern Capture Device (b) A Near Field View of the Agent Ground Pattern Capture Device Array Immediately after Testing (c) A Water Pattern Test Utilizing the Flow Deflector During Startup (d) A Semi-aerial View of an AFFF Jet Test in Progress. 
Figure 4.5: An Example Agent Capture Device Footprint Based on Firefighting Jet Delivery Geometry.

Figure 4.6: Full-Scale 1-D Agent Ground Pattern Testing 62

Figure 4.7: PDPA Profile Measurement Locations 64

Figure 4.8: Phase Doppler Data Acquisition on a Low Flow, Low Press. (a) Water Jet (b) AFFF Jet .65

Figure 5.1: The Physical Model Flow Domain 74

Figure 5.2: Continuum Phase Model Boundary Conditions. .74

Figure 6.1: Standard Far field Flow Visualization Parallel to the $y=0-m$ Plane: Low Flow, Low Press. Water Jet (Flow is from Right to Left)

Figure 6.2: Standard Far field Flow Visualization Parallel to the $y=0-m$ Plane: Low Flow, Low Press. AFFF Jet (Flow is from Right to Left)

Figure 6.3: Standard Far Field Flow Visualization Parallel to the $y=0-m$ Plane: Low Flow, High Press. Water Jet (Flow is from Right to Left)

Figure 6.4: Standard Far field Flow Visualization Parallel to the $y=0-m$ Plane: Low Flow, High Press. AFFF Jet (Flow is from Right to Left) 80

Figure 6.5: Standard Far Field Flow Visualization Parallel to the $y=0-m$ Plane: Med. Flow, Med.

Press. Water Jet (Flow is from Right to Left).

Figure 6.6: Standard Far Field Flow Visualization Parallel to the $y=0-m$ Plane: Med. Flow, Med. Press. AFFF Jet (Flow is from Right to Left)

Figure 6.7: Standard Far Field Flow Visualization Parallel to the $y=0-m$ Plane: High Flow, Low Press. Water Jet (Flow is from Right to Left).

Figure 6.8: Standard Far Field Flow Visualization Parallel to the $y=0-m$ Plane: High Flow, Low Press. AFFF Jet (Flow is from Right to Left)

Figure 6.9: Standard Far Field Flow Visualization Parallel to the $y=0-m$ Plane: High Flow, High Press. Water Jet (Flow is from Right to Left)

Figure 6.10: Standard Far Field Flow Visualization Parallel to the $y=0-m$ Plane: High Flow, High Press. AFFF Jet (Flow is from Right to Left)

Figure 6.11: Laser Sheet Far Field Flow Visualization Oblique to the $z=0-m$ Plane: (a) Low Flow, Low Press. Water Jet (b) Low Flow, Low Press. AFFF Jet (Flow is from Bottom to Top).....

Figure 6.12: Laser Sheet Far Field Flow Visualization Oblique to the $z=0-m$ Plane: (a) Low Flow, High Press. Water Jet (b) Low Flow, High Press. AFFF Jet (Flow is from Bottom to Top)

Figure 6.13: Laser Sheet Far Field Flow Visualization Oblique to the $z=0-m$ Plane: (a) Med. Flow, Med. Press Water Jet (b) Med. Flow, Med. Press. AFFF Jet (Flow is from Bottom to Top).

Figure 6.14: Laser Sheet Far Field Flow Visualization Oblique to the $z=0-m$ Plane: High Flow, Low Press. Water Jet (b) High Flow, Low Press. AFFF Jet (Flow is from Bottom to Top) .83 
Figure 6.15: Laser Sheet Far Field Flow Visualization Oblique to the $z=0-m$ Plane: High Flow, High Press. Water Jet (b) High Flow, High Press. AFFF Jet (Flow is from Bottom to Top).... .84

Figure 6.16: Laser Sheet Far Field Flow Visualization Oblique to the $x=6.1-m$ Plane: (a) Low Flow, Low Press. Water Jet (b) Low Flow, Low Press. AFFF Jet (Flow is from Bottom to Top)

Figure 6.17: Laser Sheet Far Field Flow Visualization Oblique to the $x=6.1-m$ Plane: Low Flow, High Press. Water Jet (b) Low Flow, High Press. AFFF Jet (Flow is from Right to Left) .85

Figure 6.18: Laser Sheet Far Field Flow Visualization Oblique to the $x=6.1-\mathrm{m}$ Plane: (a) Med. Flow, Med Press. Water Jet (b) Med. Flow, Med. Press. AFFF Jet (Flow is from Right to Left).

Figure 6.19: Laser Sheet Far Field Flow Visualization Oblique to the $x=6.1-\mathrm{m}$ Plane: (a) High Flow, Low Press. Water Jet (b) High Flow, Low Press. AFFF Jet (Flow is from Right to Left) 86

Figure 6.20: Laser Sheet Far Field Flow Visualization Oblique to the $x=6.1-m$ Plane: (a) High Flow, High Press. Water Jet (b) High Flow, High Press. AFFF Jet (Flow is from Right to Left)..... 86

Figure 6.21: Stroboscopic Mid Field Flow Visualization of the High Flow, Low Press. Water Jet at Different Stages of Evolution: (a) Magnification of a Pulsation (b) Pulsation Growth Near the Nozzle Exit (c) Pulsation Fully Dispersing (d) Two Pulsations in Succession (Flow is from Right to Left)

Figure 6.22: Stroboscopic Mid Field Flow Visualization Focused on the $y=0-m$ Plane of the Med. Flow, Med. Press. Water Jet: (a) Downstream of the Nozzle (b) Near the Nozzle (Flow is from Right to Left)

Figure 6.23: FFT Spectral Analysis of Nozzle Pressure Data Recorded on the High Flow, Low Press. Water Jet.

Figure 6.24: FFT Spectral Analysis of Nozzle Pressure Data Recorded on the Med. Flow, Med. Press. Water Jet.....

Figure 6.25: Standard Mid-field Flow Visualization Focused Oblique to the $y=0-m$ Plane for the (a) High Flow, Low Press. Water Jet (b) Med. Flow, Med. Press. Water Jet (Flow is from Right to Left)

Figure 6.26: High Speed Mid-field Flow Visualization of Each Jet Configuration Focused on the $\mathrm{y}=0-\mathrm{m}$ Plane (Flow is from Right to Left)

Figure 6.27: Near field View of the High Flow, Low Press. Water Jet at Select Downstream Nozzle Locations (Flow is from Right to Left)

Figure 6.28: Near field View of Jet Break-up Approximately $3 \mathrm{~m}(9.8 \mathrm{ft})$ Downstream from the Nozzle for the (a) Low Flow, Low Press. Water Jet (b) Low Flow, Low Press. AFFF Jet (Flow is from Right to Left).

Figure 6.29: Near field Flow Visualization of the High Flow, Low Press. AFFF Jet Highlighting Multi-scale Bubble Masses at Different Downstream Locations (Flow is from Right to Left)

Figure 6.30: 2-D Agent Ground Pattern Water Accumulation Contour Plots Illustrating Firefighting Jet Dependence on the Firefighting Jet Containment Zone Curtains . .95 
Figure 6.31: 2-D Agent Ground Pattern Contour Plots of Low Press. Water Jets................................... 96

Figure 6.32: 2-D Agent Ground Pattern Contour Plots of Med. Press. Water Jets ..................................97

Figure 6.33: Two-dimensional Agent Ground Pattern Contour Maps of High Press. Water Jets.............. 98

Figure 6.34: 2-D Agent Ground Pattern Contour Plots of Low Flow AFFF Jets ...................................... 100

Figure 6.35: 2-D Agent Ground Pattern Contour Plots of Med. Flow AFFF Jets .................................... 101

Figure 6.36: 2-D Agent Ground Pattern Contour Plots of High Flow AFFF Jets ..................................... 102

Figure 6.37: Agent Ground Pattern Firefighting Jet Performance Summary....................................... 103

Figure 6.38: Foam Expansion Ratio vs. Axial Location of the Med. Flow, Med. Press. AFFF Jet with Respect to Variation in AFFF Solution Concentration ........................................................... 105

Figure 6.39: Full-scale 1-D Agent Ground Pattern Results Illustrating (a) Agent Accumulation vs. Axial Location and (b) Foam Expansion Ratio vs. Axial Location .............................................. 106

Figure 6.40: A Characteristic Firefighting Jet Droplet Size Distribution Measurement with Accompanying Fit. 108

Figure 6.41: Characteristic Phase Doppler Profiles Highlighting Measurement Locations Selected to Visualize Mean Axial Droplet Velocity and Mean Droplet Diameter Sample Distribution at $\mathrm{x}=3.05 \mathrm{~m}(10 \mathrm{ft})$

Figure 6.42: Select Sampling Data of Axial Droplet Velocity vs. Droplet Diameter of the Med. Flow, Med. Press. Jet at $x=3.05 \mathrm{~m}(10 \mathrm{ft})$

Figure 6.43: A Sample of Vertical, Phase Doppler, Droplet Profile Data from the $x=6.10 \mathrm{~m}$ (20 $\mathrm{ft}$ ) Axial Downstream Location from the Med. Flow, Med. Press. Firefighting Jet

Figure 6.44: A Sample of Vertical, Phase Doppler Profile Estimates of Axial, Gaseous (Air) Velocity from the $\quad x=6.10 \mathrm{~m}(20 \mathrm{ft})$ Axial Downstream Location from the Med. Flow, Med. Press. Firefighting Jet 114

Figure 6.45: A Schematic Illustrating the Max. and Mean Phase Doppler Profile Value. 115

Figure 6.46: Firefighting Jet PDPA Summary Data in Terms of Max. and Mean Profile, Axial Droplet Velocity.

Figure 6.47: Firefighting Jet PDPA Summary in Terms of Non-Dimensional Max. Profile, Mean Axial Droplet Velocity

Figure 6.48: Firefighting Jet PDPA Summary in Terms of Dimensional Max. Profile, Mean Axial Droplet Velocity.

Figure 6.49: Firefighting Jet PDPA Summary in Terms of Mean Profile, Mean Axial Droplet Velocity; Vertical Bars Represent Mean Profile, \pm RMS Axial Droplet Velocity Magnitude. 120

Figure 6.50: Firefighting Jet PDPA Summary Data in Terms of Max. and Mean Profile, Mean Vertical Droplet Velocity.

Figure 6.51: Firefighting Jet PDPA Summary in Terms of Max. Profile, Mean Vertical Droplet Velocity 
Figure 6.52: Firefighting Jet PDPA Summary in Terms of Mean Profile, Mean Vertical Droplet Velocity; Vertical Bars Represent Mean Profile, \pm RMS Vertical Droplet Velocity Magnitude ....... 124

Figure 6.53: Firefighting Jet PDPA Summary Data in Terms of Mean Profile, Droplet Diameters .......... 127

Figure 6.54: Firefighting Jet PDPA Summary in Terms of Mean Profile, Mean Droplet Diameter .......... 128

Figure 6.55: Firefighting Jet PDPA Summary in Terms of Mean Profile, RMS Droplet Diameter ............129

Figure 6.56: Firefighting Jet PDPA Summary in Terms of Mean Profile, Sauter Mean Droplet Diameter

Figure 6.57: (a) PDPA Mean Profile Data Acquisition Rate vs. Axial Measurement Location (b) PDPA Mean Data Sample Count vs. Axial Measurement Location

Figure 6.58: Med. Flow, Med. Press. Jet Vertical Location vs. Mean Axial Droplet Velocity with Respect to Variation in AFFF concentration

Figure 6.59: Med. Flow, Med. Press. Jet Vertical Location vs. Mean Vertical Droplet Velocity with Respect to Variation in AFFF concentration

Figure 6.60: Med. Flow, Med. Press. Jet Vertical Location vs. Mean Droplet Diameter with Respect to Variation in AFFF concentration 135

Figure 6.61: Med. Flow, Med. Press. Jet Vertical Location vs. RMS Droplet Diameter with Respect to Variation in AFFF concentration.

Figure 6.62: Med. Flow, Med. Press. Jet Vertical Location vs. Sauter Mean Droplet Diameter with Respect to Variation in AFFF concentration.

Figure 6.63: CFD Model Solution Dependence on the DPM Secondary Droplet Break-up Model...........138

Figure 6.64: CFD Model Solution Dependence on the DPM Coalescence Model .................................139

Figure 6.65: CFD Model Solution Dependence on the Mesh Resolution............................................140

Figure 6.66: CFD Model Solution Dependence on the Physical Time Step

Figure 6.67: CFD Model Solution Dependence on the Presence of the Firefighting Jet

Containment Zone.....

Figure 6.68: Qualitative Flow Structure Comparison between CFD Model and Flow Visualization Results of the Low Flow, Low Press. Jet (Flow is from Right to Left)

Figure 6.69: Qualitative Flow Structure Comparison between CFD Model and Flow Visualization Results of the Med. Flow, Med. Press. Jet (Flow is from Right to Left)......

Figure 6.70: Qualitative Flow Structure Comparison between CFD Model and Flow Visualization Results of the High Flow, High Press. Jet (Flow is from Right to Left)

Figure 6.71: A Comparison of Phase Doppler and CFD Model, Mean Axial Droplet Velocity Profile Data for the Med. Flow, Med. Press. Jet.....

Figure 6.72: A Comparison of Phase Doppler and CFD Model, Mean Vertical Droplet Velocity Profile Data for the Med. Flow, Med. Press. Jet. 148 
Figure 6.73: A Comparison of Phase Doppler and CFD Model, Mean Droplet Diameter Profile Data for the Med. Flow, Med. Press. Jet 149

Figure 6.74: A Comparison of PDPA and CFD Mean Vertical Profile Data for the Low Flow, Low Press. Jet..... 150

Figure 6.75: A Comparison of PDPA and CFD Mean Vertical Profile Data for the Low Flow, High Press. Jet. 151

Figure 6.76: A Comparison of PDPA and CFD Mean Vertical Profile Data for the Med. Flow, Med. Press. Jet..... 152

Figure 6.77: A Comparison of PDPA and CFD Mean Vertical Profile Data for the High Flow, Low Press. Jet. 153

Figure 6.78: A Comparison of PDPA and CFD Mean Vertical Profile Data for the High Flow, High Press. Jet 154

Figure 6.79: CFD Model Results of Droplet Residence Time for Med. Flow, Med. Press Jets 156

Figure 6.80: CFD Model Results of Droplet Velocity Magnitude for Low Flow, Low Press. Jets...... 158

Figure 6.81: CFD Model Results of Droplet Velocity Magnitude for Low Flow, High Press. Jets 159

Figure 6.82: CFD Model Results of Droplet Velocity Magnitude for Med. Flow, Med. Press. Jets 160

Figure 6.83: CFD Model Results of Droplet Velocity Magnitude for High Flow, Low Press. Jets 161

Figure 6.84: CFD Model Results of Droplet Velocity Magnitude for High Flow, High Press. Jets 162

Figure 6.85: CFD Model Results of Droplet Diameter for Low Flow, Low Press. Jets ..... 163

Figure 6.86: CFD Model Results of Droplet Diameter for Low Flow, High Press. Jets 164

Figure 6.87: CFD Model Results of Droplet Diameter for Med. Flow, Med. Press. Jets 165

Figure 6.88: CFD Model Results of Droplet Diameter for High Flow, Low Press. Jets 166

Figure 6.89: CFD Model Results of Droplet Diameter for High Flow, High Press. Jets 167

Figure 6.90: CFD Model Results of Gaseous Weber No. for Low Flow, Low Press. Jets 168

Figure 6.91: CFD Model Results of Gaseous Weber No. for Low Flow, High Press. Jets 169

Figure 6.92: CFD Model Results of Gaseous Weber No. for Med. Flow, Med. Press. Jets 170

Figure 6.93: CFD Model Results of Gaseous Weber No. for High Flow, Low Press. Jets 171

Figure 6.94: CFD Model Results of Gaseous Weber No. for High Flow, High Press. Jets. 172

Figure 6.95: CFD Model Results of the Gaseous Continuous (Air) Phase Velocity Magnitude for Low Flow, Low Press. Jets. 173

Figure 6.96: CFD Model Results of the Gaseous Continuous (Air) Phase Velocity Magnitude for Low Flow, High Press. Jets 174

Figure 6.97: CFD Model Results of the Gaseous Continuous (Air) Phase Velocity Magnitude for Med. Flow, Med. Press Jets 175 
Figure 6.98: CFD Model Results of the Gaseous Continuous (Air) Phase Velocity Magnitude for High Flow, Low Press. Jets.

Figure 6.99: CFD Model Results of the Gaseous Continuous (Air) Phase Velocity Magnitude for High Flow, High Press. Jets .

Figure B.1: AFFF Solution Density vs. AFFF Solution Concentration 193

Figure B.2: (a) AFFF Solution Dynamic Viscosity vs. AFFF Solution Concentration (b) AFFF Solution Dynamic Viscosity vs. Shear Rate for Various AFFF Solution Concentrations. 194

Figure B.3: (a) Equilibrium Surface Tension vs. AFFF Solution Concentration (b) Dynamic Surface Tension vs. Surface Age for Various AFFF Solution Concentrations. 196

Figure B.4: (a) Degrees Brix vs. AFFF Solution Concentration (b) Index of Refraction vs. AFFF Solution Concentration. 198

Figure C.1: Near Field Low Flow, Low Press. Jet Vertical Profiles of Mean Axial Droplet Velocity..........200

Figure C.2: Far Field Low Flow, Low Press. Jet Vertical Profiles of Mean Axial Droplet Velocity 201

Figure C.3: Near Field Low Flow, Low Press. Jet Vertical Profiles of Mean Vertical Droplet Velocity 202

Figure C.4: Far Field Low Flow, Low Press. Jet Vertical Profiles of Mean Vertical Droplet Velocity........ 203

Figure C.5: Near Field Low Flow, Low Press. Jet Vertical Profiles of Mean Droplet Diameter .................204

Figure C.6: Far Field Low Flow, Low Press. Jet Vertical Profiles of Mean Droplet Diameter ...................205

Figure C.7: Near Field Low Flow, Low Press. Jet Vertical Profiles of RMS Droplet Diameter ..................206

Figure C.8: Far Field Low Flow, Low Press. Jet Vertical Profiles of RMS Droplet Diameter 207

Figure C.9: Near Field Low Flow, Low Press. Jet Vertical Profiles of Sauter Mean Droplet Diameter 208

Figure C.10: Far Field Low Flow, Low Press. Jet Vertical Profiles of Sauter Mean Droplet Diameter

Figure C.11: Low Flow, Low Press. Jet Droplet Size Distribution at the Max. Profile, Mean Axial Droplet Velocity for Select Vertical Profiles..... 210

Figure C.12: Near Field Low Flow, High Press. Jet Vertical Profiles of Mean Axial Droplet Velocity ....... 211

Figure C.13: Far Field Low Flow, High Press. Jet Vertical Profiles of Mean Axial Droplet Velocity...........212

Figure C.14: Near Field Low Flow, High Press. Jet Vertical Profiles of Mean Vertical Droplet Velocity

Figure C.15: Far Field Low Flow, High Press. Jet Vertical Profiles of Mean Vertical Droplet Velocity 214

Figure C.16: Near Field Low Flow, High Press. Jet Vertical Profiles of Mean Droplet Diameter 215

Figure C.17: Far Field Low Flow, High Press. Jet Vertical Profiles of Mean Droplet Diameter 216

Figure C.18: Near Field Low Flow, High Press. Jet Vertical Profiles of RMS Droplet Diameter 217 
Figure C.19: Far Field Low Flow, High Press. Jet Vertical Profiles of RMS Droplet Diameter

Figure C.20: Near Field Low Flow, High Press. Jet Vertical Profiles of Sauter Mean Droplet Diameter

Figure C.21: Far Field Low Flow, High Press. Jet Vertical Profiles of Sauter Mean Droplet Diameter 220

Figure C.22: Low Flow, High Press. Jet Droplet Size Distribution at the Maximum Profile, Mean Axial Droplet Velocity for Select Vertical Profiles.

Figure C.23: Near Field Med. Flow, Med. Press. Jet Vertical Profiles of Mean Axial Droplet Velocity

Figure C.24: Far Field Med. Flow, Med. Press. Jet Vertical Profiles of Mean Axial Droplet Velocity........223

Figure C.25: Near Field Med. Flow, Med. Press. Jet Vertical Profiles of Mean Vertical Droplet Velocity

Figure C.26: Far Field Med. Flow, Med. Press. Jet Vertical Profiles of Mean Vertical Droplet Velocity

Figure C.27: Near Field Med. Flow, Med. Press. Jet Vertical Profiles of Mean Droplet Diameter ..........226

Figure C.28: Far Field Med. Flow, Med. Press. Jet Vertical Profiles of Mean Droplet Diameter ...... 227

Figure C.29: Near Field Med. Flow, Med. Press. Jet Vertical Profiles of RMS Droplet Diameter 228

Figure C.30: Far Field Med. Flow, Med. Press. Jet Vertical Profiles of RMS Droplet Diameter ...... 229

Figure C.31: Near Field Med. Flow, Med. Press. Jet Vertical Profiles of Sauter Mean Droplet Diameter. 230

Figure C.32: Far Field Med. Flow, Med. Press. Jet Vertical Profiles of Sauter Mean Droplet Diameter

Figure C.33: Select Med. Flow, Med. Press. Jet Vertical Profiles of Estimated Axial Gaseous (Air) Velocity

Figure C.34: Select Med. Flow, Med. Press. Jet Vertical Profiles of Estimated Vertical Gaseous (Air) Velocity..... 233

Figure C.35: Select Med. Flow, Med. Pressure Jet Horizontal Profiles of Mean Axial Droplet Velocity .234

Figure C.36: Select Med. Flow, Med. Press. Jet Horizontal Profiles of Mean Vertical Droplet Velocity 235

Figure C.37: Select Med. Flow, Med. Press. Jet Horizontal Profiles of Mean Droplet Diameter.............236

Figure C.38: Select Med. Flow, Med. Press. Jet Horizontal Profiles of RMS Droplet Diameter. 237

Figure C.39: Select Med. Flow, Med. Press. Jet Horizontal Profiles of Sauter Mean Droplet Diameter..... 238

Figure C.40: Med. Flow, Med. Press. Jet Droplet Size Distribution at the Max. Profile, Mean Axial Droplet Velocity for Select Vertical Profiles 239 
Figure C.41: Near Field High Flow, Low Press. Jet Vertical Profiles of Mean Axial Droplet Velocity....... 240

Figure C.42: Far Field High Flow, Low Press. Jet Vertical Profiles of Mean Axial Droplet Velocity.......... 241

Figure C.43: Near Field High Flow, Low Press. Jet Vertical Profiles of Mean Vertical Droplet Velocity

Figure C.44: Far Field High Flow, Low Press. Jet Vertical Profiles of Mean Vertical Droplet Velocity

Figure C.45: Near Field High Flow, Low Press. Jet Vertical Profiles of Mean Droplet Diameter 244

Figure C.46: Far Field High Flow, Low Press. Jet Vertical Profiles of Mean Droplet Diameter 245

Figure C.47: Near Field High Flow, Low Press. Jet Vertical Profiles of RMS Droplet Diameter... 246

Figure C.48: Far Field High Flow, Low Press. Jet Vertical Profiles of RMS Droplet Diameter ..... 247

Figure C.49: Near Field High Flow, Low Press. Jet Vertical Profiles of Sauter Mean Droplet Diameter 248

Figure C.50: Far Field High Flow, Low Press. Jet Vertical Profiles of Sauter Mean Droplet Diameter.

Figure C.51: High Flow, Low Press. Jet Droplet Size Distribution at the Max. Profile, Mean Axial Droplet Velocity for Select Vertical Profiles ...... 250

Figure C.52: Near Field High Flow, High Press. Jet Vertical Profiles of Mean Axial Droplet Velocity ...... 251

Figure C.53: Far Field High Flow, High Press. Jet Vertical Profiles of Mean Axial Droplet Velocity .........252

Figure C.54: Near Field High Flow, High Press. Jet Vertical Profiles of Mean Vertical Droplet Velocity 253

Figure C.55: Far Field High Flow, High Press. Jet Vertical Profiles of Mean Vertical Droplet Velocity 254

Figure C.56: Near Field High Flow, High Press. Jet Vertical Profiles of Mean Droplet Diameter............. 255

Figure C.57: Far Field High Flow, High Press. Jet Vertical Profiles of Mean Droplet Diameter................ 256

Figure C.58: Near Field High Flow, High Press. Jet Vertical Profiles of RMS Droplet Diameter...............257

Figure C.59: Far Field High Flow, High Press. Jet Vertical Profiles of RMS Droplet Diameter .................258

Figure C.60: Near Field High Flow, High Press. Jet Vertical Profiles of Sauter Mean Droplet

Diameter

Figure C.61: Far Field High Flow, High Press. Jet Vertical Profiles of Sauter Mean Droplet

Diameter..... 260

Figure C.62: High Flow, High Press. Jet Droplet Size Distribution at the Max. Profile, Mean Axial Droplet Velocity for Select Vertical Profiles 261

Figure D.1: PDPA Droplet Size Calibration Certification Comparing MDG-100 Diameter and PDPA Mean D10. 263 


\section{LIST OF TABLES}

Table 2.1: Reported AFFF Concentrate Chemical Constituents Used in the Present Study [12] ................ 8

Table 2.2: Equilibrium Fluid Properties of Water and AFFF at Standard Conditions ............................... 13

Table 2.3: MIL-SPEC AFFF Chemical and Physical Minimum Performance Criteria [5] ........................... 16

Table 2.4: Ohnesorge's Classification of Various Liquid Jet Break-up Regimes via Weber No. [36] ..........23

Table 2.5: The Five Droplet break-up Modes Based on Weber No. [46] ..............................................2 27

Table 4.1: Firefighting Jet Test Case Summary in the Aqueous Firefighting Agent Application

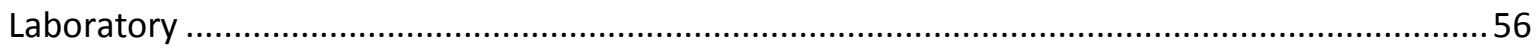

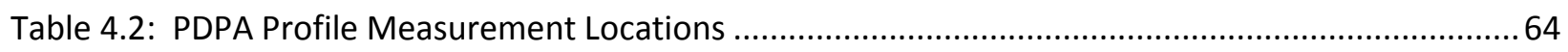

Table 5.1: The Computational Matrix Summarizing Firefighting Jet Simulation Conditions ....................66

Table 6.1: Agent Ground Pattern Mass Conservation Analysis .......................................................... 104

Table 6.2: Phase Doppler Profile Measurement Locations Containing Uncertainty Analysis ..................111

Table 6.3: Calculated Pump Oscillations per Phase Doppler Data Acquisition Sampling Period.............132

Table 6.4: Computational Firefighting Jet Flow Model Domain Droplet Summary ............................... 155

Table A.1: A Summary of the Firefighting Jet Nozzle Exit Conditions Examined ................................... 191

Table B.1: Density of Various AFFF Solution Concentrations............................................................. 192

Table B.2: Derived Dynamic Viscosity of Various AFFF Solution Concentrations ................................... 194

Table B.3: Equilibrium Surface Tension of Various AFFF Solution Concentrations................................196

Table B.4: Measured Degrees Brix and the Corresponding Index of Various AFFF Solution

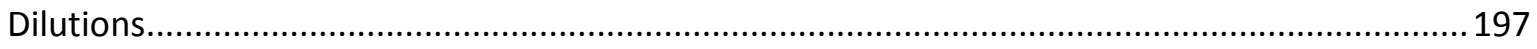

Table D.1: PDPA Droplet Size Calibration Certification Check Points .................................................263 


\section{LIST OF ACRONYMS AND ABBREVIATIONS}

Notation

$1-\mathrm{D}$

2-D

3-D

AFFF

AFRL

ARFF

ASTM

CFD

CPU

DOD

DPM

DNS

EPA

FAA

FFT

LES

MIL-SPEC

NFPA

PDPA

PFOA

PFOS

RMS

RPM

SIMPLE

SSD

$\mathrm{TAB}$

UHP

VOF

WALE

WVU

\section{Description}

One-dimensional

Two-dimensional

Three-dimensional

Aqueous film-forming foam

Air Force Research Laboratory

Airport rescue and firefighting

American Society of Testing and Materials

Computational fluid dynamics

Central processing unit

Department of Defense

Discrete phase model

Direct Numerical Simulation

Environmental Protection Agency

Federal Aviation Administration

Fast Fourier transform

Large eddy simulation

Military specification

National Fire Protection Association

Phase Doppler particle analysis

Perfluorooctanoic acid

Perfluorooctane sulfonate

Root mean square

Revolutions per minute

Semi-implicit method for pressure-linked equations

Stochastic secondary droplet

Taylor analogy break-up

Ultra high pressure firefighting

Volume of fluid

Wall-adapting local eddy-viscosity sub-grid scale model

West Virginia University 


\section{LIST OF SYMBOLS}

Symbol Definition

\begin{tabular}{|c|c|}
\hline$\beta$ & Damping coefficient \\
\hline$\gamma_{S G}$ & Specific gravity \\
\hline$\Delta t$ & Simulation time step \\
\hline$\delta$ & Fringe spacing \\
\hline$\varepsilon$ & Gas to liquid density ratio \\
\hline$\eta_{0}$ & Initial liquid jet disturbance wavelength \\
\hline$\theta$ & PDPA laser beam intersection angle \\
\hline$\phi$ & PDPA phase shift angle \\
\hline$\Phi$ & Raw data signal \\
\hline$\lambda$ & Wavelength \\
\hline$\mu$ & Dynamic viscosity \\
\hline$\mu_{G}$ & Gaseous dynamic viscosity \\
\hline$\mu_{L}$ & Liquid dynamic viscosity \\
\hline$v$ & Kinematic viscosity \\
\hline$\rho$ & Density \\
\hline$\rho_{G}$ & Gaseous density \\
\hline$\rho_{L}$ & Liquid density \\
\hline$\sigma$ & Surface tension \\
\hline$\sigma_{A F F F}$ & Surface tension of AFFF \\
\hline$\sigma_{C}$ & Surface tension of cyclohexane \\
\hline$\sigma_{I}$ & Interfacial tension between cyclohexane and AFFF \\
\hline$\sigma_{L / G}$ & Surface tension between a liquid and surrounding gas \\
\hline$\sigma_{S}$ & Spreading coefficient due to surfaced tension \\
\hline$\varphi$ & PDPA scattering angle \\
\hline$\psi$ & PDPA receiving angle \\
\hline$\omega$ & Droplet oscillation frequency \\
\hline$A$ & Amplitude of an undamped oscillation \\
\hline$C$ & Solution concentration \\
\hline$C_{\beta}$ & TAB break-up model constant \\
\hline$C_{b}$ & TAB break-up model constant \\
\hline$C_{\text {Drag }}$ & Drag coefficient \\
\hline$C_{\text {Drag,Sphere }}$ & Drag coefficient of a sphere \\
\hline
\end{tabular}

Typical SI Units

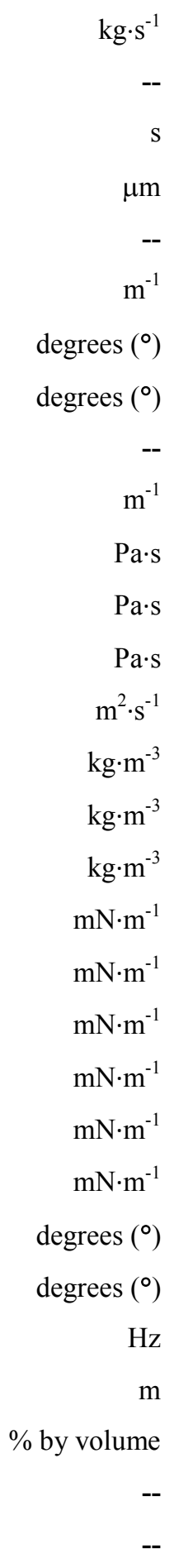




\begin{tabular}{|c|c|c|}
\hline$C_{F}$ & TAB break-up model constant & -- \\
\hline$C_{k}$ & TAB break-up model constant & -- \\
\hline$d$ & Droplet diameter & $\mu \mathrm{m}$ \\
\hline$d_{M I N}$ & Minimum droplet diameter & $\mu \mathrm{m}$ \\
\hline$d_{M A X}$ & Maximum droplet diameter & $\mu \mathrm{m}$ \\
\hline$D$ & Liquid jet or nozzle diameter & $\mathrm{mm}$ \\
\hline D10 & Mean droplet diameter & $\mu \mathrm{m}$ \\
\hline D32 & Sauter mean droplet diameter & $\mu \mathrm{m}$ \\
\hline$D_{R M S}$ & Root mean square diameter & $\mu \mathrm{m}$ \\
\hline E & $\begin{array}{l}\text { Estimated deviation between the true mean and calculated mean in a } \\
\text { measured flow parameter }\end{array}$ & $\mathrm{m} \cdot \mathrm{s}^{-1}$ or $\mu \mathrm{m}$ \\
\hline$f_{\text {Doppler }}$ & Doppler frequency & $\mathrm{Hz}$ \\
\hline$F_{E x t}$ & External Force & $\mathrm{N}$ \\
\hline$F_{\text {Drag }}$ & Drag force & $\mathrm{N}$ \\
\hline$F_{\text {Other }}$ & Other force & $\mathrm{N}$ \\
\hline$g$ & Acceleration due to gravity & $\mathrm{m} \cdot \mathrm{s}^{-2}$ \\
\hline$k$ & Spring stiffness constant & $\mathrm{N} \cdot \mathrm{m}^{-1}$ \\
\hline$m_{d}$ & Droplet mass & $\mathrm{kg}$ \\
\hline$\dot{m}_{d}$ & Droplet mass flow rate & $\mathrm{kg} \cdot \mathrm{s}^{-1}$ \\
\hline$M$ & PDPA phase-diameter slope & -- \\
\hline$O h$ & Ohnesorge or stability number & -- \\
\hline$N$ & Number of samples & -- \\
\hline$N_{\text {decorr }}$ & Number of decorrelated samples & -- \\
\hline$r$ & Droplet radius & $\mu \mathrm{m}$ \\
\hline$R$ & Nozzle radius & $\mathrm{m}$ \\
\hline$R e_{d}$ & Relative Reynolds number base on droplet diameter & \\
\hline $\operatorname{Re}_{L}$ & Reynolds number based on liquid jet characteristics & -- \\
\hline$s$ & TAB break-up model droplet distortion & $\mathrm{m}$ \\
\hline$t$ & Simulation time & $\mathrm{s}$ \\
\hline$t_{d}$ & Droplet life time & $\mathrm{s}$ \\
\hline$T$ & Temperature & K \\
\hline$V_{d}$ & Droplet velocity & $\mathrm{m} \cdot \mathrm{s}^{-1}$ \\
\hline$V_{c, r e l}$ & Relative velocity between colliding droplets & $\mathrm{m} \cdot \mathrm{s}^{-1}$ \\
\hline$V_{d, r e l}$ & Relative velocity between a droplet and the surrounding gas phase & $\mathrm{m} \cdot \mathrm{s}^{-1}$ \\
\hline$V_{d, X}$ & Axial droplet velocity & $\mathrm{m} \cdot \mathrm{s}^{-1}$ \\
\hline$V_{d, Z}$ & Vertical droplet velocity & $\mathrm{m} \cdot \mathrm{s}^{-1}$ \\
\hline
\end{tabular}




$\begin{array}{llr}V_{G, X} & \text { Axial gaseous (air) velocity } & \mathrm{m} \cdot \mathrm{s}^{-1} \\ V_{G, Z} & \text { Vertical gaseous (air) velocity } & \mathrm{m} \cdot \mathrm{s}^{-1} \\ V_{J} & \text { Jet velocity } & \mathrm{m} \cdot \mathrm{s}^{-1} \\ W e_{d} & \text { Gaseous Weber number based on liquid droplet characteristics } & -- \\ W e_{G} & \text { Gaseous Weber Number based on aerodynamic droplet characteristics } & -- \\ W e_{L} & \text { Weber number based on liquid jet characteristics } & - \\ W e_{T} & \text { Weber number based on Taylor's analogy } & - \\ x & \text { Axial direction } & - \\ x_{e} & \text { TAB break-up model droplet equator location } & \mathrm{m} \\ y & \text { Horizontal direction } & - \\ z & \text { Vertical direction } & - \\ Z & \text { Jet break-up length } & \mathrm{m}\end{array}$




\section{CHAPTER 1: INTRODUCTION}

\subsection{Background}

In recent decades, aircraft rescue and firefighting (ARFF) research as a metrological science has evolved largely due to the advancement of applicable engineering tools. Government civil and defense sectors in conjunction with academic institutions have established programs investigating all aspects of accidental fire phenomenology, including combustion mechanisms, fire mitigation strategies, and emergency response tactics. Innovation fostered in fields other than the ARFF industry has also played a major role through technology transfer. However, most fundamental research is still rooted in the principal sciences.

ARFF research is primarily driven by changes in threat level posed by technological advances in other aspects of the aircraft industry. Novel materials, fuel compositions, airport or airbase logistics, layouts, and structures, and the evolution of aircraft shape and size provide unique challenges fire safety planners must continuously address. Domestic research efforts are chiefly supported by the Department of Transportation's Federal Aviation Administration (FAA) and the Department of Defense (DOD), including relationships with national laboratories and their associated contracting agencies. In reference to aviation fire mishaps involving armed military aircraft, specialized programs have also been sponsored by the Department of Energy and the Nuclear Regulatory Commission.

As part of an interagency agreement with the Air Force Research Laboratories' (AFRL) Fire Research Group, the FAA has supported a multitude of research efforts to investigate risk assessment metrics for aircraft crash fire environments. These programs have included a combination of engineering approaches that have historically relied on the AFRL multi-scale live fire facilities located at Tyndall Air Force Base, FL. Figures 1.1 through 1.3 illustrate various research programs including characterization of combustion environments, fire suppression techniques, and emergency vehicle performance. 


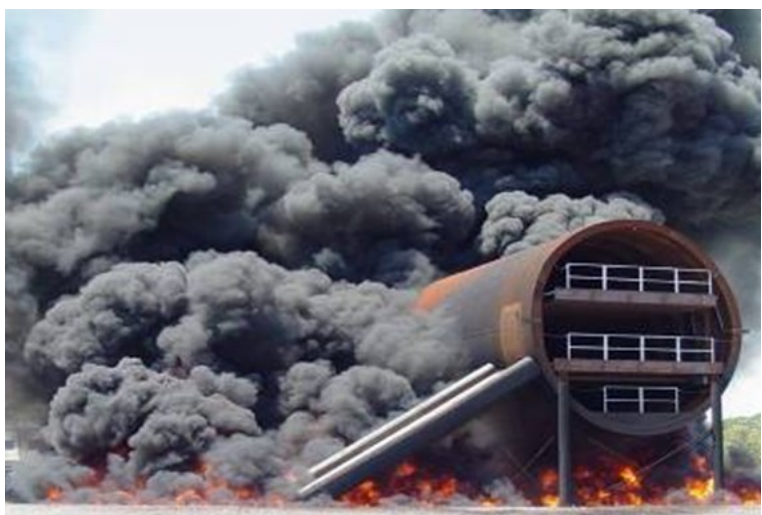

(a)

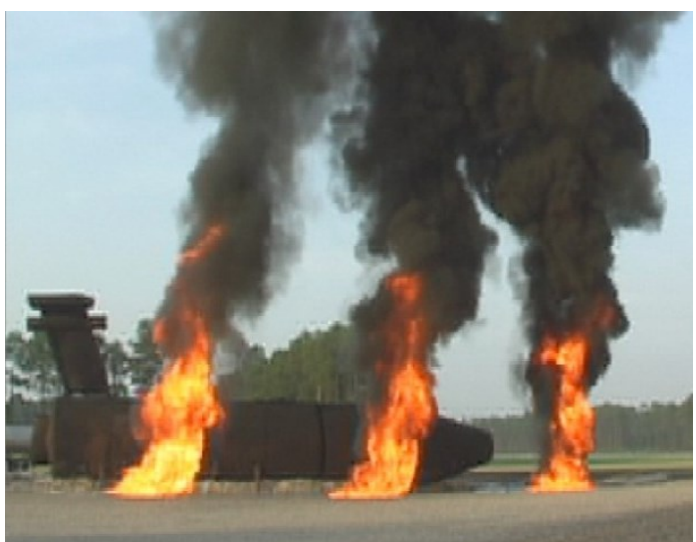

(b)

Figure 1.1: (a) A Large-frame Aircraft Mock-up Engulfed by a JP-8 Pool Fire (b) Soot Release Flow Characterization of Various Synthetic Jet Fuel Blends

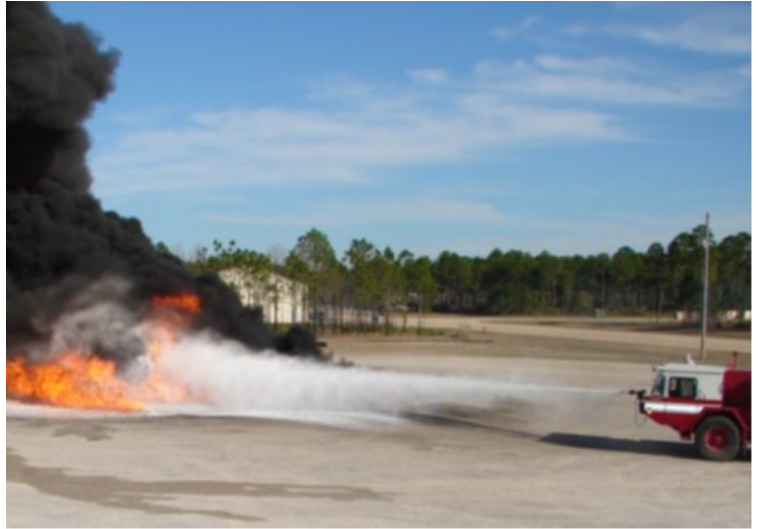

(a)

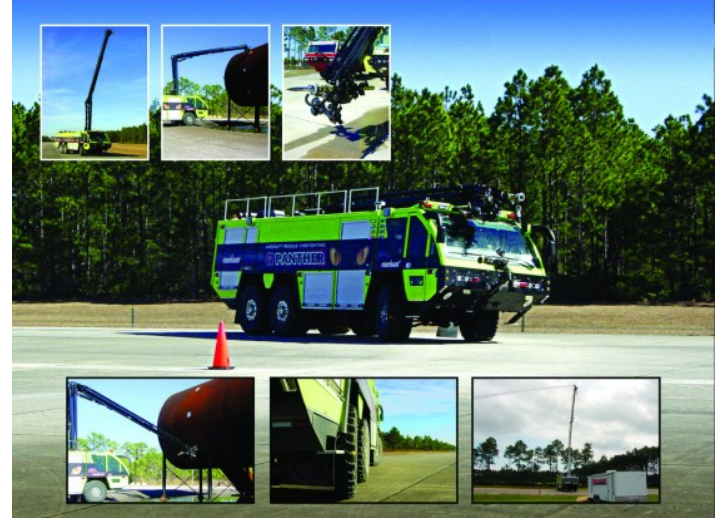

(b)

Figure 1.2: (a) A Fire Suppression Demonstration using Ultra High Pressure (UHP) Firefighting Technology (b) An ARFF Vehicle Undergoing a Mobility Performance Trial

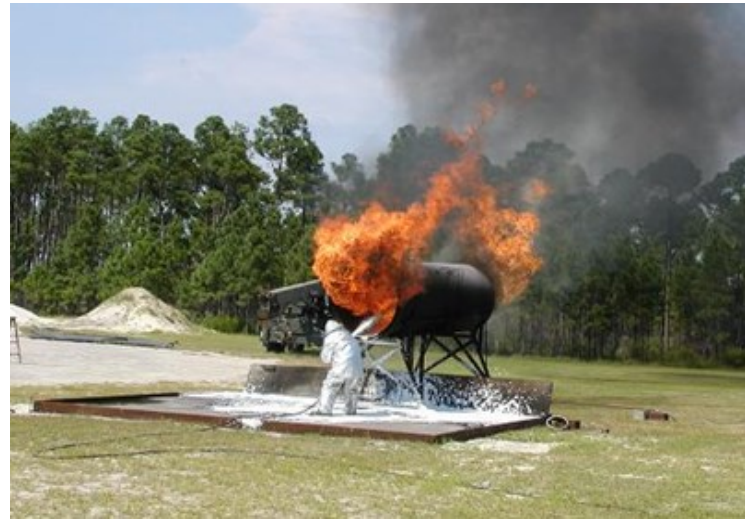

(a)

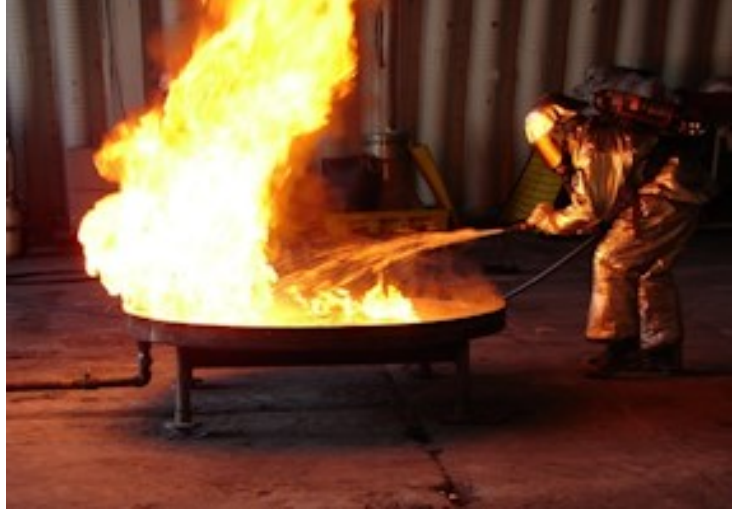

(b)

Figure 1.3: (a) Firefighting Agent Evaluation of a 3-D Running Fuel Fire from an Aircraft Engine Mock-up (b) Firefighting Agent Evaluation on a 2-D pool fire 
Although full-scale testing is critical to aviation fire research, it harbors some disadvantages. Fuel, firefighting agent, equipment, and manpower costs can be prohibitive. Outdoor studies must contend with uncontrolled and often chaotic atmospheric influences typically resulting in significant variance between repeated trials that are important to study but difficult to quantify. In addition, the destructive combustion-spray environment results in reduced life expectancy for both fabricated test and data acquisition equipment. Constantly increasing pressure from environmental regulators also threatens full-scale experimental sustainability. Efforts are further complicated by historically unreported or inexact statistics recorded for aviation fire mishaps. This makes fire emergency service readiness difficult to analyze. Experimental aviation crash data is also scarce due to expense and practicality.

To supplement testing efforts, computational methods are under investigation to further characterize the comprehensive aircraft-crash-fire-suppression event. Results from the present work are intended to provide further quantitative insight into the complex environment, as well as provide an additional set of engineering tools to predict mishap scenarios that are too expensive or impracticable to reproduce experimentally. A collaborative effort has been established on four distinct but interrelated fronts: (1) a dynamic aircraft crash analysis, (2) fuel spread analysis, (3) pool fire combustion analysis, and finally an (4) agent application fire suppression analysis. Each module presents a distinct set of modeling challenges that represent varying degrees of difficulty and progress level in their respective field. Each sector also requires a specific level of fidelity to adequately represent the bulk physical characteristics necessary for fire risk assessment. Figure 1.4 depicts the framework under investigation.

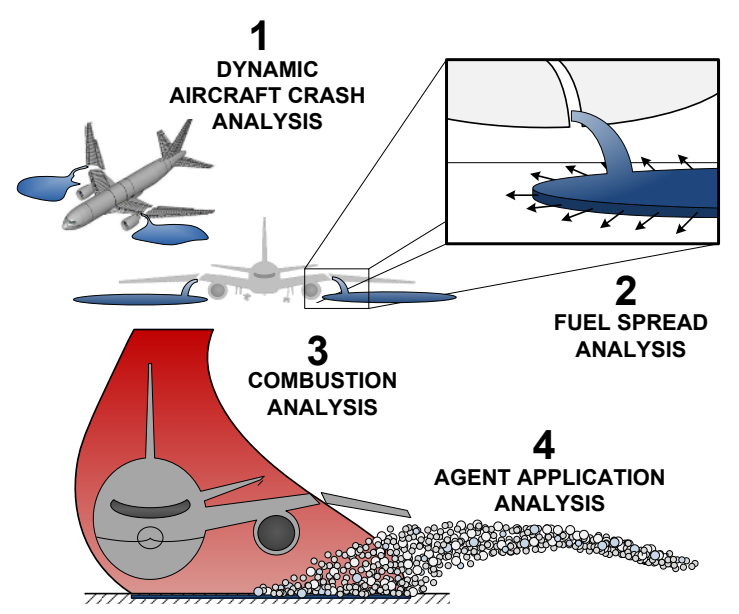

Figure 1.4: The Computational Aircraft-crash-fire-suppression Methodology 


\subsection{Terminology}

This document assumes the reader has familiarity with basic terms commonly used in the academic field of fluid mechanics and the ARFF industry. The phrase "AFFF concentrate" strictly refers to the non-diluted composition direct from the manufacturer. The term "AFFF" refers to a solution of AFFF concentrate mixed with water. The term " $n$-percent AFFF" denotes the specific percent $n$ amount of AFFF concentrate that is mixed by volume with water. The term "firefighting jet" generically refers to both AFFF and water jets. Unless otherwise specified, "water" refers to tap water. All results are reported in SI units for consistency, with dual reference to English units in some instances for added clarity.

\subsection{Scope}

The goal of the present study was to provide a better understanding of the firefighting agent application process. Its mission was to experimentally quantify the flow characteristics that differentiate water and AFFF jets and to provide the first known attempt at developing a firefighting agent application delivery model that included the influence of AFFF. Experimental efforts focused on measuring jet droplet velocity and size in addition to ground accumulation behavior. Although AFFF has served as the mainstay agent for aviation class B liquid fire suppression for several decades, its applicability in its current composition moving forward has been brought into question due to evolving environmental regulations. Even though its emulsified foam state has been well characterized, especially in the presence of a hydrocarbonfuel-air interface, very little is known about the fluid dynamics of AFFF in the turbulent liquid jet break-up flow regime en route to the flame front. Once the fire has been breached by spray impingement, AFFF acts to form a layer of insulating foam between the liquid hydrocarbon fuel surface and heated fuel vapor in order to prevent re-ignition. Of particular interest was studying the effect varying pressure, flow rate, and AFFF concentration had on the jetting process. Agent fluid dynamics altered by the presence of fire were neglected for the present study which only focused on firefighting jet flow characterization within an adiabatic environment. Research efforts were focused on analyzing the "in-flight" portion of firefighting jet flows up until initial contact with the ground is made. Post airborne interactions like liquid-liquid (i.e. agent-fuel surface) or agent surface spreading parameters were not considered. The long-term goal is to 
integrate the firefighting jet modeling approach developed in the present study with the global aircraft-crash-fire-suppression simulation methodology depicted in Figure 1.4.

The following technical objectives were identified as key milestones necessary to achieve the aforementioned goals.

- Apply experimental flow visualization techniques to provide graphical details of the in-flight firefighting jet transport process.

- Conduct experimental agent ground pattern analysis to quantify firefighting jet performance characteristics such as resultant firefighting foam quality (AFFF jets only), agent accumulation rates, and agent distribution.

- Apply experimental phase Doppler particle analysis (PDPA) to measure in-flight firefighting jet droplet velocity and droplet size distribution.

- Develop a computational fluid dynamic (CFD) modeling strategy to predict the in-flight firefighting jet characteristics measured and qualitatively corroborated by PDPA and flow visualization, respectively.

A literature review is presented in Chapter 2 to discuss the physical characteristics of AFFF in both liquid and emulsified foam forms. An introduction to turbulent round liquid jets entering still air with an extension to firefighting applications is then discussed from both an experimental and computational perspective. The aqueous firefighting agent application laboratory constructed for experimental work is overviewed in Chapter 3. Chapter 4 explains the experimental approach taken including the flow visualization process, PDPA, and agent ground pattern analysis. Chapter 5 discusses the implementation of a Lagrange-Euler CFD modeling strategy using ANSYS Fluent ${ }^{\circledR}$ v14.5 to model the firefighting jet configurations tested in the laboratory. Key results are summarized in Chapter 6 followed by conclusions and references in Chapters 7 and 8, respectively. An overall case summary is listed in Appendix A. AFFF material property data to support the aforementioned objectives is presented in Appendix B. Auxiliary PDPA data are depicted in Appendix C, and an overview on the PDPA calibration procedure carried out by the instrument's manufacturer is summarized in Appendix D. 


\subsection{Overview}

With current work investigating the effect AFFF has on conventional firefighting jet transport, a background on AFFF including its molecular composition and physical traits as both a liquid and foam is reported. Very little, if any, research exists on characterizing the role AFFF plays in influencing firefighting jet break-up. Most conventional firefighting jets are a subset of a broader phenomenology of round, turbulent, pressure-atomized liquid jets discharging into still air. Due to this, an introduction to the fundamental principles governing liquid jet instability and break-up is discussed with a look at pioneering work followed by a modern day perspective. Analysis of the fluid transport mechanisms and liquid jet break-up modes are described highlighting regimes specific to firefighting jets. Because AFFF is a surface active agent, studies examining the role surfactants play in primary liquid jet disintegration and secondary droplet break-up are also considered. Relevant analytical, experimental, as well as computational modeling methods used to characterize turbulent liquid jet break-up behavior are then discussed with extension to firefighting jet applications where available. Most firefighting jet research dating back to the 1960 s is generally limited to nozzle design and delivery improvements to water jets. The majority of contemporary fire suppression spray research focuses on sprinkler system designs for fighting Class A fires in buildings, which is outside the scope of the present effort.

\subsection{Physical Characteristics of Aqueous Film-Forming Foam (AFFF)}

\subsubsection{Background}

Initially developed for military purposes by the Naval Research Laboratory in cooperation with the fluorochemical manufacturer 3M in the early 1960s, AFFF is a highly effective, water-based class B liquid fire suppression agent. As commercial airline passenger service and military airpower demands began to increase significantly around the same time period, an alternate synthetic option was desired capable of doubling fire suppression performance in terms of reduced foam requirements over older, less efficient protein-based foams. AFFF adoption became widespread, eventually making its way into the civilian firefighting industry by the late 1970s. It has emerged as the firefighting agent of choice for fighting pool fires at domestic 
airports, military bases, oil refineries, and other outlets whose operations rely heavily on hydrocarbon consumption $[1,2]$.

Originally referred to as "light water," AFFF has the ability to cover a lower density liquid hydrocarbon fuel surface with a foam layer to act as both a thermal and evaporative barrier to hinder and ultimately extinguish combustion. Water alone is typically unsuccessful at extinguishing hydrocarbon fires because the higher density water tends to sink below the lower density fuel providing negligible firefighting benefit. Figure 2.1 depicts AFFF applied to medium and large-frame aircraft JP-8 pool fires carried out by AFRL personnel at Tyndall Air Force Base, FL.

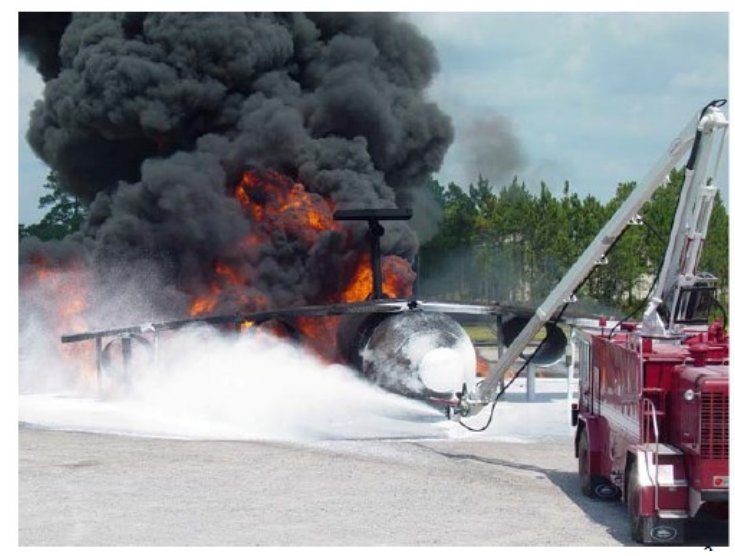

(a)

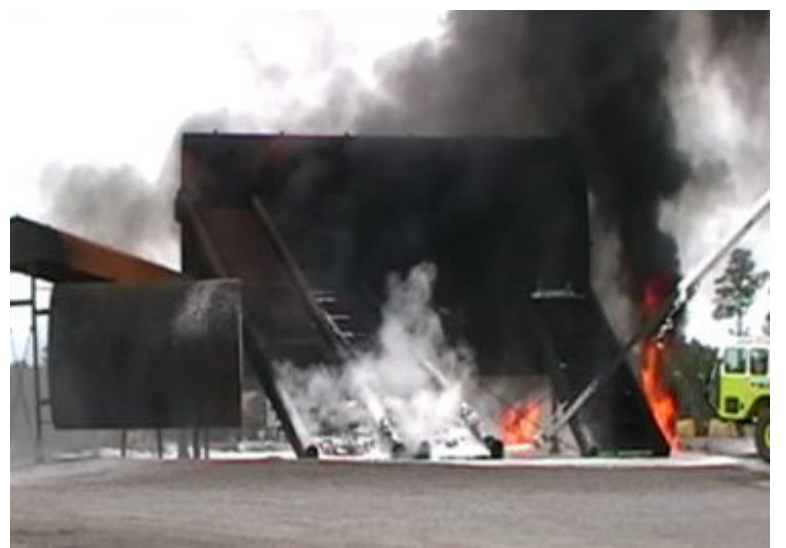

(b)

Figure 2.1: (a) AfFF Being Applied to a Medium-frame Aircraft JP-8 Pool Fire (b) AfFF Being Applied to a Largeframe Aircraft JP-8 Pool Fire

AFFF's film-forming properties are derived from its ability to sustain a thin film along the hydrocarbon fuel surface after the foam layer has collapsed, exhibiting a unique capacity to selfheal if penetrated by debris [1]. Classic work has characterized AFFF-based firefighting foam compositions in terms of agent delivery proportioning characteristics, minimum AFFF concentration needs, and nozzle discharge requirements for effective fire suppression. Foam rheological properties like yield stress, density, and viscosity have also been investigated to develop AFFF composition guidelines for use in delivery systems designed for protein foams that by comparison are heavier, more viscous, less stable, and have faster drain rates [1, 3-8]. Continued research has led to the development of a minimum set of performance standards for agent quality as well as firefighting agent delivery systems that are recognized by several key agencies including the DOD, FAA, International Civil Aviation Organization, and the National 
Fire Protection Agency (NFPA). Military Specification (MIL-SPEC) AFFF used by the DOD must conform to performance provisions outlined by Mil-F-24385 current revision $F$ [9]. Civilian firefighting practices are governed by the FAA and follow standards in NFPA 403: Standard for Aircraft Rescue and Fire-Fighting Services at Airports and NFPA 412: Standard for Evaluating Aircraft Rescue and Fire-Fighting Foam Equipment [10, 11]. Military firefighting standards often adhere to the same or similar standards as those defined by NFPA.

\subsubsection{Molecular Composition and Behavior}

MIL-SPEC C301MS 3-percent AFFF manufactured by Chemguard and Williams ${ }^{\circledR}$, a subsidiary of Tyco International, was the AFFF concentrate used exclusively for the present study. Its constituents and fluid properties are typical of most MIL-SPEC AFFF formulations. MIL-SPEC AFFF is manufactured and distributed in concentrate form in either 3-percent or 6-percent formulas. The percentage denotes the volumetric proportion at which AFFF concentrate must be mixed with water to generate a firefighting agent solution capable of meeting MIL-SPEC (or NFPA) performance criteria. In other words, 3-percent AFFF concentrate must be mixed 3 parts concentrate with 97 parts water, and a 6-percent AFFF concentrate must be mixed with 6 parts concentrate and 94 parts water for both solutions to achieve the same minimum required level of firefighting effectiveness. In practice, most modern firefighting agent delivery systems allow for variable water-AFFF concentrate proportioning to suit specific needs. Table 2.1 lists the known chemical constituents that make up the AFFF concentrate used in the present study [12]. Approximate AFFF concentrate compositions developed by other manufacturers are in the following selected references $[2,13-16]$.

Table 2.1: Reported AFFF Concentrate Chemical Constituents Used in the Present Study [12]

\begin{tabular}{|c|c|}
\hline Chemical Name & Percentage \\
\hline Diethylene glycol mono butyl ether (Butyl Carbitol $^{\mathrm{TM}}$ ) & $0-8 \%$ \\
\hline Magnesium Sulfate & $0.5-1.5 \%$ \\
\hline Ethylenediane tetra acetic acid & $0.5-1.5 \%$ \\
\hline Hydrocarbon surfactant & Proprietary \\
\hline Fluorocarbon surfactant & Proprietary \\
\hline
\end{tabular}

AFFF concentrate is primarily made up of an "active" blend of hydrocarbon and fluorocarbon surfactants, also referred to as surface active agents, in percentages known exclusively to the 
manufacturer. Modern synthesis of fluorocarbon surfactants is typically the most expensive manufacturing stage based on telomerization. Older techniques used by manufacturers like $3 \mathrm{M}$ relied on electrochemical fluorination. Because highly favorable firefighting foam properties can be achieved from a relatively low concentration of fluorocarbon surfactants, their expense is offset when mixed with more of the less expensive hydrocarbon surfactants. Both types of surfactant exhibit enhanced firefighting performance when mixed together as opposed to when used separately. AFFF concentrate also contains secondary viscosity enhancers such as Butyl Carbitol along with minor additives to optimize the final physical state of the foam like insoluble salts, thickeners, corrosion inhibitors, and stabilizers. Unfortunately, an exact explanation on the complex role each ingredient plays is publicly unavailable due to the proprietary nature of the AFFF concentrate formulation. AFFF concentrate compositions may also vary significantly from batch to batch just so long as industry specific performance requirements like the MILSPEC standard are met [2].

The surfactants in AFFF concentrate are made up of long anionic and amphoteric molecular chains of hydrophilic, hydrophobic, and oleophobic components that are barely miscible in water. They are used to lower the surface energy of the aqueous solution, generate foam, and improve spread efficiency across the fuel surface. Water soluble surfactants are typically modeled as having a hydrophilic ionic or polarized "head" along with an organic hydrophobic or oleophobic "tail." Anionic surfactants are a common sub-family of surfactants displaying properties characteristic of most soaps and detergents. These surfactants generate complicated electrical interactions in head groups along water-oil interfaces that are capable of creating regions of unbalanced charge. These intermolecular forces, largely based on the dissolved ionic concentration, can significantly affect interfacial rheology. Amphoteric surfactants are less commonly used industrially and can exhibit a positive, negative, or dual charge dependent on the solution $\mathrm{pH}$ [17]. Figure 2.2 illustrates two telomerization-based fluorocarbon surfactants identified in AFFF concentrate specific to this study [18]. 
Fluorotelomer thioether amido sulfonate

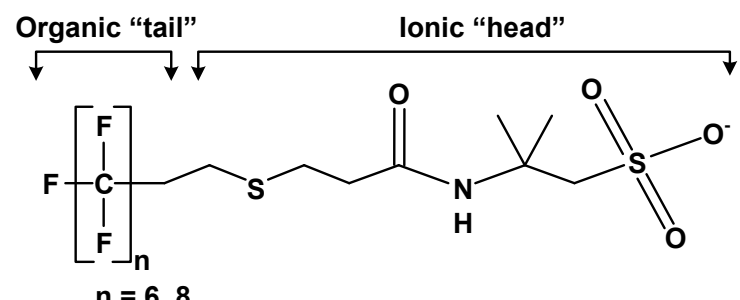

(a)
Fluorotelomer thioether amido amino carboxylic acid

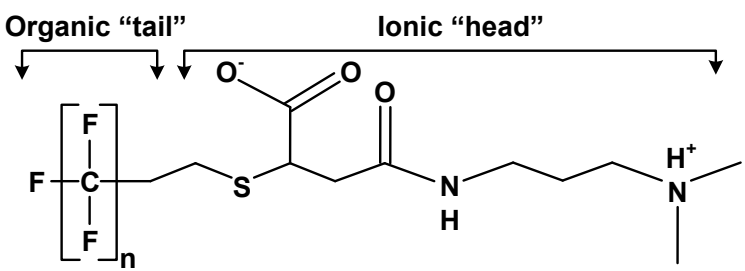

$n=6,8,10$

(b)

Figure 2.2: Examples of Fluorocarbon Surfactants Identified in AFFF [18]

AFFF surfactant molecules prefer to align along a free surface such that their hydrophilic heads migrate toward water and their hydrophobic and oleophobic tails migrate toward the fuel surface and air, respectively. Although fluorocarbon and hydrocarbon surfactant polar head groups can exhibit strong similarities, fluorocarbon surfactant tails are both hydrophobic and oleophobic by nature whereas hydrocarbon surfactant tails are only hydrophobic [13]. Figure 2.3 illustrates the favorable orientation of surfactant molecules when AFFF is situated between a fuel-air interface.
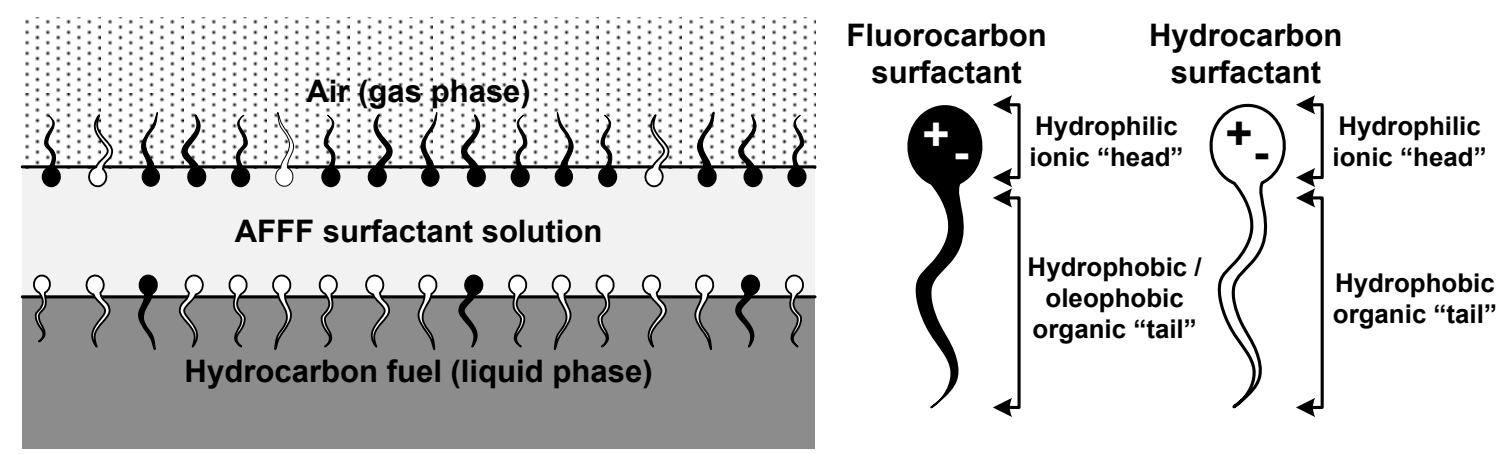

Figure 2.3: The Conceptual Alignment of Surfactants in an Air-water-fuel Environment [2, 13]

Fluorocarbon surfactants are generally characterized by outstanding film-forming traits that help lower the surface tension of AFFF. Flourocarbon surfactants also provide stronger thermal resistance and stability compared to hydrocarbon surfactants which are known for having greater foam forming capability and lowering the overall interfacial tension between AFFF and the fuel surface [17].

In dynamic scenarios such as firefighting jets where AFFF is injected into the atmosphere from a nozzle breaking up into droplets, the surfactant's preference to self-orient becomes a timedependent process based on molecular diffusion. In the initial stages of injection into air, surfactants are randomly distributed throughout the AFFF. As time progresses, the surfactant 
molecules undergo preferential alignment to create a monolayer based upon the same rules as those illustrated in Figure 2.3. The level of organization the surfactants reach increases as surface age increases. This molecular process helps define an instantaneous surface tension at a finite rate, better known as the dynamic surface tension of the fluid. Figure 2.4 depicts this morphology using the cross-section of a single airborne droplet of AFFF aging with time [2].

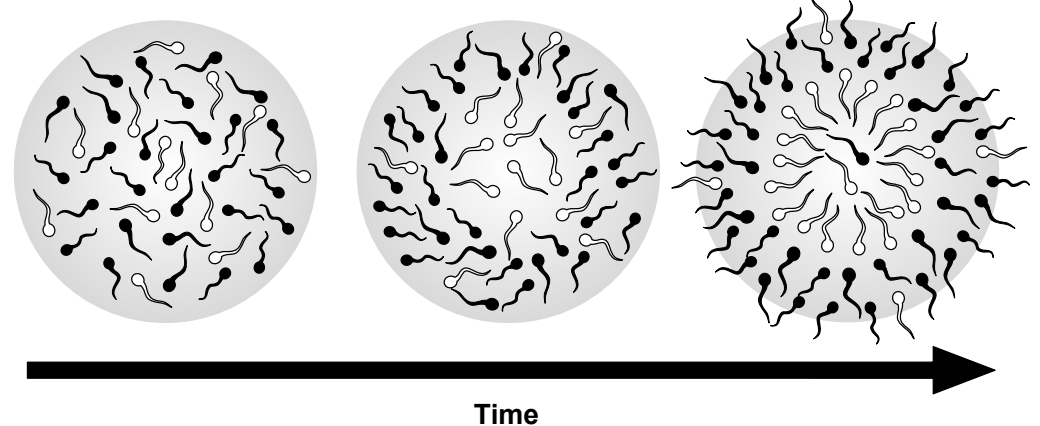

Figure 2.4: An Illustration Depicting the Tendency of Surfactant Molecules to Self-align within an Airborne AFFF Droplet as Surface Age Increases [2]

In the absence of a free surface, surfactants are inclined to self-organize if their concentration is adequately high - what is referred to as the critical micelle concentration. Beyond the critical micelle concentration, they freely combine to form spherical structures called micelles to reduce the contact between the hydrophobic tail of the molecule and the aqueous solution. Solution concentrations above the critical micelle concentration allow minimum surface and interfacial tensions to be achieved in shorter time periods that scale with an increase in concentration. However, an increase in surfactant concentration does not significantly alter the minimum values of either surface or interfacial tension. Figure 2.5a illustrates the shape of a standard water soluble micelle. This shape can evolve into complex structures like double, cylindrical, and multi-layered sheet micelles if the surfactant concentration increases significantly beyond the critical micelle concentration. Figure $2.5 \mathrm{~b}$ depicts the relationship between the surfactant's interfacial tension and the surfactant concentration $(C)$ denoting the location of the critical micelle concentration [17]. 


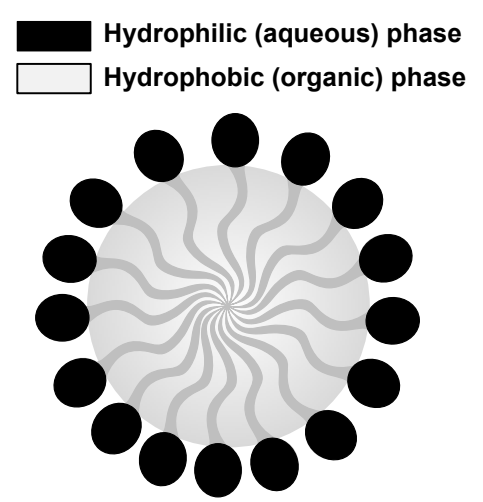

(a)

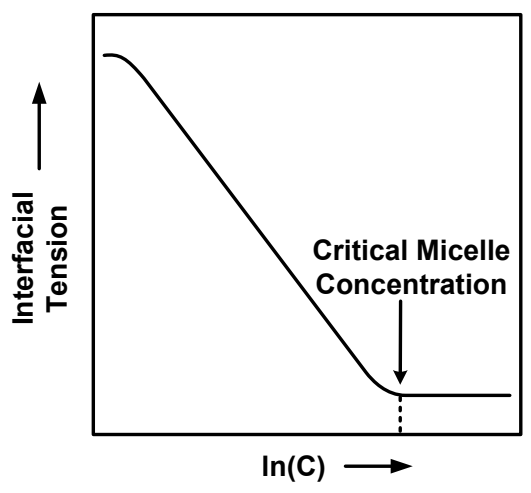

(b)

Figure 2.5: (a) Illustration of a Standard Micelle (b) Interfacial Tension vs. Surfactant Concentration [17]

Although fluorocarbon surfactants are oxidatively stable, they have been found to show up in surface and ground water near military firefighting training sites [14]. In the late 1990s, perfluorooctyl sulfonate (PFOS)-based surfactants used in early 3M-based AFFF compositions were found to be bioaccumulative, environmentally persistent, slightly toxic, and classified as an emerging contaminant by the United States Environmental Protection Agency (EPA). PFOS was subsequently replaced by presumably more environmentally friendly surfactants like perfluoroctanoic acid (PFOA) in the early 2000s. In 2012, however, PFOA was also recognized as an emerging contaminant, jeopardizing the long-term viability of current AFFF compositions. In response to the negative effect PFOS and PFOA have on human health and the environment, by 2010 the EPA's PFOA stewardship program committed eight major manufacturers to reduce PFOA and its related chemical production by 95 percent. All PFOA production is to be eliminated by 2015. These concerns over current AFFF concentrate compositions highlight the pressing need for firefighting agent research to identify and preserve key performance characteristics for next generation agents that will be subject to even more rigorous environmental regulations $[13,19]$.

\subsubsection{Liquid Continuum Characteristics}

AFFF concentrate exhibits many of the same fluid state properties as water, most notably density with others like boiling and freezing point. These properties approach the properties of water even more so once AFFF concentrate is diluted at typical proportions with water to form firefighting agent solution. AFFF concentrate tends to be slightly alkaline at approximately 7.9, but its $\mathrm{pH}$ falls within the acceptable range for tap water between 6.5 and 8.5. Besides its 
slightly yellow to amber appearance, viscosity and more so surface tension properties differentiate AFFF concentrate from water the most as alluded to in the previous section. These intrinsic properties are typically used to quantify the influence AFFF has on firefighting jet performance, especially on the continuum scale. Table 2.2 lists the equilibrium fluid properties of AFFF compared to water at standard conditions.

Table 2.2: Equilibrium Fluid Properties of Water and AFFF at Standard Conditions

\begin{tabular}{|c|c|c|}
\hline Fluid Property & Water & AFFF \\
\hline Density $\left(\mathrm{kg} \cdot \mathrm{m}^{-3}\right)$ & $997.0[20]$ & $1079[12]$ \\
\hline Dynamic Viscosity $\left(\mathrm{kg} \cdot \mathrm{m}^{-1} \cdot \mathrm{s}^{-1}\right)$ & $8.98 \times 10^{-4}[20]$ & $7.00 \times 10^{-3}[12]$ \\
\hline Equilibrium Surface Tension $\left(\mathrm{mN} \cdot \mathrm{m}^{-1}\right)$ & $72.1[20]$ & $17.4[12]$ \\
\hline
\end{tabular}

Because firefighting jets represent a dynamic process, variation of fluid properties when exposed to transient conditions or to a range of applied stresses is of further interest. Although the nonNewtonian behavior of AFFF in its emulsified foam state is well documented, details on the viscous nature of any MIL-SPEC AFFF over a range of applied shear rates was not found. Although beyond the scope of the current study, AFFF concentrate derivatives such as alcohol resistant AFFF designed for alcohol fire suppression due to the addition of polar solvents exhibits strong non-Newtonian behavior. This class of AFFF concentrates is well documented and known for demonstrating pseudoplastic, shear-thinning, and thixotropic properties due to the addition of rheological modifiers like xanthan gum resins or Carbopol ${ }^{\circledR}$ [21].

Dynamic surface tension data exists for several AFFF compositions, some of which are reproduced to illustrate the property's sensitivity to various controlled conditions [22, 23]. Dynamic surface tension is strongly dependent on factors like surfactant concentration, molecular diffusion rates, fluid temperature, and salinity, along with other variables that may affect the surfactant's relationship with the aqueous solution and surrounding atmosphere. For very low surface ages without surfactant additives, dynamic surface tension approaches the value of the solution (i.e. water). Equilibrium surface tension values are approached for high surface ages. In the case of pure liquids like water, surface tension is independent of surface age [22].

Hyland and Williams used a model Kruss BP2 maximum bubble pressure tensiometer to measure dynamic surface tension with sensitivity to AFFF composition, dilution, salinity, and 
temperature. These relationships are illustrated in Figures 2.6a, 2.6b, 2.7a, and 2.7b, respectively [22]. Figure 2.6a shows that MIL-SPEC AFFF formulations behave similar to one another as expected due to the common equilibrium surface tension values all MIL-SPEC compositions must meet. Figure 2.6b illustrates that as AFFF concentration is increased, dynamic surface tension approaches its equilibrium value more rapidly; a concept illustrated previously on the molecular level in Figure 2.5b.

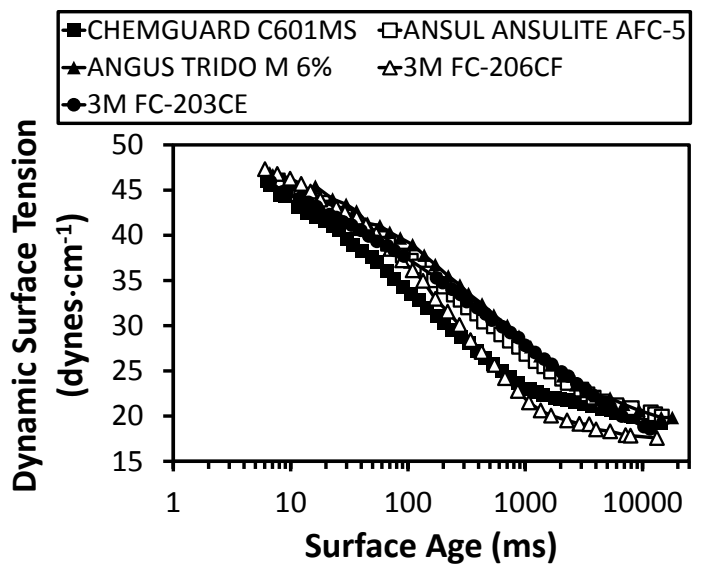

(a)

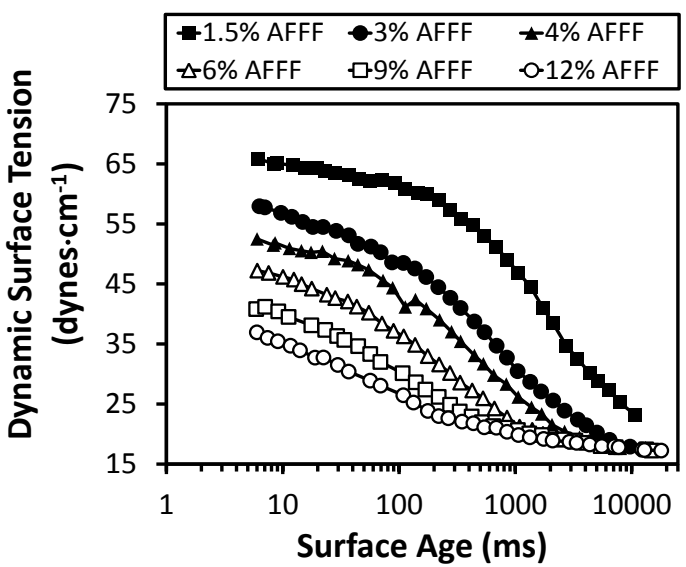

(b)

Figure 2.6: Dynamic Surface Tension vs. Surface Age for (a) Various MIL-SPEC AFFF Formulations and (b) at Different AFFF Concentration Levels [22]

Figure 2.7a shows that for certain MIL-SPEC AFFF formulations, water salinity can significantly affect dynamic surface tension whereas in others it plays a negligible role, owing to the diverse secondary (i.e. non-surfactant) constituents some makers use in the manufacturing process. The salt water solution used represents the effect of ocean water composed of $41.2 \mathrm{~g}$ of "sea salt" (as defined by the ASTM D-1141-52 standard) mixed with 11 of distilled water [24]. Figure $2.7 \mathrm{~b}$ illustrates dynamic surface tension's dependency on temperature as it lowers with increasing temperature similar to typical viscosity trends. In both Figure 2.6 and Figure 2.7, it should be noted the dynamic surface tension magnitudes of MIL-SPEC AFFF start off almost 50 percent from one another. But, by a surface age of approximately $10 \mathrm{~s}$, all values are within 25 percent or significantly less of one another along with the AFFF equilibrium surface tension value listed in Table 2.2. 


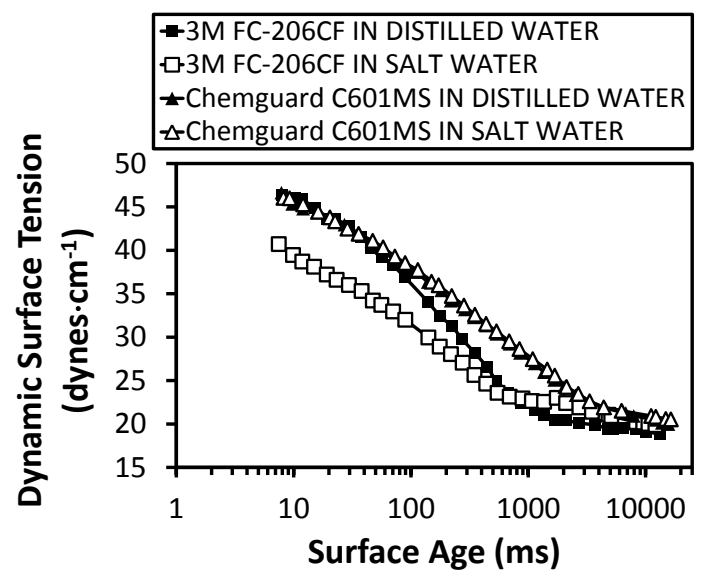

(a)

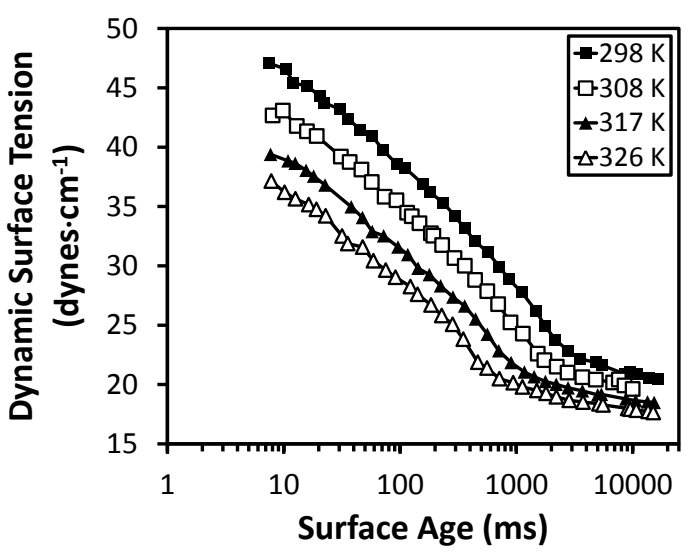

(b)

Figure 2.7: (a) Dynamic Surface Tension vs. Surface Age for Various Salinities and (b) Dynamic Surface Tension vs. Surface Age for Various Temperatures [22]

\subsubsection{Foam Continuum Characteristics}

AFFF fluid dynamics have been analyzed on multiple spatial levels spanning molecular to continuum length scales. Most literature is dedicated to AFFF's state as homogenous stabilized foam slowly spreading over top fuel layers where interacting fluid surface and interfacial molecular tension forces help drive bulk motion [26]. Very little information is available on the state of foam or foam solution during the jetting process. Specific work has focused on foam stability, rheology, viscosity, as well as its fire suppression capability [5-8, 15, 19, 23, 27-31].

Non-equilibrium foam behavior is important due to AFFF's thermodynamically unstable, timedependent life. With temporal scales on the order of a few seconds or less during the jetting process, fluid properties like surface tension, viscosity, and concentration help shape downstream foam formation characteristics. The influential degree of each property is still unknown - let alone the physical state of AFFF during the firefighting jet transport process. Increases in AFFF concentration typically shorten the non-equilibrium time scale and encourage foam growth to occur more rapidly compared to more diluted AFFF compositions as shown in Figure 2.4 and Figure 2.6b [25]. Some researchers have inferred that for particularly high speed liquid jets traveling relatively short distances made up of smaller agent droplets, quasi-equilibrium conditions can be assumed considering conventional foam evolutionary time scales are much longer on the order of several seconds to approaching minutes. This would result in the suppression of foam growth [27]. 
Table 2.3 lists some of the key chemical and physical performance requirements MIL-SPEC AFFF must meet either in concentration or solution form with water. Some performance characteristics not relevant to the present study have been omitted from this table, such as corrosion rate, biodegradability, and toxicity.

Table 2.3: MIL-SPEC AFFF Chemical and Physical Minimum Performance Criteria [5]

\begin{tabular}{|c|c|c|c|}
\hline \multirow{2}{*}{ Requirement } & \multicolumn{2}{|c|}{ Values } & \multirow{2}{*}{ Applicable Publication } \\
\cline { 2 - 4 } & Type 3\% & Type 6\% & \\
\hline Refractive Index, minimum & 1.3630 & 1.3580 & -- \\
\hline $\begin{array}{c}\text { Viscosity, centistokes } \\
\text { Maximum at 278.15 K }\end{array}$ & 20 & 20 & \\
Minimum at 298.15 K & 2 & 2 & \\
\hline Hydrogen ion concentration (pH) & 7.0 to 8.5 & 7.0 to 8.5 & \\
\hline Spreading Coefficient, minimum & 3 & 3 & NFPA 412 Paragraph 4.7.2 \\
\hline Foamability & & 5.0 & NFPA 412 \\
Minimum Foam Expansion & 5.0 & 2.5 & Paragraph 4.7.5 \\
Maximum 25-percent drainage time, \\
minutes, min
\end{tabular}

The spreading coefficient refers to the rate at which AFFF can cover a fuel surface. The spreading coefficient must be positive if spontaneous spreading is to occur. The spreading coefficient $\left(\sigma_{S}\right)$ due to surface tension is defined by the following relationship:

$$
\sigma_{S}=\sigma_{C}-\sigma_{A F F F}-\sigma_{I}
$$

where $\sigma_{C}$ is the surface tension of cyclohexane, $\sigma_{A F F F}$ is the surface tension of the AFFF, and $\sigma_{I}$ is the interfacial tension between the cyclohexane and AFFF. Foam expansion is determined from one of two methods from NFPA 412. The most common method uses a foam sampling apparatus to collect foam from a firefighting jet in $1000 \mathrm{ml}$ graduated cylinders positioned below a collecting back board. Foam expansion is calculated by the following equation:

$$
\text { Expansion }=\frac{\text { volume of foam }}{\text { volume of solution }}
$$


Foam 25-percent drainage time is calculated by monitoring the amount of time it takes for 25 percent of the agent solution to be drained from the foam captured downstream of a firefighting jet in a similar fashion to the foam expansion test mentioned above. For details on 25-percent foam drainage time testing and other AFFF foam testing procedures listed in Table 2.3, refer to either the MIL-SPEC or NFPA 412 standard $[9,11]$.

\subsection{Turbulent Round Liquid Jets Entering Still Air}

\subsubsection{Early Work on Liquid Jet Instability and Break-up Entering Still Air}

Although the study of liquid jet instability and disintegration when injected into a stagnant gaseous atmosphere has been documented since the early 1800 s, the problem of liquid jet breakup particularly in the turbulent flow regime is still not fully understood [32-36]. Early observations on low speed, laminar jet flows conducted by Savart followed by theoretical perspectives offered by Plateau resulted in some of the first impressions regarding this complex physical phenomenon. Further contributions by Bidone, Magnus, Buff, and Boussinesq helped pave the way for laminar instability theory substantiated by Rayleigh and Weber, which sets up the discussion for examining turbulent liquid jet break-up behavior [33]. Work by Chen and Davis, Grant, Phinney, Hoyt and Taylor, and Ruff et al. have provided the most useful theoretical and empirical perspectives specific to the turbulent liquid jet break-up regime leading into the 1980s [32-35, 38, 40]. Detailed reviews by Grant, McCarthy and Malloy, Lein and Reitz, Faeth et al., and most recently Birouk and Lekic in 2009 summarize the general physical mechanisms believed to be responsible for liquid jet disintegration into still air, highlighting areas where further work is still needed [32-34, 36, 37].

Rayleigh proposed that a laminar free jet surface near the nozzle in a vacuum represents a wide range of infinitesimal, symmetrical disturbances that could be mathematically represented as sinusoidal waves; a basis rooted in small perturbation theory. Through an energy balance between a disturbed and undisturbed water column and extrapolating the surface disturbance growth rate, Rayleigh derived a linearized relationship describing the break-up length for an inviscid laminar liquid jet in air as a sole function of surface tension and inertial forces, expressed as: 


$$
\frac{Z}{D}=1.03\left(\ln \frac{R}{\eta_{0}}\right) W e_{L}^{0.5}
$$

where $Z$ is the continuous jet break-up length, $D$ is the liquid jet diameter, $R$ is the liquid jet radius, $\eta_{0}$ is the infinitesimal disturbance, and $W e_{L}$ is the Weber number based on liquid jet characteristics. The Weber number is generally defined as the ratio between inertial and surface tension forces, and specifically defined in Equation 2.3 as:

$$
W e_{L}=\frac{\rho_{L} V_{J}^{2} D}{\sigma_{L / G}}
$$

where $\rho_{L}$ is the density of the jet liquid, $V_{J}$ is the jet nozzle velocity, and $\sigma_{L / G}$ is the surface tension between the liquid jet and the surrounding ambient gas. Rayleigh postulated that liquid jet break-up occurs because infinitesimal axisymmetric disturbances near the nozzle exit grow exponentially until they reach the same length scale of the liquid jet radius causing destabilization, what he referred to as varicose break-up (now referred to as Rayleigh break-up). Equation 2.3 can also be derived in a similar format using similitude analysis [32, 33].

Weber extended Rayleigh's efforts with the inclusion of viscous effects by assuming the liquid jet maintains a mean flow with surface disturbances imparted upon it. After developing a characteristic equation for the disturbance growth rate in terms of surface tension and simplifying through an order of magnitude analysis, Weber was able to expand Equation 2.3 to include viscosity $[32,33]$ :

$$
\frac{Z}{D}=\ln \frac{R}{\eta_{0}}\left\{W e_{L}^{0.5}+\frac{3 W e_{L}}{R e_{L}}\right\}
$$

where $R e_{L}$ is the Reynolds number defined as the ratio of inertial to viscous forces, and specifically in Equation 2.5 with respect to liquid jet characteristics as:

$$
R e_{L}=\frac{\rho_{L} V_{J} D}{\mu_{L}}
$$

where $\mu_{L}$ is the dynamic viscosity of the jet liquid. Equation 2.5 provides a good approximation for the break-up length for limiting cases in which the physical assumptions of an axisymmetric, 
low speed laminar Newtonian viscous jet apply, except in peculiar situations like for a mercury liquid jet where the linear slope $\ln \left(R / \eta_{0}\right)$ was approximately four times larger compared to other liquids in experiments conducted by Smith and Moss. Alternatively, Grant and Middleman state the slope in Equation 2.3 is better represented by the following relation,

$$
\ln \frac{R}{\eta_{0}}=-2.66 \ln (O h)+7.68
$$

where $\mathrm{Oh}$, referred to as the Ohnesorge or stability number, is defined as a combination of the Weber and Reynolds number:

$$
O h=W e_{L}^{0.5} R e_{L}^{-1}=\frac{\mu_{L}}{\left(\rho_{L} D \sigma_{L / G}\right)^{0.5}}
$$

where dependence on the characteristic velocity $\left(V_{J}\right)$ is removed through a combination of the Weber and Reynolds number. With the addition of Equation 2.8, Grant and Middleman re-cast Equation 2.5 with modified coefficients from their own experimental work based on a wide range of liquid viscosities but minor changes in surface tension and density [33]:

$$
\frac{Z}{D}=19.5\left\{W e_{L}^{0.5}+\frac{3 W e_{L}}{R e_{L}}\right\}^{0.85}
$$

Although linear instability theory forged a mathematical argument for laminar liquid jet breakup, it has since been repudiated by more recent experimental observations identifying irregular surface waves near the nozzle - even in the case of low Reynolds number laminar jets [36]. Phinney among others has brought attention to the lack of a theoretical framework governing turbulent liquid jet flows, exhibiting minor success in extending linearized laminar theory into the turbulent flow regime $[35,36]$. The majority of early turbulent liquid jet stability studies have resulted in empirical correlations which prove useful in a few limiting situations [33]. Owing to the physical complexities involved with describing liquid jet break-up behavior, especially in the case of transitional to fully turbulent flow, contemporary views recommend more sophisticated aerodynamic theories that also take into account non-linear interactions associated with the liquid jet's surroundings [36]. 


\subsubsection{Modern Methods of Classifying Liquid Jet Break-up}

Modern liquid jet break-up classification is based on regimes that reflect qualitative differences in appearance as the operating conditions are modified. Characteristics such as surface tension, liquid inertia, and aerodynamic forces are the measurable criteria used to quantify these regimes which are absorbed in dimensionless parameters like the Reynolds, Weber, and Ohnesorge number [34]. Fundamental principles of primary and secondary atomization are introduced followed by a more rigorous classification of each break-up regime.

A liquid jet is defined by Birouk and Lekic as a "beam of liquid emerging from a nozzle into a quiescent, gaseous atmosphere," and specifically for the current study as a firefighting jet consisting of either an AFFF or water jet discharging into still air [36]. After exiting the nozzle, the liquid disintegrates in either a single or two-stage process. Primary break-up takes place when the liquid jet core is torn into ligaments and other irregular shapes as its surface interacts in close proximity with the surrounding air to form a multiphase mixing layer. As ligament strands peel away from the liquid jet core, they morph or break off into individual droplets. Primary break-up governs the liquid jet break-up length, starts the atomization process, and facilitates initial conditions for secondary break-up to occur in the dispersed downstream region of the flow [37]. Primary break-up is also heavily dependent upon internal liquid forces derived from surface tension, velocity profile relaxation, and turbulence [33]. Secondary break-up occurs if shed satellite droplets further disintegrate into smaller droplets resulting from an even stronger influence from the surrounding air. Figure 2.8a illustrates near field representations of primary break-up from the liquid jet surface adapted from shadowgraph photography recorded by Sallam and Faeth [36]. Figure 2.8b depicts both primary and secondary break-up with respect to the liquid jet break-up length. 


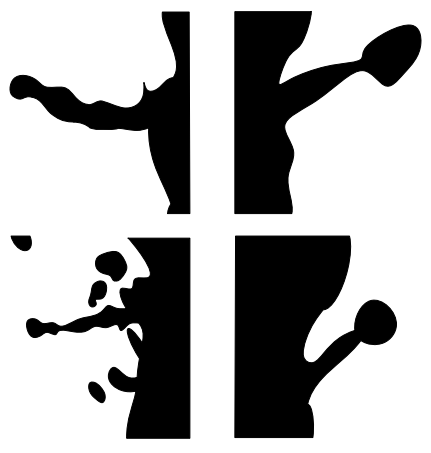

(a)

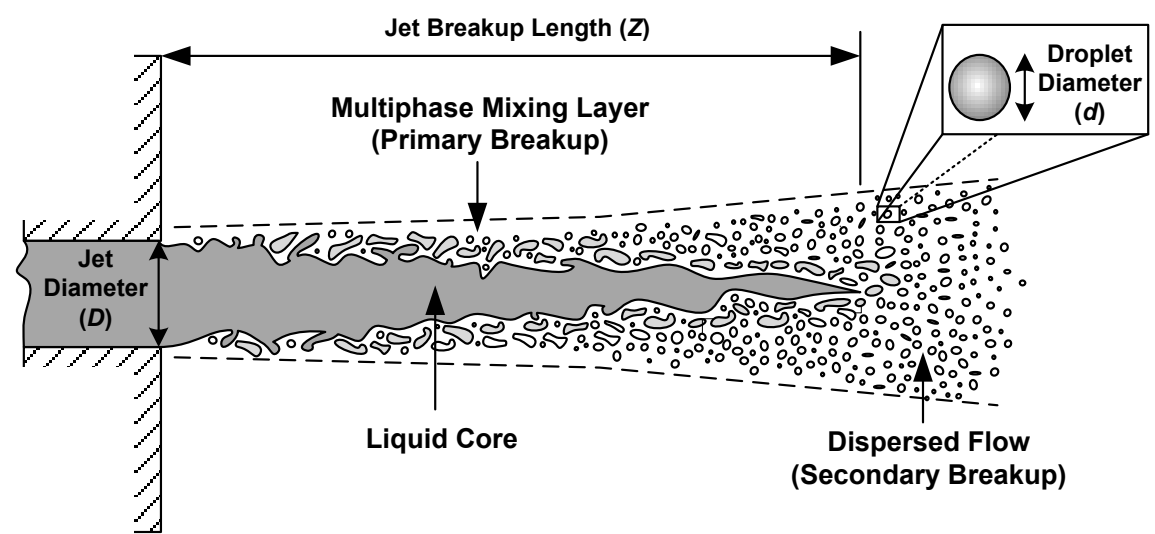

(b)

Figure 2.8: (a) Shadowgraph Illustrations of Ligaments Peeling Away from an Intact Liquid Jet Core [36] (b) Illustration of the Nozzle Exit Region Depicting Primary and Secondary Break-up [37]

Illustrated in Figure 2.9, liquid atomization can be further resolved into four liquid jet break-up regimes: the Rayleigh (varicose) break-up regime I, the first wind-induced (sinuous) break-up regime II, the second wind-induced break-up regime III, and the atomization break-up regime IV. The physical characteristics that define each regime are discussed after Figure 2.9.

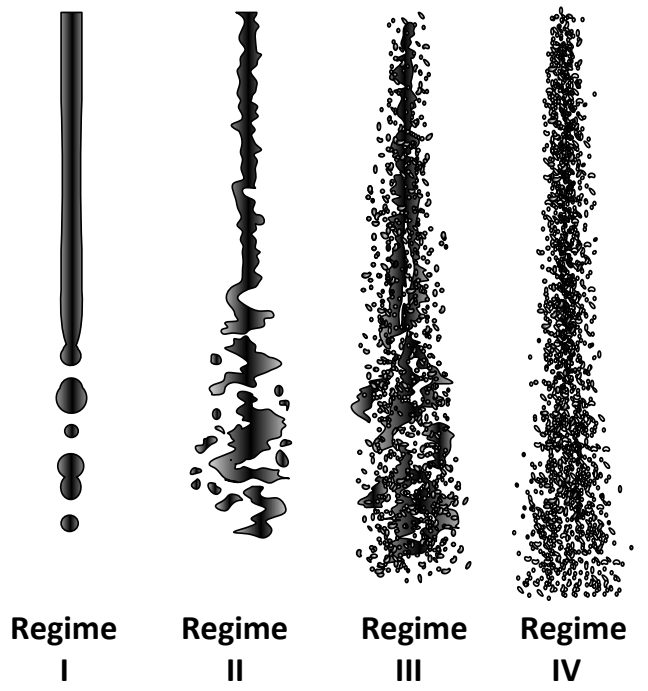

Figure 2.9: The Four Regimes of Liquid Jet Break-up [41]

1. The Rayleigh Liquid Jet Break-up Regime I - The Rayleigh or varicose regime is characteristic of laminar, low velocity inviscid liquid jets with long wavelengths and small, symmetric surface perturbations dominated by the interaction between surface tension (capillary) and inertial forces. Aerodynamic effects are considered insignificant in the Rayleigh break-up regime where surrounding air entrainment is negligible. Drop 
diameters that form downstream are sized slightly larger than that of the liquid jet diameter, exclusively characterized by primary break-up mechanisms $[34,36]$.

2. The First Wind-induced Liquid Jet Break-up Regime II - The first wind-induced, sinuous, or wavy regime are common names referring to this mode which involves slightly higher relative velocities compared to the Rayleigh regime. Liquid jet dynamics are identified by sinusoidal, or transverse vibrations that are still dilational by nature but slightly amplified by the surrounding ambient air. Similar to the Rayleigh regime, primary break-up dominates this regime as well. Resulting droplet formation is sized on the order of the liquid jet diameter $[34,36]$.

3. The Second Wind-induced Liquid Jet Break-up Regime III - The second windinduced regime has a more pronounced aerodynamic interdependency with atmosphere conditions compared to lower regimes causing more liquid jet surface irregularity to form. Primary and secondary break-up are easily visible in this mode, forcing downstream droplet formation much smaller compared to the jet diameter $[34,36]$.

4. The Atomization Liquid Jet Break-up Regime IV - The atomization regime represents liquid jets with the highest velocities and consequently the highest kinetic energy. This mode is distinguished by the formation of a dense, fully dispersed liquid jet that breaks down into fine droplets very close to the nozzle exit resulting almost exclusively in secondary break-up, often the result of cavitating nozzle conditions. The existence of a coherent liquid core is widely debated in this regime, and those that do believe in its existence agree it is very short and ends close to the nozzle exit $[34,36]$.

Historically, each break-up regime has been graphically defined using two different approaches: Ohnesorge's classification and the liquid jet stability curve. Depicted in Figure 2.10, Ohnesorge's classification provides the most reliable approach by identifying each mode within a logarithmic plot of Ohnesorge versus Reynolds number. Most conventional firefighting jets are characterized by pressure-atomized nozzle conditions that fall within the atomization breakup regime IV highlighted on Figure 2.10. Ohnesorge's classification can be similarly defined via the liquid and ambient gas Weber number, $W e_{L}$ and $W e_{G}$, respectively, listed in Table 2.4. The ambient gas Weber number is defined as follows with $\rho_{G}$ denoting the ambient gas density [36]: 


$$
W e_{G}=\frac{\rho_{G} V_{J}^{2} D}{\sigma_{L / G}}
$$

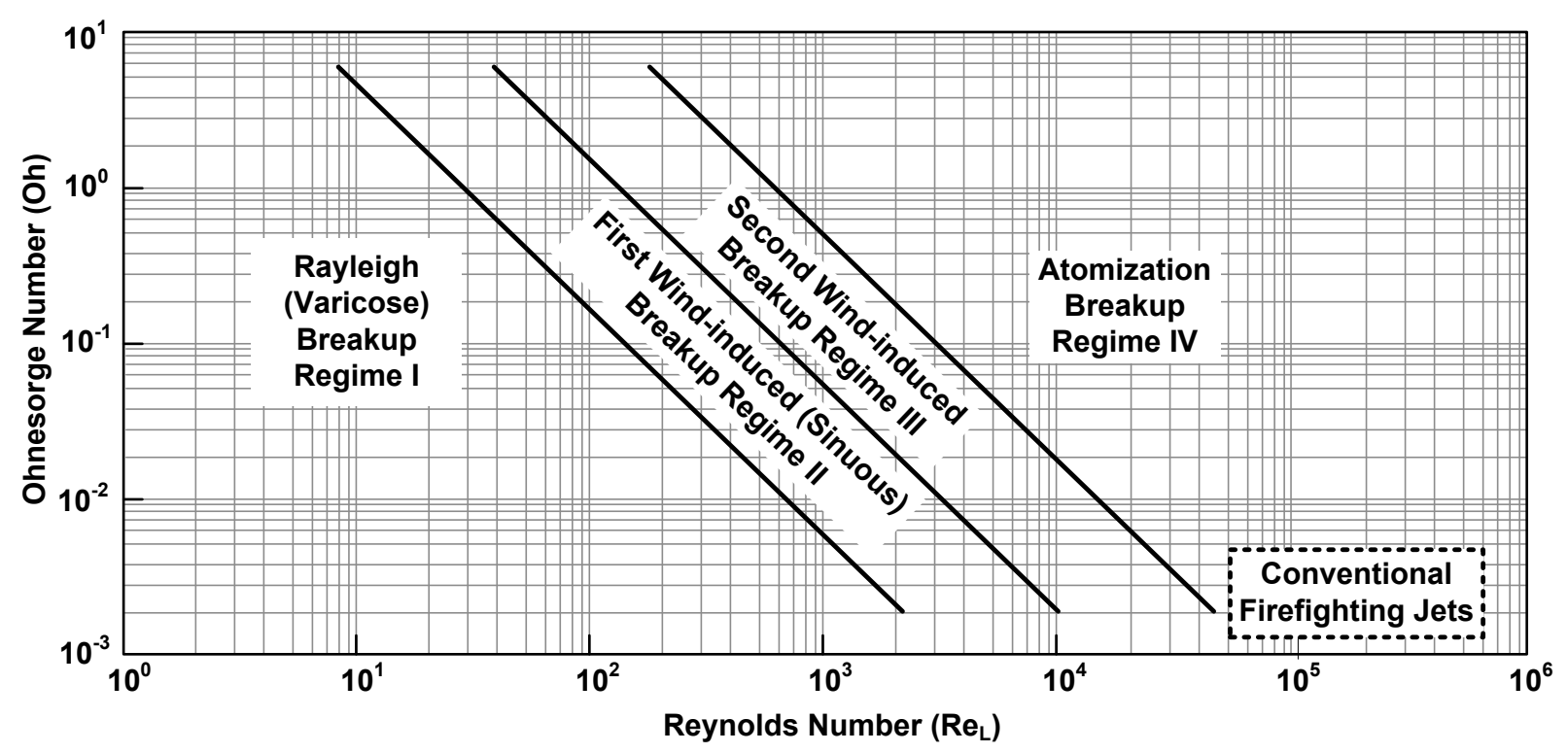

Figure 2.10: Liquid Jet Break-up Regime Classification in Quiescent Air with Respect to Reynolds No. and Ohnesorge No. [35]

Table 2.4: Ohnesorge's Classification of Various Liquid Jet Break-up Regimes via Weber No. [36]

\begin{tabular}{|c|c|}
\hline Liquid Jet Break-up Mode & Range \\
\hline Rayleigh's Break-up Regime I & $W e_{L}>8, W e_{G}<0.4$ \\
\hline First Wind-induced Break-up Regime II & $0.4<W e_{G}<13$ \\
\hline Second Wind-induced Break-up Regime III & $13<W e_{G}<40$ \\
\hline Atomization Break-up Regime IV* & $W e_{G} \sim 40$ \\
\hline
\end{tabular}

* Conventional Firefighting Jet Break-up Regime

An alternate approach to classifying the liquid break-up modes is by analyzing the ambient gas to liquid jet to ambient air density ratio $(\varepsilon)$ as a function of Reynolds number, defined as:

$$
\varepsilon=\rho_{G} \cdot \rho_{L}{ }^{-1}
$$

Figure 2.11 depicts the non-linear sensitivity the gaseous Weber number has on defining the break-up modes as this ratio approaches zero. With a ratio of about 0.001 for water ejecting into still air, the conventional firefighting jet break-up regime is again highlighted in its respective location within the atomization break-up regime. Further information on the gaseous Weber number correlation shown in Figure 2.11 separating break-up regime I from regime II are in the associated figure reference. 


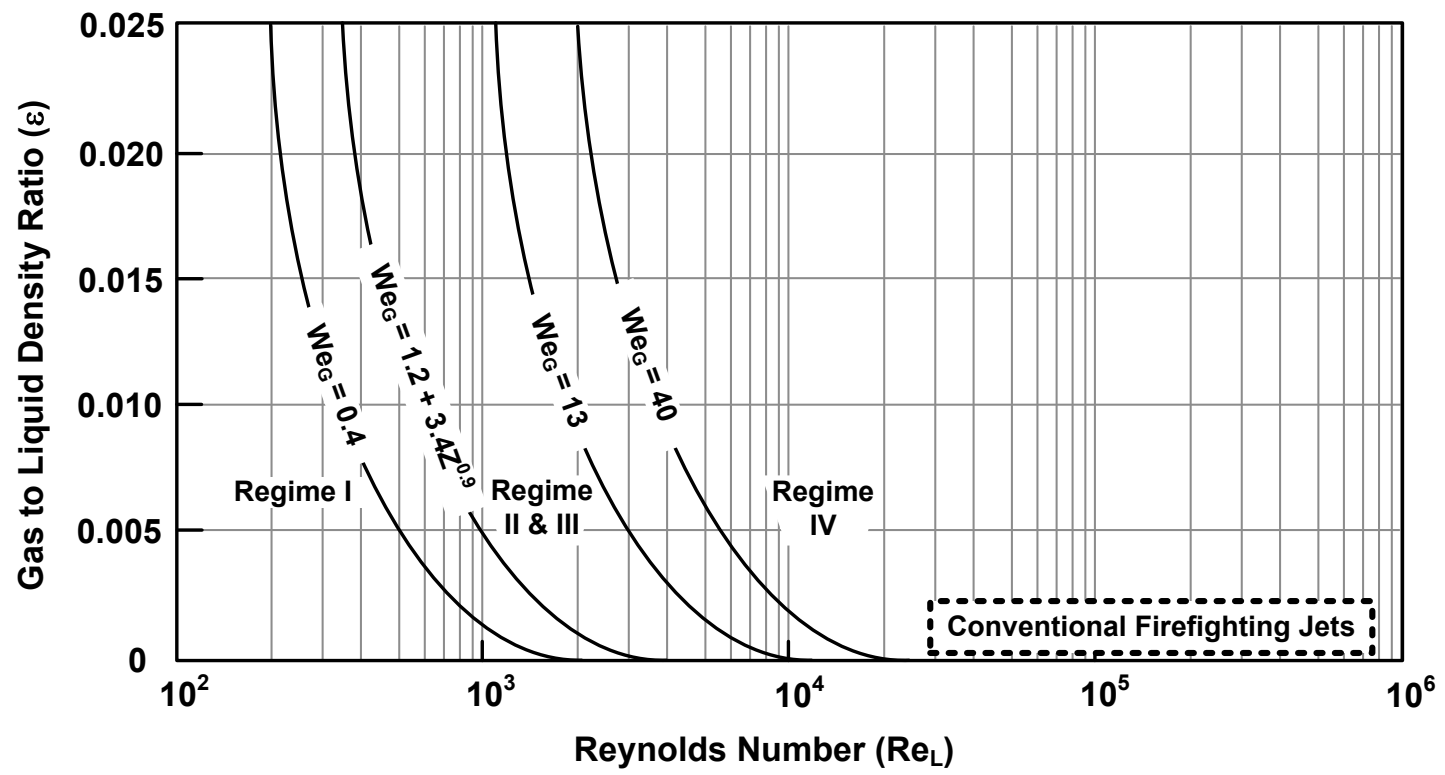

Figure 2.11: Liquid Jet Break-up Regimes Classification in Still Air with Respect to Weber No., Reynolds No., and the Gas to Liquid Density Ratio [36]

The liquid jet stability curve depicted in Figure 2.12 provides an alternate interpretation of the liquid jet break-up modes depicting liquid jet break-up length as a function of jet efflux (flow) velocity. Although qualitative trends of the liquid jet stability curve are well accepted, precise numerical bounds on the data remain a point of contention due to data collection inconsistencies among researchers. The conventional firefighting jet break-up regime in Figure 2.12 is estimated based upon values defined in Figures 2.10 and 2.11.

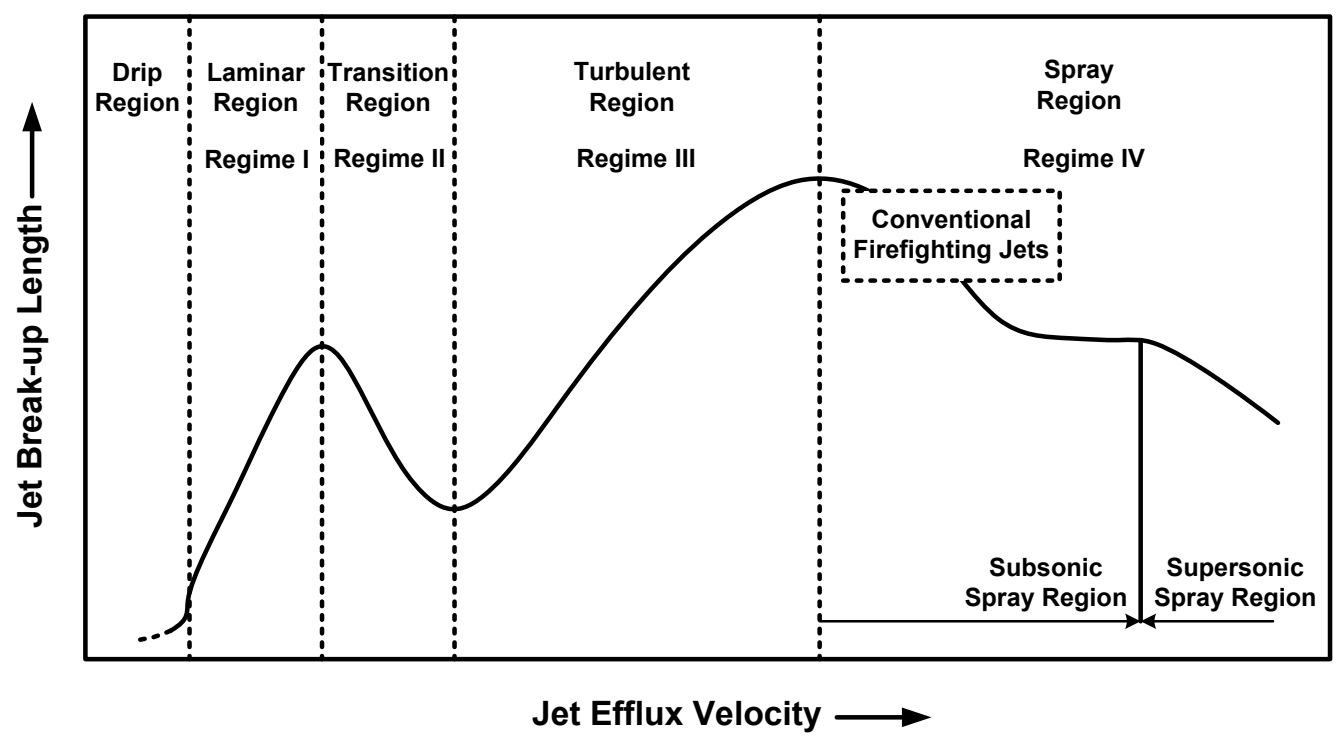

Figure 2.12: The Jet Stability Curve for Liquid Jets Entering a Gaseous Atmosphere [36] 
The liquid jet stability curve is broken down into five subsequent, interconnected regions with reference to the four liquid jet break-up modes already mentioned for comparison: the drip region, the laminar region, the transition region, the turbulent region, and the fully developed spray region. The laminar flow region is reflective of the Rayleigh break-up regime I depicting a constant increase in the liquid jet break-up length as velocity increases, and graphically representative of the classic linear instability analysis referred to earlier. The preceding drip region has a sporadic, inconsistent break-up length due to almost negligible flow velocity and is ignored for break-up mode classification. The transition region (also referred to as the wavy jet region) is representative of Ohnesorge's first wind-induced break-up regime II characterized by the production of surface transverse as opposed to symmetrical waves to dissipate energy more rapidly, but with no significant aerodynamic interactions present. The first influential non-linear interactions with the surrounding environment emerge here and overall break-up length begins to decrease with increasing velocity [36]. Grant proposed an empirical correlation for this regime based upon fully-developed parabolic profiles emanating from long tubes, expressed as:

$$
R e_{L}=3.25(O h)^{-0.28}
$$

which corresponds well with Equation 2.7, but only for liquid jets interacting with standard atmospheric conditions $[32,33,36]$. As inertial forces continue to increase with increasing jet velocity, transition to a fully turbulent flow causes shearing aerodynamic forces to dominate the liquid-stagnant air interface indicative of the second wind-induced break-up regime III. This restabilizes the flow field causing the liquid jet break-up length to increase again. The interaction of liquid jet turbulence and atmospheric effects play a paramount role in the turbulent break-up regime because ambient air properties like pressure and density can amplify or dampen surface perturbations and three-dimensional (3-D) turbulent structures resulting in drastically different break-up lengths and overall jet behavior. The physics in the fully developed subsonic spray regime are analogous to the atomization break-up regime IV. Liquid jet break-up in the supersonic spray regime introduces more complex non-linearities and is beyond the scope of inquiry for conventional firefighting jets and the present study [36].

Key elements known to significantly influence liquid jet break-up discharged into still air especially in the turbulent flow regime are summarized [34-37, 39, 42]: 
- Aerodynamic and hydrodynamic force interactions between the coherent liquid jet surface, expelled droplets, and ambient air interface

- Thermodynamic and physical states of both the liquid and ambient air

- Upstream delivery disturbances such as supply line pressure vibration and unsteady electromechanical disruption

- Nozzle internal flow characteristics like flow separation and cavitation

- Liquid jet velocity profile attributes at the nozzle exit

- Liquid jet vorticity, liquid turbulence, and ambient air turbulence magnitudes

\subsubsection{Secondary Break-up of Liquid Droplets in Still Air}

Because conventional firefighting jets are principally characterized by atomization via secondary break-up, further attention must be paid to the physical mechanisms responsible for satellite droplet disintegration due to interactions with the surrounding air. Based on Figure 2.10 and Figure 2.11, liquid jet disintegration is enhanced for higher Reynolds numbers $\left(\operatorname{Re}_{D}>10^{3}\right)$ and lower Ohnesorge number flows $(O h<1)$, or for when viscous effects are minimal. Five droplet break-up modes have been identified: vibrational, bag, multimode, sheet-thinning, and catastrophic. These break-up regimes are well defined via the gaseous Weber number based on droplet diameter $d$, defined as:

$$
W e_{d}=\frac{\rho_{G} V_{J}^{2} d}{\sigma_{L / G}}
$$

$W e_{d}$ loses its dependence on Ohnesorge number for conventional firefighting jet flow regimes, or more generally for liquid jets entering gaseous atmospheres that exhibit Ohnesorge numbers less than 0.1 [36]. The five droplet break-up modes are graphically depicted in Figure 2.13 with their respective gaseous Weber number ranges presented in Table 2.5. Each droplet break-up mode is described afterwards. 


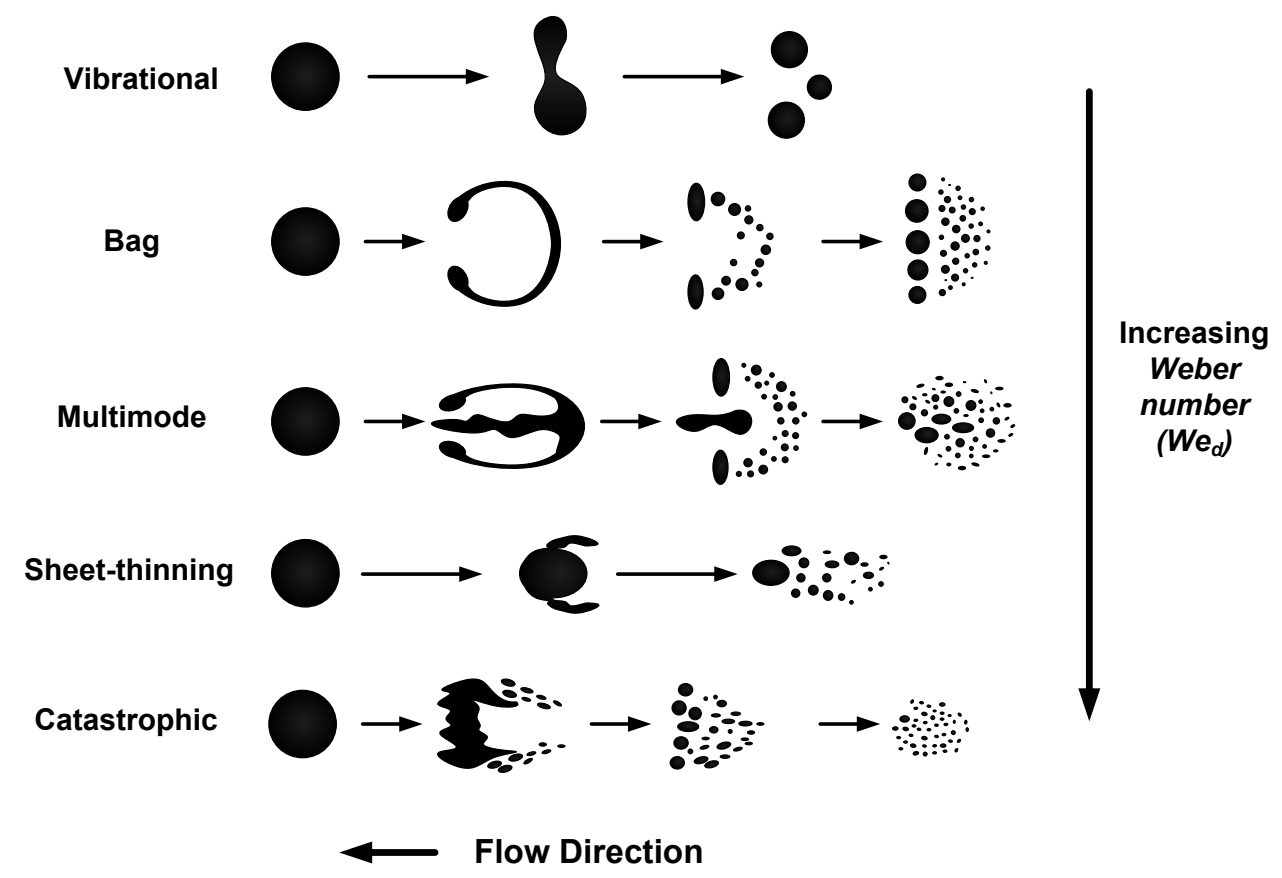

Figure 2.13: The Five Droplet Break-up Modes [46]

Table 2.5: The Five Droplet break-up Modes Based on Weber No. [46]

\begin{tabular}{|c|c|}
\hline Break-up Regime & We $\boldsymbol{e}_{\boldsymbol{d}}$ Range \\
\hline Vibrational & $W e_{d}<\sim 12$ \\
\hline Bag & $\sim 12<W e_{d}<\sim 35$ \\
\hline Multimode & $\sim 35<W e_{d}<\sim 80$ \\
\hline Sheet-thinning & $\sim 80<W e_{d}<\sim 350$ \\
\hline Catastrophic & $\sim 350>W e_{d}$ \\
\hline
\end{tabular}

1. The Vibrational Droplet Break-up Regime - The vibrational break-up regime is characterized by negligible aerodynamic stripping forces and droplet fragmentation due to destabilizing oscillations generated at the natural frequency of the parent droplet. Vibrational droplet deformation typically morphs into an oblate spheroid resulting in child droplets sized on the order of the parent droplet. This break-up regime requires longer break-up times compared to other break-up modes, and is often overlooked even though deformation originates here and significantly affects secondary break-up drag and trajectory.

2. The Bag Droplet Break-up Regime - The bag droplet break-up regime is best described in four stages where aerodynamic forces have become elevated: (1) initial deformation where spherical droplets become oblate spheroids, (2) bag growth where the drop center 
expands downstream from a ring opening into a balloon-like shape, (3) bag break-up where the bag ruptures into a large number of small droplets, and (4) ring break-up where finally the bag ring bursts into further satellite droplets forming several smaller fragments. Although the physical mechanisms that govern bag growth are still not well understood, most believe dynamics and overall break-up structure are guided by capillary instabilities as well as local air and liquid disturbances due to turbulence and particulate impurities.

3. The Multimode Droplet Break-up Regime - The multimode break-up regime, also known as the "bag-and-stamen" or "dual bag break-up" regime, is a transitional mode that exhibits characteristics of both bag and sheet-thinning droplet break-up due to further increased aerodynamic influence. This mode acts similar to bag break-up, except the central core is maintained further downstream resulting in the existence of a sustained internal plume where drops are continuously stripped reflecting sheet-thinning behavior.

4. The Sheet-thinning Droplet Break-up Regime - Sheet-thinning droplet break-up exhibits rapid shear droplet deformation due to a high relative velocity gradients induced between the droplet surface and air surrounding. Ligament stripping occurs along the droplet edge until the droplet has completely disintegrated. The physical processes responsible for this mode are generally disputed with accepted theories driving severe fragmentation based on "boundary layer stripping" or "shear-stripping" mechanisms observed along the liquid-air interface.

5. The Catastrophic Droplet Break-up Regime - Catastrophic droplet break-up is characteristic of low density liquid jets with droplets at extremely high relative velocities to that of the surrounding air. This mode is dominated by the growth of unstable surface waves along the liquid-air interface that eventually penetrate the droplet causing catastrophic shattering. Both Rayleigh-Taylor and Kelvin-Helmholtz instabilities are well represented in this break-up regime. Alternative break-up theories are offered based on shear-induced entrainment and boundary layer stripping mechanisms mentioned earlier, except droplet surface disturbances play a stronger role in determining wave propagation and final droplet fragmentation. 
Experimental efforts have shown that for liquid to ambient gas density ratios over 500 (e.g., firefighting jets), primary break-up is governed mainly by upstream liquid turbulent conditions and velocity profiles dictated by nozzle entrance and geometric conditions. Velocity profile relaxation, or the act of momentum transfer between layers normal to the direction of flow, imposes relative motion on the jet velocity profile as it expands from the confines of the nozzle. These redistributive forces are destabilizing, enhance mixing rates, and add to surface disturbances already present along the liquid-air interface. Thus, fully-developed uniform or plug profiles characteristic of turbulent jets are naturally more stable compared to laminar jets represented by more rounded, parabolic profiles. Secondary break-up is more dependent on the surrounding air turbulence and its aerodynamic interaction with individual liquid droplets $[32,33,36,43-45]$.

\subsubsection{Experimental Flow Characterization with Extension to Firefighting Jets}

Most modern experimental fire suppression research involves characterizing and enhancing water mist systems for indoor fire protection, which is beyond the scope of the present study. However, earlier work specific to firefighting jets was conducted that examined the delivery process of high flow, turbulent water jets. Few mathematical correlations exist for firefighting streams because large characteristic turbulent length scales are not accounted for in traditional break-up theories based upon surface tension effects. Aspects like nozzle discharge coefficient tuning, turbulent reduction methods, and trajectory analysis are the major emphases in this area of research, with more recent developments dedicated to investigating the role droplet size and distribution play. The average consensus from most researchers is that maximum jet throw distance is achieved by delivery mechanisms that sustain a uniform velocity profile, remove swirl, and reduce turbulence at the nozzle entrance [47]. Although flow characterization of chemical additives to alter liquid jet break-up behavior has been well-documented from a variety of industries, little information is available regarding the application of foaming surfactant additives like AFFF.

The study of firefighting jets has been an on-going effort since the late 1800s with Freeman's pioneering work analyzing the hydraulics of firefighting agent delivery systems, focusing primarily on the optimization of nozzle discharge coefficients [48]. Freeman's investigation into the physical fundamentals of firefighting streams not only helped give birth to the fire research 
community, but served as a keystone reference for decades to come. Rouse, Howe, and Metzler examined the role of turbulence nearly 70 years later through a comprehensive evaluation of high-flow water jets for naval firefighting [49]. The work of Rouse et al. provided the first detailed description of turbulent flow structures within firefighting jets by analyzing their negative impact on agent delivery efficacy in three major areas: (1) a reduction in internal flow efficiency as the agent navigates the delivery system and nozzle geometry, (2) the transverse growth of turbulent eddies interacting with the air orthogonal to the firefighting jet's trajectory, and (3) entrainment of the surrounding atmosphere forming a surge of air in the direction of the flame front. Rouse et al.'s work elevated the current state of the art in nozzle design by applying turbulent flow fundamentals to address practical firefighting suppression needs. Murakami and Katayama also examined losses in firefighting jets due to turbulence, providing a detailed review of discharge coefficient theory studying performance affecting factors like nozzle geometry, surface roughness, and flow straighteners [50].

Rouse et al.'s work included the construction of an indoor $100 \times 10 \times 14$ foot test facility where flow visualization and jet concentration measurements were conducted catching the agent normal to the oncoming flow along with full-scale trajectory experiments. Firefighting jet disintegration was studied indoors using high speed photography in conjunction with a specialized profilometer utilizing a high intensity horizontal beam of light. Figure 2.14 depicts the experimental gallery outlining the relative location of the pumping system, monitor station, and spray capture zone. Figure 2.15a shows a sample firefighting jet concentration plot, and Figure 2.15b illustrates the sampler apparatus used to record the measurements. A typical concentration plot consisted of over 900 data acquisition points [49]. 

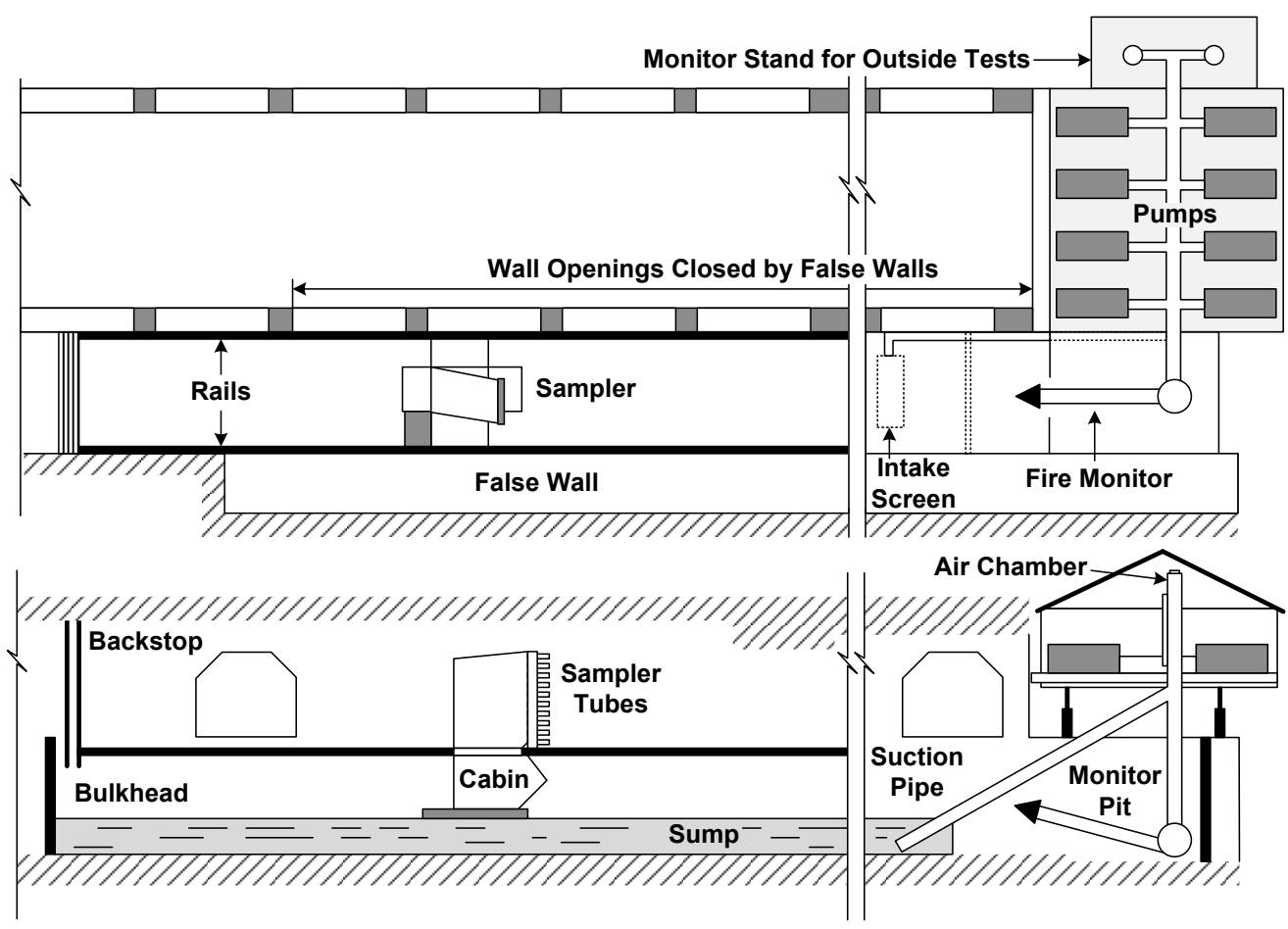

Figure 2.14: The Indoor Firefighting Jet Test Facility Layout [49]

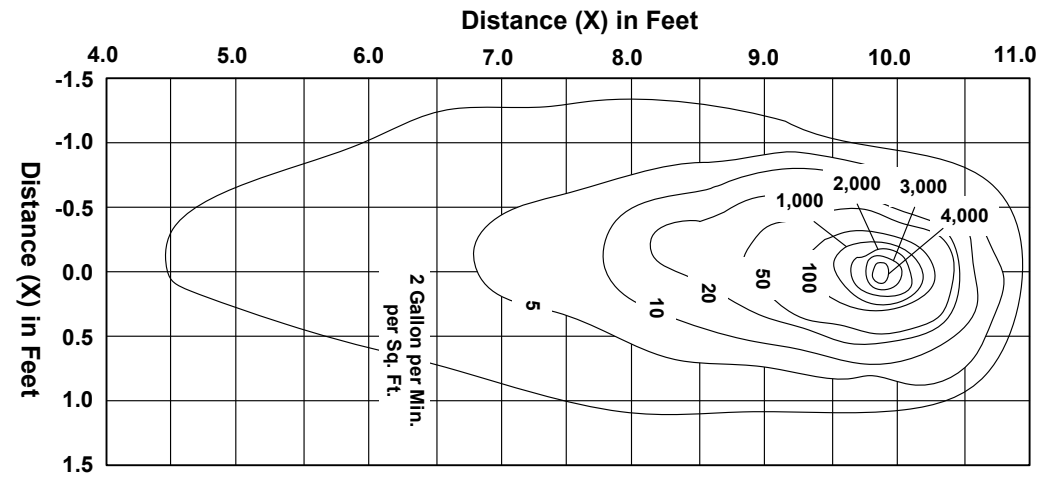

(a)

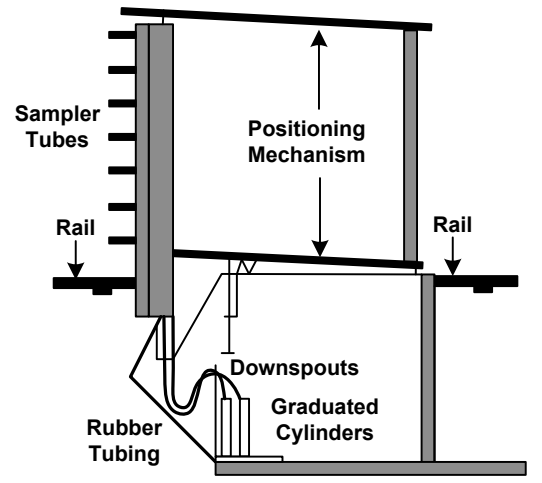

(b)

Figure 2.15: (a) A Sample Firefighting Jet Concentration Plot $27 \mathrm{~m}$ from a $30^{\circ}$ Inclined Nozzle (b) The Sampler Tube Apparatus Used to Record the Firefighting Jet Concentration Plot [49]

Testing higher flow rate firefighting jets required an exterior target stand designed similar in stature to the sampler apparatus, but instead having a general open capture area on the order of $10 \mathrm{~m}^{2}\left(100 \mathrm{ft}^{2}\right)$. Exterior trajectory measurements proved quite challenging with favorable atmospheric conditions present about 1 percent of the time [49]. Analogous water jet capture efforts were conducted later by Theobald to optimize nozzle design in terms of maximizing agent delivery quantities at particular downstream locations [51]. Figures 2.16 and 2.17 illustrate Theobald's methodology. 


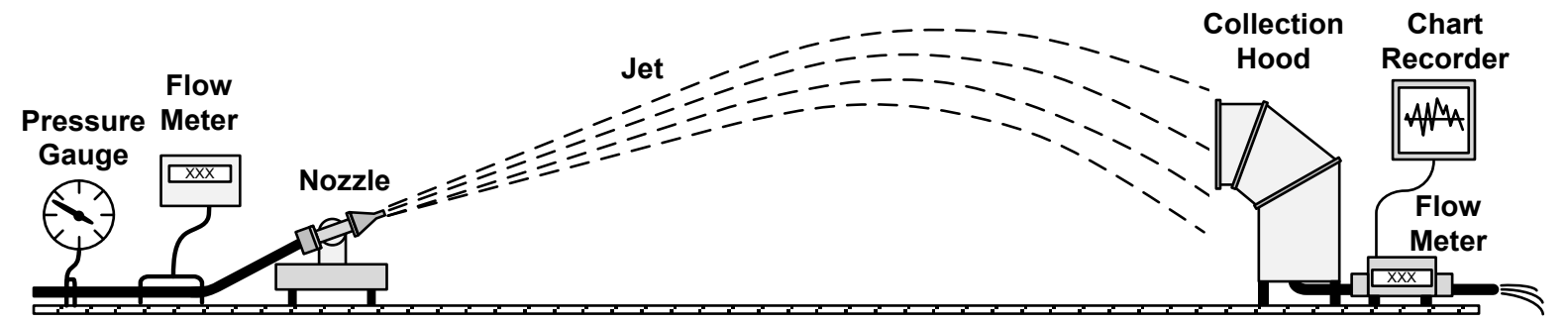

Figure 2.16: A Schematic of Theobald's Test Configuration for Measuring Downstream Nozzle Delivery Efficiency [51]

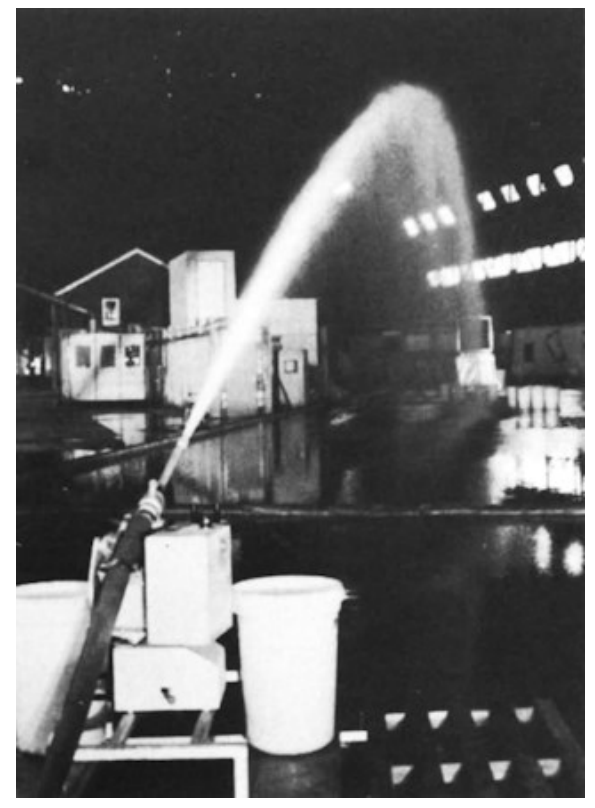

(a)

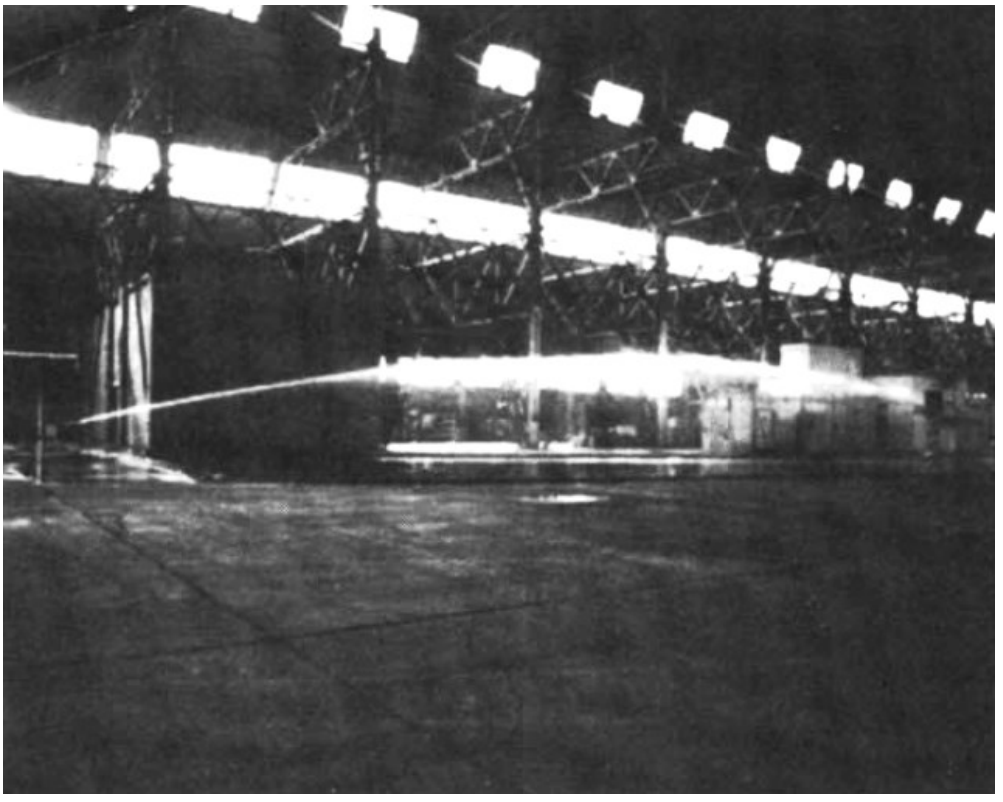

(b)

Figure 2.17: Photographs of Theobald's Test Configuration for Measuring Downstream Nozzle Delivery Efficiency (a) Axial View (b) Horizontal View [51]

In a separate study, Theobald determined the range and distribution of firefighting jets using what he referred to as a "continuous bucket" approach to analyze agent accumulation as a function of pressures ranging from approximately 200 to $400 \mathrm{kPa}$ (2 to 4 bar). Figure 2.18 depicts results from his analysis showing as pressure increases, accumulation disperses over a wider area, while the deliver area increases. 


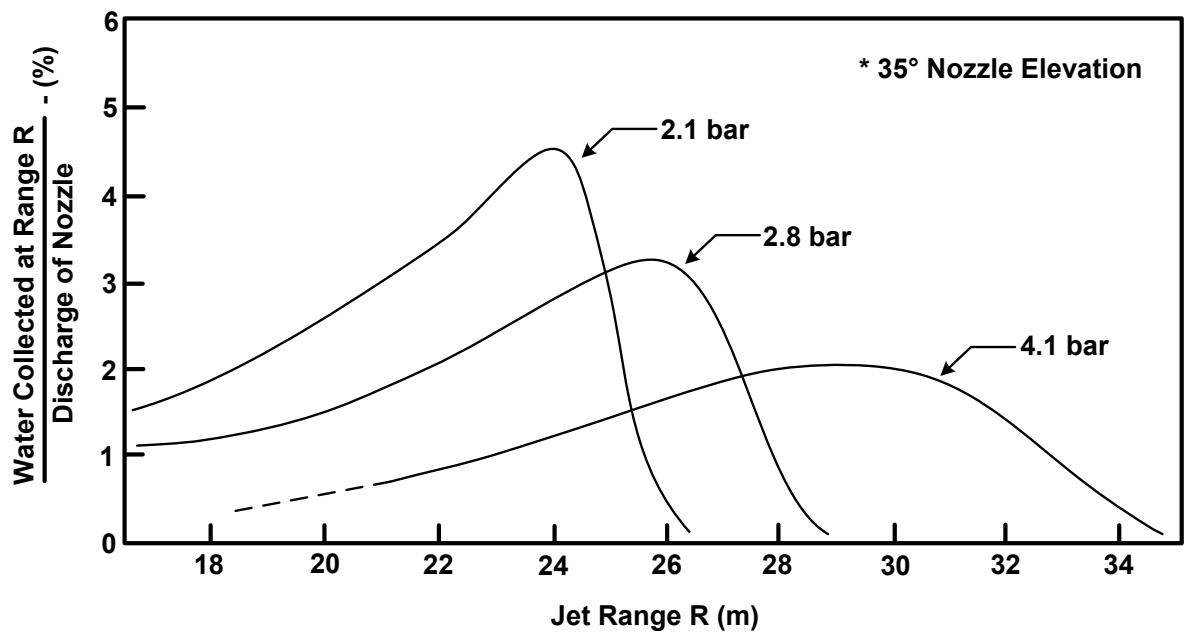

Figure 2.18: Theobald's “Continuous Bucket” Results Depicting Dispersion Variation with Nozzle Pressure [51]

Geyer conducted one of the first known studies on AFFF characterization to determine its firefighting compatibility with protein foams and prospective value to aviation fire suppression. The scope of his work involved quantifying full-scale fire suppression, fire containment, and foam characteristics from various AFFF manufacturers. Geyer measured foam quality among other bulk fluid properties, fire suppression effectiveness, as well as provided full ground pattern estimates for different nozzle designs. His work also outlined the first working guidelines for AFFF [5].

Multiple authors have utilized various methods to quantify turbulent liquid jet break-up and better define the stability curve, particularly for firefighting jet applications. The most common experimental techniques are based upon monitoring the change of electrical resistance of a conductive substrate placed normal to the oncoming firefighting jet at various axial locations downstream of the nozzle, a practice applied by Phinney, Theobald, and Hiroyasu [35, 42, 52]. Figure 23a illustrates Theobald's measurement concept, and Figure 23b depicts the firefighting jet stability's sensitivity to nozzle design. Theobald's work developing firefighting jet stability curves for a variety of firefighting nozzles proved considerably sensitive to nozzle geometry reaffirming conclusions presented earlier [52]. 


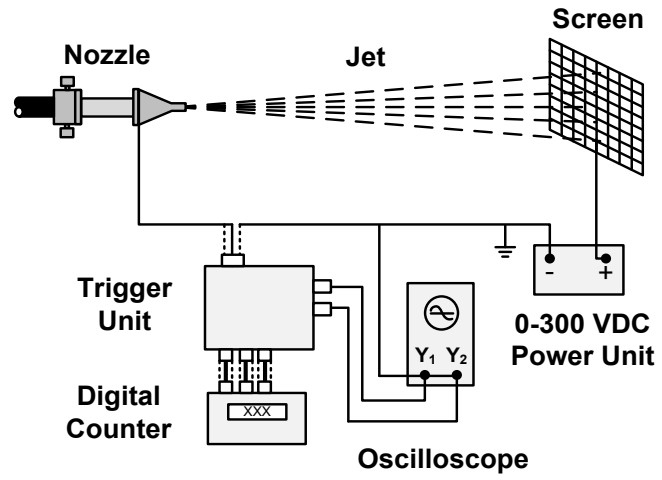

(a)

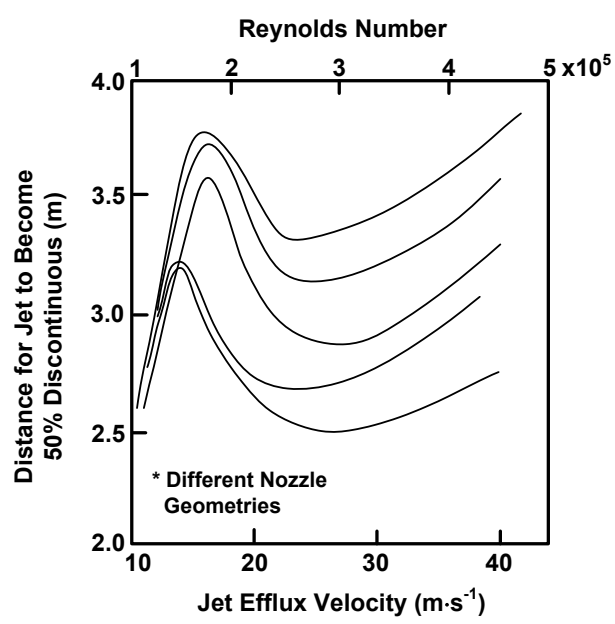

(b)

Figure 2.19: (a) A Schematic of the Firefighting Jet Continuity Measurement Apparatus. (b) A Plot Illustrating the Dependence of Firefighting Jet Stability on Nozzle Design [52]

Theobald also formed regressions correlating factors like maximum firefighting jet height and range based upon nozzle pressure and nozzle diameter, concluding primary break-up and trajectory could be approximated by the projectile equations of motion up to apogee (or maximum height) of the firefighting jet. Once secondary break-up occurs due to increased aerodynamic effects, range becomes a stronger function of droplet size [52].

\subsubsection{Computational Modeling Methods with Extension to Firefighting Jets}

Little CFD work has been conducted specific to firefighting jets. Most modeling progress in the field of turbulent liquid jets entering still air has been with respect to fuel spray model development to enhance internal combustion engine performance. Computational firefighting jet research has its roots in the late 1970s when Hatton and Osborne developed a numerical technique to predict water jet trajectories exposed to various operating conditions such as efflux velocity, flow rate, nozzle elevation, along with head and tail wind effects. They solved seven simultaneous projectile equations of motion explicitly, and simulations were validated with experimental data from Rouse et al. Results affirmed that for very large firefighting jet streams operating at moderately low nozzle pressures, the flow path can be adequately modeled with first order accuracy [47, 52]. Although Hatton and Osborne did not report results for higher nozzle pressures, it is speculated this approach would be less accurate and loses applicability because of secondary break-up domination less representative of slug or "projectile-like" jet flow. Figure 2.20 illustrates example results from Hatton and Osborne's work. 


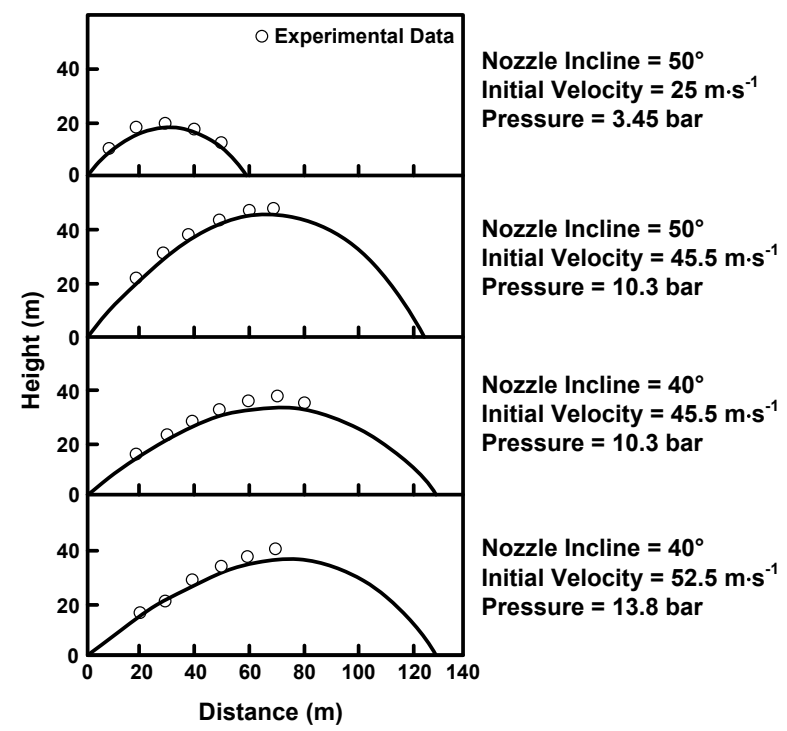

(a)

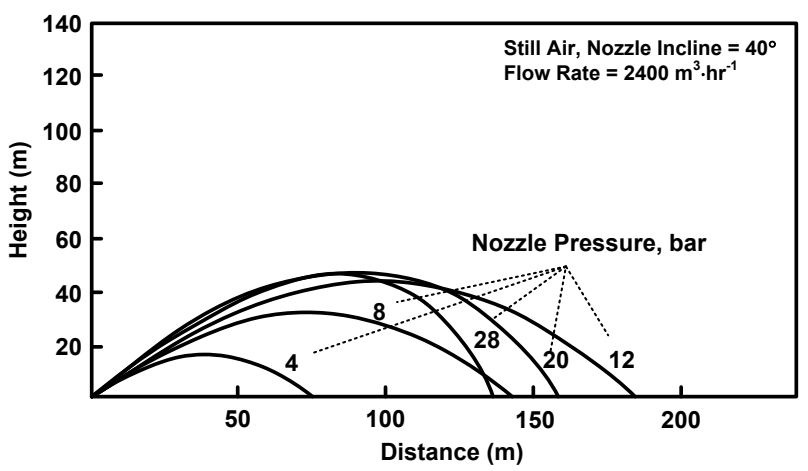

(b)

Figure 2.20: Numerical Solutions of Large-scale Firefighting Jet Trajectories [47]

Modern CFD methods have approached the challenge of predicting liquid jet break-up using a wide range of sophisticated numerical techniques. Several extensive reviews on computational gas-liquid two-phase jet flows have been conducted including works by Jiang et al., Gorokhovski and Herrmann, and most recently Archambault in 2010 [53-55]. Most CFD multiphase jet modeling flow classifications fall into one of three main categories: stochastic, Euler-Euler, or Euler-Lagrange. Stochastic or probabilistic modeling methods are often based on a probability density function that describes the indiscriminate evolution of a droplet property interacting with the gaseous phase capable of gathering a wide range of statistics at every point in the flow. They are often advantageous for modeling highly atomized, dispersed liquid jet flows that result in a very random motion [56].

Euler-Euler or dual-continuum techniques govern droplet transport by a set of conservation equations assigned to each phase whereby coupling is achieved through each phase's respective source terms [56]. Dual-continuum techniques are generally not well-suited for modeling firefighting jets because the discrete droplet phase is treated as a continuous medium not indicative of the firefighting jet atomization flow regime classification noted earlier. However, application may exist for very high flow, low pressure firefighting jets where local droplet number densities are high enough such that most of the liquid jet core stays intact so that it can be assumed continuous with negligible dependence on secondary break-up. Volume of fluid 
(VOF) modeling is a popular industrial Euler-Euler technique suitable for this type of modeling. However, VOF modeling is highly reliant on the mesh resolution interface between the two fluid mediums and not computationally efficient for modeling large-scale firefighting jets that are dominated by secondary droplet break-up mechanisms [56]. Figure 2.21 depicts a high-fidelity direct numerical simulation (DNS) using VOF modeling techniques conducted by Menard, Tanguy, and Berlement. The figure shows the details of a 3-D turbulent liquid jet atomization process including a close-up of primary ligament and droplet break-up [57].
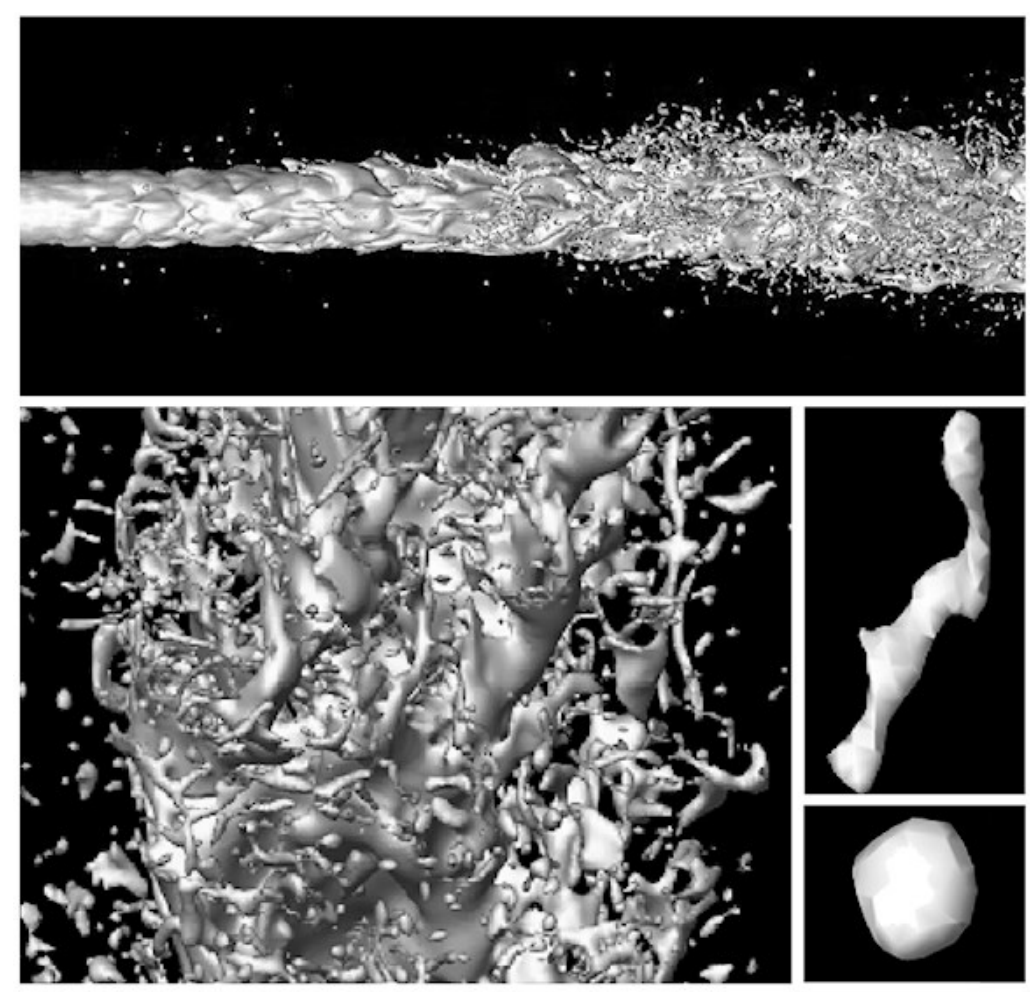

Figure 2.21: A 3-D Model of Liquid Jet Surface and Break-up Near the Nozzle using VOF Techniques [57]

Euler-Lagrange methods describe liquid jets by injecting discrete droplets into the continuous gas phase. Droplet time-accurate trajectories are calculated by numerically integrating the Lagrangian equations of motion. Reynolds-Averaged Navier-Stokes modeling is often unwisely chosen to govern the gas phase due to a conflicting time-accurate versus time-averaged description of the interacting discrete and continuous phases, respectively. However, its implementation is often the rule rather than the exception due to computational resource limitations. A more appropriate physical treatment of the Eulerian phase is to use an inherently unsteady method like large eddy simulation (LES) or DNS to match the unsteady nature of the 
Lagrangian phase. For most time and length scales associated with industrial engineering problems, DNS computational costs are too prohibitive. Thus, LES methods with well-described spatial filters provide a workable compromise. The common limiting factor with Euler-Lagrange techniques is the number of droplet trajectories required to resolve a typical firefighting jet especially for large length scales indicative of real world firefighting agent delivery systems. To reduce computational overhead, success has been shown grouping droplets into parcels, each of which are tracked separately. However, accurate coupling of the interphase exchange terms and sub-modeling of the discrete phase interaction with itself can be critical depending on the dominating flow physics within the problem [55]. It should be noted that several hybrid methods of the three aforementioned classes exist whereby detailed exchange must occur between primary and secondary break-up mechanisms. Most of these methods are still relatively novel and have yet to make their way into commercial codes for interaction with other sub-modeling methods such as combustion modeling [56]. Figure 2.22 visualizes the evolution of a high velocity turbulent liquid jet injected into still air using the Euler-Lagrange approach from a simulation conducted by Shi and Kleinstreuer. The figure shows the change in overall liquid jet shape and droplet diameter over time [58].

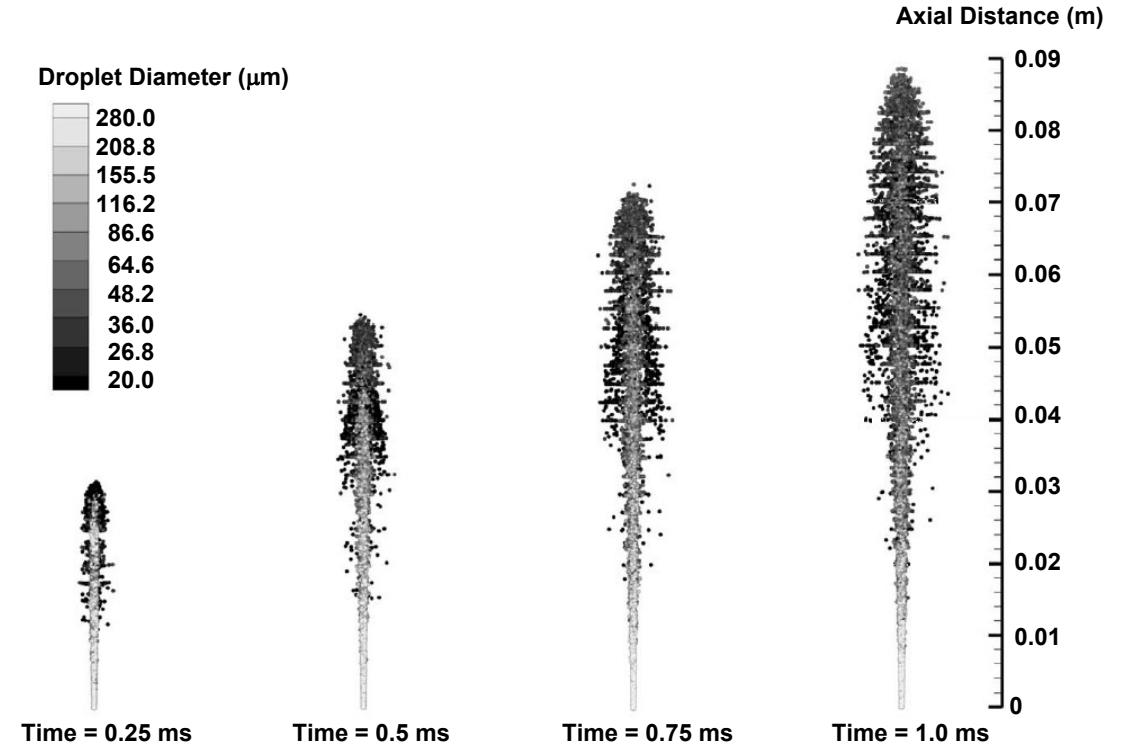

Figure 2.22: Time-Evolved CFD Results from an Euler-Lagrange Liquid Jet Flow Model [58]

Similar to experimental research, little work exists on the general application of CFD modeling of large-scale water jets, particularly those designed for fire suppression or interaction with other combustion sub-modeling techniques. Most work in this area involves modeling sprinkler sprays 
for the use inside of residential buildings to quantify aspects such as nozzle discharge coefficients, water mist spray optimization, and to address the general problem of predicting the amount of fire suppression agent required to suppress a unique indoor fire threat. The majority of these approaches apply Euler-Lagrange techniques in commercial CFD packages referred to as either discrete phase modeling (DPM) or Particle Tracking Modeling (PTM) methods [59-63]. High-fidelity fire suppression applications used to model firefighting jet interactions with larger, outdoor fire environments have been successfully implemented by DesJardin and Gritzo on the SANDIA in-house CFD code named VULCAN for simulating cooling jet interaction with LES fires [64]. 


\section{CHAPTER 3: AQUEOUS FIREFIGHTING AGENT APPLICATION LABORATORY}

\subsection{Overview}

In order to experimentally characterize firefighting jets, a flow parameter envelope was selected to accommodate practical, small-scale experimental conditions while not significantly sacrificing relational validity to a full-scale firefighting jet environment. To accomplish this task, an indoor test facility was constructed to maximize firefighting jet dimension while eliminating outdoor atmospheric influences. A $25 \times 9-\mathrm{m}(80 \times 30-\mathrm{ft})$ mezzanine storage space in the West Virginia University (WVU) Hangar at the Morgantown, WV Municipal Airport (Hart Field) was converted into an aqueous firefighting agent application laboratory for the experimental phase of this study. Figure 3.1 depicts most of the major laboratory features.

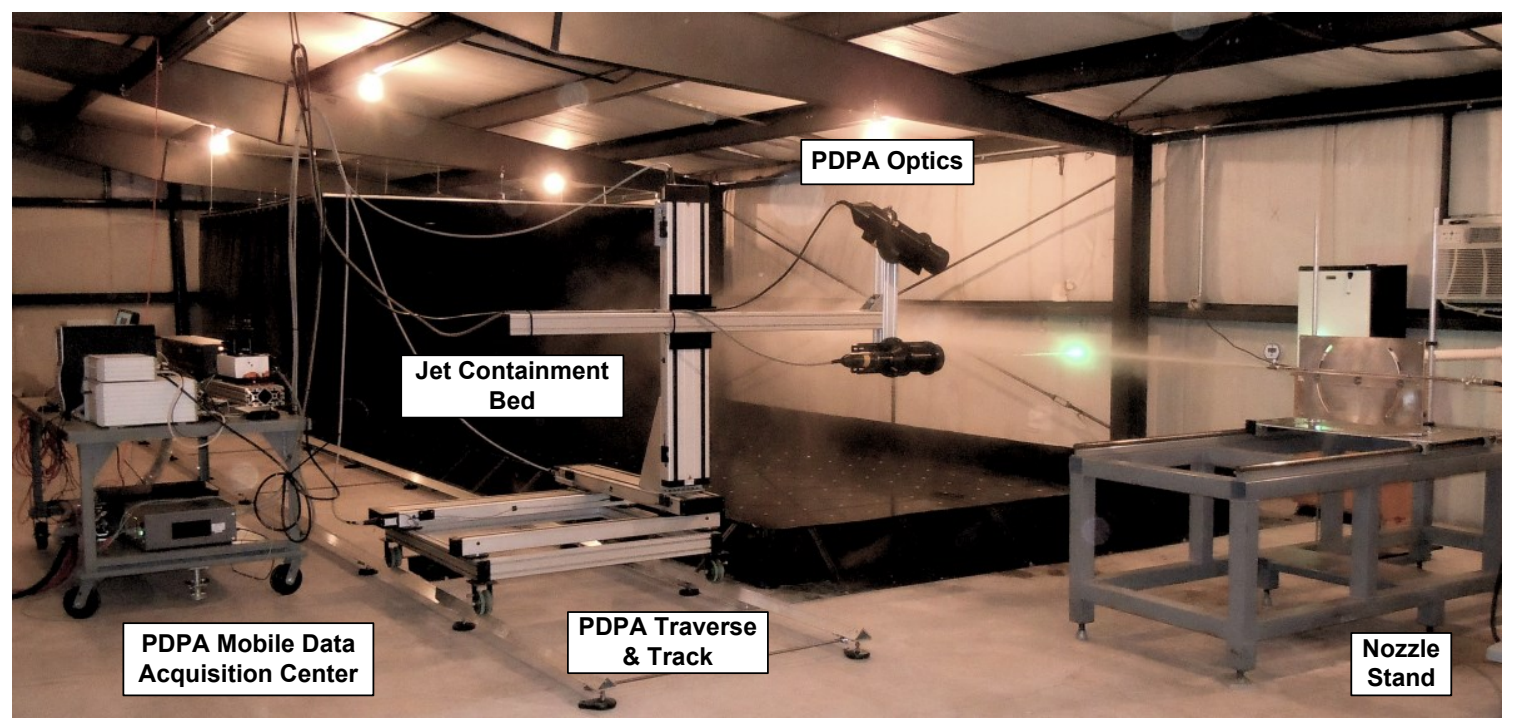

Figure 3.1: Key Components of the Aqueous Firefighting Agent Application Laboratory

Besides essential features required for wet laboratory operation, a custom firefighting agent delivery system, nozzle apparatus, firefighting jet containment bed, backdrop curtain for flow visualization, and mobile PDPA were key additions to the transformation. All components were custom fabricated unless otherwise noted. To manage spray drift, dilute AFFF droplet emissions, and help maintain a constant relative humidity, a $221-\mathrm{m}^{3} \cdot \mathrm{min}^{-1}\left(7,800-\mathrm{ft}^{3} \cdot \mathrm{min}^{-1}\right)$ exhaust fan was installed to exchange laboratory air about every $3.5 \mathrm{~min}$. A 51/34-MJ (48,000/32,000-BTU) heating and cooling system was added to air condition the space. Standard $110-\mathrm{V}$ electrical service was also installed throughout the laboratory along with 208 and $480 \mathrm{~V}$ 
to power the air conditioning units, 5-Watt PDPA laser, and firefighting jet delivery system. Figure 3.2 shows a schematic of the laboratory depicting the overall layout in addition to all critical components. Figure 3.3 depicts key laboratory dimensions in addition to the origin and orientation of the coordinate system employed throughout this effort.

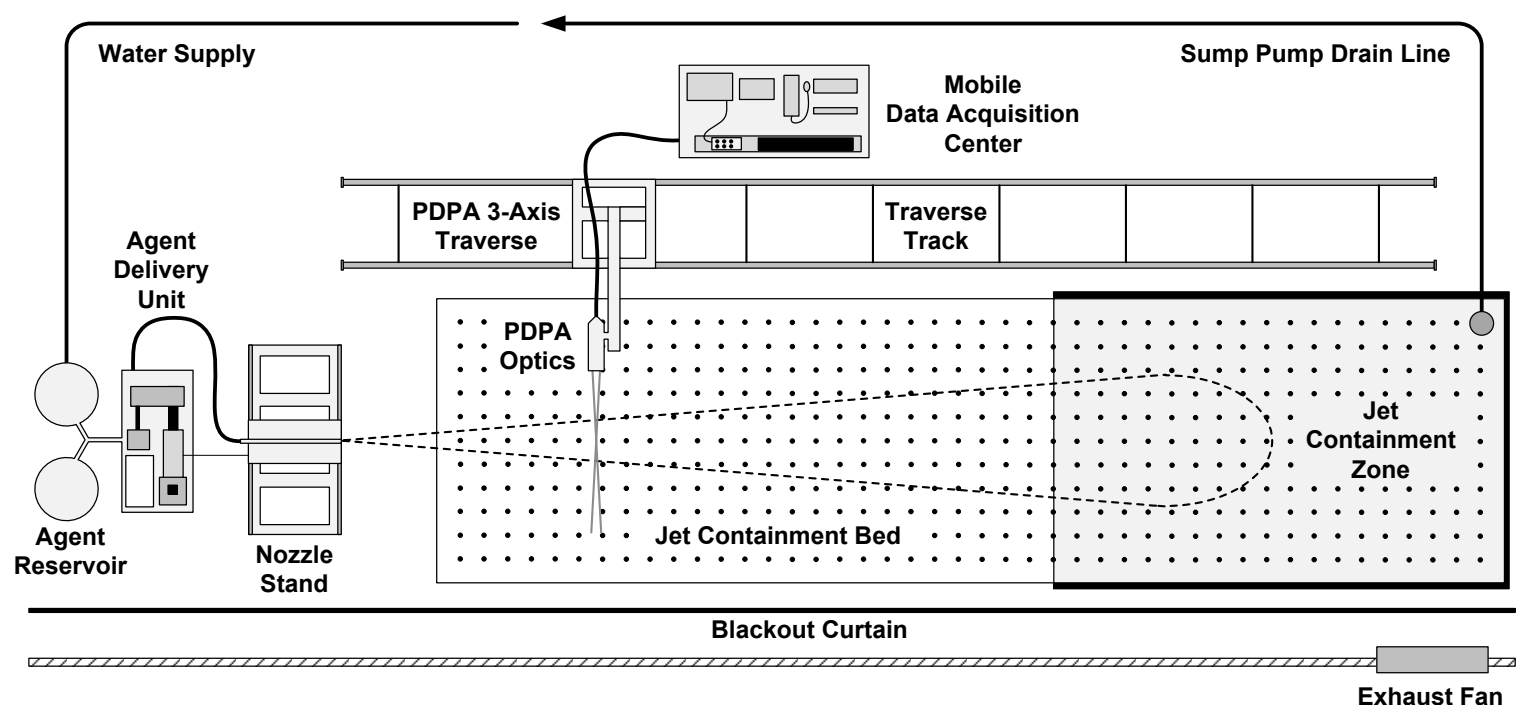

Figure 3.2: Key Components of the Aqueous Firefighting Agent Application Laboratory
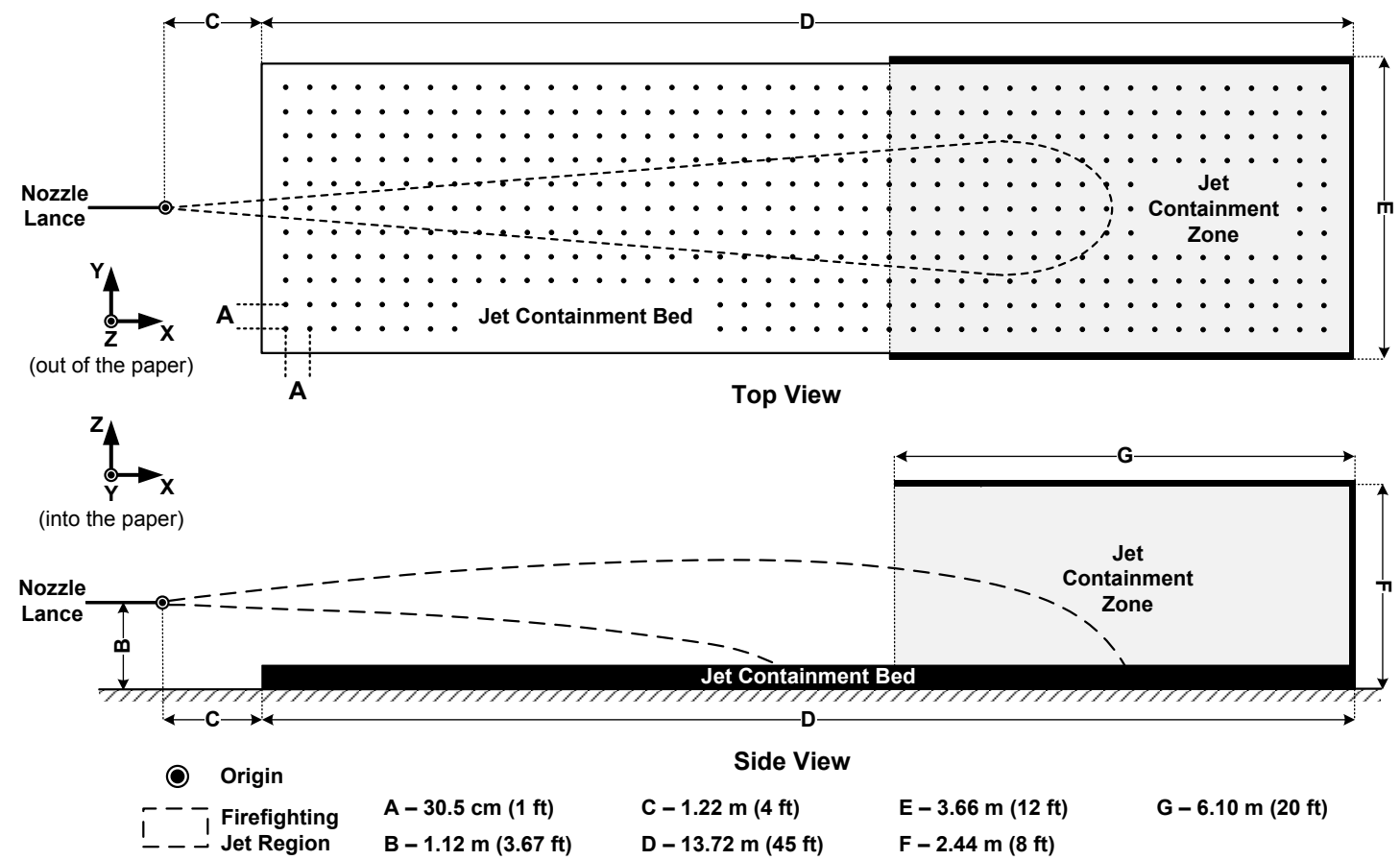

Figure 3.3: The Aqueous Firefighting Agent Application Laboratory Identifying Key Dimensions 


\subsection{Firefighting Agent Delivery System}

The firefighting agent delivery system was designed and fabricated by AFRL personnel. The firefighting jet was generated using a small-scale variable speed agent delivery system capable of dispensing approximately 4 to $251 \cdot \mathrm{min}^{-1}\left(1\right.$ to $\left.6 \mathrm{gal} \cdot \mathrm{min}^{-1}\right)$ of agent from 0 to $21 \mathrm{MPa}$ (0 to $3000 \mathrm{lbf} \cdot \mathrm{in}^{-2}$ ). Due to laboratory electrical power constraints, nozzle pressures were limited to half the aforementioned range at maximum flow rate. Maximum axial throw and horizontal span based on conventional nozzles of about 15 and $3 \mathrm{~m}$ (50 and $10 \mathrm{ft}$ ), respectively, were achievable within the bounds of the test facility. The system was powered by a reciprocating piston pump directly driven through a $1.5: 1$ gear ratio by a $480-\mathrm{V}$ AC $3.7-\mathrm{kW}$ (5-hp) electric motor to maintain highly repeatable nozzle entrance conditions. A digital interface provided manual or automatic control through variable frequency regulation while reporting current draw, voltage, and motor speed in revolutions per minute (RPM). Figure 3.4 illustrates the torque and power curves as a function of shaft motor speed for the electric motor. Because available power beyond the motor speed midpoint range began to decay with increasing speed, nozzle performance envelopes were non-uniform and limited in high flow, high pressure regimes requiring maximum power. Peak performance was also a function of other variables like plumbing configuration, pump resistance due to temperature-dependent lubricating oil, and thermal losses incurred by the electric motor during operation.

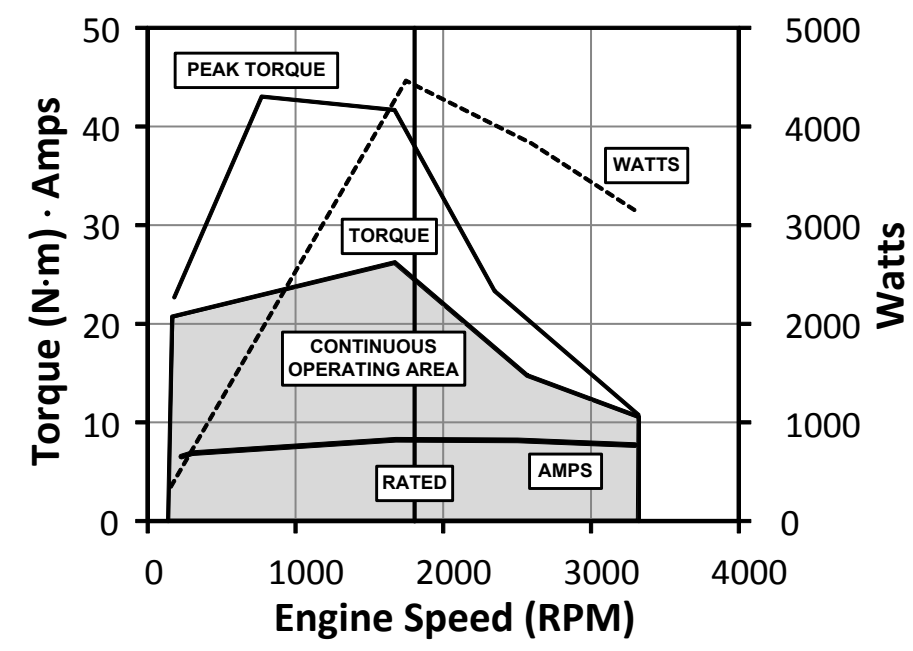

Figure 3.4: Firefighting Agent Delivery System Electric Motor Performance [65] 
The firefighting agent delivery system was fitted with a supplemental agent reservoir designed to provide 757-1 (200-gal) capacity for sustained periods of low flow rate testing voiding the use of an original 132-1 (35-gal) onboard tank. Agent was fed individually or simultaneously from two separate 379-1 (100-gal) tanks through a wye valve connected to a small centrifugal pump priming the main reciprocating pump. A 3.79 to $37.9 \pm 0.76-1 \cdot \mathrm{min}^{-1}$ ( 1 to $10 \pm 0.2$-gal $\cdot \mathrm{min}^{-1}$ ) turbine flow meter with digital display was installed between the two pumps to report agent flow rate. For the present work, one tank exclusively held tap water and the other tank held premixed AFFF to reduce firefighting jet contamination from leftover residue. An accumulator was installed downstream of the main pump to dampen high frequency acoustics due to periodic pulsations induced by pump operation. A 6-m (20-ft) high-pressure rubber hose delivered agent to a 1-m (3-ft) long, 0.25 -in diameter pipe. The 1-m entrance pipe was followed by a pipe cross to accommodate static pressure measurements using a 0 to $21 \pm 0.05-\mathrm{MPa}\left(0\right.$ to $3000 \pm 7.5-1 \mathrm{bf} \cdot \mathrm{in}^{-2}$ ) digital display pressure gauge and 0 to $14 \pm 0.06$-MPa ( 0 to $\left.2000 \pm 8-\mathrm{lbf} \cdot \mathrm{in}^{-2}\right)$ pressure transducer for data acquisition. A multipurpose high speed USB dual data acquisition and analog control module capable of recording and function generation at a rate up to one million samples $\cdot \mathrm{s}^{-1}$ was used to capture the signal output from the pressure transducer. A low pressure loss Swagelok ${ }^{\circledR}$ fitting immediately downstream of the pipe cross allowed for easy nozzle exchange. Although the nozzle entrance length was not perfectly conditioned to achieve fully-developed flow according to academic standards because of the pipe cross fitting, the firefighting agent delivery system was typical of fire suppression field units exhibiting flow discontinuities typically all the way up to the nozzle entrance. This is generally due to design compromises based on competing factors like multiple delivery technologies feeding a single nozzle or configuration conflicts with other platform subsystems, especially with respect to deployable fire suppression systems where weight and space are at a premium.

A ball valve installed on the low pressure side of the reciprocating pump controlled recirculation back to the AFFF tank to unload system pressure. It also provided flow logic to premix the AFFF before testing. A pressure relief valve pre-set to $24 \mathrm{MPa}\left(3500 \mathrm{lbf} \cdot \mathrm{in}^{-2}\right)$ was installed for safety, and an analog 0 to $35 \pm 0.34-\mathrm{MPa}$ ( 0 to $5000 \pm 50-\mathrm{lbf}^{-\mathrm{in}^{-2}}$ ) Bourdon-style gauge was stationed on the high pressure side of the reciprocating pump to monitor pump exit pressure. All plumbing hardware was made of high grade stainless steel to withstand high pressure agent flows 
and to avoid corrosion. All low pressure hardware and agent storage tanks were forged from polypropylene derivatives to also resist corrosion. Figure $3.5 \mathrm{a}$ depicts the firefighting agent delivery system highlighting the major features discussed, and Figure 3.5b shows the system accompanied by the agent reservoir addition. Figure 3.6 illustrates the firefighting agent delivery system's flow control schematic.

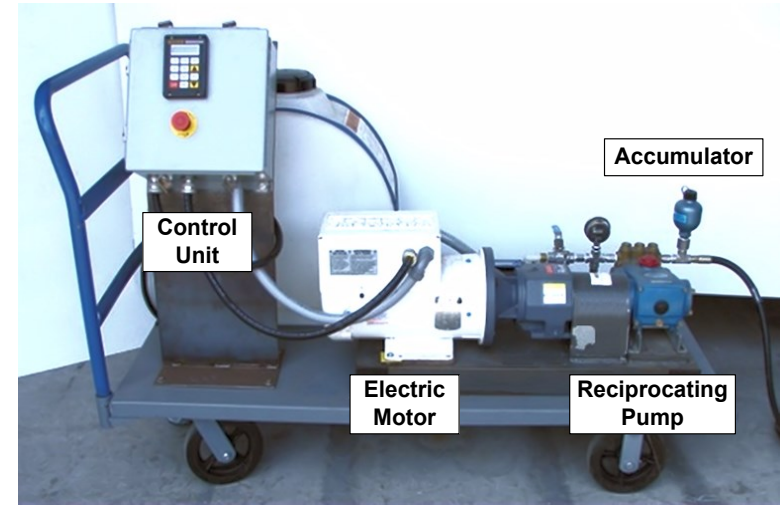

(a)

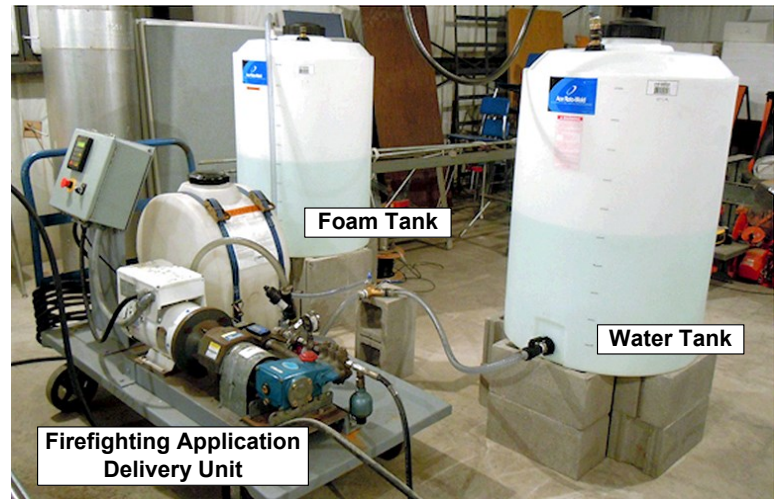

(b)

Figure 3.5: The Firefighting Agent Delivery System

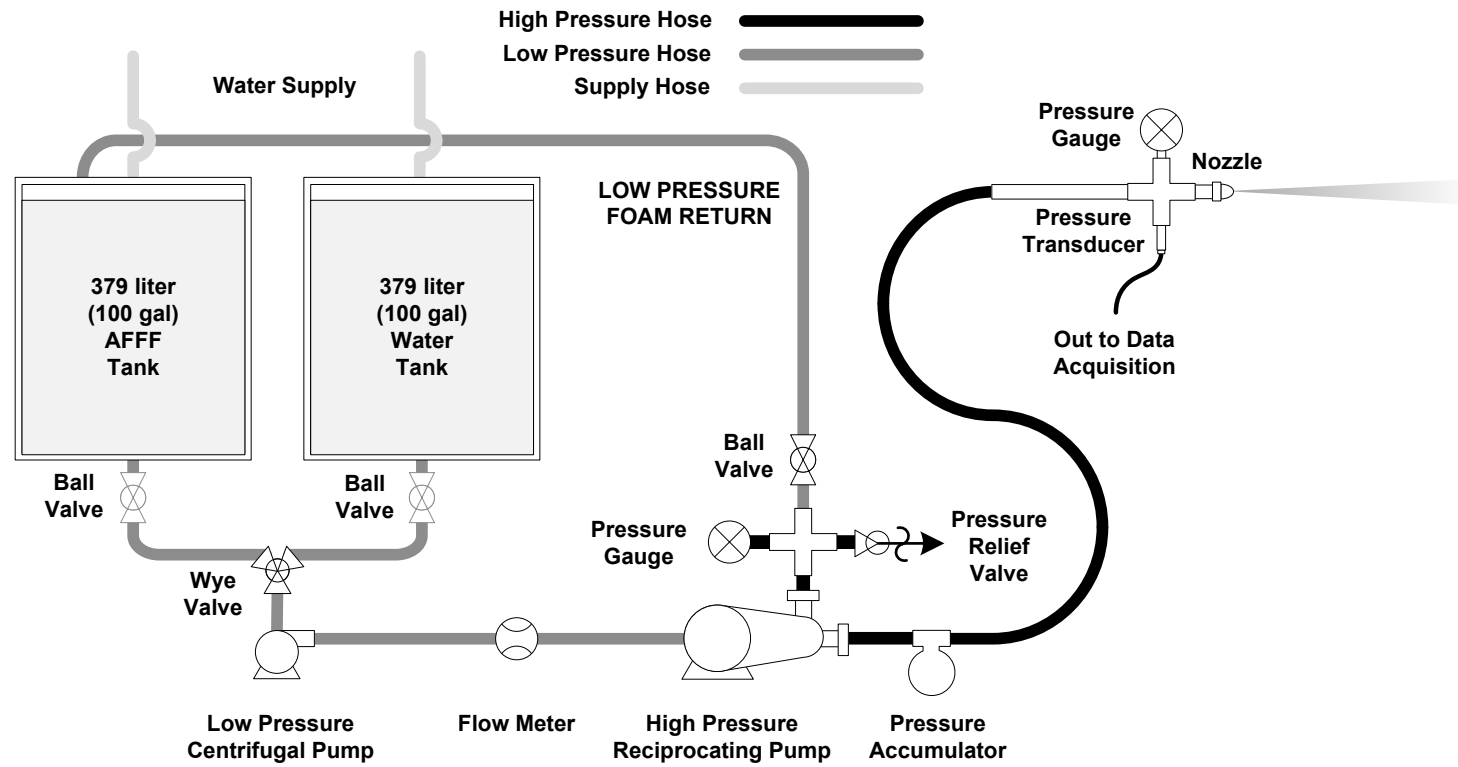

Figure 3.6: The Firefighting Agent Delivery Flow Control Schematic

The nozzle stand consisted of a $2.4 \times 1.2 \times 0.9-\mathrm{m}(8 \times 4 \times 3-\mathrm{ft})$ linear rail table which allowed the nozzle to be swept horizontally parallel with the firefighting jet containment bed. The nozzle lance (1-m long entrance pipe) was supported by a rectangular platform resting on four linear bearing pillow blocks riding the table rails. The platform hosted two masts, with the forward one 
fixed and the rear one free to rotate for fine adjustment in the transverse plane. The nozzle lance rested directly on a sub-platform attached to a vertical plate supported by the two masts allowing for rotation and fine adjustment in the vertical plane. Nozzle platform adjustment along with the linear motion table provided three degrees of freedom for nozzle positioning. Figure 3.7 illustrates various aspects of the nozzle stand and surrounding accessories.

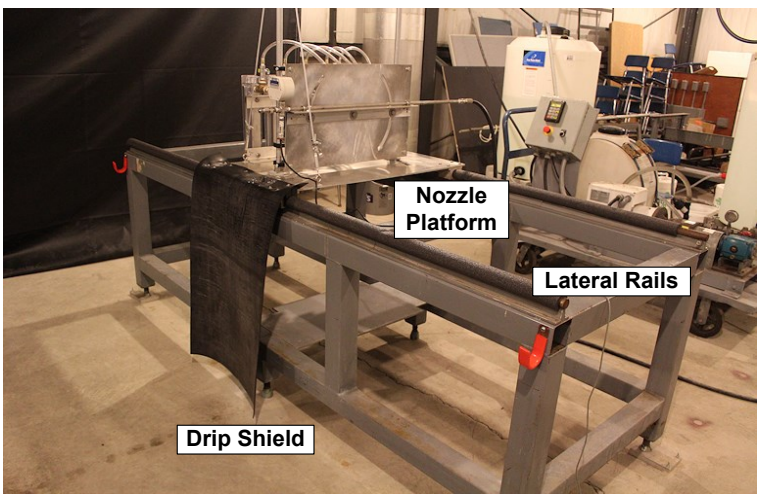

(a)

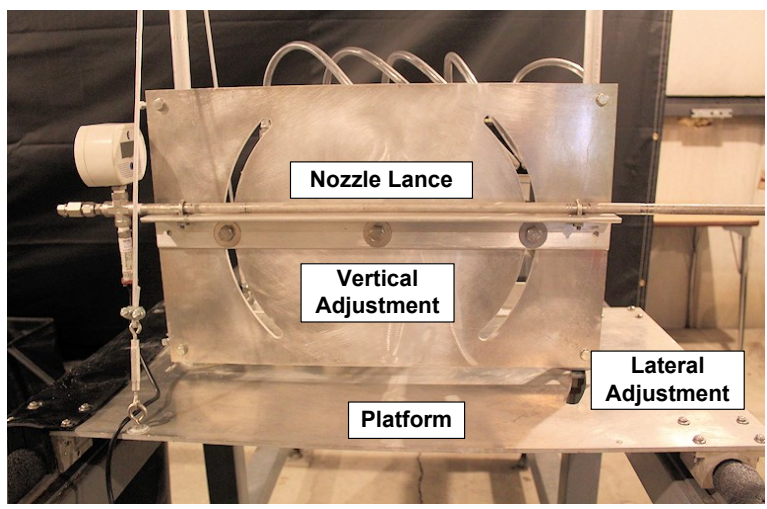

(c)

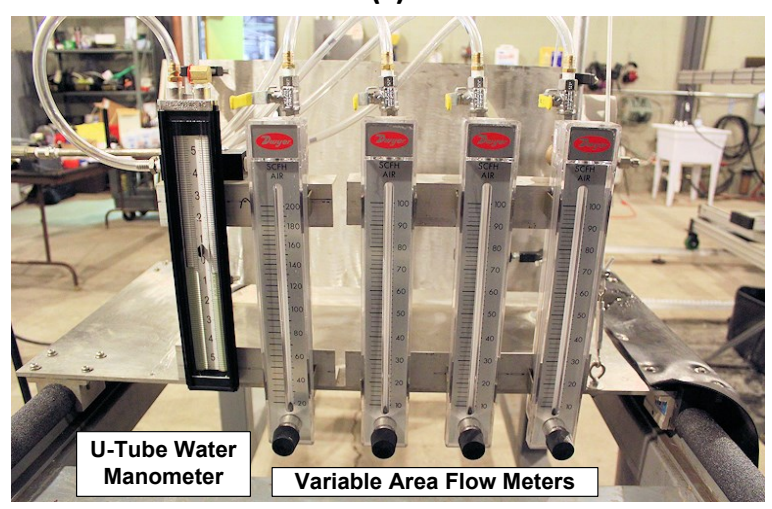

(e)

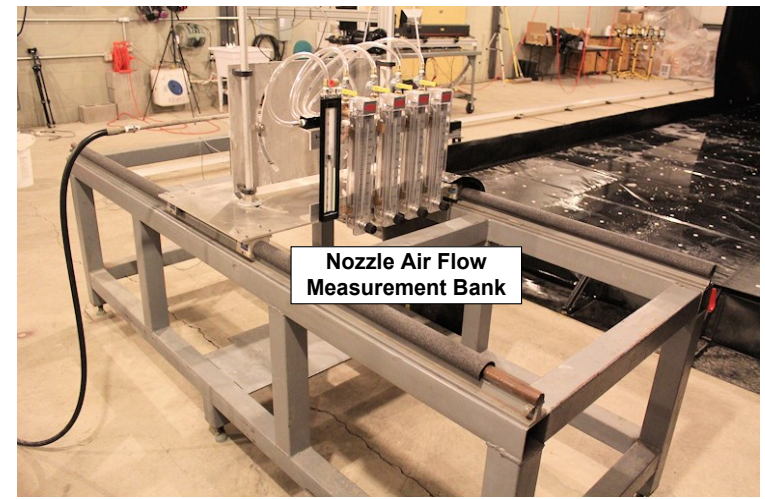

(b)

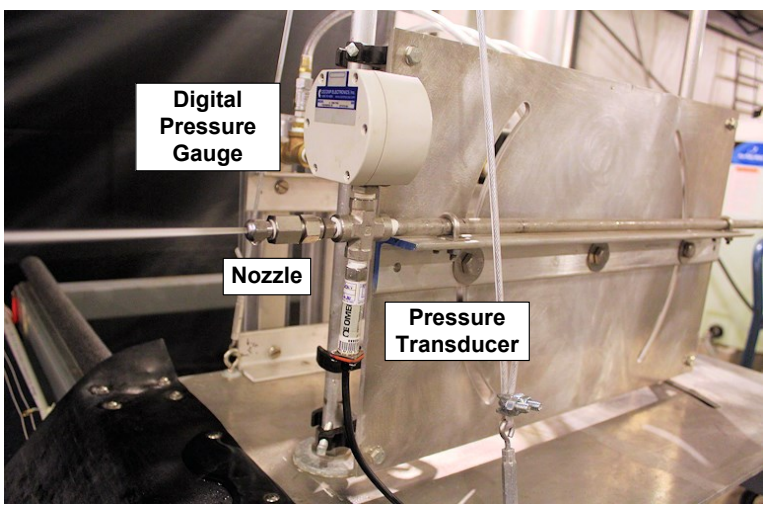

(d)

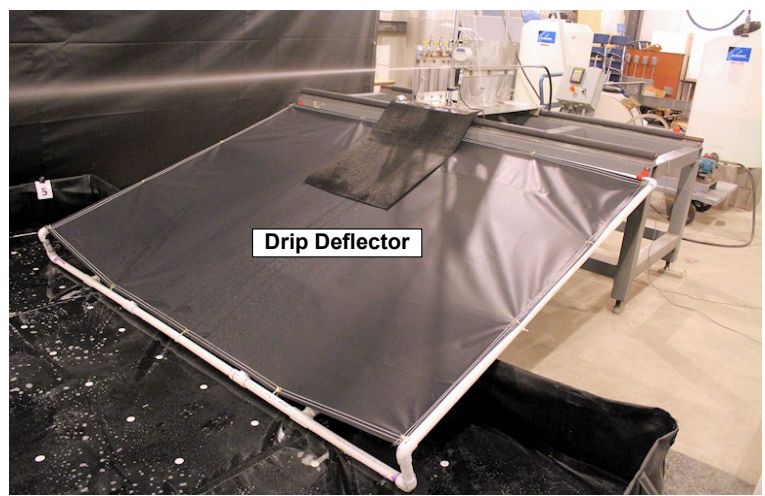

(f)

Figure 3.7: The Nozzle Stand and Accompanying Accessories 
Although not utilized in the present study, the nozzle platform was also fitted with a nozzle air flow measurement bank consisting of a U-tube water manometer and a series of variable area flow meters to monitor air ingestion pressure and flow for air aspirating nozzles. To contain drip run-off generated by the firefighting jet during sustained test periods, a drip shield and deflector were installed to bridge the gap between the nozzle stand and firefighting jet containment bed.

\subsection{Jet Containment Bed}

The firefighting jet containment bed consisted of a floor anchored, vinyl-coated polyester $13.7 \times 3.7 \times 0.3-\mathrm{m}(45 \times 12 \times 1-\mathrm{ft})$ spill berm manufactured by Seattle Tarp and used traditionally as a protective environmental barrier for heavy-duty trucks undergoing maintenance. With minor

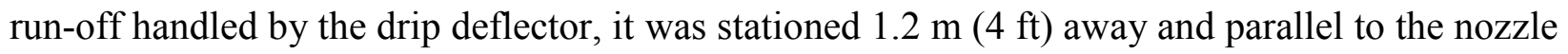
stand yielding an effective longitudinal capture zone of about $15 \mathrm{~m}(50 \mathrm{ft})$. Although the containment bed was designed to hold up to 15,142 1 (4000 gal) of liquid, agent levels seldom

surpass a few hundred gallons at a time due to an on-demand $34-1 \cdot \mathrm{min}^{-1}\left(9\right.$-gal $\left.\cdot \mathrm{min}^{-1}\right)$ sump pump that was installed to continuously drain the bed while testing was in progress. The bed floor was outfitted with painted $2.5-\mathrm{cm}(1-\mathrm{in})$ diameter white dots orthogonally spaced $30.5 \mathrm{~cm}(1 \mathrm{ft})$ apart throughout the floor to form a Cartesian grid serving as a location reference for agent ground pattern analysis.

A $6.1 \times 3.7 \times 2.4-\mathrm{m}(20 \times 12 \times 8-\mathrm{ft})$ jet containment zone riding a U-shaped galvanized curtain track suspended from the ceiling protected the rear laboratory walls from jet drift. The vertical containment curtain was made of similar corrosion-resistant material to that of the bed, and was weighted and stiffened by metallic chain sewn into its base to reduce flapping. The rear containment zone was completed using similar material to form a roof over the track centrally supported by a "tent-like" suspension system from structural ceiling beams. Containment zone roof edges were secured through industrial grade Velcro ${ }^{\circledR}$ along the track and canvas periphery. Roller track as opposed to a more permanent fixture was installed to easily alter the containment zone wall configuration for jet interaction studies and add optical access for flow visualization and PDPA. Figure 3.8a shows the firefighting jet containment bed in standard deployment, and Figure $3.8 \mathrm{~b}$ shows the rear containment zone with its curtain sides drawn demonstrating its versatility. 


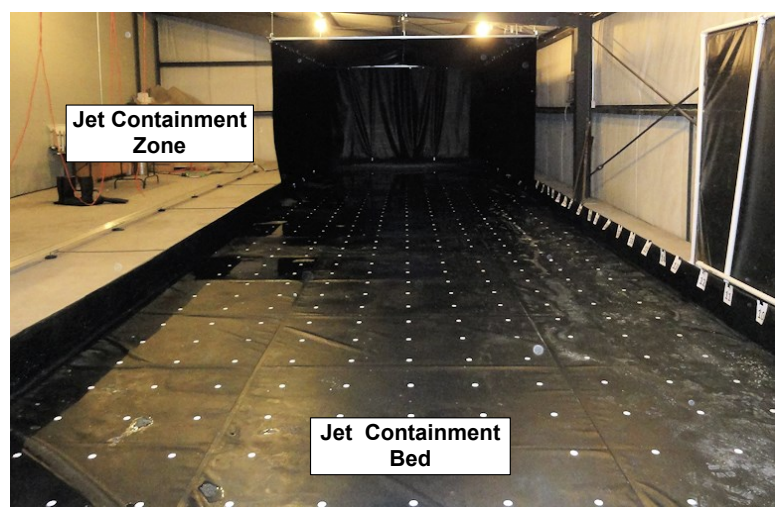

(a)

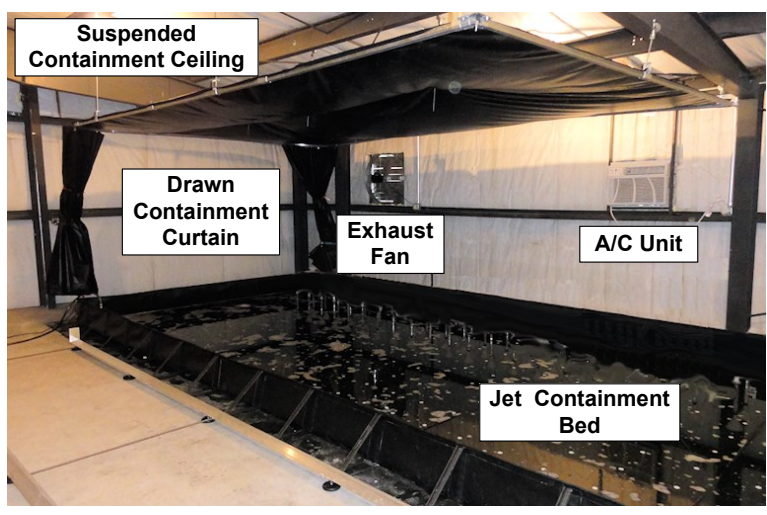

(b)

Figure 3.8: Various Views of the Firefighting Jet Containment Bed

\subsection{Flow Visualization System}

Firefighting jet flow visualization was available using a variety of capture techniques involving high speed videography, standard digital photography with laser sheeting, as well as stroboscopic lighting techniques. A stowable backdrop curtain running along the jet containment bed lip opposite the PDPA traverse track was used along with the jet containment zone track to alter the optical access for different photographic conditions. Firefighting jet high speed video was available with the aid of a Photron SA5 color camera capable of 7,500 frames $\cdot \mathrm{s}^{-1}$ at 1-megapixel resolution. Although the camera could reach recording speeds up to 1 million frames $\cdot \mathrm{s}^{-1}$ at a reduced resolution, indoor lighting limited recording speeds to between 5,000 and 7,500 frames $\cdot \mathrm{s}^{-1}$. Figure $3.9 \mathrm{a}$ depicts the camera, and Figure $3.9 \mathrm{~b}$ shows it in operation with industrial work lighting.

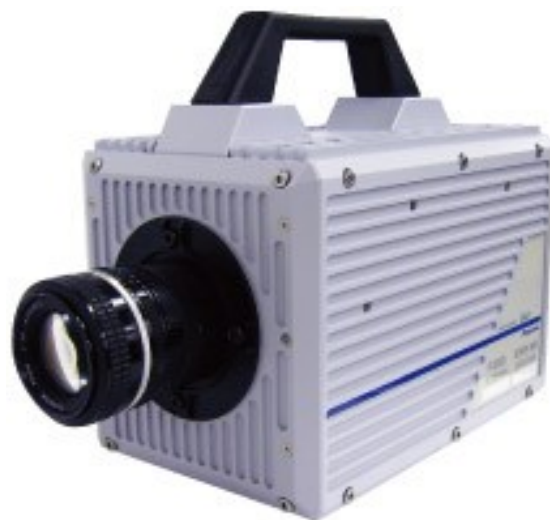

(a)

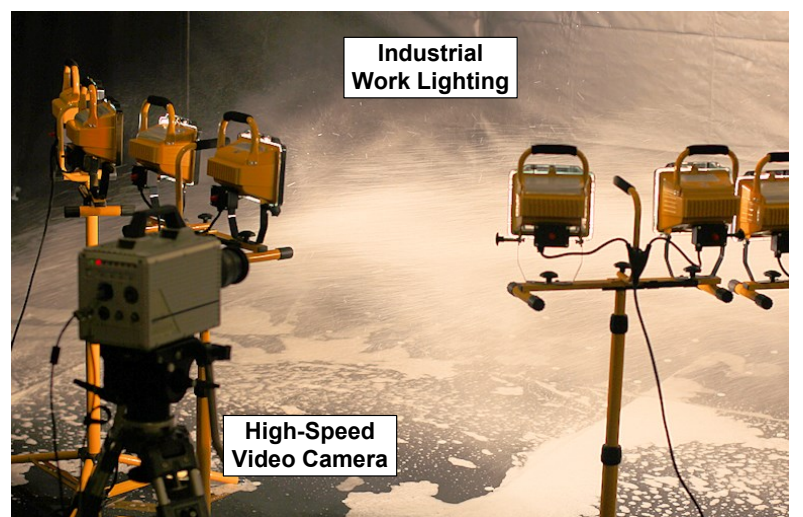

(b)

Figure 3.9: The High Speed Video Camera in Operation 
Laser sheet photography was conducted through the use of an XY galvanometer scanner utilizing laser light from the 5-Watt PDPA argon-ion laser. Key components were supplied by Cambridge Technology with system integration executed in-house. The galvanometer was mounted on the optical bench intercepting the laser beam before entering the beam separator used for PDPA. By oscillating one mirror using a sine wave voltage signal applied to the galvanometer and holding the opposing mirror at a constant deflection angle, the scanner was able to produce a uniform intensity horizontal or vertical laser sheet at a maximum $40^{\circ}$ angular excursion operating at speeds on the order of $500 \mathrm{~Hz}$. Both mirrors were driven by a function generator powered through the high speed multipurpose USB module also utilized for pressure transducer data acquisition. Figure 3.10a depicts the galvanometer scanner mounted on the PDPA optical bench in operation. It was outfitted with a heat sink to dissipate heat away from the mirror drive galvanometers. Figure 3.10 b shows the scanner creating a vertical laser sheet.

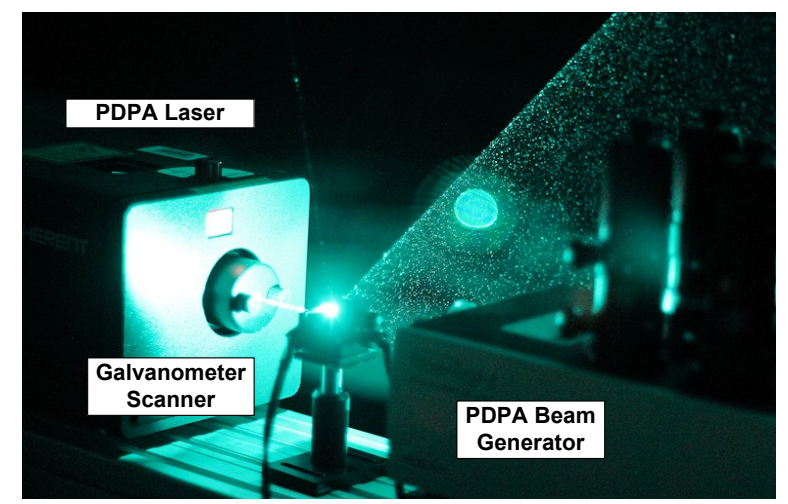

(a)

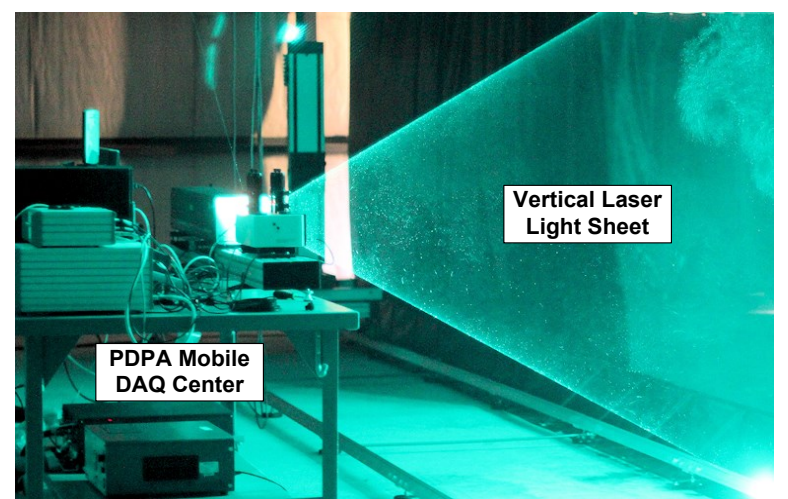

(b)

Figure 3.10: The XY Galvanometer Scanner in Operation

For jets that exhibited strong periodicity, a stroboscope can be used to highlight the pulsation frequency to allow conventional photography techniques to capture flow details. An 18.1 megapixel Canon Rebel T3i digital single-lens reflex camera with an 18 to 55 and 50-mm fixed focal length lens was available to take standard still photographs and low-speed high definition (1080p) video. A 60-mm macro (zoom) lens was also used to resolve small-scale break-up dynamics at a safe distance away from the jet. 


\subsection{Phase Doppler Particle Analyzer}

To quantify firefighting jet spray characteristics, a PDPA manufactured by TSI, Inc. was acquired to measure the axial ( $x$-direction) velocity, vertical ( $z$-direction) velocity, and droplet size distribution. PDPA is a high-fidelity experimental flow characterization tool based on similar operating principles to that of laser Doppler velocimetry with the inclusion of recording particle size. By assuming the particles (or droplets/small bubbles) are small, on the order of microns to sub-millimeter in size and spherical in shape, PDPA was used to quantify their velocity and size by measuring the scattered light off of them. The PDPA data acquisition process is explained as follows. The intersection of two laser beams for each velocity component generates a probe (or measuring) volume. Each probe volume is comprised of a bright and dark fringe pattern such that when a droplet passed through the probe volume, light scattered from crossing a bright fringe can be collected by a photo detector via optics and converted to a velocity knowing the fringe geometry and laser beam wavelength $(\lambda)$. The axial droplet velocity component $\left(V_{d, x}\right)$, for example, is determined by the following linear relationship [66]:

$$
V_{d, X}=\delta f_{\text {Doppler }}
$$

where $\delta$ is the fringe spacing and $f_{\text {Doppler }}$ is the Doppler frequency, also known as the temporal frequency, for that velocity component measured by the PDPA. Fringe spacing is calculated from the following equation:

$$
\delta=\frac{\lambda}{2 \sin (\theta / 2)}
$$

where $\theta$ is the laser beam intersection angle [66]. Figure 3.11 depicts the geometric relationship between the intersecting beam angle and the measuring volume [67]. 


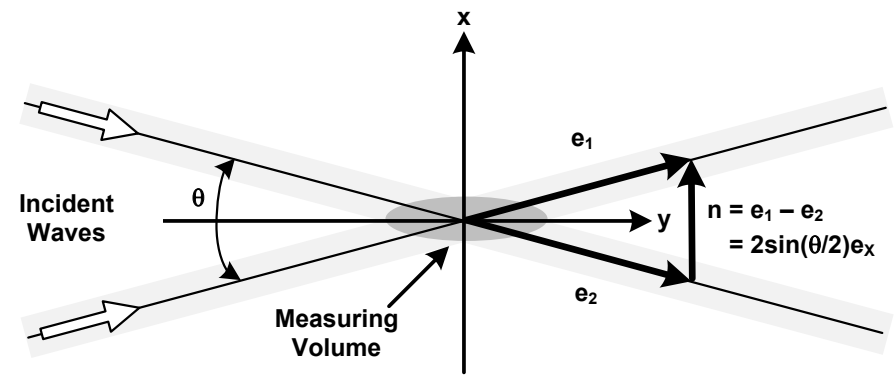

Figure 3.11: Relevant Vector Relations for Determining Droplet Velocity [67]

To discern flow direction and measure droplets at or near zero velocity, one of the two intersecting beams for each velocity component is frequency shifted by a Bragg cell which effectively generated moving fringes at $40 \mathrm{Mhz}$. If a droplet is moving against the fringe pattern motion, its frequency is $40 \mathrm{Mhz}$ minus the Doppler frequency. If a droplet is moving with the fringe pattern, its frequency is $40 \mathrm{Mhz}$ plus the Doppler frequency. Photomultiplier tubes convert collected scattered light from fringes in the probe volume to electrical current pulses. Figure 3.12 depicts a typical light scattering pattern recorded by a droplet passing through the laser beam intersection or measuring volume. Its envelope shape is Gaussian in nature due to laser beam Gaussian light intensity across the beam width [66].

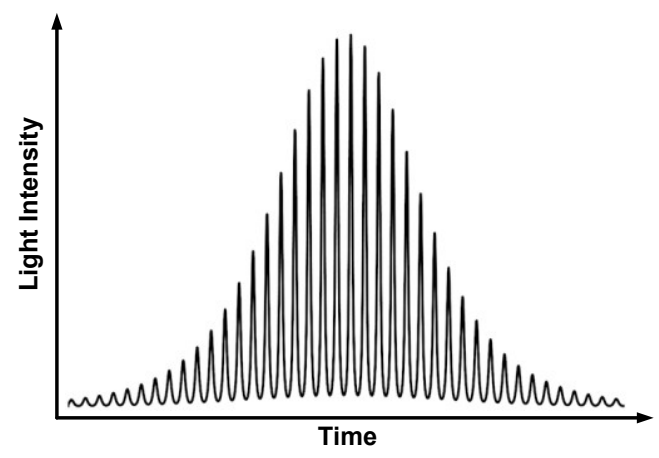

Figure 3.12: Light Intensity Scattered by a Particle Passing through the Probe Volume [66]

The photomultiplier tube electrical output is passed through a high-pass filter to remove the low frequency from the signal generating the low frequency envelope, or what is commonly referred to as the pedestal. The signal is then further filtered through a series of bandpass filters and amplified [66].

Whereas particle velocity is proportionally based on a temporal frequency, particle size is proportionally based on the spatial frequency of the scattered light fringe pattern. Scattered light rays are composed primarily of reflection and refraction. An exact description of scattered light 
by a homogeneous sphere for the case of wavelength comparable to or much smaller than the particle size is given by the Mie solution to Maxwell's equations. In addition to the intersection angle of the two laser beams and their wavelength, scattered light measurements to calculate particle size are also dependent on particle liquid index of refraction, the receiving angle ( $\psi)$, and the scattering angle $(\varphi)$. Particle diameter measurements are also reliant on the polarization or scattering plane orientation with respect to the photo detector as well as the shape and size of the photo detector aperture. As a particle scatters light from the two incident laser beams, two different photo detectors receive the scattered light ray resulting in a measurement of the shifted Doppler phase. Each phase is defined by the scattered light's optical path length. The phase shift angle $(\phi)$ between these two signals is proportional to the particle surface curvature, which effectively is a measure of particle diameter based on the assumption that the particles are perfectly spherical. Figure 3.13a illustrates how incident laser beam light reflects and refracts off of a particle, and Figure $3.13 \mathrm{~b}$ depicts the orientation of each optical parameter involved with measuring the phase shift. The particle or droplet diameter $(d)$ is derived from its relationship with the phase shift from the following equation:

$$
\frac{\phi}{360}=M \times \frac{\text { Photo Detector Separation }}{\text { Receiver Focal Length }} \times \frac{d}{\delta}
$$

where $M$ is the slope of the phase-diameter relationship estimated based on Mie theory and constants defined by the PDPA optics and the droplet fluid medium [66, 67].

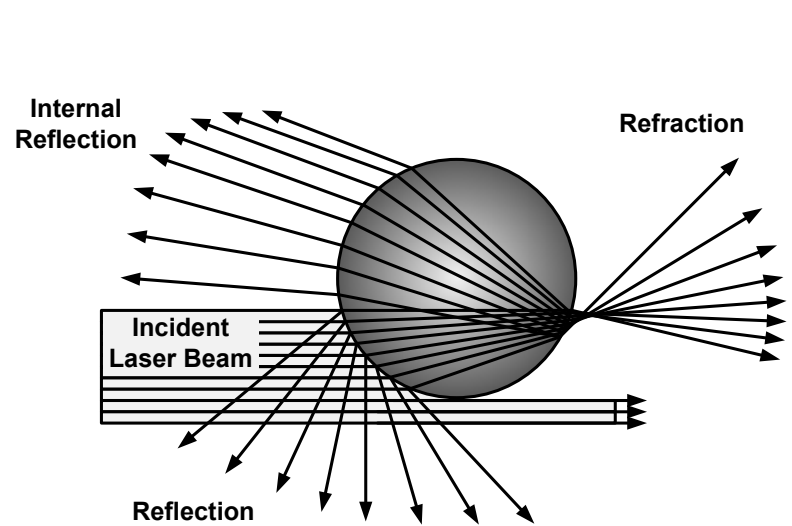

(a)

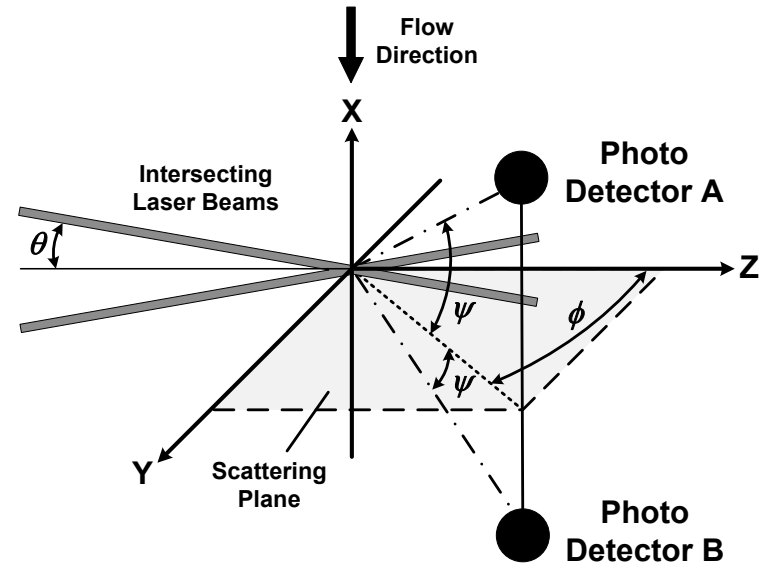

(b)

Figure 3.13: (a) Methods of Light Scatter off of a Particle Based on Mie Theory (b) The Orientation of Each Optical Parameter Involved in Measuring Phase Shift $[66,68]$ 
To improve measurement confidence, three photo detectors were used to generate a correlation based upon two independent droplet size measurements. Both Fast Fourier Transform (FFT) and autocorrelation techniques were employed to obtain the droplet temporal frequency (velocity) and phase (diameter) measurement [66]. Error in both the velocity and droplet size measurement is difficult to determine precisely as it is a function of not only hardware resolution but also system spatial alignment. Taking these factors into account, velocity measurement uncertainty is typically less than 0.50 percent of the measured velocity. Droplet diameter measurement uncertainty has similar dependencies and is generally less than one percent of the maximum measurable droplet diameter plus one percent of the actual measured droplet diameter [69].

A software compatible tri-axis traverse was installed to translate the PDPA optics with submillimeter resolution over one metric volume. The traverse could be controlled automatically through PDPA data acquisition software, or manually positioned using a hand control unit. The traverse was customized with a V-groove wheel base to travel along a 13.7-m (45-ft) long, 1-m (3.3-ft) wide aluminum linear V-track fabricated in-house to run adjacent against the jet containment bed. The track was supported in approximate $1.8-\mathrm{m}(6-\mathrm{ft})$ intervals with adjustable floor levelers to make up for known sag in the suspended laboratory floor.

The rest of the PDPA components trail the traverse on a nearby mobile table accommodating a water-cooled 5-Watt argon-ion continuous laser and multicolor beam separator aligned on an optical bench. The beam separator was responsible for splitting the primary laser beam into different wavelengths for each velocity component, as well as creating a shifted and un-shifted beam per wavelength via an internal Bragg cell (green $-514.5 \mathrm{~nm}$ for the axial or $x$-direction velocity component and blue $-488 \mathrm{~nm}$ for the vertical or $z$-direction velocity component). Although the beam separator could accommodate three component velocity measurements, the configuration used for the current study was only set up for two. To complete the system, the table also housed a photo detector module containing the photomultiplier tubes and 175-Mhz signal analyzer to interpret droplet velocity and size, a desktop computer to command the PDPA data acquisition software, the laser power supply, and traverse controller. The FSA 4000 signal analyzer was chosen based upon its ability to resolve high speed velocities expected near the nozzle for high pressure firefighting jets with exit velocities in excess of $100 \mathrm{~m} \cdot \mathrm{s}^{-1}$. The signal analyzer had a maximum sampling rate of $800 \mathrm{Mhz}$. Signals were digitized at numerous 
sampling rates to allow automatic dynamic selection of the appropriate sampling rate to measure droplet size and velocity accurately. A digital high speed oscilloscope was also available to conduct independent PDPA diagnostics.

The PDPA transmitter probe and optical receiver were mounted on the traverse to communicate transmitted and reflected laser light via fiber optic cable from the beam separator and to the photo detector module, respectively. Fiber optic as well as traverse axis power cables were suspended from the ceiling to reduce floor hazards. Figure 3.14 depicts a schematic showing main PDPA components in forward scatter mode along with their associated connectivity.

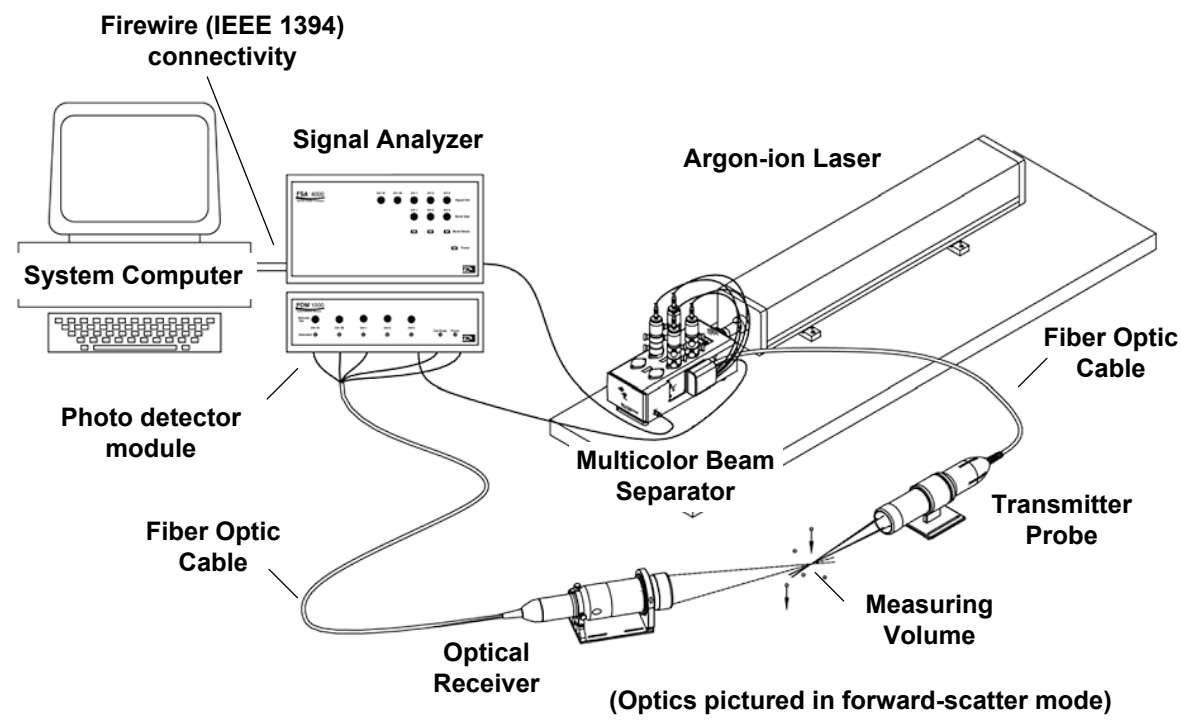

Figure 3.14: A Schematic of PDPA Components in Forward Scatter Mode [70]

To accommodate the horizontal expanse of the jet containment bed, PDPA optics were arranged in backward scatter mode positioning the optical receiver on the same side of the measuring volume as the horizontal transmitter probe. Following Figure 3.13a as an orientation guide with respect to the incident laser beam and light scatter direction, forward scatter mode measures light scattered due to refraction, and back scatter mode measures light scattered due to internal reflection. The optical receiver was stationed about 30 degrees off axis from the transmitting probe to optimize the reception of scattered light reflected back from the jet droplets. A 2-m (6.6-ft) T-rail supported the optics secured against the vertical traverse axis. Due to the substantial moment arm imposed by the cantilevered configuration, the traverse base was counter-weighted opposite the optics to reestablish stability. Using a 1-m focal length lens on the transmitter probe and receiver, from traverse (track) center, measurements were taken 
approximately 2.6-m (8.5-ft) away just beyond the containment bed longitudinal centerline. Alternate optical configurations were available, but this layout accommodated the laboratory design the best. Figure 3.15 depicts major PDPA system components while in operation.

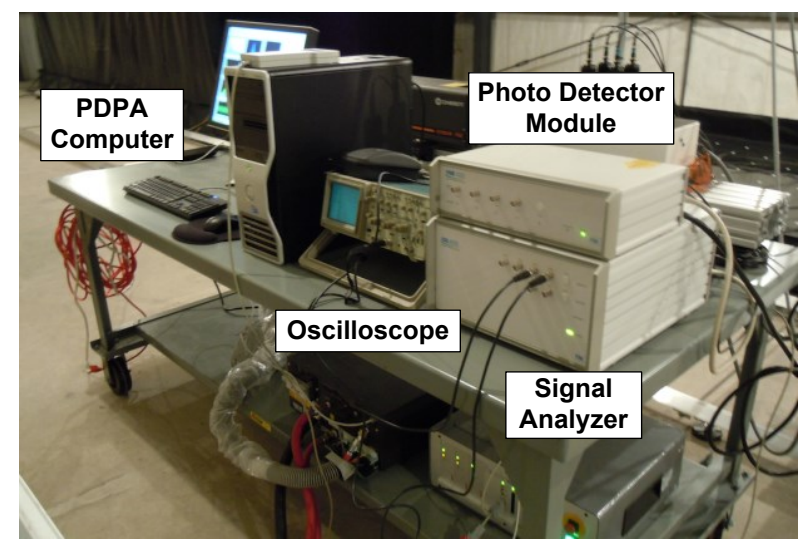

(a)

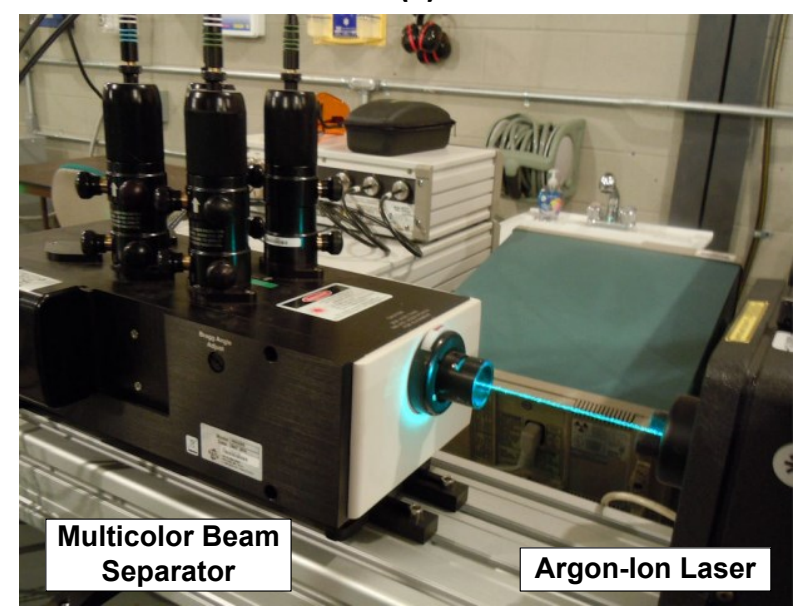

(c)

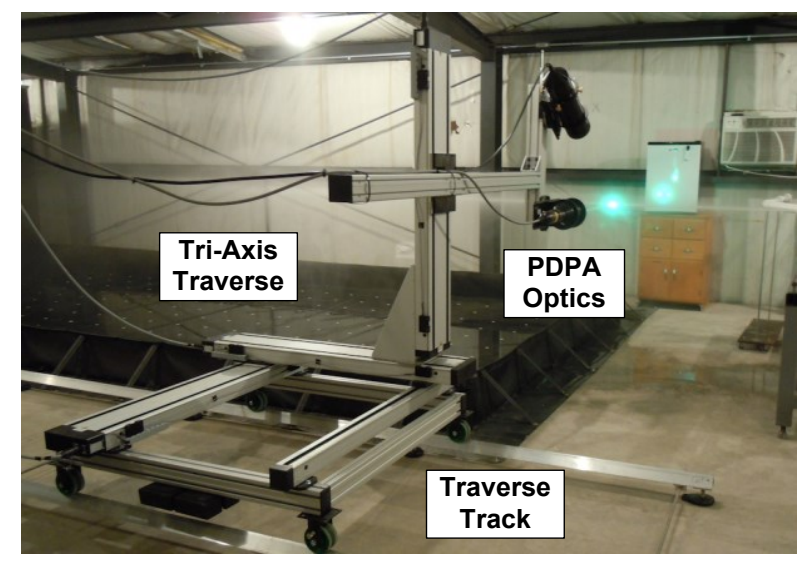

(e)

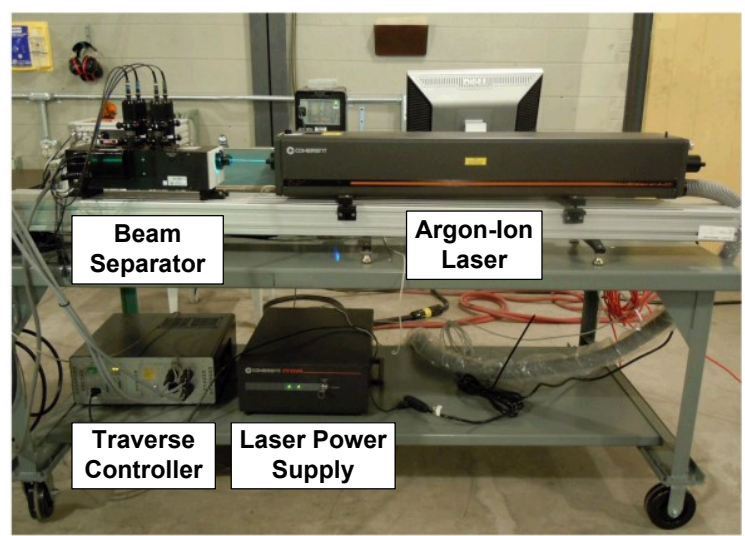

(b)

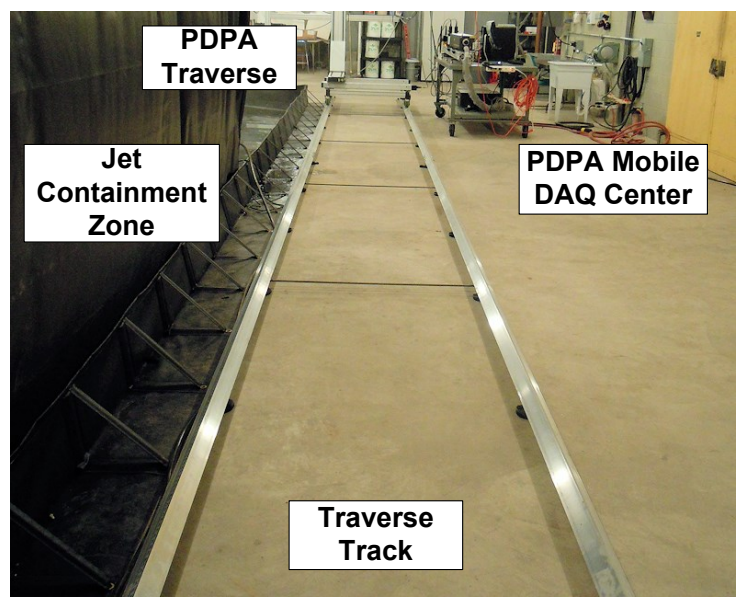

(d)

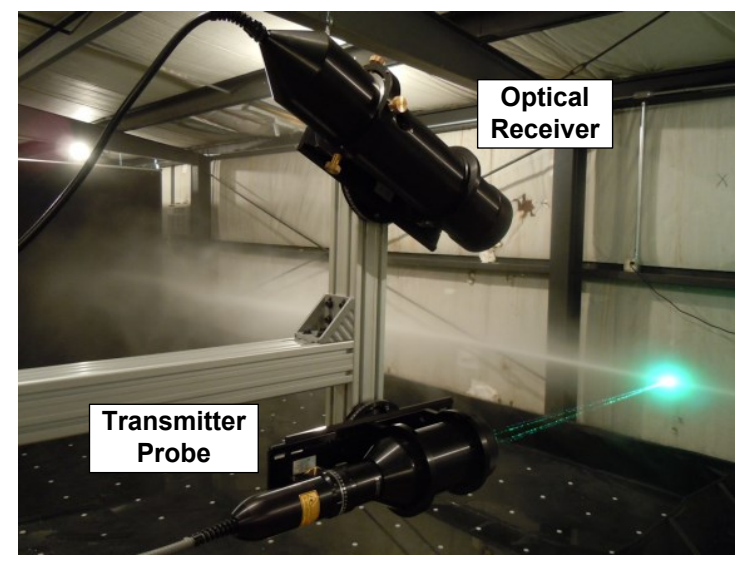

(f)

Figure 3.15: An Overview of the PDPA System 


\section{CHAPTER 4: EXPERIMENTAL APPROACH}

\subsection{Overview}

Experiments were conducted in the aqueous firefighting agent application laboratory to characterize firefighting jet performance with respect to change in nozzle pressure, flow rate, and AFFF concentration. Measured output parameters included jet velocity, droplet size, ground accumulation pattern, and foam quality. Initial strides were taken to characterize a commercial firefighting nozzle designed to operate within the firefighting agent delivery system performance envelope while meeting minimum foam quality standards as outlined by the NFPA [11]. Further constraints on nozzle selection dictated the use of conventional channel flow nozzle profiles to reduce geometric complexity to simplify the translation process to a CFD model environment. A firefighting nozzle meeting all of these criteria did not exist during the time of testing. All commercially available firefighting nozzles designed for $37.81 \cdot \mathrm{min}^{-1}\left(10 \mathrm{gal} \cdot \mathrm{min}^{-1}\right)$ or lower operation reported a maximum foam expansion of approximately 3:1 which is below the minimum accepted NFPA value of 5:1 for ARFF use [11]. Several nozzles designed for alternative applications like industrial cleaning were examined. Each failed at least one of the aforementioned criteria. In following the tendency of most manufacturers, high foam quality was forfeited to meet the other requirements in this study. The 6.4-mm (0.25-in) AP4TM Attack Tip (AP4) developed by Stoneage Waterblast Tools ${ }^{\circledR}$ was chosen due to its precise manufacturing standards, the availability of its family of self-similar simple nozzle designs to span the entire firefighting agent delivery system performance envelope, along with its capacity to produce reasonable foam quality. The AP4 nozzle is a single bore nozzle with a step-reducing channel. Figure 4.1 illustrates various views of the AP4 nozzle with $D$ denoting nozzle diameter.
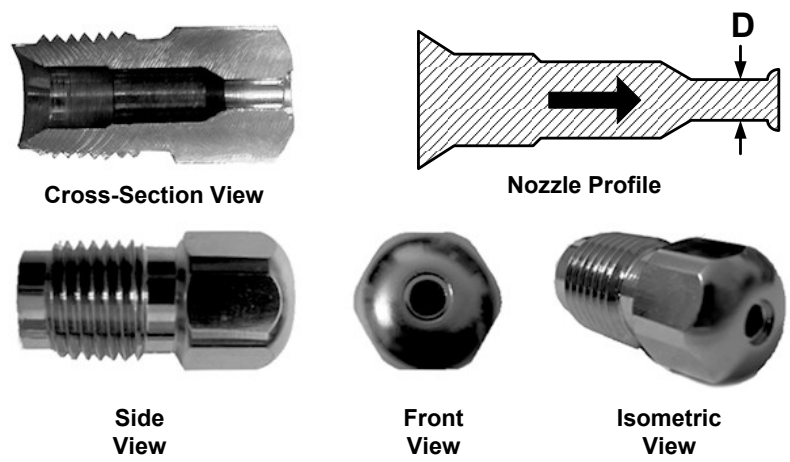

Nozzle Profile

Figure 4.1: The Stoneage Waterblast Tools AP4 Nozzle 
Figure 4.2 provides a visual overview of all experiments conducted in the laboratory using the AP4 nozzle family in terms of nozzle exit pressure and flow rate combinations. Each of the 18 dotted lines represents the performance of an individual AP4 nozzle with a unique exit diameter. All nozzle performance profiles were measured using water-only because the addition of AFFF negligibly affected nozzle pressure and flow rate measurements. Firefighting jet flow rates were chosen to fill out the firefighting agent delivery performance envelope with respect to nozzle pressures regimes commonly applied in the ARFF industry. Three nozzle pressure and flow rate magnitudes were chosen spaced equally apart to designate a low, medium, and high condition for pressure and flow rate. Studies that involved varying AFFF concentration were carried out in increments of 3-percent ranging from water-only up to a maximum of 12-percent AFFF concentrate by volume mixed with water.
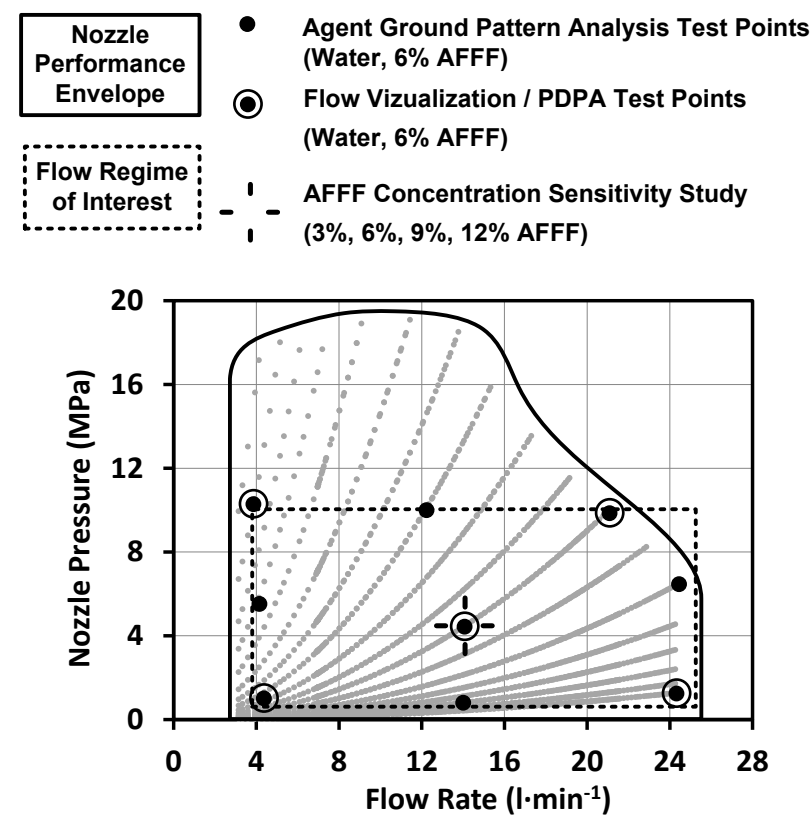

Figure 4.2: The Firefighting Agent Delivery System Performance Envelope and Associated Firefighting Jet Experiments

Two-dimensional agent ground pattern analysis was conducted using water and 6-percent AFFF at the nine pressure-flow rate combinations shown in Figure 4.2. Due to the expense and high consumption rate of AFFF during testing, the number of experimental conditions conducted for flow visualization and phase Doppler measurements were reduced from nine to five by removing the pressure-flow rate border mid-points. PDPA as well as flow visualization was conducted using water and 6-percent AFFF at the five reduced pressure-flow rate combinations highlighted 
in Figure 4.2. Pre-sampled flow visualization results ranging from 3 to 12-percent AFFF also showed negligible difference to warrant a more thorough study. A one-dimensional (1-D) AFFF concentration sensitivity study was conducted at the pressure-flow rate center point for both the agent ground pattern analysis and PDPA, ranging from 3 to 9-percent AFFF and 3 to 12-percent AFFF, respectively. Twelve-percent AFFF was removed from the PDPA concentration sensitivity study because a negligible change in results was observed beyond 6-percent AFFF.

A total of ten tests total were conducted for flow visualization, 20 tests were conducted for agent ground pattern analysis, and thirteen tests were conducted for PDPA. Table 4.1 summarizes all of the aforementioned experiments that were carried out in the aqueous firefighting agent application laboratory.

Table 4.1: Firefighting Jet Test Case Summary in the Aqueous Firefighting Agent Application Laboratory

\begin{tabular}{|c|c|c|c|c|c|c|}
\hline & \multicolumn{3}{|c|}{ Water Jet } & \multicolumn{3}{|c|}{ AFFF Jet } \\
\hline Flow Visualization & \multicolumn{3}{|c|}{ Nozzle Flow Rate } & \multicolumn{3}{|c|}{ Nozzle Flow Rate } \\
\hline Nozzle Pressure & Low & Medium & High & Low & Medium & High \\
\hline Low & $\bullet$ & & $\bullet$ & $\mathbf{0}$ & & $\mathbf{0}$ \\
\hline Medium & & $\bullet$ & & & $\mathbf{0}$ & \\
\hline High & - & & - & $\mathbf{0}$ & & 0 \\
\hline Agent Ground Pattern Analysis & \multicolumn{3}{|c|}{ Nozzle Flow Rate } & \multicolumn{3}{|c|}{ Nozzle Flow Rate } \\
\hline Nozzle Pressure & Low & Medium & High & Low & Medium & High \\
\hline Low & $\bullet$ & $\bullet$ & $\bullet$ & $\mathbf{0}$ & 0 & $\mathbf{0}$ \\
\hline Medium & $\bullet$ & $\bullet$ & • & 0 & $\square$ & 0 \\
\hline High & $\bullet$ & $\bullet$ & $\bullet$ & 0 & 0 & $\mathbf{0}$ \\
\hline Phase Doppler Particle Analysis & \multicolumn{3}{|c|}{ Nozzle Flow Rate } & \multicolumn{3}{|c|}{ Nozzle Flow Rate } \\
\hline Nozzle Pressure & Low & Medium & High & Low & Medium & High \\
\hline Low & $\bullet$ & & $\bullet$ & 0 & & 0 \\
\hline Medium & & $\bullet$ & & & $\diamond$ & \\
\hline High & - & & • & 0 & & 0 \\
\hline
\end{tabular}

Key operating conditions for the test cases presented in Figure 4.2 and Table 4.1 are in Appendix A: Case Study Summary. It should be noted that Reynolds, Weber, and Ohnesorge 
numbers reported in Appendix A are consistent with firefighting jet regimes highlighted in classifying liquid jet break-up in Section 2.3.2.

Additional 1-D jet centerline ground pattern measurements were also recorded at similar laboratory pressures, but at full-scale flow rates approximately three times larger than the laboratory high flow rate condition. Results from this particular analysis are presented separately from the core experiments conducted in the aqueous firefighting agent application laboratory.

In an ancillary effort, several AFFF material properties were quantified due to insufficient data available in the literature. Vital to nearly all phases of research, their collection focused on the classification of Chemguard C301MS 3-percent AFFF in various dilutions with water for which all experimental work was conducted. Density, dynamic viscosity, equilibrium and dynamic surface tension, in addition to index of refraction were either recorded or extrapolated from relationships to measurements of other properties. Details of each property characterization effort are summarized in Appendix B: AFFF Material Property Data.

Proper proportioning of AFFF concentrate with water was critical for producing consistent foam quality and for ensuring repeatability throughout the experimental phase. All experimental AFFF solutions were premixed in storage tanks via closed-loop feedback to the firefighting agent delivery pumps with dilution levels adjusted based upon test condition requirements. The firefighting agent delivery system was flushed prior to testing whenever AFFF proportion levels had to be modified between experiments. Output from AFFF jets was periodically sampled to ensure AFFF concentrate was proportioned within \pm 0.25 percent of the specified value. AFFF proportion levels were calibrated and monitored using refractive techniques. Details of this process are in Section B.4 where results of AFFF index of refraction are presented. All results from the experimental effort including flow visualization, agent ground pattern analysis, and PDPA are presented in Sections 6.2, 6.3, and 6.4, respectively.

\subsection{Flow Visualization}

Flow visualization was conducted on five pressure-flow rate combinations using water and 6percent $\mathrm{AFFF}$ to record a total of ten unique firefighting jet configurations. As mentioned earlier, 6-percent AFFF was used solely for flow visualization because variation in AFFF 
concentration was qualitatively difficult to perceive. Conventional photography with standard lighting as well as laser sheet lighting, high speed photography, and stroboscopic (pulsed lit) photography techniques were all used to characterize various aspects of the firefighting jet environment. Details of these flow visualization methods are discussed in Section 3.4. Standard, wide, and macro (zoom) lenses were used depending on the subject size and relative range to the camera. Figure 4.3 depicts the three main focal planes from a top view where far field flow visualization was recorded along with the approximate location of the camera. Each plane is defined based on the direction it is normal to via the coordinate system. The $x$-plane refers to $x=$ $6.09-\mathrm{m}(20-\mathrm{ft})$ downstream of the nozzle running vertically upward in the $z$-direction and was chosen as an approximate mid-plane for all firefighting jets investigated. The $y$-plane refers to the $y=0$-m $(0-\mathrm{ft})$ plane intersecting the nozzle vertically along the $z$-direction, and the $z$-plane refers to the $z=0-\mathrm{m}(0-\mathrm{ft})$ plane intersecting the nozzle horizontally along the $x$-direction. The blackout curtain adjacent to the laboratory wall was dropped to provide contrast for the photographs. The jet containment zone walls were partially exposed to allow extended optical access for $y$-plane far field photography. Near field imagery was taken at various locations along the $y$-plane. All results from flow visualization are presented in Section 6.2.

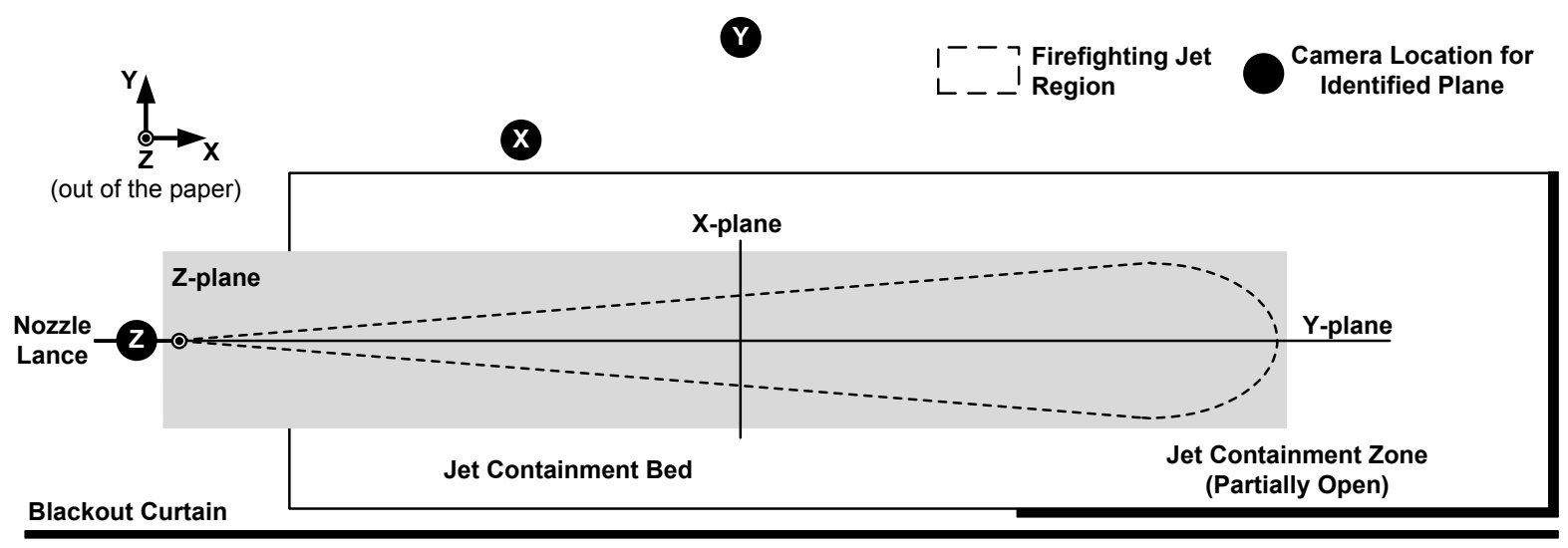

Figure 4.3: The Main Focal Planes Used for Firefighting Jet Flow Visualization

\subsection{Agent Ground Pattern Analysis}

Agent ground pattern analysis was conducted to describe agent accumulation, foam quality, ground coverage area, reach, and maximum span. Utilizing the containment bed's floor reference system, modified $100 \pm 2-\mathrm{mL}$ graduated cylinders were placed along the footprint of the jet to measure agent accumulation. The adapted capture device consisted of a standard 
polypropylene cylinder outfitted with a 127-mm (5-in) diameter funnel attached to the cylinder mouth via a holed rubber stopper slightly bored out to friction fit the funnel's neck. The funnel was necessary to extend the effective agent capture rate range without compromising measurement resolution. A steel puck was fixed to the cylinder's underside to add extra weight and keep the device upright when exposed to the oncoming force of the jet. The entire base was dipped in a plasticizer to avoid corrosion. Agent accumulation was recorded by manually reading the agent height within the cylinder. Foam quality, or foam expansion ratio, defined by Equation 2.2 as the volumetric ratio of the emulsified foam relative to the same quantity collapsed to a pure liquid, was determined by a more involved method. Once the agent volume was read, the capture device was weighed and recorded using a $1000 \pm 0.2$-g scale. Knowing the dry weight of each capture device beforehand via pre-numbered identification, the expansion ratio was calculated by dividing the difference in the capture base's wet and dry weight into the agent volume. This process was justified irrespective of foam concentration percentage because the density of AFFF is similar to that of water $\left(1 \mathrm{~g} \cdot \mathrm{ml}^{-1}\right)$ as noted in Section B.1.

A standard agent ground pattern test was conducted by operating the firefighting agent delivery system at one of the jet conditions illustrated in Figure 4.2 and listed in Table 4.1 in Section 4.1. To avoid transience during developing jet conditions prior to each experiment, a flow deflector was positioned in front of the jet to divert the flow out of the path of the floor capture devices to a location near the corner of the containment bed. Once the firefighting agent delivery system reached steady-state operation, the deflector was removed and the agent capture devices were allowed to fill up. Firefighting jet delivery periods were determined based on the observed time it took for at least one capture device in the entire footprint to consistently become 90 percent full over repeated trials. Due to different nozzle and agent composition conditions affecting jet trajectory, accumulation times were optimized for each case. This method was enforced to maximize measurement accuracy for each configuration. Results were reported nondimensionally for consistent comparison and to reduce nozzle condition dependence. This method was reminiscent of Theobald's more simplified testing of high flow firefighting jets discussed in Section 2.3.4 [50, 51]. Because there were 454 grid floor locations and only about 150 capture devices available, most full two-dimensional (2-D) agent ground pattern investigations were completed using multiple runs to populate the entire floor pattern. For large 
foam ground patterns generated by medium and high flow rate and pressure AFFF jets, a staggered measurement pattern was enforced everywhere but along the centerline. Skipped measurement locations were interpolated from surrounding measurement locations. Three replicated measurements were taken and averaged for each location due to the laborious nature of the data acquisition process. Although foam drainage, or foam breakdown time was on the order of minutes, it did not strongly influence measurement accuracy because the foam's dry cell-structure was still visibly resident in the same suspended cylinder location even hours after testing. Sample agent ground pattern tests were conducted using $1000 \mathrm{~mL}$ graduated cylinders to confirm foam accumulation was not dependent upon capture vessel design or scale. For highly expanded (dryer) foams, funnel drain size could become a limiting factor for this particular design as the drainage rate into the graduated cylinder may be slower than the rate at which the funnel fills up with foam.

A jet containment zone sensitivity study was initially performed to quantify the curtain's effect on agent ground pattern measurements. The high flow, high pressure jet was selected because it was the largest sized jet and presumed to have the most interdependency with the curtains and other local surroundings. Agent ground coverage area was calculated by summing all agent capture devices that recorded at least $2 \mathrm{ml}$ of agent. A 1-D ground centerline AFFF concentration sensitivity study ranging from 3 to 12-percent AFFF was conducted at the medium flow, medium pressure jet condition illustrated in Figure 4.2 and listed in Table 4.1 in Section 4.1. One-dimensional agent ground pattern testing was approached similarly as 2-D testing except data was recorded exclusively along the jet centerline. Figure 4.4a depicts the capture device, and Figures $4.5 \mathrm{~b}$ through Figure $4.5 \mathrm{~d}$ show various aspects of a conventional ground pattern test conducted in the laboratory. Figure 4.5 depicts the coverage area for a typical ground pattern measurement based upon the firefighting jet spray region. 


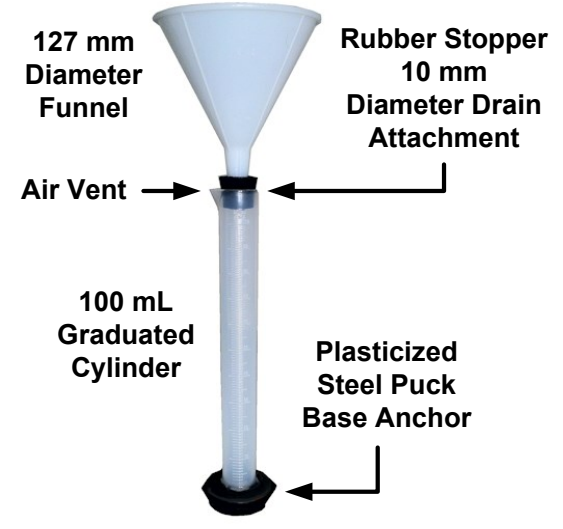

(a)

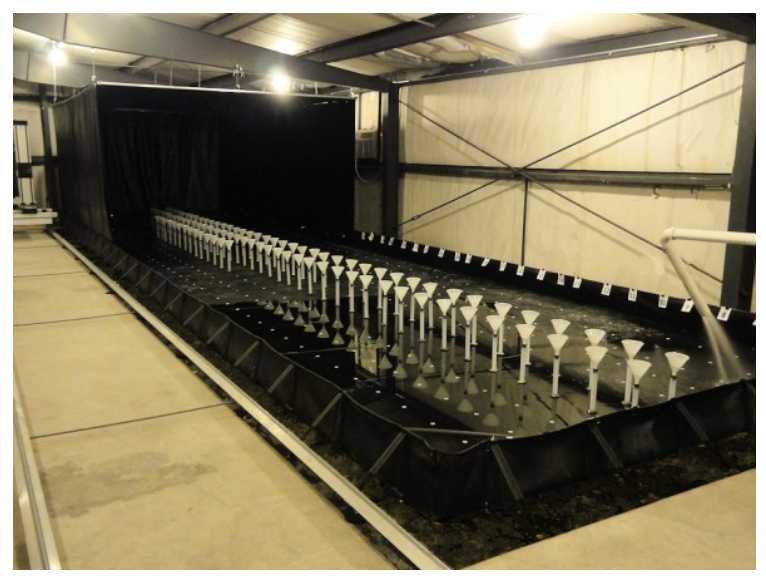

(c)

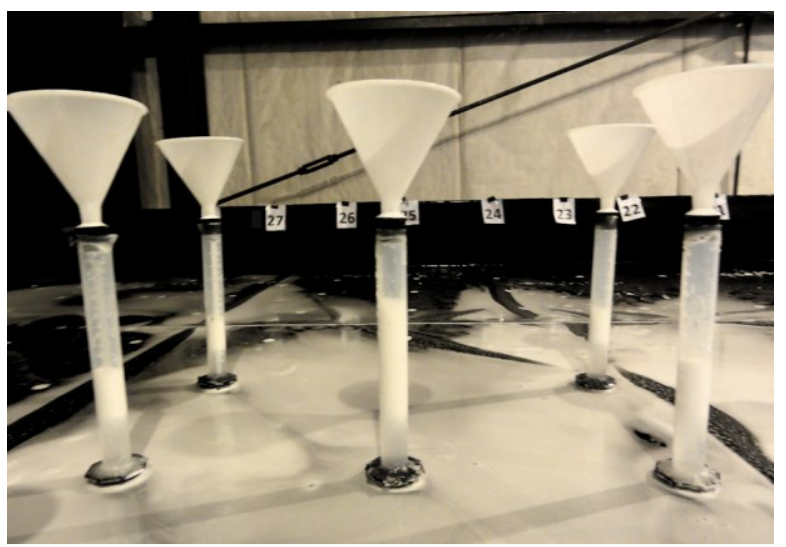

(b)

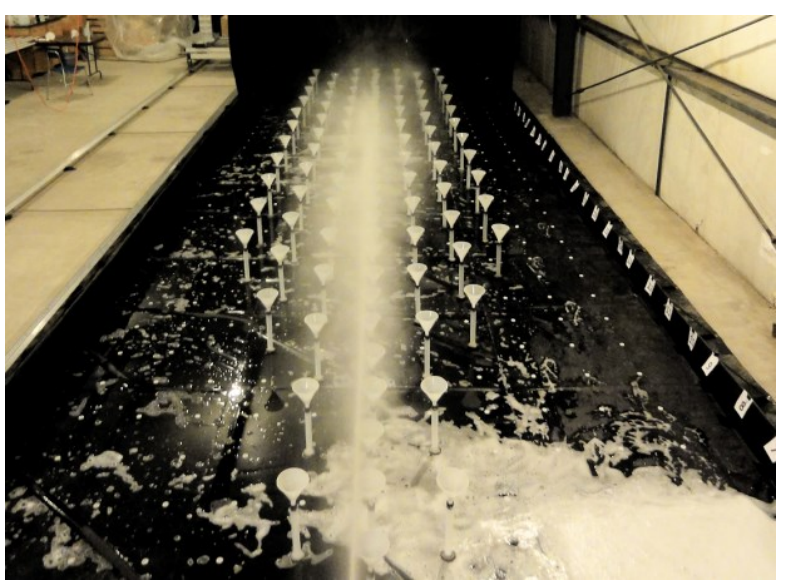

(d)

Figure 4.4: (a) The Agent Ground Pattern Capture Device (b) A Near Field View of the Agent Ground Pattern Capture Device Array Immediately after Testing (c) A Water Pattern Test Utilizing the Flow Deflector During Startup (d) A Semi-aerial View of an AfFF Jet Test in Progress

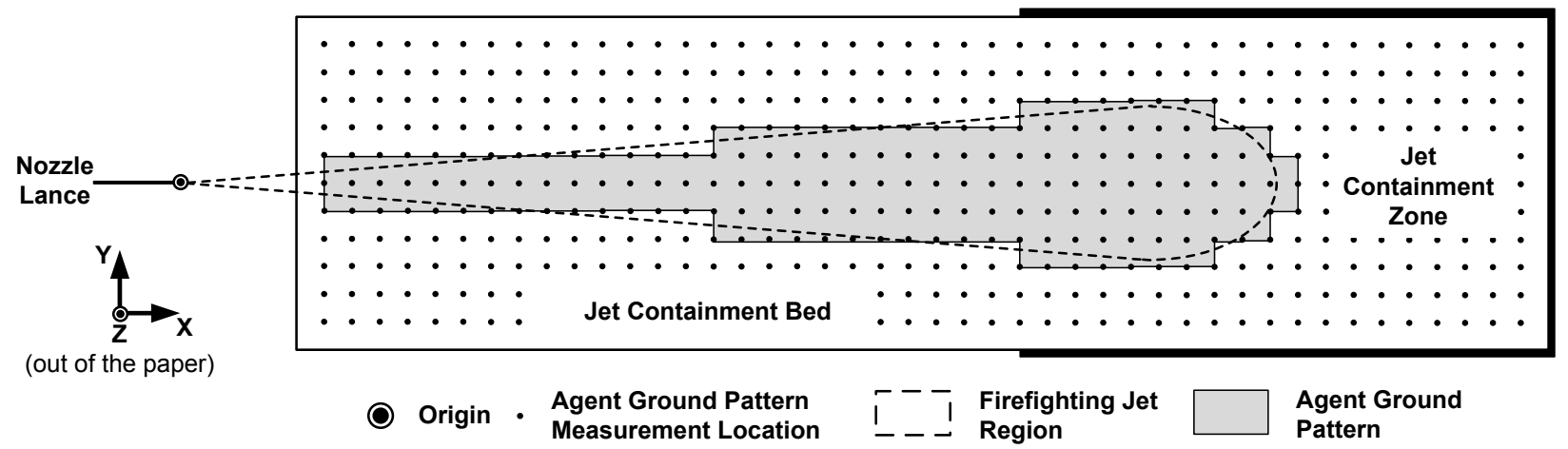

Figure 4.5: An Example Agent Capture Device Footprint Based on Firefighting Jet Delivery Geometry

Full-scale 1-D jet centerline agent ground pattern experiments were conducted in the Sky X Hangar test facility located at Tyndall Air Force Base, FL with the aid of AFRL personnel to supplement laboratory testing. Water accumulation and 6-percent AFFF foam expansion ratio 
patterns were recorded using the same AP4 nozzle family. The nozzles were modified to support a flow rate of $75.71 \cdot \mathrm{min}^{-1}\left(20 \mathrm{gal} \cdot \mathrm{min}^{-1}\right)$ representative of a full-scale firefighting hand line. Nozzle pressures were tested at the same approximate low, medium, and high pressure magnitudes as those in the laboratory. Full-scale test procedures were analogous to laboratory tests except all aspects were scaled upwards to accommodate larger jets including agent capture device volume (100 to $1000 \mathrm{ml}$ ), distance between capture devices (30 to $60 \mathrm{~cm}$ ), and overall measurement length $(15.2$ to $23.2 \mathrm{~m})$. Figures $4.6 \mathrm{a}$ through $\mathrm{d}$ illustrates the full-scale nozzle stand, a water jet and AFFF jet test in progress, and representative agent capture devices filled with foam after an AFFF jet test was completed, respectively.

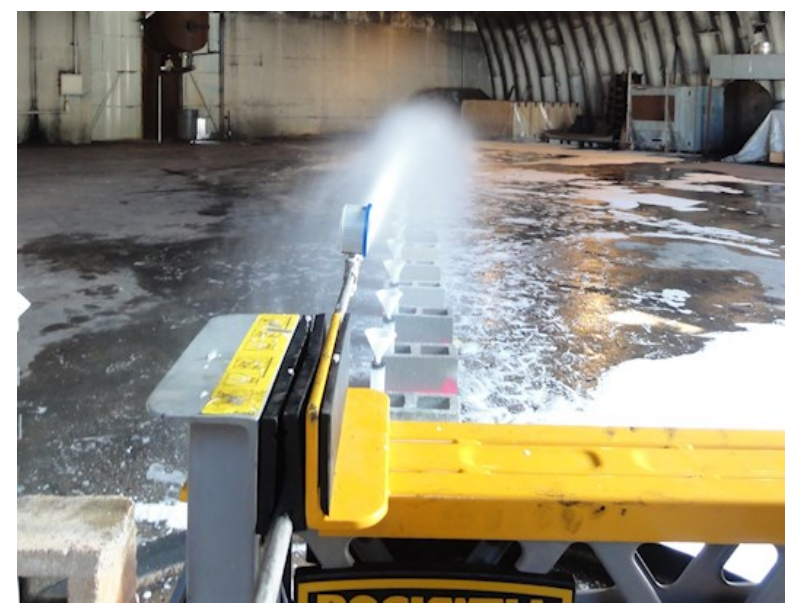

(a)

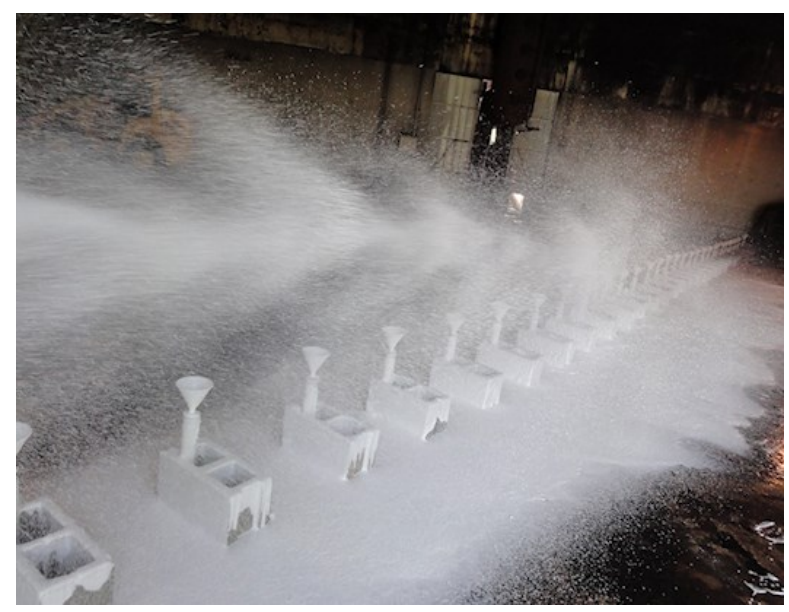

(c)

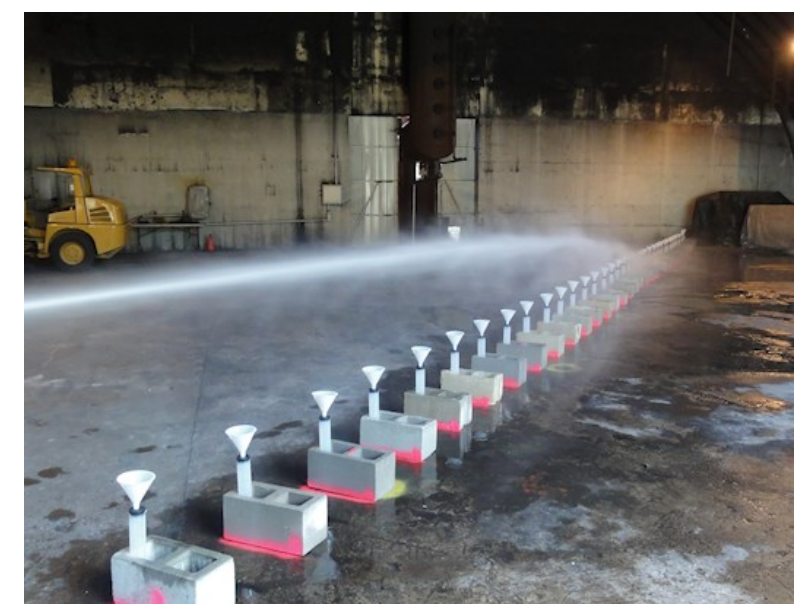

(b)

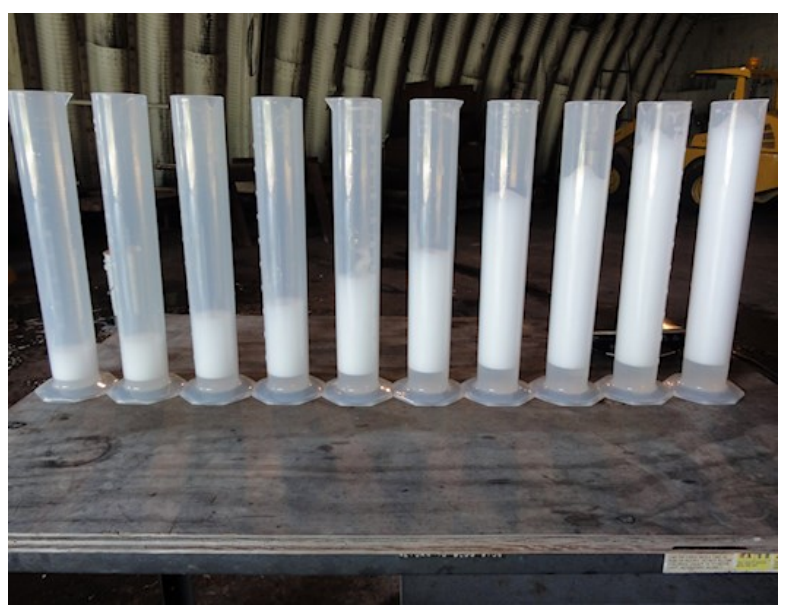

(d)

Figure 4.6: Full-Scale 1-D Agent Ground Pattern Testing 


\subsection{Phase Doppler Particle Analysis}

PDPA was used to measure 2-D (axial $x$-direction and vertical $z$-direction) droplet velocity and droplet size of the firefighting jet configurations illustrated in Figure 4.2 and listed in Table 4.1 in Section 4.1. PDPA data acquisition details are discussed in Section 3.5. For each firefighting jet configuration, nine to eleven 1-D vertical (z-direction) profiles were recorded depending on jet reach. For the medium flow, medium pressure jet, an additional reduced set of 1-D horizontal (y-direction) profiles was also recorded. PDPA profile data was collected first at a specific location on a fully-developed water jet. Once complete, the firefighting agent delivery system was manually transitioned to dispense AFFF while maintaining pump RPM. While the AFFF jet was fully-developing, the PDPA traverse re-traced its steps in space to repeat the measurement process for the AFFF jet at the same discrete point locations where water jet data was just recorded. The tri-axis traverse in tandem with the traverse track allowed profiles to be measured from the nozzle exit to a distance of about 13.7-m (45-ft) downstream. However, the maximum range of all firefighting jet configurations considered for the present study ended at approximately $x=9.14 \mathrm{~m}(30 \mathrm{ft})$, so PDPA data acquisition ceased at that location. Twenty-five discrete points were recorded for each 1-D profile. Points were located close together near the jet axial centerline to resolve steep velocity gradients, and then were rapidly expanded outward to measure as much of the jet profile as possible within the range of the traverse stationed on the track. Uneven time-sampled data were recorded for twenty seconds at each point or a combined 50,000 valid droplet velocity and diameter measurement count, whichever limit was reached first. Vertical profiles were spaced in close proximity to the nozzle to capture large jet velocity gradients and expanded further downstream to increments of about $1.5-\mathrm{m}$ (5-ft) beyond the $x=1.5-\mathrm{m}(5-\mathrm{ft})$ location where changes in jet velocity were less drastic. Vertical profiles were also subject to variation in relative distance from the ground due to profile relaxation from droplet dispersion and overall jet sag due to gravity. These aspects varied for each pressure-flow rate combination. To characterize the horizontal expanse of the medium flow, medium pressure jet, horizontal profiles were measured at four axial locations that coincided with vertical profile locations. These same four axial stations were also chosen to conduct an AFFF solution concentration sensitivity study on medium flow, medium pressure jet conditions from 3 to 9-percent AFFF. Figure 4.7 depicts the location of each PDPA profile and Table 4.2 lists the $x$-axis location of each profile shown along with the agent composition recorded. 

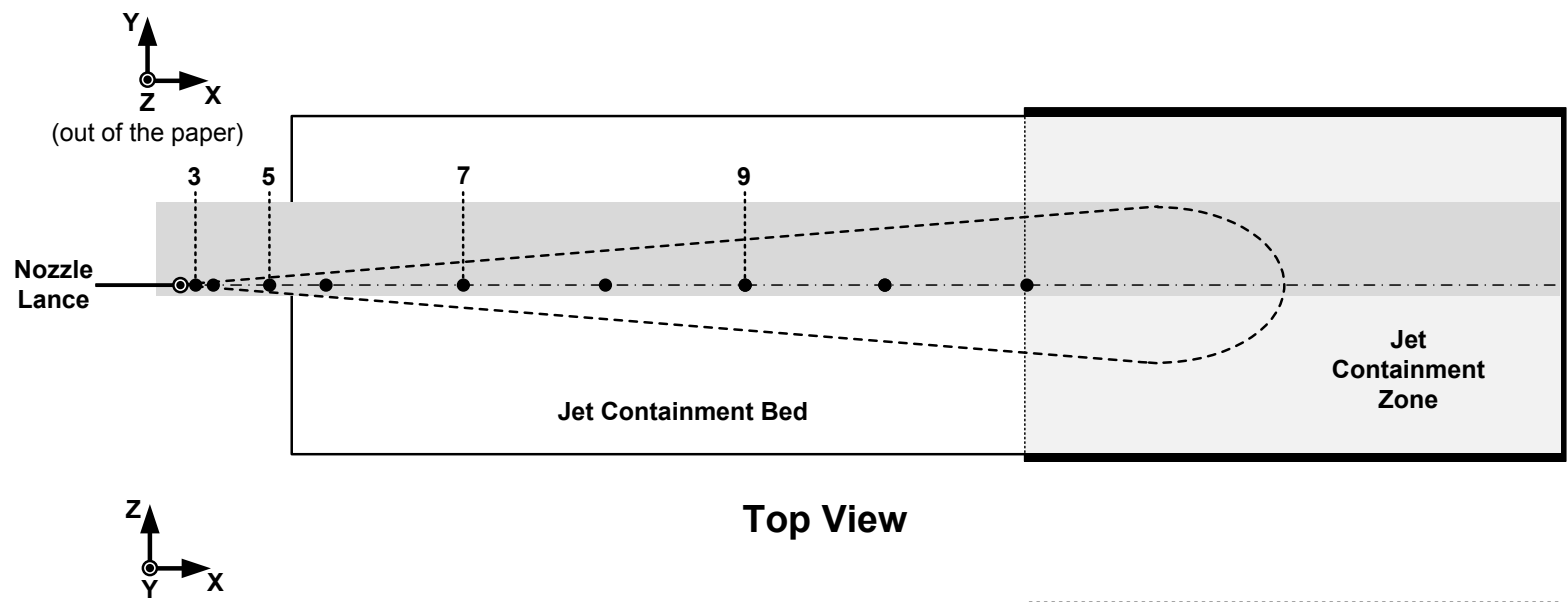

Top View

(into the paper)

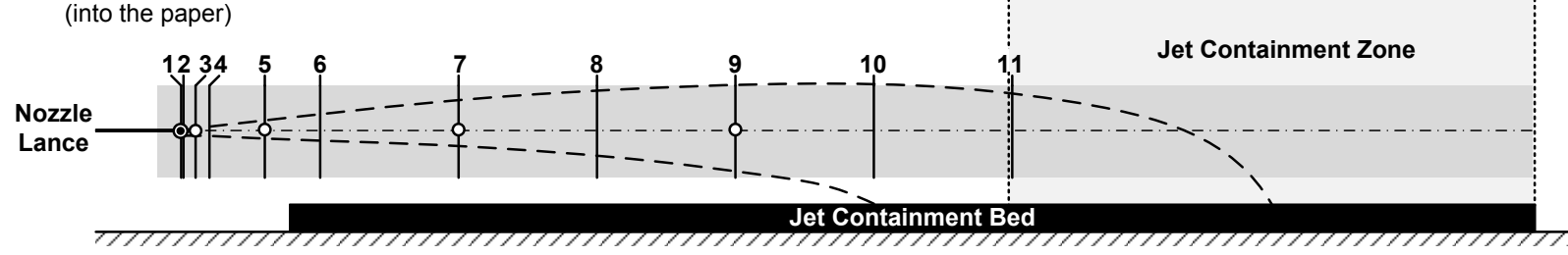

Side View

Origin $\quad$ Y-direction Horizontal PDPA Profiles

Z-direction Vertical

PDPA Profiles
PDPA Measurement

Envelope $\perp-\neg$ Firefighting Jet
$\left\llcorner \_\_\right.$Region

Figure 4.7: PDPA Profile Measurement Locations

Table 4.2: PDPA Profile Measurement Locations

\begin{tabular}{|c|c|c|c|c|c|}
\hline \multirow[b]{2}{*}{$\begin{array}{c}\text { Station } \\
\text { No. }\end{array}$} & \multirow[b]{2}{*}{$\begin{array}{c}\text { X-Axis } \\
\text { Location }\end{array}$} & \multicolumn{2}{|c|}{ Water Jet } & \multicolumn{2}{|c|}{ AFFF Jet } \\
\hline & & $\begin{array}{l}\text { 1-D Vertical } \\
\text { Z-axis Profile }\end{array}$ & $\begin{array}{l}\text { 1-D Horizontal } \\
\text { Y-axis Profile }\end{array}$ & $\begin{array}{l}\text { 1-D Vertical } \\
\text { Z-axis Profile }\end{array}$ & $\begin{array}{l}\text { 1-D Horizontal } \\
\text { Y-axis Profile }\end{array}$ \\
\hline 1 & $6.4 \mathrm{~mm}$ (0.25 in) & $\bullet$ & & 0 & \\
\hline 2 & $25.4 \mathrm{~mm}$ (1 in) & • & & 0 & \\
\hline 3 & $0.152 \mathrm{~m}$ (6 in) & $\bullet$ & $\bullet$ & $\diamond$ & 0 \\
\hline 4 & $0.305 \mathrm{~m}(1 \mathrm{ft})$ & $\bullet$ & & 0 & \\
\hline 5 & $0.914 \mathrm{~m}(3 \mathrm{ft})$ & $\bullet$ & $\bullet$ & $\diamond$ & 0 \\
\hline 6 & $1.52 \mathrm{~m}(5 \mathrm{ft})$ & $\bullet$ & & $\mathbf{0}$ & \\
\hline 7 & $3.05 \mathrm{~m}(10 \mathrm{ft})$ & $\bullet$ & $\bullet$ & $\diamond$ & o \\
\hline 8 & $4.57 \mathrm{~m}(15 \mathrm{ft})$ & • & & 0 & \\
\hline 9 & $6.10 \mathrm{~m}(20 \mathrm{ft})$ & $\bullet$ & • & $\diamond$ & 0 \\
\hline 10 & $7.62 \mathrm{~m}(25 \mathrm{ft})$ & $\bullet$ & & 0 & \\
\hline 11 & $9.14 \mathrm{~m}(30 \mathrm{ft})$ & $\bullet$ & & 0 & \\
\hline
\end{tabular}


PDPA data acquisition was a challenge in peripheral areas of the jet where droplet concentrations were sparse making it difficult to record a large number of samples. Special care was required in conserving AFFF to keep operating costs manageable. Even-time data sampling considerations were universally abandoned because of severely limited data acquisition rates in sparsely populated droplet locations in the flow. Firefighting jet unsteadiness, particularly for low pressure jets where droplet velocities were significantly lower in the far downstream reach of the jet, provided the additional challenge of recording consistent, repeatable data.

Figures $4.8 \mathrm{a}$ and $4.8 \mathrm{~b}$ show the PDPA recording an automated vertical far field profile of both a water and AFFF jet, respectively.

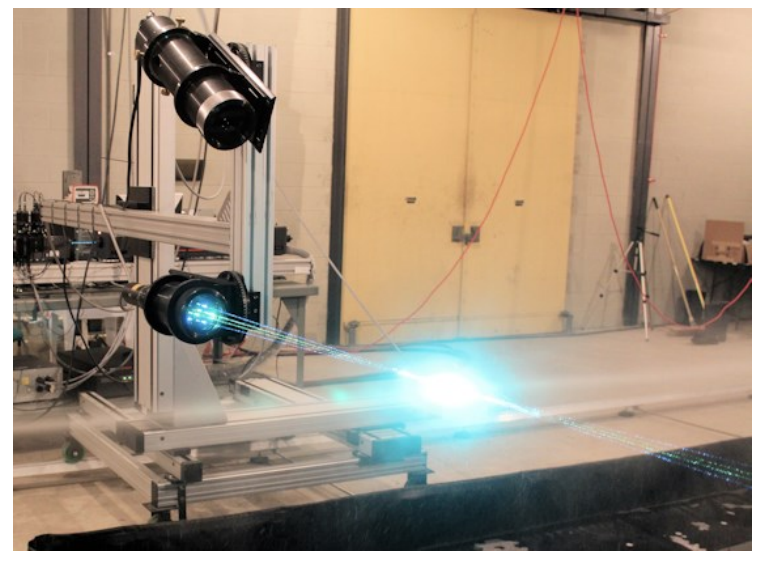

(a)

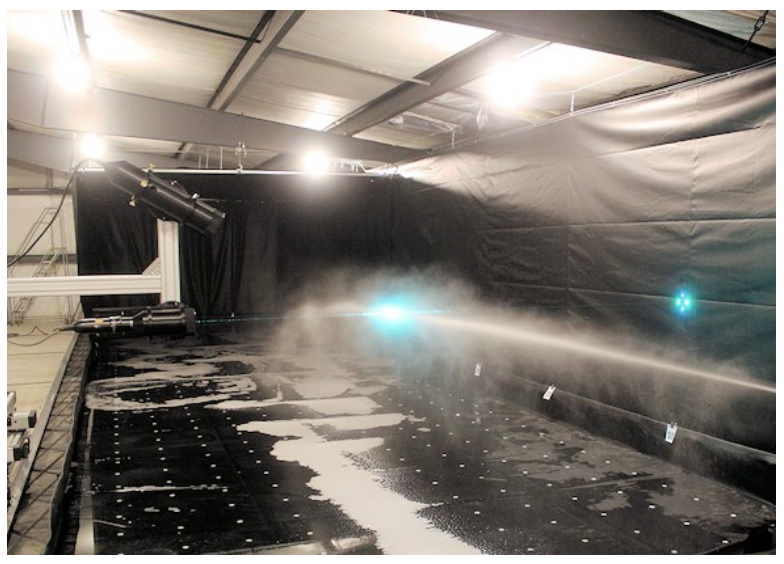

(b)

Figure 4.8: Phase Doppler Data Acquisition on a Low Flow, Low Press. (a) Water Jet (b) AFFF Jet

\subsection{Measurement Error and Uncertainty Analysis}

Measurement error and uncertainty analysis for all quantitative data are addressed in Chapter 6 in context with the results presented. Supplemental commentary on PDPA measurement error and uncertainty is generalized in Section 3.5 using methods developed by the manufacturer, and information regarding measurement uncertainty and instrument calibration specific to the PDPA system used in the current study is in Appendix D: PDPA Calibration. 
CHAPTER 5: COMPUTATIONAL MODELING APPROACH

\subsection{Overview}

CFD models were developed to predict general firefighting jet transport behavior with special attention paid to the influence AFFF has on droplet dynamics. Overall simulation strategy was shaped by the firefighting jet break-up atomization regime classification coupled with data analysis from experiments. Developing a set of consistent modeling techniques for all of the flow regimes examined proved challenging due to the wide range of time and length scales associated with each nozzle condition. Other goals included developing a computational method that was compatible with combustion models to eventually examine scenarios involving aircraft fire and firefighting jet interaction. Model dependence on parameters such as certain CFD physical sub-models, the modeling environment, and on temporal and spatial mesh resolution were also investigated. Solidworks ${ }^{\circledR} 2012$ and Pointwise ${ }^{\circledR}$ v17.0 were used for solid modeling of the flow environment and mesh generation, respectively [71, 72]. ANSYS Fluent ${ }^{\circledR}$ v14.5 was chosen as the CFD modeling software due to its extensive array of multiphase modeling tools and their ease of integration with combustion modeling methods already in use for aircraft hydrocarbon pool fires [56]. ANSYS Fluent ${ }^{\circledR}$ was also used for flow visualization and postprocessing. The CFD simulation strategy and technical details of each CFD sub-model selected, construction of the physical modeling environment, boundary and initial conditions, numerical methods, and overall solution strategy are discussed in the following sections. Table 5.1 lists the CFD case results reported which reflects key PDPA results that were used for model development and comparison. For further details on any of the CFD sub-models presented, refer to the associated reference.

Table 5.1: The Computational Matrix Summarizing Firefighting Jet Simulation Conditions

\begin{tabular}{|c|c|c|c|c|c|c|}
\cline { 2 - 7 } \multicolumn{1}{c|}{} & \multicolumn{3}{c|}{ Water Jet Models } & \multicolumn{3}{c|}{ AFFF Jet Models } \\
\hline CFD & \multicolumn{3}{c|}{ Nozzle Flow Rate } & \multicolumn{3}{c|}{ Nozzle Flow Rate } \\
\hline Nozzle Pressure & Low & Medium & High & Low & Medium & High \\
\hline Low & $\bullet$ & & $\bullet$ & 0 & & 0 \\
\hline Medium & & $\bullet$ & & & 0 & 0 \\
\hline High & $\bullet$ & & $\bullet$ & 0 & & 0 Water $06 \%$ AFFF \\
\hline
\end{tabular}




\subsection{Computational Physical Sub-models}

\subsubsection{Multiphase Flow}

Conventional firefighting jet transport for the flow regimes under investigation is principally dominated by secondary droplet disintegration mechanisms with little evidence to suggest the existence of a continuous liquid core. This infers firefighting jet flow dynamics are governed primarily by droplet trajectories interacting with the surrounding air as well as themselves in terms of collision and break-up. An Euler-Lagrange multiphase modeling architecture best supports these assumptions by treating the atmosphere as a continuum (Eulerian) field by solving the Navier-Stokes equations while tracking firefighting jet droplets as a dispersed (Lagrangian) phase through the calculated flow field. Referred to discrete phase modeling or DPM in ANSYS Fluent $^{\circledR}$, limitations on this computational approach exist when the local volume of dispersed droplets approaches the volume of the discretized continuum field (i.e. local mesh cell volume $<<$ local droplet volume, or about 10 to 12-percent droplet-to-cell volume). However, large droplet-to-air mass ratios are acceptable and still allow for numerically robust simulations. Similar modeling approaches like dense discrete phase modeling remove the limitation of secondary phase high volume loading and may be more accurate for simulating higher flow rates where near-nozzle jet disintegration is not as distinct. However, this method is currently incompatible with combustion models actively used to predict aircraft fire behavior and was thus not considered for the present study [56].

Discrete droplet trajectories were predicted by a force balance integration equating the droplet inertia with the surrounding forces acting on the droplet from the continuous gas phase such as gravity, atmospheric pressure gradients, and aerodynamic drag. The general force balance can be written as [55]:

$$
\frac{d V_{d}}{d t}=F_{D r a g} \cdot V_{d, r e l}+\frac{g\left(\rho_{L}-\rho_{G}\right)}{\rho_{L}}+F
$$

where $V_{d}$ is the droplet velocity, $V_{d, r e l}$ is the relative velocity between the droplet and gas phase, $g$ is the acceleration due to gravity, $t$ is the simulation time, and $F_{D r a g} \cdot V_{d, r e l}$ is the drag force per unit particle mass. The $F$ term is a general acceleration per unit mass term that accounts for 
additional forces such as flow field pressure gradients and virtual mass effects. The drag force is defined as [56]:

$$
F_{\text {Drag }}=\frac{18 \mu_{G}}{\rho_{L} d^{2}} \frac{C_{\text {Drag }} R e_{d}}{24}
$$

where $\mu_{G}$ is the gas phase dynamic viscosity. The $C_{\text {Drag }}$ term is the drag coefficient, and $R e_{d}$ is the relative Reynolds number based on the droplet diameter, defined as [56]:

$$
R e_{d}=\frac{\rho_{G} d\left|V_{d, r e l}\right|}{\mu_{G}}
$$

Droplets were modeled in the present study as adiabatic (non-evaporating) inert particles undergoing collision and break-up due to two-way coupled momentum exchange with the surrounding environment. Momentum exchange was calculated by inspecting the forces imposed on the droplet as it passed through each mesh cell, or control volume, computed as:

$$
F=\sum\left(\frac{18 \mu_{G} C_{\text {Drag }} R e_{d}}{\rho_{L} d^{2} 24} V_{d, r e l}+F_{\text {other }}\right) \dot{m}_{d} \Delta t
$$

where $\dot{m}_{d}$ is the mass flow rate of the droplets and $\Delta t$ is the simulation time step. The $F_{\text {Other }}$ term represents per unit mass forces generated from other CFD sub-models such as turbulence that can play a significant role in altering overall droplet momentum. Mass and heat exchange effects were assumed negligible primarily due to relative humidity measurements approaching 100 percent, particularly in the local jet flow region in the laboratory. This simplification was further supported based on expectedly short in-flight droplet lifetimes coupled with larger droplet diameters with relatively low evaporation rates. Unsteady droplet trajectory calculations were resolved coincidentally with the transient continuous phase solution. If droplets collided, they either bounced off one another or coalesced to form a single droplet. Solution time step size was limited primarily by numerical stability requirements from two-phase solution coupling and secondarily by accuracy requirements induced by droplet collision and break-up sub-models. Too coarse of a time step or mesh resolution caused collision and break-up models to falsely steer the solution. Time step size also affected trajectory accuracy by controlling the accuracy of the direction and distance a droplet must travel with respect to its velocity [56]. 


\section{Droplet Drag}

Droplet drag estimation was approximated using a dynamic drag law that accounted for the effects of droplet distortion observed to be significant in certain circumstances from near field flow visualization photography. Distortion effects are important because drastic changes in droplet shape can affect drag values by as much as 50 percent or more. The dynamic drag law varies the drag between the value for a sphere $\left(C_{\text {Drag,Sphere }}\right)$ and a value of 1.54 corresponding to the shape of a disk parallel to the oncoming flow. The drag coefficient is derived through empirical correlation and defined by [56]:

$$
C_{\text {Drag }}=C_{\text {Drag, Sphere }}(1+2.632 s)
$$

where $C_{\text {Drag,sphere }}$ is determined by:

$$
C_{\text {Drag,Sphere }}=\left\{\begin{array}{cl}
0.424 & R e_{d}>1000 \\
\frac{24}{R e_{d}}\left(1+\frac{1}{6} R e_{d}^{2 / 3}\right) & R e_{d} \leq 1000
\end{array}\right.
$$

The $s$ term is droplet distortion derived from the solution of:

$$
\frac{d^{2} s}{d t^{2}}=\frac{C_{F}}{C_{b}} \frac{\rho_{G}}{\rho_{L}} \frac{V_{d, r e l}^{2}}{r^{2}}-\frac{C_{k} \sigma_{L / G}}{\rho_{L} r^{3}} s-\frac{C_{\beta} \mu_{L}}{\rho_{L} r^{2}} \frac{d s}{d t}
$$

where $C_{F}, C_{b}, C_{k}$, and $C_{\beta}$ terms are dimensionless model constants associated with Taylor's analogy. The $r$ term represents the droplet radius. At maximum distortion $(s=1)$, the drag coefficient asymptotes to a disk. In the limit of no distortion $(s=0)$, the drag coefficient of a sphere is recovered [56].

\section{Droplet Break-up}

Break-up model selection was governed by the jet's characteristic gaseous Weber number regime. Firefighting jets exhibit gaseous Weber numbers typically less than 100 indicating a low gaseous Weber number model was best suited for this study. Vibrational and bag break-up typically govern low gaseous Weber number jet disintegration. The classic Taylor Analogy Break-up (TAB) model was selected due to model implementation simplicity, acceptable 
accuracy for a wide range of industrial flows including the firefighting jets presently studied, as well as numerical speed to achieve a solution compared to alternate break-up models. The TAB model is based on Taylor's analogy between a distorting and oscillating droplet and a spring mass system. The equation governing a damped, forced oscillator is:

$$
F_{E x t}-k x_{e}-\beta \frac{d x_{e}}{d t}=m_{d} \frac{d^{2} x_{e}}{d t^{2}}
$$

where $x_{e}$ is the droplet equator placement from its spherical or undisturbed position, $\beta$ is a damping coefficient, and $m_{d}$ is the mass of the droplet. Model coefficients are derived from Taylor's analogy:

$$
\begin{aligned}
& \frac{F_{E x t}}{m_{d}}=C_{F} \frac{\rho_{G} V_{d, r e l}^{2}}{\rho_{L} r} \\
& \frac{k}{m_{d}}=C_{k} \frac{\sigma_{L / G}}{\rho_{L} r^{3}} \\
& \frac{\beta}{m_{d}}=C_{\beta} \frac{\mu_{L}}{\rho_{L} r^{2}}
\end{aligned}
$$

where droplet drag, surface tension, and viscosity forces are analogous to the restoration of an externally applied aerodynamic force $\left(F_{E x t}\right)$, a spring force defined via a spring stiffness constant $(k)$, and a damping force. The droplet is assumed to break-up if the distortion grows to a critical ratio of the droplet radius defined by:

$$
x_{e}>C_{b} r
$$

Where $C_{b}$ is a constant equal to 0.5 indicating break-up occurs when droplet distortion is equal to half of the droplet radius. This approach assumes the droplet undergoes a single or fundamental oscillation mode. Once the amplitude $(A)$ for an undamped oscillation is calculated using the following equation,

$$
A=\left[\left(s-W e_{T}\right)^{2}+\left(\frac{d s \cdot d t^{-1}}{\omega}\right)^{2}\right]^{0.5}
$$


a solution for determining droplet break-up can be found. By non-dimensionalizing the droplet distortion term $s=x_{e} \cdot C_{b} r^{-1}$, substituting back into Equation 5.6 to yields:

$$
\begin{aligned}
s(t)=W e_{T} & +e^{-\left(t \cdot t_{d}^{-1}\right)}\left[\left(s(0)-W e_{T}\right) \cos (\omega t)\right. \\
& \left.+\frac{1}{\omega}\left(\frac{d s(0)}{d t}+\frac{s(0)-W e_{T}}{t_{d}}\right) \sin (\omega t)\right]
\end{aligned}
$$

For $s>1$, droplet break-up occurs. The term $W e_{T}$ is the modified gaseous Weber number based on droplet radius and Taylor's analogy model break-up coefficients defined as:

$$
W e_{T}=\frac{C_{F}}{C_{k} C_{b}} \frac{\rho_{g} V_{d, r e l}^{2} r}{\sigma_{L / G}}
$$

where droplet lifetime $\left(t_{d}\right)$ and oscillation frequency $(\omega)$ are defined, respectively, as:

$$
\begin{aligned}
& t_{d}=\frac{2}{C_{\beta}} \frac{\rho_{L} r^{2}}{\mu_{L}} \\
& \omega=\left(C_{k} \frac{\sigma_{L / G}}{\rho_{L} r^{3}}-\frac{1}{t_{d}^{2}}\right)^{0.5}
\end{aligned}
$$

The model constants $C_{k}, C_{\beta}$, and $C_{F}$ are a combination of both empirically and theoretically derived terms taken to be 8,5 , and 0.33 , respectively [56].

As droplet oscillations grow toward a critical value, the "parent" droplet breaks up into a number of smaller "child" droplets. As droplets distort from a spherical shape, the dynamic drag law accounts for modifications to the drag coefficient. The size of the child droplet is determined by equating the total energy of the parent drop due to distortion and oscillation to the combined energy of the child droplets, all while enforcing mass conservation. The TAB model is customized to the jet in terms of child droplet resolution when break-up does occur, and can become a limiting factor in terms of defining a smooth child droplet diameter distribution [56].

An alternative break-up model referred to as the Stochastic Secondary Droplet (SSD) model also applicable to low gaseous Weber number flows was also available within ANSYS Fluent ${ }^{\circledR}$. It treats break-up as a discrete random event resulting in a distribution of diameter scales over a 
range independent of parent droplet size. The SSD model is sampled from an analytical solution to the Fokker-Planck equation for the probability distribution of break-up with droplet size distribution based on local conditions. The SSD model provided nearly identical results to that of the TAB model, but was not selected due to reduced computational efficiency [56].

\section{Droplet Collision}

Because firefighting jets consist of billions of droplets, the computational cost to explicitly calculate droplet collision from first principles with respect to the large length scales specifically involved with this study was still prohibitive. To circumvent this issue, parcels or a statistical representation of a number of individual droplets, were used to simulate droplet collision as well as break-up mechanisms. A second order accurate collision algorithm developed by O'Rourke was employed. Its implementation was based on the relationship between a pair of parcels located within the same computational mesh cell. Collision stemmed from a probability distribution based on a Poisson distribution. Collision estimates were calculated with respect to the parcel containing the larger diameter droplet. If both parcels were on a head-on collision or approaching one another at an obtuse angle, collision tended to result in coalescence. If the collision angle was acute, parcels tended to deflect or bounce off one another. Once parcels impacted one another, trajectory was altered regardless of collision type. Collision calculations were based on the collisional Weber number $\left(W e_{C}\right)$, defined as follows [56]:

$$
W e_{C}=\frac{\rho_{G} V_{c, r e l}^{2} D 10}{\sigma}
$$

where $V_{c, \text { rel }}$ is the relative velocity between colliding parcels and D10 is the mean droplet diameter of the involved parcel groups [56].

\subsubsection{Turbulence}

All jet configurations investigated were high Reynolds number flows consistent with conventional firefighting jet classification, thus the role of turbulence was considered. The large eddy simulation (LES) turbulent model was selected for its ability to explicitly resolve largescale eddies primarily dependent on boundary conditions, simulation geometry, and droplet-air interactions. Small-scale eddies were mathematically modeled which tend to be geometrically 
independent, more isotropic, and therefore more universal. However, complex multiphase fluid interactions between the liquid jet and surrounding air can be a viable source of anisotropic turbulence. LES models typically demand more refined meshing requirements due to near wall effects often limiting its industrial application by substantially increasing computational overhead. However, most wall effects were neutralized in the present work because the physical domain of interest had minimal relative wall influence in critical areas of the flow [56].

LES models use a sub-grid scale model to mathematically represent the finer small-scale turbulent eddies in the continuous phase. It should be noted that the effect of droplets have not been included in the sub-grid scale model which can be significant for the flow regimes in the present study. The Wall-Adapting Local Eddy-Viscosity (WALE) sub-grid scale model was employed due to its wide applicability to an assortment of jet flows. The WALE model provided further advantage by returning zero turbulent viscosity in pure laminar areas of the flow field. Alternative sub-grid scale modeling options were incapable of approaching exclusive laminar flow behavior. The WALE model also provided greater numerical stability when solving the firefighting jet flow field compared to other sub-grid scale models [56].

\subsection{Physical Domain}

The physical domain was modeled to represent the extent of the jet containment bed and nozzle stand region. Geometries were kept simple to maintain high grid quality and to focus refinement in areas exposed to high jet shear flow rates particularly local and axially downstream from the nozzle. The nozzle, its stand, and downstream jet containment zone were not explicitly modeled. This approach resulted in a fully orthogonal structured mesh topology illustrated in Figure 5.1. The mesh consisted of approximately 411,000 cells ranging from $2 \mathrm{~cm}$ in the near vicinity of the nozzle to $10 \mathrm{~cm}$ in size in far downstream reaches. The origin, located at the nozzle exit, along with directional orientation was kept consistent with the experimental study. Details on the firefighting jet injection settings into the physical domain are discussed with boundary conditions in Section 5.4. 


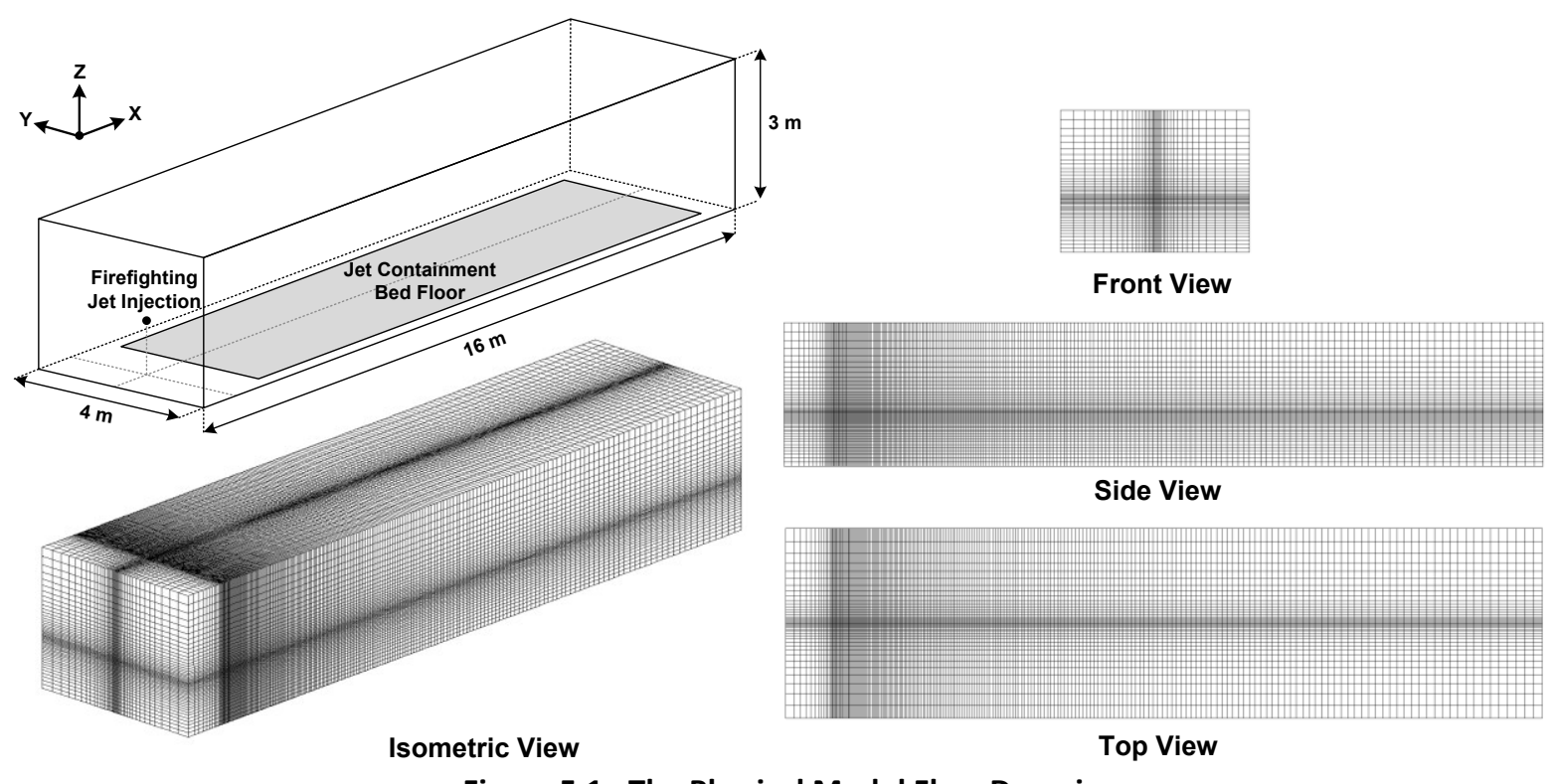

Figure 5.1: The Physical Model Flow Domain

\subsection{Boundary Conditions}

Individual boundary conditions were assigned for the continuous (Eulerian) phase representing the atmosphere as well as the discrete (Lagrangian) phase representing firefighting jet droplet dispersion. The continuous phase ceiling and sides were defined as subsonic pressure outlet boundaries, and the continuous phase floor was defined as a no-slip wall with respect to the air. With air expected to flow in and out of the domain due to jet entrainment, the pressure outlet boundary was selected because the flow direction was allowed to float either inward or outward based on local changes in total pressure. The boundary static pressure was set to $101.3 \mathrm{kPa}$. Figure 5.2 depicts boundary condition assignments for the continuous phase.

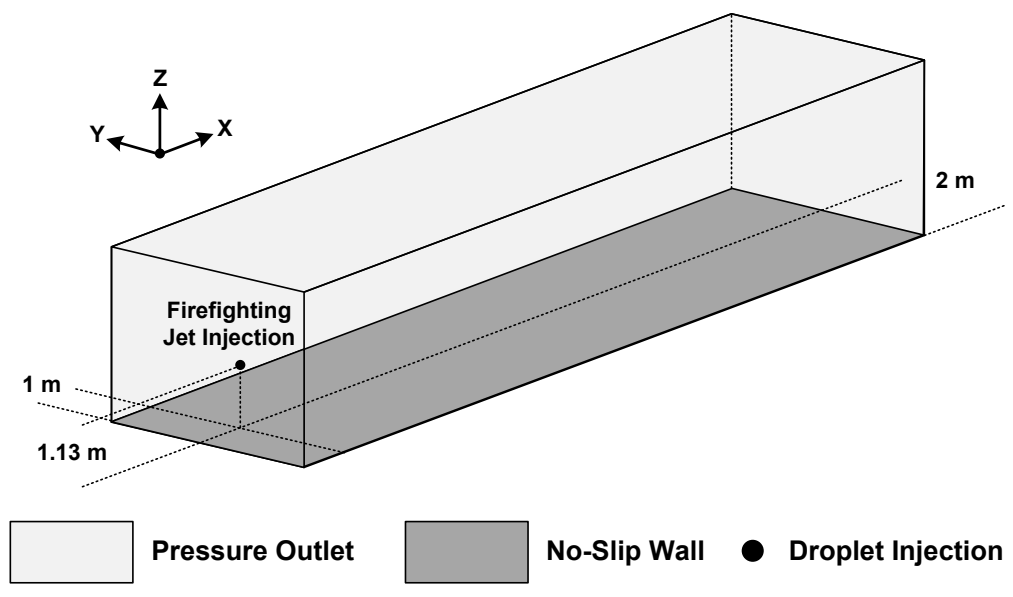

Figure 5.2: Continuum Phase Model Boundary Conditions 
Although the experimental set-up included a containment zone along the back half of the firefighting jet containment bed, it was not integrated into final model results. Its influence was estimated to be minimal based on agent ground pattern testing in comparing results with and without containment zone curtains. These results are reported in Section 6.2.1. A CFD model sensitivity study with and without the presence of the rear jet containment zone was conducted to further examine this issue.

An escape condition was applied for the discrete phase to all domain boundaries. Although in reality the droplets landing on the floor either formed a liquid pool or foam pattern depending on agent composition, this detail was disregarded as the goal of this modeling effort was to analyze the flow dynamics of the firefighting jetting process only. No significant coupling between the firefighting jet and ground pooling behavior was observed experimentally during flow visualization.

Firefighting jet entry into the domain was defined in terms of a single point DPM injection where the nozzle was located in space similar to how experiments were conducted. Unique injection conditions were defined for each firefighting jet condition listed in Table 5.1. Phase Doppler, 1-D vertical profile data recorded closest to the nozzle exit ( $x=6.4-\mathrm{mm}$ or 0.25 in plane) was used to define constant injection conditions. Near nozzle exit perturbation effects were not considered. Droplet injection velocity for all diameter bins was approximated by the maximum profile, mean axial droplet velocity from the $x=6.4-\mathrm{mm}$ plane which was typically located at the central point of where phase Doppler data were collected. Droplet size distribution was approximated using measurements from the same profile point discretized over 25 equal width diameter bins. Mean axial droplet velocities recorded by the PDPA were corroborated by readings from the firefighting agent delivery system flow meter and knowledge of the nozzle diameter for each test case. Flow meter results were also used to define the firefighting jet total mass flow injection rate.

\subsection{Material Properties}

Although all CFD simulations were adiabatic, material properties were defined at $298 \mathrm{~K}$ consistent with the approximate mean laboratory temperature during phase Doppler testing. Compressibility effects were negligible as air flow velocities stayed well below Mach 0.3 and the 
liquid firefighting agent density was assumed to be constant. All material properties were defined as constant values for both the continuous air phase as well as the discrete phase representing the liquid droplets. Material properties were defined uniquely for both water and AFFF including parameters such as density, dynamic viscosity, and surface tension. The differences in these properties, particularly with regard to surface tension, provided the distinguishing influence of AFFF in the computational study. Although AFFF surface tension varies as a function of surface age, due to current CFD sub-modeling software incompatibilities this property was defined as its equilibrium constant. This was considered a reasonable simplification given the average firefighting jet droplet spent most of its in-flight lifetime which was on the order of $1 \mathrm{~s}$ near its equilibrium surface tension value. See Figure 6.78 in Section 6.4.3 for droplet resident time results. For low pressure firefighting jets with low nozzle exit velocities, this argument is strengthened, and vice versa for high pressure firefighting jets with high nozzle exit velocities. All AFFF material properties used in the present study are in Appendix B.

\subsection{Numerics}

All simulations were executed using an implicit, segregated pressure-based solver employing the Semi-Implicit Method for Pressure-Linked Equations (SIMPLE) pressure-velocity coupling algorithm to solve the continuous phase. DPM droplet trajectories were solved using the default automated tracking scheme and employed both a high order trapezoidal and low order implicit discretization method. This approach was favored over alternatives because it handled nonequilibrium flow conditions with greater accuracy. This method also maintains compatibility with the largest number of CFD sub-models capable of imparting forces on droplet parcels likely to be employed in future stages of model development. By default, the CFD software applies the influence of the DPM parcel only to the control volume or mesh cell containing the parcel. As an alternative approach, a high order Gaussian node-based averaging scheme was used to incorporate the influence of surrounding mesh cells on the droplet parcel. This method which employed default scheme values also helped reduce mesh dependence particularly on collision and break-up calculations [55].

Spatial discretization of gradients, pressure, and momentum were enforced using least squares cell-based, second order, and bounded central differencing methods, respectively. Transient 
formulations were carried out using bounded second order implicit techniques. Bounded methods are preferred over unbounded methods due to their added numerical stability. Underrelaxation factors were kept at default software values. For additional details regarding aspects of the numerical strategy, the reader is referred to the ANSYS Fluent Theory Guide [55].

\subsection{Solution Strategy}

Pre-processing, preliminary CFD calculations, and post-processing were conducted on a 3.46-Ghz Intel Xeon ${ }^{\circledR}$ Westmere-EP 12-core Linux server with 48 GB of shared memory located in the WVU Engineering Data Center. Final production computations were carried out at the U.S. Army Engineering Research and Development Center DOD Supercomputing Resource Center on a SGI Altix Ice 8200. The SGI is capable of 172 teraflops operating 1,920 2.8-Ghz Intel Xeon ${ }^{\circledR}$ (Nehalem-EP) nodes, 8-cores per node, with 48-GB of shared memory per node. The SGI uses 4X DDR Infiniband for distributed (parallel) computing across nodes.

The physical domain was initialized to static pressure and zero velocity conditions. For each firefighting jet configuration, droplet injections were enforced at each time step to match the total mass flow rate of the jet at the location shown in Figure 5.2. After a temporal sensitivity study was conducted, A 1-ms fixed time step was chosen for all firefighting jet simulation cases with the continuous and discrete phase coupled after every time step. Inner temporal iterations were limited to 30 with convergence set at the scaled residual default of 0.001 . Conservation of the coupled droplet-air mass and momentum convergence was achieved on most occasions but not for every single time step. However, residuals dropped a minimum of three orders of magnitude every time step exhibiting excellent convergence consistency and numerical stability. Simulations were solved for $10 \mathrm{~s}$ in physical time to ensure each firefighting jet was fully developed for analysis and comparison to phase Doppler data. Droplet velocity and size data were tabulated as they passed through virtual planes positioned normal to the jet centerline axis defined in the same axial location as phase Doppler vertical profiles. Solutions took about 375 central processing unit (CPU) hours to complete. Sixty-four to 128 parallel CPUs per simulation on DOD SGI resources were utilized which equated to about four to six hours of real time based on machine load to solve each CFD model case. 
CHAPTER 6: RESULTS

\subsection{Experimental Firefighting Jet Flow Visualization Results}

Flow visualization was recorded on the ten firefighting jet configurations presented in Section 4.1. Each configuration is illustrated in Figure 4.2 and listed in Table 4.1 in Section 4.1. Details on the experimental approach are in Section 4.2. The ten configurations represented five nozzle pressure-flow rate combinations using water and 6-percent AFFF as the agent. Images were recorded at far, middle, and near field points of observation. All photographs were converted to grey scale with their brightness and contrast optimized to better distinguish firefighting jet features from their surroundings. Standard photographs taken of middle and high pressure jets were slightly blurry compared to low pressure jets at the expense of camera shutter speed to allow for great illumination of the subject matter. Although laser sheet lighting methods alleviated most of these issues, this process was limited by the maximum sweep speed achievable by the galvanometer generating the laser sheet which was still lower than jet speeds reached near the nozzle for medium and high pressure jets. However, galvanometer speeds were adequate for all firefighting jets flow visualization further downstream.

\subsubsection{Far Field Flow Visualization Results}

Figures 6.1 through 6.10 illustrate the firefighting jet far field in the axial direction using standard photography methods. White square makers were stationed along the lip of the jet

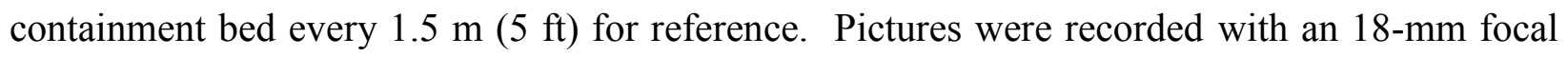
length for wide angle capture of the jet parallel to the $y=0$-m plane. The camera was positioned against the opposing laboratory wall to maximize viewing area. The effective reach of all firefighting jets fell within the bounds of the frame except for high flow rate, low pressure jets which extended 1 to $2-\mathrm{m}$ beyond.

Based on these photographs, low pressure jets stayed intact demonstrating a more cohesive stream compared to medium and high pressure jets which exhibited enhanced break-up and relatively larger-scale turbulent eddy formation and dispersion due to an increase in jet Reynolds number. As flow rate was increased, an increase in jet reach was observed due to an increase in axial momentum. High pressure jets were less affected by gravity and exhibited more axial 
symmetry transporting smaller, less massive droplets at higher axial velocities compared to low pressure droplets composed of larger, more massive droplets at lower axial velocities.

Qualitative structural differences between water and AFFF jets were difficult to perceive because AFFF jets were significantly more opaque, even when nozzle conditions (i.e. pressure and flow rate) were held constant. The blackout curtain added to provide background contrast remedied this issue somewhat, but improvements were marginal. AFFF jets appeared fuller or thicker which was assumed to be due to a larger concentration of droplets forming downstream compared to water jets. Figures 6.11 through 6.15 illustrate the firefighting jet far field in the axial direction, which were photographs taken at an oblique angle from behind and above the nozzle focused on the $z=0-\mathrm{m}$ plane and illuminated by a horizontal laser light sheet. Figures 6.16 through 6.20 depict an oblique horizontal view across the $y$-direction of each firefighting jet along the $x=6.1-\mathrm{m}(20-\mathrm{ft})$ plane and were also illuminated by a vertical laser light sheet. This plane approximates the axial midpoint for medium and high flow rate jets. Figures 6.11 through 6.20 support the same observations as those stated for Figures 6.1 through 6.10.

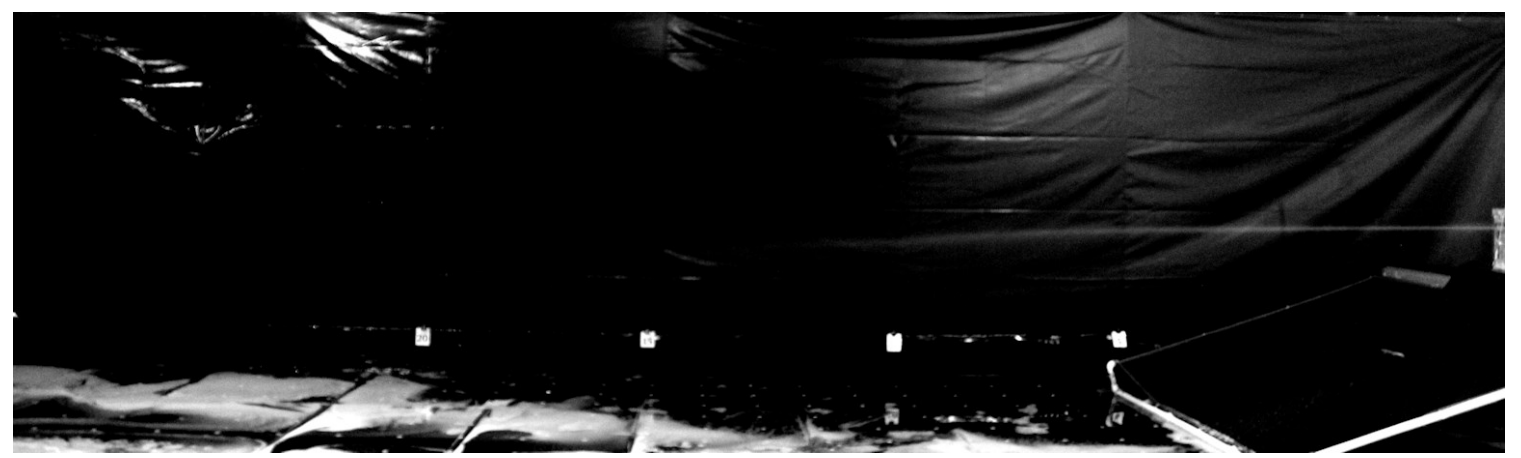

Figure 6.1: Standard Far field Flow Visualization Parallel to the $y=0-m$ Plane: Low Flow, Low Press. Water Jet (Flow is from Right to Left)

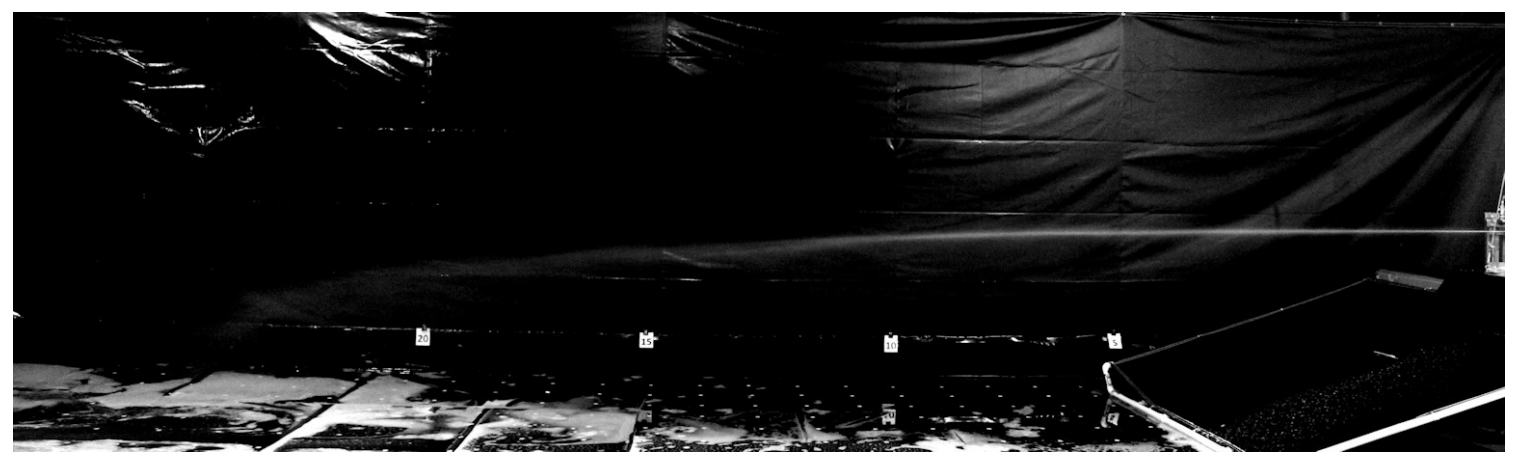

Figure 6.2: Standard Far field Flow Visualization Parallel to the $y=0-m$ Plane: Low Flow, Low Press. AFFF Jet (Flow is from Right to Left) 


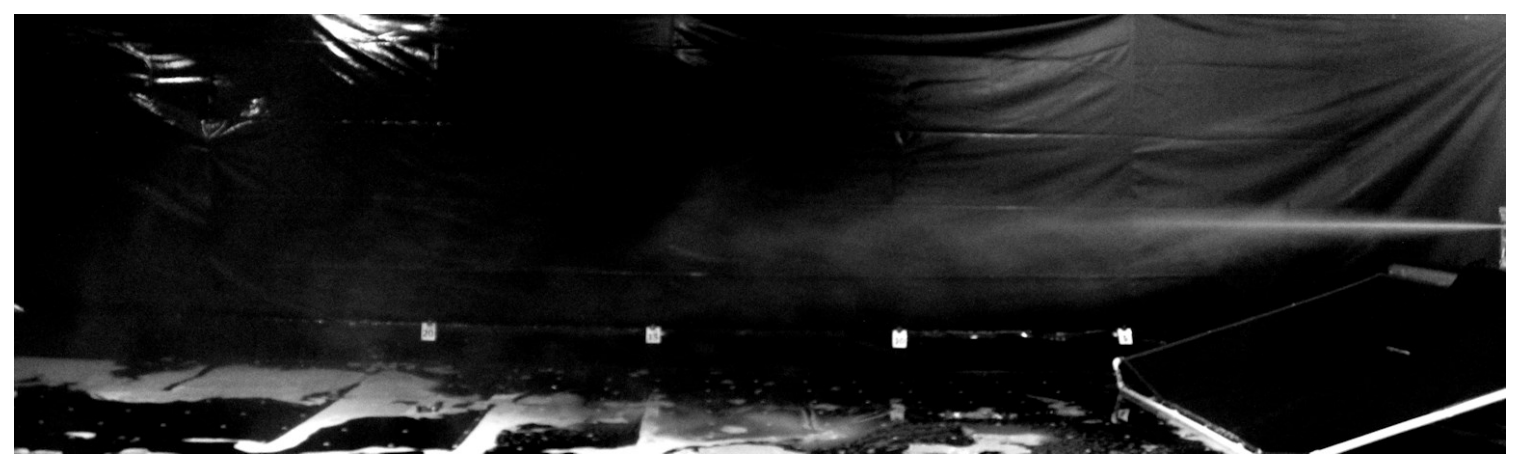

Figure 6.3: Standard Far Field Flow Visualization Parallel to the $y=0-m$ Plane: Low Flow, High Press. Water Jet (Flow is from Right to Left)

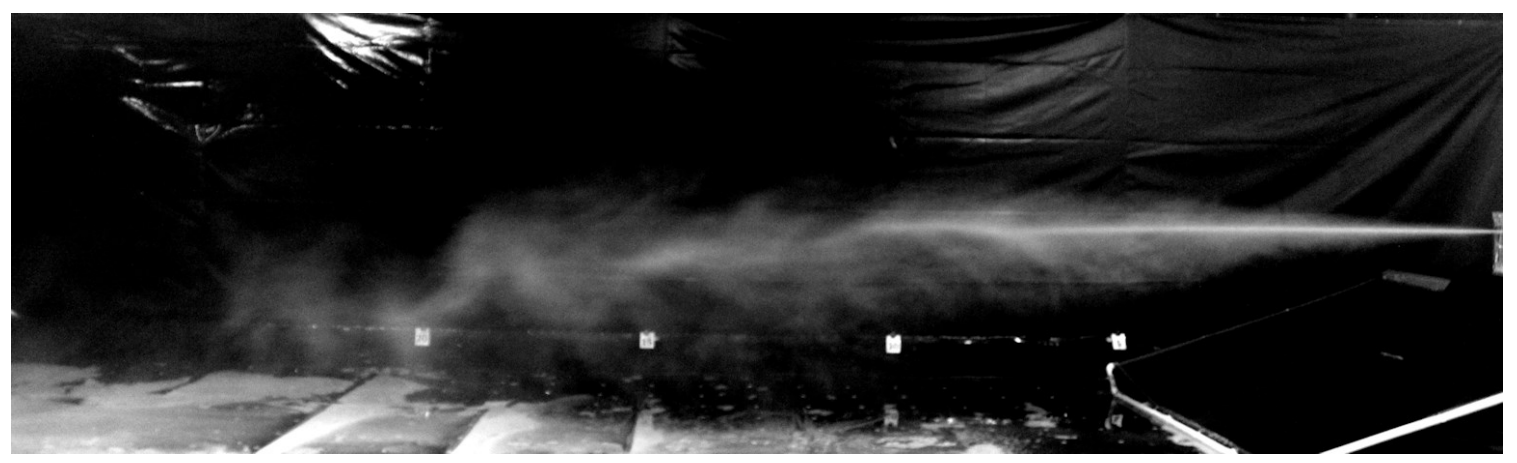

Figure 6.4: Standard Far field Flow Visualization Parallel to the $y=0-m$ Plane: Low Flow, High Press. AFFF Jet (Flow is from Right to Left)

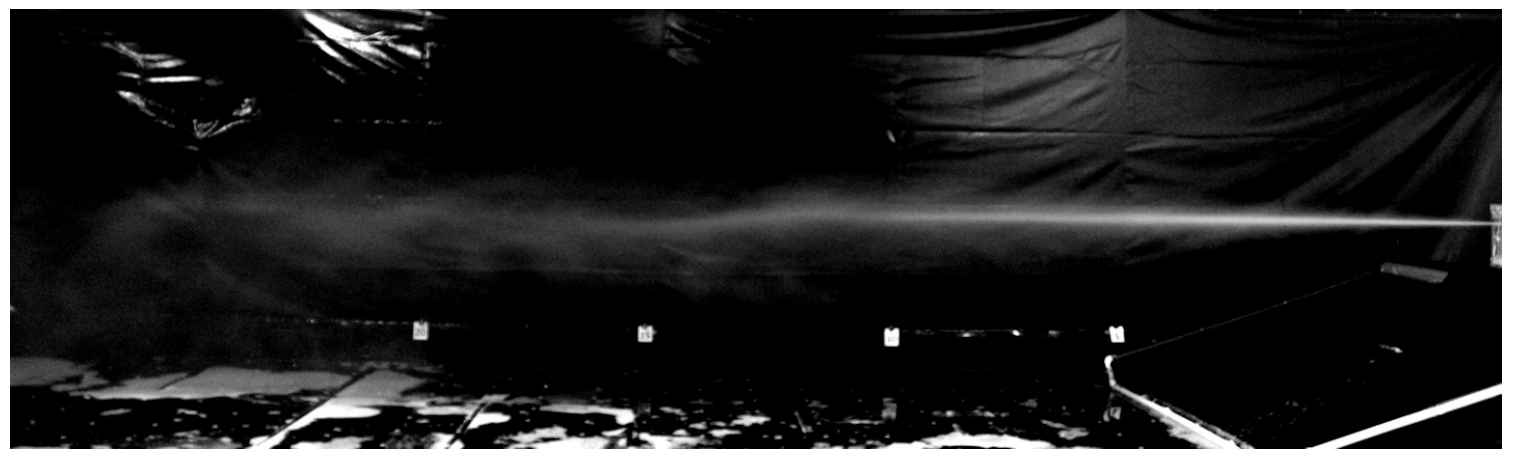

Figure 6.5: Standard Far Field Flow Visualization Parallel to the $y=0-m$ Plane: Med. Flow, Med. Press. Water Jet (Flow is from Right to Left)

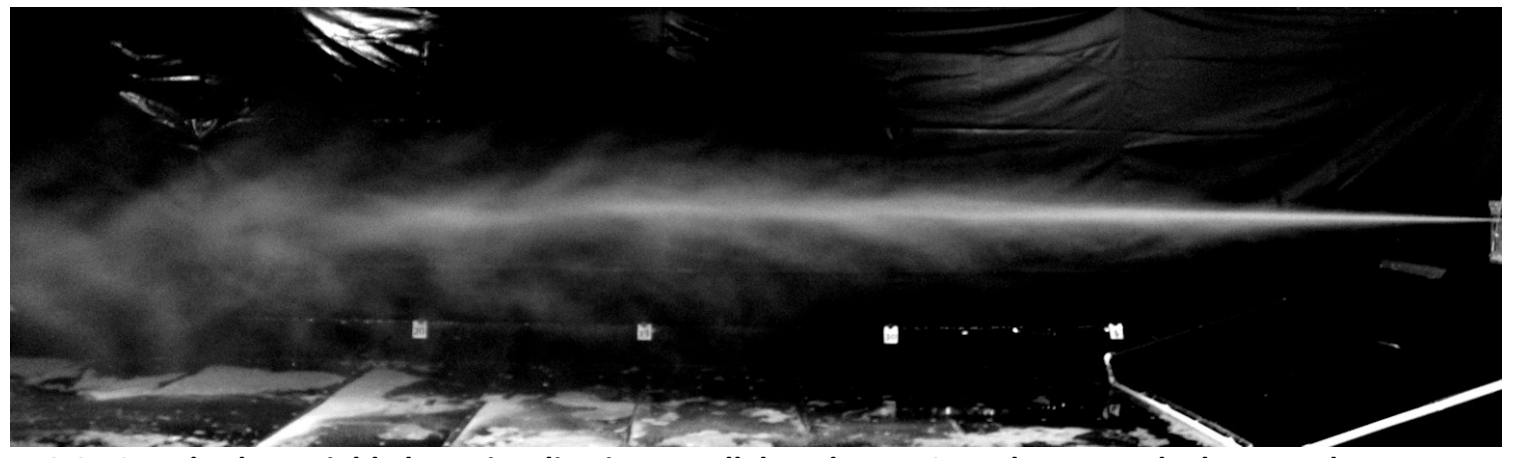

Figure 6.6: Standard Far Field Flow Visualization Parallel to the $y=0-m$ Plane: Med. Flow, Med. Press. AfFF Jet (Flow is from Right to Left) 


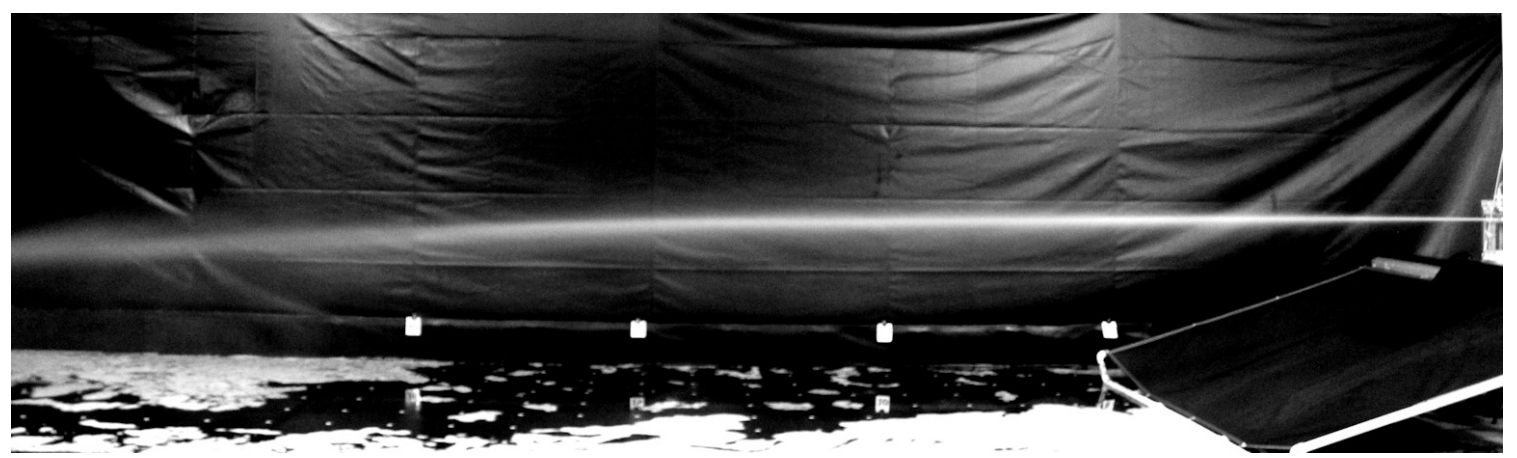

Figure 6.7: Standard Far Field Flow Visualization Parallel to the $y=0-\mathrm{m}$ Plane: High Flow, Low Press. Water Jet (Flow is from Right to Left)

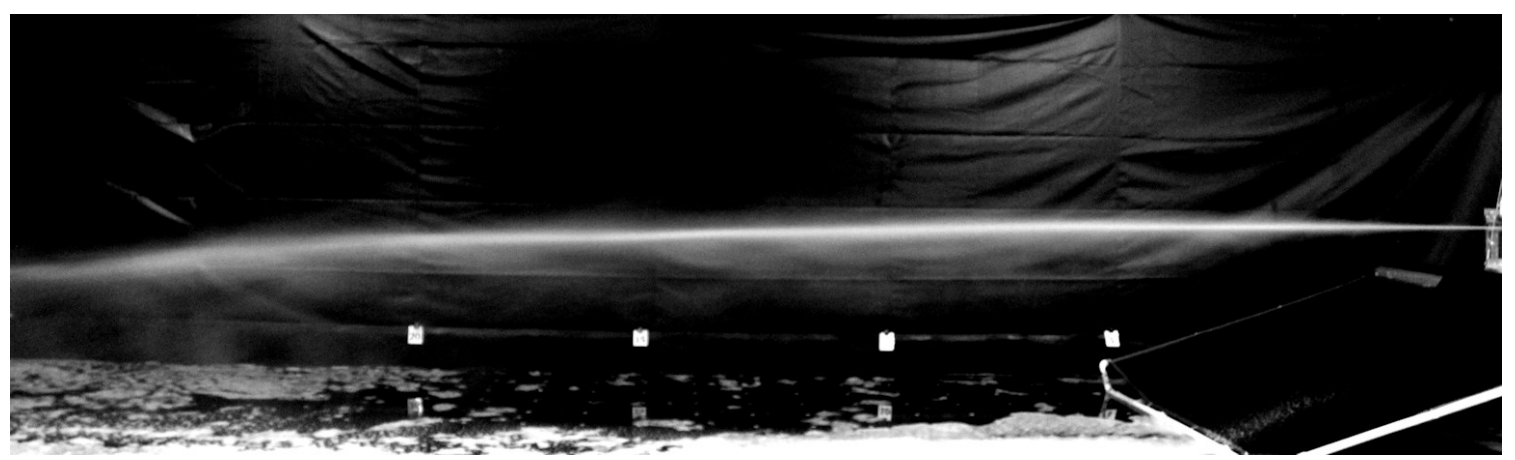

Figure 6.8: Standard Far Field Flow Visualization Parallel to the $y=0-m$ Plane: High Flow, Low Press. AfFF Jet (Flow is from Right to Left)

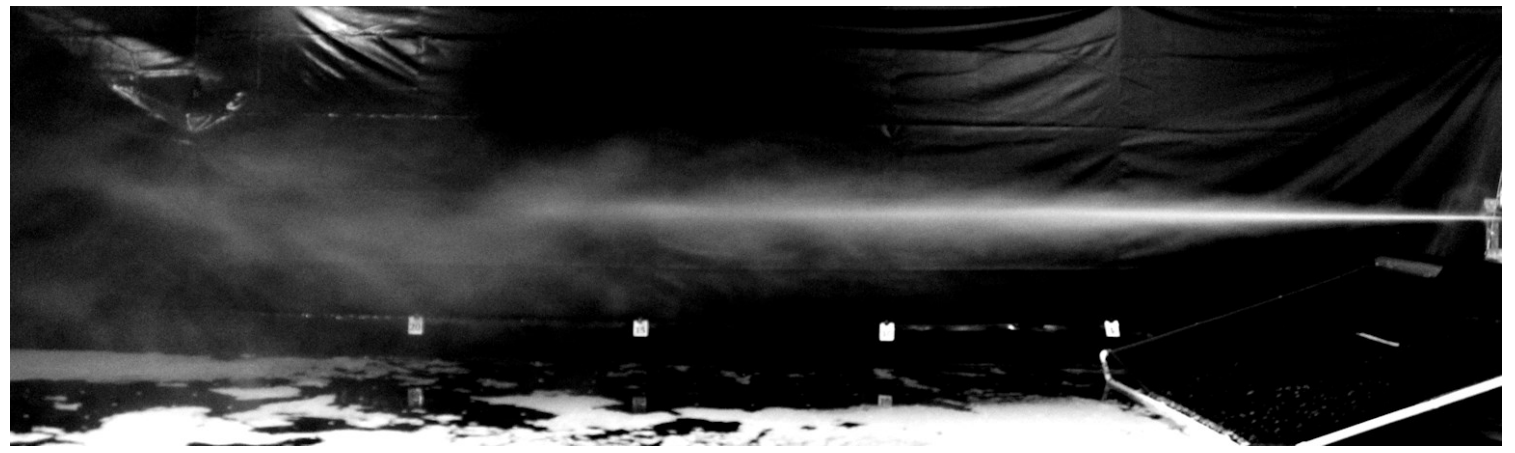

Figure 6.9: Standard Far Field Flow Visualization Parallel to the $y=0-m$ Plane: High Flow, High Press. Water Jet (Flow is from Right to Left)

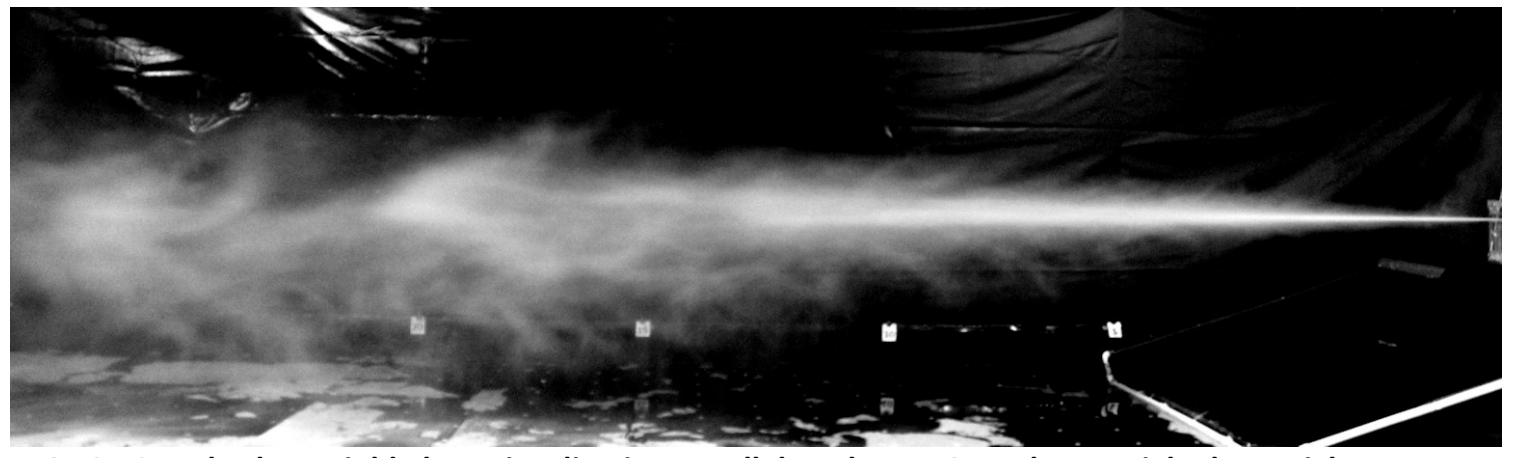

Figure 6.10: Standard Far Field Flow Visualization Parallel to the $y=0-m$ Plane: High Flow, High Press. AffF Jet (Flow is from Right to Left) 


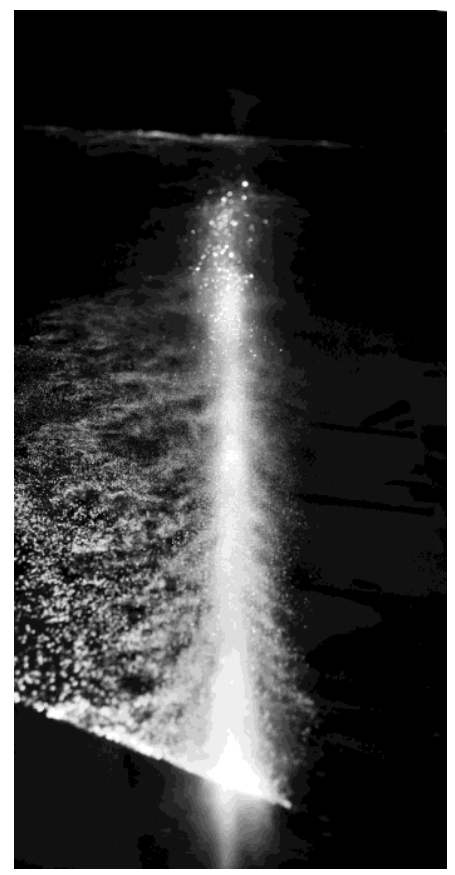

(a)

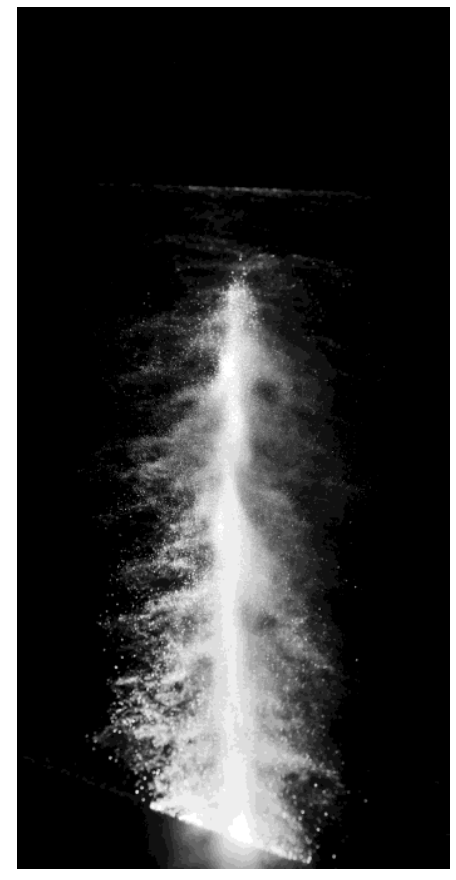

(b)

Figure 6.11: Laser Sheet Far Field Flow Visualization Oblique to the $z=0-m$ Plane: (a) Low Flow, Low Press. Water Jet (b) Low Flow, Low Press. AFFF Jet (Flow is from Bottom to Top)

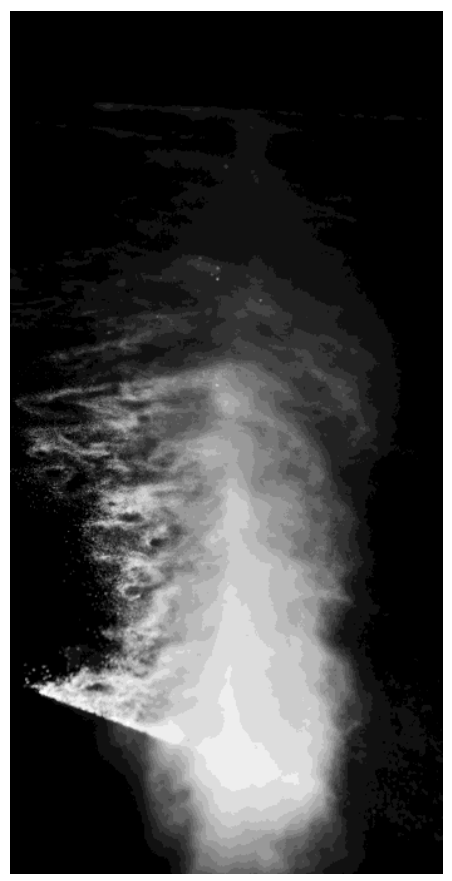

(a)

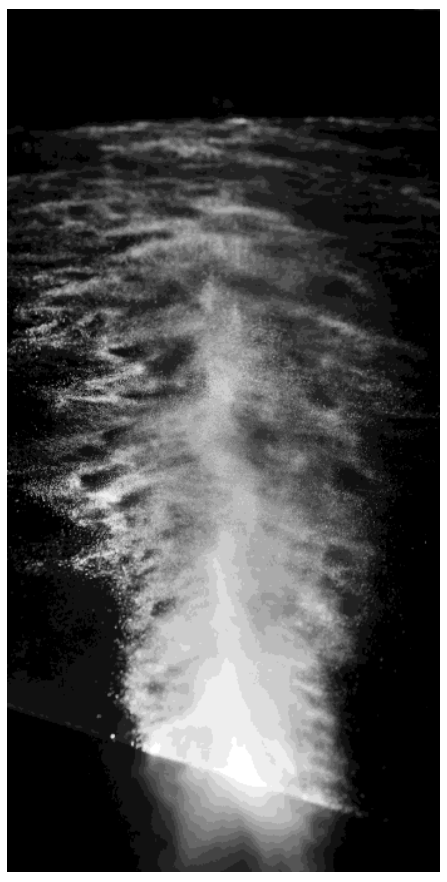

(b)

Figure 6.12: Laser Sheet Far Field Flow Visualization Oblique to the $z=0-m$ Plane: (a) Low Flow, High Press. Water Jet (b) Low Flow, High Press. AFFF Jet (Flow is from Bottom to Top) 


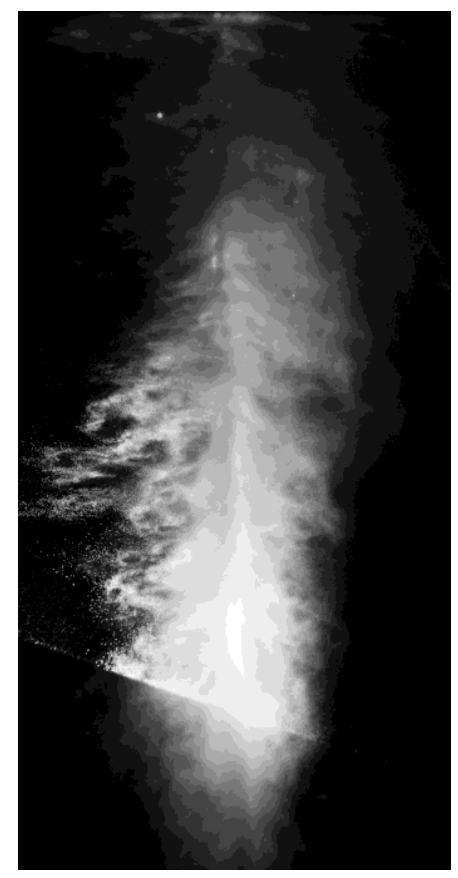

(a)

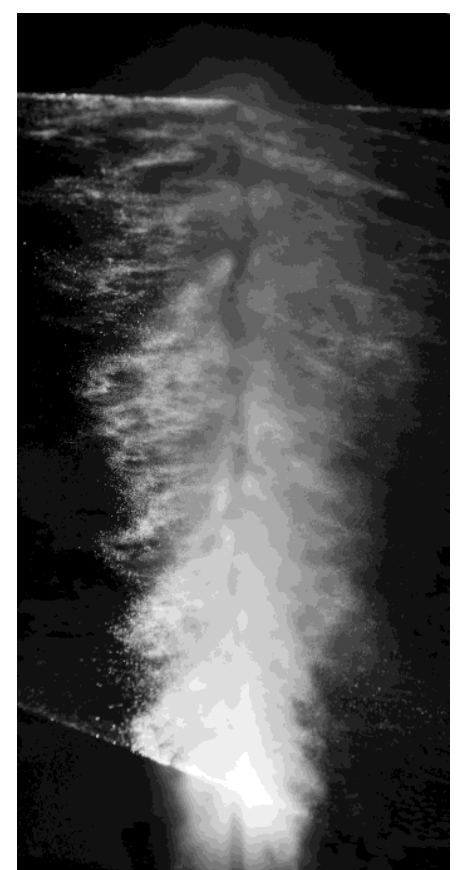

(b)

Figure 6.13: Laser Sheet Far Field Flow Visualization Oblique to the $z=0-m$ Plane: (a) Med. Flow, Med. Press Water Jet (b) Med. Flow, Med. Press. AFFF Jet (Flow is from Bottom to Top)

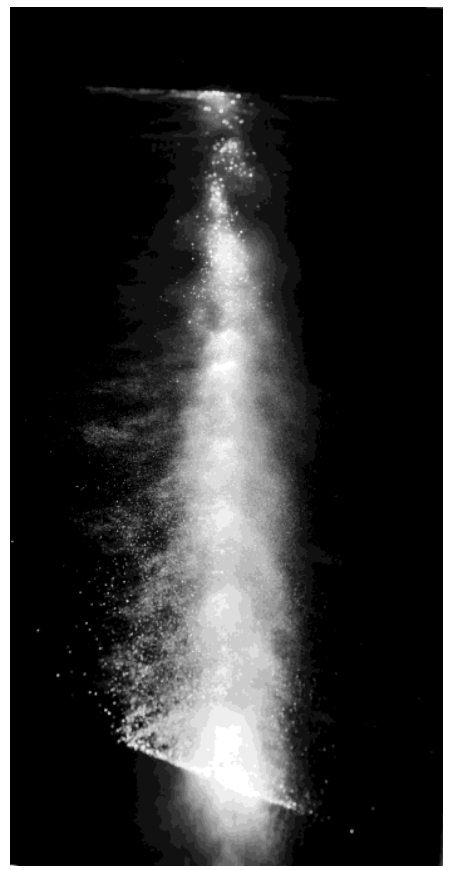

(a)

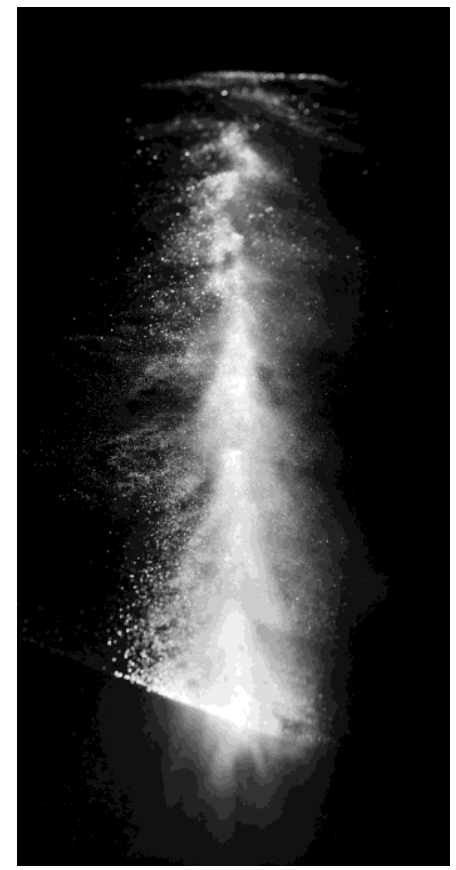

(b)

Figure 6.14: Laser Sheet Far Field Flow Visualization Oblique to the $z=0-m$ Plane: High Flow, Low Press. Water Jet (b) High Flow, Low Press. AFFF Jet (Flow is from Bottom to Top) 


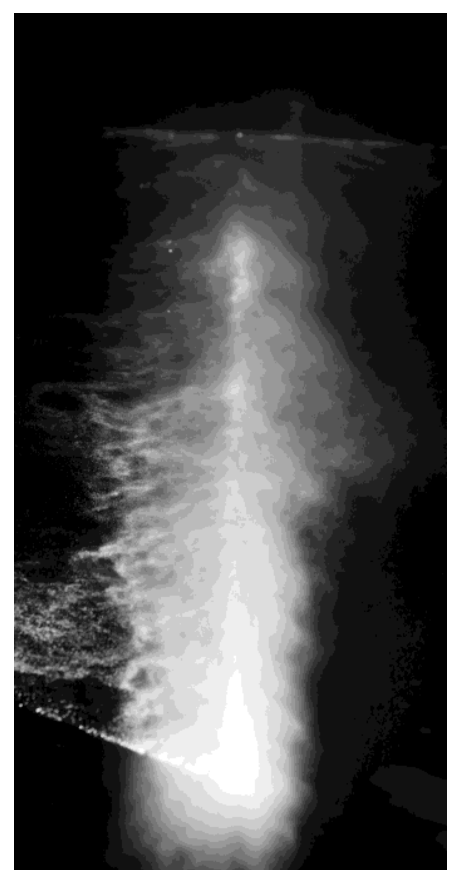

(a)

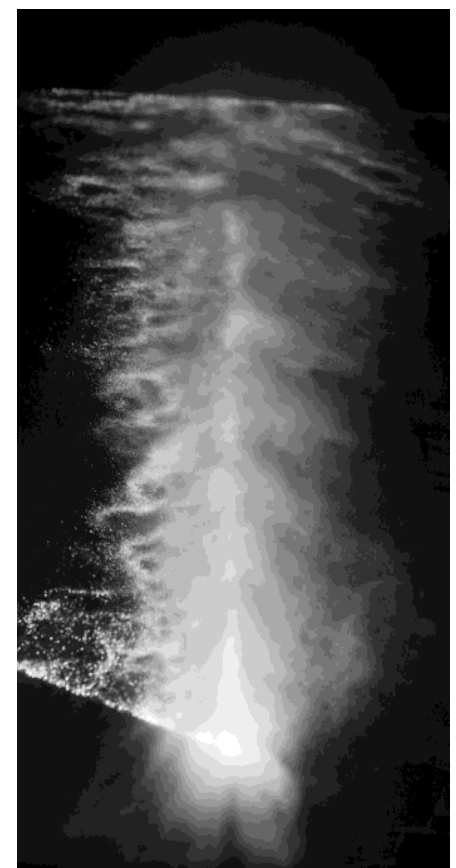

(b)

Figure 6.15: Laser Sheet Far Field Flow Visualization Oblique to the $z=0-m$ Plane: High Flow, High Press. Water Jet (b) High Flow, High Press. AFFF Jet (Flow is from Bottom to Top)

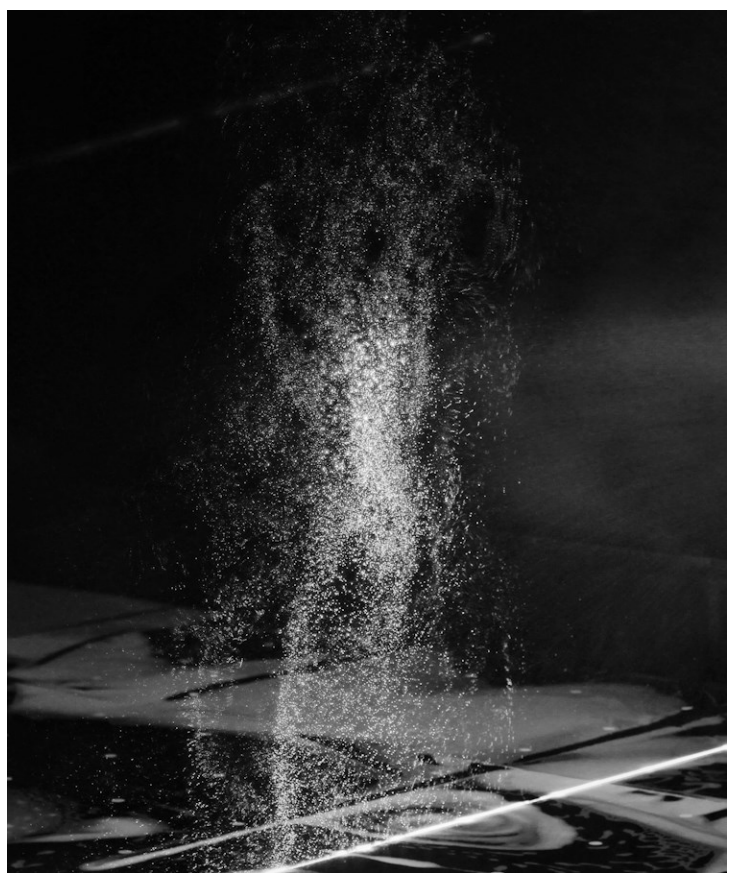

(a)

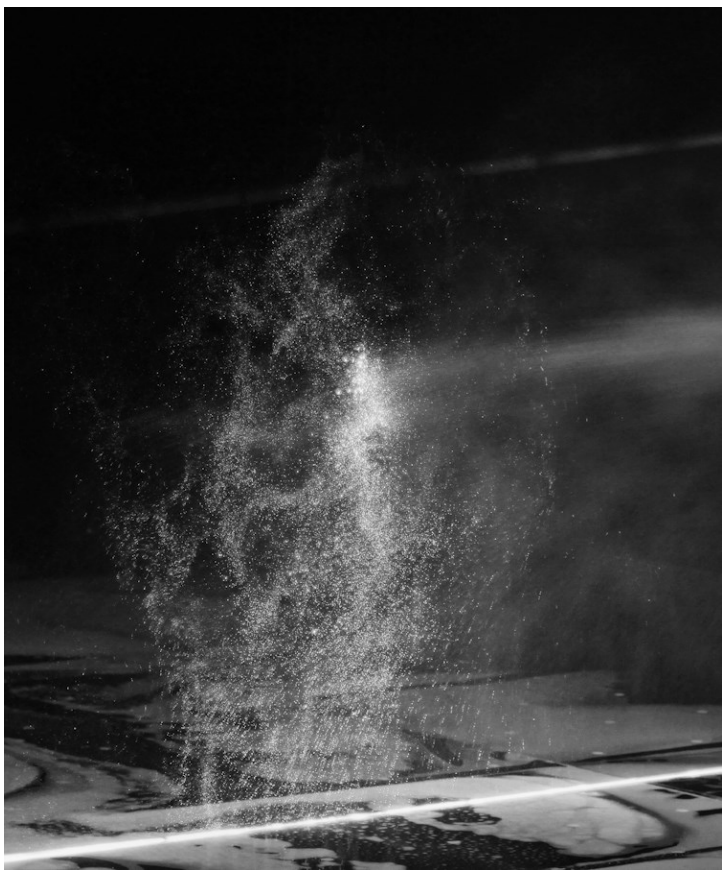

(b)

Figure 6.16: Laser Sheet Far Field Flow Visualization Oblique to the $x=6.1-m$ Plane: (a) Low Flow, Low Press. Water Jet (b) Low Flow, Low Press. AFFF Jet (Flow is from Bottom to Top) 


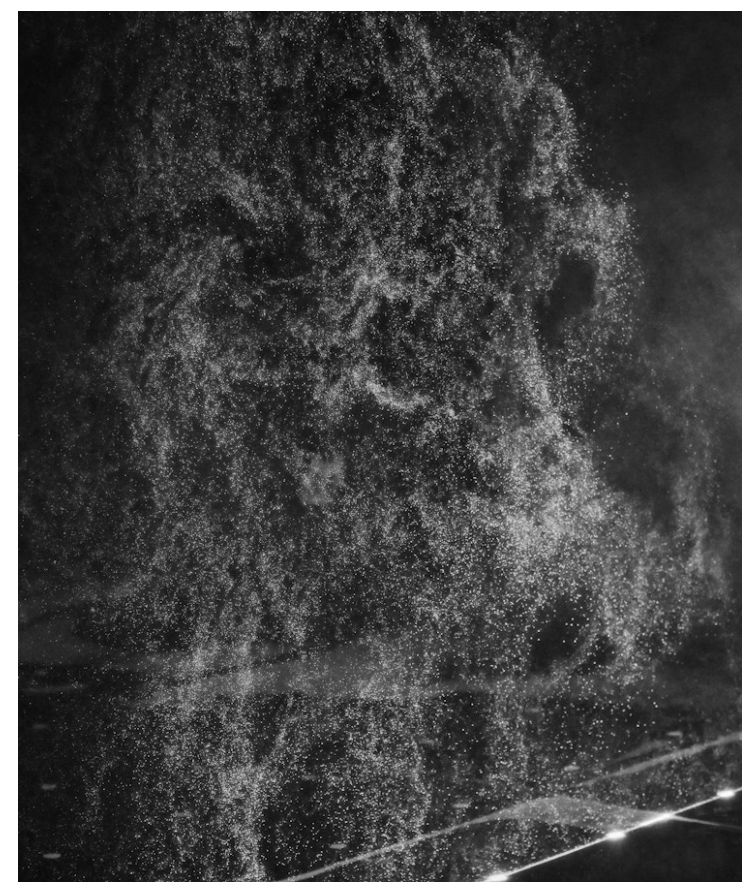

(a)

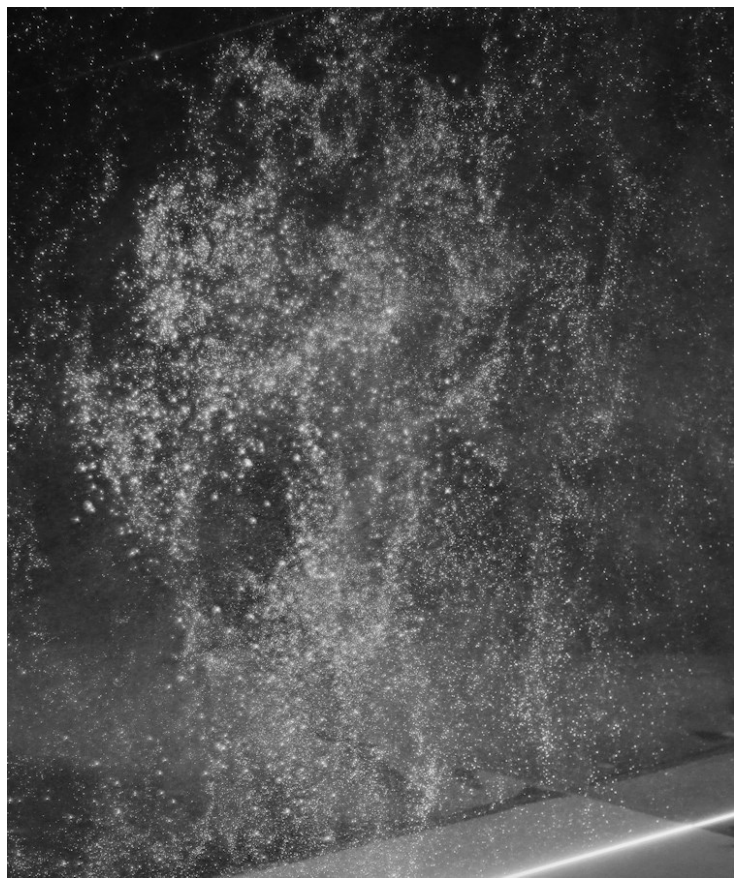

(b)

Figure 6.17: Laser Sheet Far Field Flow Visualization Oblique to the $x=6.1-\mathrm{m}$ Plane: Low Flow, High Press. Water Jet (b) Low Flow, High Press. AFFF Jet (Flow is from Right to Left)

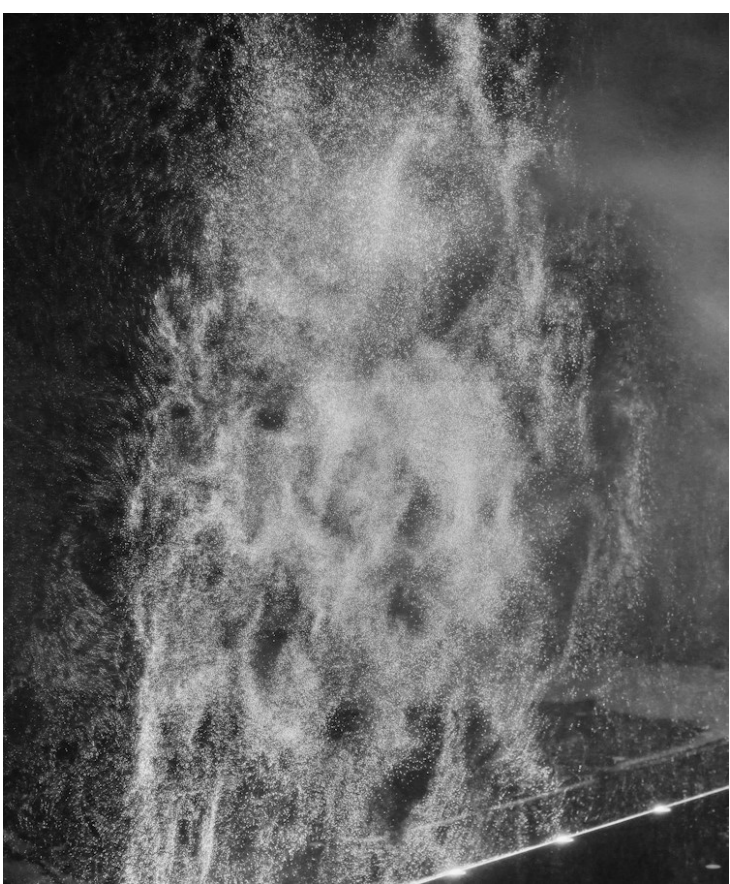

(a)

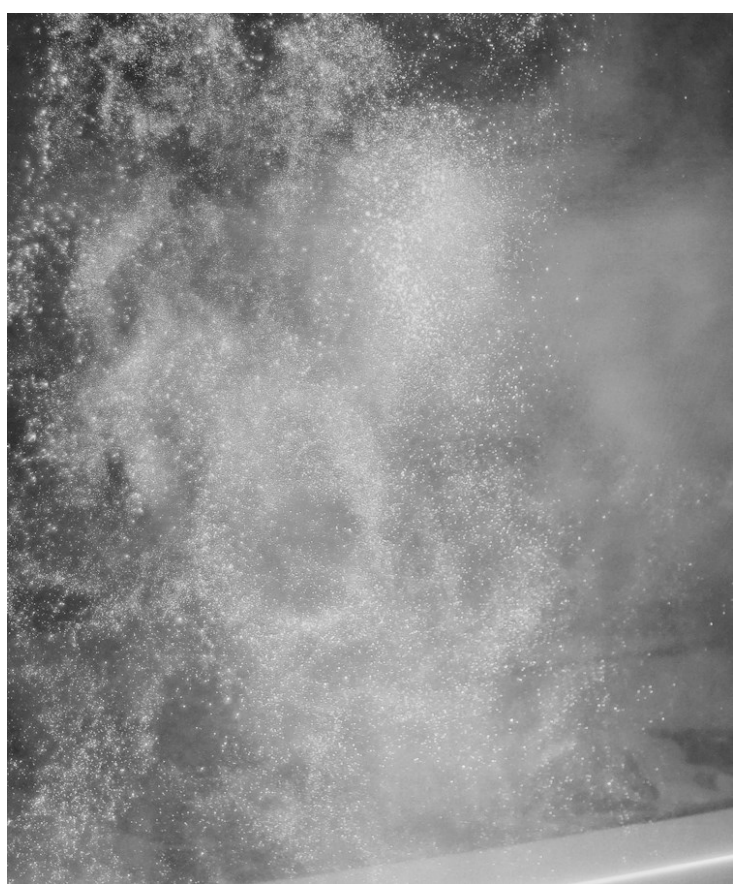

(b)

Figure 6.18: Laser Sheet Far Field Flow Visualization Oblique to the $x=6.1-\mathrm{m}$ Plane: (a) Med. Flow, Med Press. Water Jet (b) Med. Flow, Med. Press. AFFF Jet (Flow is from Right to Left) 


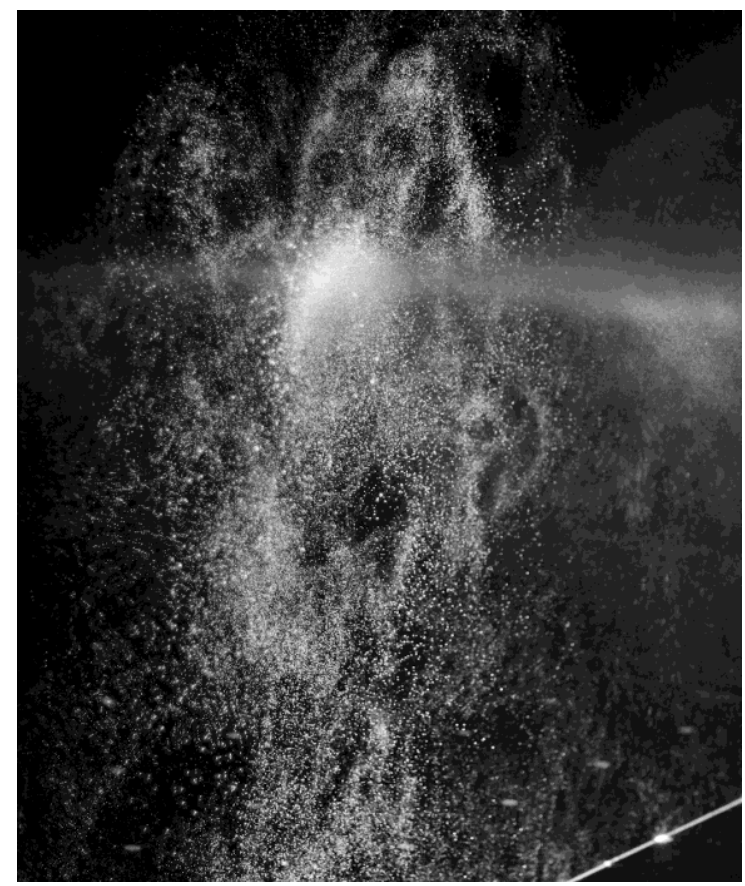

(a)

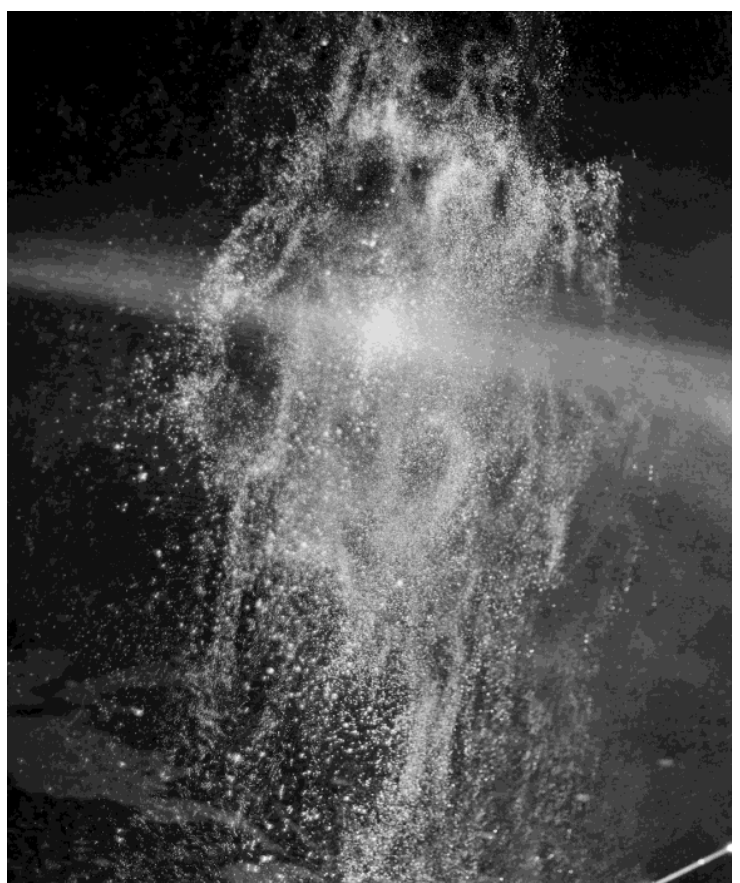

(b)

Figure 6.19: Laser Sheet Far Field Flow Visualization Oblique to the $x=6.1-\mathrm{m}$ Plane: (a) High Flow, Low Press. Water Jet (b) High Flow, Low Press. AFFF Jet (Flow is from Right to Left)

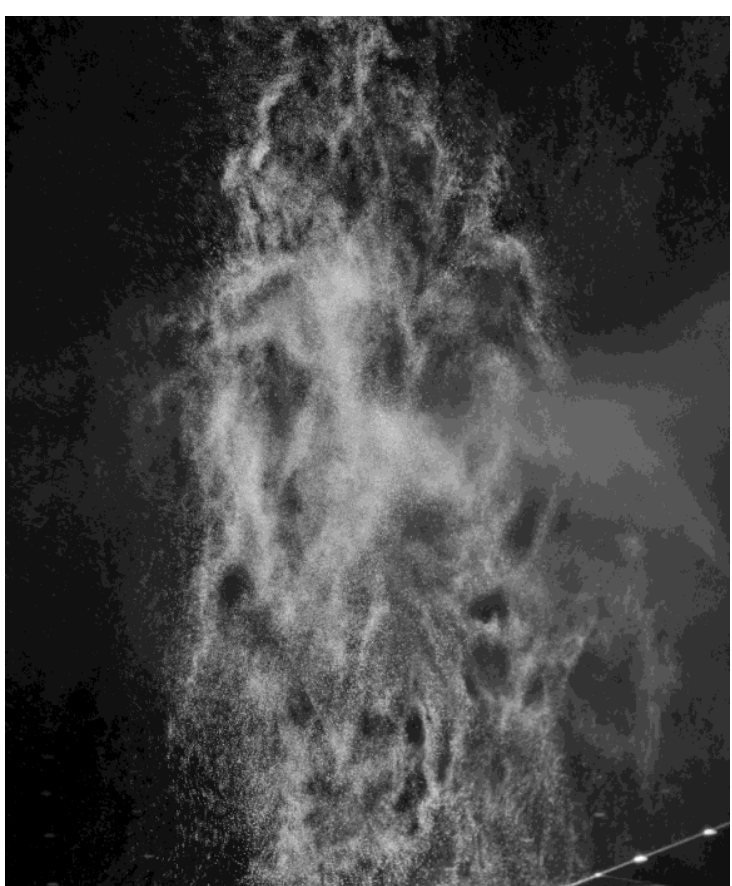

(a)

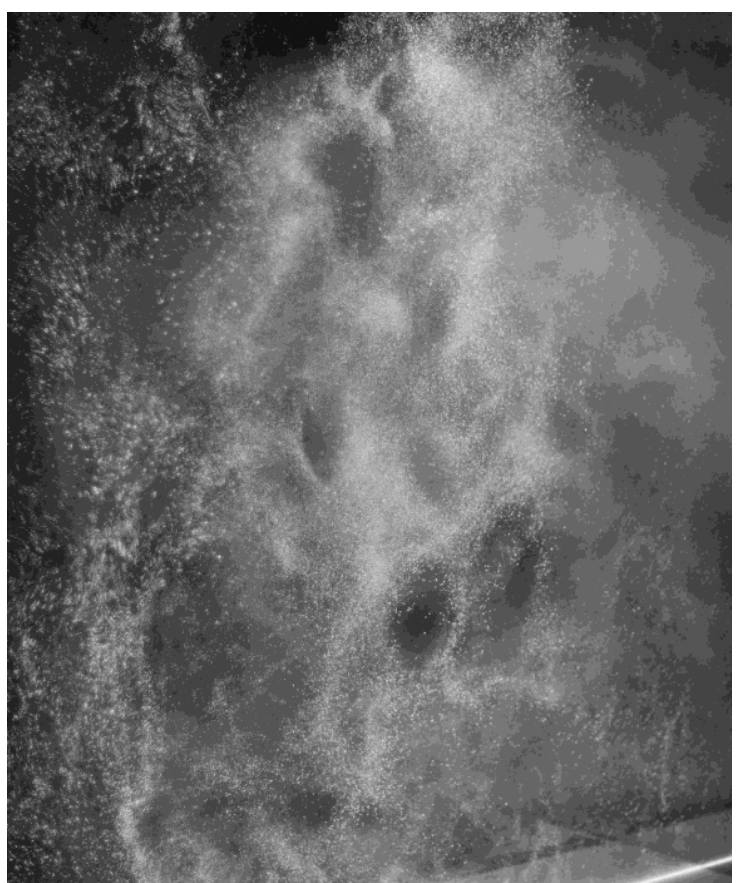

(b)

Figure 6.20: Laser Sheet Far Field Flow Visualization Oblique to the $x=6.1-\mathrm{m}$ Plane: (a) High Flow, High Press. Water Jet (b) High Flow, High Press. AFFF Jet (Flow is from Right to Left) 


\subsubsection{Mid Field Flow Visualization Results}

Mid field flow visualization illustrated the relationship between overall jet flow dynamics and near field droplet interactions. Mid-field image capture was a challenge compared to far field capture due to the 3-D downstream jet spread. This made focal plane alignment sporadic and difficult thus limiting most imagery to areas close to the nozzle near the $y=0-\mathrm{m}$ plane where the jet path was more reliable. High quality mid-field and near field image capture was mostly restricted to low pressure jets where camera shutter speed was not a limiting factor, especially for standard photographic techniques. High speed video was recorded and stills extracted for presentation. However, frame rates were limited to a maximum of 7500 frames $\cdot \mathrm{s}^{-1}$ due to poor subject illumination even after the addition of significant lighting. With a DSLR camera shutter speed of 4000 frames $\cdot \mathrm{s}^{-1}$, high speed video results were marginally better compared to conventional methods for high pressure jets. Electrical safety and water damage risk to photography equipment was an equal concern that limited access to downstream medium and high pressure jets where agent dispersion was the greatest.

Figure 6.21 depicts various evolutionary stages of the high flow, low pressure water jet near the nozzle using stroboscopic photography techniques. High quality images were possible due to the strong periodic nature observed with this particular pressure-flow rate combination. The second strongest periodicity was witnessed from the medium flow, medium pressure water jet with photographs of its mid-field shown in Figure 6.22. The other jets exhibited some but much less definitive periodicity. Qualitative difference between water and AFFF jets was unremarkable using stroboscopic techniques due to the saturated lighting conditions masking fine droplet details. The Strobe flash was set at $2350 \mathrm{~Hz}$ and $5100 \mathrm{~Hz}$ for the high flow, low pressure and medium flow, medium pressure water jet, respectively. The high flow, low pressure water jet pulsation frequency corresponded to the oscillation frequency of the three pump pistons based on the firefighting agent delivery system's motor speed. The medium flow, medium pressure water jet pulsation frequency was more difficult to associate with mechanical pump oscillation, although motor speeds were operating at almost half the RPM of the high flow, low pressure water jet indicating a possible shared harmonic. The medium flow, medium pressure water jet pulsation frequency could have also been influenced by the accumulator located immediately 
downstream of the pump. It was configured to suppress higher frequency (i.e. higher pressure) pulsations for which the firefighting agent delivery system was originally designed.

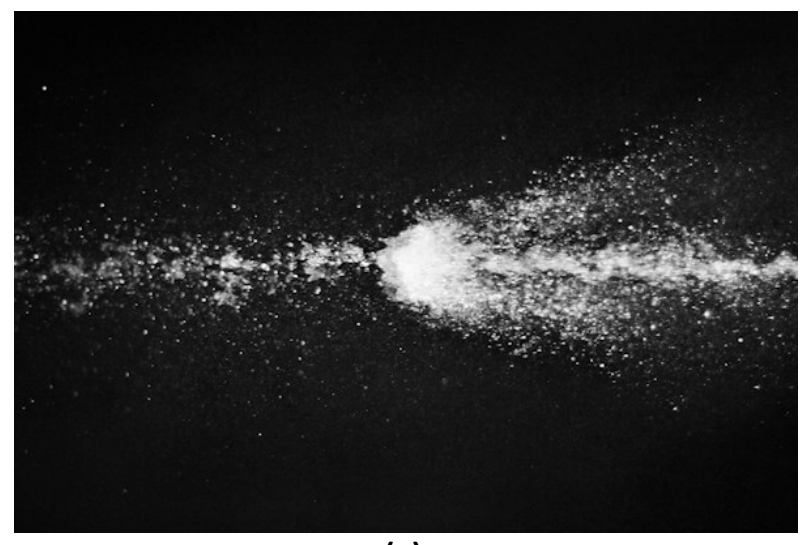

(a)

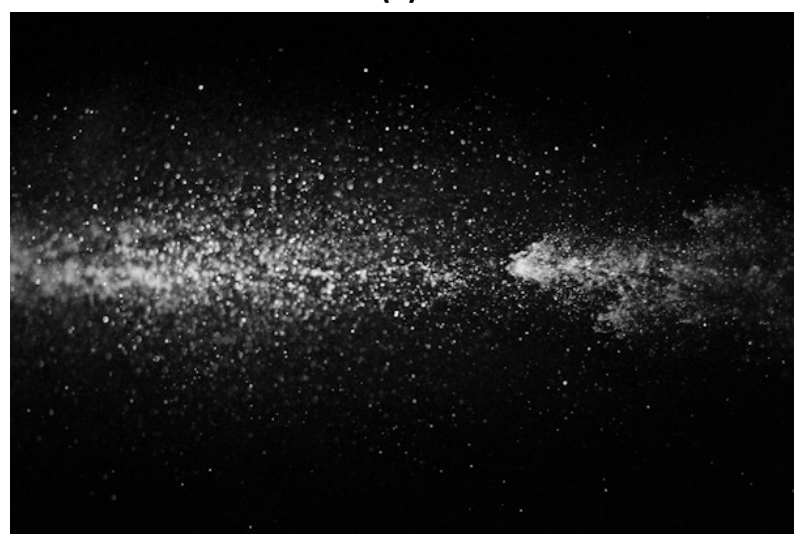

(c)

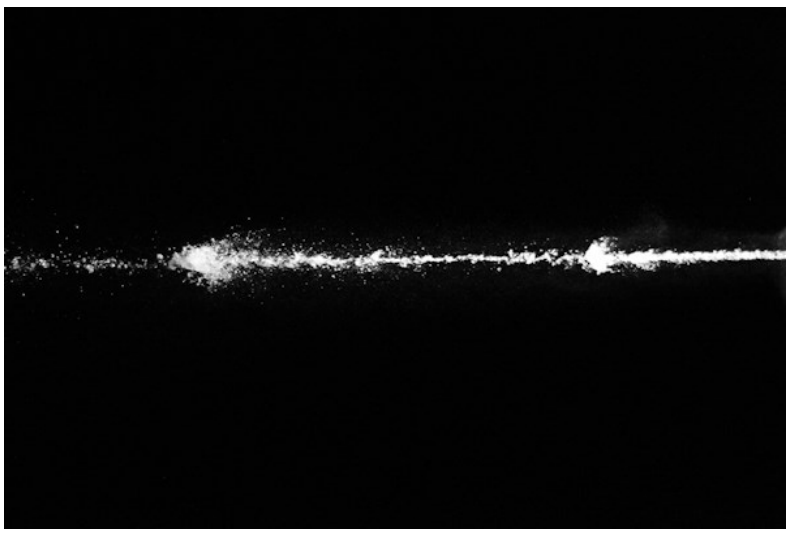

(b)

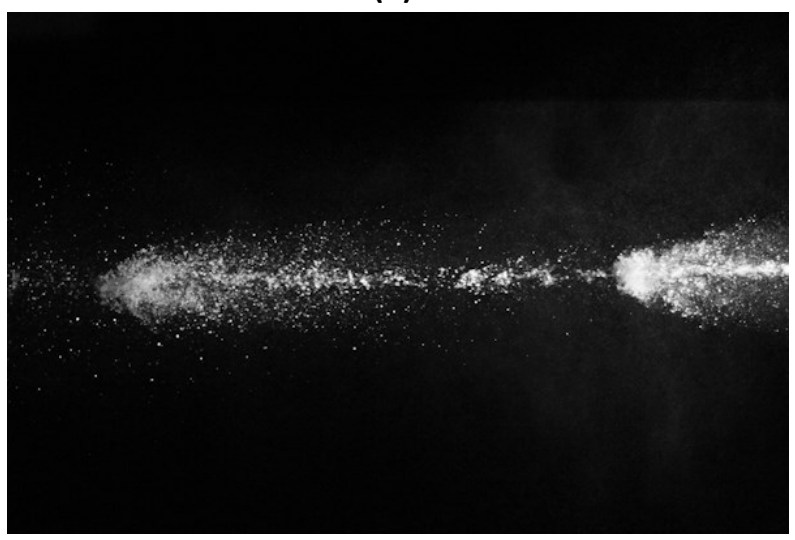

(d)

Figure 6.21: Stroboscopic Mid Field Flow Visualization of the High Flow, Low Press. Water Jet at Different Stages of Evolution: (a) Magnification of a Pulsation (b) Pulsation Growth Near the Nozzle Exit (c) Pulsation Fully Dispersing (d) Two Pulsations in Succession (Flow is from Right to Left)

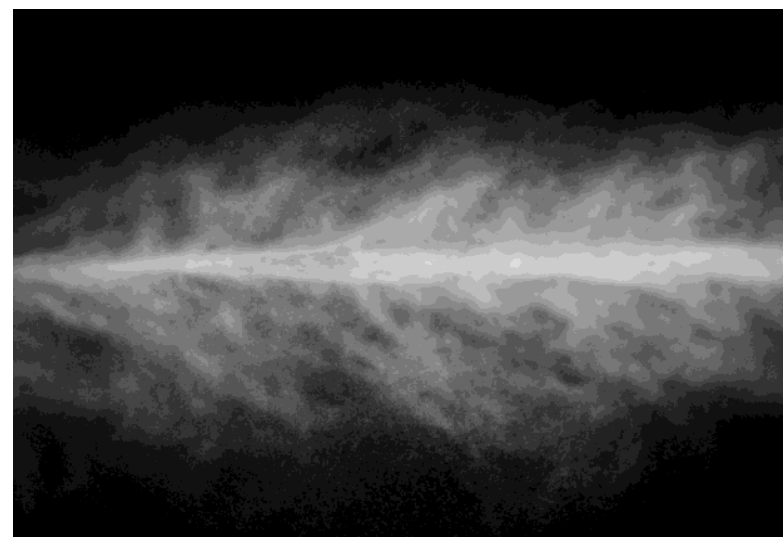

(a)

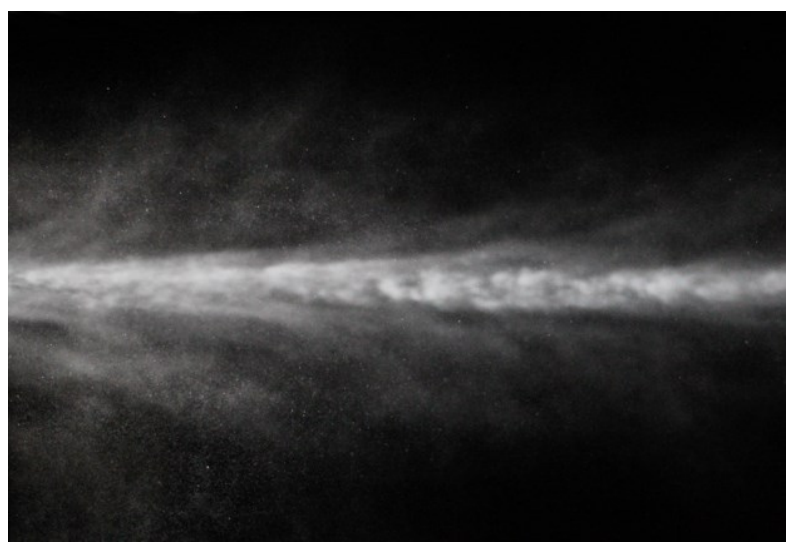

(b)

Figure 6.22: Stroboscopic Mid Field Flow Visualization Focused on the $y=0-m$ Plane of the Med. Flow, Med. Press. Water Jet: (a) Downstream of the Nozzle (b) Near the Nozzle (Flow is from Right to Left) 
Figure 6.23 and 6.24 depict a normalized FFT spectral analysis on pressure data recorded by the nozzle pressure transducer for the high flow, low pressure and medium flow, medium pressure water jet configurations, respectively. The FFT analysis indicates a dominant frequency mode at about $124 \mathrm{~Hz}$ corresponding to the pump piston oscillation frequency for the high flow, low pressure water jet, with a less dominant but similar frequency at around $122 \mathrm{~Hz}$ for the medium flow, medium pressure water jet. Stronger frequencies in the vicinity of the dominant mode in the medium flow, medium pressure water jet may indicate why stroboscopic pulsations were not as well-defined for the high flow, low pressure water jet.

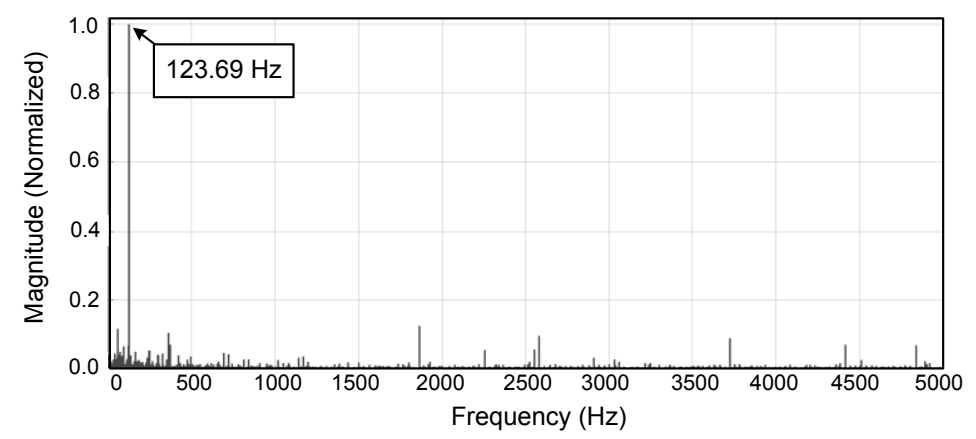

Figure 6.23: FFT Spectral Analysis of Nozzle Pressure Data Recorded on the High Flow, Low Press. Water Jet

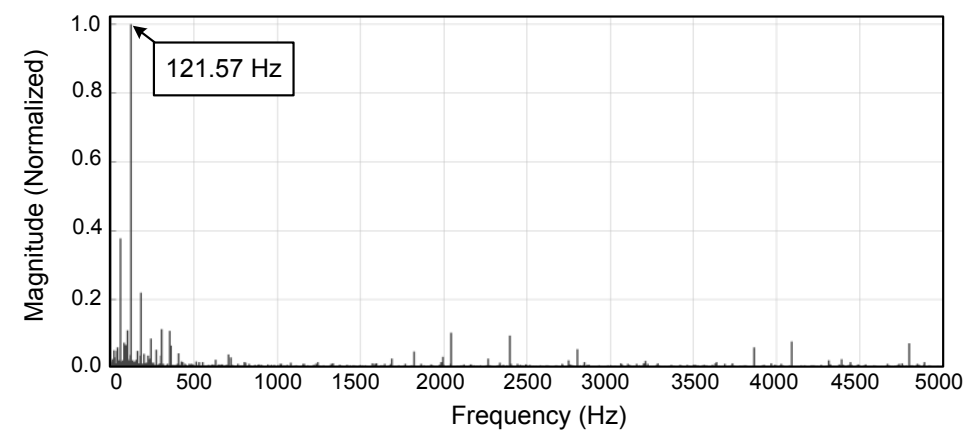

Figure 6.24: FFT Spectral Analysis of Nozzle Pressure Data Recorded on the Med. Flow, Med. Press. Water Jet

Figure 6.25 compares conventional images of the high flow, low pressure and medium flow, medium pressure water jet recorded at a shutter speed of $4000^{-1} \mathrm{~s}$. High flow, low pressure pulsations were easily identified at these camera speeds, but became less apparent for the medium flow, medium pressure water jet. Figure 6.26 depicts high speed photographic stills from high speed video recorded using a 24-mm lens for both water and AFFF jets from the midfield near the nozzle along the $y=0$-m plane. The images depict similar trends as those taken with conventional photography techniques, except the elevated high speed camera frame rate was able to resolve a few extra lower frequency modes. 


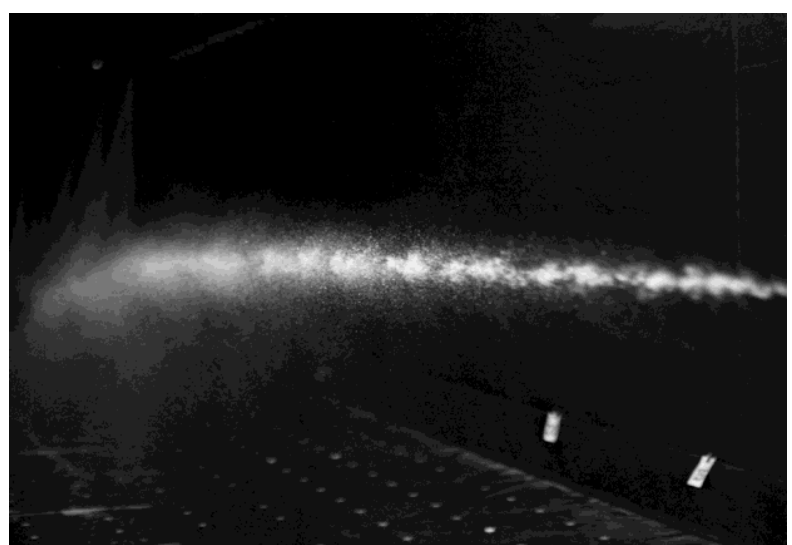

(a)

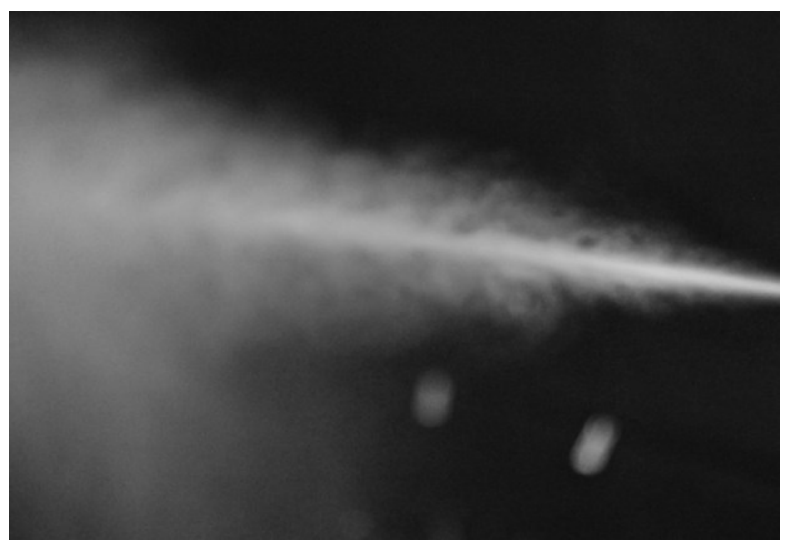

(b)

Figure 6.25: Standard Mid-field Flow Visualization Focused Oblique to the $y=0-m$ Plane for the (a) High Flow, Low Press. Water Jet (b) Med. Flow, Med. Press. Water Jet (Flow is from Right to Left)

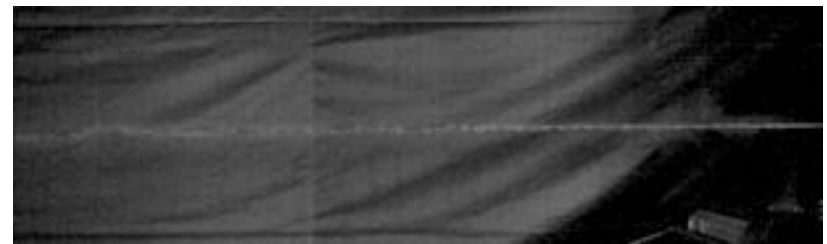

(a) Low Flow, Low Press. Water Jet

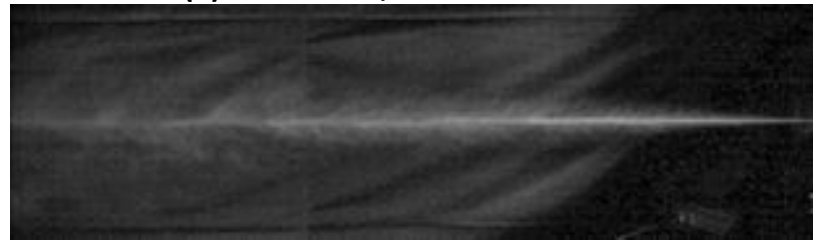

(c) Low Flow, High Press. Water Jet

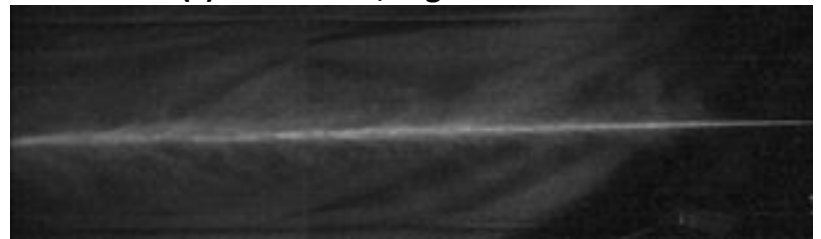

(e) Med. Flow, Med. Press. Water Jet

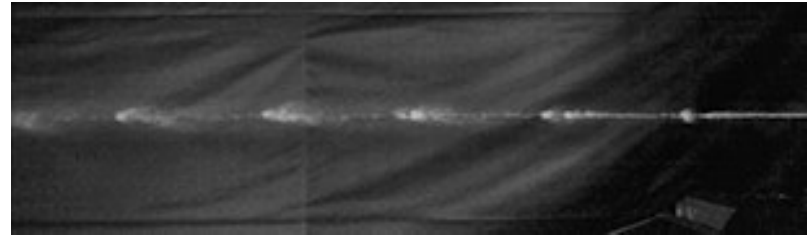

(g) High Flow, Low Press. Water Jet

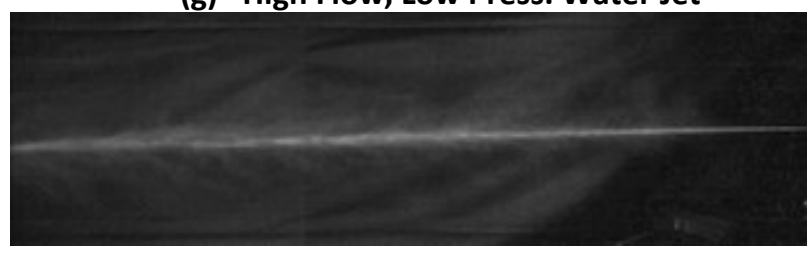

(i) High Flow, High Press. Water Jet

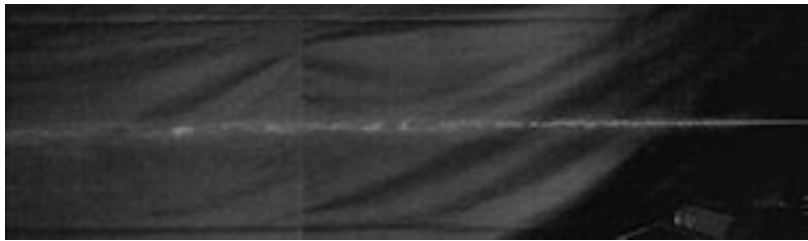

(b) Low Flow, Low Press. AFFF Jet

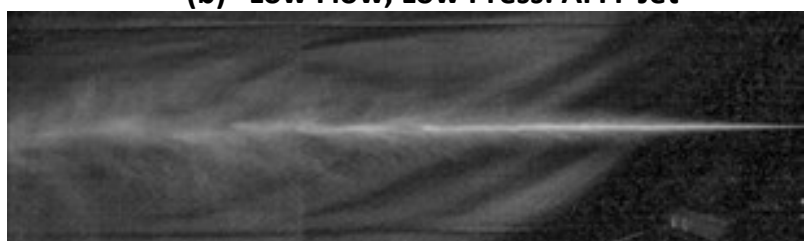

(d) Low Flow, High Press. AFFF Jet

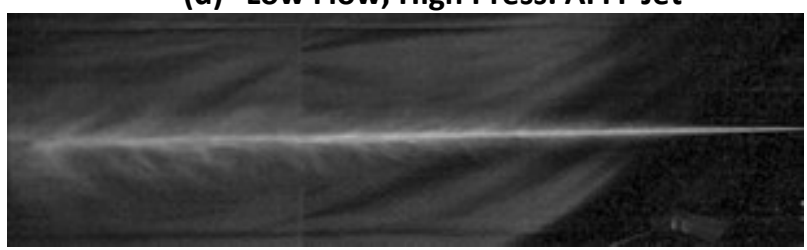

(f) Med. Flow, Med. Press. AfFF Jet

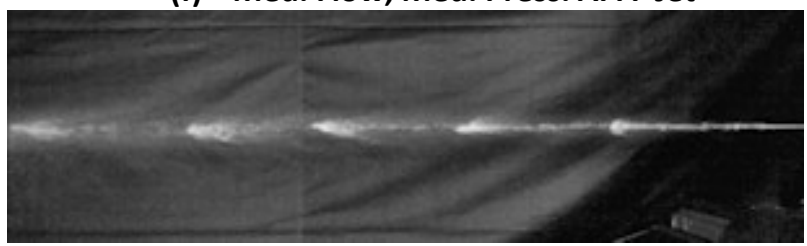

(h) High Flow, Low Press. AFFF Jet

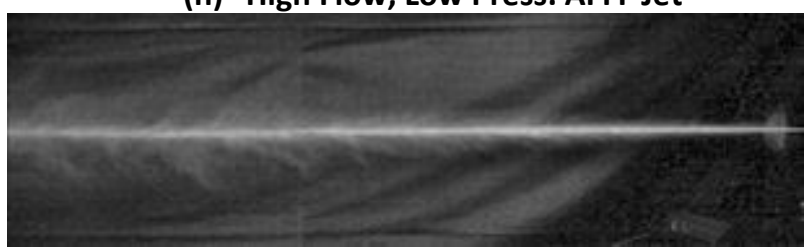

(j) High Flow, High Press. AFFF Jet

Figure 6.26: High Speed Mid-field Flow Visualization of Each Jet Configuration Focused on the $y=0-m$ Plane (Flow is from Right to Left) 


\subsubsection{Near Field Flow Visualization Results}

Near field photography of droplet break-up posed similar challenges to those encountered while recording mid-field imagery, except issues were amplified due to the relatively smaller length scales involved. Macro or zoom lenses ranging from 100 to $180 \mathrm{~mm}$ in focal length were used. High quality images were restricted to low pressure jets as the required combination of high intensity lighting and camera speeds made fine droplet flow visualization details from medium to high pressure jets unfeasible, especially near the nozzle where jet velocities were the greatest.

Figure 6.27 illustrates the standard evolution of a droplet emanating from the nozzle of a high flow, low pressure water jet at incremental distances downstream. Secondary jet break-up was detected throughout the entire flow field leading up to the nozzle exit plane. This phenomenon was similarly witnessed for the low flow, low pressure water jet and was more readily apparent for higher pressure jets, both utilizing water and AFFF as the agent. This observation supports the validity that the Reynold, Weber, and Ohnesorge classification of firefighting jets illustrated in Figures 2.10 and 2.11 in Section 2.3.2 largely inhabits the atomization break-up regime.

Qualitative differences between low flow, low pressure water and AFFF jet break-up were easiest to discern at 3-m (10-ft) downstream shown in Figures $6.28 \mathrm{a}$ and $6.28 \mathrm{~b}$, respectively. Figure 6.28 shows that a higher concentration of satellite droplets formed for the AFFF jet compared to the water jet, however the primary flow structures making up most of the jet mass did not significantly change. This trend held for higher pressure jet configurations, although overall droplet size tended to decrease from visual inspection. 


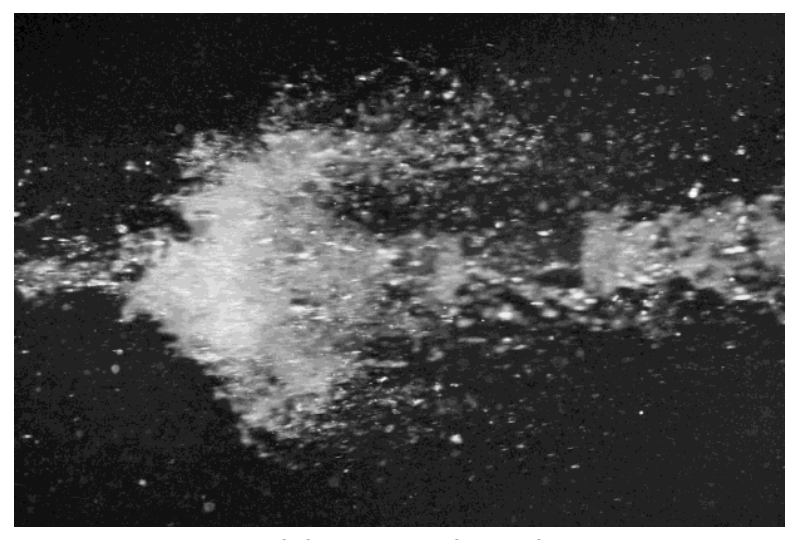

(a) $x=1 \mathrm{~m}(3.3 \mathrm{ft})$

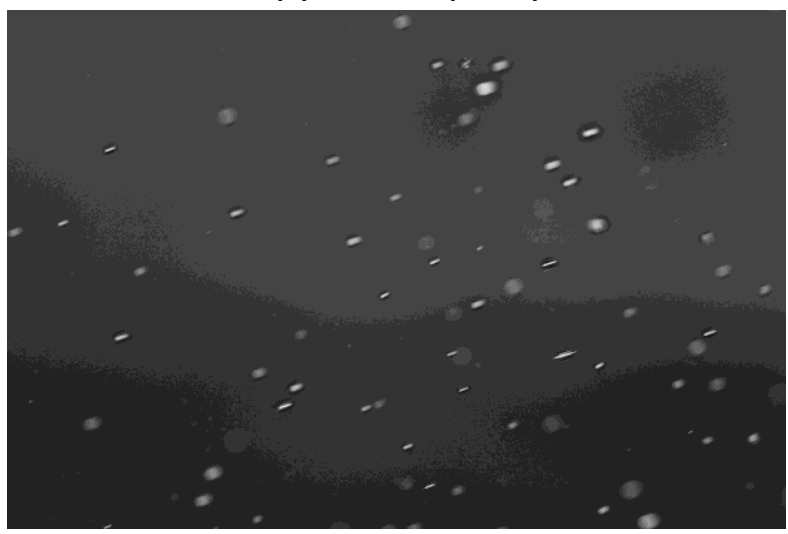

(c) $x=6 \mathrm{~m}(19.7 \mathrm{ft})$

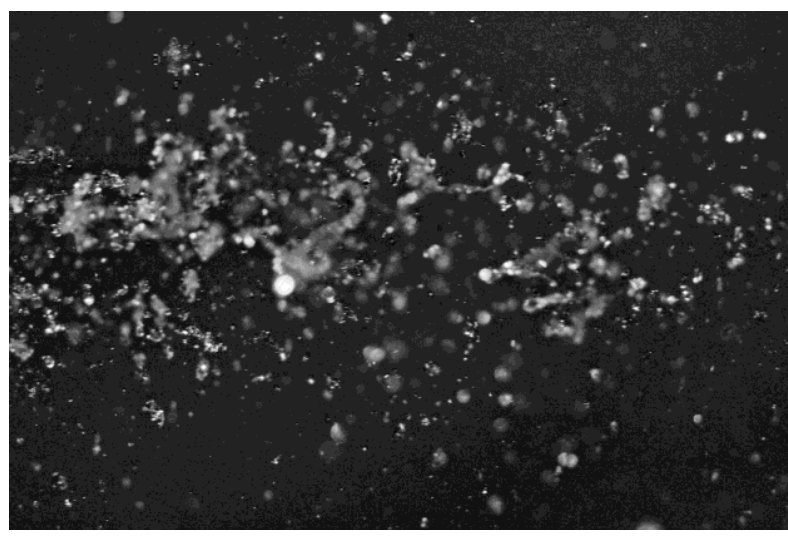

(b) $x=3 \mathrm{~m}(9.8 \mathrm{ft})$

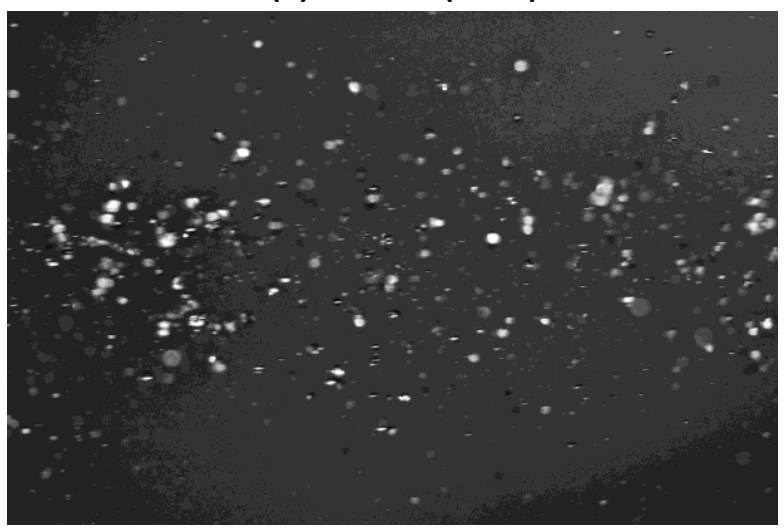

(d) $x=10 \mathrm{~m}(32.8 \mathrm{ft})$

Figure 6.27: Near field View of the High Flow, Low Press. Water Jet at Select Downstream Nozzle Locations (Flow is from Right to Left)

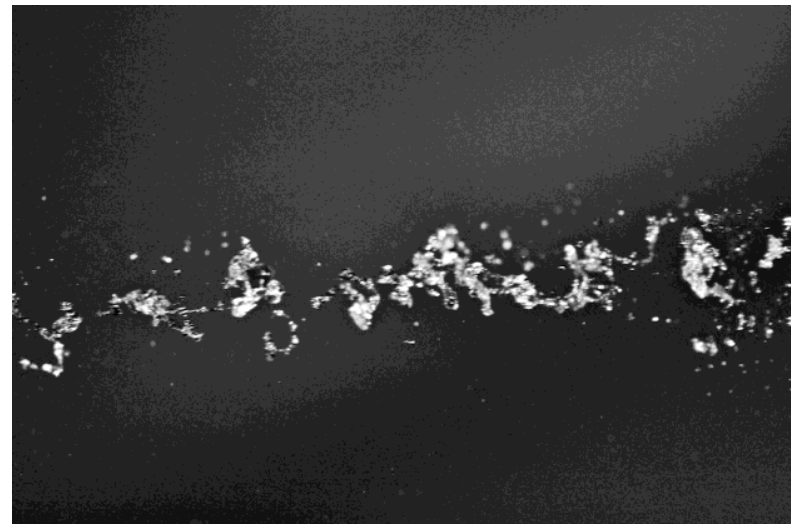

(a)

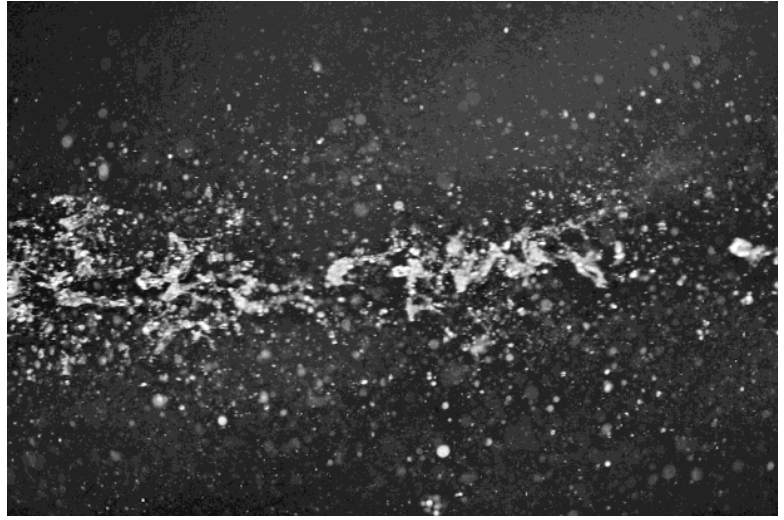

(b)

Figure 6.28: Near field View of Jet Break-up Approximately $3 \mathrm{~m}(9.8 \mathrm{ft})$ Downstream from the Nozzle for the (a) Low Flow, Low Press. Water Jet (b) Low Flow, Low Press. AFFF Jet (Flow is from Right to Left)

Contrary to other jet configurations, near field imagery of the high flow, low pressure AFFF jet identified unique multi-scale bubble formations along the leeward side of decaying droplet groupings from the pulsations found approximately halfway downstream from the nozzle. 
Figures $6.29 \mathrm{a}, 6.29 \mathrm{~b}$, and $6.29 \mathrm{c}$ illustrate various examples of this phenomenon significant bubble growth in areas associated with the largest concentration of droplets. These bubble growth structures appear similar to droplet multi-mode break-up and bag break-up discussed in Section 2.3.3 and illustrated in Figure 2.13. Figure 6.29d illustrates the more common occurrence of sparse sporadic bubble formation found throughout the jet.

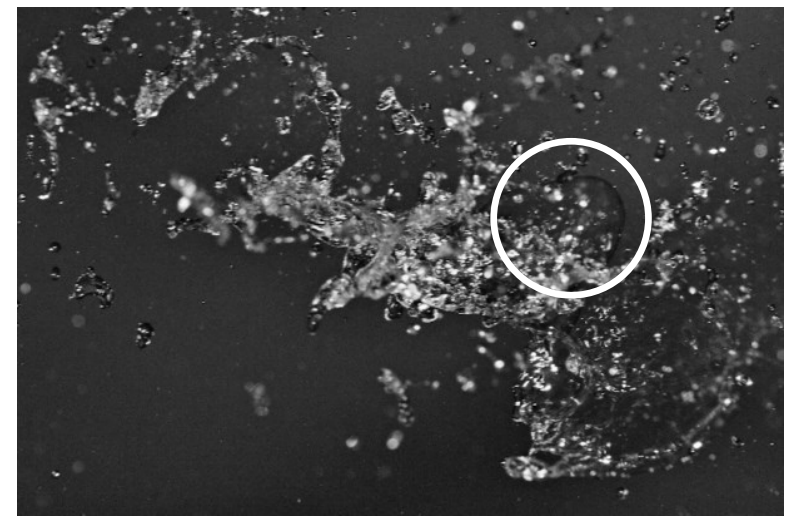

(a)

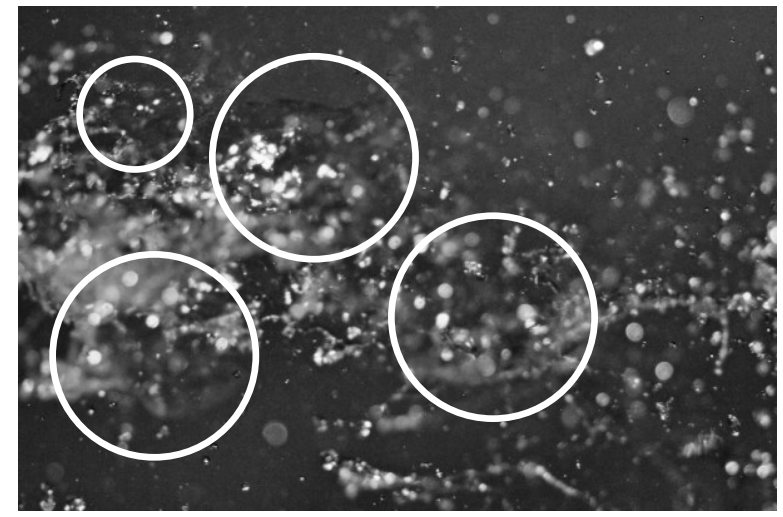

(c)

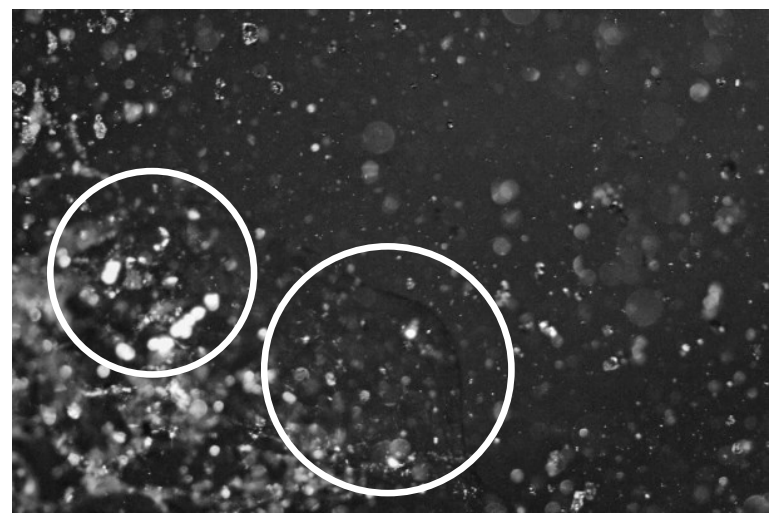

(b)

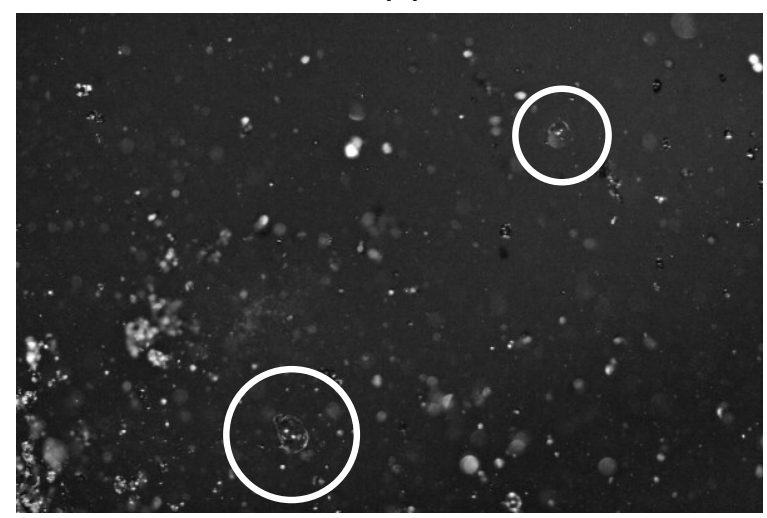

(d)

Figure 6.29: Near field Flow Visualization of the High Flow, Low Press. AFFF Jet Highlighting Multi-scale Bubble Masses at Different Downstream Locations (Flow is from Right to Left)

\subsection{Experimental Firefighting Jet Ground Pattern Results}

Agent ground pattern analysis was conducted on 21 different firefighting jet configurations overviewed in Section 4.1. Each configuration is illustrated in Figure 4.2 and listed in Table 4.1 in Section 4.1. Details of the experimental approach are in Section 4.3. Two-dimensional agent ground pattern measurements are presented on nine pressure-flow rate combinations using water and 6-percent AFFF as the agent. A brief study was also conducted to examine the influence the 
firefighting jet containment zone had on results. One-dimensional centerline agent ground pattern measurements were also recorded on the medium flow, medium pressure jet to determine the agent ground pattern sensitivity to AFFF concentration ranging from 3 to 12-percent AFFF. Agent ground pattern reach, coverage area, and span results for all 2-D firefighting jet work is then summarized along followed by a mass conservation analysis. In addition, six 1-D centerline agent ground pattern experiments were recorded to examine how full-scale flow rates alter the agent ground pattern compared to those tested at a smaller-scale in the laboratory.

\subsubsection{2-D Water Jet Containment Zone Sensitivity Ground Pattern Results}

A 2-D water ground pattern sensitivity analysis was conducted to determine the jet containment zone's influence on firefighting jet flow behavior. For spatial reference, Figure 4.5 in Section 4.3 illustrates the relative size and location of a medium-sized firefighting jet with respect to the downstream containment zone. Agent ground pattern 2-D accumulation studies were conducted on the largest (high flow, high pressure) water jet tested with and without the curtains drawn in the downstream portion of the jet containment bed. Testing was not carried out with AFFF jets for this particular study. Figure 6.30 compares the agent ground pattern results for both circumstances. Results are presented non-dimensionally as a percentage of the total agent dispensed for all agent ground pattern studies to remove the dependency on jet flow rate. The dimensions shown in Figure 6.30 reflect those of the firefighting jet containment bed

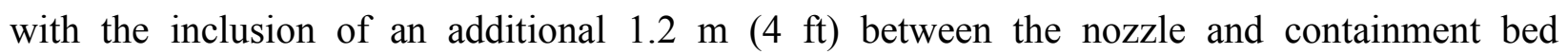
boundary where accumulation was negligible.

The test case with containment zone curtains open shows the firefighting jet body tapering slightly towards the negative $y$-direction indicating interaction with the adjacent laboratory wall parallel to the bed. The proximity of this wall generated a lower pressure region which caused the firefighting jet to bend toward it resulting in asymmetric flow along the $x$-axis, or axial centerline of the jet. The test case with containment zone curtains closed depicts a more axisymmetric jet from the pseudo wall effect imposed by the curtains located equidistant from the axial jet centerline. Firefighting jet characteristics were slightly affected by the presence of the containment curtains by limiting reach about 5 percent and inflating the coverage area by 8 percent. This was determined to be an acceptable consequence as protecting the laboratory environment from daily moisture deposition and potential mildew growth was paramount. 


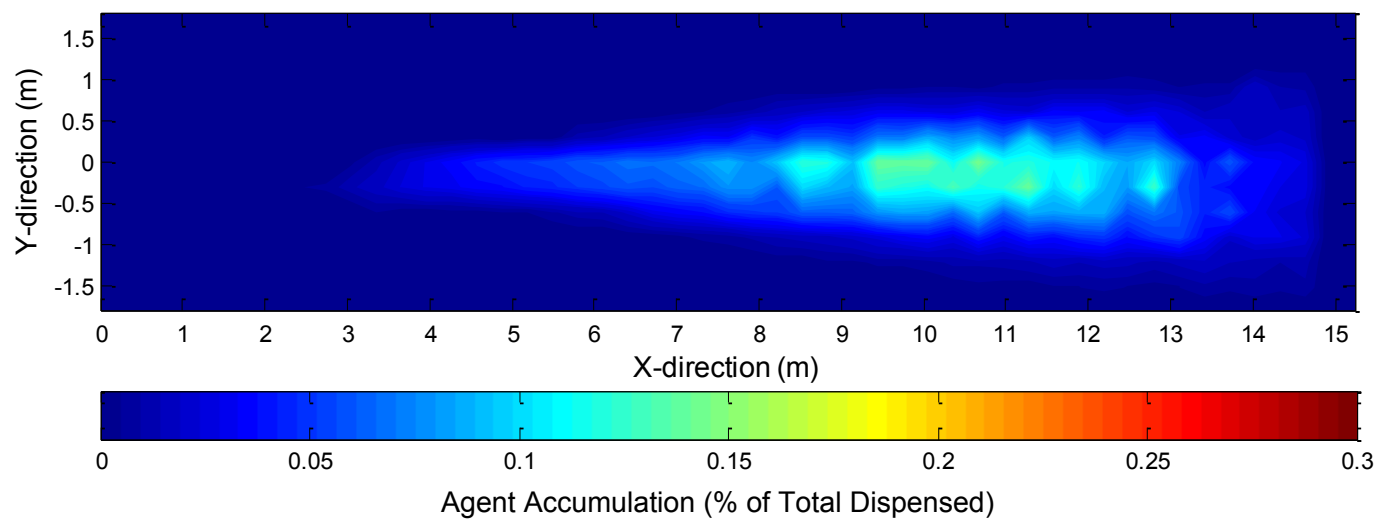

(a) Containment Curtains Open

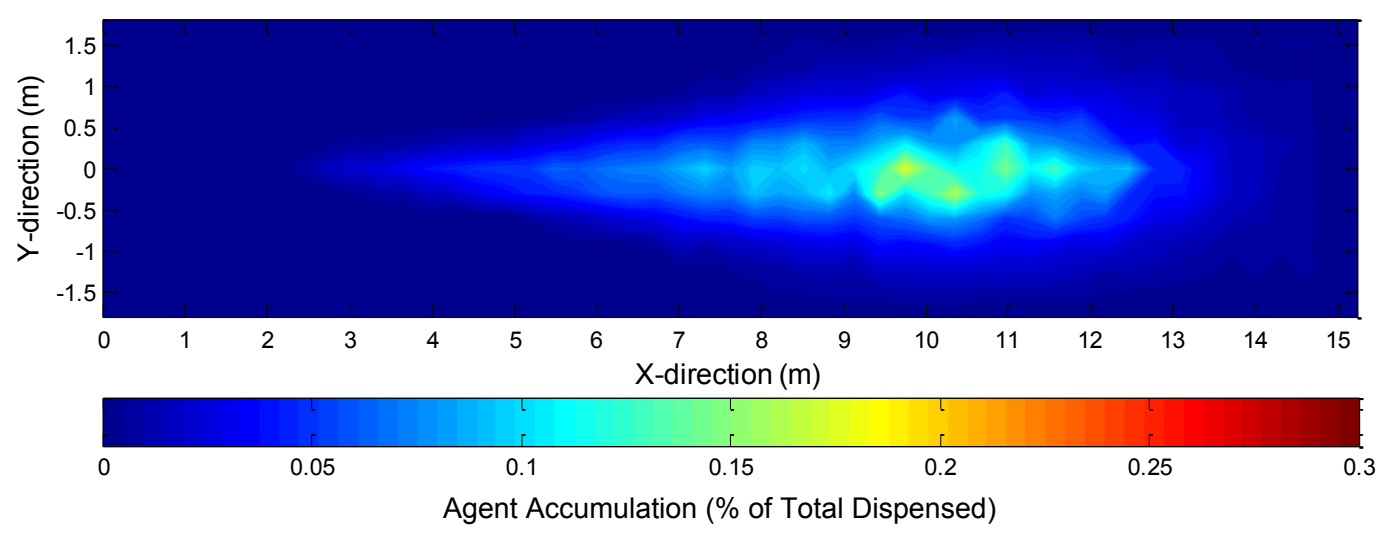

(b) Containment Curtains Closed

Figure 6.30: 2-D Agent Ground Pattern Water Accumulation Contour Plots Illustrating Firefighting Jet Dependence on the Firefighting Jet Containment Zone Curtains

\subsubsection{2-D Water Jet Ground Pattern Results}

Two-dimensional water ground pattern accumulation results were recorded on nine firefighting jet pressure-flow rate combinations. Figures 6.31 through 6.33 depict 2-D water accumulation contour plots grouped by nozzle pressure to show trends based on increasing flow rate from (a) to (c) for each figure. Results are presented similar to Figure 6.30. Accumulation levels were greatest for low pressure jets because they exhibited the least amount of jet dispersion with a peak of 1.8 percent of total agent dispensed, whereas high pressure jets exhibited the most spread peaking at 0.3 percent. All ground pattern results showed that most accumulation fell along the jet centerline and downstream following the airborne jet trajectory. An increase in ground reach was shown to be strongly dependent on an increase in jet flow rate with less of an impact on maximum ground span, which was dominated by nozzle pressure similar to coverage area. 


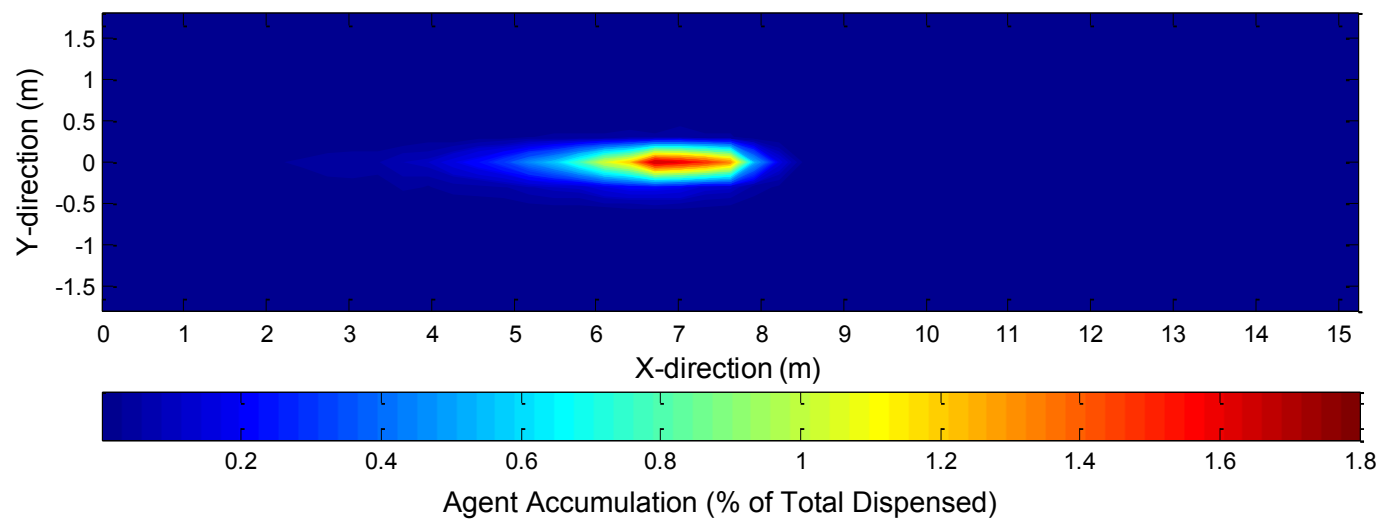

(a) Low Flow, Low Press. Water Jet

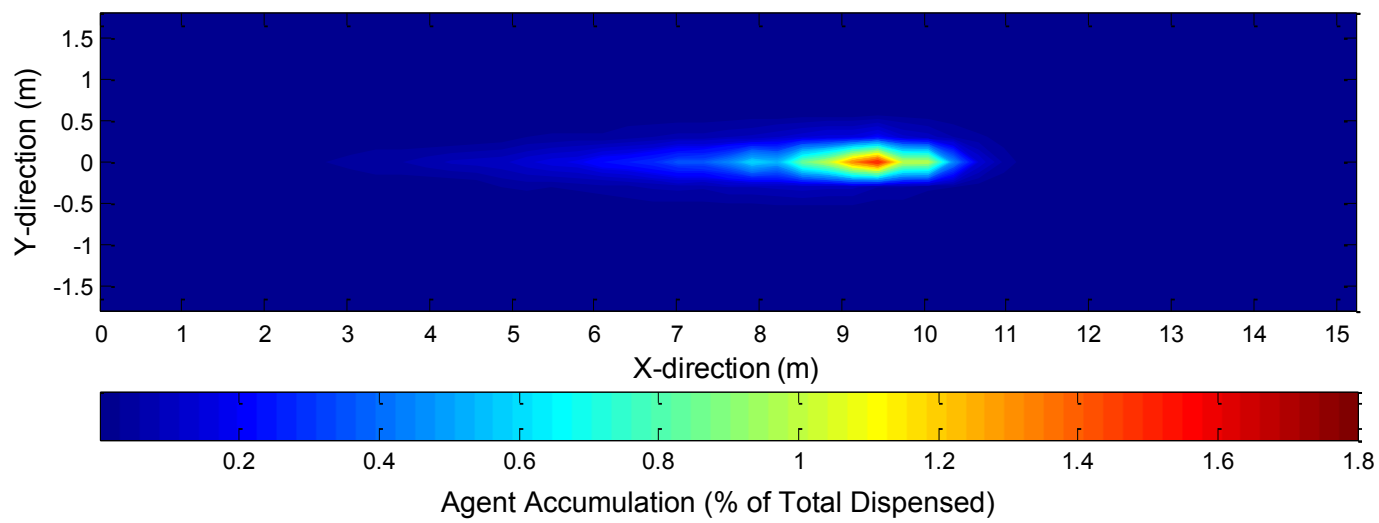

(b) Med. Flow, Low Press. Water Jet

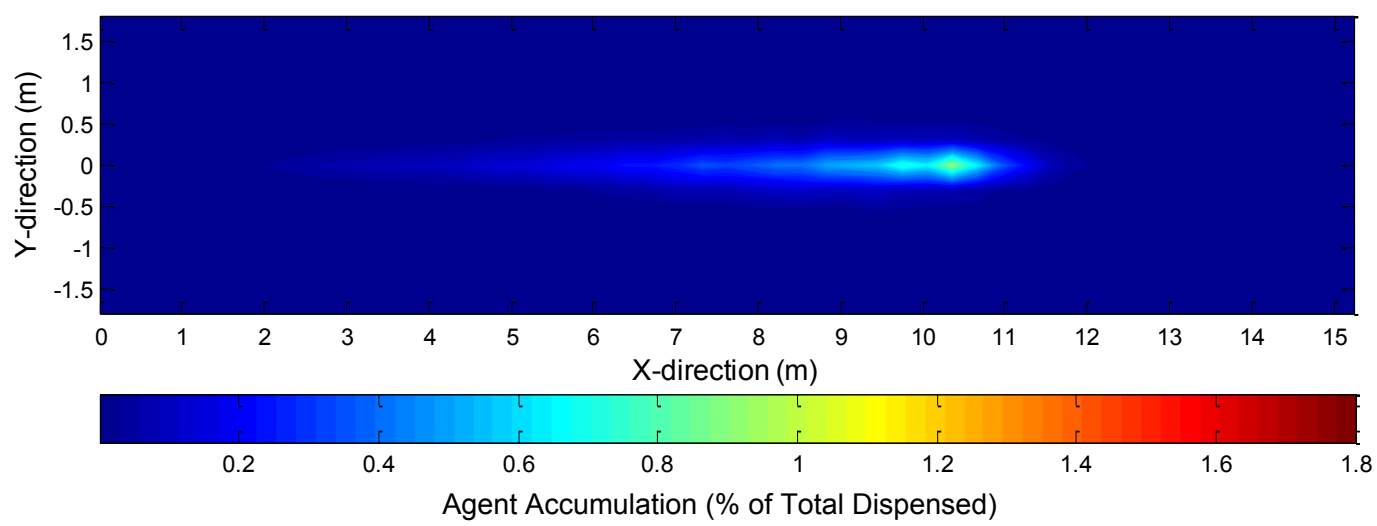

(c) High Flow, Low Press. Water Jet

Figure 6.31: 2-D Agent Ground Pattern Contour Plots of Low Press. Water Jets 


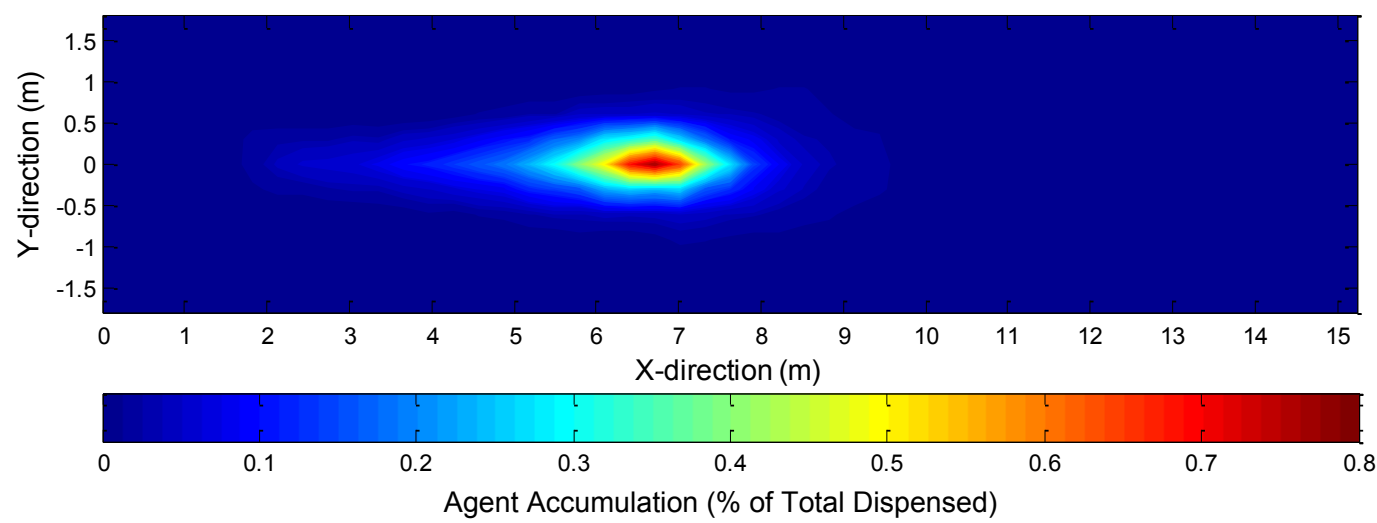

(a) Low Flow, Med. Press. Water Jet

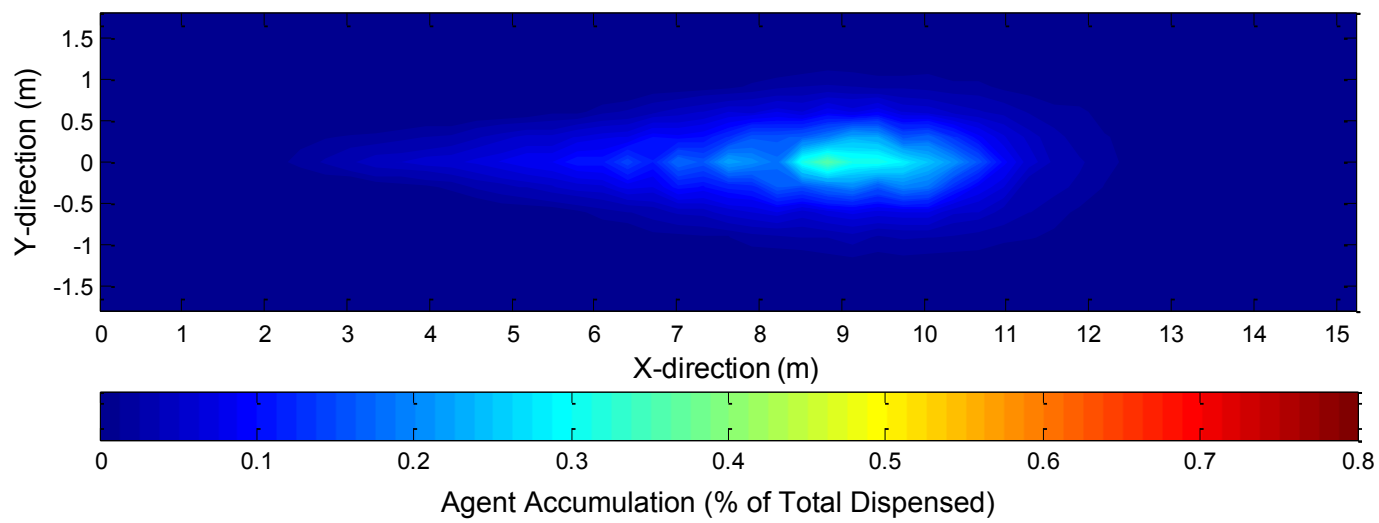

(b) Med. Flow, Med. Press. Water Jet

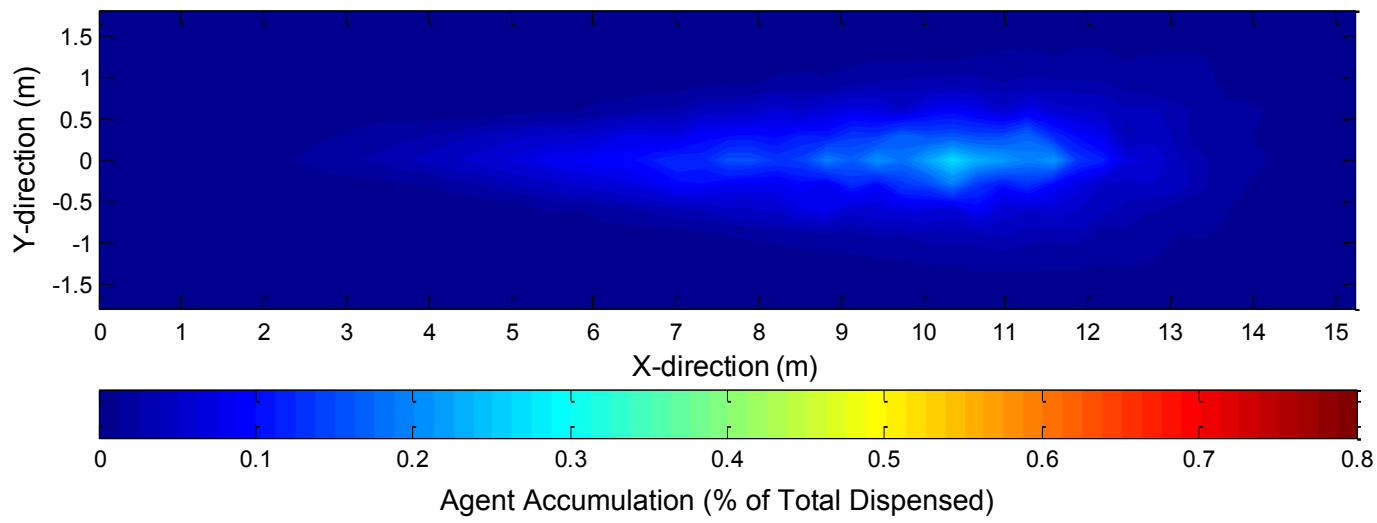

(c) High Flow, Med. Press. Water Jet

Figure 6.32: 2-D Agent Ground Pattern Contour Plots of Med. Press. Water Jets 


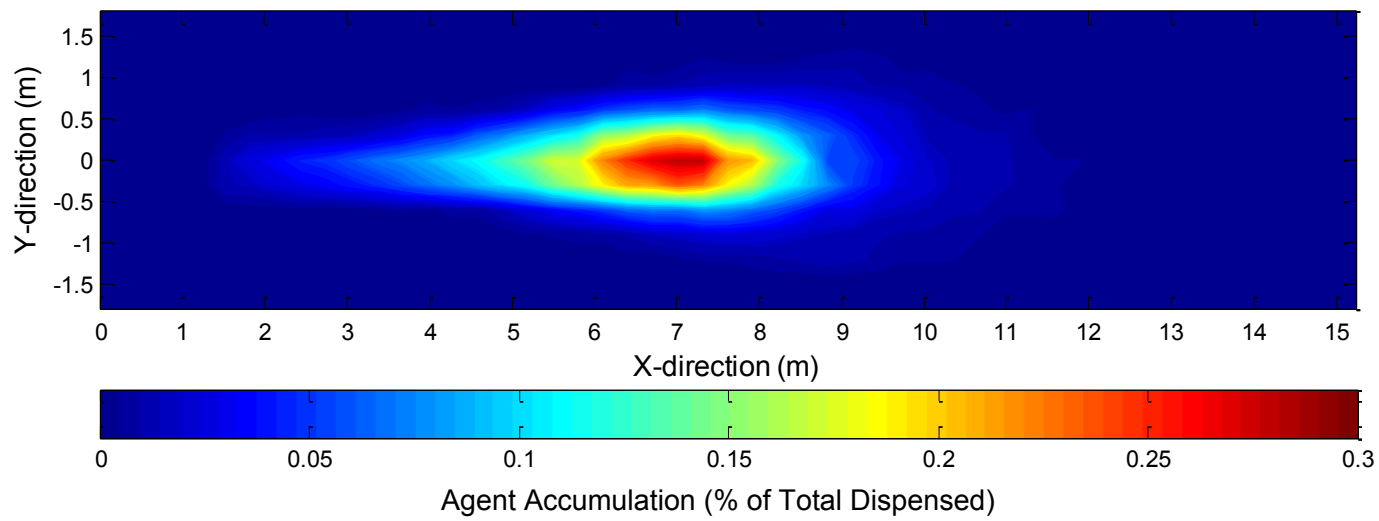

(a) Low Flow, High Press. Water Jet

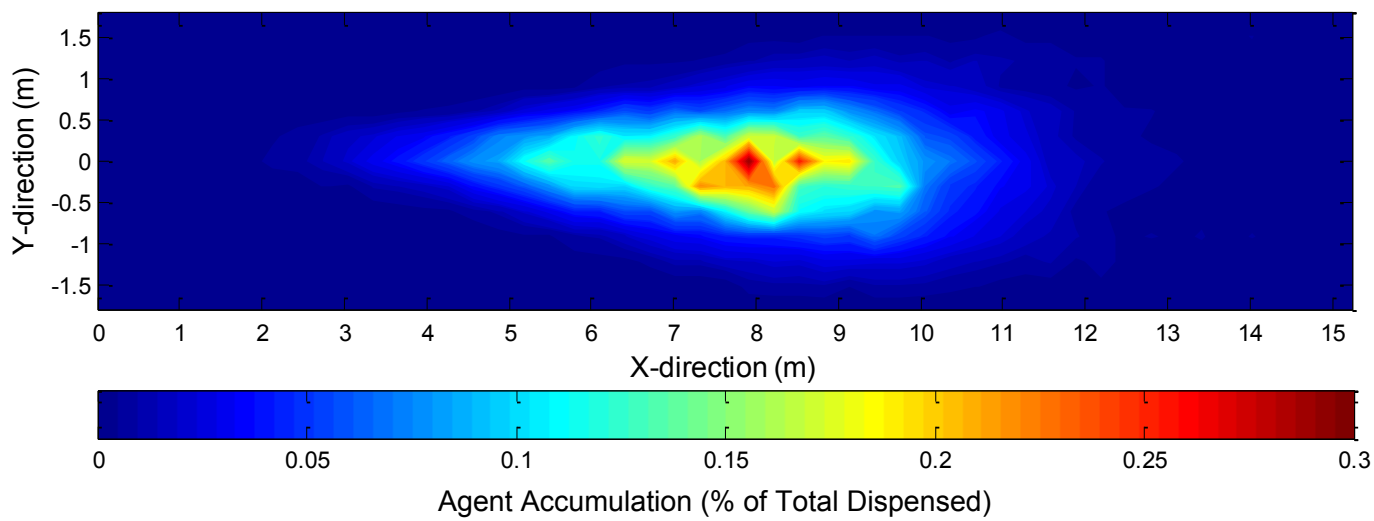

(b) Med. Flow, High Press. Water Jet

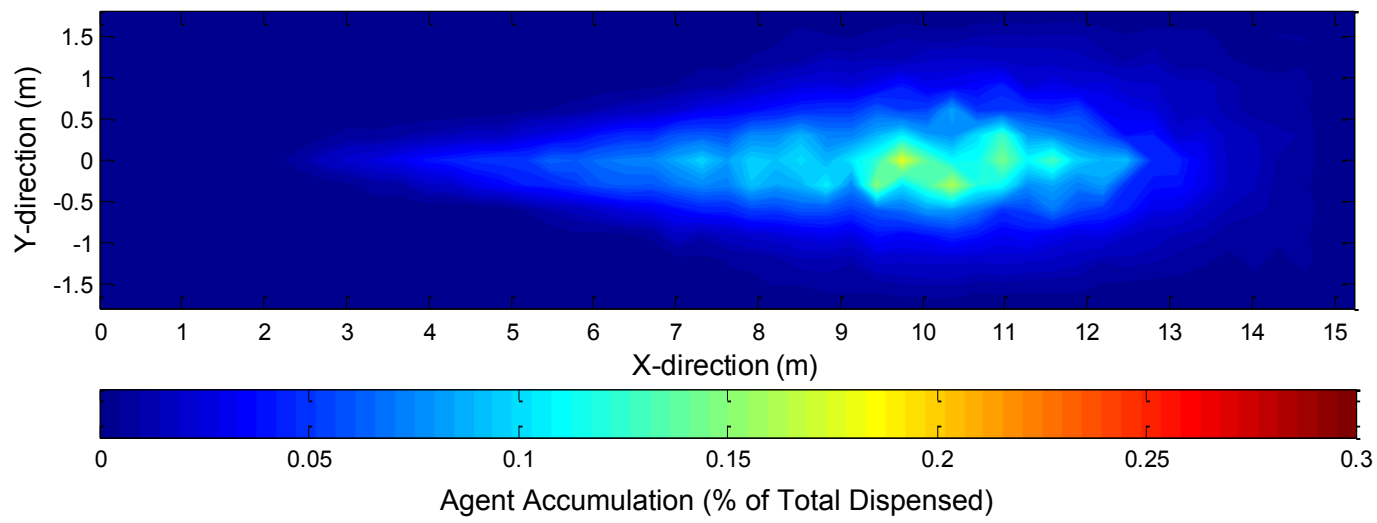

(c) High Flow, High Press. Water Jet

Figure 6.33: Two-dimensional Agent Ground Pattern Contour Maps of High Press. Water Jets 


\subsubsection{2-D AFFF Jet Ground Pattern Results}

Two-dimensional AFFF ground pattern accumulation results were recorded on the same nine firefighting jet pressure-flow rate combinations as those conducted with water. Figures 6.34 through 6.36 depict 2-D foam expansion ratio (or foam quality) contour plots grouped by flow rate. Six-percent AFFF was used for all results shown. Trends based on an increase in nozzle pressure are shown from (a) to (c) for each respective figure. AFFF was not collected in zones showing a foam expansion ratio of zero. Low flow rate and medium flow rate AFFF jets peaked with foam qualities of about 2.5:1. Foam expansion ratio increased for the high flow rate AFFF jet peaking at a foam quality of approximately 3:1. For low flow rate AFFF jets, foam quality increased as nozzle pressure increased. However, this trend was not consistent for medium and high flow rate AFFF jets as foam expansion ratio maintained a constant value across all nozzle pressure settings resulting in an inconclusive relationship between the two factors, at least for the parameter space examined in the laboratory.

The ground pattern shapes generated by AFFF mostly resembled those of the water ground patterns illustrated in Section 6.2.2, except with a minor inflation in coverage area and span along with a minor reduction in jet reach. In general, this suggests foam expansion ratio may be dependent on local agent accumulation. The largest pattern discrepancy was observed to be in areas closer to the nozzle where AFFF jet distribution appeared more dispersive as evidenced by flow visualization results reported in Section 6.1.3 and specifically illustrated in Figure 6.28. 


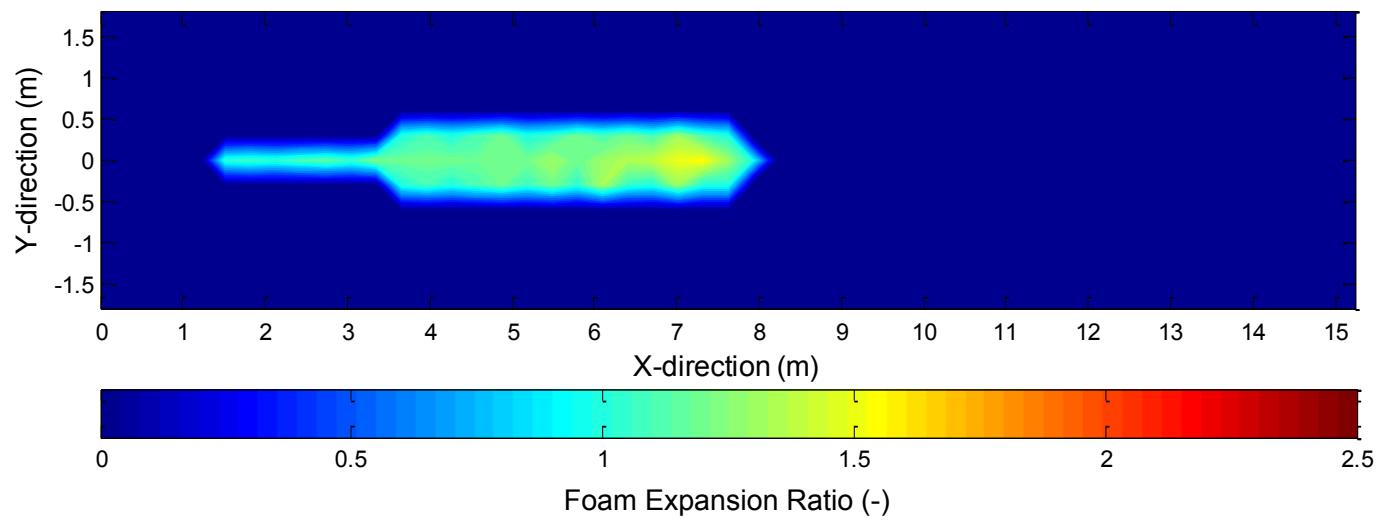

(a) Low Flow, Low Press. AFFF Jet

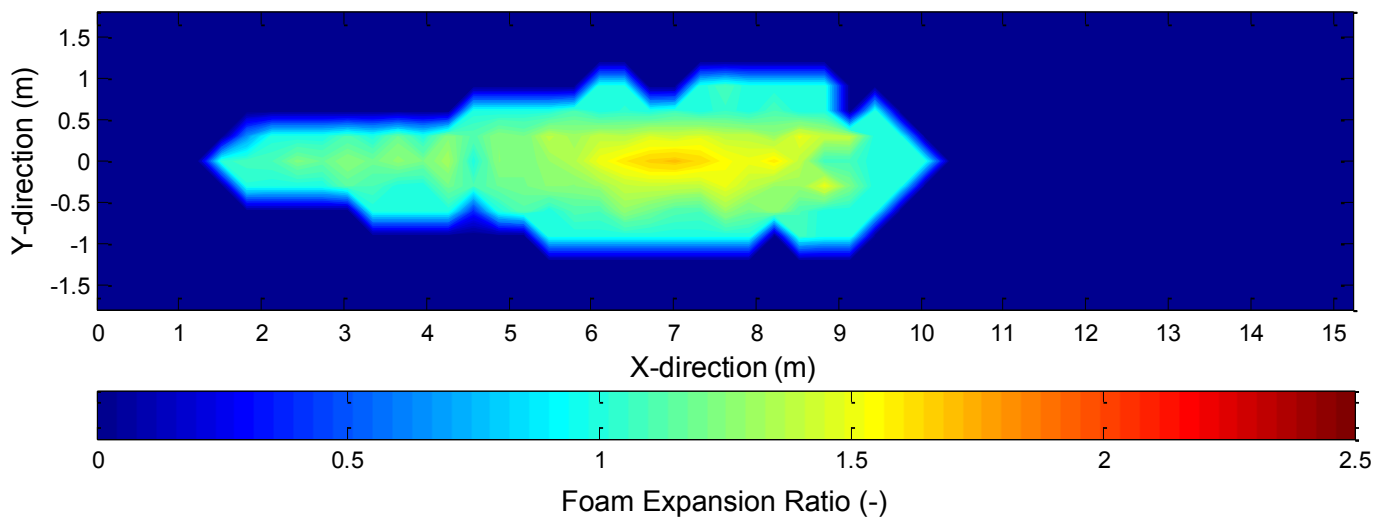

(b) Low Flow, Med. Press. AfFF Jet

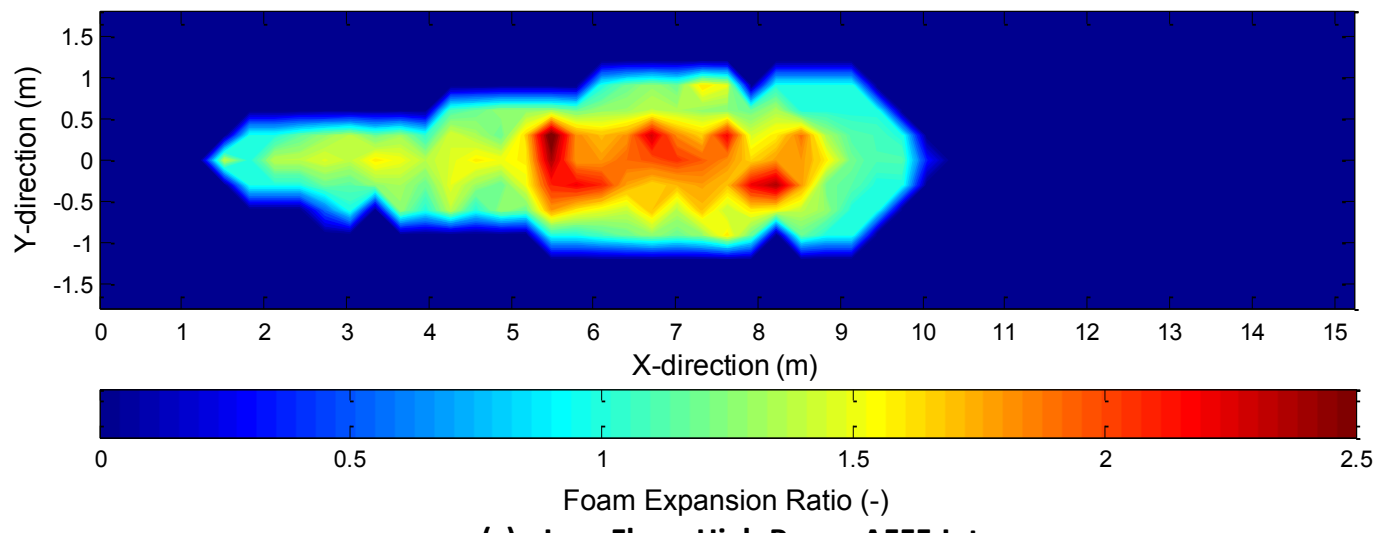

(c) Low Flow, High Press. AFFF Jet

Figure 6.34: 2-D Agent Ground Pattern Contour Plots of Low Flow AFFF Jets 


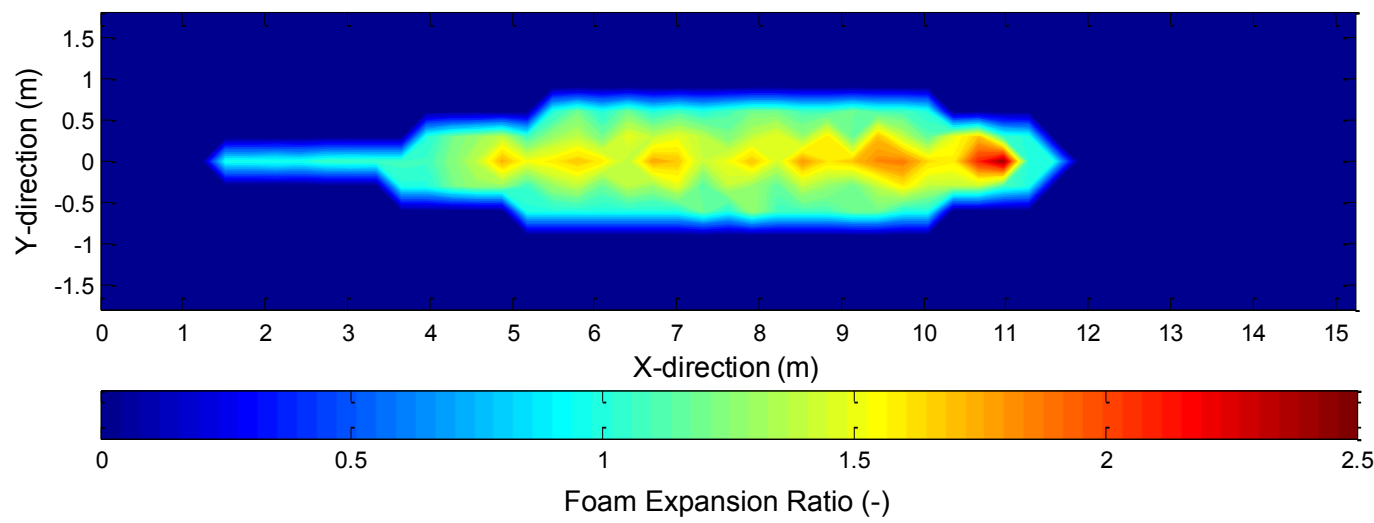

(a) Med. Flow, Low Press. AFFF Jet

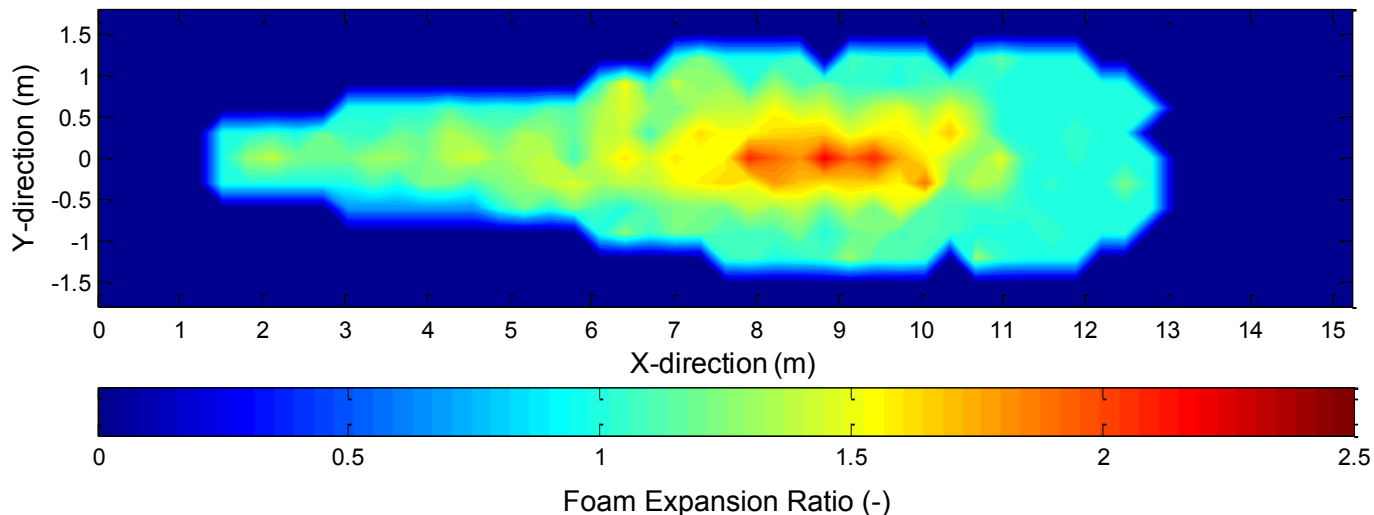

(b) Med. Flow, Med. Press. AfFF Jet

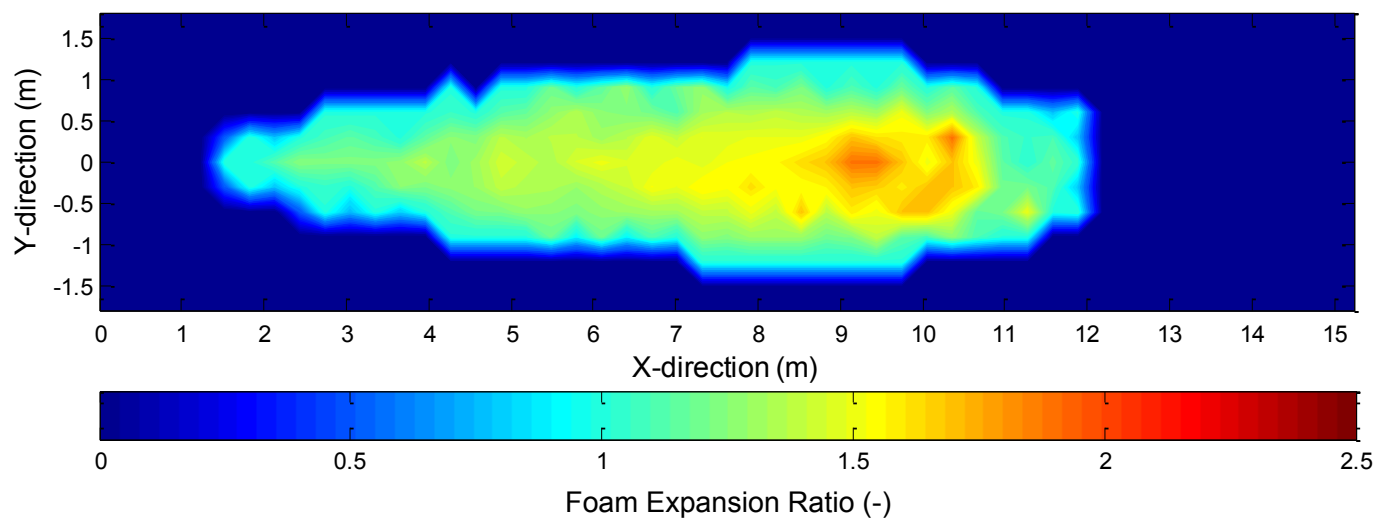

(c) Med. Flow, High Press. AfFF Jet

Figure 6.35: 2-D Agent Ground Pattern Contour Plots of Med. Flow AFFF Jets 


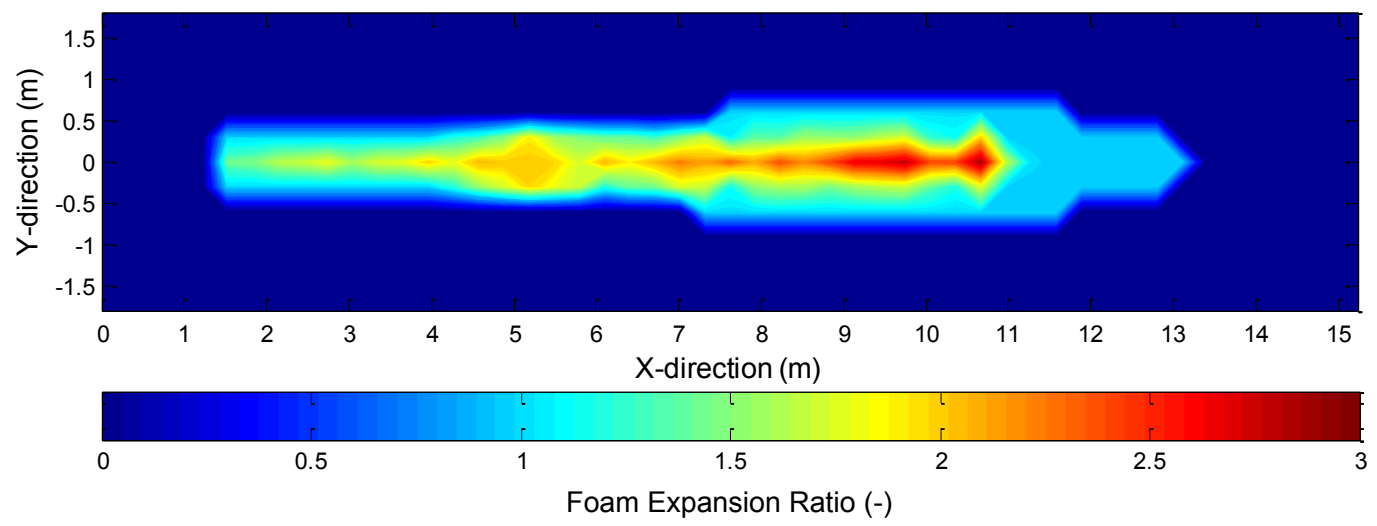

(a) High Flow, Low Press. AFFF Jet

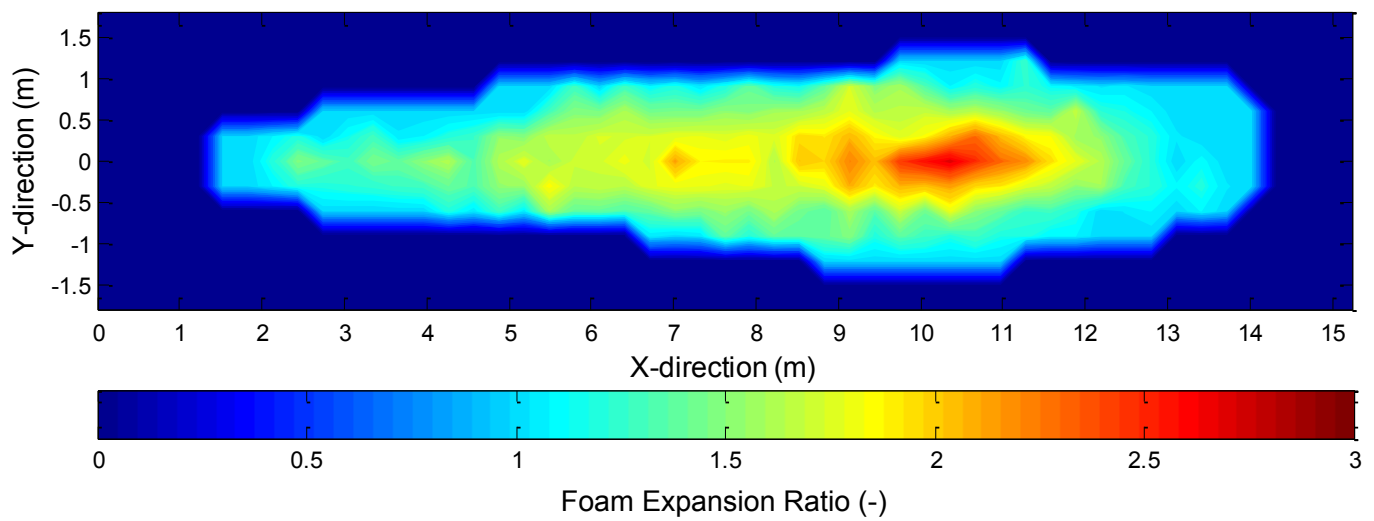

(b) High Flow, Med. Press. AFFF Jet

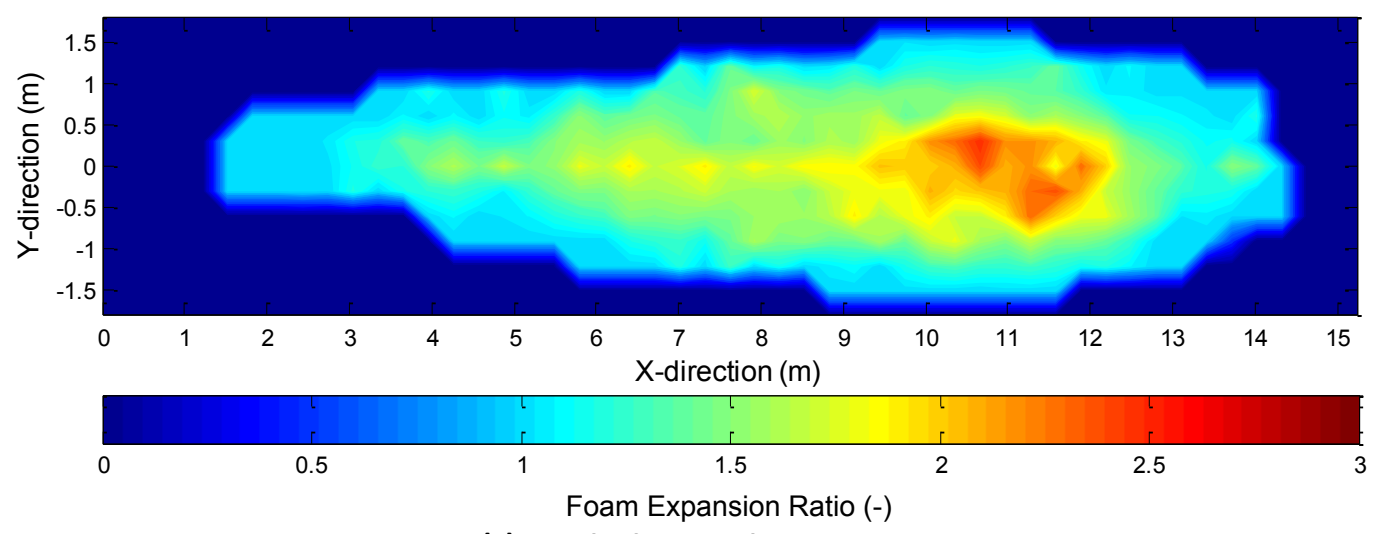

(c) High Flow, High Press. AFFF Jet

Figure 6.36: 2-D Agent Ground Pattern Contour Plots of High Flow AFFF Jets

\subsubsection{2-D Firefighting Jet Ground Pattern Results Summary}

Figure 6.37 summarizes all 2-D agent ground pattern testing results presented in Sections 6.2.2 and 6.2.3 for water and 6-percent AFFF jets, respectively. Firefighting jet performance 
parameters are presented in terms of total ground coverage area, maximum reach, and maximum span as a function of flow rate. Data is grouped illustrating linear trends based on nozzle pressure. AFFF jets exhibited a mean 3.3-percent increase in coverage area efficiency and a mean 0.08-percent decrease in maximum reach efficiency per unit increase in flow rate compared to water jets. AFFF jets demonstrated a mean 1.5-percent gain in maximum span efficiency per unit increase in flow rate compared to water jets. These subtle distinctions are attributed to enhanced AFFF droplet break-up generating slightly greater jet dispersion. Droplet dynamics and their influence on global jet behavior are explored further in Section 6.3 as part of the PDPA. Error estimates are not shown in Figure 6.37 because agent capture devices remained in a static position for each flow configuration measured. This was due to little change between each firefighting jet ground pattern measurement coupled with the coarseness of the floor grid. Firefighting jet agent ground pattern uncertainty is addressed in Sections 6.2.5 and 6.2.6.

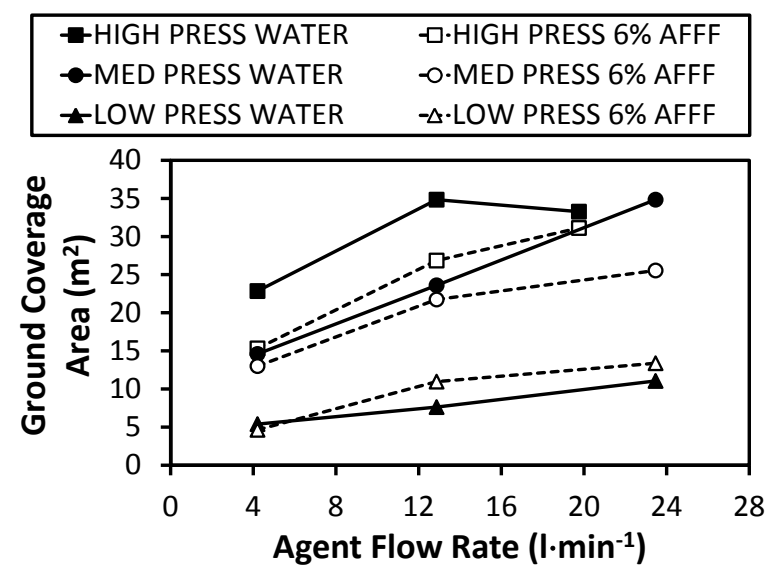

(a)

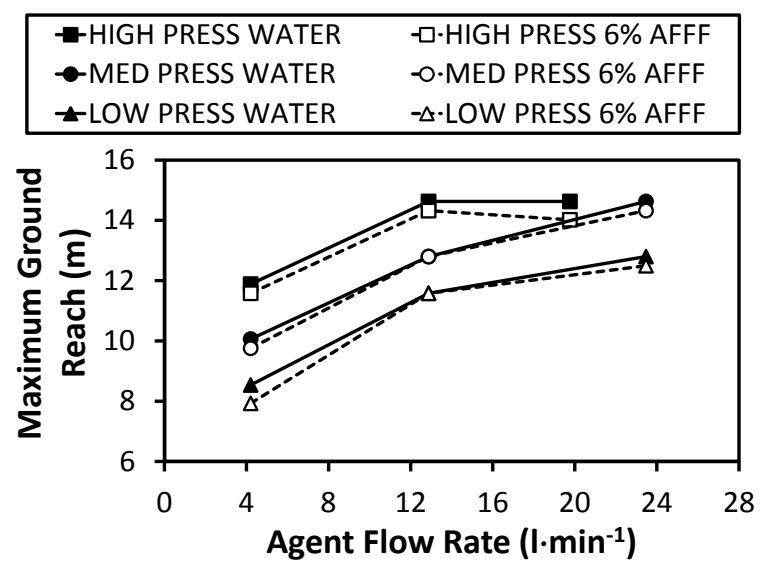

(b)

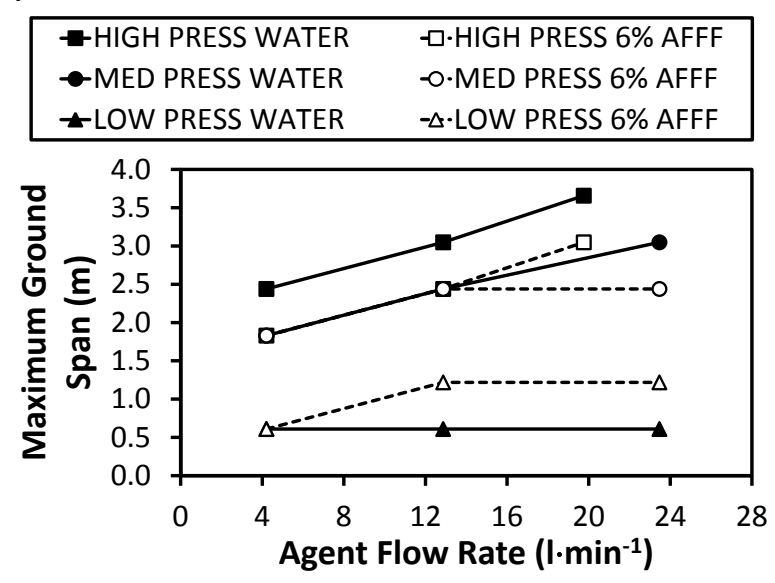

(c)

Figure 6.37: Agent Ground Pattern Firefighting Jet Performance Summary 
Ground pattern data was used in conjunction with agent capture assumptions to estimate the amount of firefighting jet mass conserved between the nozzle and the jet containment bed. Assuming each firefighting jet uniformly distributed agent across the containment bed floor, the ideal agent captured was about 13.63 percent. This value is based on the percent area all agent capture devices occupied with respect to the entire containment bed floor area. The method neglects loss due to evaporation and droplets transported outside of the containment bed. Table 6.1 lists the mean actual agent captured for all ten 2-D agent ground pattern tests recorded along with their associated differences compared to ideal settings. An over-prediction as high as 6.60 percent was recorded for the low flow, low pressure water jet, and an under-prediction as low as 19.44 percent was recorded for the low flow, low pressure jet for all cases considered. Over-predictions occurred for low pressure jets as the majority of agent landed along the axial centerline where agent capture devices were located, leaving less agent to fall on the remaining floor area. Conversely, medium and high pressure jets were under-predicted because of their much greater horizontal spread couples with excess mass loss due to evaporation and/or drift out of the jet containment bed. Medium and high pressure jets were prone to increased evaporation due to the generation of smaller, more concentrated droplets compared to a lesser number of larger droplets created from low pressure jets. In general, AFFF jets demonstrated increased mass loss compared to water jets due to the enhanced secondary droplet formation caused by the surfactants in AFFF.

Table 6.1: Agent Ground Pattern Mass Conservation Analysis

\begin{tabular}{|c|c|c|c|c|c|}
\hline $\begin{array}{c}\text { Nozzle } \\
\text { Flow Setting }\end{array}$ & $\begin{array}{c}\text { Ideal Agent } \\
\text { Captured } \\
\mathbf{( \% )}\end{array}$ & $\begin{array}{c}\text { Actual Water } \\
\text { Captured } \\
\mathbf{( \% )}\end{array}$ & $\begin{array}{c}\text { Actual Water } \\
\text { Difference } \\
\mathbf{( \% )}\end{array}$ & $\begin{array}{c}\text { Actual AFFF } \\
\text { Captured } \\
\text { (\%) }\end{array}$ & $\begin{array}{c}\text { Actual AFFF } \\
\text { Difference } \\
\text { (\%) }\end{array}$ \\
\hline Low Flow, Low Press. & 13.63 & 14.53 & 6.60 & 13.89 & 1.91 \\
\hline Low Flow, Med. Press. & 13.63 & 12.40 & -9.02 & 11.76 & -13.72 \\
\hline Low Flow, High Press. & 13.63 & 11.13 & -18.34 & 10.98 & -19.44 \\
\hline Med. Flow, Low Press. & 13.63 & 14.80 & 8.58 & 13.97 & 2.49 \\
\hline Med. Flow, Med. Press. & 13.63 & 13.55 & -0.59 & 12.74 & -6.53 \\
\hline Med. Flow, High Press. & 13.63 & 13.01 & -4.55 & 13.03 & -4.40 \\
\hline High Flow, Low Press. & 13.63 & 14.67 & 7.63 & 13.94 & 2.27 \\
\hline High Flow, Med. Press. & 13.63 & 13.40 & -1.69 & 12.89 & -5.43 \\
\hline High Flow, High Press. & 13.63 & 11.88 & -12.84 & 11.93 & -12.47 \\
\hline
\end{tabular}




\subsubsection{1-D Firefighting Jet Ground Pattern AFFF Concentration Sensitivity Results}

A 1-D AFFF concentration sensitivity ground pattern analysis was conducted using medium flow, medium pressure nozzle settings to examine how foam expansion ratio changed with respect to AFFF solution concentration. Figure 6.38 illustrates the results from this study comparing foam quality distribution from 3 to 12-percent AFFF in 3-percent increments as a function of jet axial $(x)$ location downstream non-dimensionalized in terms of nozzle diameter $D$. The two vertical bars shown for each AFFF concentration plotted in Figure 6.38 represents the minimum and maximum uncertainty based on two standard deviations, or a 95.5-percent confidence level, that the data falls within that range. Per 3-percent increase in AFFF concentration, the AFFF jet foam expansion ratio proportionally increased by about 25 percent. These results followed the same accumulation trend as the 2-D AFFF jet ground pattern results presented in Section 6.2.3 by generating peak foam expansion ratio magnitudes where maximum agent also accumulated along the centerline.

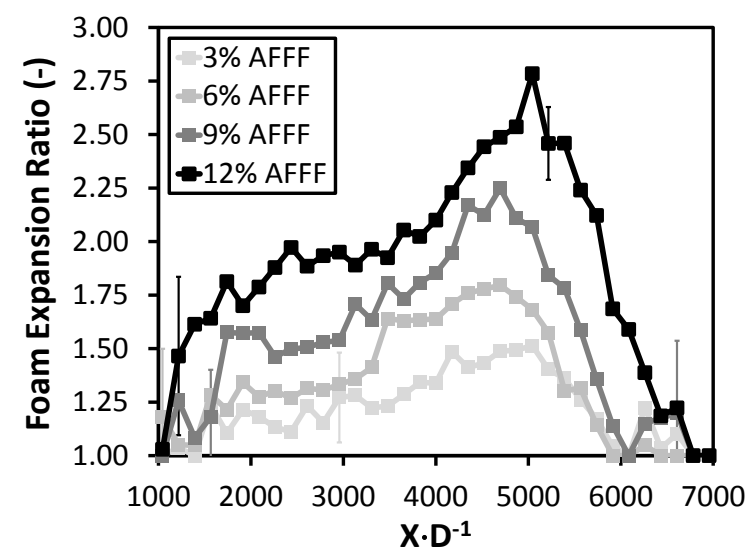

Figure 6.38: Foam Expansion Ratio vs. Axial Location of the Med. Flow, Med. Press. AFFF Jet with Respect to Variation in AFFF Solution Concentration

\subsubsection{Full-Scale 1-D Firefighting Jet Ground Pattern Results}

Full-scale 1-D firefighting jet ground pattern experiments were conducted at Tyndall Air Force Base, FL using the AP4 nozzle family at higher flow rates but similar low, medium, and high pressure settings as those tested in the aqueous firefighting agent application laboratory. Details on the experimental approach are in Figure 4.6 in Section 4.3. Data were recorded for water and 6-percent AFFF jets at a flow rate applicable to real world firefighting conditions of $75.71 \cdot \mathrm{min}^{-1}$ $\left(20 \mathrm{gal} \cdot \mathrm{min}^{-1}\right)$. Figure $6.39 \mathrm{a}$ and $6.39 \mathrm{~b}$ report water accumulation and foam expansion ratio 
magnitudes, respectively, as a function of axial $(x)$ downstream location similar to Figure 6.38 in Section 6.2.5. Vertical bars represent the same uncertainty conditions as those in Figure 6.38. Figure $6.39 \mathrm{a}$ shows that water jet accumulation peaks at low pressure and decreases as nozzle pressure is increased. This is expected as an increase in nozzle pressure enhances jet spread which causes droplets to disperse more widely and thus reduce centerline accumulation. Figure $6.39 \mathrm{a}$ also shows how water jet reach is significantly increased as nozzle pressure is increased across the test range by about a factor of two. Figure $6.39 \mathrm{~b}$ confirms a similar effect on jet reach as Figure 6.39a but for the AFFF jet instead. Foam expansion ratio measurements illustrate that foam quality has a stronger dependence on nozzle pressure at full-scale flow rates compared to lower flow rates measured within the laboratory. Peak foam expansion ratios of 4.8:1 were recorded resulting in an approximate 50-percent increase over laboratory measurements at the same pressure. It is also shown by comparison with small-scale laboratory results that foam expansion ratio increased as flow rate increased. Results further confirm foam quality is significantly dependent on agent accumulation as both figures depict a similar trend between the two parameters.

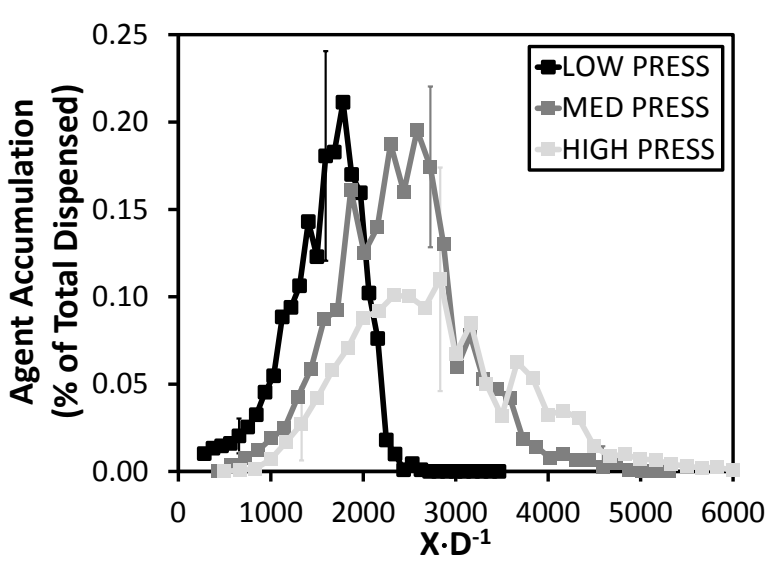

(a) Water Jets

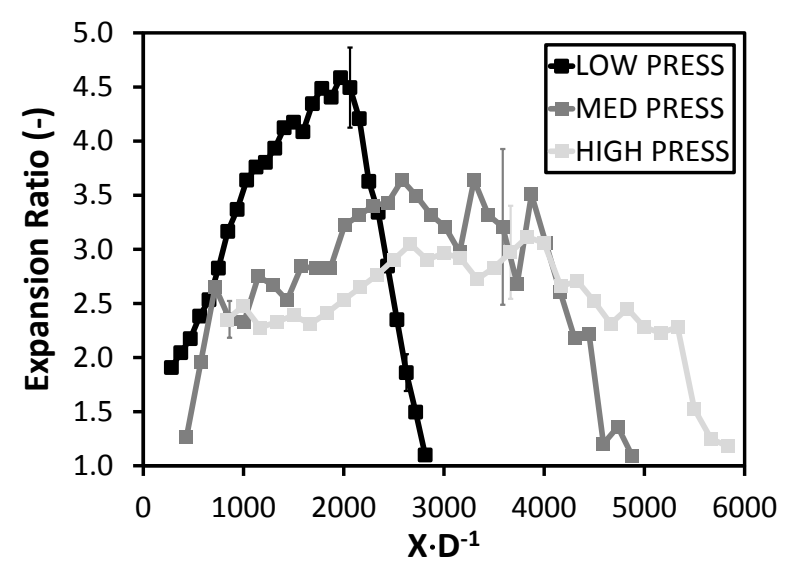

(b) $6 \%$ AFFF Jets

Figure 6.39: Full-scale 1-D Agent Ground Pattern Results Illustrating (a) Agent Accumulation vs. Axial Location and (b) Foam Expansion Ratio vs. Axial Location

\subsection{Experimental Firefighting Jet Phase Doppler Results}

PDPA was conducted on 12 firefighting jet configurations presented in Section 4.1. Each configuration is depicted in Figure 4.2 and outlined in Table 4.1 in Section 4.1. Details on the experimental approach are in Section 4.4. The 12 configurations represented five nozzle 
pressure-flow rate combinations using water and 6-percent AFFF as the agent. Additional phase Doppler data was recorded for 3 and 9-percent AFFF at the pressure-flow rate center point, namely the medium flow, medium pressure jet, to examine the sensitivity of phase Doppler results on AFFF concentration. Characteristic droplet size distribution results and their characteristic relationship to droplet velocity are presented initially. A substantial amount of phase Doppler data were recorded resulting in approximately 300 figures to represent all firefighting jet flow conditions tested. Select firefighting jet phase Doppler results from the central nozzle configuration are shown to introduce all of the flow parameters measured, outline the presentation style of the data, and to discuss trends common to all firefighting jet conditions. The full catalog of phase Doppler results is in Appendix C: Phase Doppler Firefighting Jet Data. All phase Doppler profile data listed in Appendix C is averaged and summarized into what are referred to as firefighting jet mean profile results to analyze global trends in the phase Doppler data in a condensed format.

\subsubsection{Firefighting Jet Phase Doppler Droplet Size Distribution Results}

Consistent with spray types involving hole-type fuel injectors or pressure atomizers, the AP4 nozzle family was best represented by Gaussian or normal drop size distribution laws that employed the logarithm of the droplet diameter as part of the fitting function. This class of droplet size distribution is referred to as the log normal distribution. Of the log normal-based fitting functions available in the Phase Doppler data acquisition software Flowsizer ${ }^{\mathrm{TM}}$, the Nukiyama-Tanasawa distribution function provided the best fit for all phase Doppler firefighting jet points measured. The Nukiyama-Tanasawa distribution is defined as follows:

$$
f(d)=d^{2} \exp -(b d)^{q}
$$

where $f(d)$ is a number distribution function describing the number of droplets of a given diameter $d$. The parameters $b$ and $q$ are fit variables. The characteristic droplet size distribution measured is illustrated in Figure 6.40 showing the fit comparison for both water and a 6-percent AFFF jet over a range of 25 discrete, equal width droplet size bins. Results represent the droplet size distribution of a measurement point that recorded approximately 50,000 measurements. Water and AFFF jets have similar distributions except water jets exhibited larger mean droplet diameters. This pattern was consistent for all firefighting jet pressure-flow rate combinations 
considered. More droplet size distribution data presented similarly to Figure 6.40 are in Appendix C.

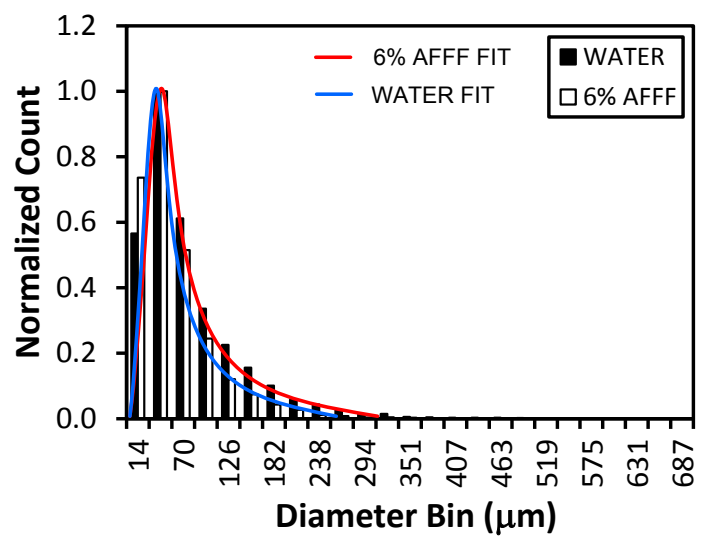

Figure 6.40: A Characteristic Firefighting Jet Droplet Size Distribution Measurement with Accompanying Fit.

Phase Doppler data of the mean axial droplet velocity $\left(V_{d, X}\right)$ as a function of droplet diameter $d$ were examined for both a water jet and 6-percent AFFF jet to analyze the sample distribution. Five locations spread across a 25 point 1-D profile were chosen for each firefighting jet. A downstream location from the central nozzle configuration is selected for presentation which demonstrates the characteristic trends from most data acquired. The ten locations are highlighted in Figure 6.41a and $\mathrm{b}$ illustrating the mean axial droplet velocity and mean droplet diameter $(D 10)$ as a function of vertical location. The points in Figure 6.41 represent values based on an average of approximately 50,000 samples recorded at that particular location.

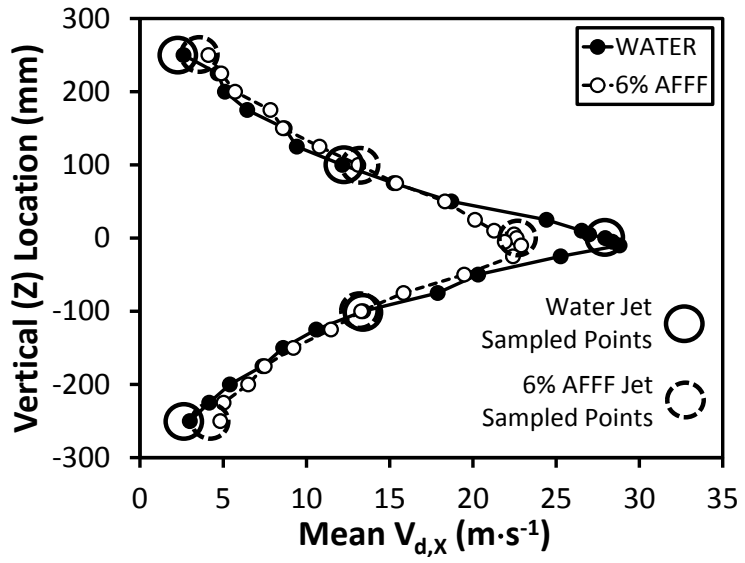

(a)

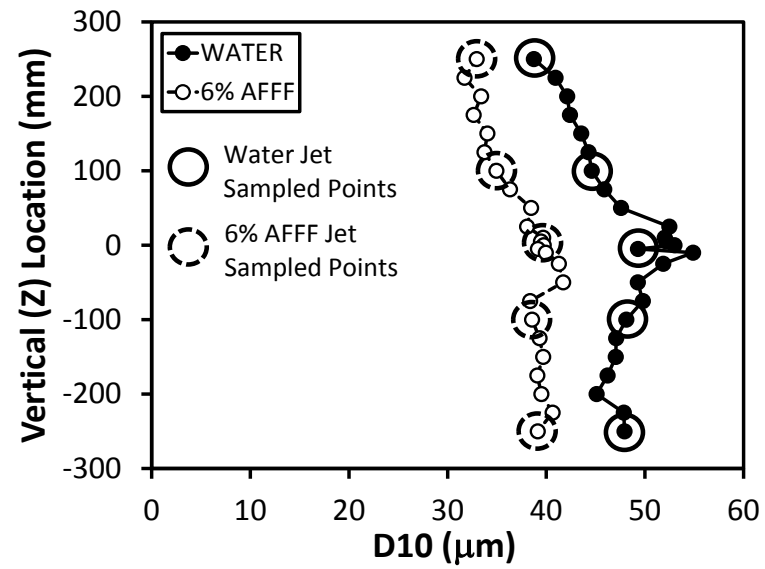

(b)

Figure 6.41: Characteristic Phase Doppler Profiles Highlighting Measurement Locations Selected to Visualize Mean Axial Droplet Velocity and Mean Droplet Diameter Sample Distribution at x = $3.05 \mathrm{~m}(10 \mathrm{ft})$ 
Raw sample distributions are depicted in Figure 6.42, and align well with data from Figure 6.41. All AFFF jet sample data is plotted in front of water jet sample data.

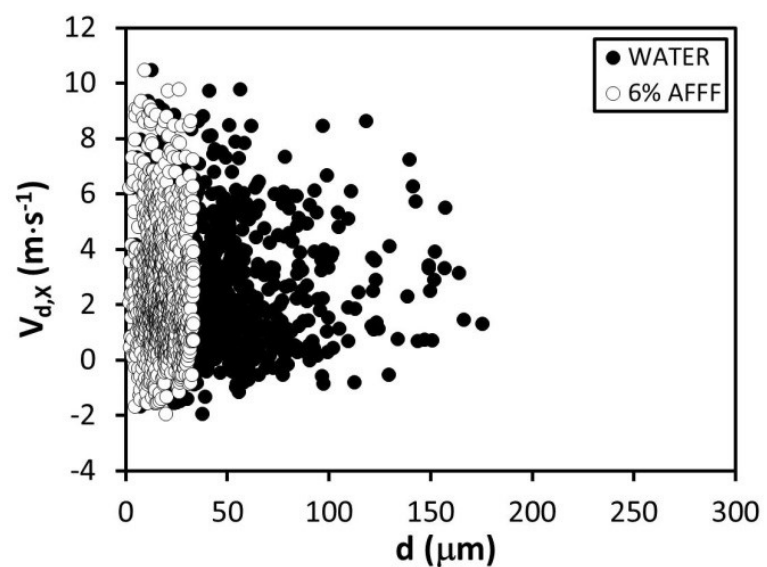

(a) Sampling Point 1 (Profile Top)

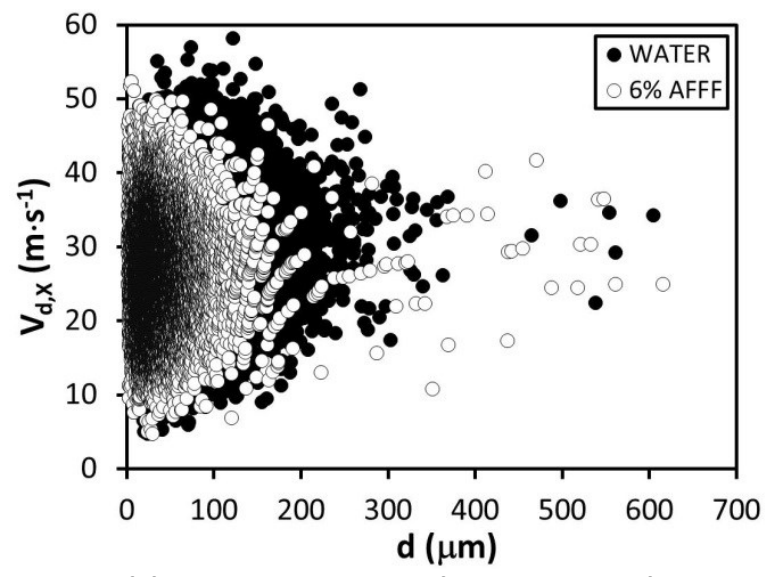

(c) Sampling Point 13 (Profile Center)

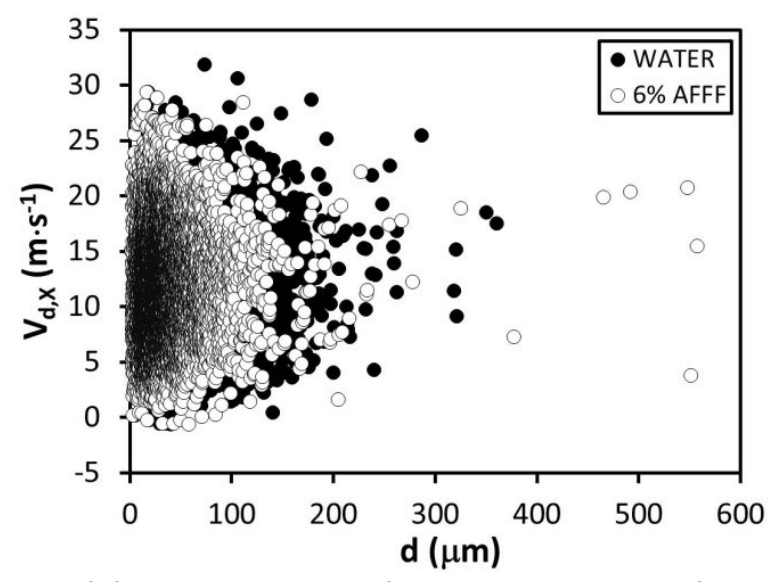

(b) Sampling Point 7 (Profile Upper Middle)

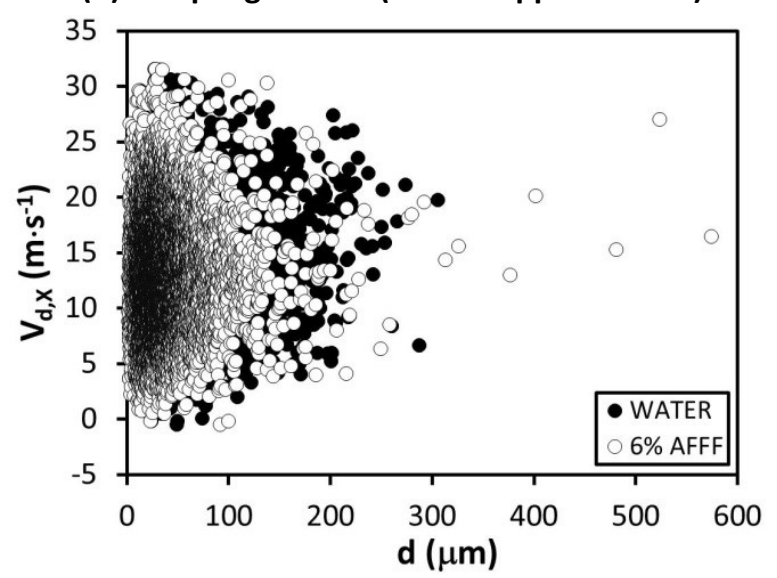

(d) Sampling Point 19 (Profile Lower Middle)

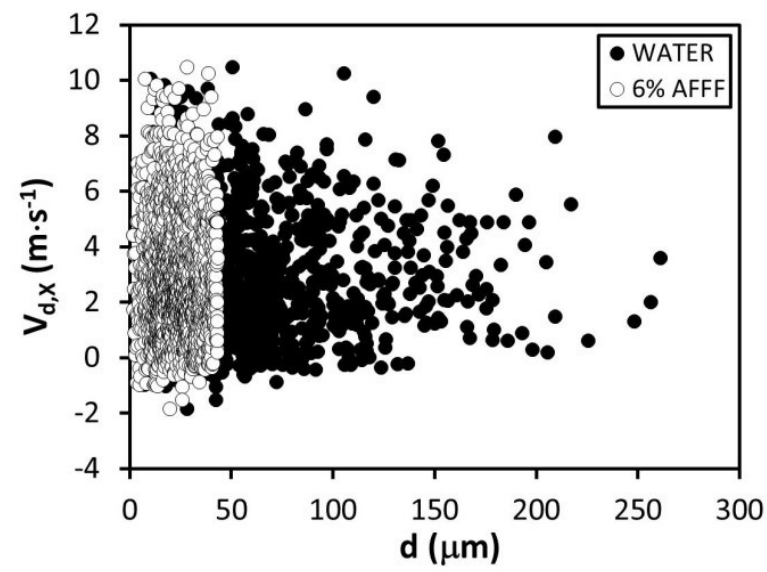

(e) Sampling Point 25 (Profile Bottom)

Figure 6.42: Select Sampling Data of Axial Droplet Velocity vs. Droplet Diameter of the Med. Flow, Med. Press. Jet at $x=3.05 \mathrm{~m}(10 \mathrm{ft})$ 
The most apparent observation in Figure 6.42 is the reduction in droplet size with the addition of AFFF. Minor differences in velocity spread between AFFF and water reflect the results shown in Figure 6.42. No major clipping was observed due to PDPA hardware limitations. Edge profile locations shown in Figure 6.42a and e exhibited a minor asymmetric distribution of 75 to $100-\mu \mathrm{m}$ sized droplets moving at lower velocities compared to other points of observation.

\subsubsection{Select Firefighting Jet Phase Doppler Results}

Main phase Doppler results are presented using the following five flow parameters:

$$
\begin{array}{ll}
\text { Mean Axial Droplet Velocity: } & V_{d, X}=\frac{1}{N} \sum_{i=1}^{N} v_{d, X_{i}} \\
\text { Mean Vertical Droplet Velocity: } & V_{d, Z}=\frac{1}{N} \sum_{i=1}^{N} v_{d, Z_{i}} \\
\text { Mean Droplet Diameter: } & D 10=\frac{1}{N} \sum_{i=1}^{N} d_{i} \\
\text { Root Mean Square Droplet Diameter: } & D_{R M S}=\sqrt{\frac{1}{N} \sum_{i=1}^{N} d_{i}^{2}} \\
\text { Sauter Mean Droplet Diameter: } & D 32=\left[\frac{\sum_{i=1}^{N} N_{i} d_{i}^{3}}{\sum_{i=1}^{N} N_{i} d_{i}^{2}}\right]
\end{array}
$$

where $N$ is the total number of samples recorded, and $v_{d, X_{i}}, v_{d, Z_{i}}$, and $d_{i}$ are the instantaneous axial droplet velocity, vertical droplet velocity, and droplet diameter, respectively. The mean droplet diameter is the standard method for comparing the relative difference between two droplet samples. The root mean square (RMS) droplet diameter is a measure of diameter spread about the mean. Mean axial and vertical droplet velocity is reported with minimum and maximum \pm RMS velocity for each profile shown via two horizontal bars. These bars are a visual characterization of the level of variability that occurs versus time due to the turbulence and not due to error bias. The Sauter mean droplet diameter represents the volume-to-surface ratio of the mean droplet diameter and typically defines the fineness of the jet spray. It is commonly used in efficiency studies involving mass transfer and chemical reactions making it a popular metric for characterizing firefighting agent application and combustion-related events. 
Filled (black) symbols and solid lines are used in all phase Doppler plots to refer to water jet data, and open (white) symbols and dashed lines are used to refer to AFFF jet data. A statistically unbiased, order of magnitude estimate $(E)$ of the deviation between the calculated mean droplet velocity and diameter values from their true mean values was found using autocorrelation techniques. By autocorrelating the raw data signal $(\Phi)$ using $\operatorname{MATLAB}^{\circledR}$, the number of samples it took for the signal to decorrelate $\left(N_{\text {decorr }}\right)$ was calculated. This information, the RMS and mean value from the sampled data, in addition to the total number of $N$ samples recorded was used to estimate $E$ from the following relationship:

$$
E=\frac{\Phi_{R M S}}{\sqrt{N \cdot N_{\text {decorr }}^{-1}}}
$$

Equation 6.7 dictates that as total sample size increased and the number of samples it took to decorrelate the signal decreased, the smaller the error estimate. This uncertainty analysis was applied to the five unique sampling locations shown in the phase Doppler profiles illustrated in Figure 6.41. The process was executed on select phase Doppler vertical profile locations listed in Table 6.2 for each of the five firefighting jet nozzle pressure-flow rate combinations utilizing water and 6-percent AFFF as the agent. The five uncertainty estimates from each profile are averaged with the results annotated on each respective profile plot.

Table 6.2: Phase Doppler Profile Measurement Locations Containing Uncertainty Analysis

\begin{tabular}{|c|c|c|c|}
\cline { 2 - 4 } \multicolumn{2}{c|}{} & Water Jet & AFFF Jet \\
\hline $\begin{array}{c}\text { Station } \\
\text { No. }\end{array}$ & $\begin{array}{c}\text { X-Axis } \\
\text { Location }\end{array}$ & $\begin{array}{c}\text { 1-D Vertical } \\
\text { Z-axis Profile }\end{array}$ & $\begin{array}{c}\text { 1-D Vertical } \\
\text { Z-axis Profile }\end{array}$ \\
\hline 3 & $0.152 \mathrm{~m}(6 \mathrm{in})$ & $\bullet$ & $\mathbf{0}$ \\
\hline 5 & $0.914 \mathrm{~m}(3 \mathrm{ft})$ & $\bullet$ & $\mathbf{0}$ \\
\hline 7 & $3.05 \mathrm{~m}(10 \mathrm{ft})$ & $\bullet$ & $\mathbf{0}$ \\
\hline 9 & $6.10 \mathrm{~m}(20 \mathrm{ft})$ & $\bullet$ & $\mathbf{0}$ \\
\hline
\end{tabular}

Auxiliary information regarding PDPA velocity and diameter measurement uncertainty based on hardware resolution and spatial system alignment from manufacturer experience is located in Section 3.5. Information on the manufacturer's calibration certification procedure for measuring particle diameter for the PDPA used specifically for this work is in Appendix D: PDPA 
Calibration. In general, all methods of determining PDPA measurement error reported uncertainties on the order of one percent or less which was often much less than the statistical variation in the flow parameters.

Figure 6.43 illustrates sample results of the five aforementioned measured flow parameters as a function of vertical profile location for the medium flow, medium pressure jet. All phase Doppler data shown in Figure 6.43 are from the axial downstream $x=6.10 \mathrm{~m}(20 \mathrm{ft})$ profile location. For spatial reference, Figure 4.7 and Table 4.2 in Section 4.4 illustrate and outline all PDPA profile measurement locations, respectively. Figure 6.43a represents a downstream mean axial droplet velocity profile exhibiting asymmetric spread directionally-biased toward the floor due to gravity. Axial AFFF droplet velocity slightly lagged axial water droplet velocity due to the presence of smaller mean AFFF droplet diameters with less momentum which are illustrated in Figure 6.43c. Figure 6.43b represents a mean vertical droplet velocity profile showing symmetry about the $z=0-\mathrm{m}$ plane. Negligible difference was observed between vertical AFFF and water droplet velocities. Figure $6.43 \mathrm{~d}$ shows RMS droplet diameters similar in magnitude to the mean droplet diameters presented in Figure 6.43c, supporting the observations in Figures 6.40 and 6.42 that a wide distribution of droplet sizes are present. AFFF RMS droplet diameter magnitudes are slightly smaller compared to water jet RMS droplet diameters, which is likely an extended consequence of smaller mean droplet sizes. Figure 6.43e shows that the AFFF jet exhibits larger Sauter mean droplet diameters in comparison to the water jet, but results are noticeably more scattered and less conclusive compared to mean droplet diameter profile results. In general, Sauter mean diameter is larger than the mean droplet diameter. Further discussion of firefighting jet PDPA flow parameter patterns as they relate to all firefighting jet configurations is in Section 6.3.3. Phase Doppler flow parameter profile data from all firefighting jet conditions examined are in Appendix C. 


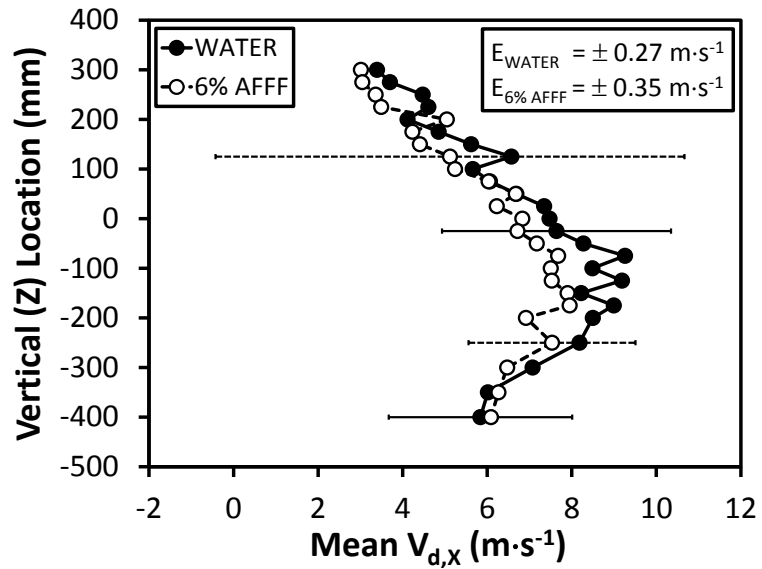

(a)

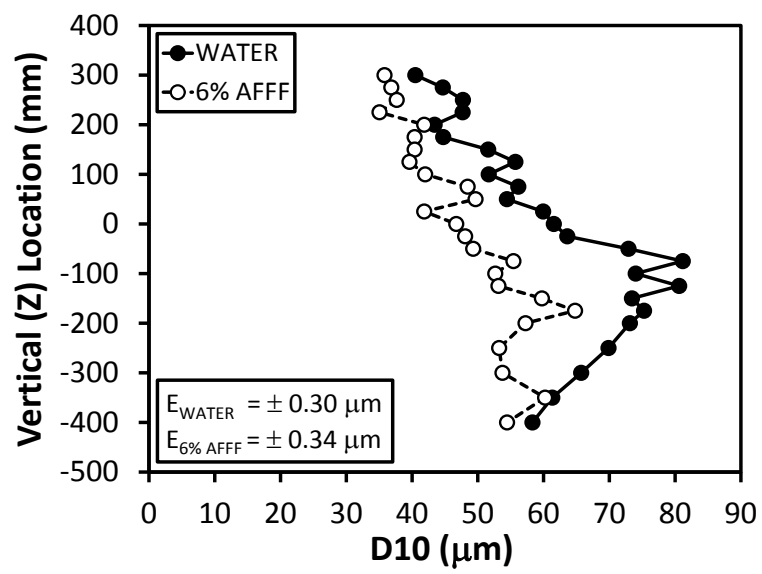

(c)

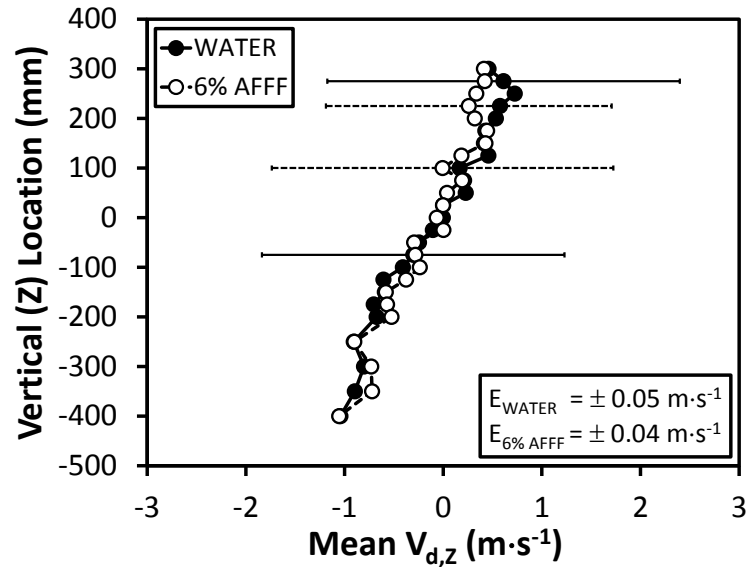

(b)

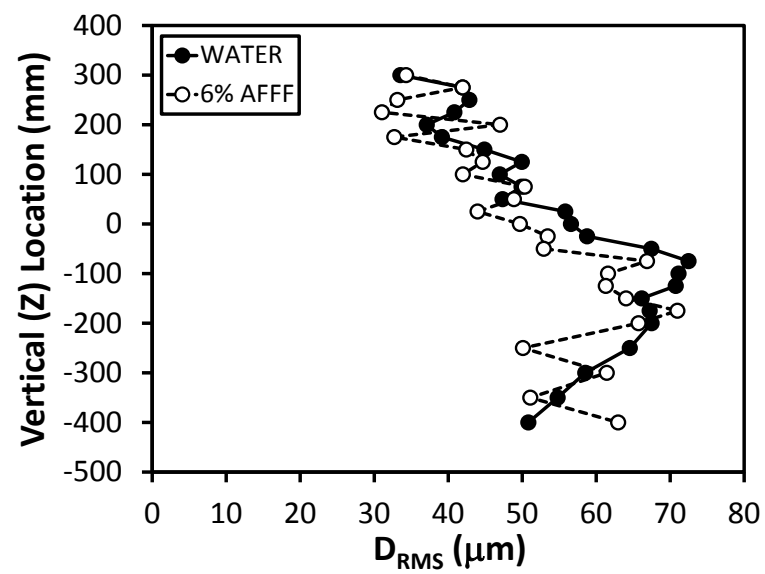

(d)

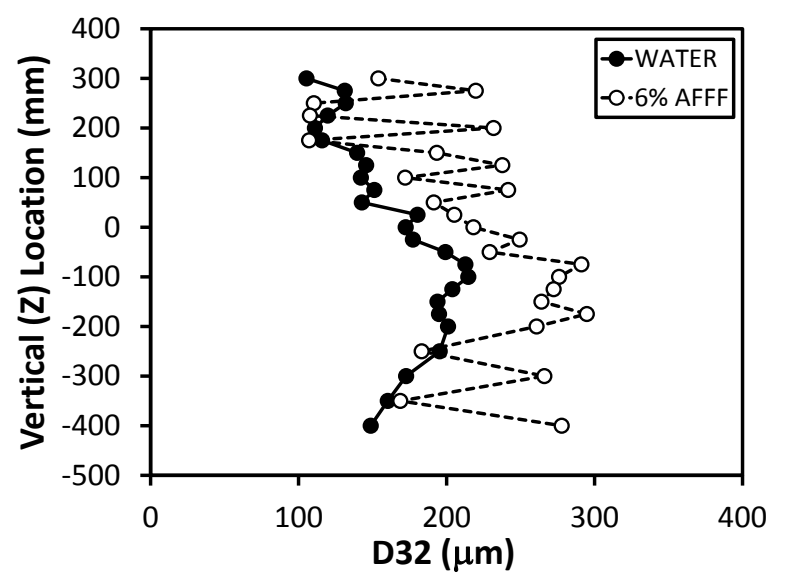

(e)

Figure 6.43: A Sample of Vertical, Phase Doppler, Droplet Profile Data from the $x=6.10 \mathrm{~m}(20 \mathrm{ft})$ Axial Downstream Location from the Med. Flow, Med. Press. Firefighting Jet

Figure 6.44a and b depict sample axial $\left(V_{G, X}\right)$ and vertical $\left(V_{G, Z}\right)$ mean, gaseous (air) vertical velocity profile estimates for the medium flow, medium pressure jet, respectively. Horizontal bars represent the \pm RMS velocity magnitude. Data are presented at the same axial downstream 
location used in Figure 6.43. These values were approximated based on phase Doppler data recorded on droplets $10 \mu \mathrm{m}$ or less in diameter assuming their trajectories followed air streamlines. Results are presented at the five measurement locations highlighted in Figure 6.41. Based on the ratio of RMS to mean air velocity fluctuations, turbulence intensity values ranged from about 35 to 90 percent indicating highly turbulent flow. This was a consistent observation for all firefighting jet configurations. Mean air velocity estimates are close but slightly less in value to those shown in Figure 6.43a and $\mathrm{b}$ which take the entire droplet diameter range measured into account. The similarity is due to fully-entrained air flow particularly downstream where droplet slip velocities subsequently reduced in magnitude as distance from the nozzle increased. AFFF jet, axial air velocities also slightly lagged water jet, axial air velocities similar to the trends and reasons observed in Figure 6.43a. More axial and vertical air velocity profile data estimates from the medium flow, medium pressure jet are in Appendix C in Section C.3.

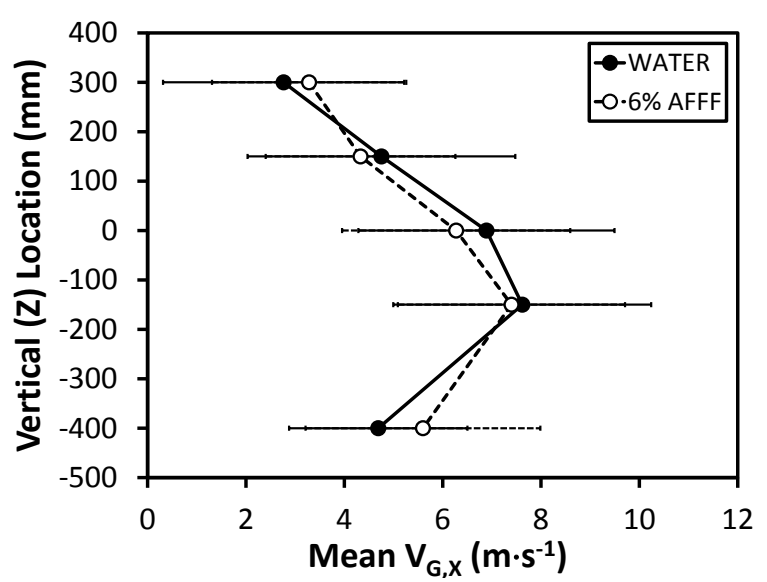

(a)

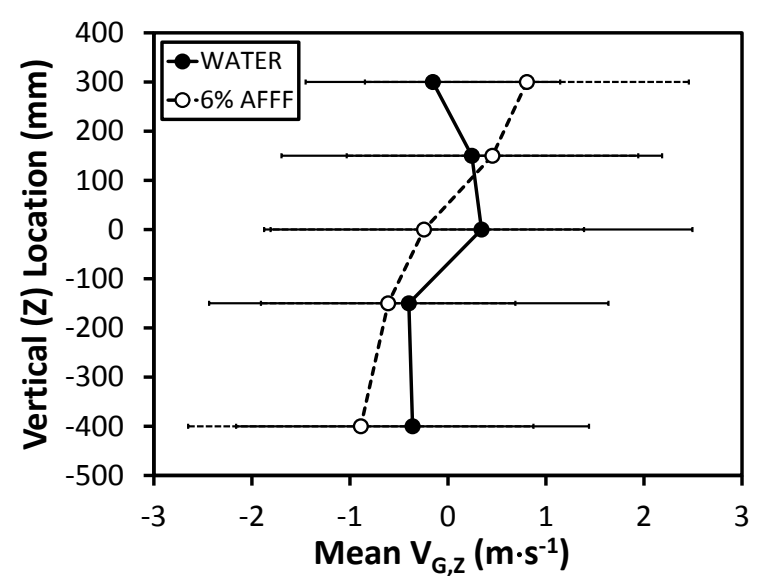

(b)

Figure 6.44: A Sample of Vertical, Phase Doppler Profile Estimates of Axial, Gaseous (Air) Velocity from the $x=6.10 \mathrm{~m}(20 \mathrm{ft})$ Axial Downstream Location from the Med. Flow, Med. Press. Firefighting Jet

\subsubsection{Firefighting Jet Phase Doppler Results Summary}

Phase Doppler results have been condensed to summarize the global patterns of each of the five main flow parameters presented for every firefighting jet configuration analyzed. All individual phase Doppler profile results are located in Appendix C. Each 1-D vertical profile composed of 25 discrete locations was averaged to generate a single mean profile value for every flow parameter. Maximum, mean axial and mean vertical droplet velocity values were also extracted from each profile to provide another point of comparison. Figure 6.45 illustrates this process. 


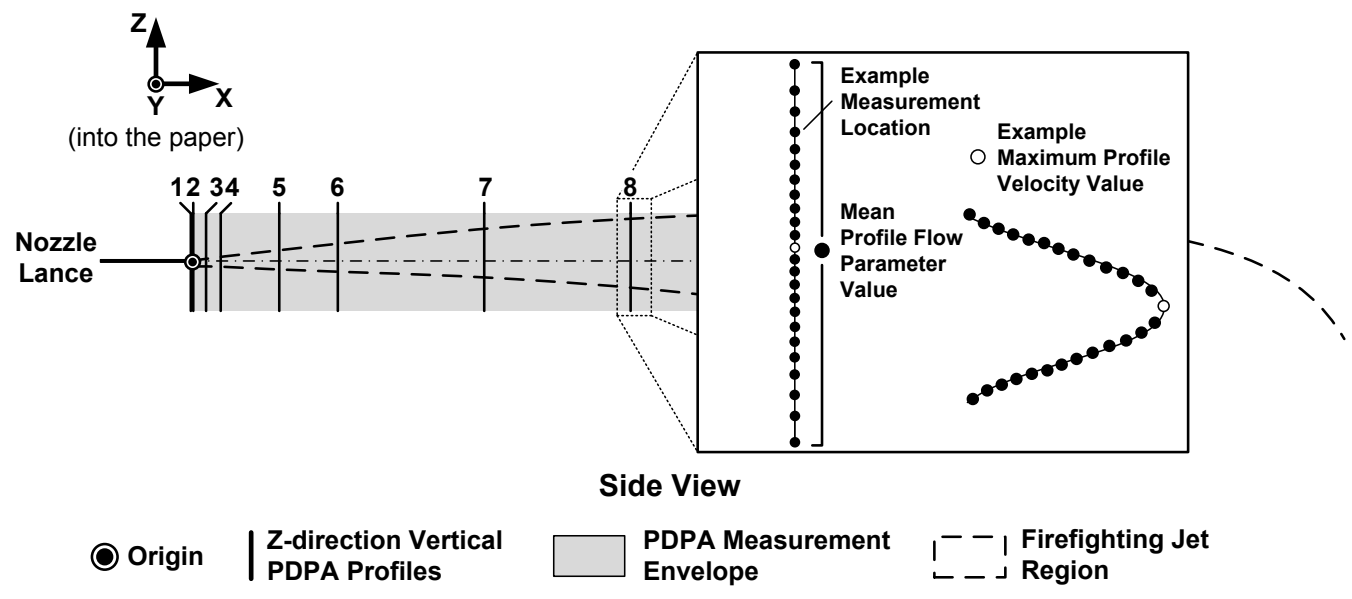

Figure 6.45: A Schematic Illustrating the Max. and Mean Phase Doppler Profile Value

Data are presented to describe the mean or maximum profile flow parameter as a function of axial downstream jet location for each firefighting jet configuration. Profile data for each flow parameter consisted of nine to eleven points depending on the number of vertical profiles recorded. The downstream $x$-axis axial jet location is reported non-dimensionally with respect to the nozzle diameter analogous to how 1-D agent ground pattern results are presented in Sections 6.2.5 and 6.2.6.

\section{Phase Doppler Axial Velocity Results Summary}

Figure 6.46 summarizes the phase Doppler, mean axial droplet velocity measurements for all firefighting jet configurations analyzed. Figure 6.46a illustrates the maximum profile, mean axial water droplet velocity non-dimensionalized with respect to the maximum profile, mean axial water droplet velocity measured adjacent to the nozzle exit (i.e. Station 1 in Figure 4.7 and Table 4.2 in Section 4.4). Figure 6.46c depicts the maximum profile, mean axial water droplet velocity but in dimensional form, and Figure 6.46e shows the mean profile, axial water droplet velocity. Figures $6.46 \mathrm{~b}, \mathrm{~d}$, and e illustrate the same scenarios as in Figure 6.46a, c, and e, respectively, but instead use 6-percent AFFF as the agent.

Maximum, mean axial droplet velocity did not occur at the measurement plane adjacent to the nozzle, but rather anywhere from approximately 2 to $15 \mathrm{~cm}$ downstream depending on the nozzle pressure-flow rate combination. This was due to a phenomenon referred to as vena contracta where the fluid stream continued to contract downstream of the orifice causing the jet to further accelerate beyond the nozzle exit plane. Water jet and AFFF jet maximum, mean axial droplet 
velocity magnitudes were similar to one another with near nozzle velocities ranging from about 45 to $138 \mathrm{~m} \cdot \mathrm{s}^{-1}$ for low flow, low pressure and low flow, high pressure firefighting jets, respectively. All firefighting jet axial velocities exhibited classic non-linear decay as downstream distance increased. Mean profile, mean axial droplet velocity followed the same trend with respect to nozzle pressure and flow rate as the highest velocities ranged from approximately 35 to $85 \mathrm{~m} \cdot \mathrm{s}^{-1}$ near the nozzle to as low as 3 to $7 \mathrm{~m} \cdot \mathrm{s}^{-1}$ at the far reaches of each firefighting jet.

Figures 6.47 through 6.49 reproduce the same data shown in Figure 6.46, but highlight the effect AFFF had on each nozzle pressure-flow rate combination compared to water. Figure 6.47 expands non-dimensional, maximum profile, mean axial droplet velocities illustrated in Figure 6.46a and $\mathrm{b}$. Figure 6.48 expands the dimensional form of the maximum profile, mean axial droplet velocities depicted in Figure 6.46c and d, and Figure 6.49 expands mean profile, mean axial droplet velocities shown in Figure 6.46e and f. In addition to reporting mean profile, mean axial droplet velocity, Figure 6.49 depicts the mean profile, \pm RMS axial droplet velocity magnitude via vertical bars. Mean profile turbulence intensities were reported up to 40 percent in near field nozzle areas and approached zero percent in some locations measured furthest downstream. Individual profiles presented in Appendix C commonly depict turbulence intensities approaching 100 percent for many cases.

Figures $6.47,6.48$, and particularly, 6.49 more clearly illustrated vena contracta exhibiting up to a 17-percent velocity increase just downstream of the nozzle for the medium flow, medium pressure firefighting jet. The high flow, low pressure firefighting jet appeared least affected by the phenomena with a near negligible increase in velocity beyond the nozzle.

Figures 6.47 through 6.49 also depict AFFF mean profile, mean axial droplet velocity equaling or slightly lagging water mean profile, mean axial droplet velocity universally for every nozzle pressure-flow rate combination by as much as 10 percent in certain circumstances. This observation was acknowledged in discussing Figure 6.43a in Section 6.3.2. It is presumed due to the additional momentum loss exhibited by AFFF jets from an aerodynamic drag increase caused by enhanced break-up generated by the surfactants in AFFF leading to greater diffusion and turbulent mixing. 


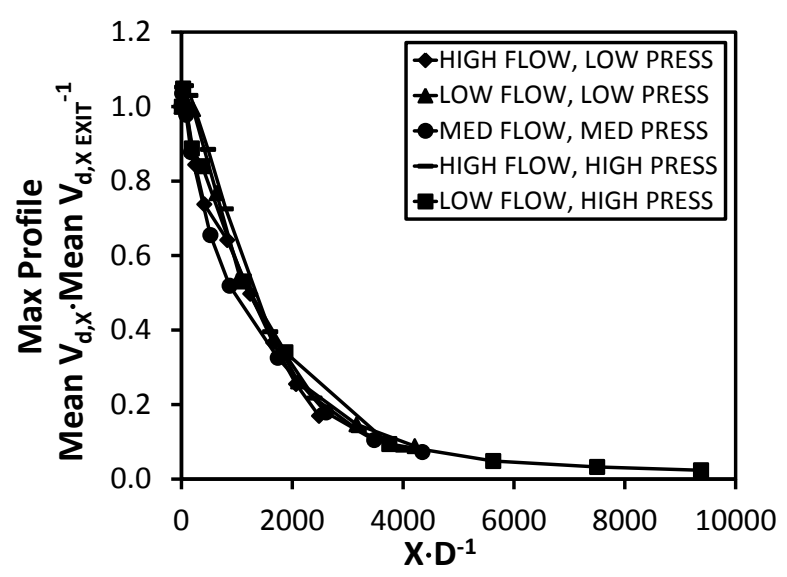

(a) Water Jets

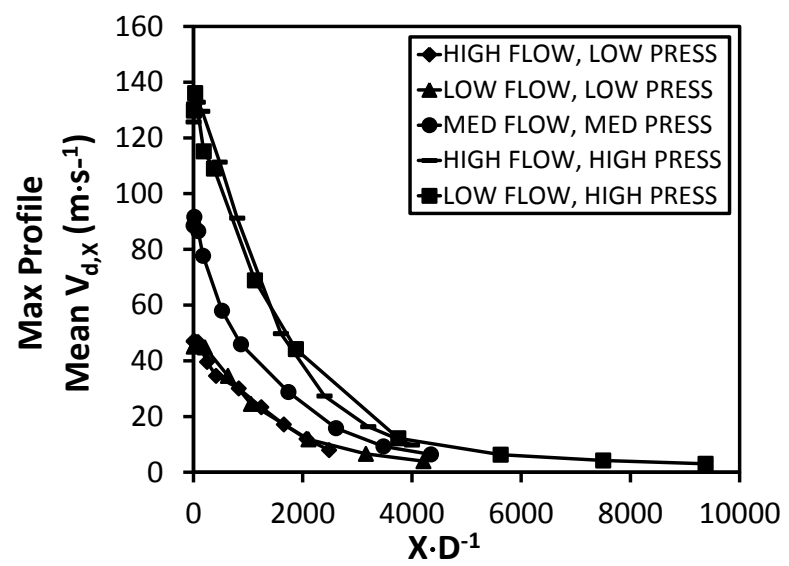

(c) Water Jets

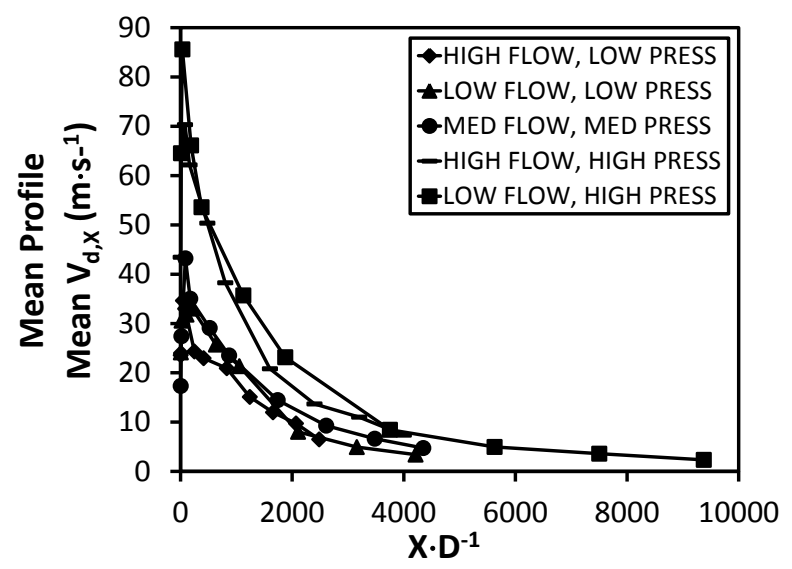

(e) Water Jets

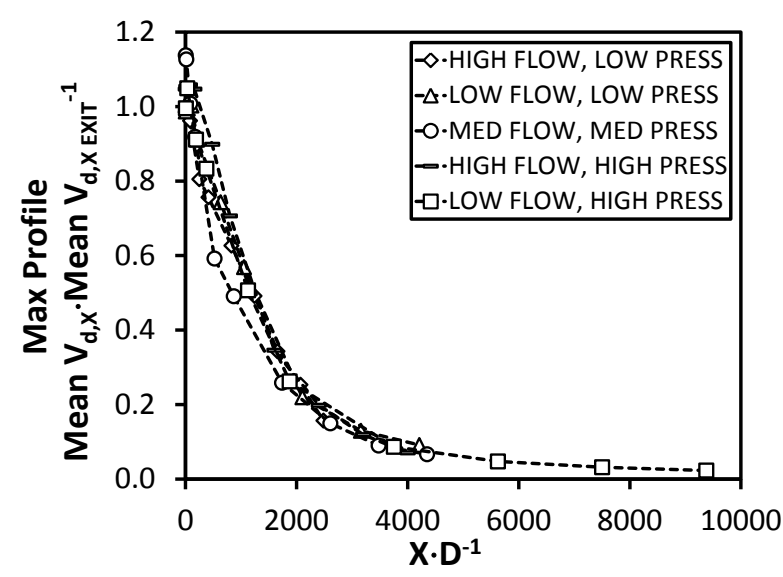

(b) $6 \%$ AFFF Jets

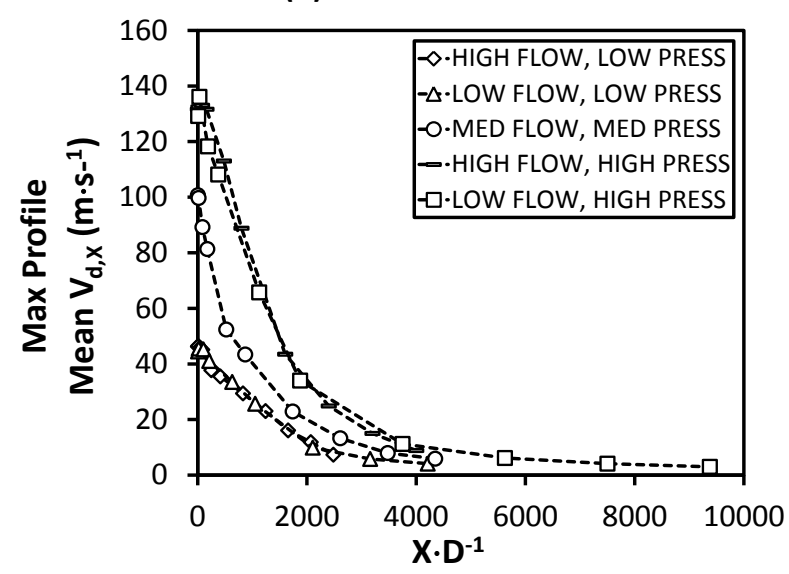

(d) $6 \%$ AFFF Jets

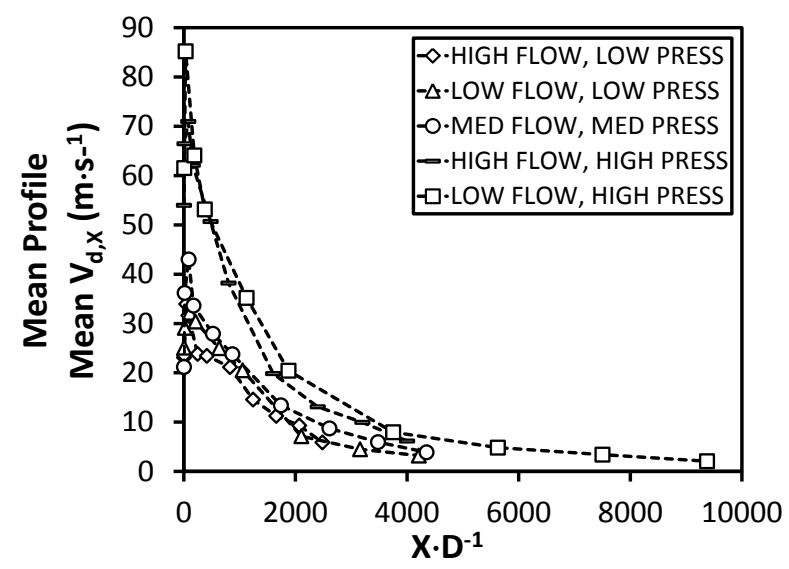

(f) $6 \%$ AFFF Jets

Figure 6.46: Firefighting Jet PDPA Summary Data in Terms of Max. and Mean Profile, Axial Droplet Velocity 


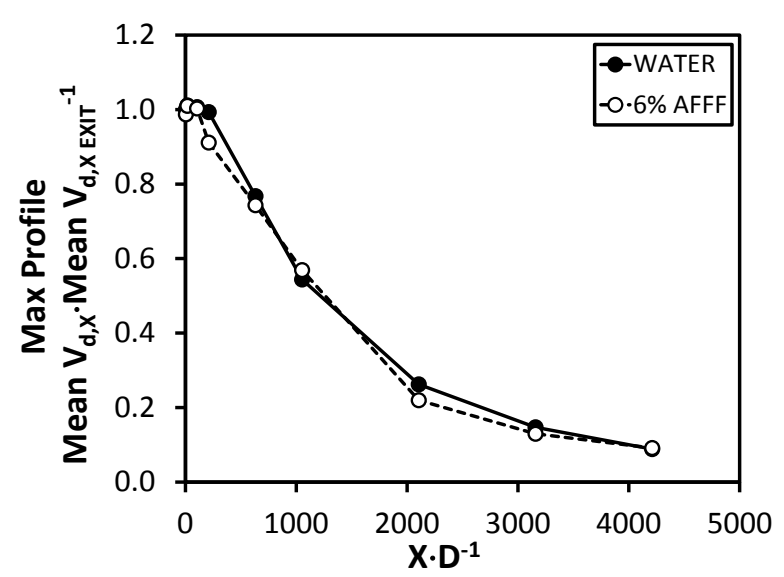

(a) Low Flow, Low Press. Jets

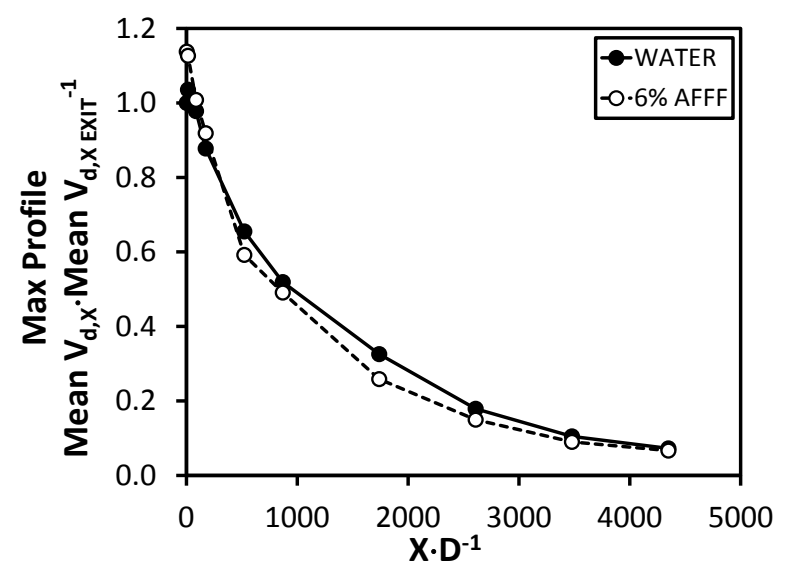

(c) Med. Flow, Med. Press. Jets

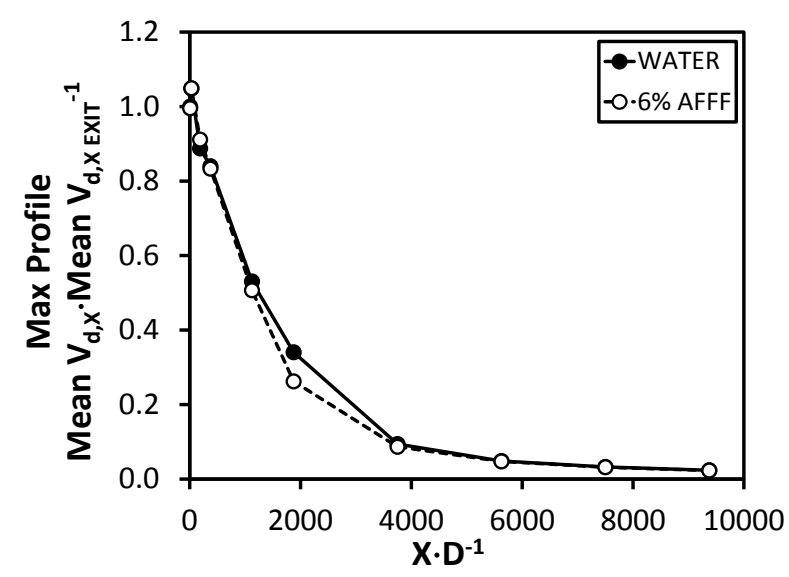

(b) Low Flow, High Press. Jets

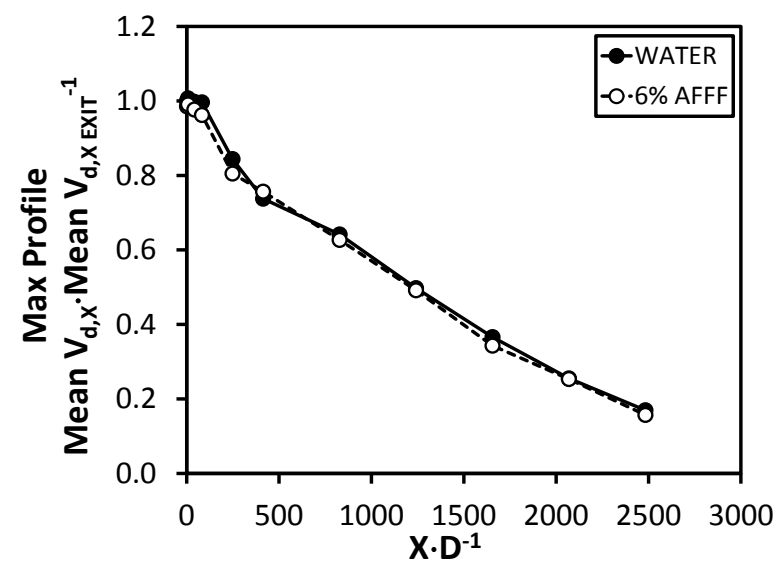

(d) High Flow, Low Press. Jets

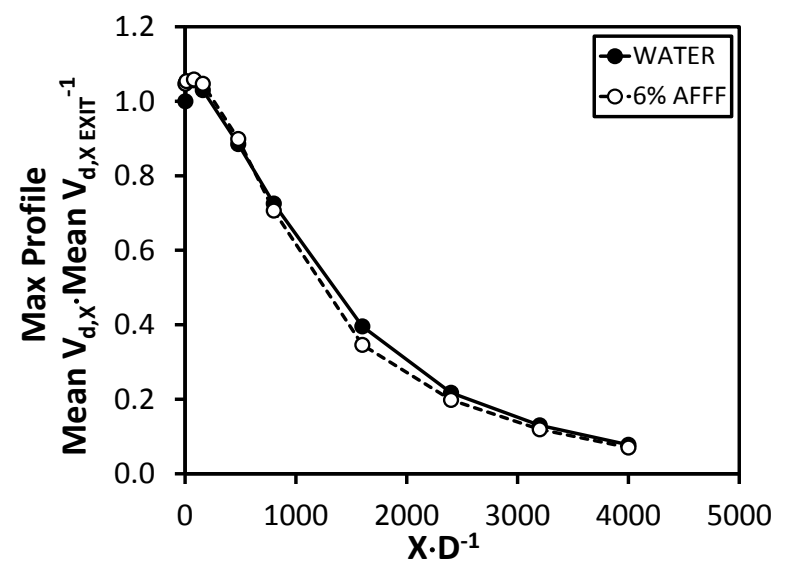

(e) High Flow, High Press. Jets

Figure 6.47: Firefighting Jet PDPA Summary in Terms of Non-Dimensional Max. Profile, Mean Axial Droplet Velocity 


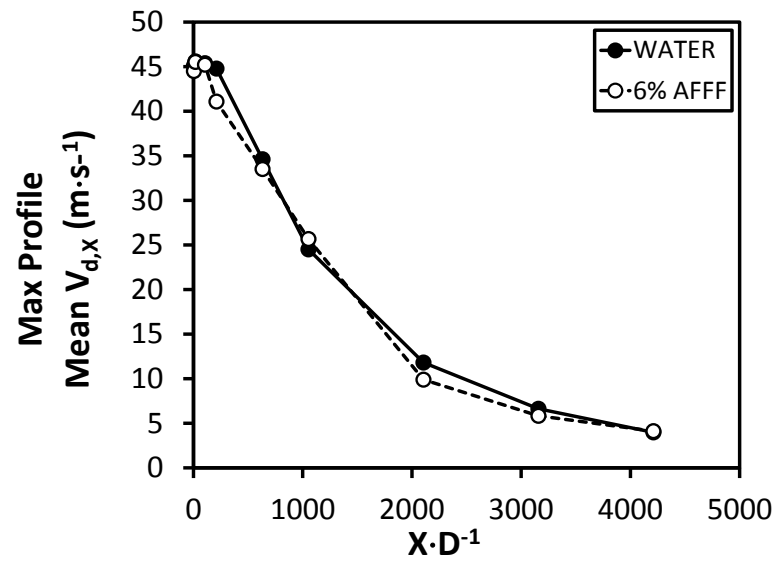

(a) Low Flow, Low Press. Jets

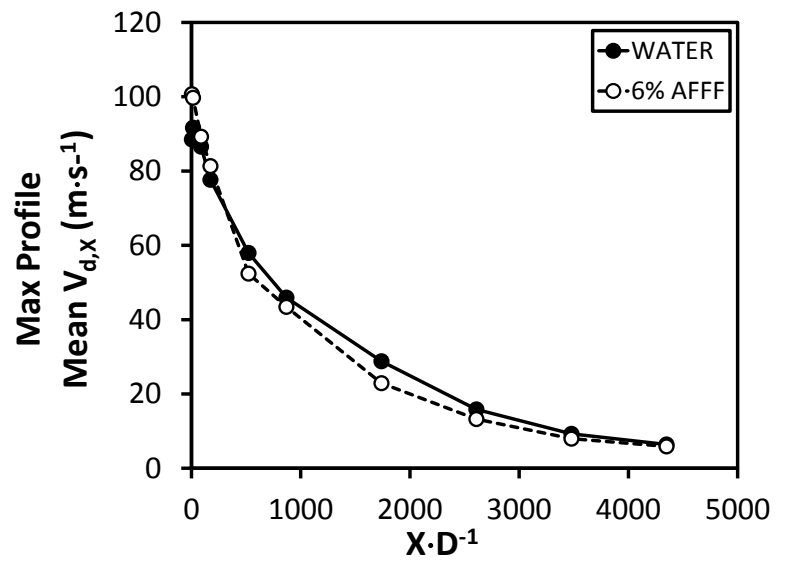

(c) Med. Flow, Med. Press. Jets

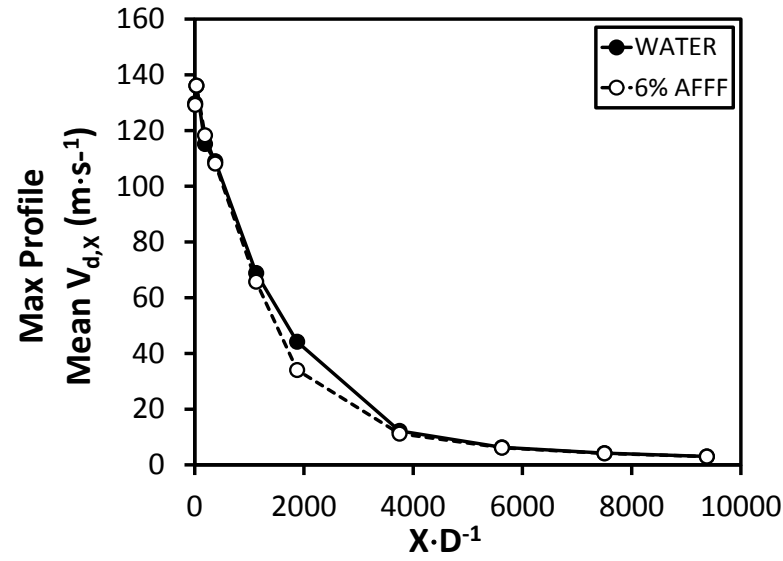

(b) Low Flow, High Press. Jets

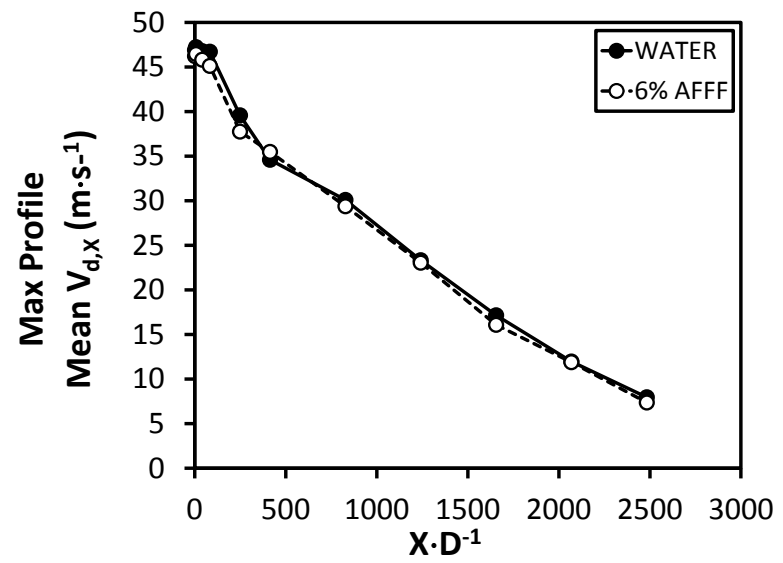

(d) High Flow, Low Press. Jets

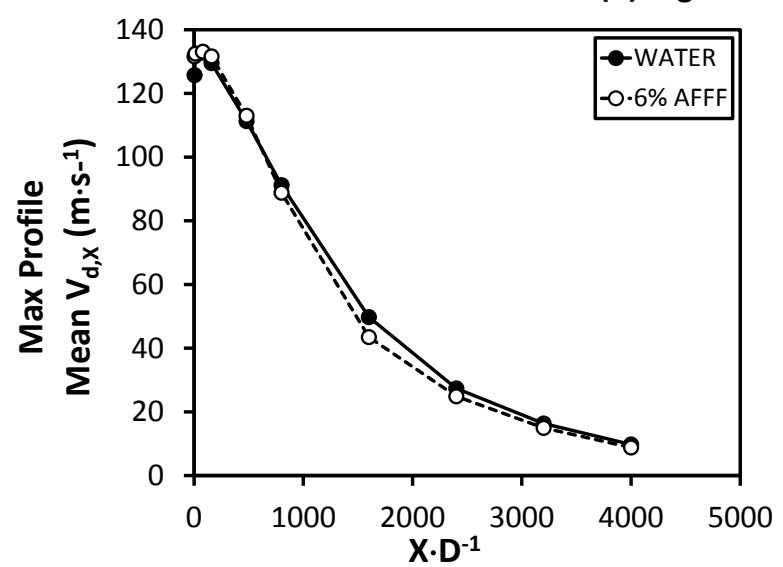

(e) High Flow, High Press. Jets

Figure 6.48: Firefighting Jet PDPA Summary in Terms of Dimensional Max. Profile, Mean Axial Droplet Velocity 


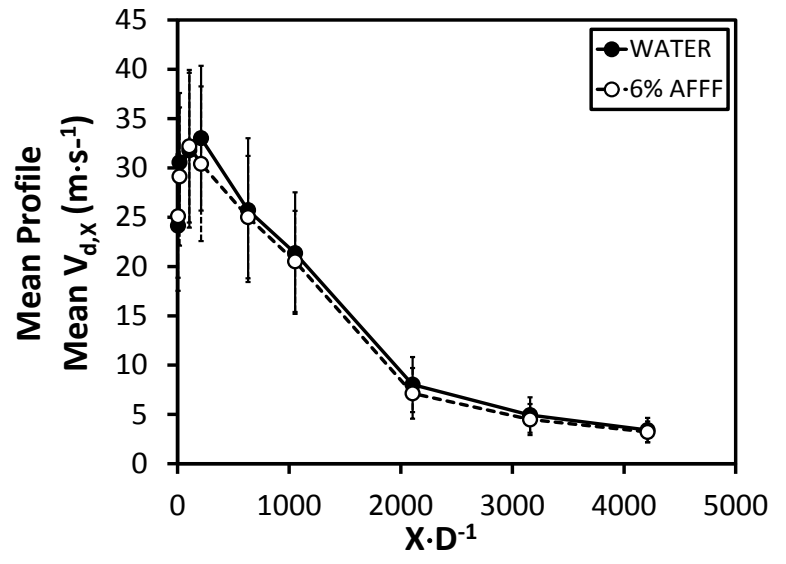

(a) Low Flow, Low Press. Jets

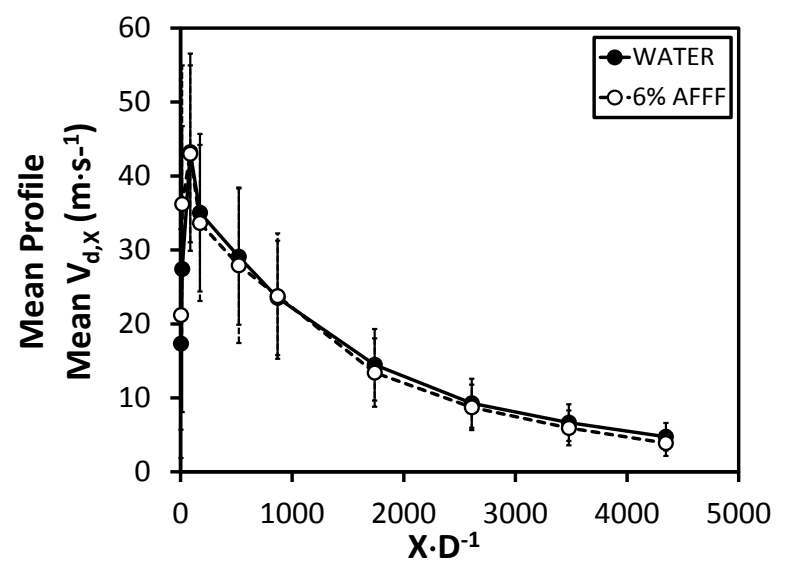

(c) Med. Flow, Med. Press. Jets

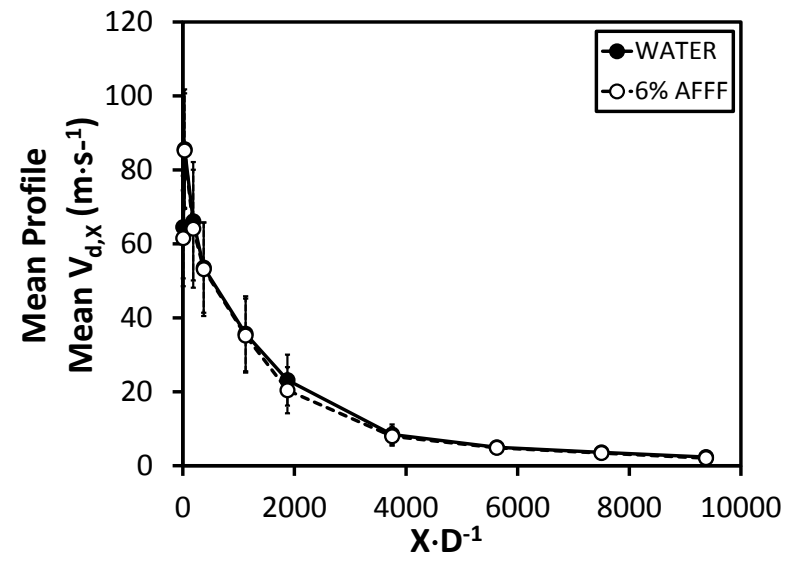

(b) Low Flow, High Press. Jets

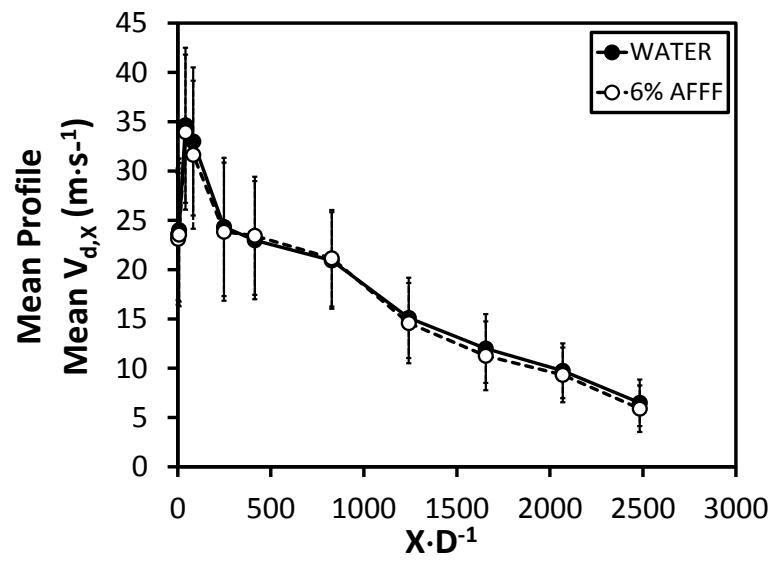

(d) High Flow, Low Press. Jets

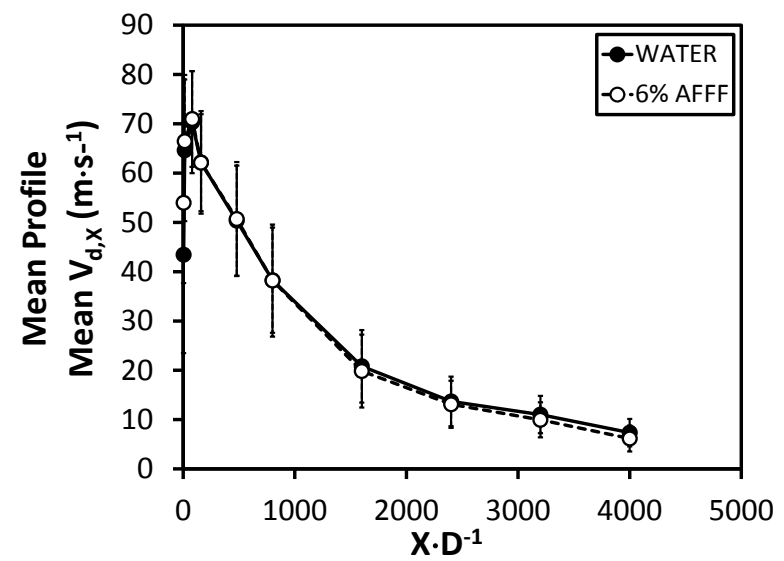

(e) High Flow, High Press. Jets

Figure 6.49: Firefighting Jet PDPA Summary in Terms of Mean Profile, Mean Axial Droplet Velocity; Vertical Bars Represent Mean Profile, \pm RMS Axial Droplet Velocity Magnitude

\section{Phase Doppler Vertical Velocity Results Summary}

Figure 6.50 summarizes the phase Doppler, mean vertical droplet velocity measurements for all firefighting jet configurations analyzed. Figure 6.50a illustrates the maximum profile, mean 
vertical water droplet velocity, and Figure $6.50 \mathrm{c}$ depicts the mean profile, mean vertical water droplet velocity. Figures $6.50 \mathrm{~b}$ and $\mathrm{d}$ depict the same scenarios as Figure $6.50 \mathrm{a}$ and $\mathrm{c}$, respectively, but instead use 6-percent AFFF as the agent.

For all firefighting jet configurations, maximum profile, mean vertical droplet velocity magnitude increased as nozzle pressure increased illustrating the effect pressure had on dispersion strength and overall momentum exchange in the vertical direction. Maximum profile, mean vertical droplet velocity magnitude also tended to decrease as flow rate increased, indicating the loss in vertical jet momentum as axial jet momentum increased. Maximum profile, mean vertical droplet velocity magnitudes ranged as high as $8 \mathrm{~m} \cdot \mathrm{s}^{-1}$ for the low flow, high pressure jet to as low as $2 \mathrm{~m} \cdot \mathrm{s}^{-1}$ for the high flow, low pressure jet. Mean profile, mean vertical droplet velocities followed the same trend as max profile data, ranging from a high of about $1.75 \mathrm{~m} \cdot \mathrm{s}^{-1}$ to a low of approximately $0.75 \mathrm{~m} \cdot \mathrm{s}^{-1}$.

Figures 6.51 and 6.52 reproduce the same data shown in Figure 6.50, but highlight the effect AFFF had on each nozzle pressure-flow rate combination compared to water. Figure 6.51 expands the maximum profile, mean vertical droplet velocities illustrated in Figure 6.50a and b, and Figure 6.52 expands the mean profile, mean vertical droplet velocities depicted in Figure $6.50 \mathrm{c}$ and d, respectively. Figure 6.52 shows the mean profile, \pm RMS vertical droplet velocity via vertical bars in addition to reporting the mean profile, mean vertical droplet velocity. Insignificant differences were observed between AFFF and water jet, mean vertical droplet velocities regardless of the nozzle pressure-flow rate combination. RMS vertical droplet velocity magnitudes with mean values typically around zero ranged as high as $5 \mathrm{~m} \cdot \mathrm{s}^{-1}$ in some instances reinforcing the turbulent nature of the firefighting jet flow field.

The results presented in Figure 6.52 also serve as a PDPA system alignment check to ensure mean profile, mean vertical droplet velocities fell at or near zero, particularly for lower flow, higher pressure firefighting jets with strong symmetry about the jet axial centerline. Low flow, high pressure and medium flow, medium pressure jets exhibited the best vertical symmetry as expected. High flow, low pressure jets showed the most significant asymmetry in Figure 6.52d with the largest observed mean droplet diameters under the strongest influence of gravity over the furthest axial reach. 


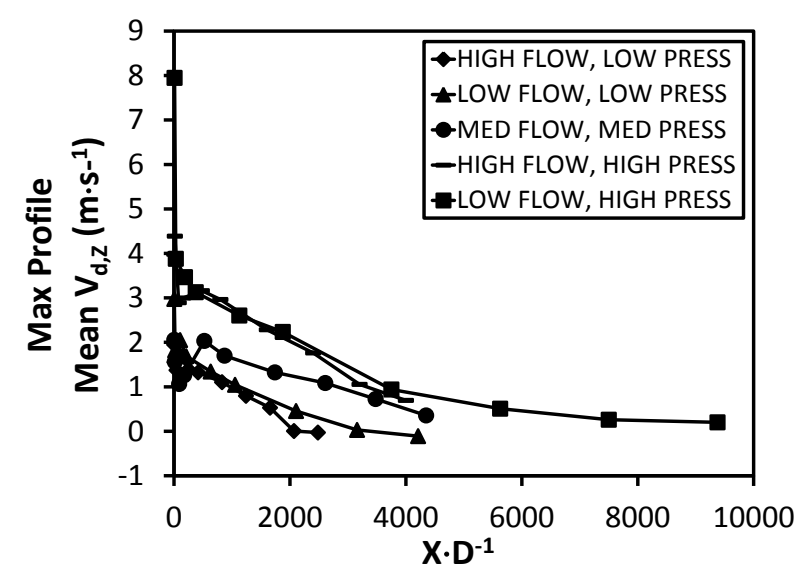

(a) Water Jets

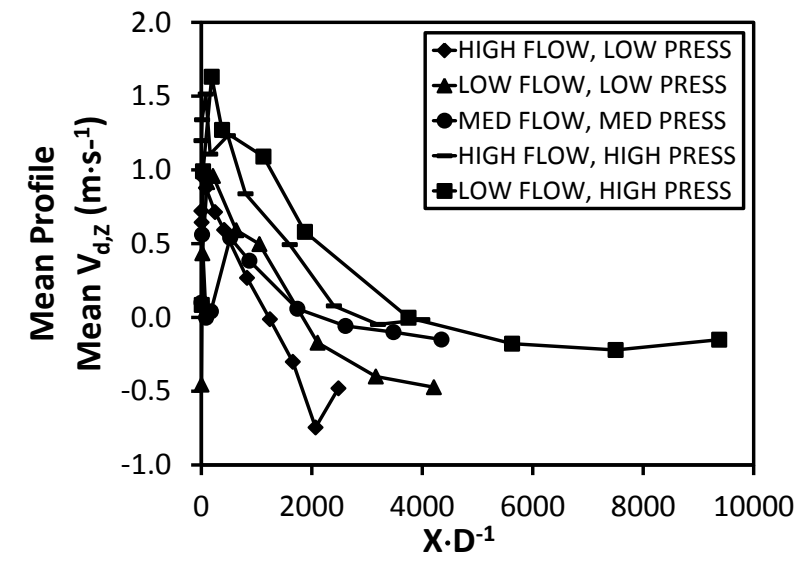

(c) Water Jets

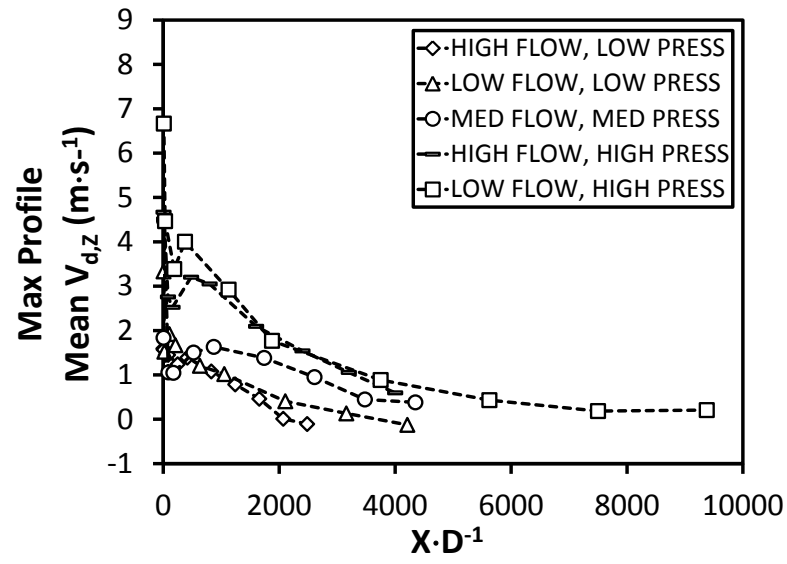

(b) $6 \%$ AFFF Jets

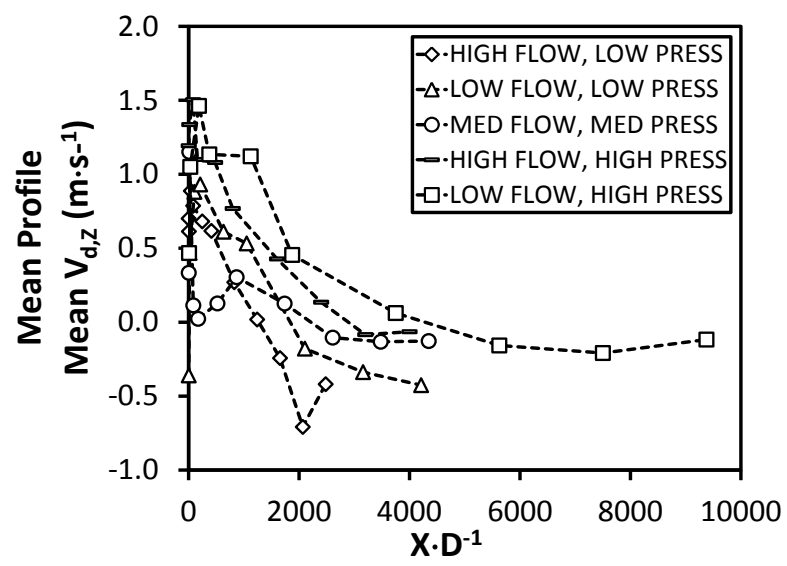

(d) $6 \%$ AFFF Jets

Figure 6.50: Firefighting Jet PDPA Summary Data in Terms of Max. and Mean Profile, Mean Vertical Droplet Velocity 


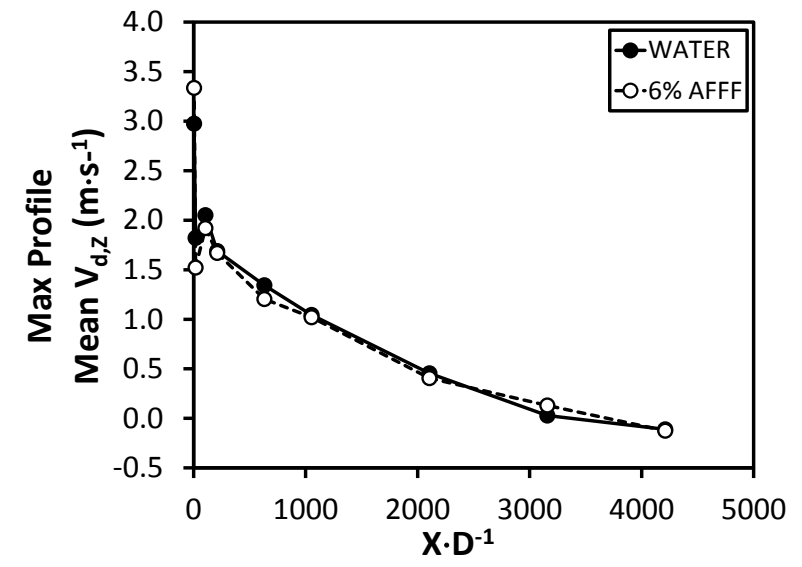

(a) Low Flow, Low Press. Jets

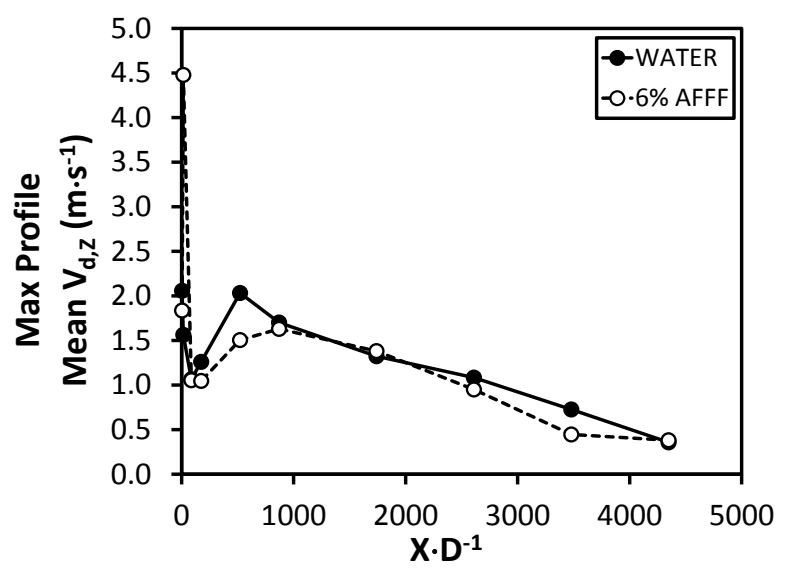

(c) Med. Flow, Med. Press. Jets

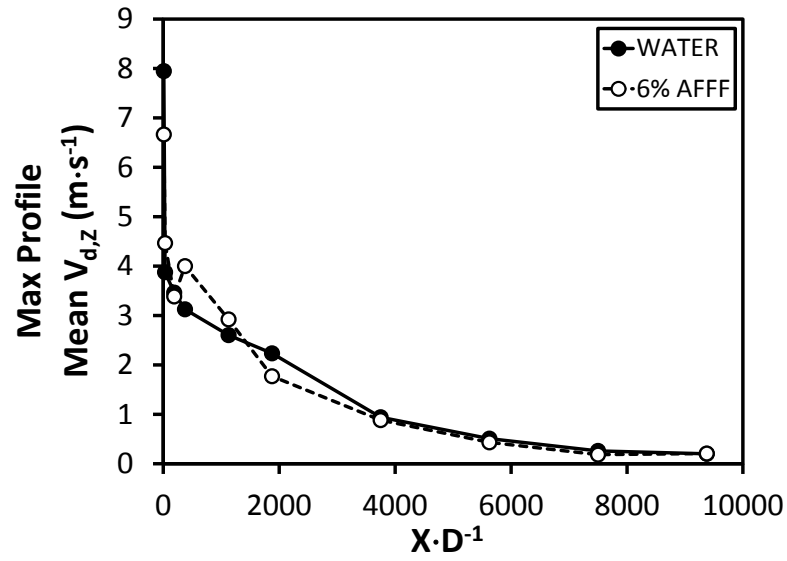

(b) Low Flow, High Press. Jets

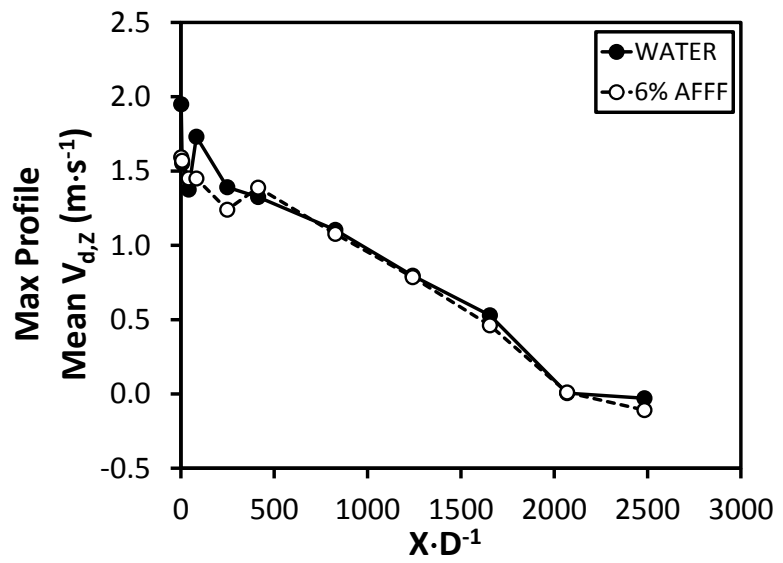

(d) High Flow, Low Press. Jets

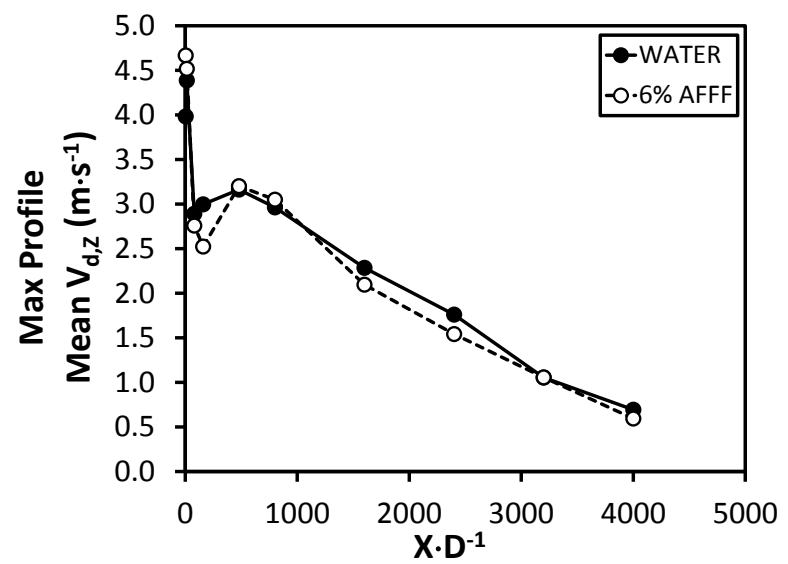

(e) High Flow, High Press. Jets

Figure 6.51: Firefighting Jet PDPA Summary in Terms of Max. Profile, Mean Vertical Droplet Velocity 


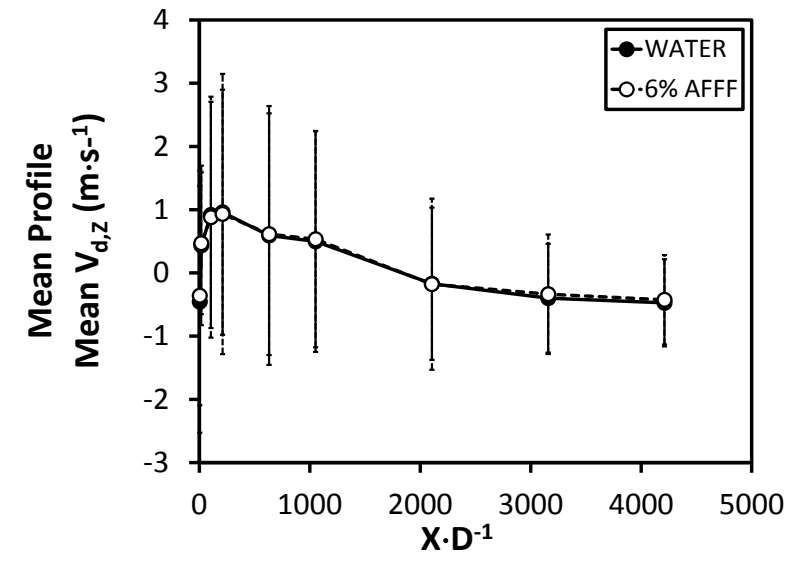

(a) Low Flow, Low Press. Jets

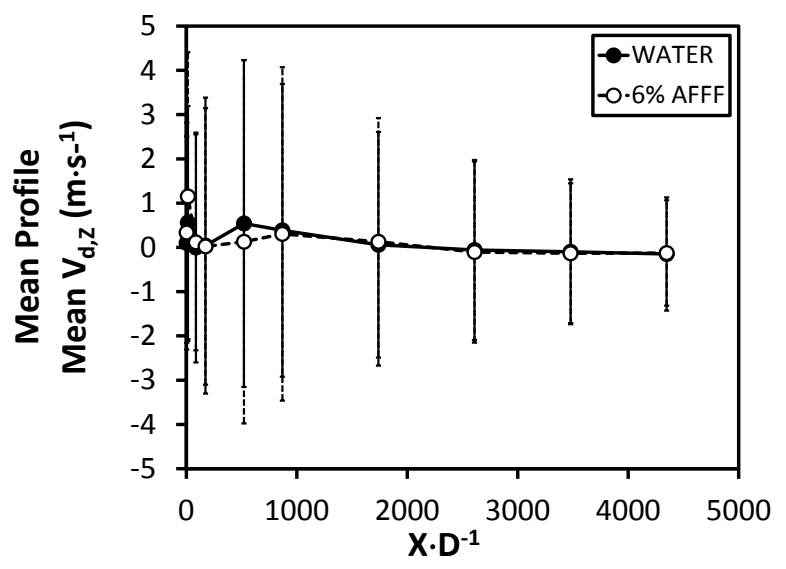

(c) Med. Flow, Med. Press. Jets

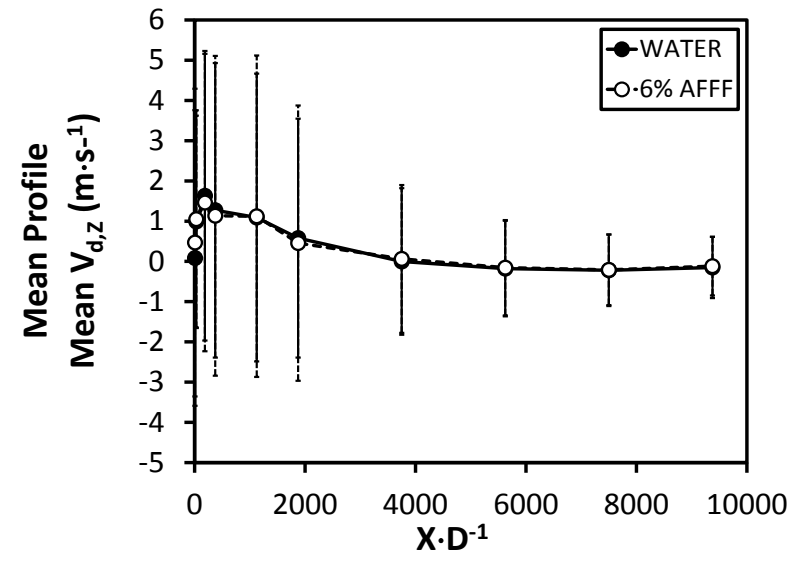

(b) Low Flow, High Press. Jets

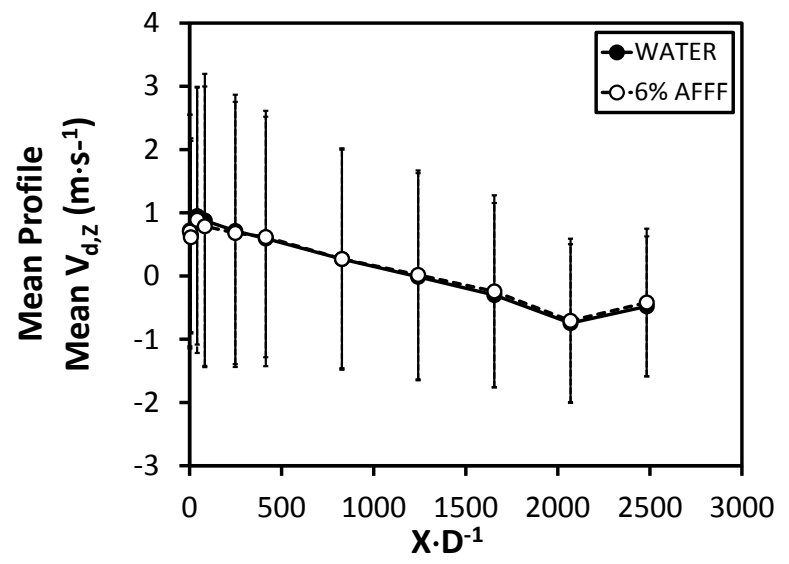

(d) High Flow, Low Press. Jets

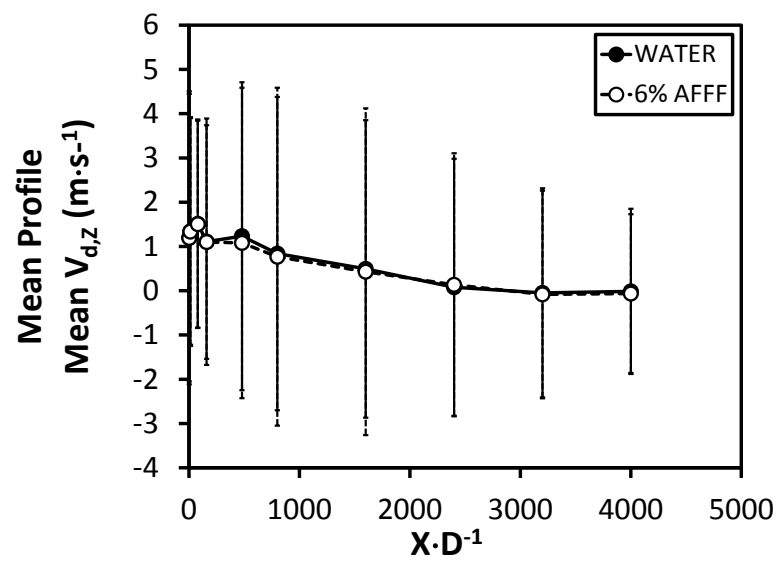

(e) High Flow, High Press. Jets

Figure 6.52: Firefighting Jet PDPA Summary in Terms of Mean Profile, Mean Vertical Droplet Velocity; Vertical Bars Represent Mean Profile, \pm RMS Vertical Droplet Velocity Magnitude

\section{Phase Doppler Diameter Results Summary}

Figure 6.53 summarizes phase Doppler droplet diameter measurements for all firefighting jet configurations analyzed. Figure $6.53 \mathrm{a}$ shows the mean profile, mean water droplet diameter, 
Figure $6.53 \mathrm{c}$ illustrates the mean profile, RMS water droplet diameter, and Figure 6.53e depicts the mean profile, Sauter mean water droplet diameter. Figures $6.53 \mathrm{~b}, \mathrm{~d}$, and e illustrate the same scenarios as in Figure 6.53a, c, and e, respectively, but instead use 6-percent AFFF as the agent.

Mean profile, mean droplet diameters for all nozzle pressure-flow rate combinations were globally reduced by 25 to 100 percent with the addition of AFFF resulting in the most definitive measure of difference between water and AFFF jets. The reduction in mean droplet size was due to the surface tension-lowering surfactant additives which improved overall jet break-up. Mean droplet diameter increased as flow rate increased with the high flow, low pressure water jet registering the largest magnitude at $90 \mu \mathrm{m}$. Mean droplet diameter decreased as flow rate decreased with the low flow, high pressure AFFF jet generated droplets recording sizes as low as $17 \mu \mathrm{m}$. Nozzle pressure increases also decreased mean droplet diameter, which was expected as jet atomization and secondary break-up was further enhanced.

Mean profile, RMS droplet diameter results observed the same trends as those of the mean profile mean droplet diameter results, most notably showing a wide droplet size distribution irrespective of nozzle pressure-flow rate combination. Mean profile, RMS droplet diameter magnitudes were also consistently similar to mean profile, mean droplet diameter magnitudes which indicated droplet distribution range was proportional to mean droplet size. This assumption was reasonable because the larger the parent droplet, the greater the opportunity for successive break-up resulting in a larger distribution of droplets.

Mean profile, Sauter mean droplet diameter results followed a similar, definitive trend to those of mean profile, mean droplet diameter results for three out of the five nozzle pressure-flow rate combinations. However, the other two nozzle settings generated conflicting results. A peak magnitude of about $365 \mu \mathrm{m}$ was exhibited by the high flow, low pressure water jet and magnitudes as low as $50 \mu \mathrm{m}$ were observed for the low flow, high pressure AFFF jet.

Figures 6.54 through 6.56 reproduce the same data shown in Figure 6.53, but highlight the effect AFFF had on each nozzle pressure-flow rate combination compared to water. Figure 6.54 expands the mean profile, mean droplet diameters illustrated in Figure 6.53a and b, and Figure 6.55 expands the mean profile RMS droplet diameters shown in Figure 6.53c and d, respectively. 
Figure 6.56a and $\mathrm{b}$ expands the mean profile, Sauter mean diameters depicted in Figure 6.53e and $\mathrm{f}$.

Figure 6.54 depicts the decisive reduction in mean profile, mean droplet diameter AFFF jets in comparison to water jets. The ability of AFFF to lower mean droplet diameters decreased as the flow rate increased, although the loss in efficiency was minor. This was likely due to the increased presence of jet ligaments as illustrated in Figure 2.8a in Section 2.3.2 compared to satellite droplets where surfactant effectiveness was reduced. The change in mean droplet diameter as nozzle pressure increased was less apparent. According to Figure 6.54, enhanced break-up due to AFFF had the greatest effect in the nozzle near field within the first one thousand nozzle diameters with its influence gradually decaying for the remainder of the effective jet length. This observation is supported by Figures 2.6 and 2.7 in Section 2.23 where AFFF has the greatest affect in lowering surface tension within the first second of droplet life.

Figure 6.55 shows mean profile, RMS droplet diameters are consistently lower for AFFF jets as compared to water jets similar to mean droplet diameter trends, with this margin decreasing as flow rate increased. The low flow, low pressure jet exhibited the largest disparity with an approximate 50 percent reduction in RMS droplet diameter.

Figure 6.56 illustrates mean profile, Sauter mean droplet diameters with inconsistent results regarding the influence of AFFF. Low flow, high pressure jets and medium flow, medium pressure jets did not follow the trend of water jet mean profile, Sauter mean droplet diameters exceeding AFFF jet values as in the other three nozzle settings. A closer inspection of individual Sauter mean diameter profiles in Appendix C shows a large amount of scatter between AFFF jet sampling points for the two outlying nozzle settings, particularly in the downstream region of each jet. The reason behind this contrast is not absolutely known. Based on the AFFF concentration sensitivity in Section 6.3 .4 on the medium flow, medium pressure jet, it is speculated Sauter mean diameter profile scatter was amplified as AFFF solution concentration was increased. This indicates a non-negligible number of non-spherical, bubble-like AFFF formations much larger than water droplets may have been validated by the PDPA skewing Sauter mean droplet diameter values for these profile locations. Very few (e.g. 10 or less) 
droplets measured in the upper threshold of the PDPA measurement envelope in the field of 50,000 samples were also capable of altering Sauter mean diameter by 10 percent or more.

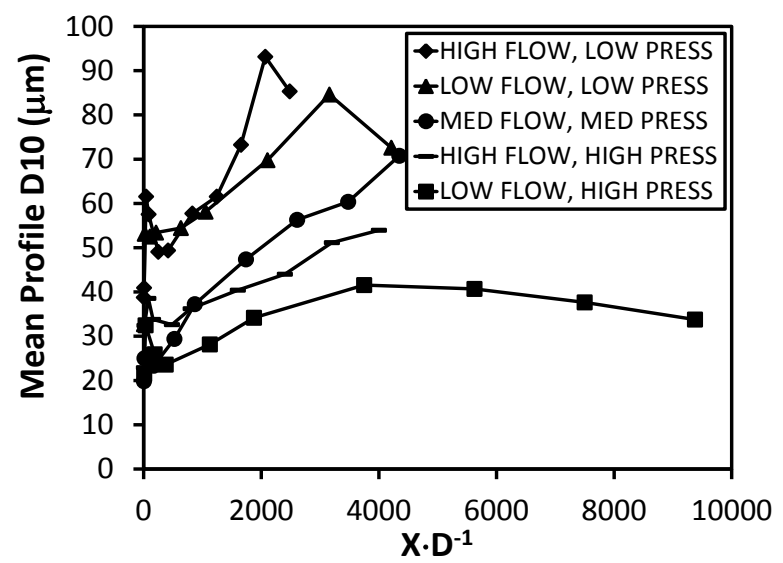

(a) Water Jets

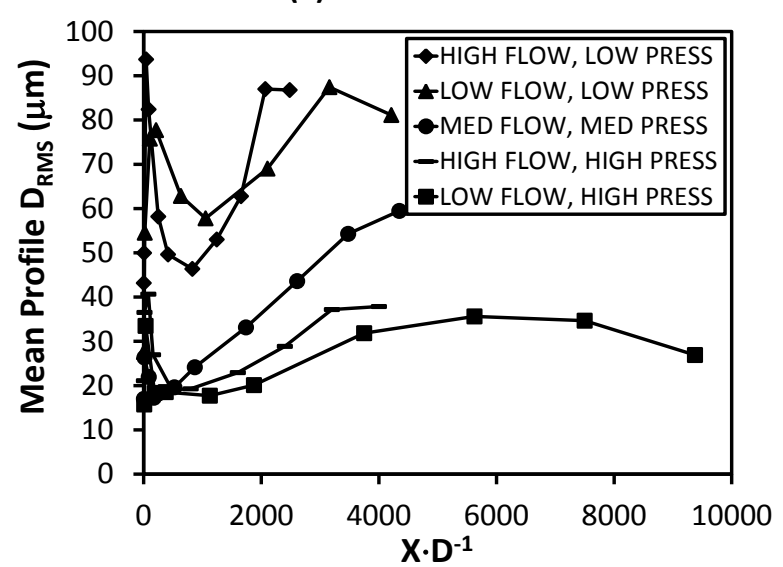

(c) Water Jets

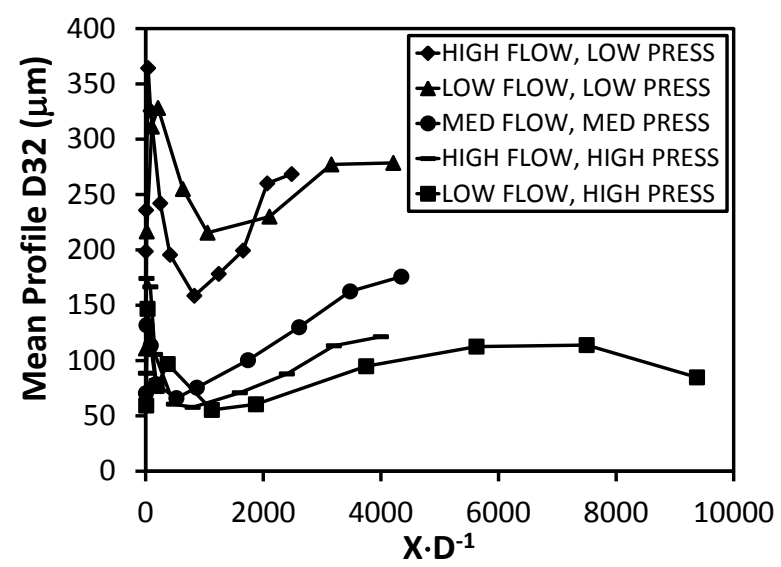

(e) Water Jets

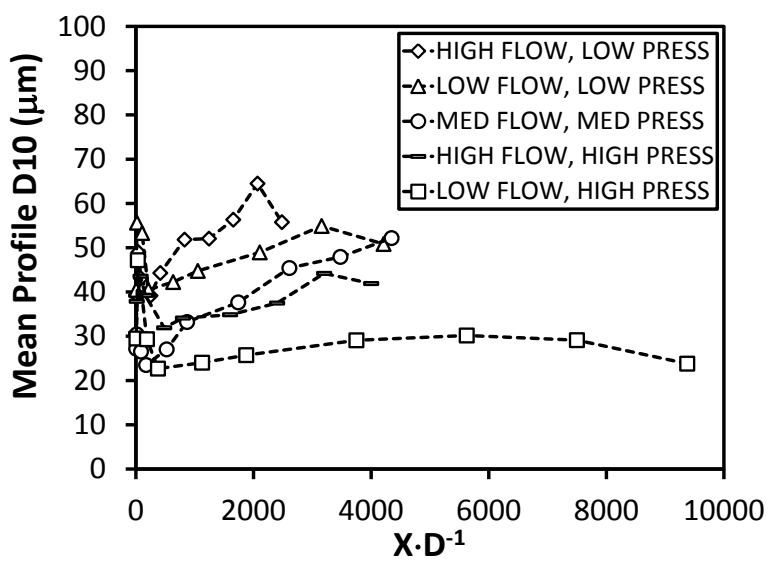

(b) $6 \%$ AFFF Jets

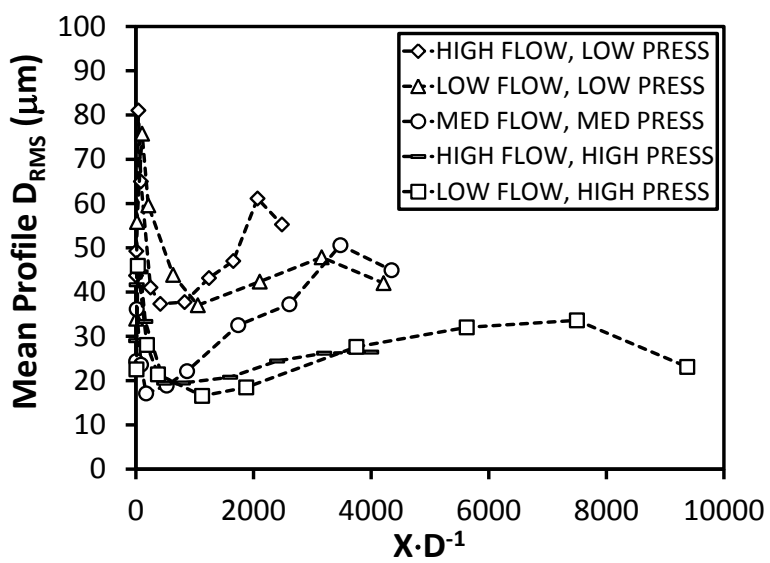

(d) $6 \%$ AFFF Jets

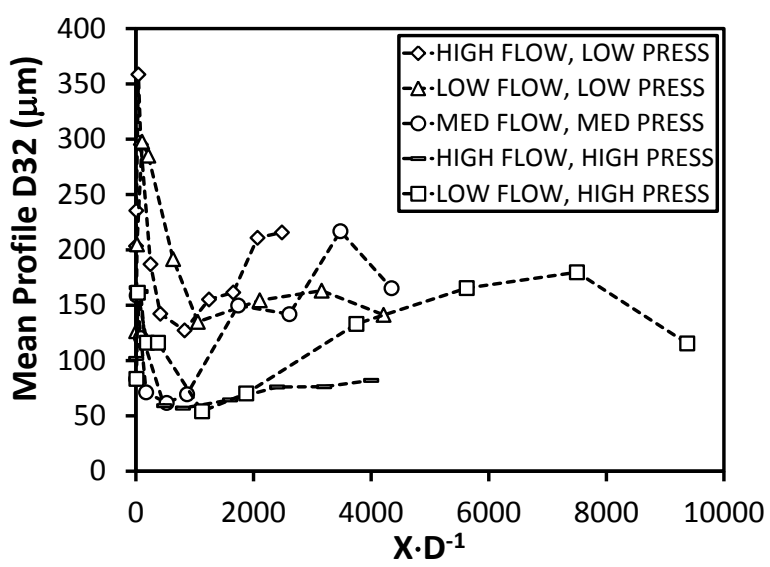

(f) $6 \%$ AFFF Jets

Figure 6.53: Firefighting Jet PDPA Summary Data in Terms of Mean Profile, Droplet Diameters 


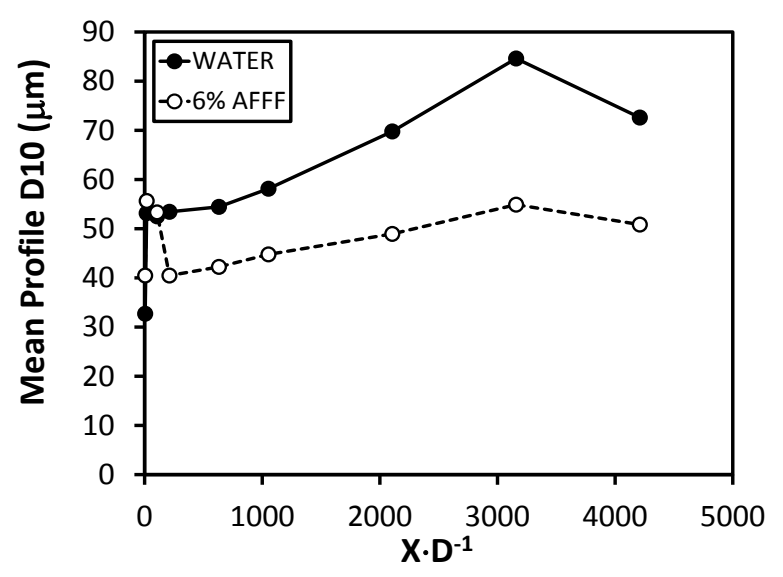

(a) Low Flow, Low Press. Jets

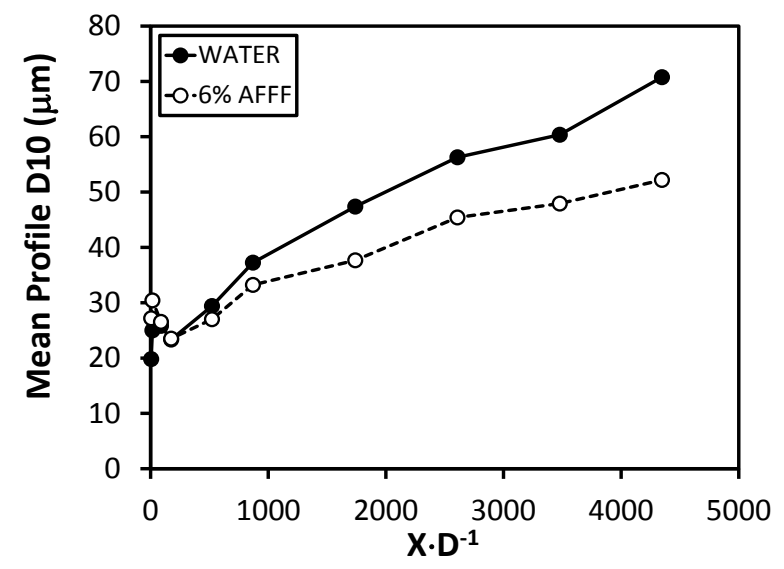

(c) Med. Flow, Med. Press. Jets

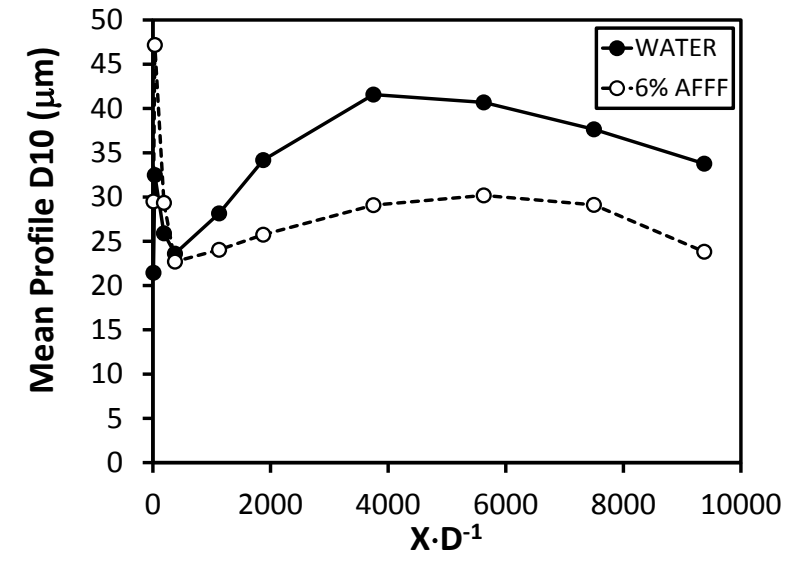

(b) Low Flow, High Press. Jets

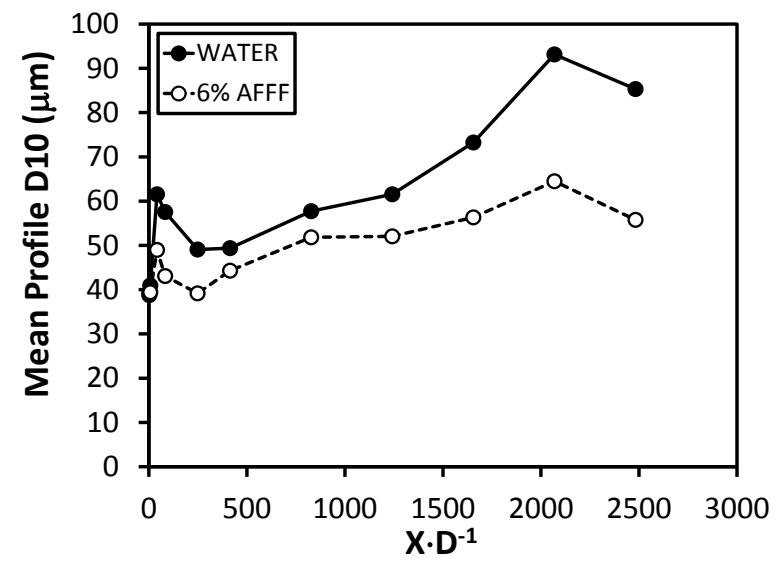

(d) High Flow, Low Press. Jets

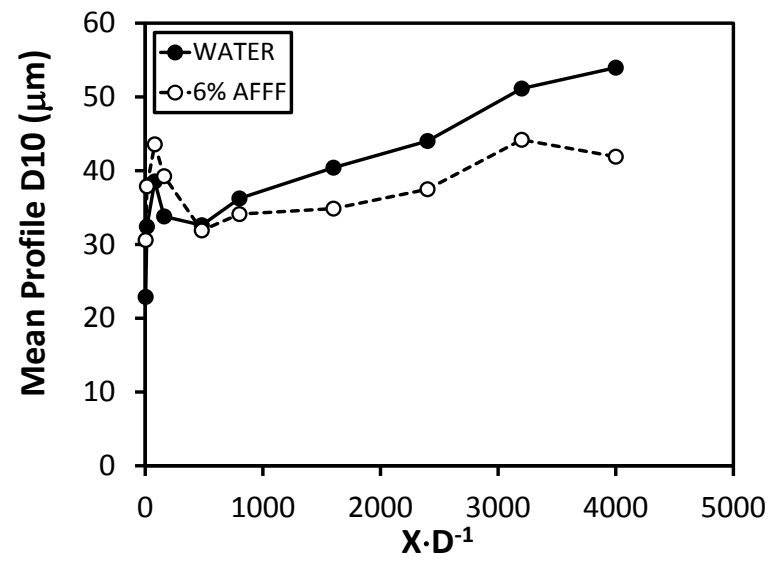

(e) High Flow, High Press. Jets

Figure 6.54: Firefighting Jet PDPA Summary in Terms of Mean Profile, Mean Droplet Diameter 


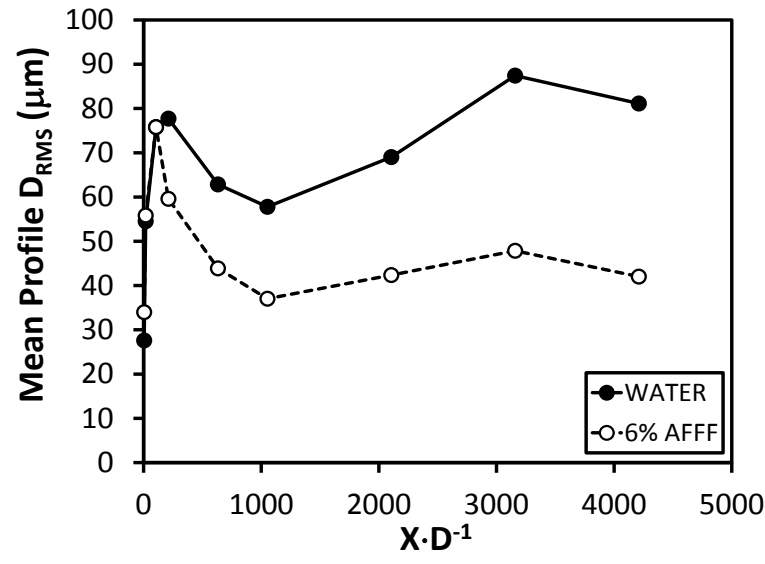

(a) Low Flow, Low Press. Jets

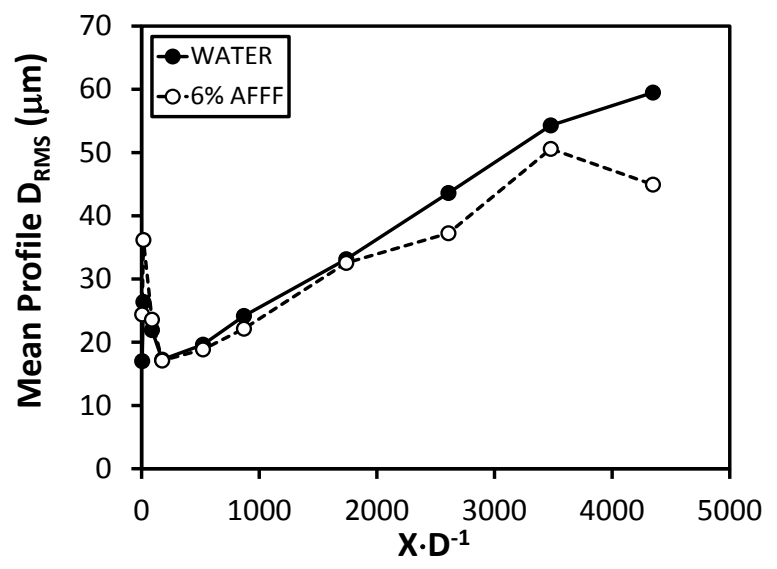

(c) Med. Flow, Med. Press. Jets

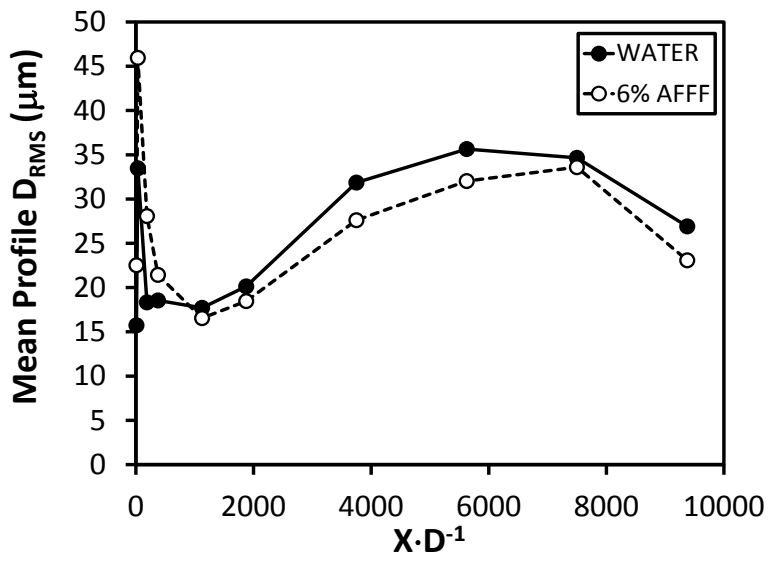

(b) Low Flow, High Press. Jets

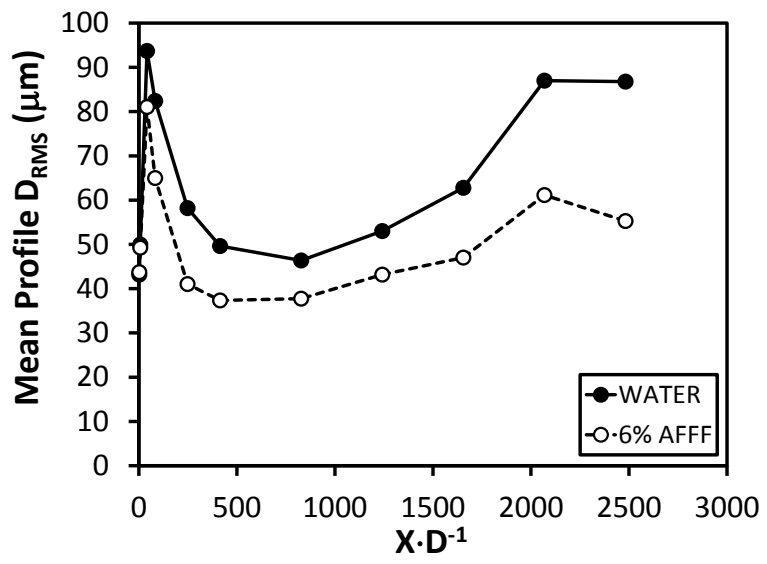

(d) High Flow, Low Press. Jets

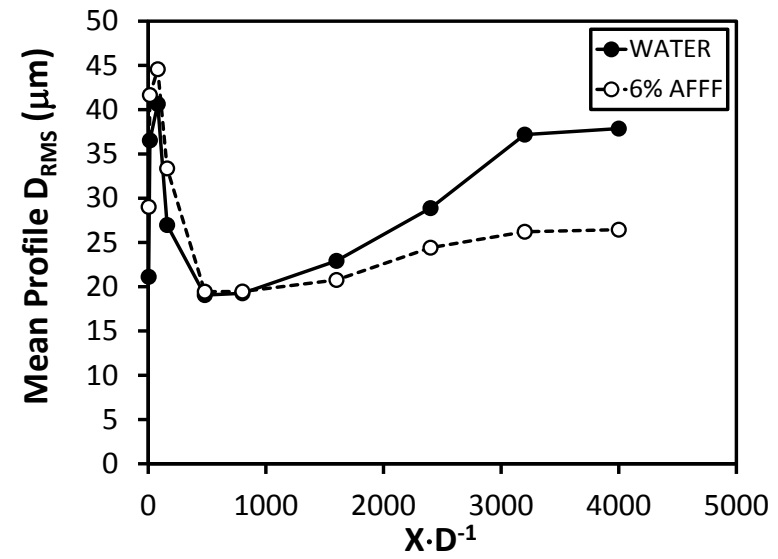

(e) High Flow, High Press. Jets

Figure 6.55: Firefighting Jet PDPA Summary in Terms of Mean Profile, RMS Droplet Diameter 


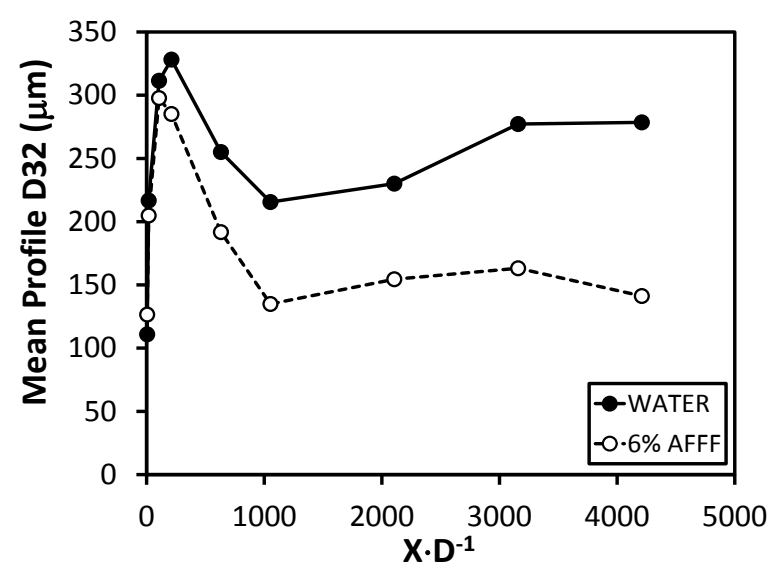

(a) Low Flow, Low Press. Jets

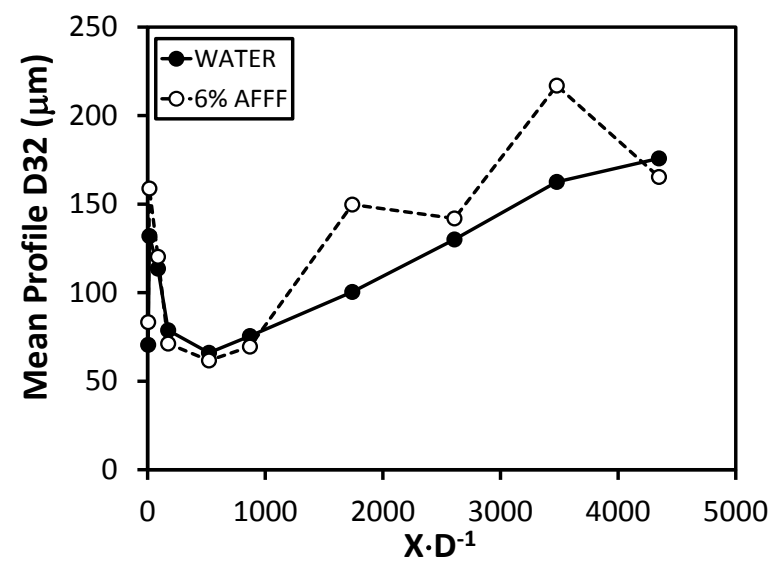

(c) Med. Flow, Med. Press. Jets

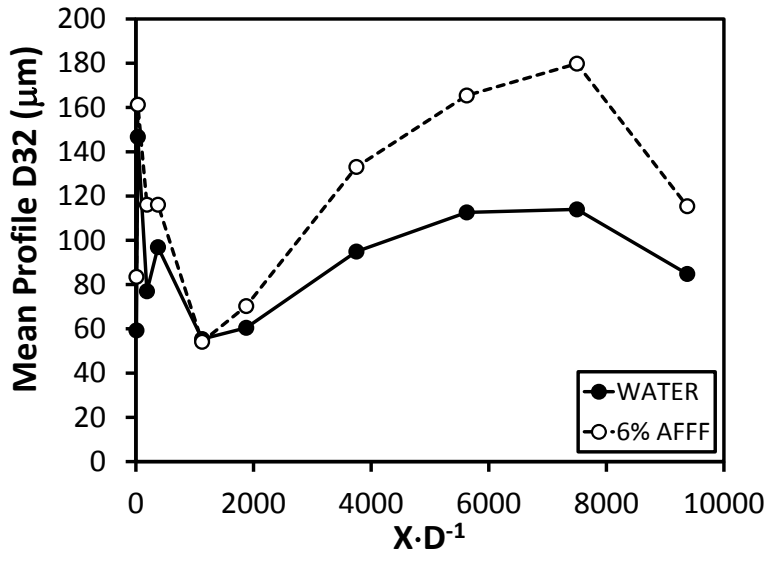

(b) Low Flow, High Press. Jets

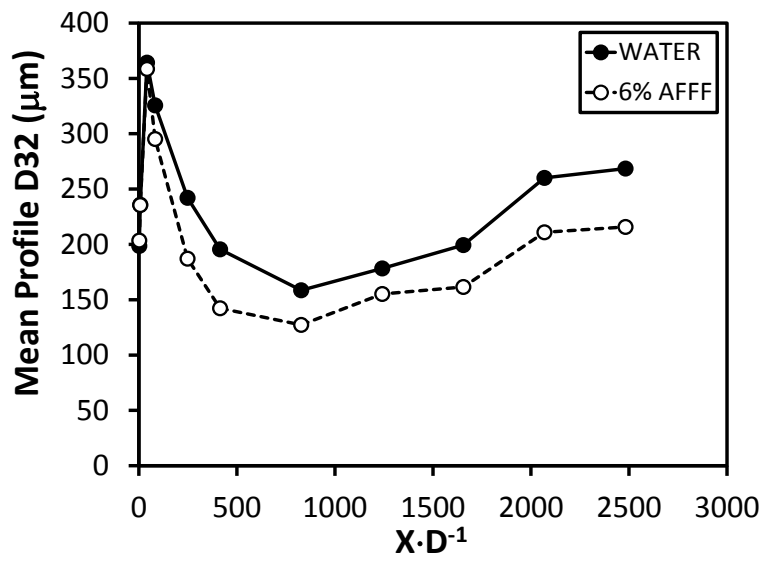

(d) High Flow, Low Press. Jets

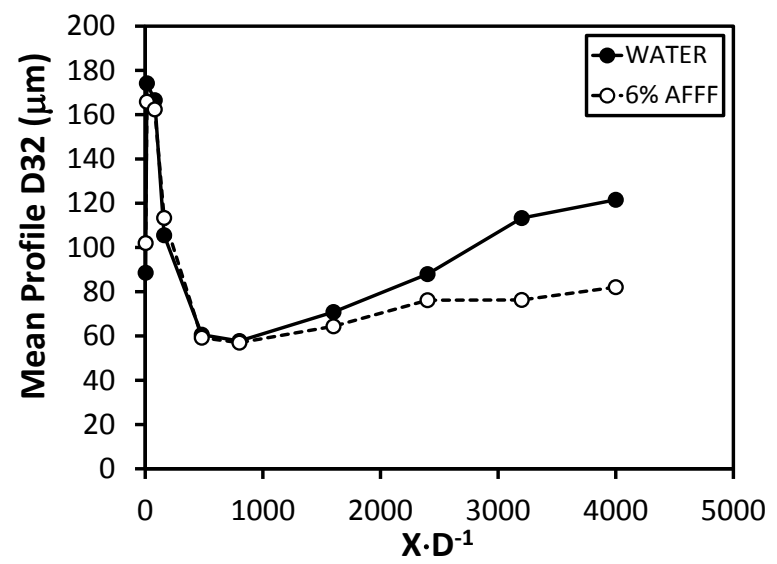

(e) High Flow, High Press. Jets

Figure 6.56: Firefighting Jet PDPA Summary in Terms of Mean Profile, Sauter Mean Droplet Diameter

\section{Phase Doppler Sampling Statistics Results Summary}

Phase Doppler sampling statistics are presented to examine broad trends in the data acquisition process. They are also investigated to determine if any bias or dependency exists in the results with respect to agent delivery pump pulsations known to affect the flow physics of certain 
firefighting jet configurations. These pulsations are discussed in detail in Section 6.1.2. PDPA data acquisition rate and mean sample count are presented in Figure $6.57 \mathrm{a}$ and $\mathrm{b}$, respectively. The data presented represent the mean profile value averaged for all five firefighting jet nozzle pressure-flow rate combinations. It is shown that in the nozzle near field, the mean profile data acquisition rate ranged from approximately 3,000 to 4,500 samples per second, but tapered to as low as 100 samples per second in the far axial reaches of each jet. Mean profile sample count followed a similar trend totaling about 42,000 samples per measurement location in the near field of the nozzle, but dropped to about 1,000 samples in the jet far field. It should be noted that PDPA data acquisition rates and sample counts shown in Figure 6.57 are biased towards a lower value. This is due to measurements purposefully taken up to and slightly beyond the outer boundary of each firefighting jet to ensure the flow was fully-described at each profile location.

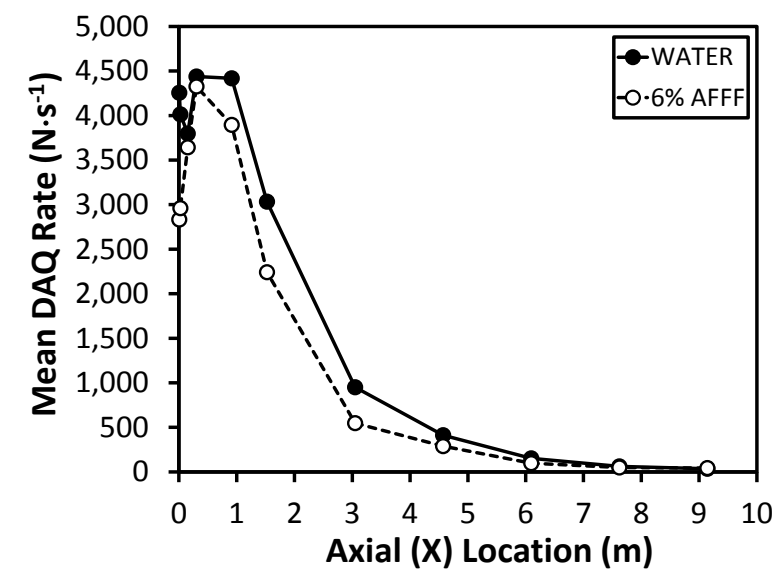

(a)

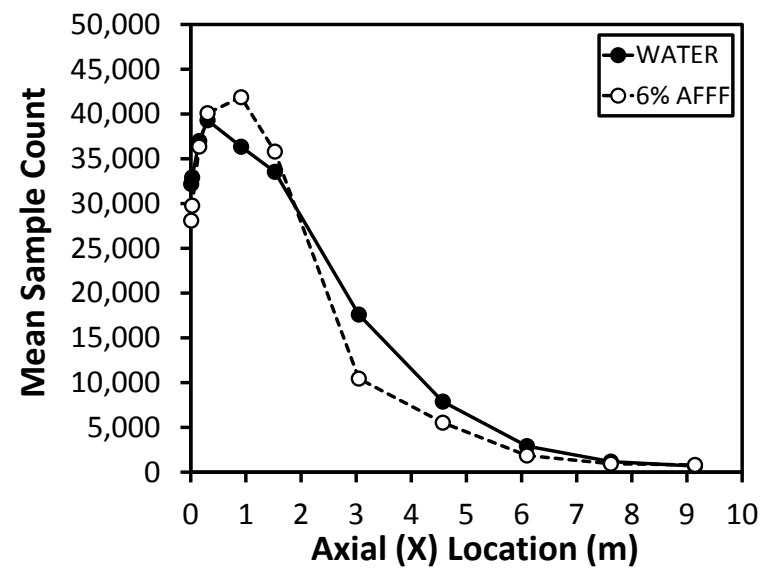

(b)

Figure 6.57: (a) PDPA Mean Profile Data Acquisition Rate vs. Axial Measurement Location (b) PDPA Mean Data Sample Count vs. Axial Measurement Location

Based on the agent delivery pump oscillation frequency and the mean elapsed time per sampling point recorded for each firefighting jet flow configuration, the number of pump oscillations per point sampling period was calculated. Results are listed in Table 6.3. A range of about 6,000 to 40,000 pump oscillations were generated while measuring each point from the low flow, high pressure jet to the high flow, low pressure jet, respectively. The phase Doppler data likely exhibited some bias towards recording higher velocity versus lower velocity droplets due to uneven time sampling of the PDPA system. Even-time sampling would have eliminated most of this bias, but was not practical due to the reduced data acquisition rates that would have accompanied this technique coupled with the expense associated with AFFF testing. 
Table 6.3: Calculated Pump Oscillations per Phase Doppler Data Acquisition Sampling Period

\begin{tabular}{|l|c|c|c|}
\hline \multicolumn{1}{|c|}{ Test Case } & $\begin{array}{c}\text { Pump Oscillation } \\
\text { Frequency } \\
\text { (Hz) }\end{array}$ & $\begin{array}{c}\text { Mean Elapsed Time } \\
\text { Per Sampling Point } \\
(\mathbf{s})\end{array}$ & $\begin{array}{c}\text { Pump Oscillations Per } \\
\text { Point Sampling Period } \\
(-)\end{array}$ \\
\hline Low Flow, Low Press. Water Jet & 389 & 17.53 & 6,819 \\
\hline Low Flow, Low Press. 6\% AFFF Jet & 389 & 16.67 & 6,485 \\
\hline Low Flow, High Press. Water Jet & 389 & 15.34 & 5,967 \\
\hline Low Flow, High Press. 6\% AFFF Jet & 389 & 17.03 & 6,625 \\
\hline Med. Flow, Med Press. Water Jet & 1,151 & 15.29 & 17,600 \\
\hline Med. Flow, Med Press. 6\% AFFF Jet & 1,151 & 16.94 & 40,500 \\
\hline High Flow, Low Press. Water Jet & 2,340 & 17.15 & 36,480 \\
\hline High Flow, Low Press. 6\% AFFF Jet & 2,340 & 15.59 & 29,930 \\
\hline High Flow, High Press. Water Jet & 1,852 & 16.16 & 32,210 \\
\hline High Flow, High Press. 6\% AFFF Jet & 1,852 & 17.39 & 40 \\
\hline
\end{tabular}

\subsubsection{Firefighting Jet Phase Doppler AFFF Concentration Sensitivity Results}

A phase Doppler AFFF concentration sensitivity study was conducted on the medium flow, medium pressure jet to quantify changes in measured firefighting jet flow parameters with respect to AFFF concentration. The concentration range spanned from 3 to 9-percent AFFF. A fourth concentration (12-percent AFFF) was not tested because an interim analysis determined a negligible difference existed between 6 and 9-percent AFFF phase Doppler results. Figures 6.58 through 6.62 show mean axial droplet velocity, mean vertical droplet velocity, mean droplet diameter, RMS droplet diameter, and Sauter mean droplet diameter profile results, respectively, for profile locations illustrated in Figure 4.7 and listed in Table 4.2. Plots are presented similarly to those in Section 6.3.2.

Mean axial droplet velocity was affected by the addition of AFFF in a similar manner as discussed in Section 6.3.3 with minor (10 percent or less) lag observed globally from water jet to 6-percent AFFF profiles. However, change in the axial velocity profile among all AFFF profiles was negligible. Mean vertical droplet velocity results behaved similarly to mean axial droplet velocity results, except differences between AFFF and water jet profiles were less significant and the difference among AFFF results was even less indiscernible. Mean droplet diameter results 
exhibited the largest variation with AFFF droplets reduced by as much as fifty percent compared to water droplets. AFFF mean droplet diameter shrank as AFFF concentration increased from 3 to 6-percent AFFF, but negligibly decreased between 6 and 9-percent AFFF. The size differential between AFFF and water droplets grew as the distance downstream increased. An increase in AFFF concentration showed a distinguishable increase in RMS droplet diameter, particularly for 9-percent AFFF as downstream distance increased similar to mean droplet diameter results, likely due to the onset of enhanced break-up earlier in the transport process. Sauter mean droplet diameter results produced analogous results to those of mean droplet diameter, except diameter magnitudes were amplified and AFFF profile data had more scatter in downstream profiles consistent with the low flow, high pressure jet studied solely at 6-percent AFFF.
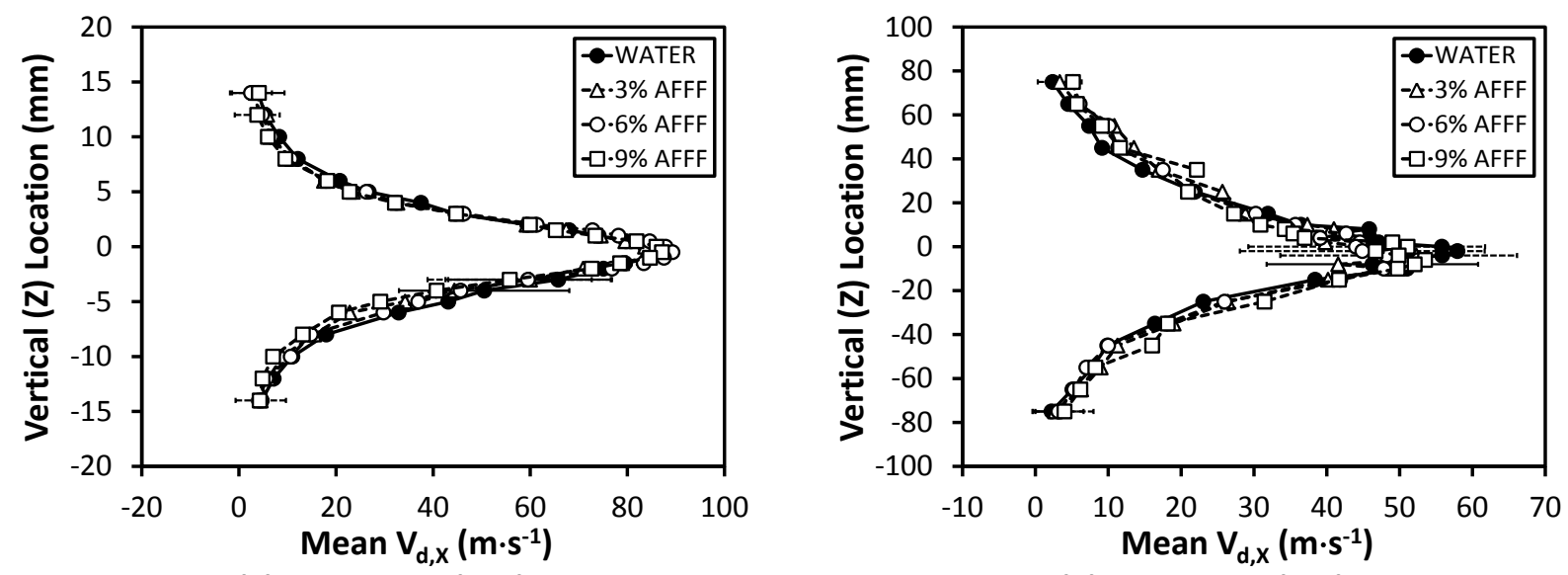

(a) $x=0.152 \mathrm{~m}(6 \mathrm{in})$

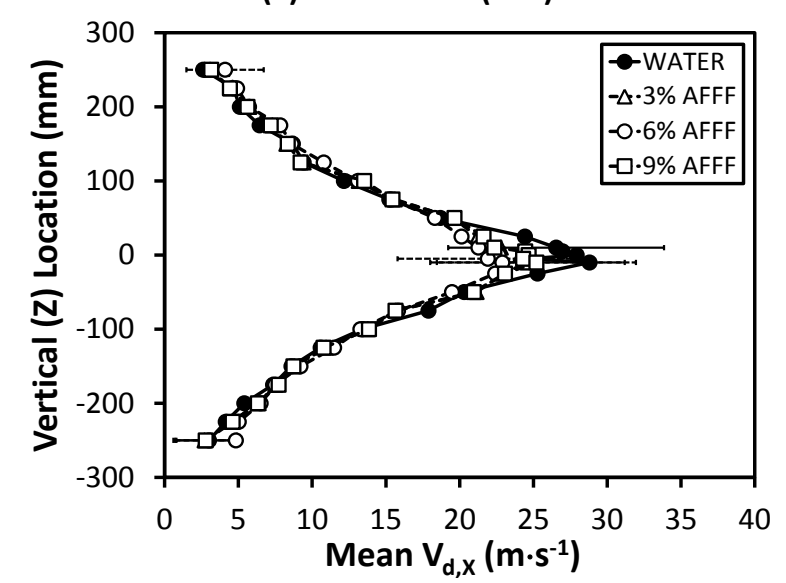

(c) $x=3.05 \mathrm{~m}(10 \mathrm{ft})$

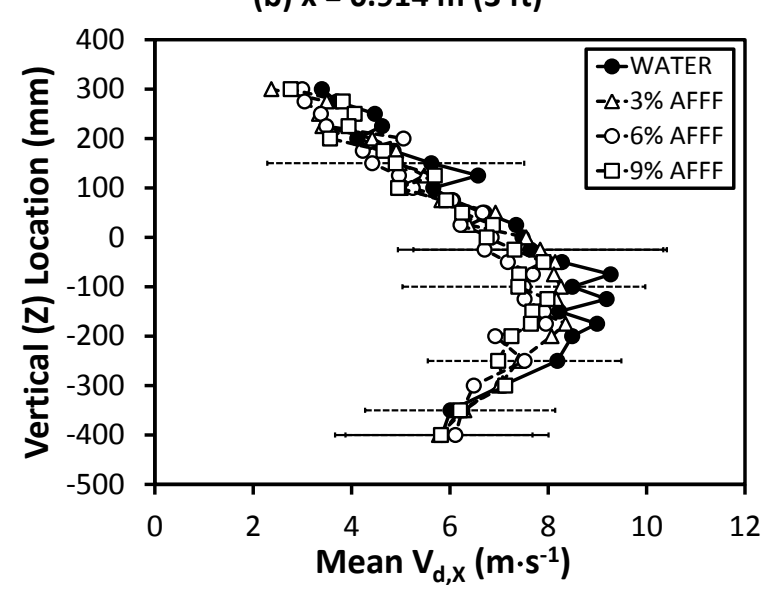

(d) $x=6.10 \mathrm{~m}(20 \mathrm{ft})$

Figure 6.58: Med. Flow, Med. Press. Jet Vertical Location vs. Mean Axial Droplet Velocity with Respect to Variation in AFFF concentration 

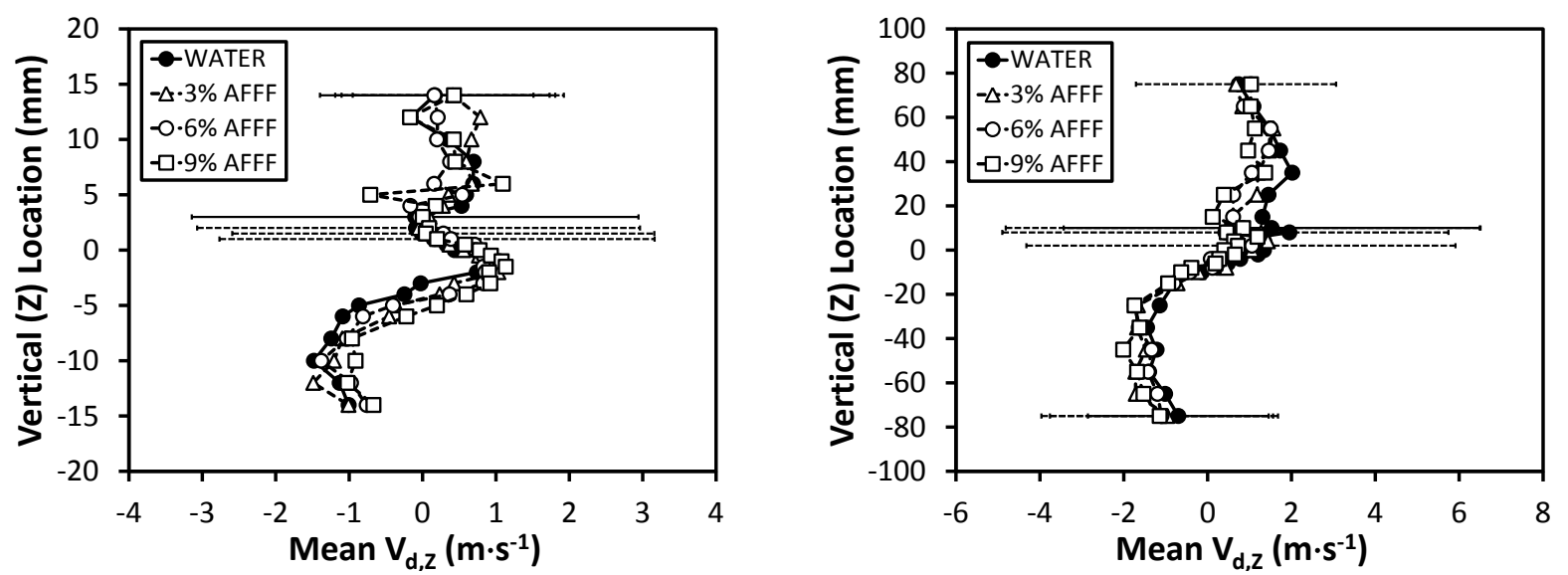

(a) $x=0.152 \mathrm{~m}(6 \mathrm{in})$

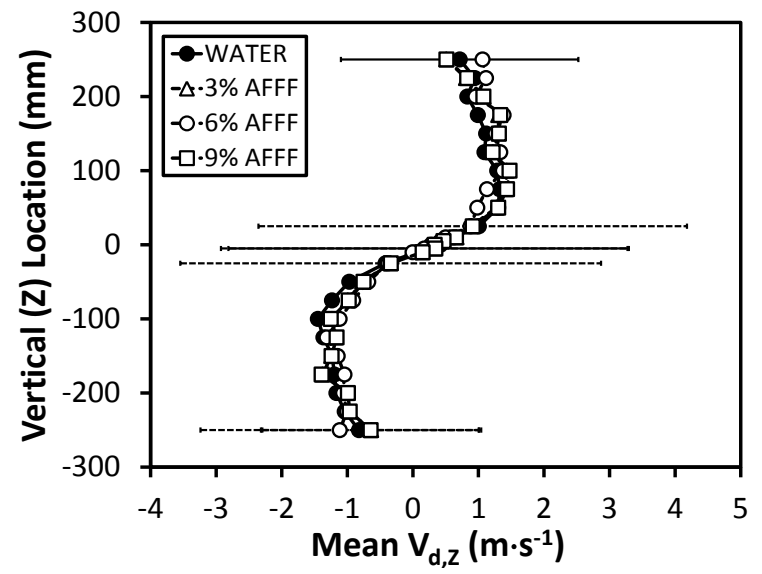

(c) $x=3.05 \mathrm{~m}(10 \mathrm{ft})$

(b) $x=0.914 \mathrm{~m}(3 \mathrm{ft})$

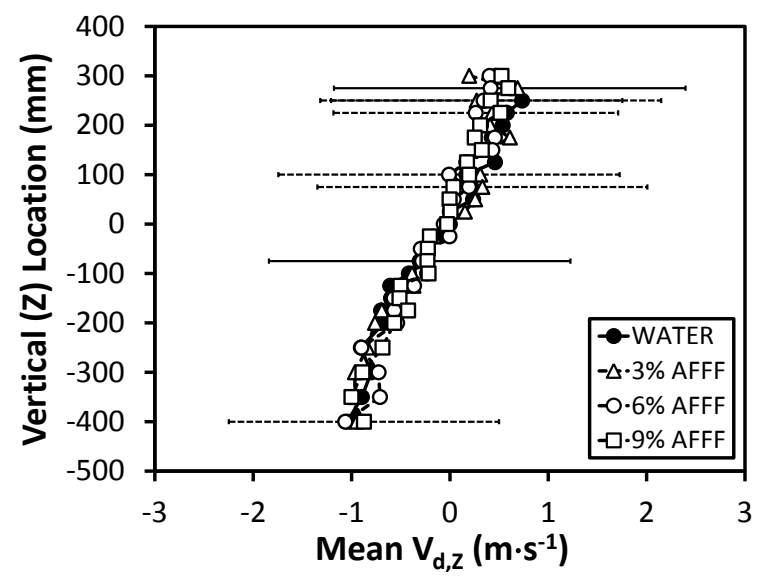

(d) $x=6.10 \mathrm{~m}(20 \mathrm{ft})$

Figure 6.59: Med. Flow, Med. Press. Jet Vertical Location vs. Mean Vertical Droplet Velocity with Respect to Variation in AFFF concentration 

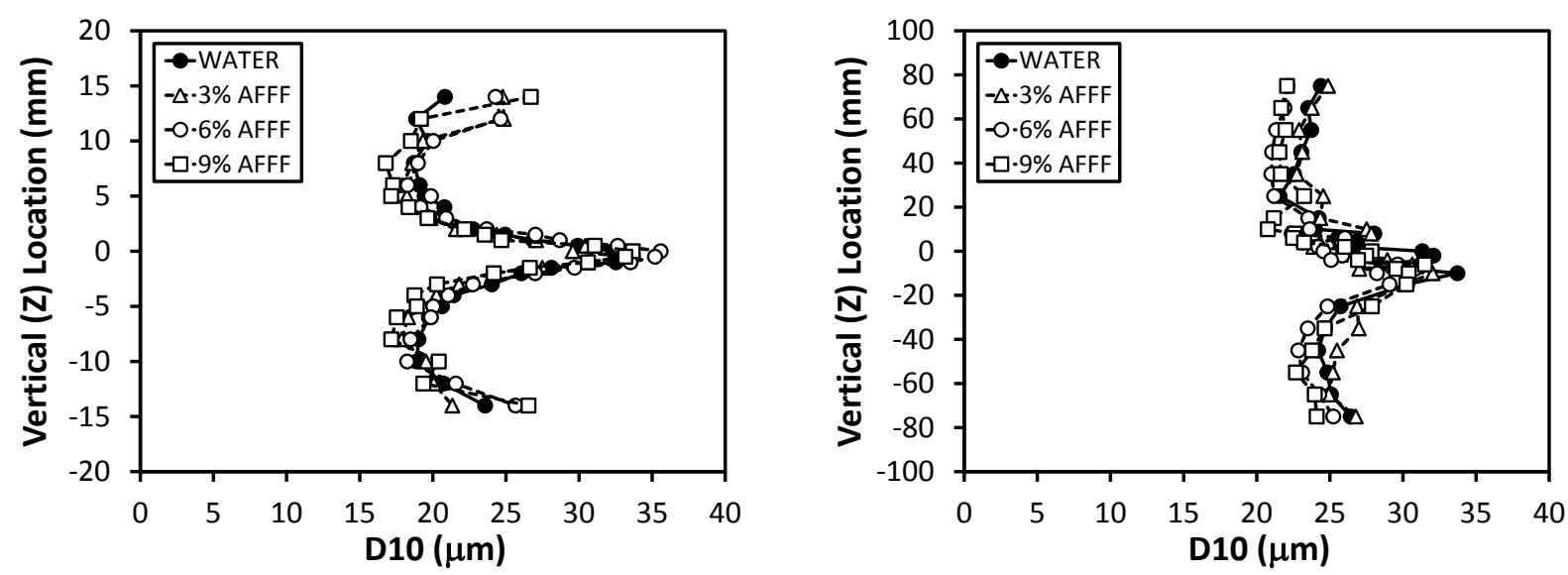

(a) $x=0.152 \mathrm{~m}(6 \mathrm{in})$

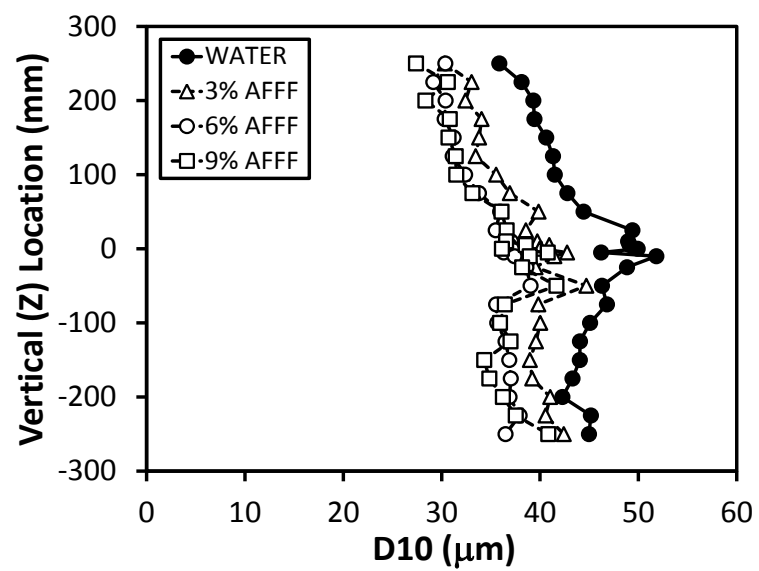

(b) $x=0.914 \mathrm{~m}(3 \mathrm{ft})$

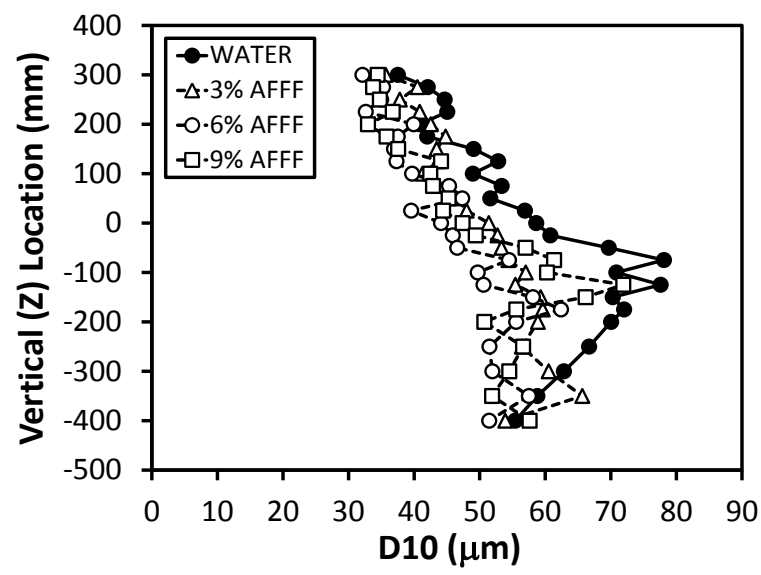

(d) $x=6.10 \mathrm{~m}(20 \mathrm{ft})$

Figure 6.60: Med. Flow, Med. Press. Jet Vertical Location vs. Mean Droplet Diameter with Respect to Variation in AFFF concentration 

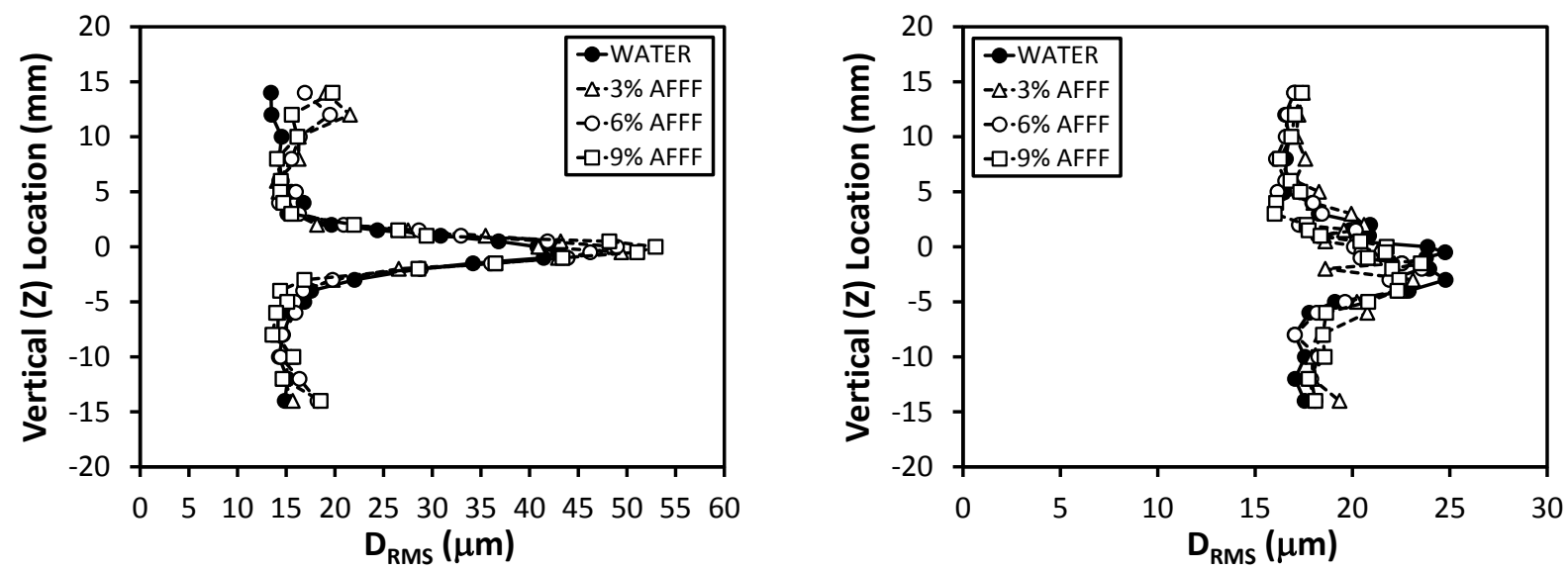

(a) $x=0.152 \mathrm{~m}(6 \mathrm{in})$

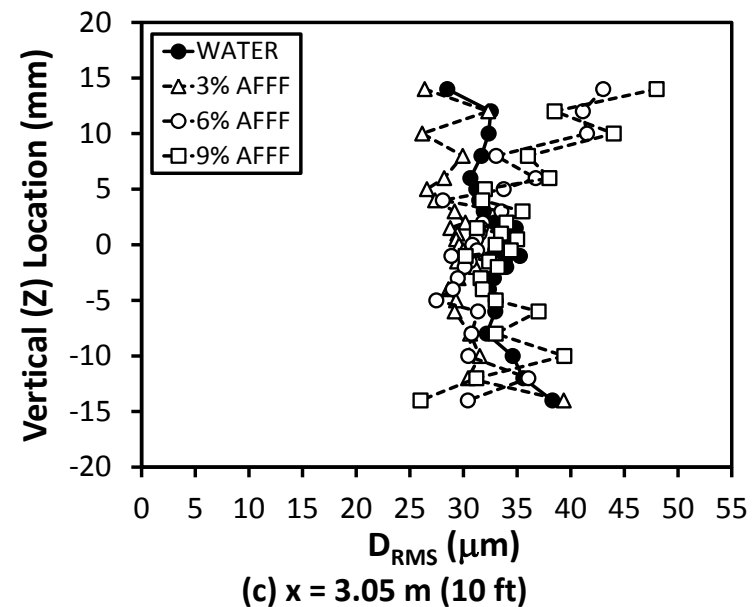

(b) $x=0.914 \mathrm{~m}(3 \mathrm{ft})$

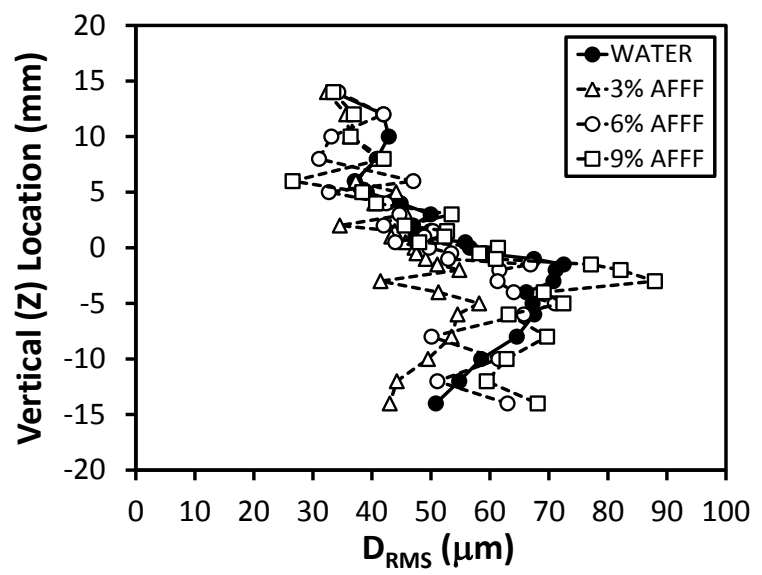

(d) $x=6.10 \mathrm{~m}(20 \mathrm{ft})$

Figure 6.61: Med. Flow, Med. Press. Jet Vertical Location vs. RMS Droplet Diameter with Respect to Variation in AFFF concentration 

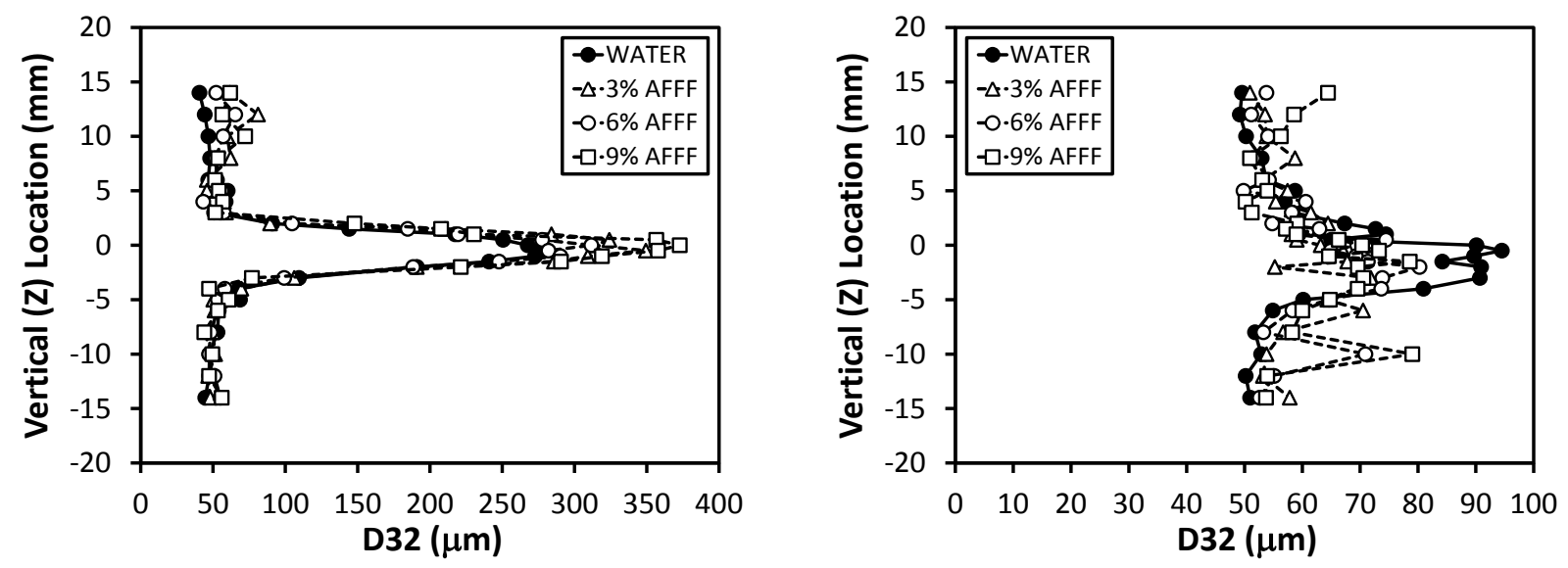

(a) $x=0.152 \mathrm{~m}(6 \mathrm{in})$

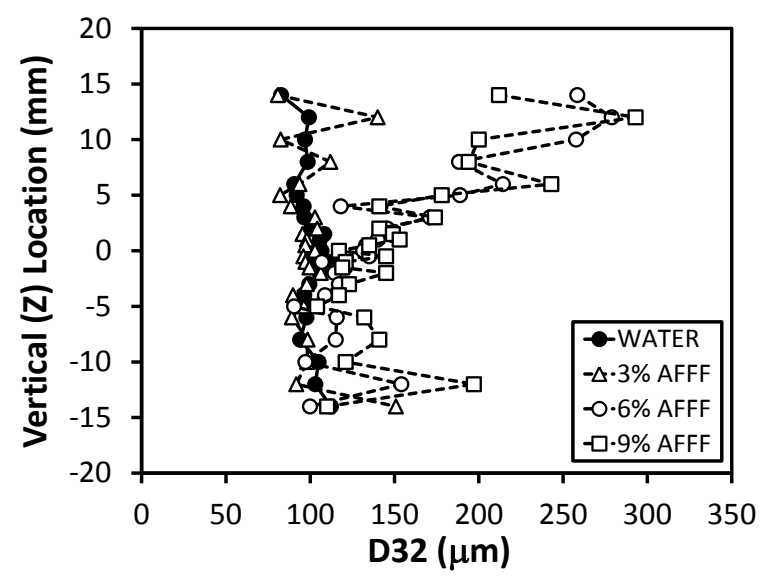

(c) $\mathrm{x}=3.05 \mathrm{~m}(10 \mathrm{ft})$

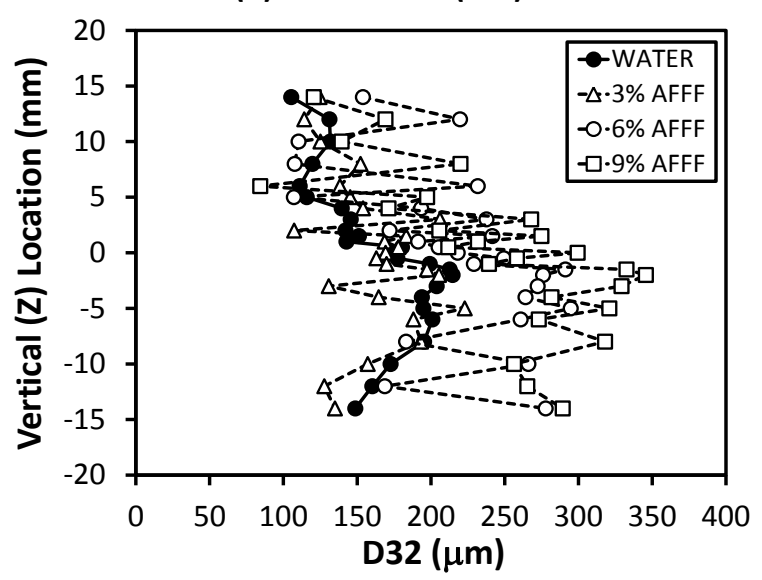

(d) $x=6.10 \mathrm{~m} \mathrm{(20 \textrm {ft } )}$

Figure 6.62: Med. Flow, Med. Press. Jet Vertical Location vs. Sauter Mean Droplet Diameter with Respect to Variation in AFFF concentration

\subsection{Computational Firefighting Jet Flow Model Results}

CFD models results were computed for the 10 firefighting jet configurations listed in Table 5.1 in Section 5.1. The same five firefighting jet pressure-flow rate combinations investigated for flow visualization and PDPA were also modeled using water and 6-percent AFFF. A model parameter dependence study is presented which helped to shape the overall computational strategy. Select CFD model results are then compared to experiments in terms of qualitative flow features, select vertical flow parameter profiles similar to the presentation in Section 6.3.2, and mean profile data similar to the presentation in Section 6.3.3. A firefighting jet flow characterization study of all CFD model solutions is then presented illustrating various aspects of the simulated firefighting jets. 


\subsubsection{Computational Firefighting Jet Flow Model Parameter Dependence Results}

A CFD model parameter dependence study was conducted to determine how variation in certain factors affected results. The key parameters examined were the DPM break-up model selection, spatial mesh resolution, temporal resolution, and the impact of the firefighting jet containment zone. Unless otherwise noted, all computational results utilized the TAB collision and coalescence CFD physical-sub models. Similar to sensitivity studies conducted experimentally, each analysis utilized the medium flow, medium pressure water jet condition for the baseline. Mean axial droplet velocity and mean droplet diameter data were the flow parameters used for comparison. The CFD model results represent each flow parameter averaged over the entire physical domain at a constant $x$-plane as a function of axial downstream location nondimensionalized with respect to the nozzle diameter. All parameter dependence studies followed the same solution strategy as that used CFD model results reported in Sections 6.4.2 and 6.4.3.

\section{Physical Sub-Model Conditions}

Two DPM break-up models were applicable for low $(<100)$ Gaseous Weber number jet flows within ANSYS Fluent ${ }^{\circledR}$ : the TAB model and the SSD model. Figure 6.63 illustrates a negligible difference in overall firefighting jet performance in terms of mean plane, mean axial droplet velocity and mean plane, mean droplet diameter over the entire length of the jet. The TAB model was selected versus the SSD model for the final solution strategy because speed to solution was 25 to 50 percent faster by comparison.

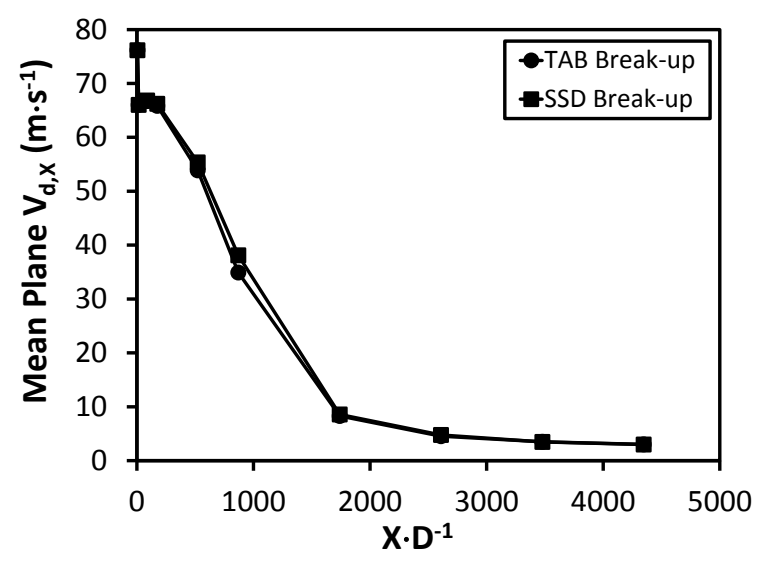

(a)

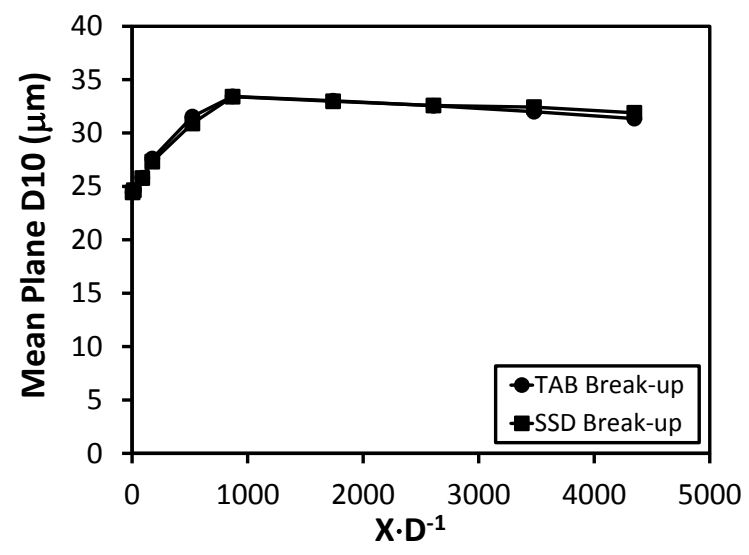

(b)

Figure 6.63: CFD Model Solution Dependence on the DPM Secondary Droplet Break-up Model 
Model sensitivity to coalescence was examined by comparing DPM TAB model solutions with and without the coalescence physical sub-model activated. Figure 6.64 illustrates the results. Coalescence modeling added approximately five percent more time per iteration to the overall computation. The collision model was active for both cases presented. Mean plane, mean axial droplet velocity magnitudes depicted mild dependence indicating coalescence modeling allowed droplet size families or parcels to enlarge and effectively alter trajectories primarily in the midfield of the firefighting jet. Mean plane, mean droplet diameter comparisons show significant divergence between model selections illustrating the flow parameter can increase by almost 50 percent. The collision only model depicted no significant growth in mean droplet diameter.

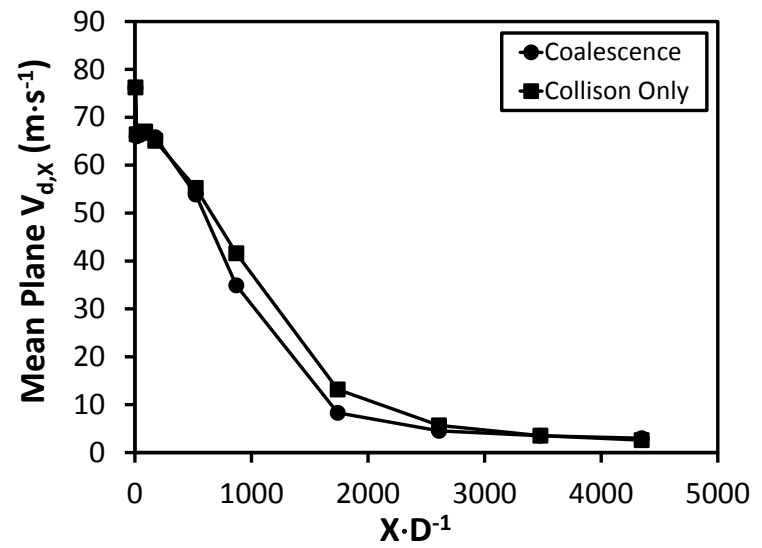

(a)

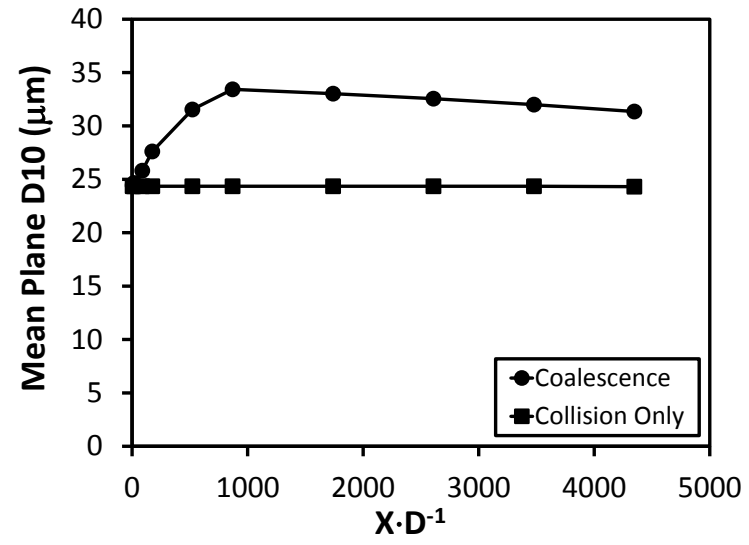

(b)

Figure 6.64: CFD Model Solution Dependence on the DPM Coalescence Model

\section{Mesh Resolution}

A spatial mesh resolution study was conducted to determine the solution dependence on three different grids ranging from a $254,000,411,000$, up to a 3.46 million cell grid shown in Figure 6.65. Grid dependence studies regarding DPM flows are complex due to DPM (droplet) volume-to-cell volume ratio thresholds that must be maintained to preserve model applicability as discussed in Section 5.2.1. Dimensions of the structured topology were linearly scaled to achieve all three grid sizes. Mesh resolution in the near nozzle injection field was maintained at $2 \mathrm{~cm}$ to maintain numerical stability. Both mean plane, mean axial droplet velocity and mean plane, mean droplet diameter results showed some dependence on the coarse mesh with values asymptoting to similar values between the regular and fine mesh. The regular mesh was employed for the resultant modeling strategy. A maximum, CFD spatial discretization error of 
\pm 1.03 and \pm 0.67 percent was calculated for the mean plane, mean axial droplet velocity and mean plane, mean droplet diameter, respectively. These errors are based on the Richardson extrapolation method, and are representative of all CFD model results in the current study [73].

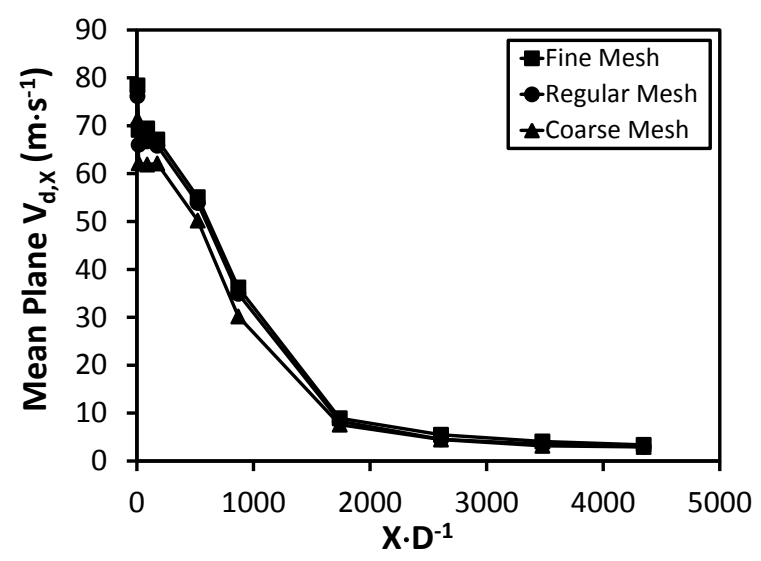

(a)

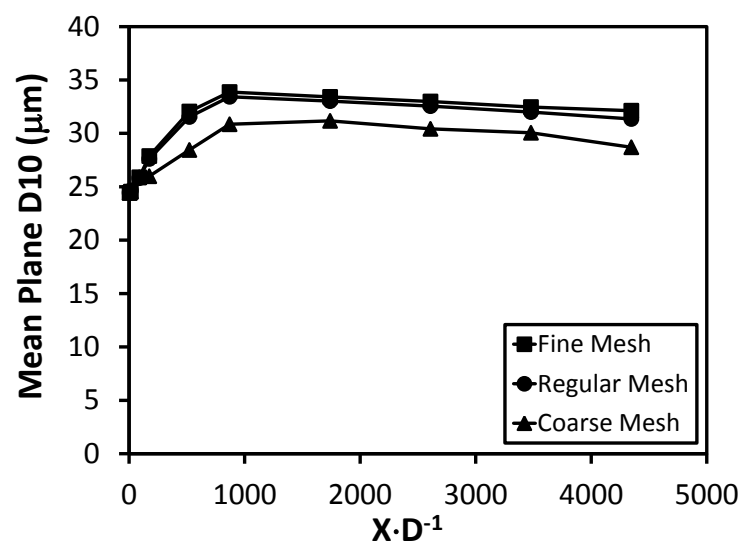

(b)

Figure 6.65: CFD Model Solution Dependence on the Mesh Resolution

\section{Temporal Resolution}

A temporal resolution study was executed to determine the solution dependence on the unsteady physical time step. Developing a CFD modeling strategy capable of generating reliable solutions with $1 \mathrm{~ms}$ time steps or larger was critical to future applications regarding integrated aircraft combustion models where scenarios of interest may last several tens of seconds to minutes. Figure 6.66 shows the results.

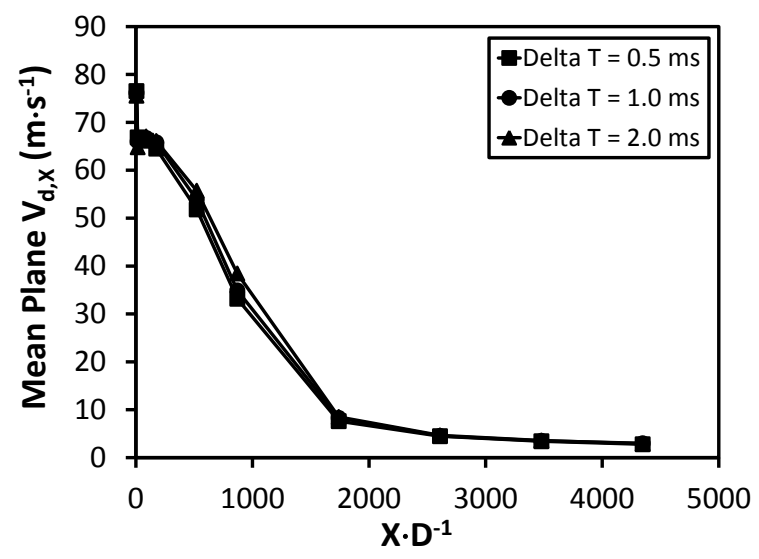

(a)

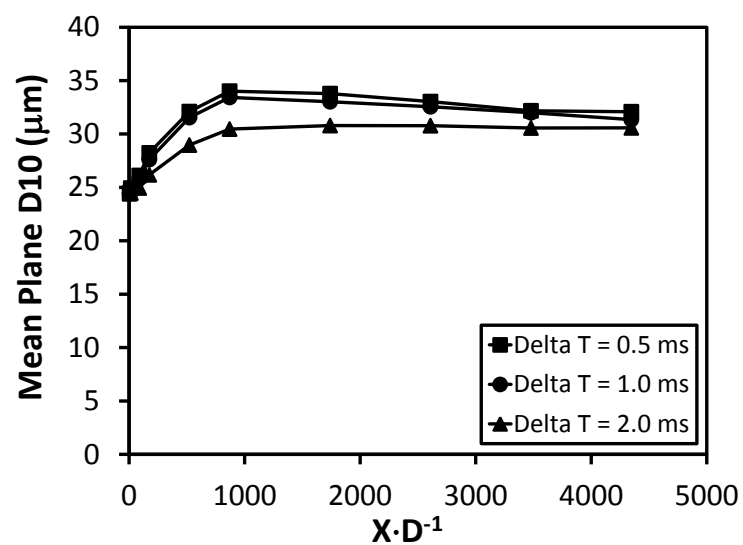

(b)

Figure 6.66: CFD Model Solution Dependence on the Physical Time Step 
A total of three time steps ranging from 0.5 to $2 \mathrm{~ms}$ were examined. Mean plane, mean axial droplet velocity magnitudes showed minor dependence on time step with noticeable dependence in mean plane, mean droplet diameter results occurring for the largest time step. Negligible change was observed between the 0.5 and $1 \mathrm{~ms}$ time step. The $1 \mathrm{~ms}$ time step was employed for all firefighting jet CFD model results.

\section{Firefighting Jet Containment Zone Influence}

A computational study was conducted to determine the impact modeling the firefighting jet containment zone located at the rear of the containment bed would have on CFD model results. The containment zone was modeled as a no-slip wall similar to how the floor is modeled for all other computational results. The results are presented in Figure 6.67. The containment bed starts at an $x \cdot D^{-1}$ of about 3500 . The containment zone was shown to alter the effective mean plane, mean axial droplet velocity by as much as 12 percent upstream of the containment zone approximately halfway between it and the nozzle. A consistent gradual loss in firefighting jet momentum was observed as the stream approached and entered the containment zone, likely due to the flow resistance (pressure increase) imparted by the containment zone curtains. Mean plane, mean droplet diameters were reduced by as much as 10 percent with the addition of the containment zone due to airborne droplet lifetimes being extended within the flow domain allowing for secondary droplet break-up to continue for a longer period of time.

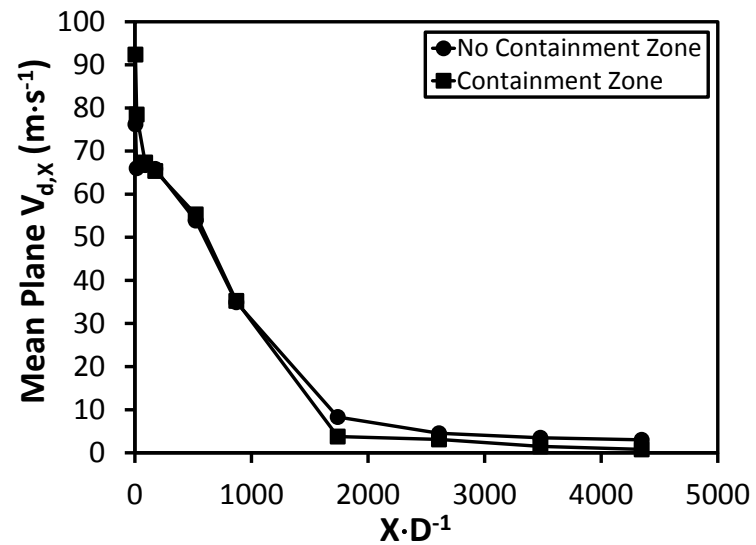

(a)

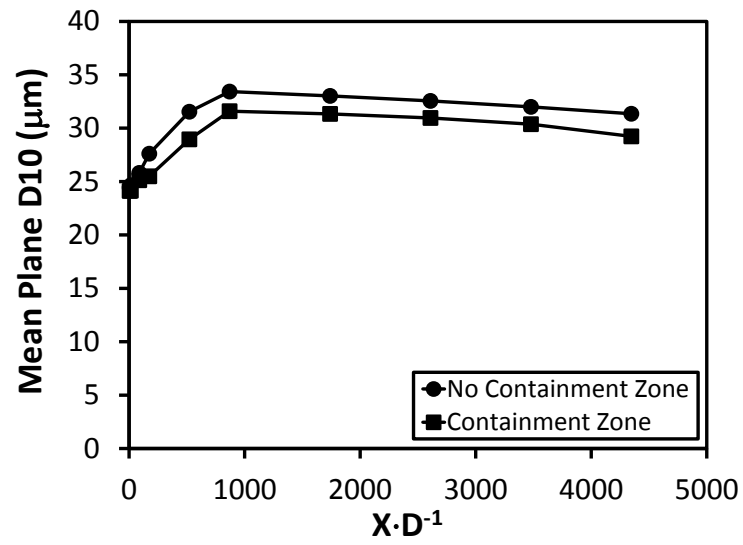

(b)

Figure 6.67: CFD Model Solution Dependence on the Presence of the Firefighting Jet Containment Zone 


\subsubsection{Select Computational Firefighting Jet Flow Model Results Compared to Experiments}

CFD model, flow visualization, and phase Doppler results are compared to determine the ability of the computational solution strategy to reproduce the major firefighting jet physical features and flow parameter trends observed in experiments. Figures 6.68 through 6.70 compare the qualitative, instantaneous firefighting jet flow structure of the low flow, low pressure jet, the medium flow, medium pressure jet, and the high flow, high pressure jet of flow visualization (labeled Flow Viz) photography and computational (labeled CFD) model results, respectively. Flow visualization illustrations are sized such that the spatial scales between experimental and computational results are equivalent. Water jet and 6-percent AFFF jet results are presented showing a side and top view of each firefighting jet. Laboratory lighting and camera lens range limited full firefighting jet trajectory comparisons. Although the visual differences between these three nozzle conditions shown are significant, the firefighting jet Reynolds number range is relatively narrow spanning from $6.9 \times 10^{4}$ to $2.47 \times 10^{5}$ and defining fully turbulent jet flow. For a summary of all case conditions examined in the present study, refer to Appendix A.

Experimental firefighting jet trajectories were reproduced by the CFD model in terms of jet reach, spread, and axial centerline decay angle under the influence of gravity. Large-scale turbulent eddies were also qualitatively similar in terms of size and structure, and became more visible for medium and high flow, high pressure jets operating at higher Reynolds numbers particularly in the downstream wake region. CFD model results also predicted the generation of more AFFF droplets compared to water droplets due to enhanced break-up from the surfactant agents, a trend consistent for every nozzle pressure-flow rate combination which was also confirmed by photography. 


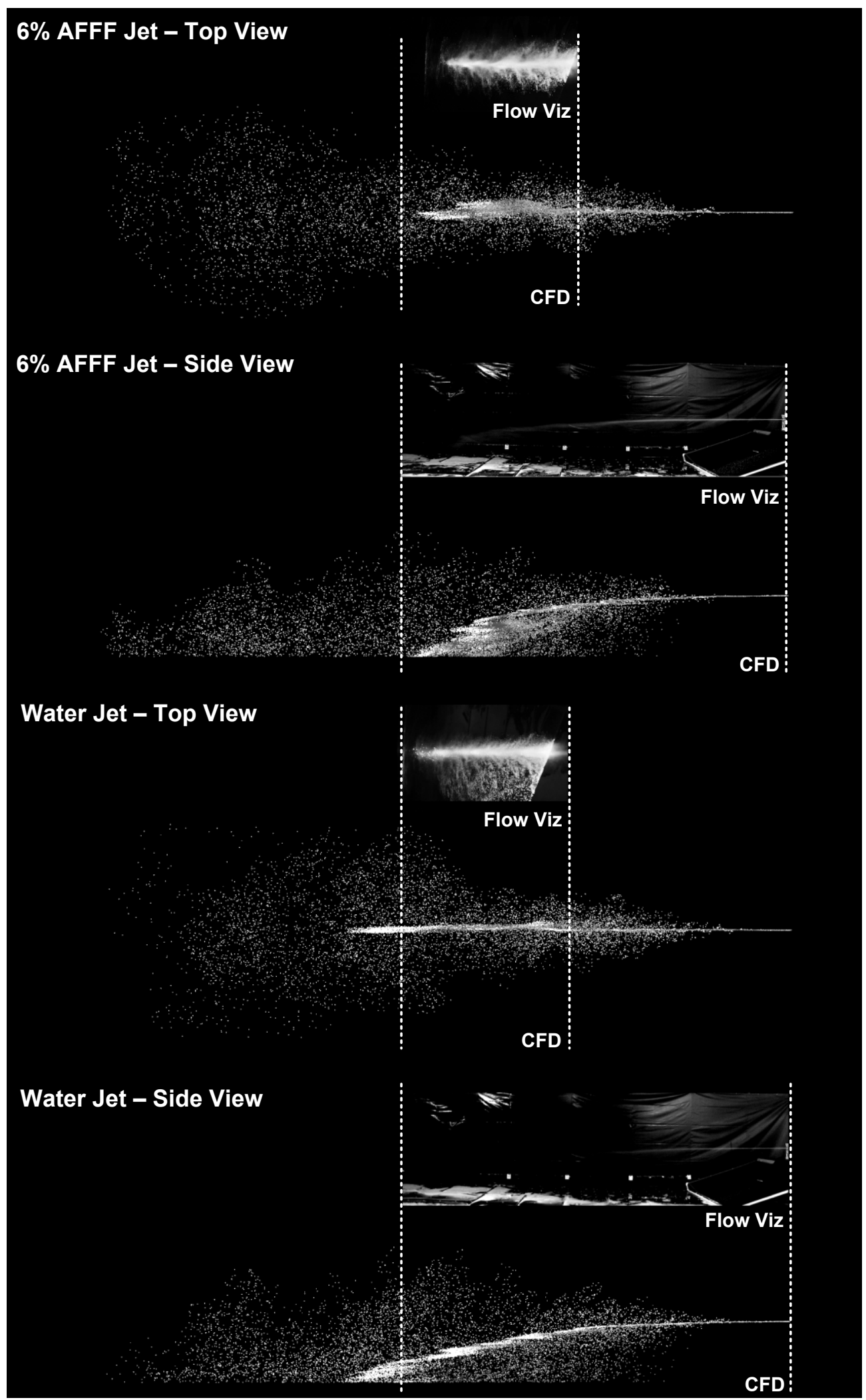

Figure 6.68: Qualitative Flow Structure Comparison between CFD Model and Flow Visualization Results of the Low Flow, Low Press. Jet (Flow is from Right to Left) 


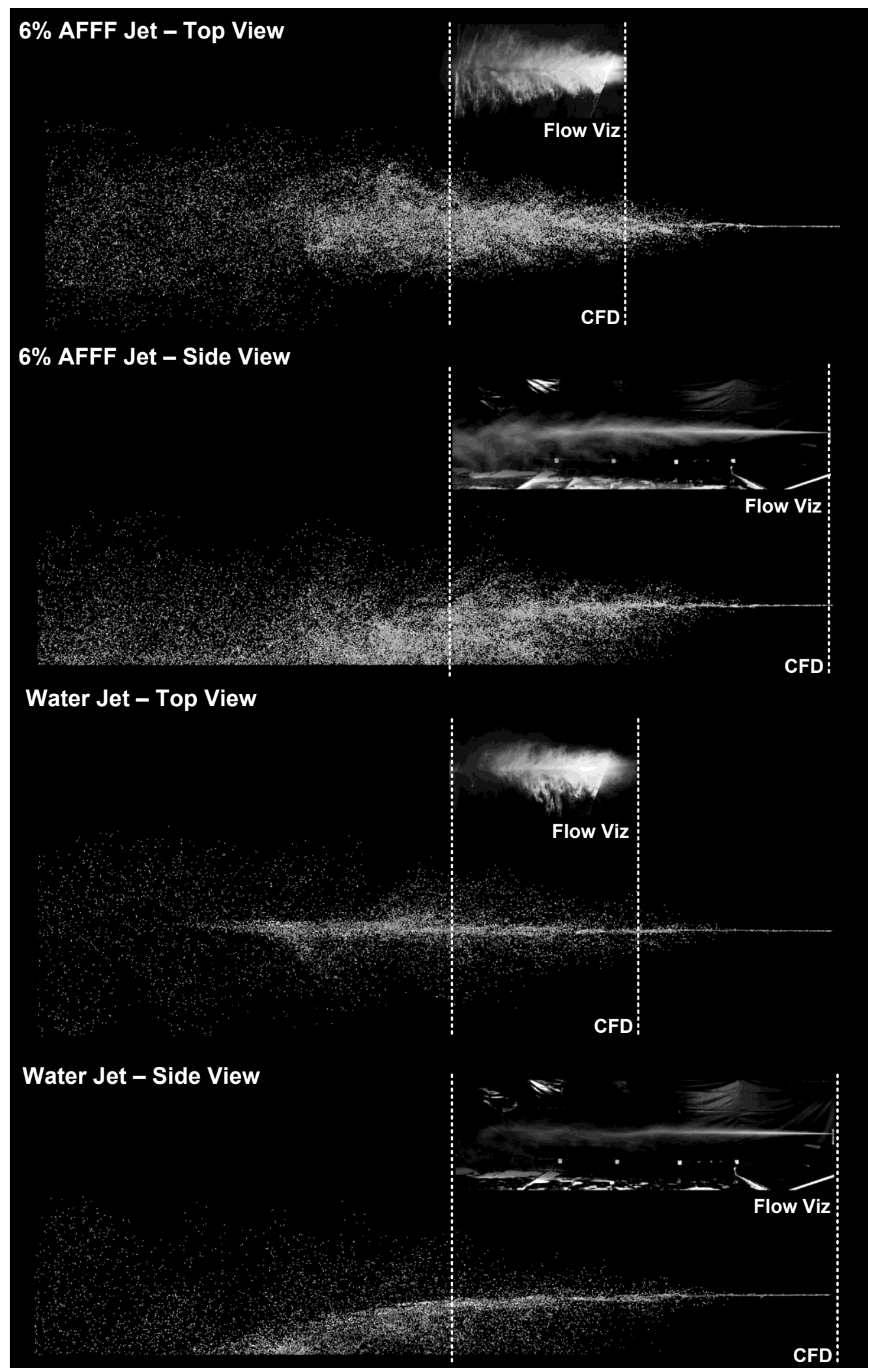

Figure 6.69: Qualitative Flow Structure Comparison between CFD Model and Flow Visualization Results of the Med. Flow, Med. Press. Jet (Flow is from Right to Left) 


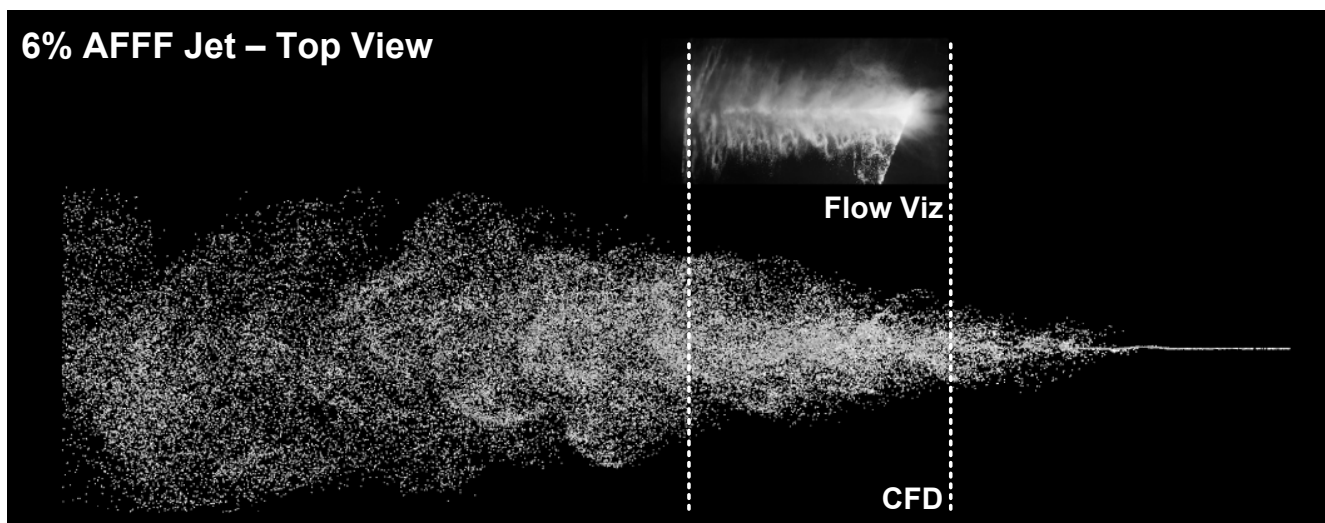

6\% AFFF Jet - Side View

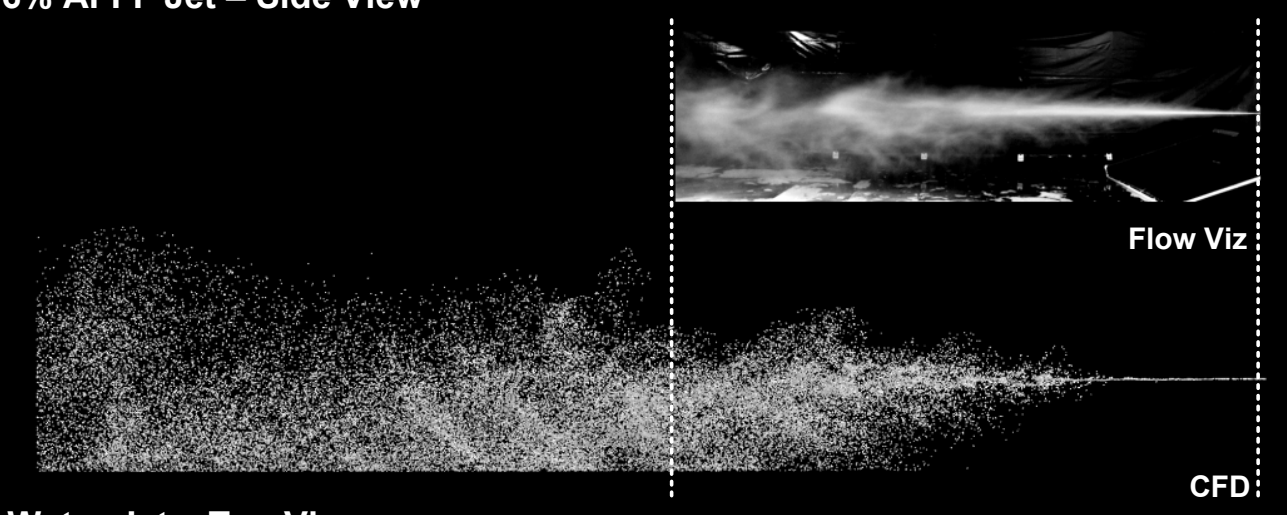

Water Jet - Top View
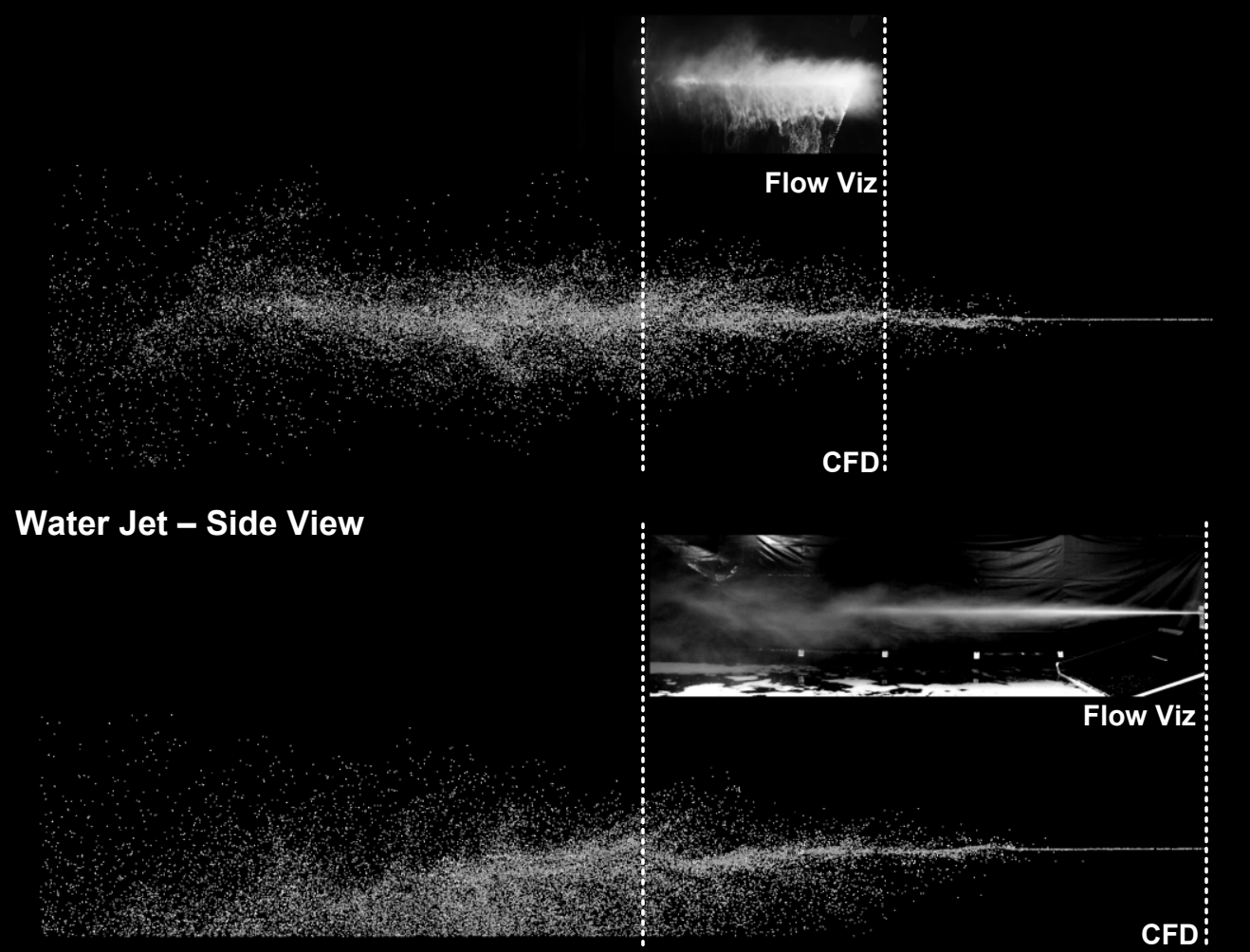

Figure 6.70: Qualitative Flow Structure Comparison between CFD Model and Flow Visualization Results of the High Flow, High Press. Jet (Flow is from Right to Left) 
Figures 6.71 through 6.73 compare CFD model and phase Doppler, vertical profile results at select jet axial downstream locations for the medium flow, medium pressure jet. Water and 6-percent AFFF jets are presented examining mean axial droplet velocity, mean vertical droplet velocity, and mean droplet diameter, respectively. Phase Doppler, mean droplet velocity profile results in Figure 6.71 and 6.72 are accompanied by two horizontal bars illustrating the minimum and maximum \pm RMS velocity for each profile in a similar style consistently used throughout the text. Both CFD model and phase Doppler, mean axial droplet velocity and mean droplet diameter data follow the same trend in terms of water jet values leading AFFF jet values with mean profile magnitudes nearly equivalent. Mean vertical droplet velocity magnitudes are also similar, with no significant change between CFD model water jet and AFFF jet results supporting observations made in Section 6.3.2 and 6.3.3. The largest disparity is how much narrower each CFD profile is compared to the phase Doppler profile. Divergence is the worst nearest to the nozzle. This is due to simplifications and assumptions mainly related to the DPM injection condition. The DPM injection into the continuous phase is prescribed as a single, nonperturbed point injection with velocity defined exclusively in the axial direction. No horizontal or vertical velocity was considered. In reality, the agent is exiting a finite, 2-D circular area while being perturbed by a 3-D flow environment. In addition, CFD profile data are derived based on a moving average of droplets that penetrated virtual planes of finite height and width defined to represent the approximate profile window measured by the PDPA. Because a moving average was employed, CFD model profile edge data were artificially clipped. The CFD solution process did not record RMS droplet velocity data to provide another means of comparison to phase Doppler experiments. The difference between CFD model and phase Doppler profile results is lessened as axial distance downstream proportionally increases due to growing 3-D droplet-air interaction. Other limitations are placed on the comparability of CFD model and phase Doppler results, particularly for the droplet size distribution where the PDPA had a measurement envelope of about 2 to $685 \mu \mathrm{m}$ whereas the CFD model had no upper or lower limit on droplet size. 

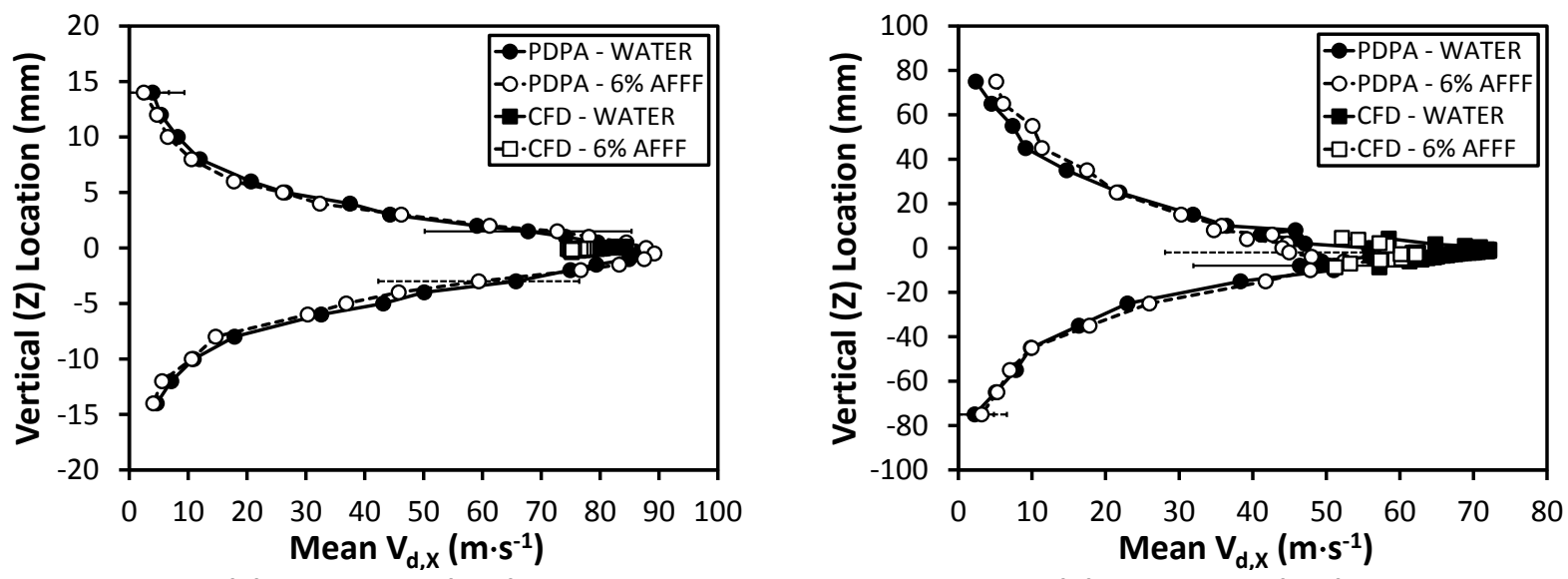

(a) $x=0.152 \mathrm{~m}(6 \mathrm{in})$

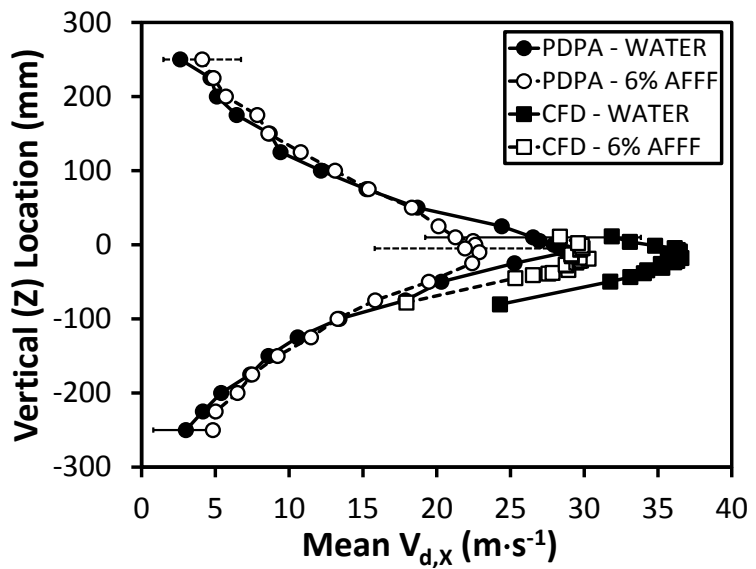

(c) $x=3.05 \mathrm{~m}(10 \mathrm{ft})$

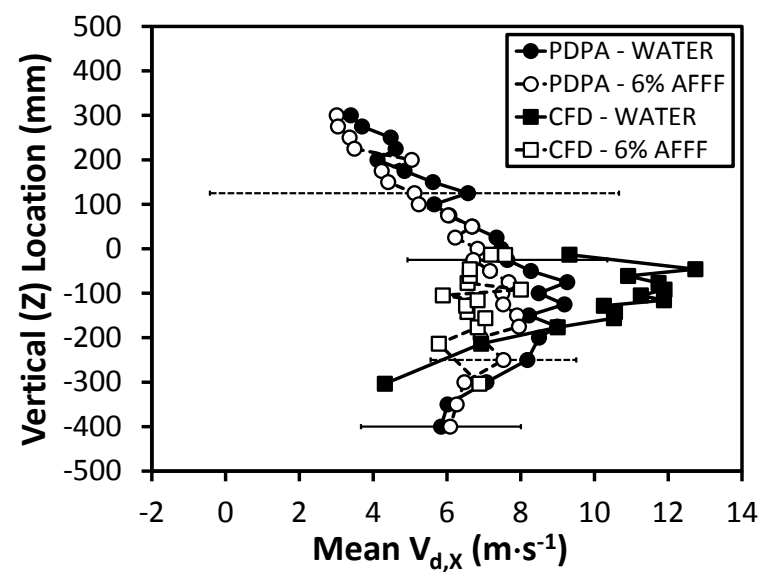

(d) $x=6.10 \mathrm{~m}(20 \mathrm{ft})$

Figure 6.71: A Comparison of Phase Doppler and CFD Model, Mean Axial Droplet Velocity Profile Data for the Med. Flow, Med. Press. Jet 

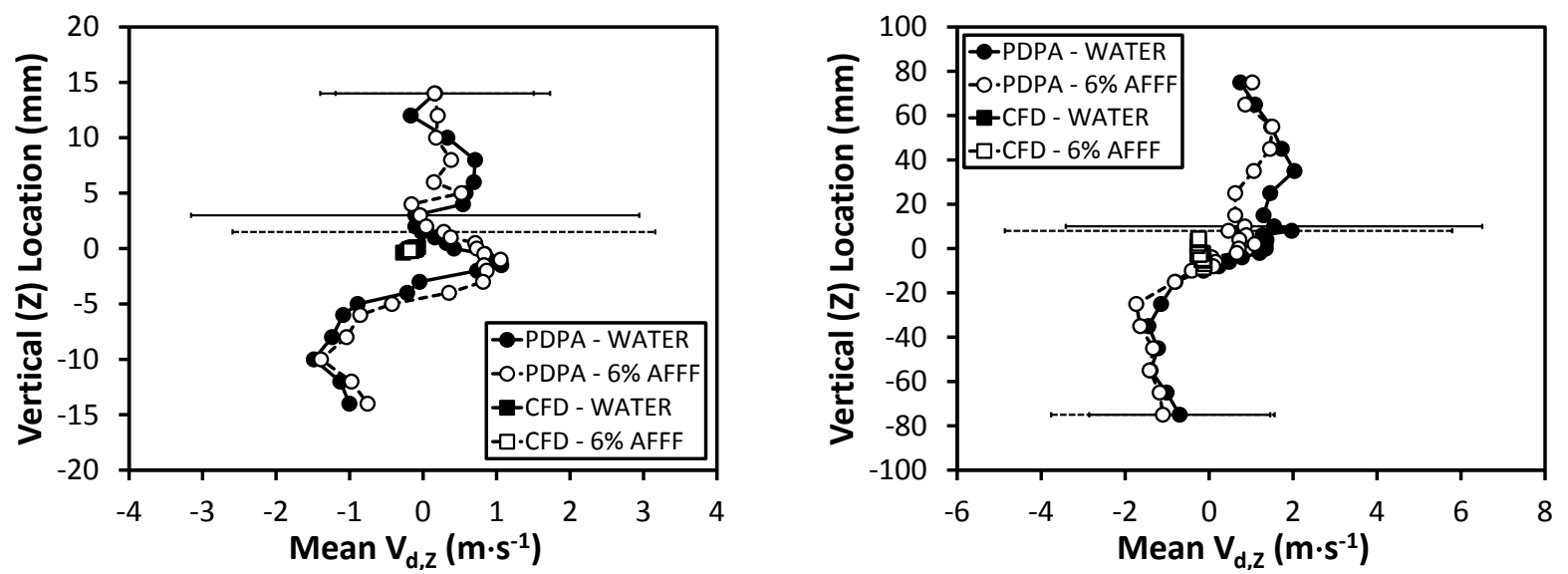

(a) $x=0.152 \mathrm{~m}(6 \mathrm{in})$

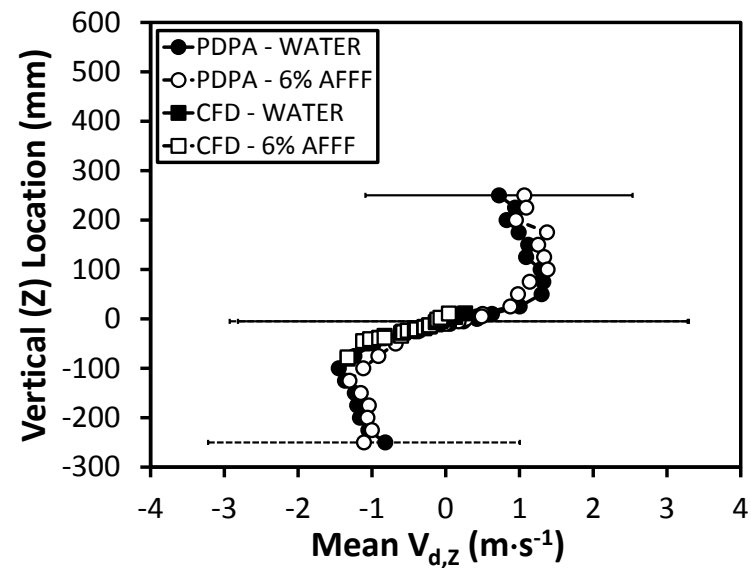

(c) $x=3.05 \mathrm{~m}(10 \mathrm{ft})$

(b) $x=0.914 \mathrm{~m}(3 \mathrm{ft})$

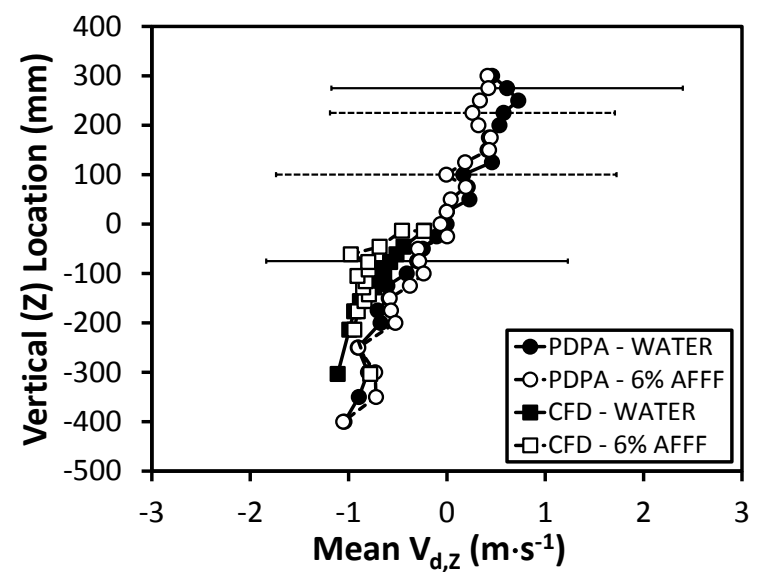

(d) $x=6.10 \mathrm{~m}(20 \mathrm{ft})$

Figure 6.72: A Comparison of Phase Doppler and CFD Model, Mean Vertical Droplet Velocity Profile Data for the Med. Flow, Med. Press. Jet 

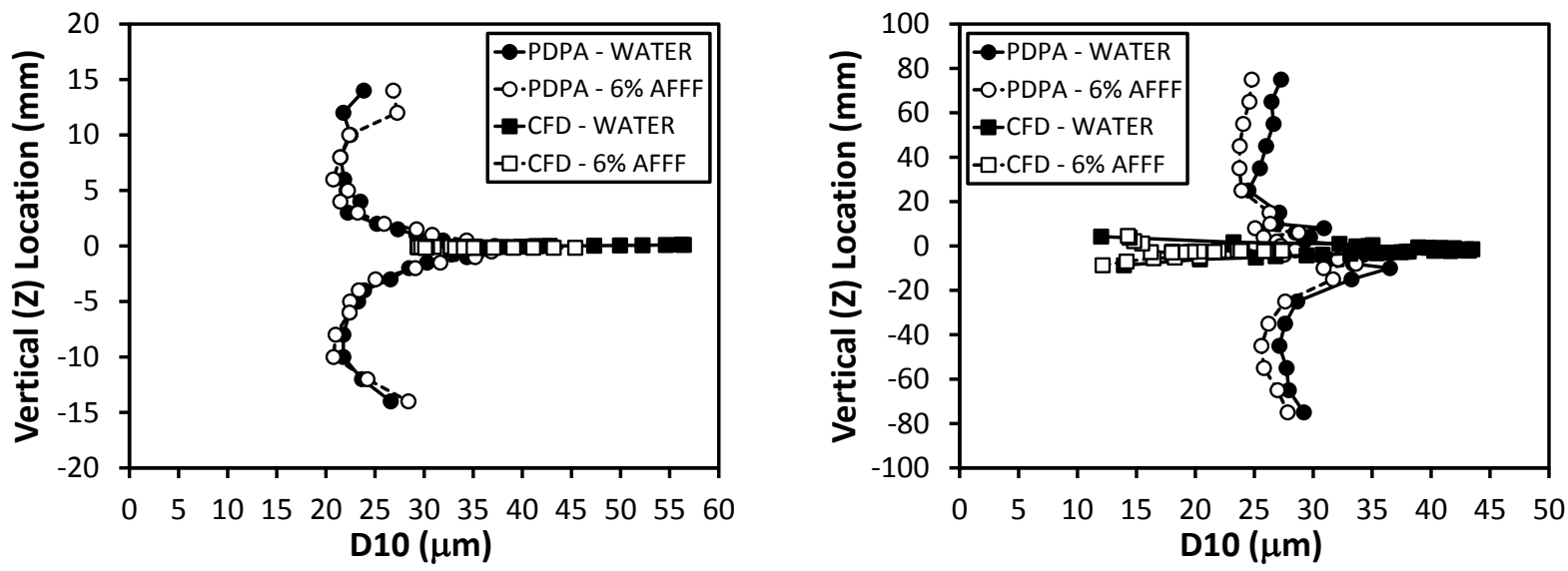

(a) $x=0.152 \mathrm{~m}(6 \mathrm{in})$

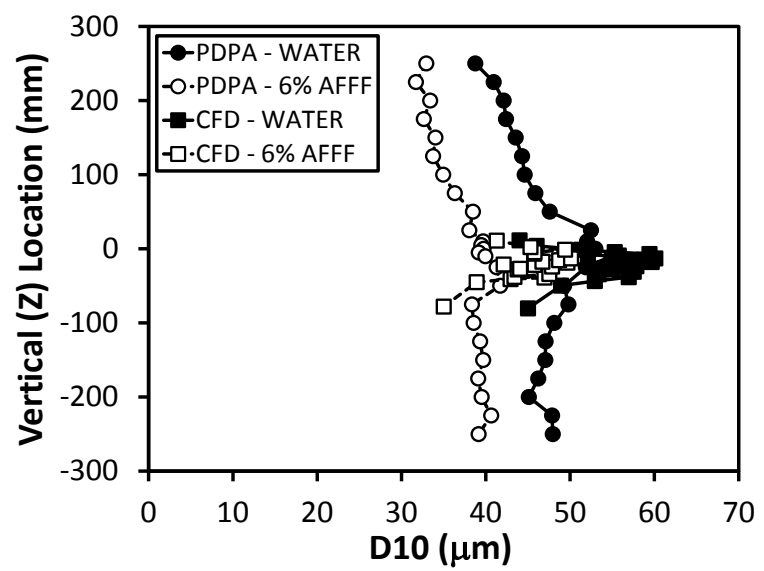

(c) $x=3.05 \mathrm{~m}(10 \mathrm{ft})$

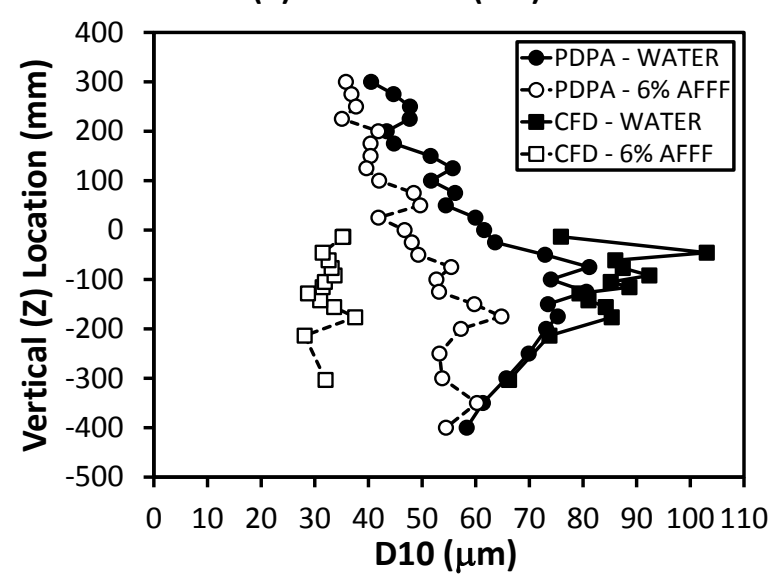

(d) $x=6.10 \mathrm{~m}(20 \mathrm{ft})$

Figure 6.73: A Comparison of Phase Doppler and CFD Model, Mean Droplet Diameter Profile Data for the Med. Flow, Med. Press. Jet

Figures 6.74 through 6.78 compare CFD model and phase Doppler results in terms of mean and maximum vertical flow parameter profiles in order to illustrate global trends in the data. Data presentation is similar to how phase Doppler summary results are presented in Section 6.3.3. Maximum profile, mean axial droplet velocity, mean profile, mean axial droplet velocity, mean profile, mean droplet diameter, and mean profile, Sauter mean diameter are chosen for comparison. Each of the aforementioned figures represents a firefighting jet pressure-flow rate combination analyzed.

In general maximum and mean profile, mean axial droplet velocity CFD model and phase Doppler results were in reasonable agreement with each other with most profile points within 10 percent of one another. CFD model results not only followed experimental trends, but the relative difference in flow parameter values between water jets and AFFF jets were similar in 
magnitude to those reported by phase Doppler experiments. For some firefighting jet configurations, larger discrepancies were observed and believed to be the result of DPM droplet injection simplifications discussed earlier. Most CFD model mean profile, mean droplet diameter results drifted higher than PDPA values. This is likely due to the PDPA being unable to record droplets larger than $685 \mu \mathrm{m}$ and the CFD model had no such upper limitation. Another theory for the observed differences could be the firefighting jet containment zone had a more significant impact on results than anticipated as mean droplet diameters tended to increase when this influence was taken into account in the CFD model. Overall, firefighting jet flow parameter characteristics of interest were predicted adequately enough by the CFD model to rely on other parameter estimations predicted by flow calculations that were too difficult or impossible to quantify experimentally.

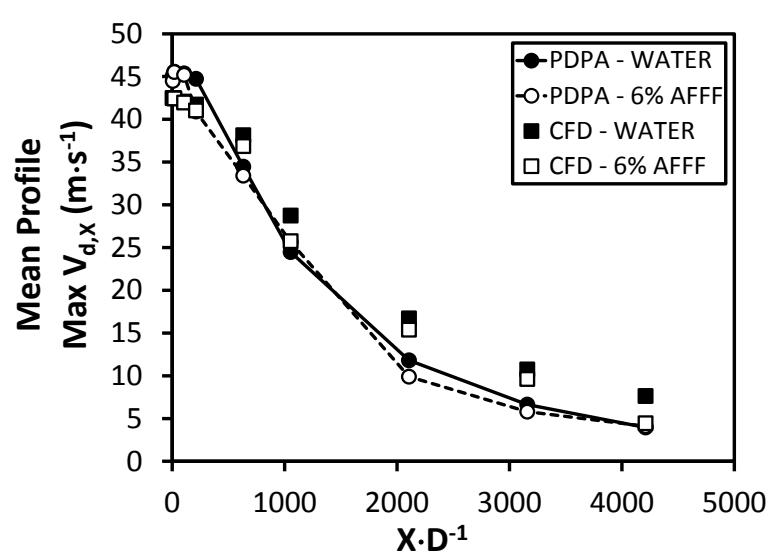

(a) Maximum Axial Droplet Velocity

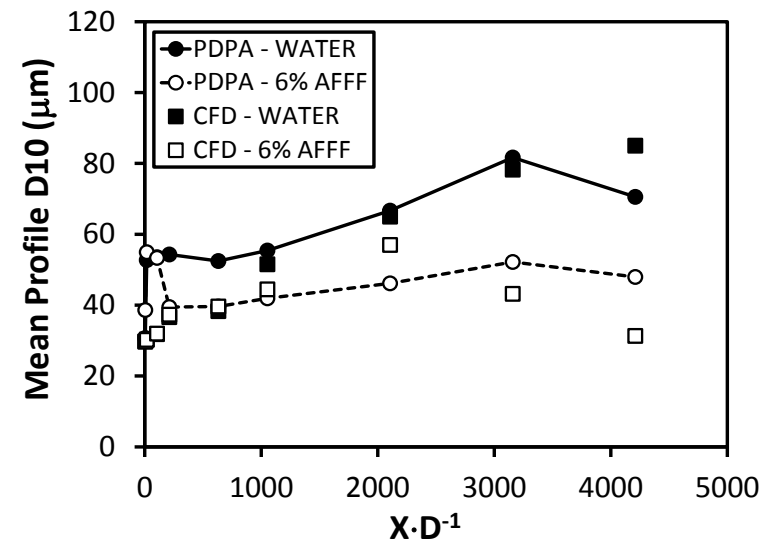

(c) Mean Droplet Diameter

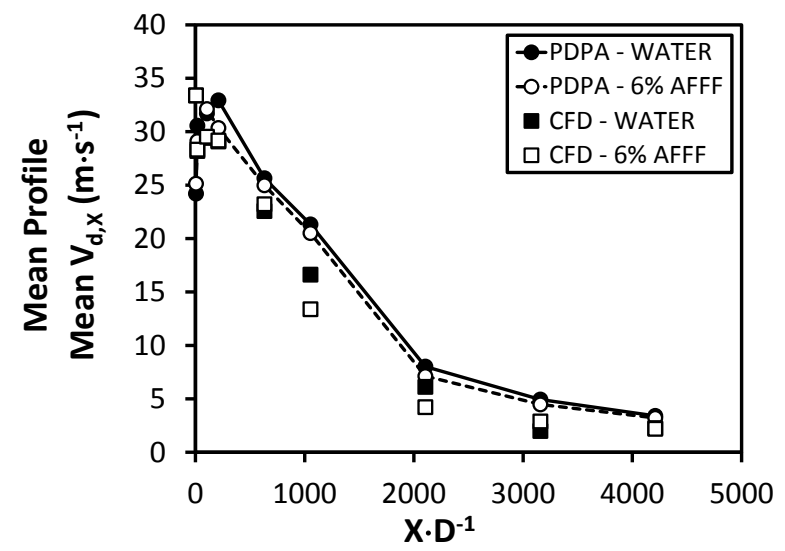

(b) Mean Axial Droplet Velocity

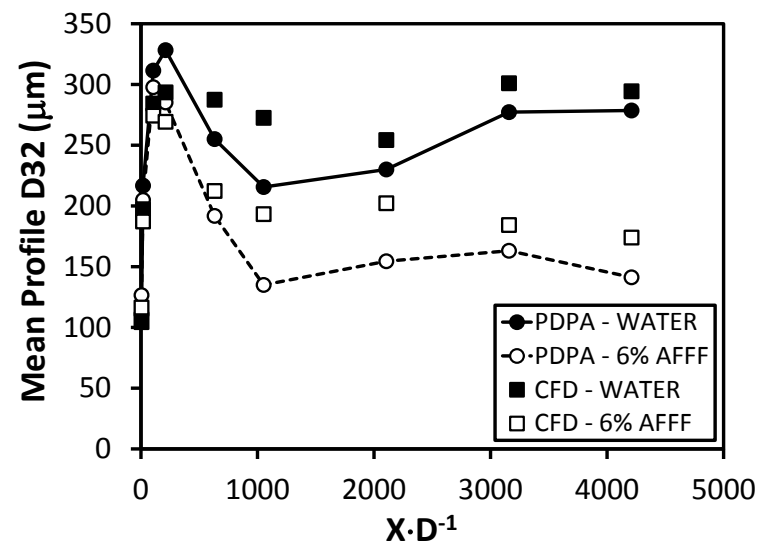

(d) Sauter Mean Droplet Diameter

Figure 6.74: A Comparison of PDPA and CFD Mean Vertical Profile Data for the Low Flow, Low Press. Jet 


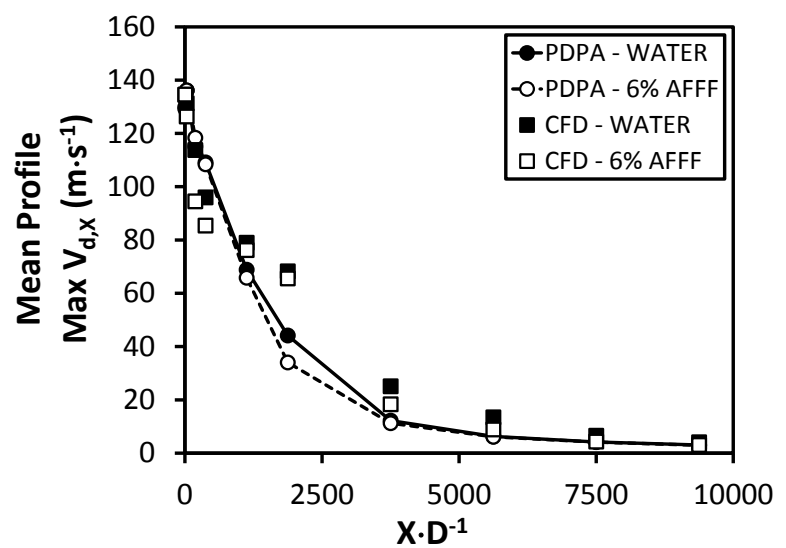

(a) Maximum Axial Droplet Velocity

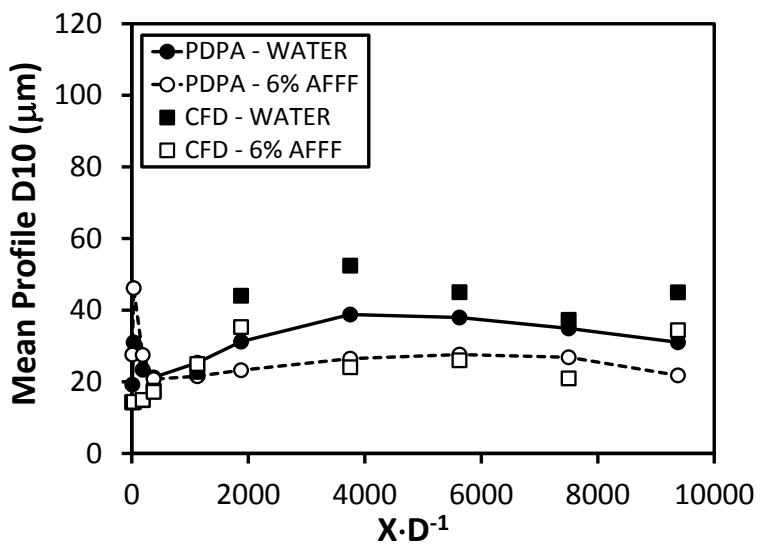

(c) Mean Droplet Diameter

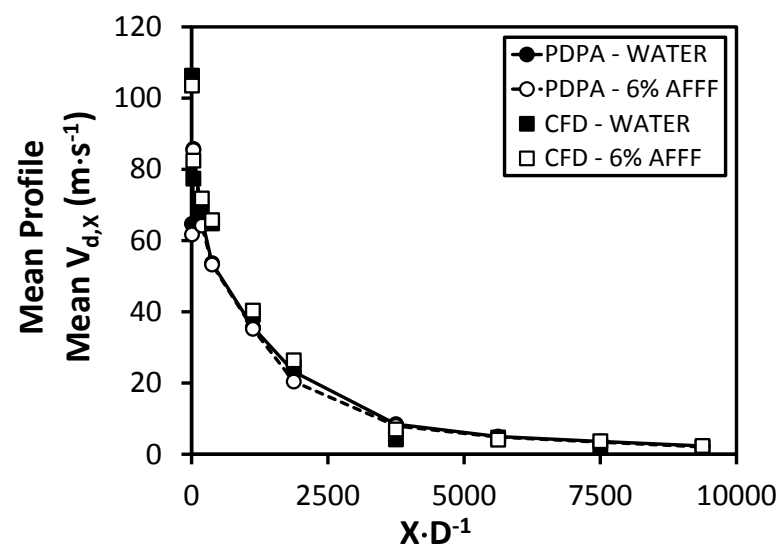

(b) Mean Axial Droplet Velocity

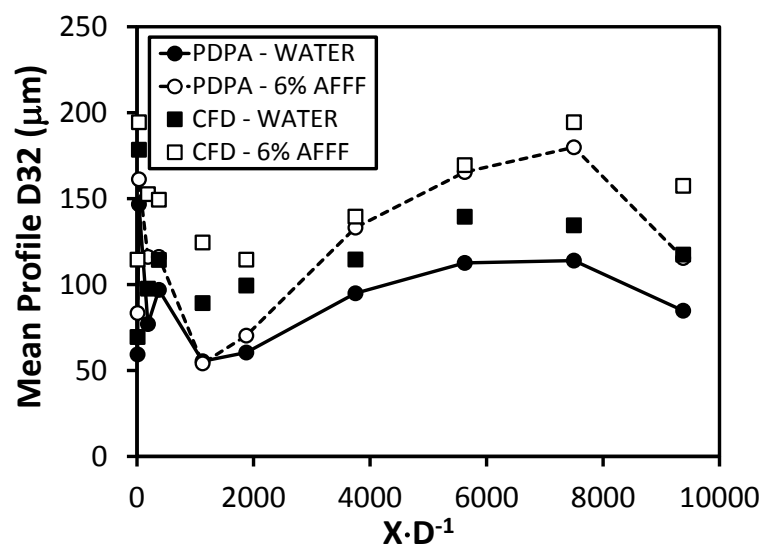

(d) Sauter Mean Droplet Diameter

Figure 6.75: A Comparison of PDPA and CFD Mean Vertical Profile Data for the Low Flow, High Press. Jet 


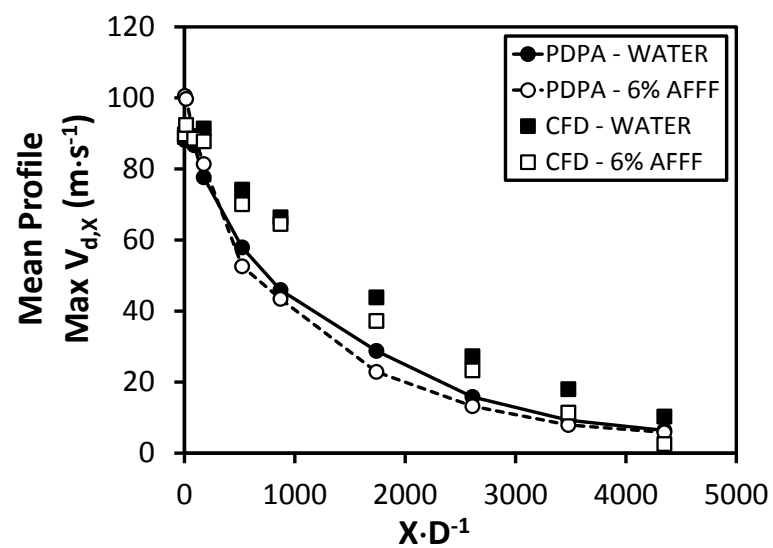

(a) Maximum Axial Droplet Velocity

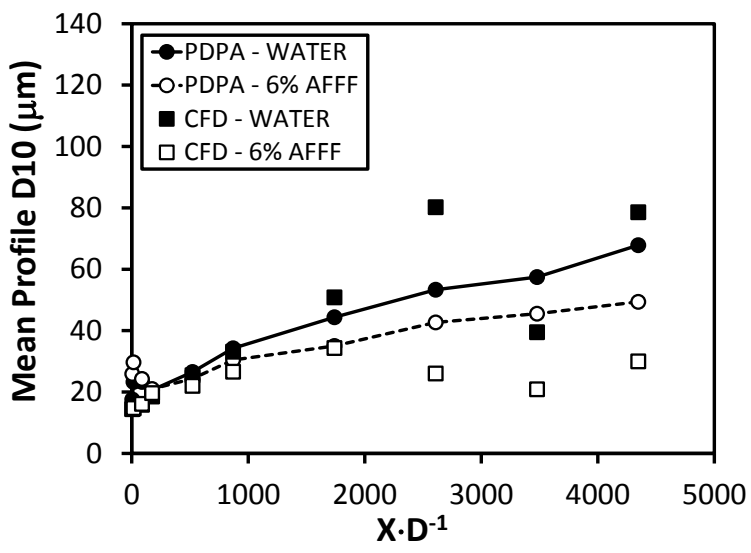

(c) Mean Droplet Diameter

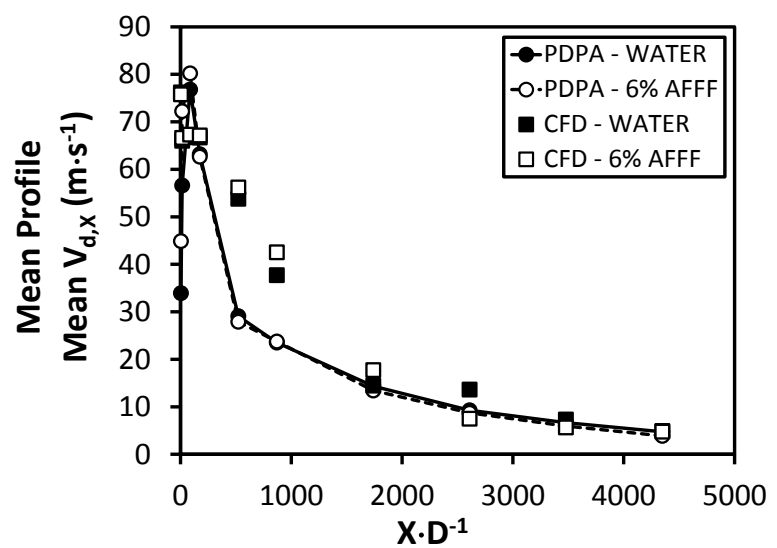

(b) Mean Axial Droplet Velocity

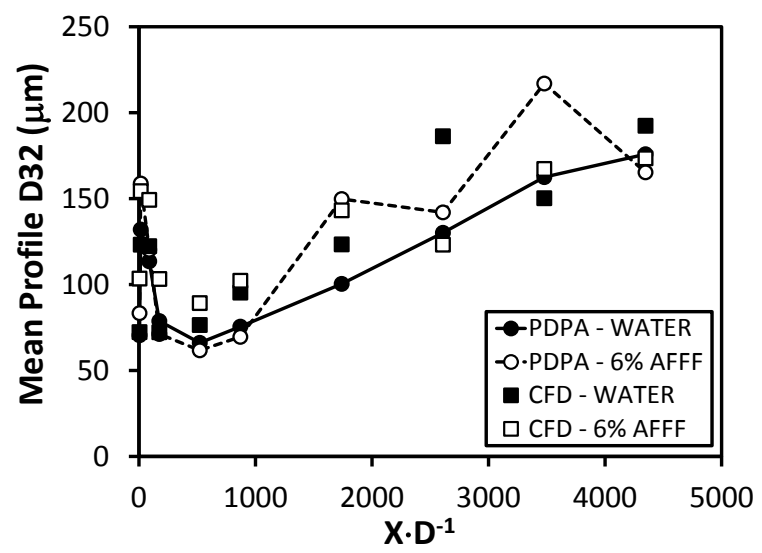

(d) Sauter Mean Droplet Diameter

Figure 6.76: A Comparison of PDPA and CFD Mean Vertical Profile Data for the Med. Flow, Med. Press. Jet 


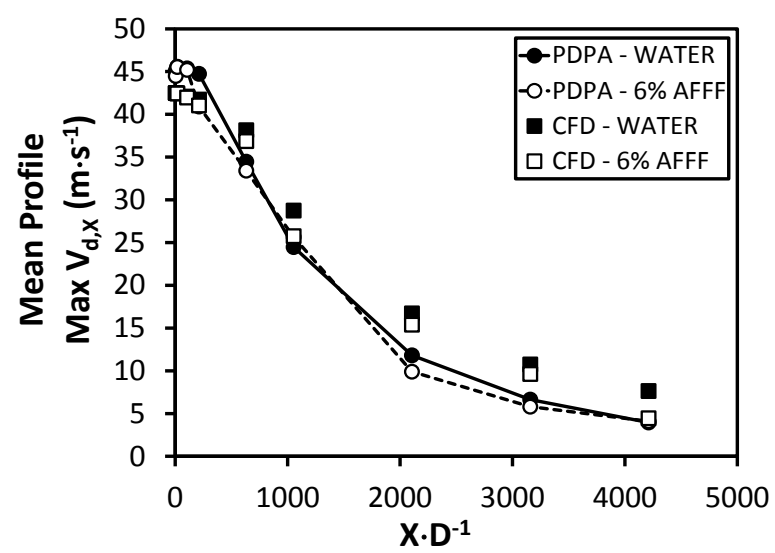

(a) Maximum Axial Droplet Velocity

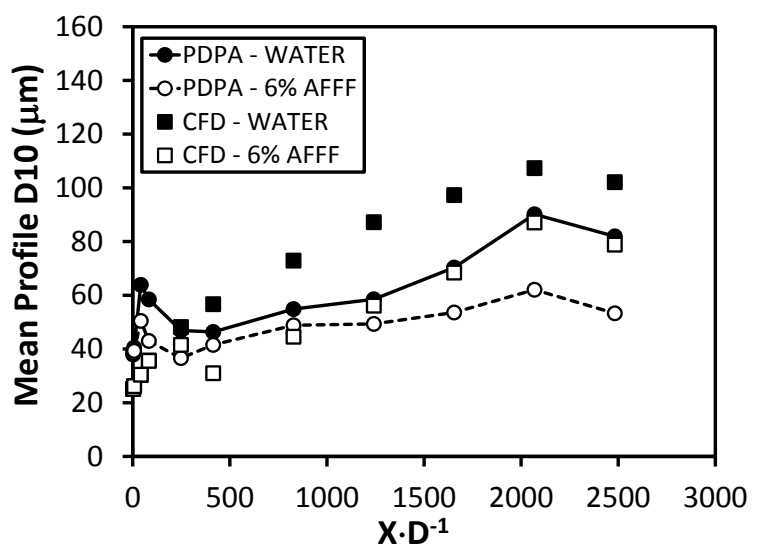

(c) Mean Droplet Diameter

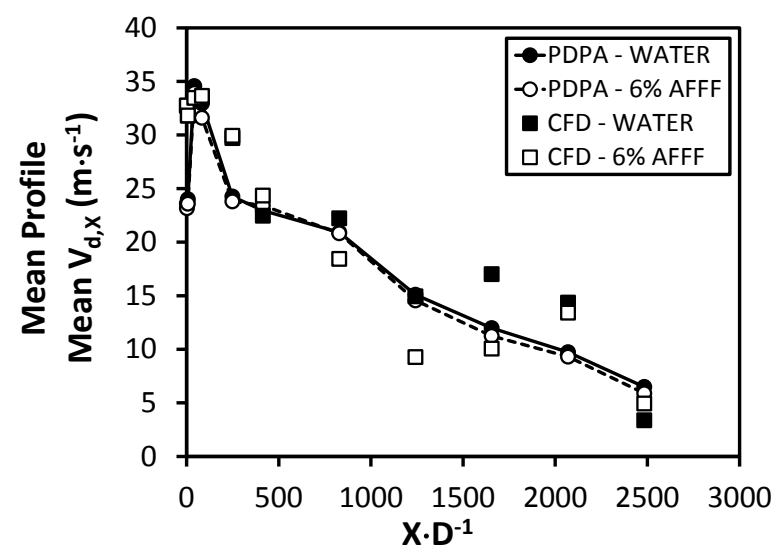

(b) Mean Axial Droplet Velocity

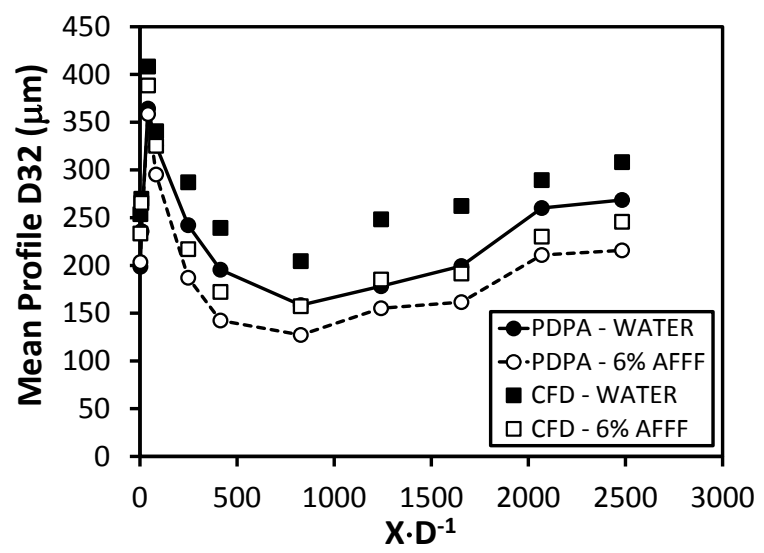

(d) Sauter Mean Droplet Diameter

Figure 6.77: A Comparison of PDPA and CFD Mean Vertical Profile Data for the High Flow, Low Press. Jet 


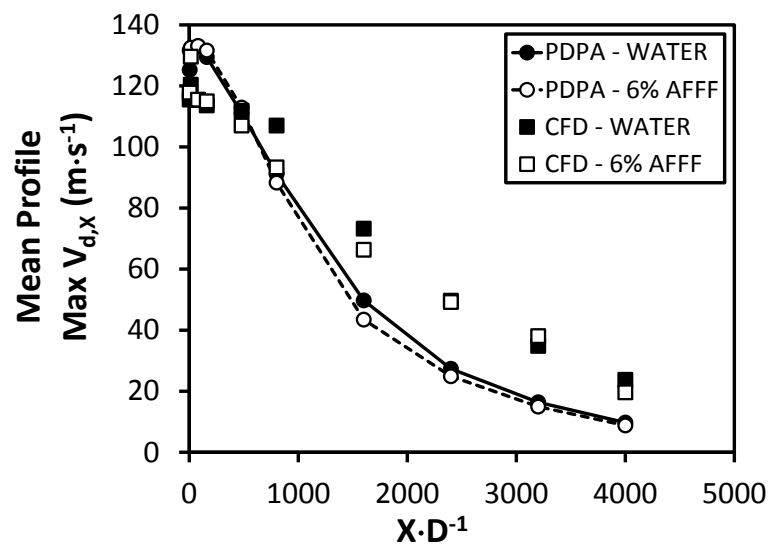

(a) Maximum Axial Droplet Velocity

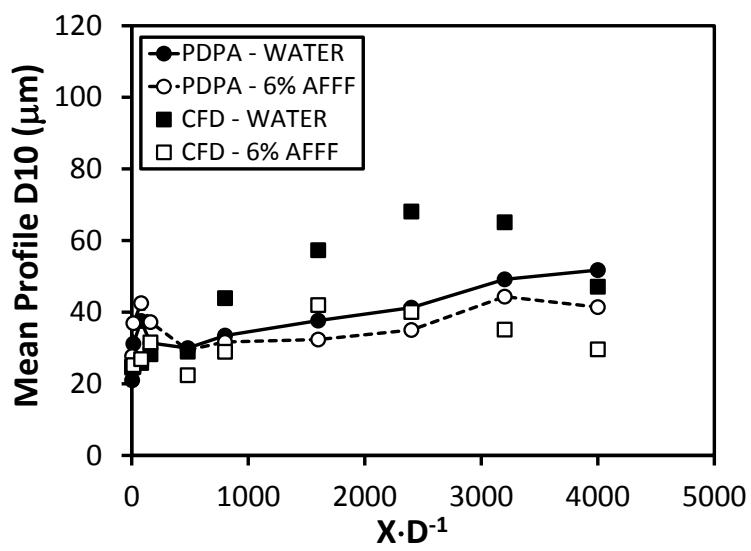

(c) Mean Droplet Diameter

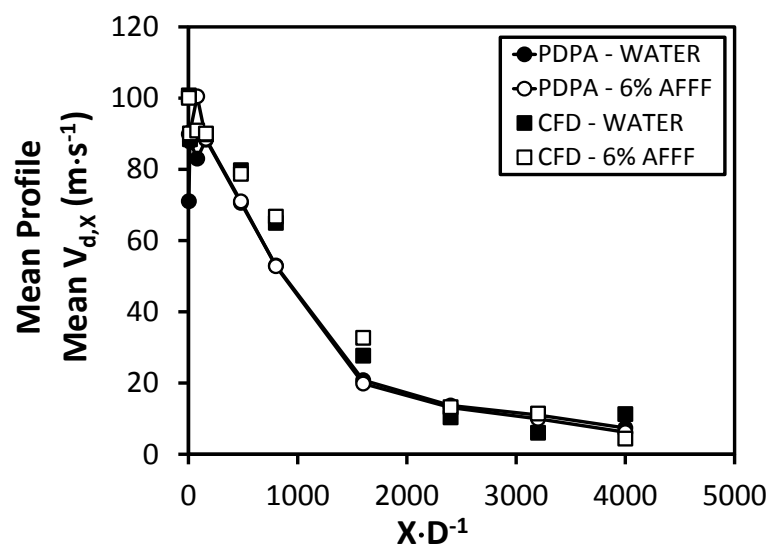

(b) Mean Axial Droplet Velocity

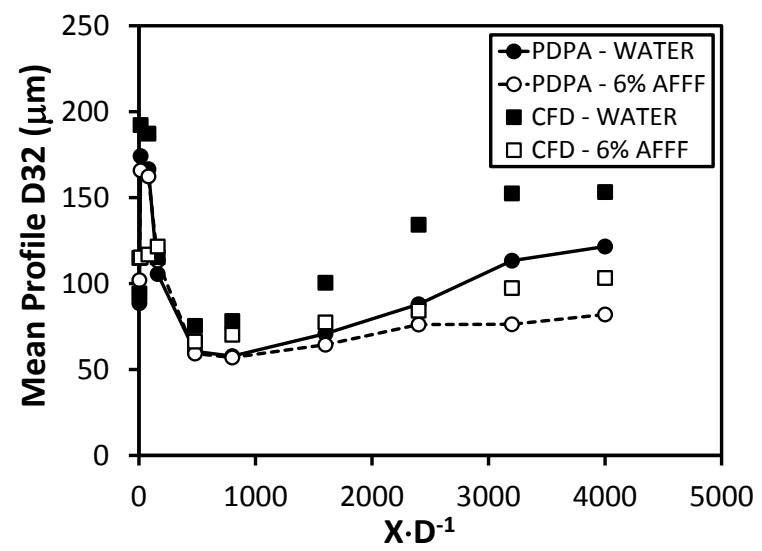

(d) Sauter Mean Droplet Diameter

Figure 6.78: A Comparison of PDPA and CFD Mean Vertical Profile Data for the High Flow, High Press. Jet

\subsubsection{Computational Firefighting Jet Flow Model Results Summary}

CFD model results are presented for the five firefighting jet pressure-flow rate combinations listed in Table 5.1 in Section 5.1. The data presented in this section describe instantaneous firefighting jet flow parameters from a fully-developed flow field. Table 6.4 summarizes the overall, globally averaged droplet size information for each flow domain. Each droplet parcel represents a group of about 50,000 droplets. The minimum droplet diameter $\left(d_{\text {min }}\right)$, maximum droplet diameter $\left(d_{\max }\right)$, mean droplet diameter, and Sauter mean droplet diameter are listed. For all instances, the amount of 6-percent AFFF jet droplet parcels were larger for every CFD model case compared to the amount of water jet droplet parcels due to the enhanced break-up induced by the surfactants in AFFF. As expected, low flow, low pressure jets generated the least amount of droplets and high flow, high pressure jets created the most droplets based on each jet's respective degree of atomization. The minimum droplet diameter is the minimum droplet 
diameter bin for the DPM injection condition which for all CFD model cases was $13.72 \mu \mathrm{m}$. The range in maximum droplet diameter followed the same trend observed in phase Doppler data. The largest-sized droplets were generated by the high flow, low press water jet, and the smallestsized droplets were produced by the low flow, high pressure 6-percent AFFF jet. The CFD models report a large disparity between low pressure water and 6-percent AFFF jets in terms of mean droplet diameter. The difference in some cases, particularly the low flow jet cases, was negligible. Conversely, medium and high pressure jets exhibited distinct mean droplet diameter divergence by as much as 28 percent. Sauter mean droplet diameter comparisons between water and 6-percent AFFF jets were less consistent across the nozzle pressure-flow rate combinations. Most CFD model results showed a reduction in Sauter mean diameter from the water jet to the 6percent AFFF jet, a similar pattern also observed in phase Doppler Sauter mean droplet profiles.

Table 6.4: Computational Firefighting Jet Flow Model Domain Droplet Summary

\begin{tabular}{|l|c|c|c|c|c|}
\hline \multicolumn{1}{|c|}{$\begin{array}{c}\text { Model } \\
\text { Test Case }\end{array}$} & $\begin{array}{c}\text { Total No. of } \\
\text { Droplet Parcels }\end{array}$ & $\begin{array}{c}\mathbf{d}_{\text {MIN }} \\
(\mu \mathrm{m})\end{array}$ & $\begin{array}{c}\mathbf{d}_{\text {MAx }} \\
(\mathrm{mm})\end{array}$ & $\begin{array}{c}\text { D10 } \\
(\mu \mathrm{m})\end{array}$ & $\begin{array}{c}\text { D32 } \\
(\mu \mathrm{m})\end{array}$ \\
\hline Low Flow, Low Press. Water Jet & 19,454 & 13.72 & 4.079 & 20.11 & 154.0 \\
\hline Low Flow, Low Press. 6\% AFFF Jet & 26,504 & 13.72 & 2.497 & 19.38 & 157.0 \\
\hline Low Flow, High Press. Water Jet & 22,246 & 13.72 & 1.005 & 28.94 & 93.92 \\
\hline Low Flow, High Press. 6\% AFFF Jet & 28,977 & 13.72 & 0.5851 & 17.86 & 88.86 \\
\hline Med. Flow, Med Press. Water Jet & 16,059 & 13.72 & 3.725 & 22.91 & 137.7 \\
\hline Med. Flow, Med Press. 6\% AFFF Jet & 38,916 & 13.72 & 1.988 & 19.81 & 170.0 \\
\hline High Flow, Low Press. Water Jet & 10,061 & 13.72 & 5.690 & 21.78 & 455.4 \\
\hline High Flow, Low Press. 6\% AFFF Jet & 13,541 & 13.72 & 3.267 & 21.66 & 422.3 \\
\hline High Flow, High Press. Water Jet & 29,334 & 13.72 & 3.326 & 28.20 & 190.6 \\
\hline High Flow, High Press. 6\% AFFF Jet & 58,722 & 13.72 & 1.547 & 20.27 & 172.7 \\
\hline
\end{tabular}

Figure 6.79 shows particle traces of firefighting jet droplets colored by residence time for the medium flow, medium pressure firefighting jet. This figure is intended to provide an estimate of the average droplet lifetime for both water and AFFF jets. Droplet lifetimes for AFFF jets appear slightly longer compared to water jets due to the break-up and more intense dispersion of smaller, less massive droplets which are prone to stay airborne longer. The effective jet core for both agents was observed to have a lifetime on the order of 1 to $2 \mathrm{~s}$ with peripheral and far downstream droplets on the order of 3 to $5 \mathrm{~s}$. Low pressure jets generate droplets with lifetimes 
about twice as long, and high pressure jets generate droplet with lifetimes about half as long. From Figure 2.6 in Section 2.2, it is shown AFFF has approached its equilibrium surface tension value by about $1 \mathrm{~s}$. This indicates that prescribing surface tension dynamically as a function of time similarly to Figure 2.6 may impact results particularly for high pressure AFFF jets, but negligibly influences low pressure AFFF jets.
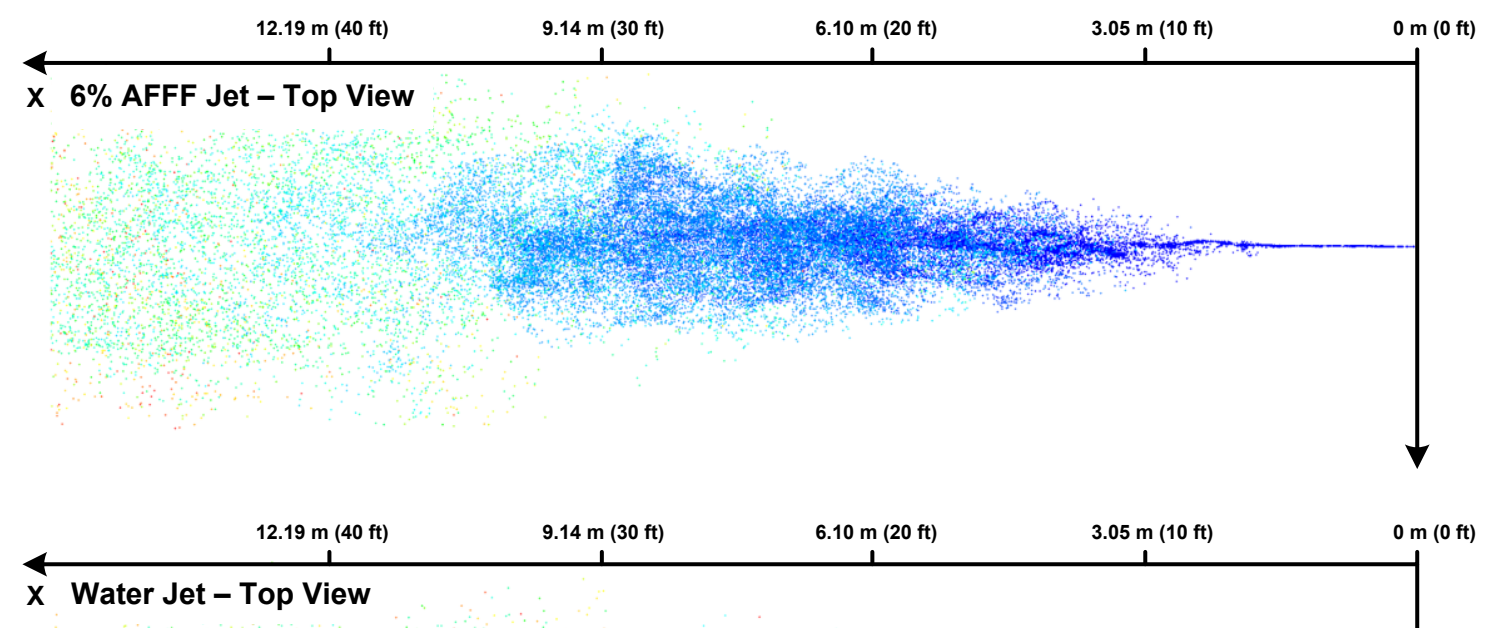

$\begin{array}{llllllllllllllllllllllllll}0.0 & 0.4 & 0.8 & 1.2 & 1.6 & 2.0 & 2.4 & 2.8 & 3.2 & 3.6 & 4.0 & 4.4 & 4.8 & 5.2 & 5.6 & 6.0 & 6.4 & 6.8 & 7.2 & 7.6 & 8.0 & 8.4 & 8.8 & 9.2 & 9.6 & 10.0\end{array}$ Particle Traces Colored by Residence Time (s)

Figure 6.79: CFD Model Results of Droplet Residence Time for Med. Flow, Med. Press Jets 
Figures 6.80 through 6.84 illustrate particle traces of firefighting jet droplets colored by velocity magnitude for each nozzle-pressure combination modeled. The non-linear velocity decay in the near field region of the nozzle is best illustrated in these figures particularly for the medium and high pressure jets. Nozzle exit velocity ranged from approximately 42 to $116 \mathrm{~m} \cdot \mathrm{s}^{-1}$. Most satellite droplets away from the jet core exhibited velocities on the order of $10 \mathrm{~m} \cdot \mathrm{s}^{-1}$ or much less.

Figure 6.85 through 6.89 depict particle traces of firefighting jet droplets colored by droplet diameter using a logarithmic scale. The ranges shown in each figure reflect the minimum and maximum droplet diameter ranges shown in Table 6.4. Finer droplet diameter details were difficult to graphically resolve while illustrating the firefighting jet in its entirety with respect to the far field - especially alongside larger diameter droplets. Hence, local average droplet diameters particularly in areas of higher droplet concentrations may appear skewed towards higher mean droplet diameters.

Figures 6.90 through 6.94 show particle traces of firefighting jet droplets colored by gaseous Weber number illustrating the effect droplet size and surface tension have on universal jet dynamics. Gaseous Weber number distinction between water and AFFF jets is more pronounced for some CFD model cases compared to others, with all values staying less than 100 , indicating the TAB break-up model was a good selection given nozzle exit flow physics.

Figures 6.95 through 6.99 illustrate velocity magnitude of the gaseous continuous (air) phase spanning from approximately $5 \mathrm{~m} \cdot \mathrm{s}^{-1}$ in the jet far field up to $45 \mathrm{~m} \cdot \mathrm{s}^{-1}$ near the nozzle exit for high pressure jets. This results in a firefighting jet droplet slip velocity of about 60 to 80 percent that of reported droplet velocities shown in Figures 6.79 through 6.83. As expected, near nozzle air velocities are higher for firefighting jets with higher pressures and higher droplet velocities due to enhanced entrainment whereas low pressure jets exhibited lower droplet velocities and in turn lower air entrainment velocities. Large-scale turbulent 3-D eddies are also well described in these figures which are also prominent in all mid-field and far field flow visualization imagery. 


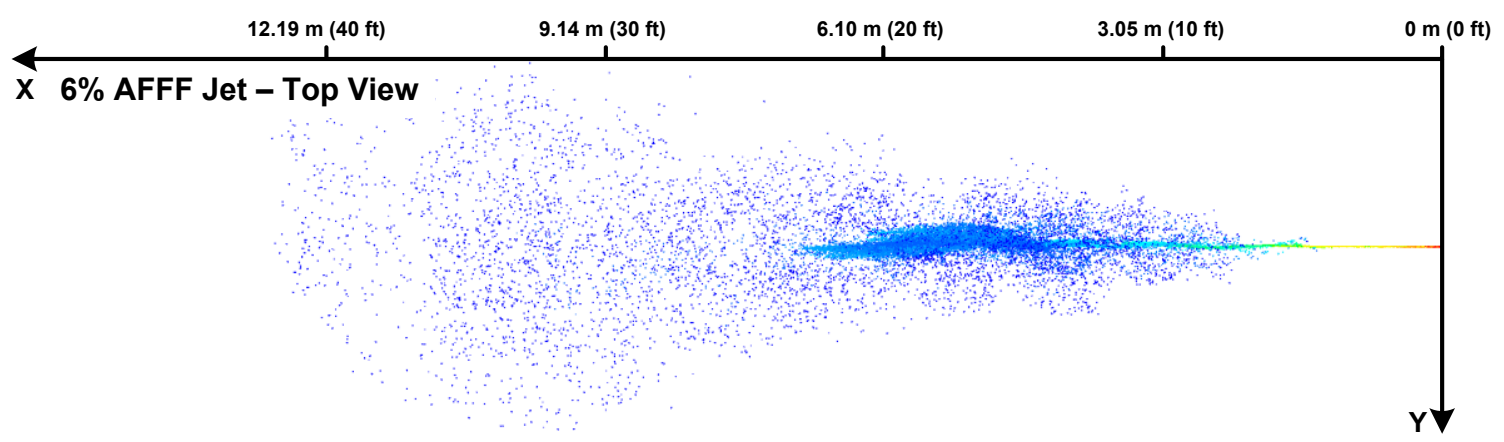

6\% AFFF Jet - Side View
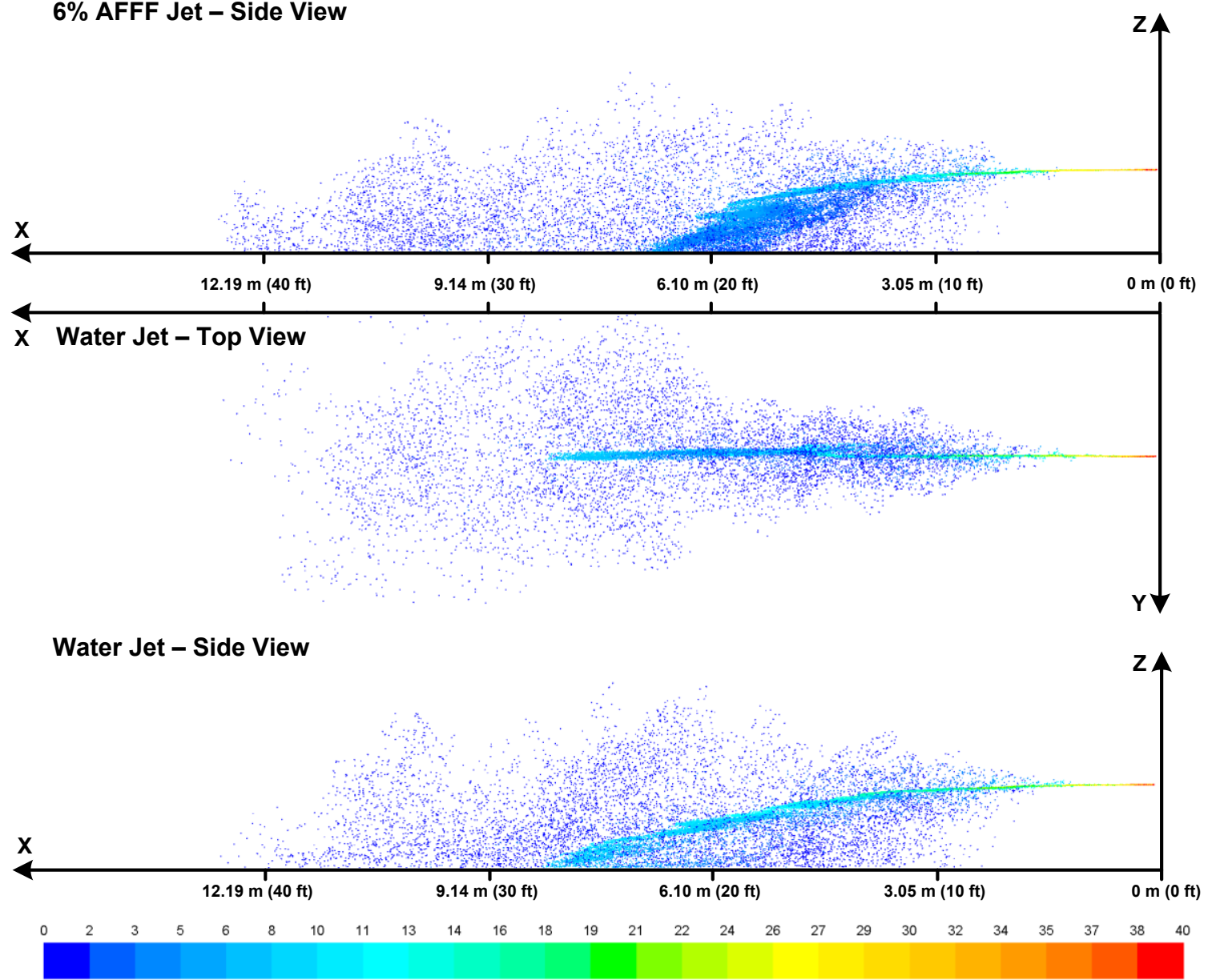

Particle Traces Colored by Velocity Magnitude $\left(\mathrm{m} \cdot \mathrm{s}^{-1}\right)$

Figure 6.80: CFD Model Results of Droplet Velocity Magnitude for Low Flow, Low Press. Jets 

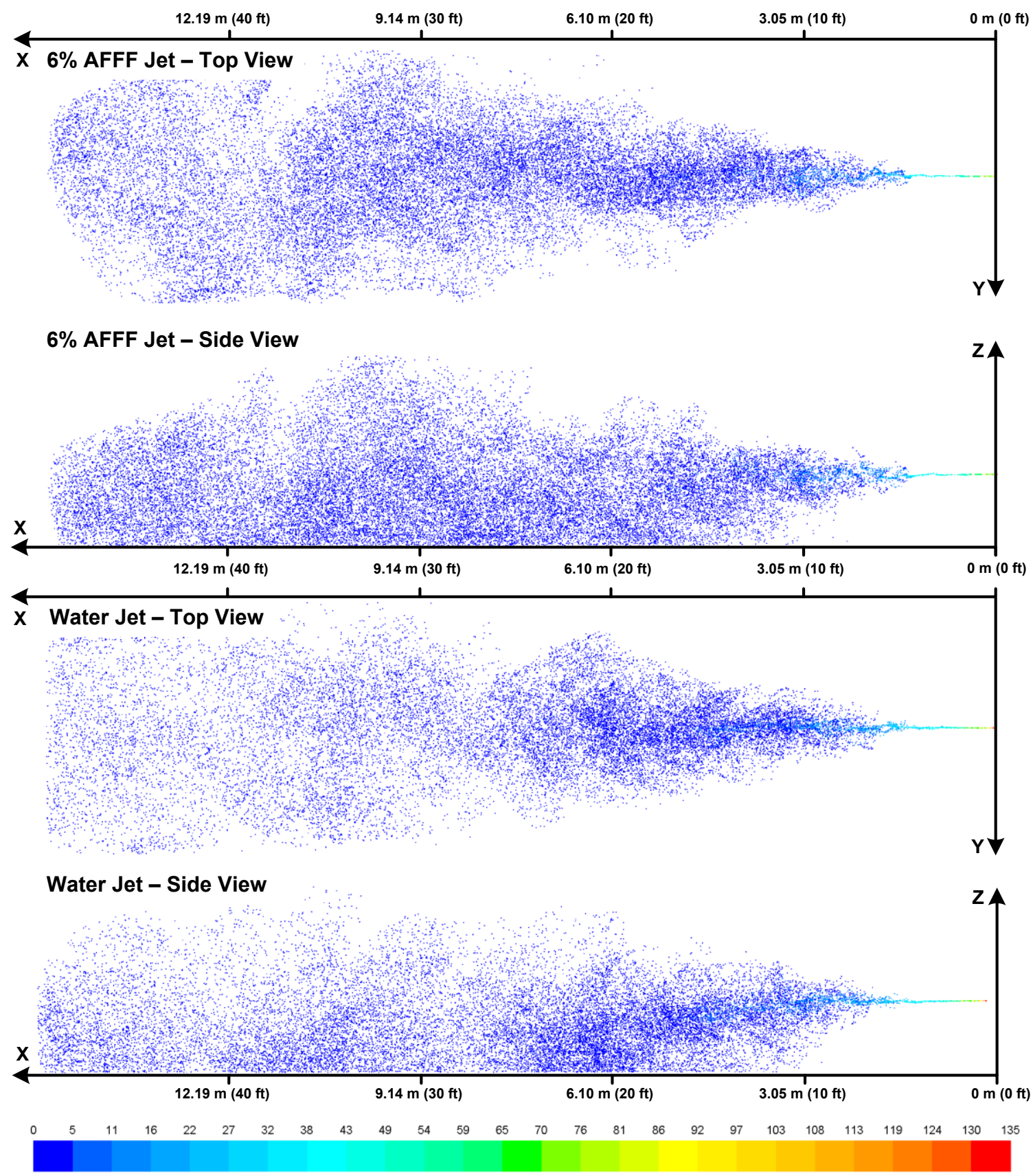

Particle Traces Colored by Velocity Magnitude $\left(\mathrm{m} \cdot \mathrm{s}^{-1}\right)$

Figure 6.81: CFD Model Results of Droplet Velocity Magnitude for Low Flow, High Press. Jets 

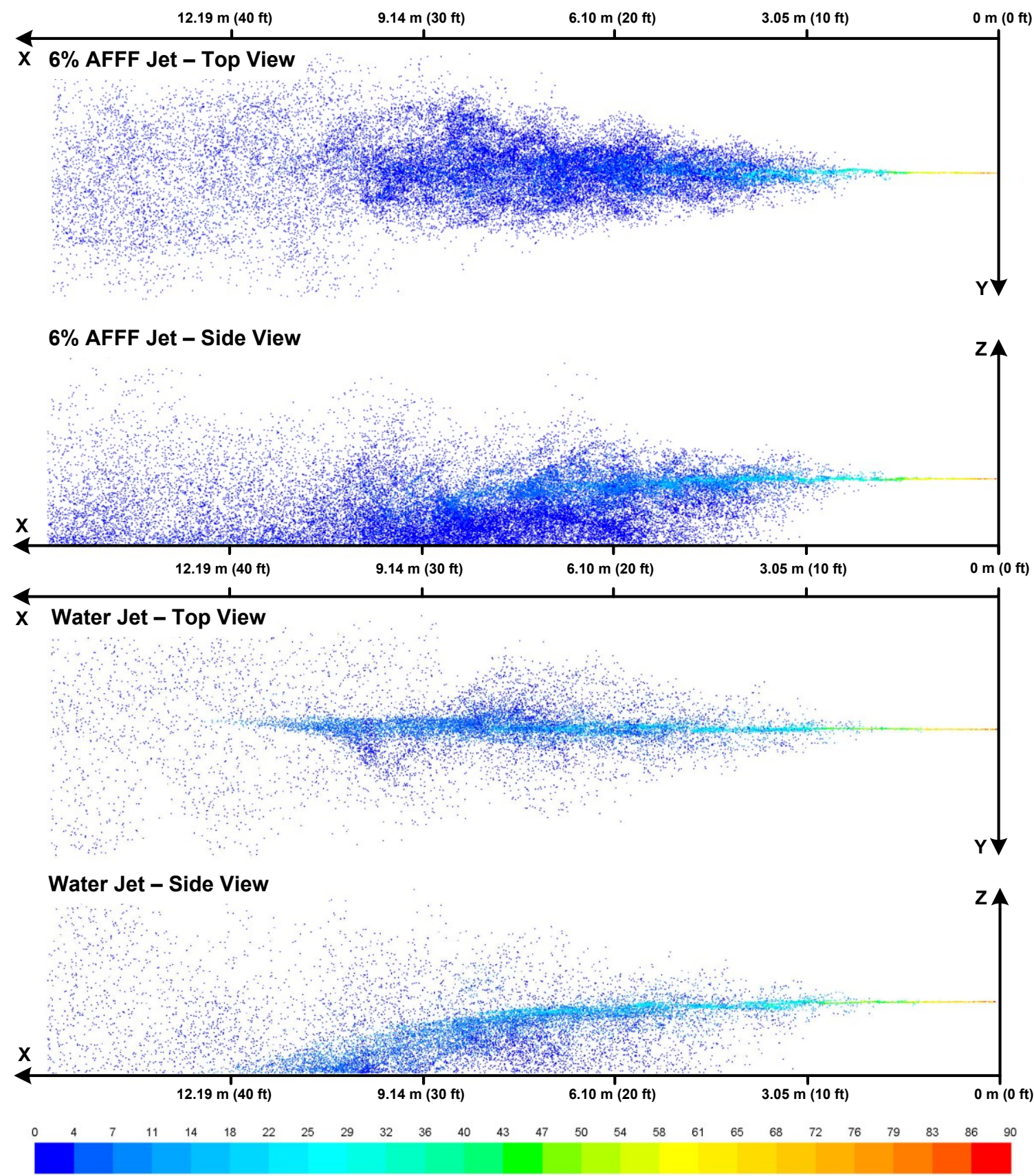

Particle Traces Colored by Velocity Magnitude $\left(\mathrm{m} \cdot \mathrm{s}^{-1}\right)$

Figure 6.82: CFD Model Results of Droplet Velocity Magnitude for Med. Flow, Med. Press. Jets 

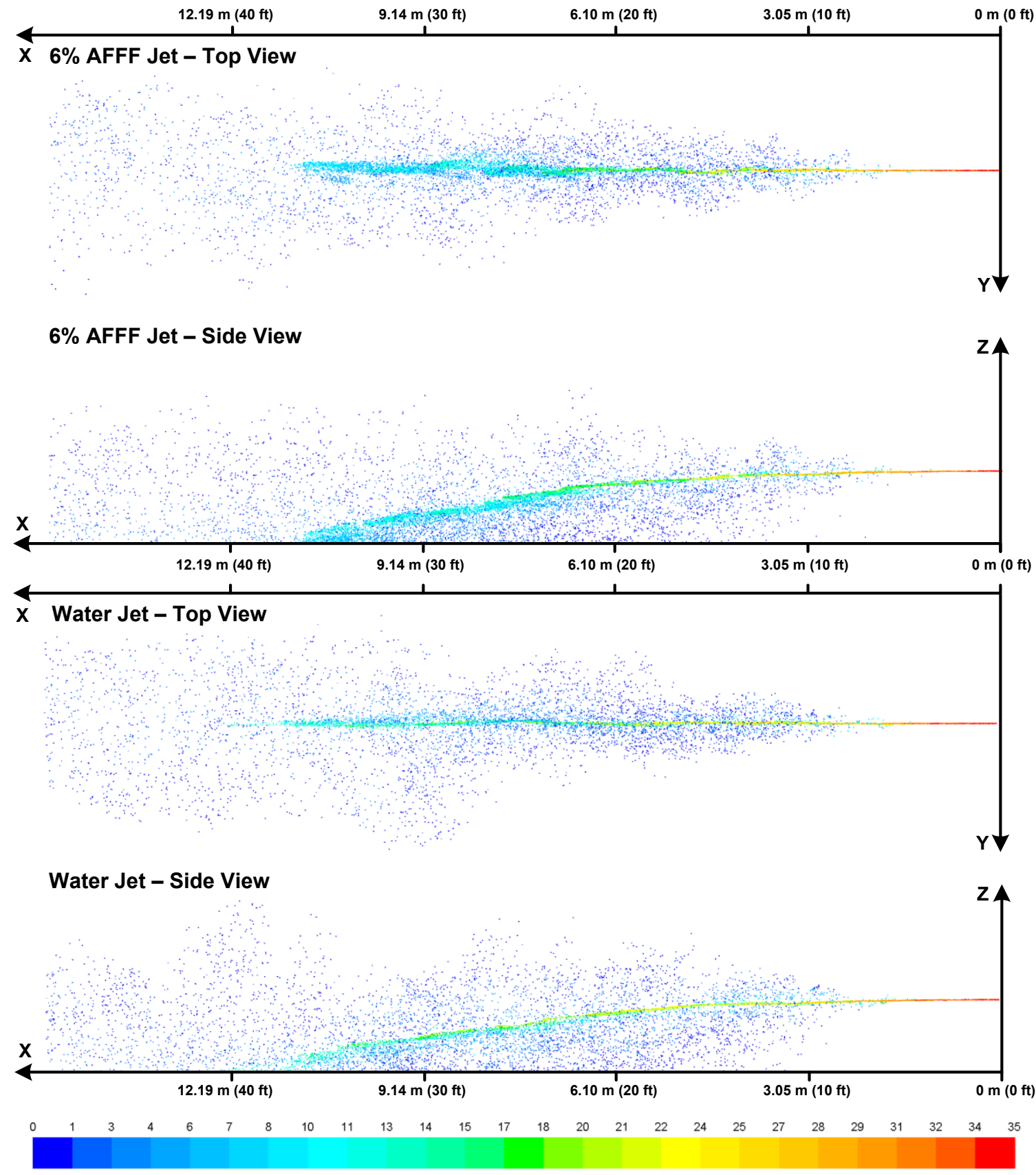

Particle Traces Colored by Velocity Magnitude $\left(\mathrm{m} \cdot \mathrm{s}^{-1}\right)$

Figure 6.83: CFD Model Results of Droplet Velocity Magnitude for High Flow, Low Press. Jets 


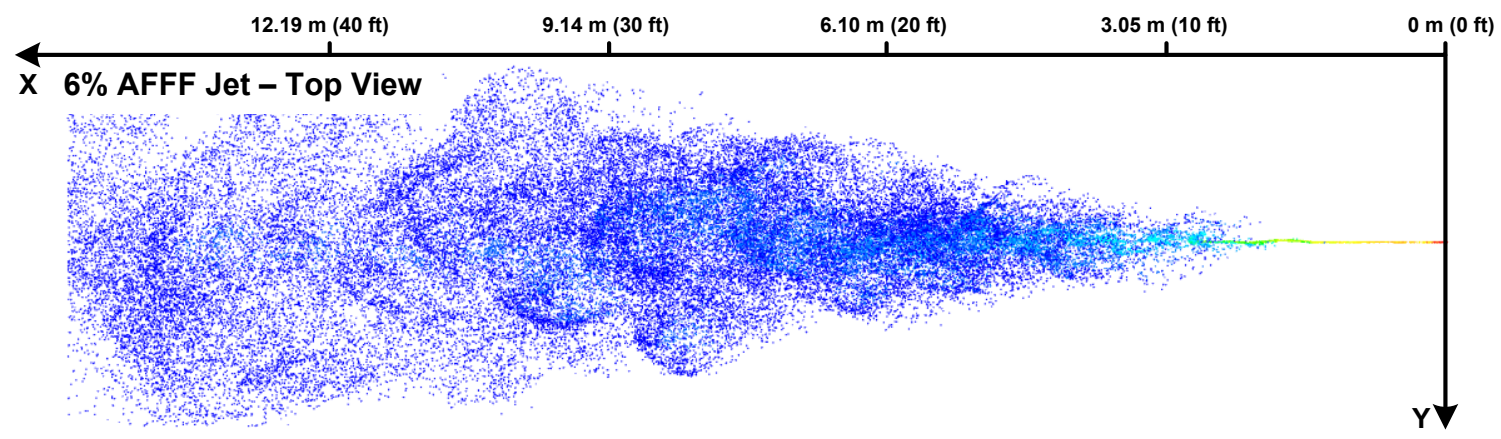

6\% AFFF Jet - Side View
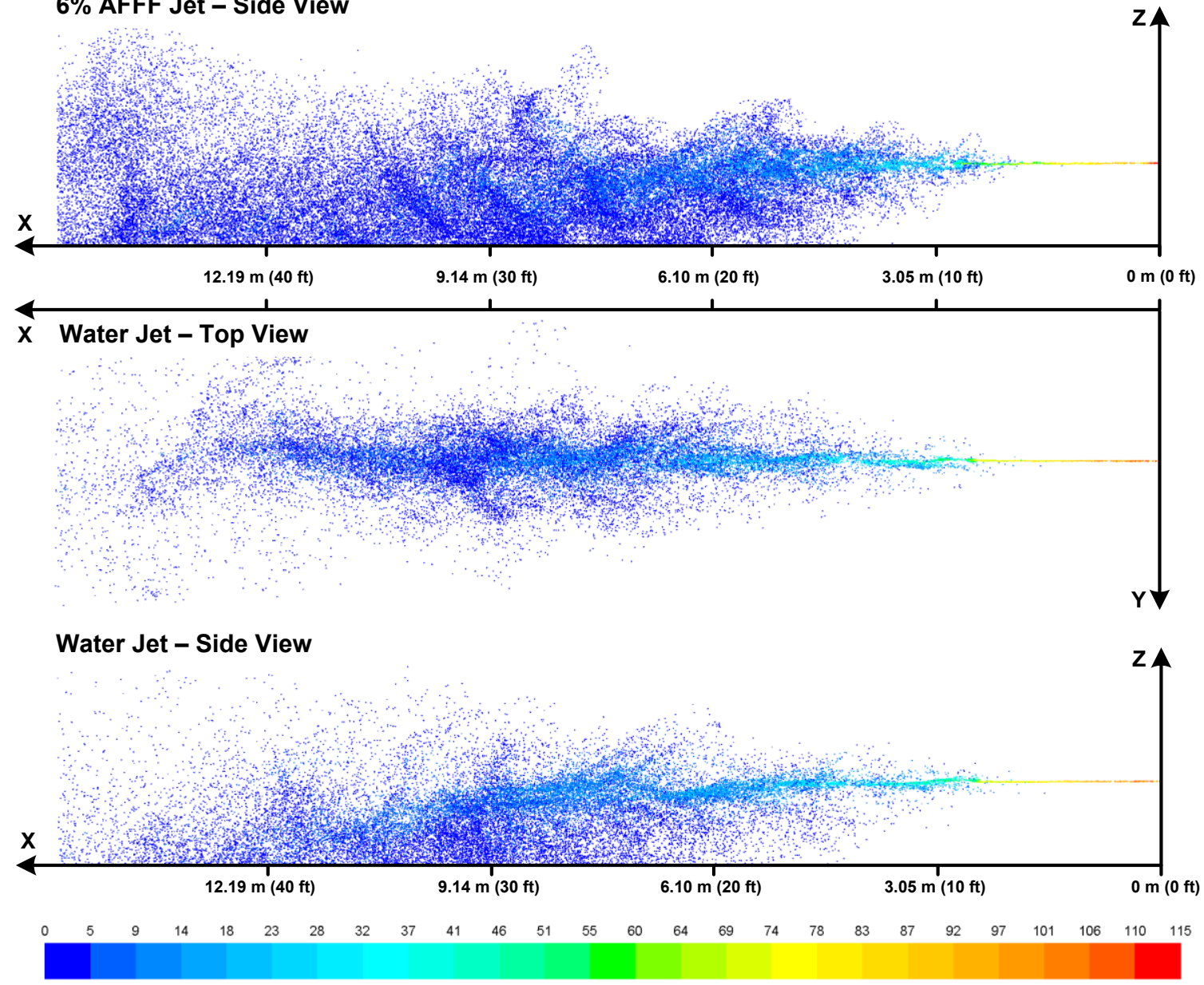

Droplets Colored by Velocity Magnitude $\left(\mathrm{m} \cdot \mathrm{s}^{-1}\right)$

Figure 6.84: CFD Model Results of Droplet Velocity Magnitude for High Flow, High Press. Jets 

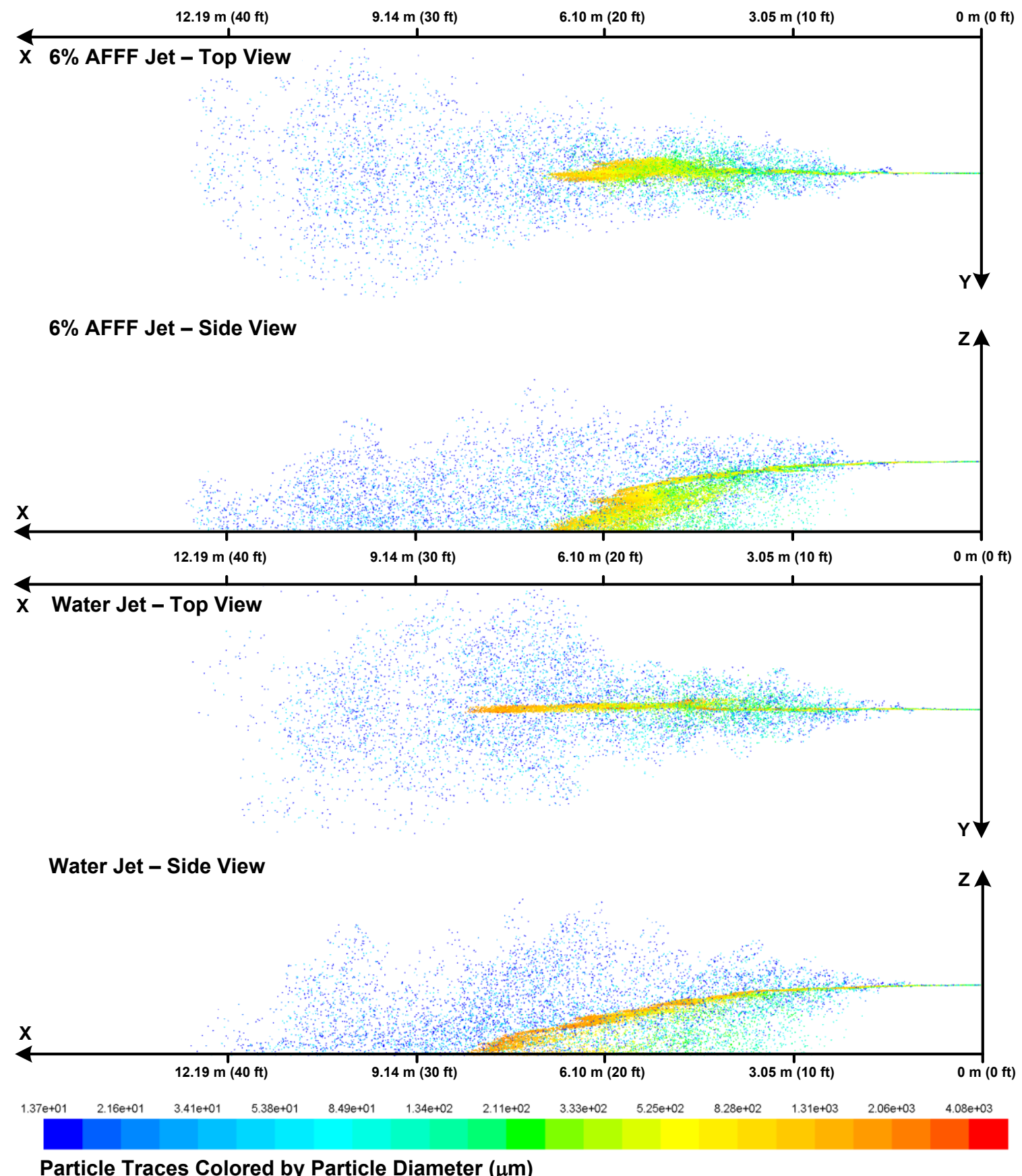

Particle Traces Colored by Particle Diameter $(\mu \mathrm{m})$

Figure 6.85: CFD Model Results of Droplet Diameter for Low Flow, Low Press. Jets 


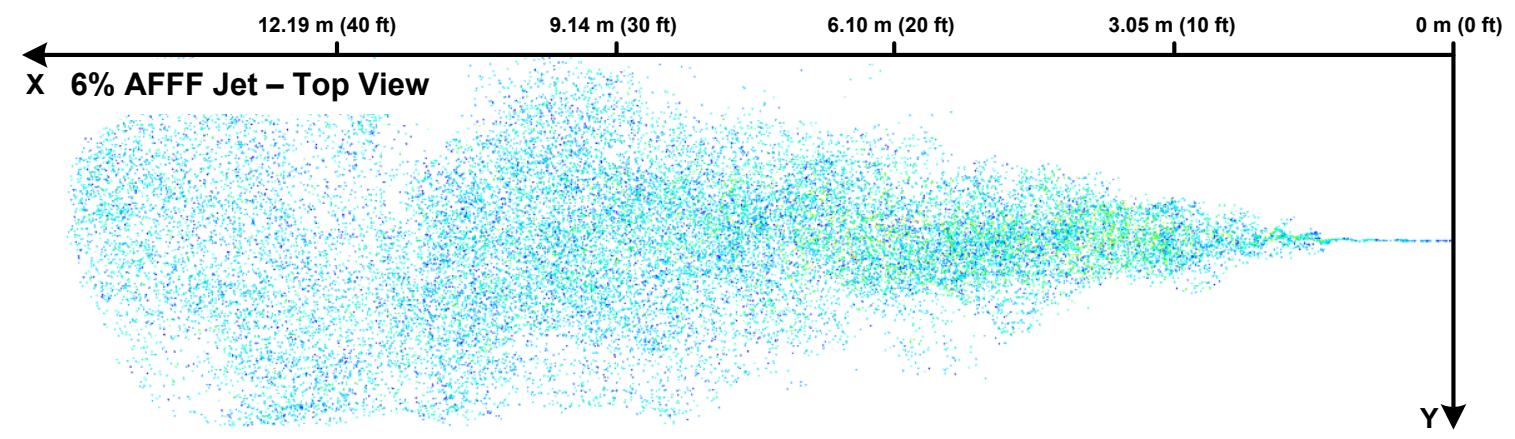

$6 \%$ AFFF Jet - Side View
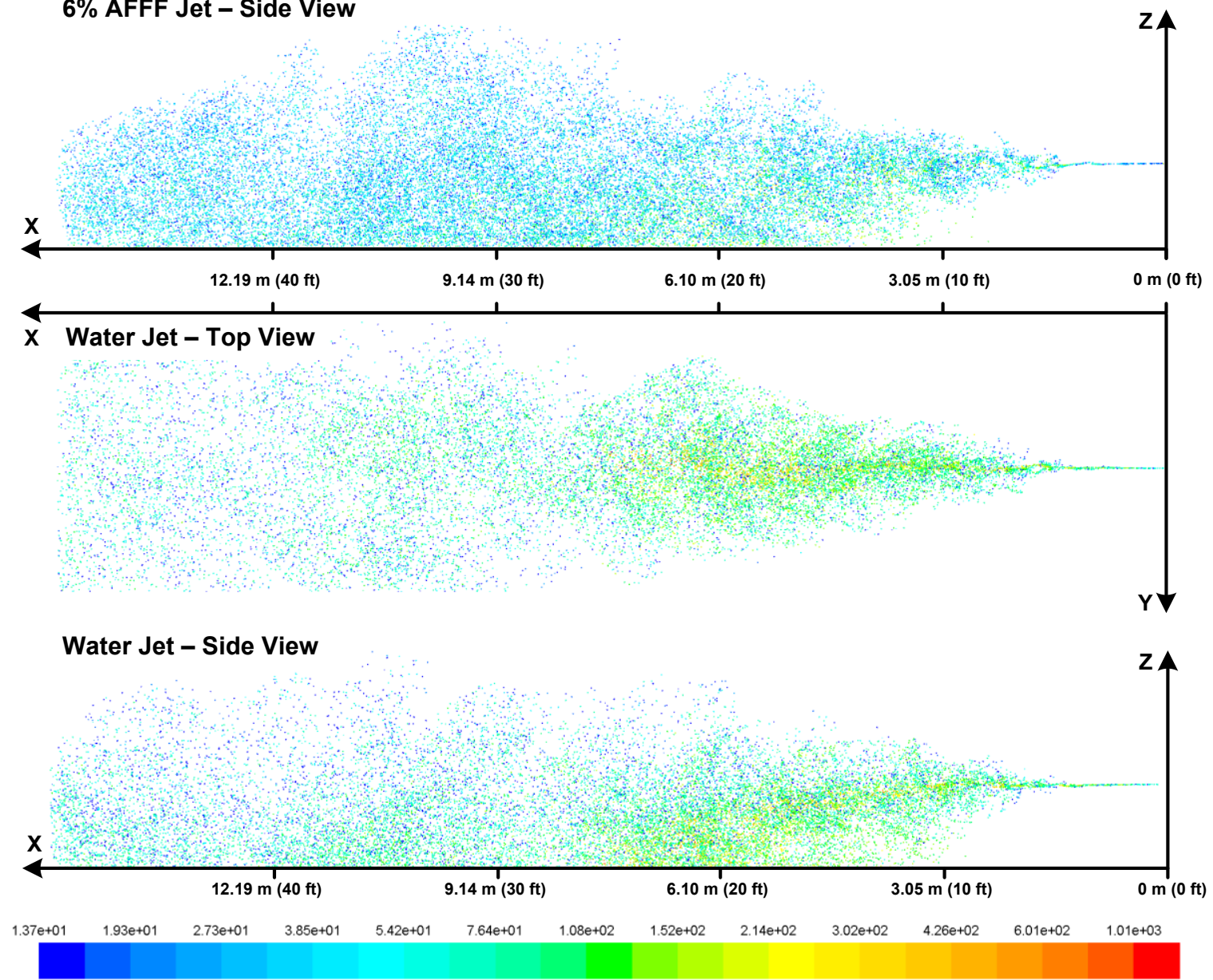

Particle Traces Colored by Particle Diameter $(\mu \mathrm{m})$

Figure 6.86: CFD Model Results of Droplet Diameter for Low Flow, High Press. Jets 


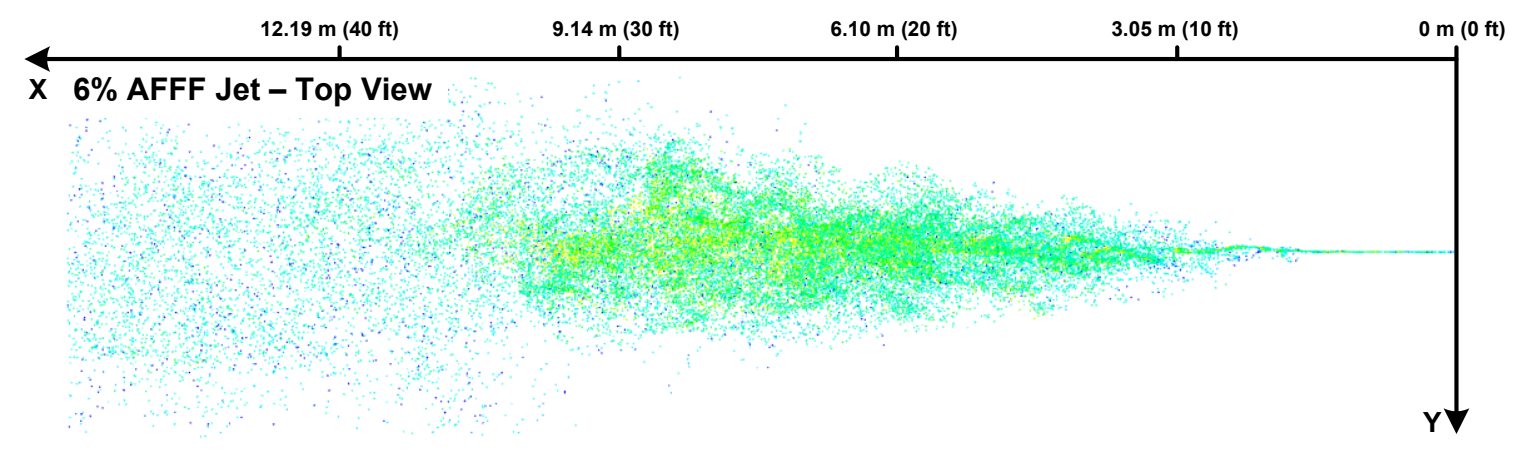

$6 \%$ AFFF Jet - Side View
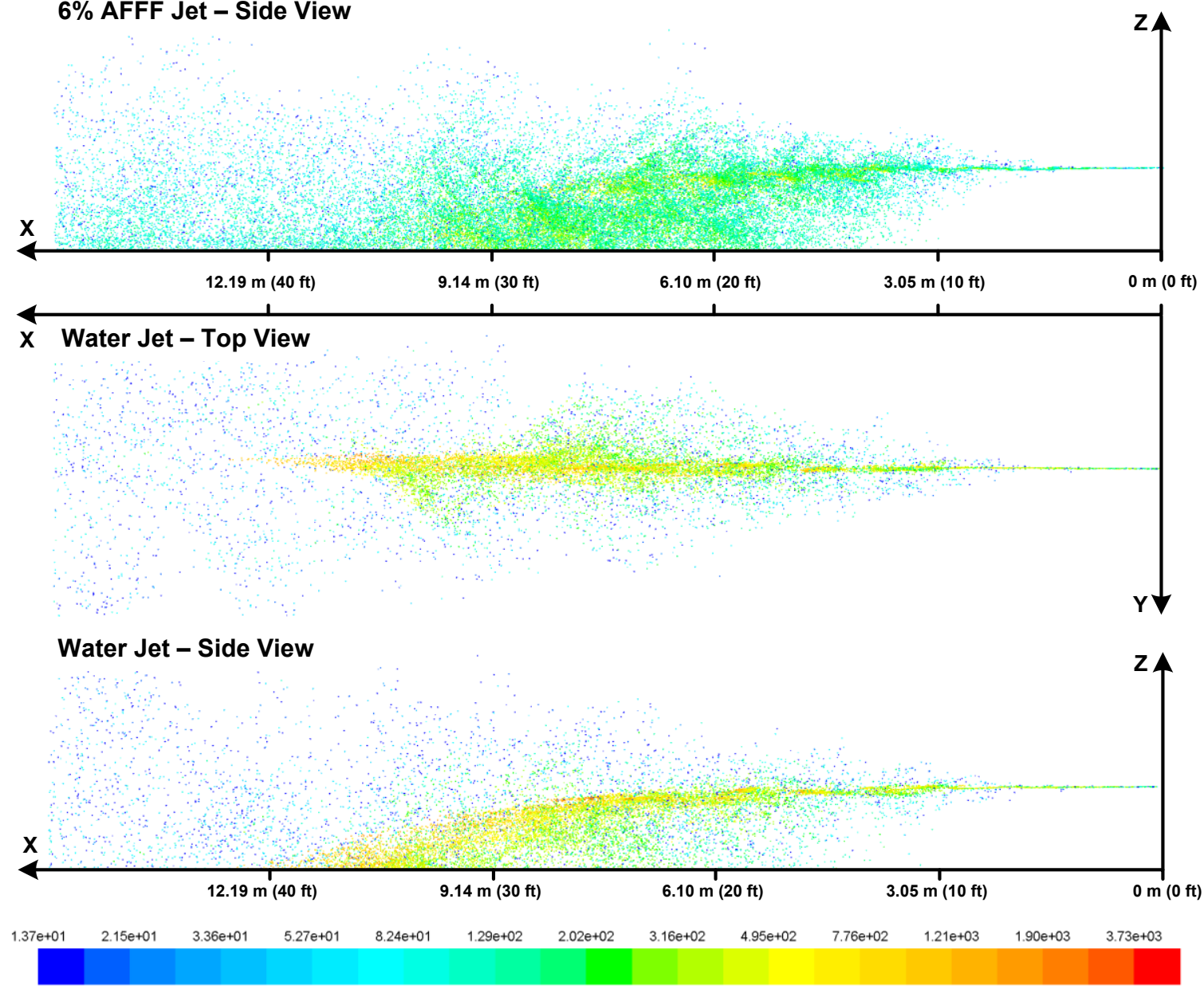

Particle Traces Colored by Particle Diameter $(\mu \mathrm{m})$

Figure 6.87: CFD Model Results of Droplet Diameter for Med. Flow, Med. Press. Jets 


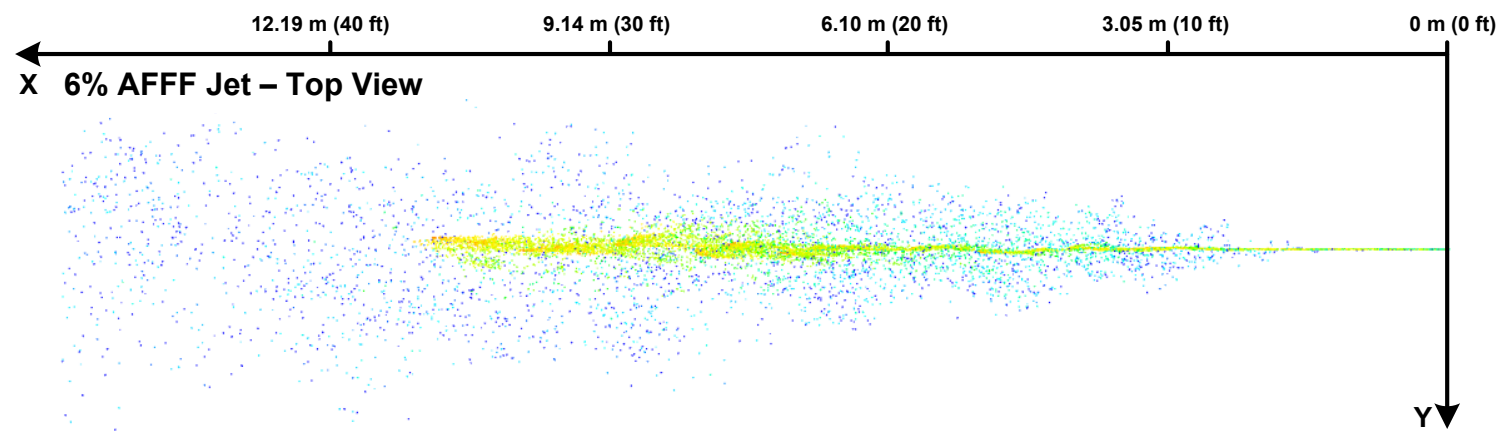

6\% AFFF Jet - Side View
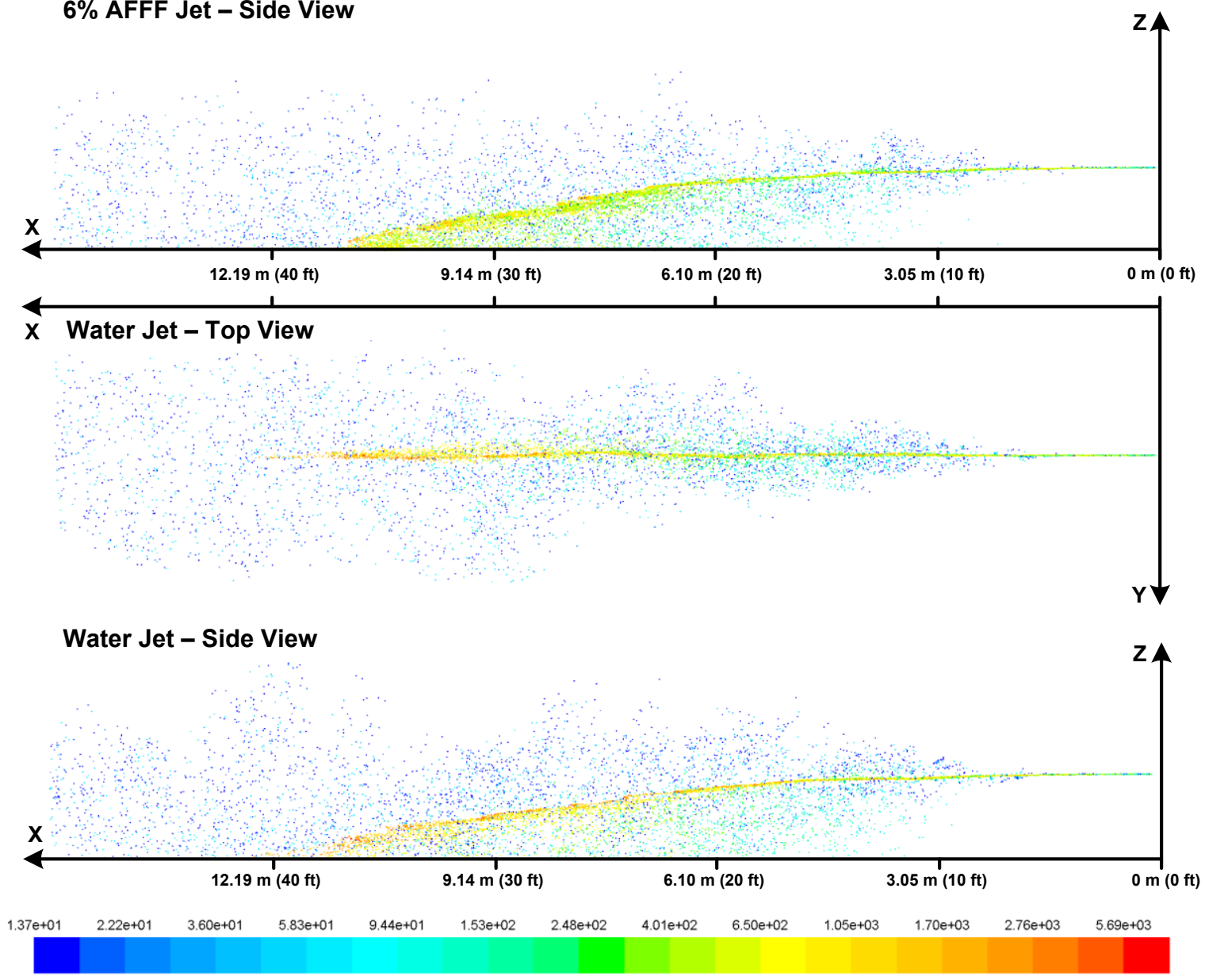

Particle Traces Colored by Particle Diameter $(\mu \mathrm{m})$

Figure 6.88: CFD Model Results of Droplet Diameter for High Flow, Low Press. Jets 

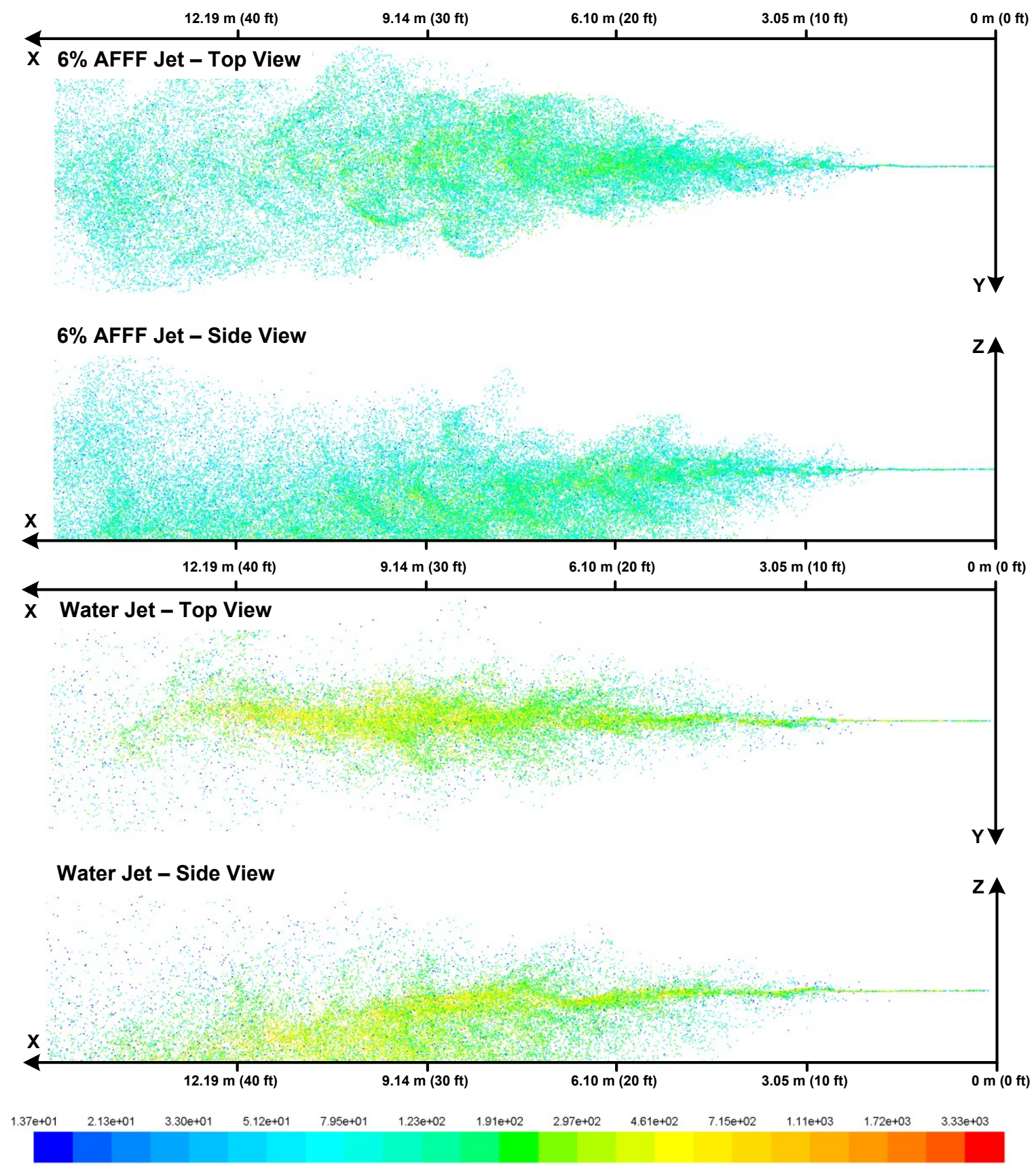

Particle Traces Colored by Particle Diameter $(\mu \mathrm{m})$

Figure 6.89: CFD Model Results of Droplet Diameter for High Flow, High Press. Jets 

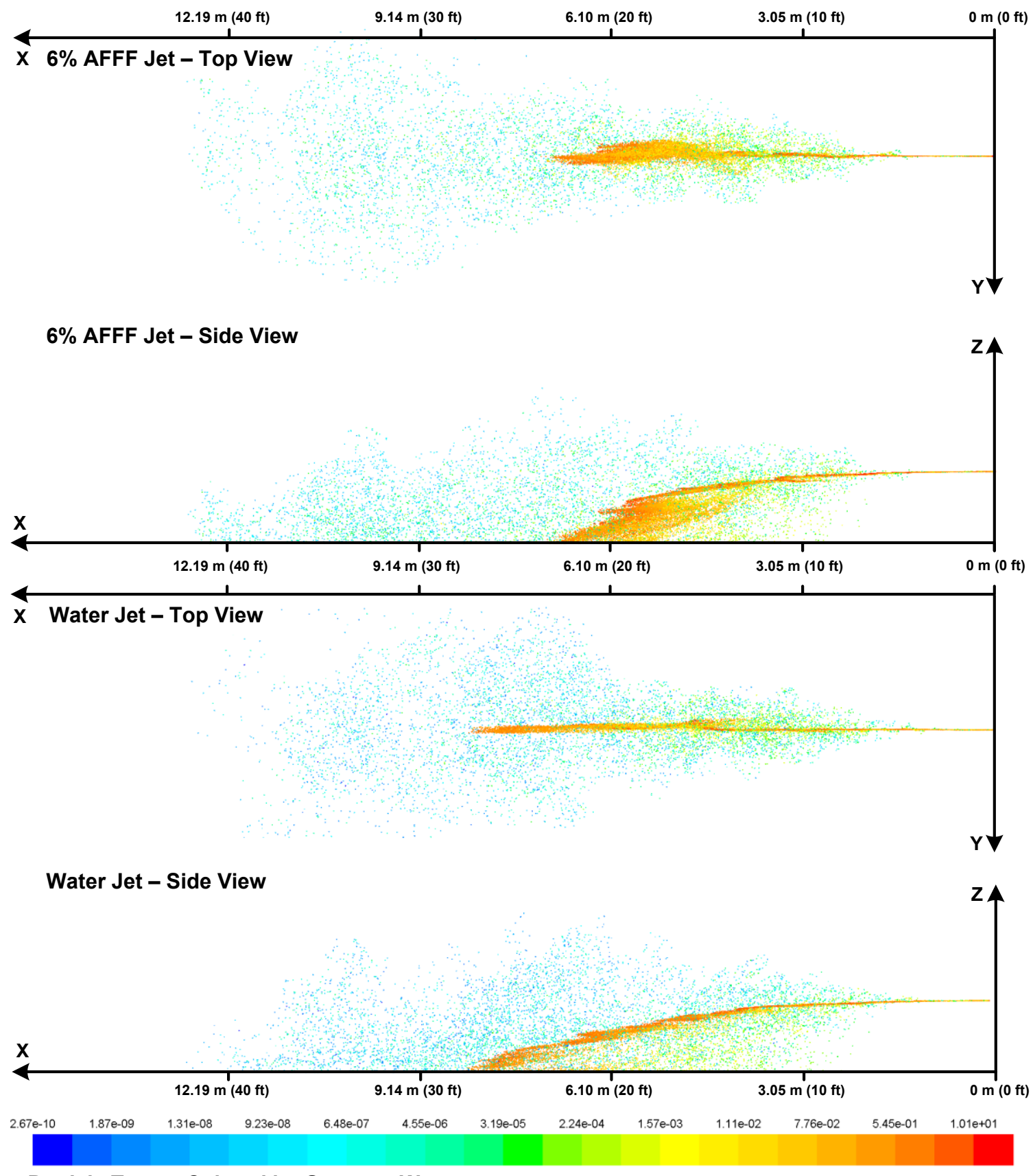

Particle Traces Colored by Gaseous We

Figure 6.90: CFD Model Results of Gaseous Weber No. for Low Flow, Low Press. Jets 

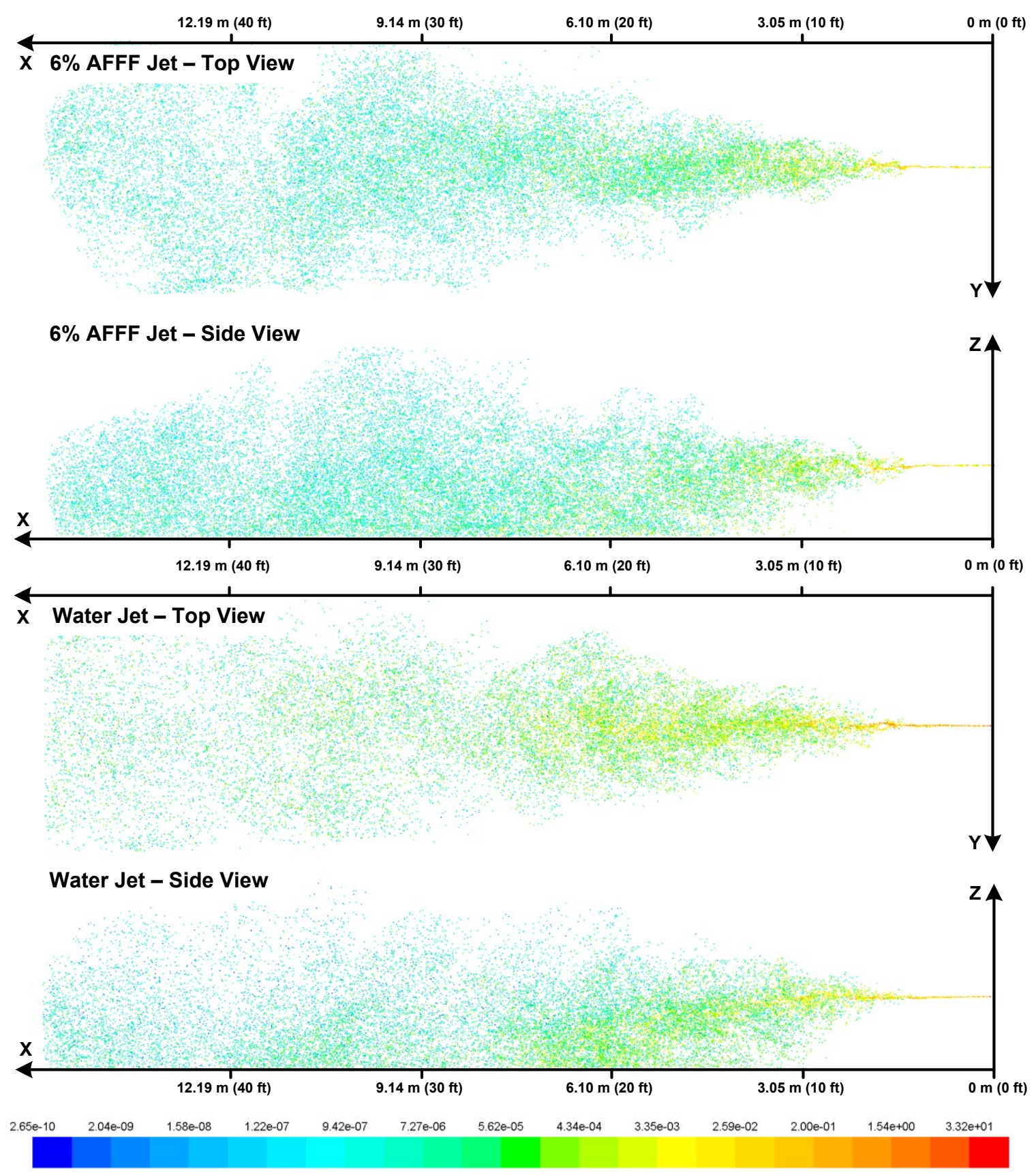

Particle Traces Colored by Gaseous We

Figure 6.91: CFD Model Results of Gaseous Weber No. for Low Flow, High Press. Jets 


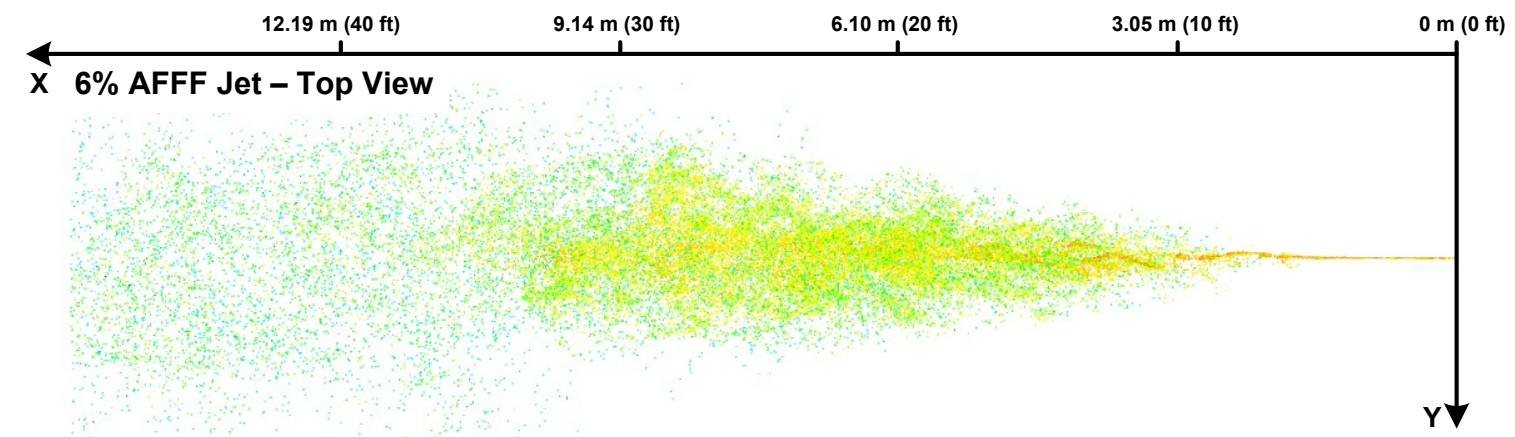

$6 \%$ AFFF Jet - Side View
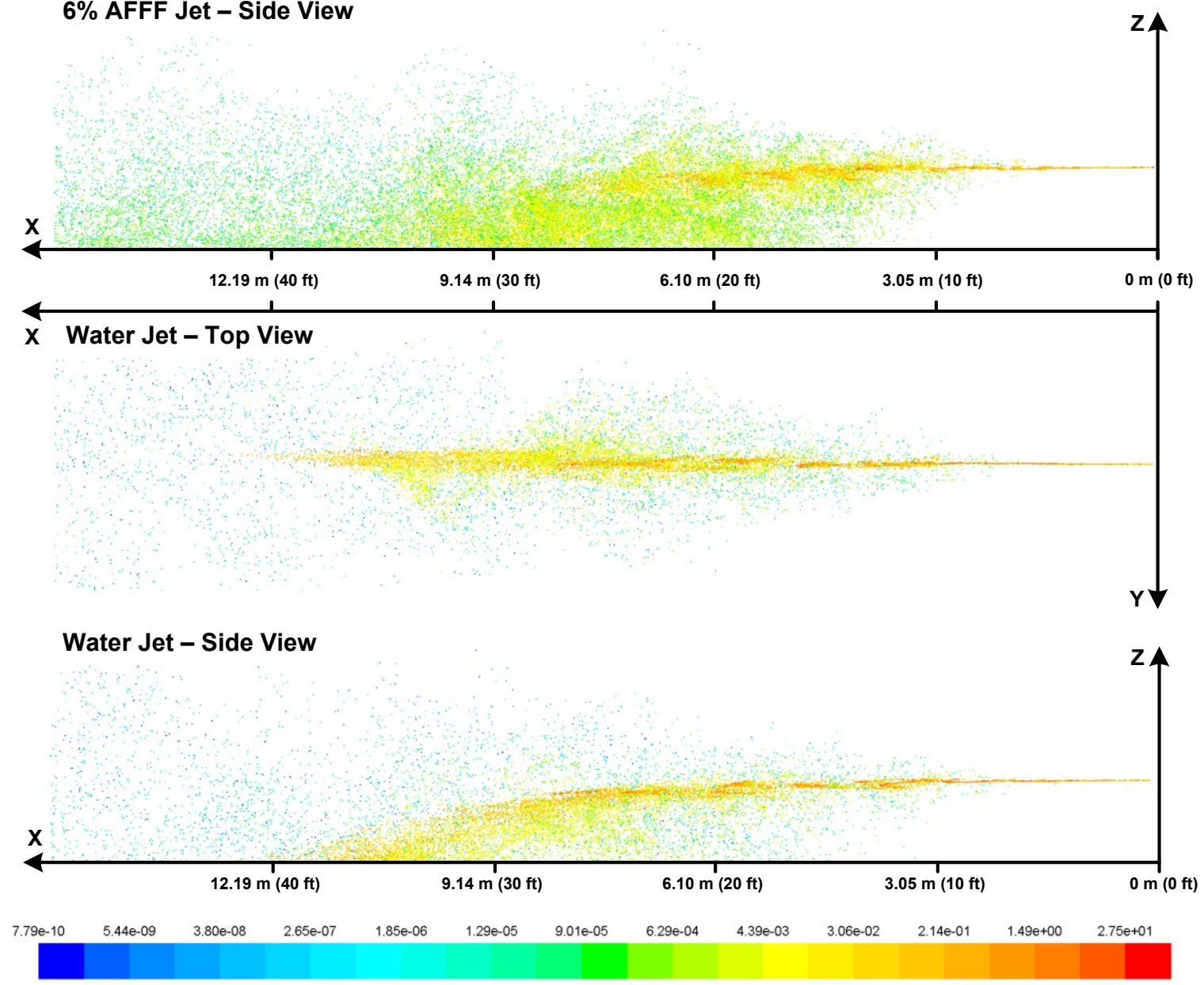

Particle Traces Colored by Gaseous We

Figure 6.92: CFD Model Results of Gaseous Weber No. for Med. Flow, Med. Press. Jets 

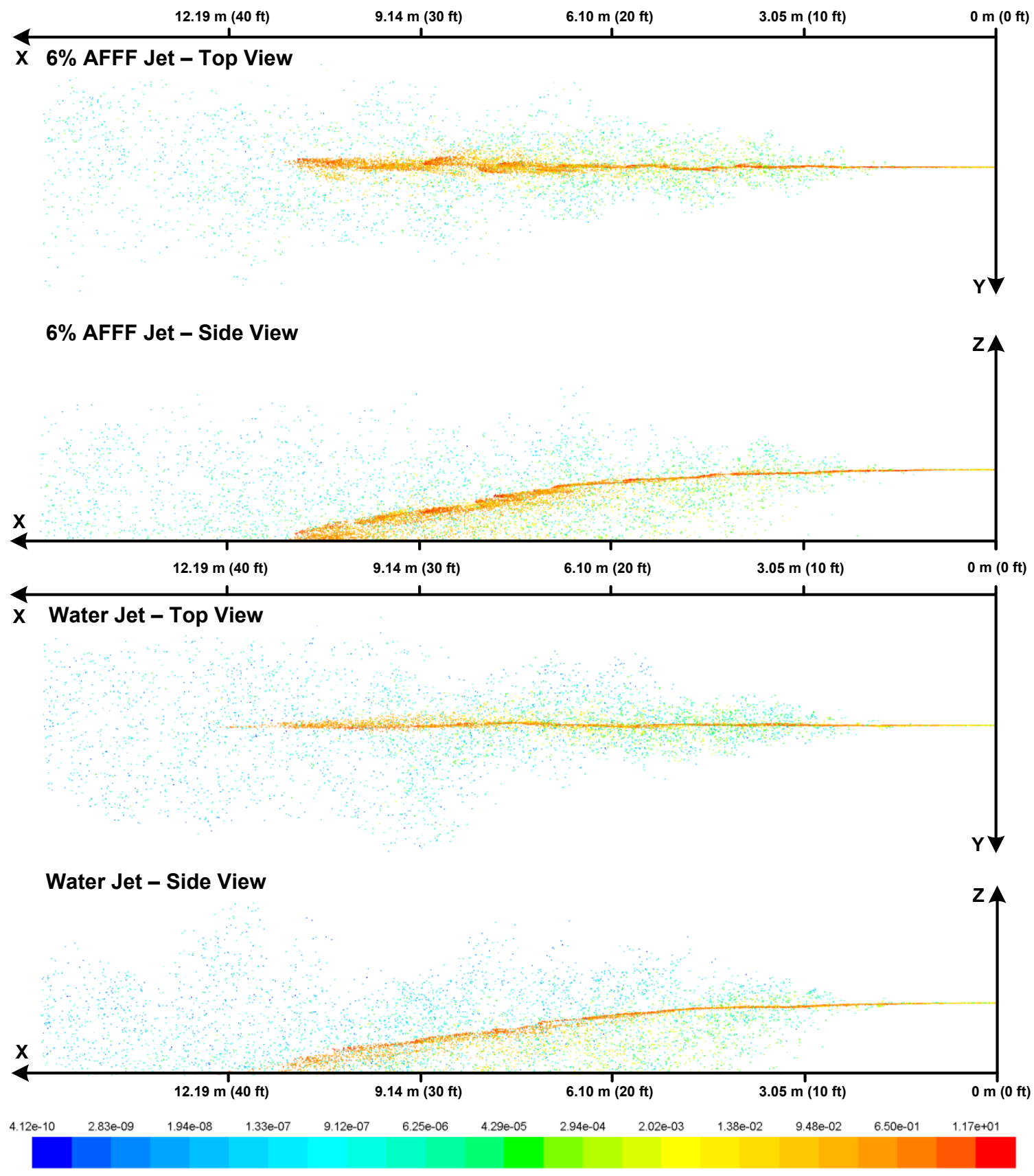

Particle Traces Colored by Gaseous We

Figure 6.93: CFD Model Results of Gaseous Weber No. for High Flow, Low Press. Jets 

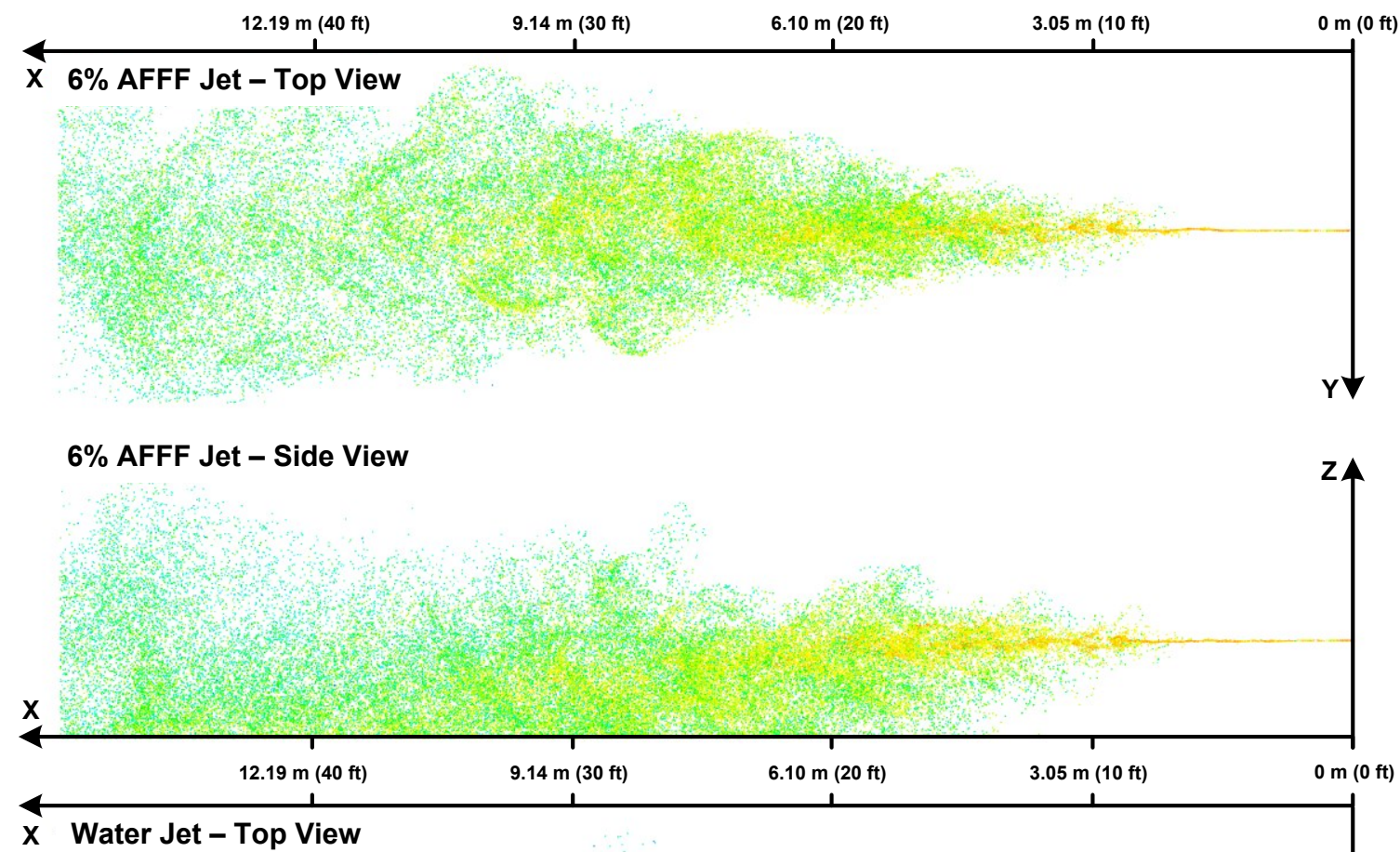

X Water Jet - Top View
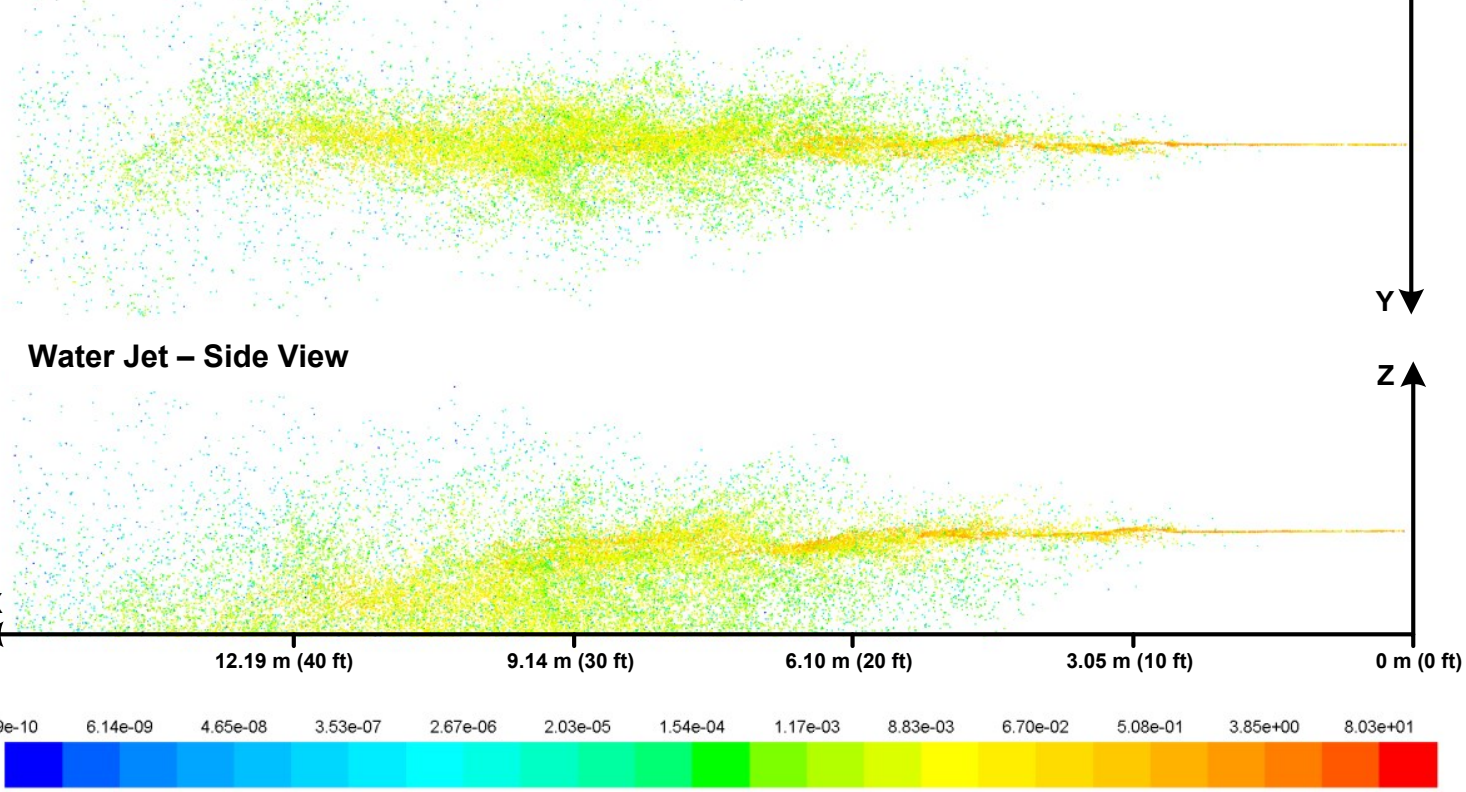

Particle Traces Colored by Gaseous We

Figure 6.94: CFD Model Results of Gaseous Weber No. for High Flow, High Press. Jets 


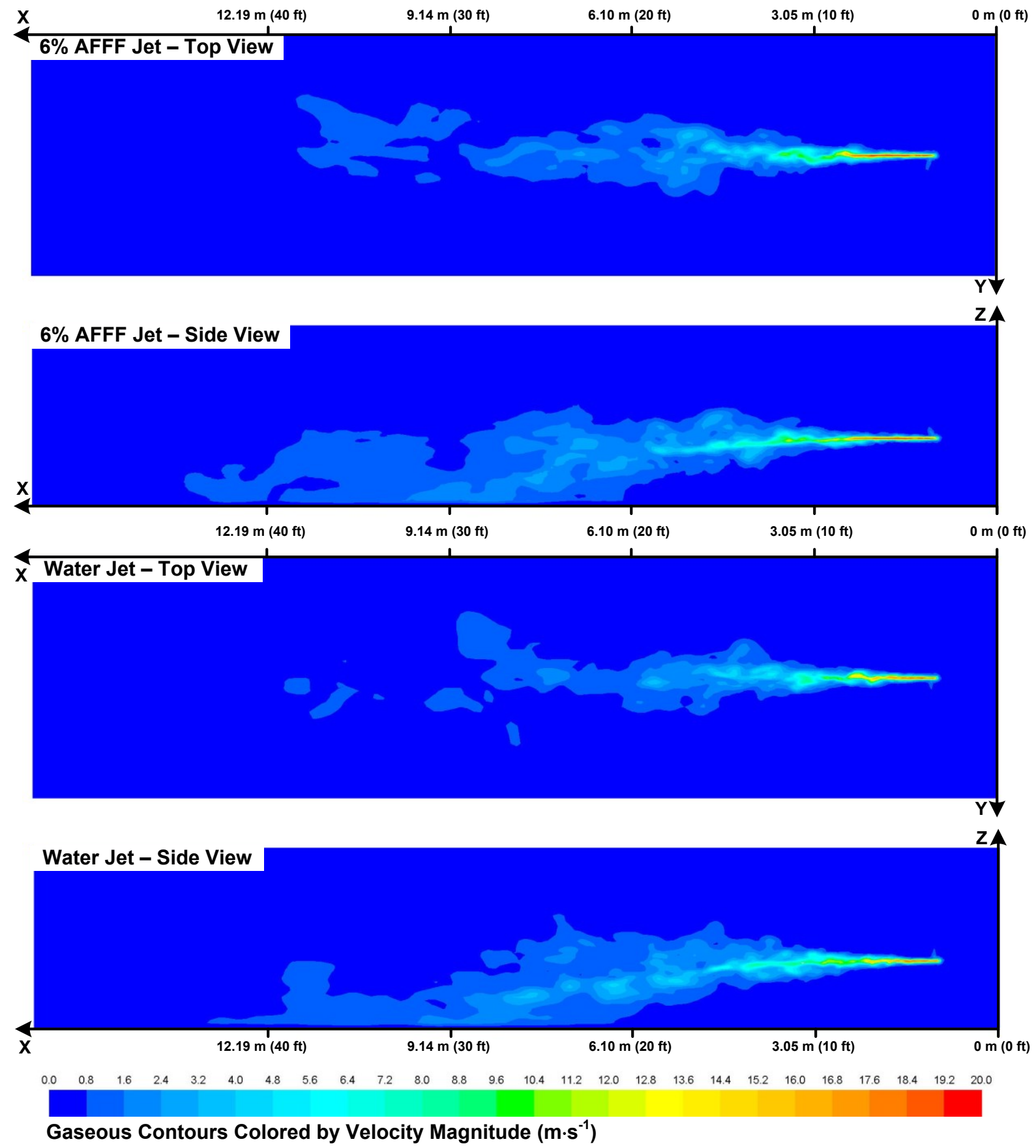

Figure 6.95: CFD Model Results of the Gaseous Continuous (Air) Phase Velocity Magnitude for Low Flow, Low Press. Jets 

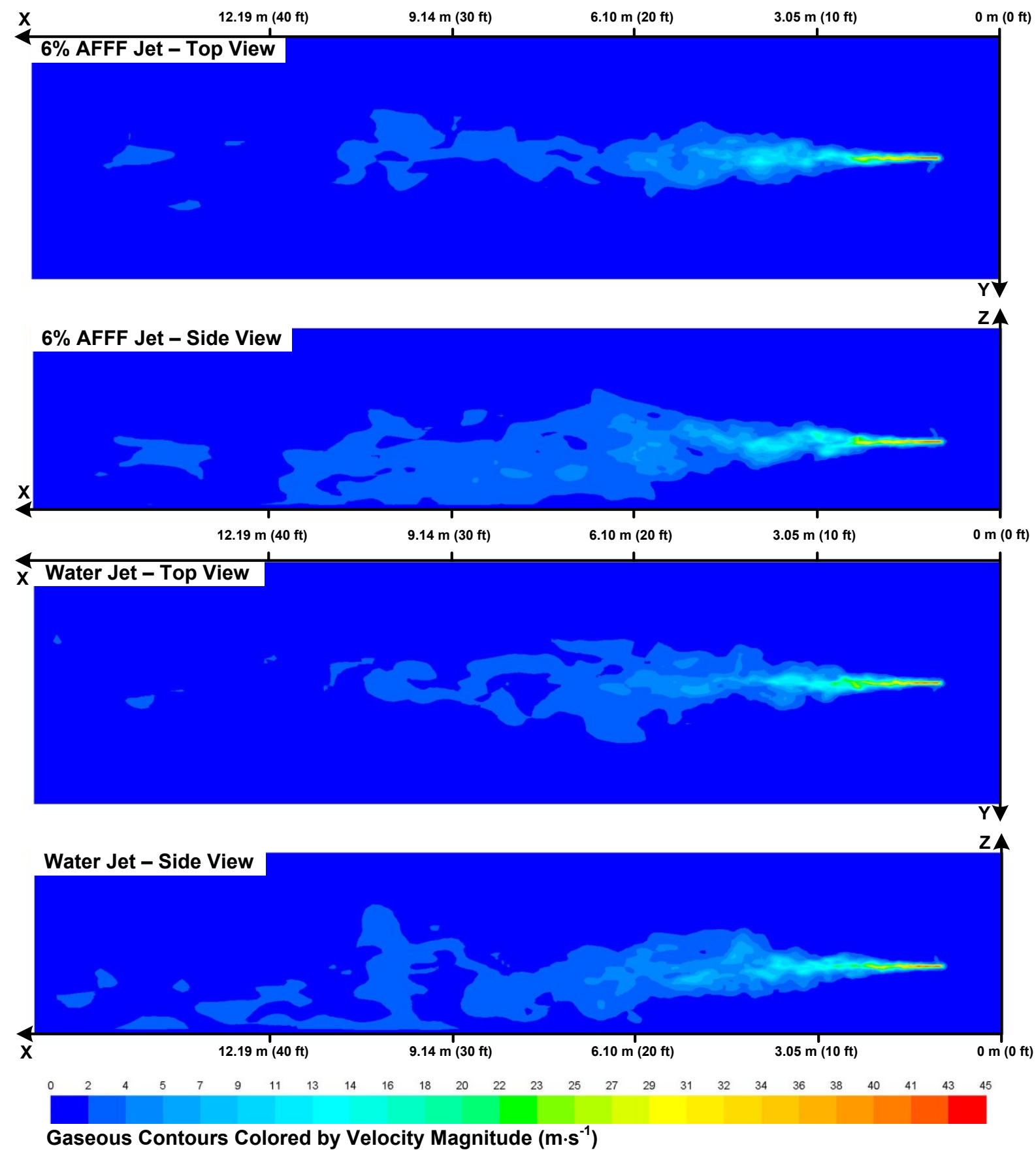

Figure 6.96: CFD Model Results of the Gaseous Continuous (Air) Phase Velocity Magnitude for Low Flow, High Press. Jets 

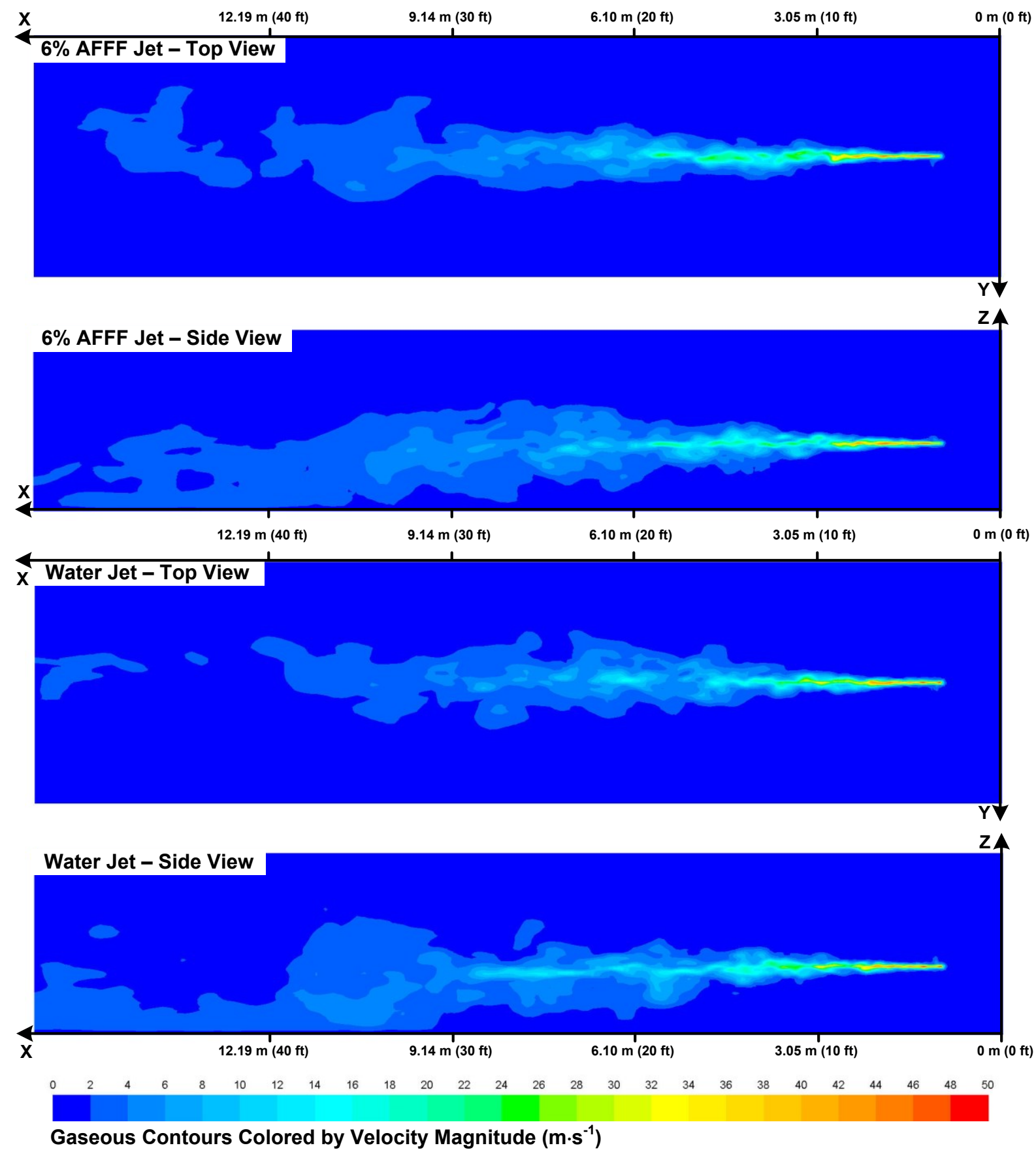

Figure 6.97: CFD Model Results of the Gaseous Continuous (Air) Phase Velocity Magnitude for Med. Flow, Med. Press Jets 

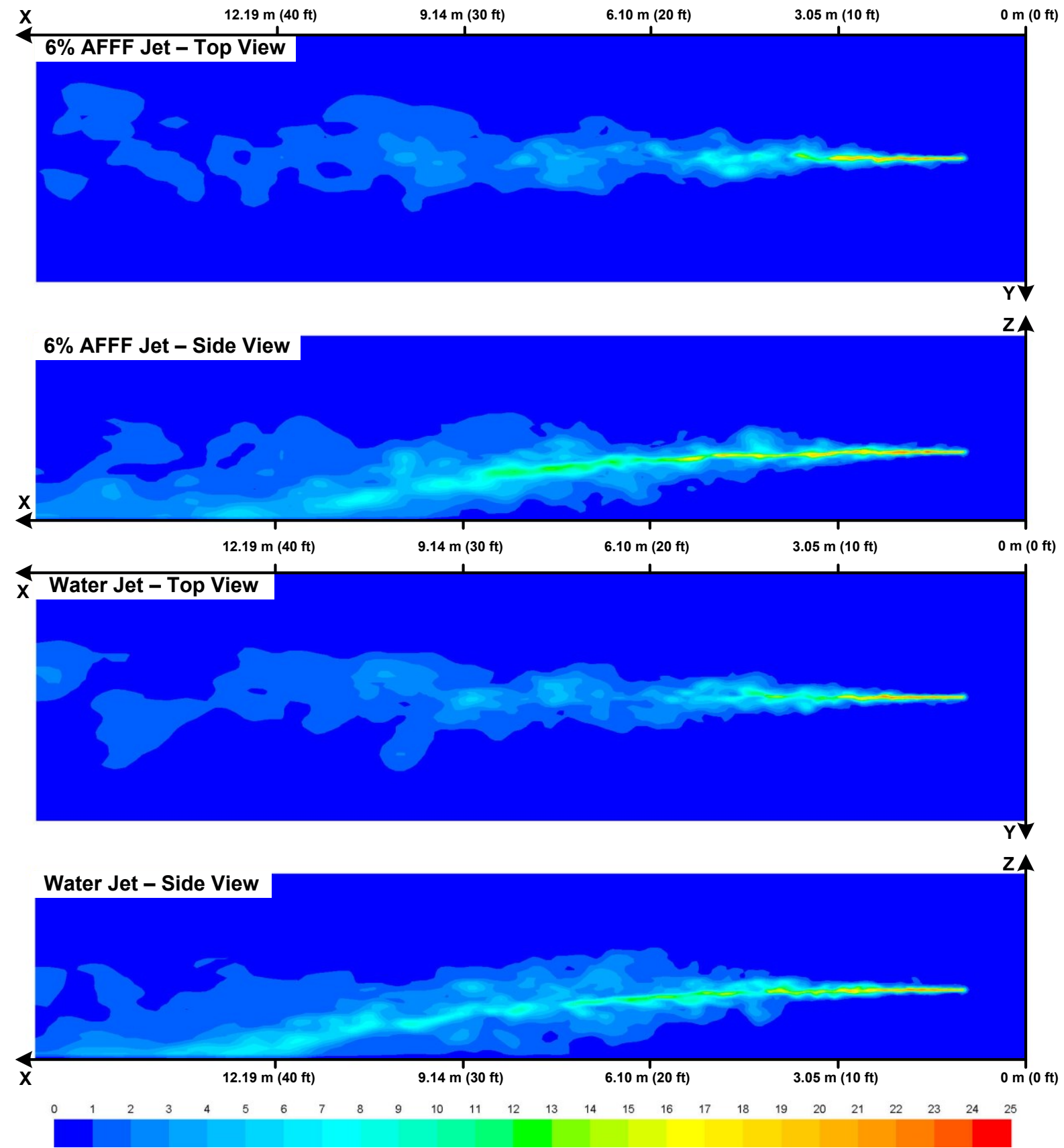

Gaseous Contours Colored by Velocity Magnitude $\left(\mathrm{m} \cdot \mathrm{s}^{-1}\right)$

Figure 6.98: CFD Model Results of the Gaseous Continuous (Air) Phase Velocity Magnitude for High Flow, Low Press. Jets 

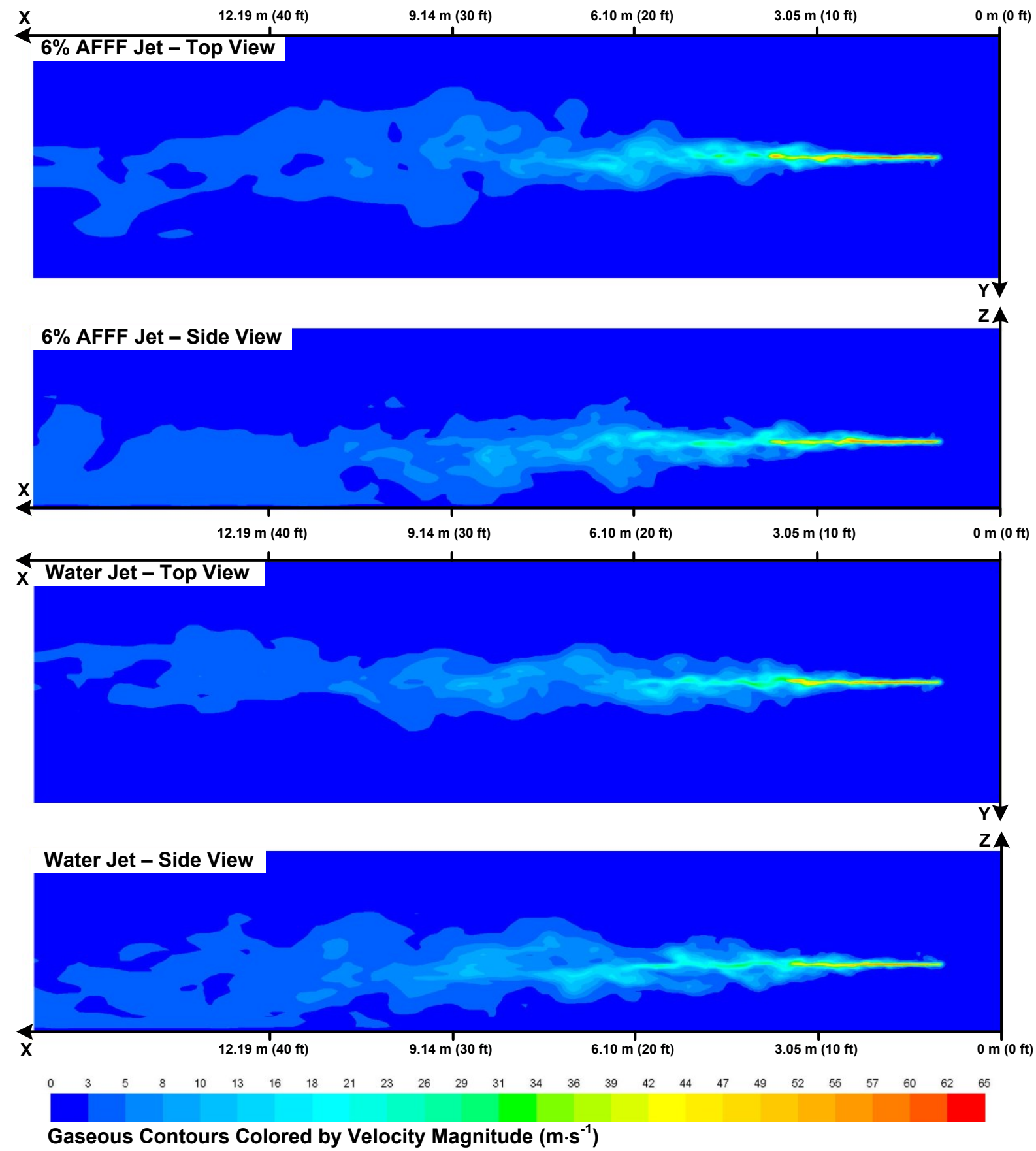

Figure 6.99: CFD Model Results of the Gaseous Continuous (Air) Phase Velocity Magnitude for High Flow, High Press. Jets 


\subsection{Overview}

All key experimental and computational research milestones defined in Section 1.3 were achieved. Construction of the aqueous firefighting agent application laboratory provided the unique infrastructure necessary to achieve all of the experimental tasks, and a CFD modeling strategy to predict the bulk in-flight flow characteristics of firefighting jets influenced by AFFF was completed. Although the foam expansion ratio for experiments fell below the NFPA recommended level of 5:1 for most applications, the AP4 nozzle family provided adequate distinction over the span of firefighting jet flow parameters analyzed to provide meaning and relevance to the results. Experimental flow visualization and PDPA provided qualitative as well as quantitative details, respectively, on the in-flight flow features that distinguish water and AFFF jets from one another. However, measurable change between the two agents was less significant than anticipated. CFD model results were able to reproduce the majority of flow trends observed in experiments, but comparisons were less agreeable. This was because of simplifications made in the modeling environment coupled with indirect comparisons between phase Doppler and CFD model data due to computational post-processing limitations.

In addition to the primary objectives, determining AFFF fluid properties in liquid solution form was of high interest as available information was previously sparse at best. It was important AFFF flow properties such as viscosity and surface tension were determined so that material properties were well defined for the CFD firefighting jet model. It was found that AFFF solution exhibits Newtonian fluid properties over the entire shear range of interest for use in firefighting agent delivery systems, a plausible outcome based on the mild flow behavior differences recorded from phase Doppler experiments between water and AFFF jets.

\subsection{Experimental Conclusions}

Most firefighting jet flow visualization showed unremarkable differences between water and AFFF jets in terms of global shape and structure. Enhanced photography methods provided better clarity compared to standard techniques, but fine, near field details of the entire pressureflow rate envelope was unable to be documented. This was due to restricted indoor lighting conditions, the physical scale and composition of the subject matter, and lack of a well-defined 
focal plane downstream in high interest areas of the firefighting jet. AFFF jets underwent enhanced break-up compared to water jets due to the surface tension-lowering effect AFFF surfactants had on firefighting jet droplets. This generated greater jet atomization and caused more light to scatter making AFFF jets appear more dense and fuller compared to water jets. Non-spherical shaped droplets and disks were also observed in several near field photographs. This was particularly evident with the high flow, low pressure AFFF jet where bubble-like formations were observed in the downstream wake region. All other AFFF jet pressure-flow rate combinations behaved similar to water jets by exhibiting discrete, spherical droplets more suited for PDPA data acquisition and more in-line with the simulation strategy employed for this study.

Agent ground pattern analysis related firefighting jet control factors like nozzle pressure, flow rate, and AFFF concentration to flow performance parameters like foam quality, ground coverage area, ground reach, and maximum ground span. Ground coverage area, reach, and maximum span demonstrated a mean, minor dependence on water versus AFFF jets. All flow performance parameters increased with an increase in flow rate. Ground coverage area benefited the most with a mean increase of $0.70 \mathrm{~m}^{2}$ per liter per minute increase in flow rate. Ground reach benefited with a mean increase of $21 \mathrm{~cm}$ per liter per minute increase in flow rate, and maximum ground span benefited the least with a mean increase of about $5 \mathrm{~cm}$ per liter per minute increase in flow rate. Flow performance parameters were aided by nozzle pressure by a similar effective range, except foam quality measured on the laboratory scale showed little dependency on either nozzle pressure or flow rate. However, full-scale agent ground pattern analysis showed definitive foam quality decline as nozzle pressure increased by as much as 50 percent from about 1 to $10 \mathrm{MPa}$ (150 to $1500 \mathrm{lbf} \cdot \mathrm{in}^{-2}$ ). Foam distribution from AFFF jets also closely tracked agent accumulation patterns from water jets demonstrating higher local agent volumes generated higher foam qualities. This observation was also supported by full-scale agent ground pattern results.

Firefighting jet mass conservation was inspected using data from the agent ground pattern analysis based on uniform agent ground accumulation. Results showed an over-shoot, or a net gain in mass, by as much as 8.58 percent to an under-shoot, or net loss in mass, by as much as 19.44 percent. Net losses were expected due to evaporation for most nozzle pressure-flow rate combinations, particularly for low flow, high pressure jets that exhibited greater atomization and 
exposed more droplet surface area to air. Net gains were anticipated for high flow, low pressure jets where most firefighting jet spray volume landed exclusively in agent capture devices placed along the jet containment bed centerline. Averaging the mass balance across all firefighting jet cases resulted in a mean net loss of 4.4 percent. In general, this indicated droplet evaporation was a factor, albeit not a very significant one.

Although PDPA had its limitations in terms of discrete point measurements, spherical particle assumptions, and possessing a finite droplet diameter measurement range, most flow fields generated a favorable environment for the data acquisition process to be effective. All firefighting jets exhibited log-normal droplet distribution behavior consistent with pressure atomizer nozzles, and the Nukiyama-Tanasawa method provided the best fit for all nozzle pressure-flow rate combinations. RMS droplet diameters consistently reflected a diameter spread of the same order of magnitude as the mean droplet diameter. PDPA reported AFFF jet, mean droplet diameters universally lower compared to water jet, mean droplet diameters by 25 to 100 percent in the effective range of the jet. However, the difference in mean axial droplet velocity profile measurements was minor with AFFF jets slightly lagging water jets - a result due to momentum loss from the transport of smaller, less massive AFFF droplet due to enhanced diffusion and greater turbulent mixing. For some firefighting jet profiles the velocity lag measured was close to or equal to zero, and in others the differential was as much as 10 percent. All firefighting jets exhibited large RMS velocity fluctuations generating turbulence intensities in several cases on the order of the mean velocity resulting in a turbulent flow classification for all flow regimes considered. Sauter mean droplet diameters followed trends consistent with mean droplet diameter measurements with relative size off-sets between AFFF and water droplets also in the range of 25 to 100 percent. However, inconsistencies were reported for a minority of nozzle settings due to extensive scatter observed in a few individual profile measurements, particularly downstream in the far reaches of each jet. The PDPA system may have recorded invalid, non-spherical, and/or bubble-like spray formations in these locations resulting in a misrepresentative, Sauter mean diameter calculation. Because Sauter mean diameter measurements weight the presence of large droplets significantly more than smaller droplets, a very few (e.g. 10 or less in a sample field of 50,000) droplets recorded along the upper threshold of the PDPA measurement envelope were capable of altering the Sauter mean diameter measurement on the order of ten percent of more. PDPA data on the order of 500,000 
samples would have to be recorded to provide good statistical convergence for this particular type of data, which was impractical due to the expense of AFFF testing.

Phase Doppler and agent ground pattern data showed unique responses to variation in AFFF solution concentration. The only flow parameter measured by the PDPA that exhibited a dependence on AFFF solution concentration was mean droplet diameter where a mean 12-percent magnitude reduction was measured between 3-percent and 6-percent AFFF. Above 6-percent AFFF, measured phase Doppler velocities and droplet sizes reported a mean negligible change of less than 1 percent. This outcome is plausible as AFFF compositions are designed for peak performance based on a particular water dilution level. Although this data were not collected for all nozzle settings, these results are likely indicative of all pressure-flow rate combinations. However, it is reasonable to assume that high flow, AFFF jets with relatively larger initial droplets would exhibit greater sensitivity to AFFF concentration as a wider range of droplet break-up would incur before reaching a potential low-end droplet size threshold. For agent ground pattern analysis, foam quality increased quasi-linearly proportional with AFFF concentration up to the highest tested concentration of 12-percent AFFF. The AFFF jet foam expansion ratio proportionally increased by about 25 percent per 3-percent increase in AFFF solution concentration. This suggests AFFF influence is more apparent in the presence of continuous or semi-continuous liquids with larger surface areas where surface tension forces dominate compared to the aerodynamic forces imparted on in-flight droplets. These results also suggest a more complex relationship between AFFF concentration diffusion rates and droplet size, or perhaps a loss in PDPA measurement accuracy due to the physical changes undergone by droplets at high AFFF concentration levels in terms of shape and refractive index properties.

\subsection{Computational Firefighting Jet Flow Modeling Conclusions}

A firefighting jet CFD flow modeling strategy was developed based on liquid jet break-up classification from the literature, basic knowledge of the firefighting agent delivery system and nozzle, and droplet size distribution information pertaining to the nozzle near field exit region from phase Doppler measurements. Qualitative CFD model results reproduced firefighting jet trajectory, reach, span, and spread well when compared to flow visualization results. CFD model flow parameter results compared better to phase Doppler results in regions further downstream in contrast to the nozzle near field due to an over-simplification of the DPM nozzle injection 181 
condition. It only accounted for the axial component of velocity in terms of a constant value at a single point in space. Nozzle perturbations and turbulent droplet dispersion was not accounted for at the injection point and thus firefighting jet characteristics were dependent on downstream droplet-air interactions to provide progressively more plausible flow physics as the jet flowed downstream. Despite these inconsistencies, the CFD model showed reasonable agreement with phase Doppler results in terms of overall flow parameter trends, magnitudes, and the influence of AFFF. The employment of a constant, model equilibrium surface tension value for AFFF increased droplet break-up which was consistent with measurements made by the PDPA. Most mean profile, axial and vertical droplet velocity CFD model and phase Doppler result comparisons were within 10 percent of one another with a minority of points deviating by as much as 50 percent. Mean droplet diameter and Sauter mean droplet diameter comparisons exhibited greater divergence with most data within 25 percent, with a minority of points differing by as much as 100 percent. The majority of CFD droplet diameters were reportedly larger than phase Doppler droplet diameters because model evaporation was neglected. Further discrepancy between phase Doppler and CFD model results was likely due to a multitude of factors, including differences in droplet size and window range reporting, droplet shape restrictions, possible alignment inconsistency between experiments and the model environment, and dropletair interactions unaccounted for in the physical sub-models. Examples of other errors induced by physical CFD sub-models include isotropic turbulence assumptions, 3-D liquid jet ligaments simulated as solid, discrete droplets (particles), and droplet parcel fidelity limited by the efficiency of numerical solution techniques and computational resources. CFD model droplets were also not allowed to directly interact with the non-permeable surfaces such as the modeled laboratory floor or firefighting jet containment zone. Their presence was only accounted for in part via the continuous (air) phase. The ANSYS Fluent ${ }^{\circledR}$ DPM simulation strategy performs best for firefighting jets that do not have a continuous liquid core and experience enhanced atomization either due to lower flow rates, higher pressures, or a combination thereof based on the modeling assumptions employed. For firefighting jets with a substantial, contiguous liquid core, alternative modeling methods such as dense discrete phase modeling must be considered.

This work describes the first known, comprehensive effort to quantify flow characteristics and properties that differentiate water and AFFF firefighting jets using high-fidelity experimental techniques. This work also includes the first known iteration of a firefighting agent application 
CFD model designed for use in the ARFF industry that takes into account the influence of AFFF. Data collected from this study can be used to develop future, more eco-friendly alternatives to AFFF, optimize current AFFF compositions and application techniques for greater effectiveness, and aid the on-going construction of a simulation framework to provide scientifically-based risk assessment for aircraft-crash-fire suppression scenarios of interest.

\subsection{Recommendations for Future Work}

A knowledge gap was revealed on the physical understanding of firefighting jet flow mechanics in the near field of surfaces, particularly for AFFF jets in terms of agent deposition and foam growth patterns. Near field experimental flow characterization of small-scale firefighting jets interacting with surfaces is needed to resolve fine details of this phenomenon. Progress in this field of study would also help evolve future generations of a firefighting agent application CFD model where wall contact can be considered for eventual use within aircraft combustion models where interaction with engulfed aircraft structures is necessary.

Upgraded laboratory lighting equipment is needed to properly resolve the flow details of high pressure firefighting jets. Little is still known about the qualitative shape of firefighting jet droplets over the majority of the pressure-flow rate regimes examined. Better optimized flow visualization techniques to remedy issues already mentioned with the present work in conjunction with enhanced video image processing may lead to a more precise quantification of droplet shapes and fluid dynamics.

Flow characterization of droplet size distributions on full-scale firefighting jet flow rates should be conducted to determine how well laboratory results scale for all flow parameters of interest. Current PDPA optics can either be modified to record a larger droplet diameter range, or alternative spray analysis methods could be employed that is more conducive to a full-scale test environment.

Follow-on generations of the firefighting jet CFD model should define the DPM injection condition with higher fidelity exploring methods to resolve nozzle transience, upstream nozzle flow turbulence, multi-component velocity, and the 2-D nozzle exit area shape. Alternative turbulence models better equipped to handle droplet-air interactions should be considered, as 
well as AFFF droplet-surface interactions where little information exists. Although the time scales associated with most firefighting jet transport processes are on the order of $10 \mathrm{~s}$ or much less, defining AFFF surface tension as a function of time should also be considered - particularly in the case of UHP firefighting jets where effective jet core droplet lifetimes are approximately $1 \mathrm{~s}$. Other improvements include thermodynamic and heat transfer effects to account for droplet evaporation and eventual firefighting jet interaction with aircraft fire scenarios. 
1. D.W. Fittes, D.J. Griffiths, and P. Nash, The Use of Light Water for Major Aircraft Fires, Fire Technology, vol. 5, no. 4, pp. 284-298, 1969.

2. M.A. Enlow, D.S. Dierdorf, and R. Campbell, Time Dependent Analysis of Hydrocarbon and Fluorocarbon Surfactant Solutions, AFRL Report AFRL-RX-TY-TR-2010-0075, Air Force Research Laboratory, Tyndall AFB, FL, 2011.

3. C.S. Grove, G.E. Wise, W.C. Marsh, and J.B. Gray, Viscosity of Fire-Fighting Foam, Journal of Industrial \& Engineering Chemistry, vol. 43, no. 5, pp. 1120-1122, 1951.

4. R.L. Gipe and H.B. Peterson, Proportioning Characteristics of Aqueous Film-Forming Foam Concentrates, NRL Report 7437, Naval Research Laboratory, Washington, D.C., 1972.

5. G.B. Geyer, Firefighting Effectiveness of Aqueous Film-Forming Foam (AFFF) Agents, Technical Report ASD-TR-73-13, National Aviation Facilities Experimental Center, Atlantic City, NJ, 1973.

6. D.E. Elliott and P.J. Chiesa, Jr., A New Foam Rheometer for Studying Fire Fighting Foams, Fire Technology, vol. 12, no. 1, pp. 66-69, 1976.

7. D.E. Elliott and P.J. Chiesa, Jr., Rheological Properties of Fire Fighting Foams, Fire Technology, vol. 12, no. 2, pp. 141-150, 1976.

8. P.J. Chiesa and R.S. Alger, Severe Laboratory Fire Tests for Fire Fighting Foams, Fire Technology, vol. 16, no. 1, pp. 12-21, 1980.

9. U.S. Military Specification MIL-F 24385F, Firefighting Extinguishing Agent, Aqueous Film-Forming Foam (AFFF) Liquid Concentrate for Fresh and Sea Water, Naval Sea Systems Command, 1992.

10. NFPA 403: Standard for Aircraft Rescue and Fire Fighting Services at Airports (2009 Edition), National Fire Protection Association (NFPA), Quincy, MA, 2009.

11. NFPA 412: Standard for Evaluating Aircraft Rescue and Fire-Fighting Foam Equipment (2009 Edition), National Fire Protection Association (NFPA), Quincy, MA, 2009.

12. Chemguard C301MS, Material Safety Data Sheet, Chemguard, Inc., Mansfield, TX, January 2011. www.chemguard.com

13. C.A. Moody and J.A. Field, Per-fluorinated Surfactants and the Environmental Implications of their Use in Fire-Fighting Foams, Environmental Science \& Technology, vol. 34, no. 18, 2000. 
14. C.D. Vecitis, Y. Wang, J. Cheng, H. Park, B.T. Mader, and M.R. Hoffmann, Sonochemical Degradation of Perfluorooctanesulfonate in Aqueous Film-Forming Foams, Environmental Science \& Technology, vol. 44, no. 1, pp. 432-438, 2010.

15. S. A. Magrabi, B. Z. Dlugogorski, and G.J. Jameson, A Comparative Study of Drainage Characteristics in AFFF and FFFP Compressed-air Fire-Fighting Foams, Fire Safety Journal, vol. 37, pp. 21-52, 2002.

16. M.L. Carruette, H. Persson, and M. Pabon, New Additive for Low Viscosity of AFFF/AR Concentrates - Study of the Potential Fire Performance, Fire Technology, vol. 40, pp. 367-384, 2004.

17. J.R. Walker, The Effect of Surfactants on the Breakup of an Axisymmetric Laminar Liquid Jet, Ph.D. Thesis, University of Maryland, College Park, MD, 2012.

18. B.J. Place and J.A. Field, Identification of Novel Fuorochemicals in Aqueous FilmForming Foams Used by the US Military, Environmental Science \& Technology, vol. 46, no. 13, pp. 7120-7127, 2012.

19. United States Environmental Protection Agency, Emerging Containments Perfluorooctane Sulfonate (PFOS) and Perfluoroctanoic Acid (PFOA): Emerging Contaminants Fact Sheet - PFOS an PFOA, Solid Waste and Emergency Response (5106P), EPA 505-F-11-002, May 2012.

20. United States: National Bureau of Standards, Journal of Physical and Chemical Reference Data, American Chemical Society, vol. 7, no. 3, 1978.

21. R.C.R. Figueredo and E. Sabadini, Firefighting Foam Stability: The Effect of the Drag Reducer Poly(ethylene) Oxide, Colloids and Surfaces A: Physiochem. Eng. Aspects, vol. 215, pp. 77-86, 2003.

22. E.K. Hyland and B.A. Williams, Characterization of the Dynamic Surface Tension of Aqueous Film-Forming Foam, NRL Report NRL/MR/6180--04-8749, Naval Technology Center for Safety and Survivability, Washington, D.C., 2004.

23. E. Meissner, B. Twardochleb, E. Milchert, A. Wroblewska, and J. Szymanowski, Estimation of Foam Forming Properties and Surface Tension of Fire-Extinguishing Agents, Journal of Tensile Surfactants Detergents, vol. 40, no. 6, pp. 353-360, 2003.

24. ASTM Standard D1141-98, 2013, Standard Practice for the Preparation of Substitute Ocean Water, ASTM International, West Conshohocken, PA, DOI: 10.1520/D1141-98, www.astm.org, 2013. 
25. R.S. Sheinson, B.A. Williams, C. Green, J.W. Fleming, R. Anleitner, S. Ayers, and A. Maranghides, The Future of Aqueous Film Forming Foam (AFFF): Performance Parameters and Requirements, NIST Special Publication 984, Proceedings of the HOTWC-2002 $12^{\text {th }}$ Halon Options Technical Working Conference, Albuquerque, NM, April 30-May 2, 2002.

26. B. Persson, A. Lonnermark, and H. Persson, FOAMSPEX: Large Scale Foam Application - Modeling of Foam Spread and Extinguishment, Fire Technology, vol. 39, pp. 347-362, 2003.

27. G. Lefort, A.W. Marshall, and M. Pabon, Evaluation of Surfactant Enhanced Water Mist Performance, Fire Technology, vol. 45, no. 3, pp. 341-354, 2008.

28. G. Timms and P. Haggar, Foam Concentration Measurement Techniques, Fire Technology, vol. 26, no. 1, pp. 41-50, 1990.

29. J.L. Scheffey, R.L. Darwin, and J.T. Leonard, Evaluating Firefighting Foams for Aviation Fire Protection, vol. 31, no. 3, pp. 224-243, 1995.

30. B.S. Gardiner, B.Z. Dlugogorski, and G.J. Jameson, Rheology of Fire-Fighting Foams, Fire Safety Journal, vol. 31, pp. 61-75, 1998.

31. A.J. Laundess, M.S. Rayson, B.Z. Dlugogorski, and E.M. Kennedy, Small-Scale Test Protocol for Firefighting Foams DEF(AUST) 5706: Effect of Bubble Size Distribution and Expansion Ratio, Fire Technology, vol. 47, pp. 149-162, 2011.

32. R.P. Grant, Newtonian Jet Stability, Ph.D. Thesis, University of Rochester, NY, University Microfilms No. 65-12006, Ann Arbor, MI, 1965.

33. M.J. McCarthy and N.A. Malloy, Review of Stability of Liquid Jets and the Influence of Nozzle Design. The Chemical Engineering Journal, vol. 7, pp. 1-20, 1974.

34. S.P. Lin and R.D. Reitz, Drop and Spray Formation from a Liquid Jet. Annual Review of Fluid Mechanics, vol. 30, pp. 85-105, 1998.

35. R.E. Phinney, The Break-up of a Turbulent Liquid Jet in a Gaseous Atmosphere. Journal of Fluid Mechanics, vol. 60, part 4, pp. 689-701, 1973.

36. M. Birouk and N. Lekic, Liquid Jet Break-up in Quiescent Atmosphere: Annual Review, Atomization and Sprays, vol. 19, pp. 501-528, 2009.

37. G.M. Faeth, L.P. Hsiang, and P.K. Wu, Structure and Breakup Properties of Sprays, International Journal of Multiphase Flow, vol. 21, Supplement. pp. 99-127, 1995.

38. T.F. Chen and J.R. Davis, Disintegration of a Turbulent Water Jet, Journal of the Hydraulics Division, vol. 90, no. 1, pp. 175-206, 1964. 
39. J.W. Hoyt and J.J. Taylor, Turbulence Structure in a Water Jet Discharging in Air, The Physics of Fluids, vol. 20, no. 10, part II, 1977.

40. G.A. Ruff, A.D. Sagar, and G.M. Faeth, Structure and Mixing Properties of PressureAtomized Sprays, AIAA Journal, vol. 27, no. 7, Jul. 1989.

41. J. Delteil, S. Vincent, A. Erriguible, and P. Subra-Paternault, Numerical Investigations in Rayleigh Breakup of Round Liquid Jets with VOF Methods, Computers \& Fluids, vol. 50, pp. 10-23, 2011.

42. H. Hiroyasu, Spray break-up Mechanism from the Hole-Type Nozzle and Its Applications, Atomization and Sprays, vol. 10, pp. 511-527, 2000.

43. P.K. Wu, R.F. Miranda, and G.M. Faeth, Effects of Initial Flow Conditions on Primary Breakup of Nonturbulent and Turbulent Liquid Jets, AIAA 95-0561, AIAA $32^{\text {nd }}$ Aerospace Sciences Meeting \& Exhibit, Reno, NV, January 10-13, 1944.

44. R.D. Reitz, Atomization and Other Breakup Regimes of a Liquid Jet, Ph.D. Thesis, Princeton University, Princeton, NJ, 1978.

45. P.K. Wu, Liquid Surface Breakup of Nonturbulent and Turbulent Liquids, Ph.D. Thesis, The University of Michigan, Ann Arbor, MI, 1992.

46. D.D. Joseph, J. Belanger, and G.S. Beavers, Breakup of a Liquid Drop Suddenly Exposed to a High-speed Airstream, International Journal of Multiphase Flow, vol. 25, no. 6-7, pp. 1263-1303, 1999.

47. A.P. Hatton and M.J. Osbourne, The Trajectories of Large Fire Fighting Jets, International Journal of Heat and Fluid Flow, vol. 1, issue 1, pp. 37-41, 1979.

48. J.R. Freeman, Experiments Relating to Hydraulics of Fire Streams, Transactions ASCE, vol. 21, pp. 303-461, 1889.

49. H. Rouse, J.W. Hoe, and D.E. Metzler, Experimental Investigation of Fire Monitors and Nozzles, Transactions ASCE, vol. 77, issue 92, pp. 1147-1188, 1952.

50. M. Murakami and K. Katayama, Discharge Coefficients of Fire Nozzles, Transactions ASME, Journal of Basic Engineering, vol. 88, pp. 706-716, 1966.

51. C.R. Theobald, The Design of a General Purpose Fire-Fighting Jet and Spray Branch, Fire Safety Journal, vol. 7, pp. 177-190, 1984.

52. C.R. Theobald, The Effect of Nozzle Design on the Stability and Performance of Turbulent Water Jets, Fire Safety Journal, vol. 4, pp. 1-13, 1981. 
53. X. Jiang, G.A. Siamas, K. Jagus, and T.G. Karayiannis, Physical Modeling and Advanced Simulations of Gas-Liquid Two-Phase Jet Flows in Atomization and Sprays, Progress in Energy and Combustion Science, vol. 36, pp. 131-167, 2010.

54. M. Gorokhovski and M. Herrmann, Modeling Primary Atomization, Annual Review of Fluid Mechanics, vol. 40, pp. 343-366, 2008.

55. M.R. Archambault, Stochastic Spray Flow Models: A Review, ILASS-Americas $22^{\text {nd }}$ Annual Conference on Liquid Atomization and Spray Systems, Cincinnati, OH, 2010.

56. ANSYS Fluent v14.5 Theory Guide, www.ansys.com, Accessed 8 April 2013.

57. T. Menard, S. Tanguy, and A. Berlement, Coupling Level Set/VOF/Ghost Fluid Methods: Validation and Application to 3D Simulation of the Primary Break-up of a Liquid Jet, International Journal of Multiphase Flow, vol. 33, pp. 510-524, 2007.

58. H. Shi and C. Kleinstreuer, Simulation and Analysis of High-speed Droplet Spray Dynamics, Journal of Fluids Engineering, vol. 129, pp. 621-633, 2007.

59. A.F. Blum, Discharge Characteristics of Canonical Sprinkler Sprays, M.S. Thesis, University of Maryland, College Park, 2007.

60. K.C. Adiga, R.F. Hatcher, Jr., R.S. Sheinson, F.W. Williams, and S. Ayers, A Computational and Experimental Study of Ultra Fine Water Mist as a Total Flooding Agent, Fire Safety Journal, vol. 42, pp. 150-160, 2007.

61. S.S. Yoon, J.C. Hewson, P.E. DesJardin, D.J. Glaze, A.R. Black, and R.R. Skaggs, Numerical Modeling and Experimental Measurements of a High Speed Solid-Cone Water Spray for Use in Fire Suppression Application, International Journal of Multiphase Flow, vol. 30, pp. 1369-1388, 2004.

62. Y. Sinai, P. Stopford, M. Edwards, and S. Watkins, CFD Modeling of Fire Suppression by Water Spray: Sensitivity and Validation for a Pool Fire in a Room, Eighth International IBPSA Conference, Eindhoven, Netherlands, pp. 1217-1220, August 11-14, 2003.

63. G. Tanner, W. Kalata, K. Brown, and R.J. Schick, Computational Study Effects of Drop Size Distribution in Fire Suppression Systems, ILASS-Americas $22^{\text {nd }}$ Annual Conference on Liquid Atomization and Spray Systems, Cincinnati, OH, 2010.

64. P.E. DesJardin and L.A. Gritzo, A Dilute Spray Model for Fire Simulations: Formulation, Usage, and Benchmark Problems, SAND2002-3419, Sandia National Laboratories, 2002.

65. Baldor 456 Smartmotor Operations Manual, www.baldor.com, Accessed 28 March 2013. 
66. TSI Incorporated, Phase Doppler Particle Analyzer (PDPA)/Laser Doppler Velocimeter Operations Manual, P/N 1990048, Revision D, 2005.

67. H.E. Albrecht, Laser Doppler and Phase Doppler Measurement Techniques, Springer Online Library, 2003.

68. Dantec Measurement Technology, Principles of Phase Doppler Anemometry, www.dantecmt.com, 2013.

69. W. Lai, J. Shakal, and D. Troolin, Accuracy, Resolution, and Repeatability of Powersight PDPA and LDV Systems, TSI Technical Note P/N 5001520 (A4), TSI Incorporated, Shoreview, MN, USA, 2013.

70. TSI Incorporated, Phase Doppler Particle Analyzer (PDPA)/Laser Doppler Velocimeter Installation Manual, P/N 1990024, Revision B, February 2005.

71. Solidworks $2012^{\circledR}$, www.solidworks.com, Accessed 8 April 2013.

72. Pointwise v17.0 ${ }^{\circledR}$, www.pointwise.com, Accessed 8 April 2013.

73. I.B. Celik, U. Ghia, P.J. Roache, C.J. Freitas, H. Coleman, and P.E. Raad, Procedure for Estimating and Reporting of Uncertainty Due to Discretization in CFD Applications, ASME Journal of Fluids Engineering (Special Publication), vol. 130, 2008.

74. ASTM Standard D445-11a, 2013, Historical Standard: ASTM D445-11a Standard Test Method for Kinematic Viscosity of Transparent and Opaque Liquids (and Calculation of Dynamic Viscosity), ASTM International, West Conshohocken, PA, DOI: 10.1520/D445-11a, www.astm.org, 2013.

75. United States Department of Agriculture, Sucrose Conversion Table: Technical Inspection Procedures, Processed Products Branch, File Code 135-A-50, 1970. 
Table A.1 summarizes the operating parameters for both the experimental as well as the CFD modeling cases executed during the present study. All cases listed in Table A.1 represent all experimental case conditions. Cases highlighted in light gray are those also investigated computationally. Refer to Chapter 2 for details on how the specific Reynolds, Weber, and Ohnesorge numbers are formulated.

Table A.1: A Summary of the Firefighting Jet Nozzle Exit Conditions Examined

\begin{tabular}{|c|c|c|c|c|c|c|c|c|c|}
\hline Case & $\begin{array}{c}\text { Nozzle } \\
\text { Diameter } \\
(\mathrm{mm})\end{array}$ & $\begin{array}{c}\text { Nozzle } \\
\text { Flow Rate } \\
\left(1 \cdot \mathrm{min}^{-1}\right)\end{array}$ & $\begin{array}{c}\text { Nozzle } \\
\text { Velocity } \\
\left(\mathrm{m} \cdot \mathrm{s}^{-1}\right)\end{array}$ & $\begin{array}{c}\text { Nozzle } \\
\text { Pressure } \\
(\mathrm{MPa})\end{array}$ & $\begin{array}{c}\operatorname{Re}_{L} \\
\left(\times 10^{5}\right)\end{array}$ & $\begin{array}{c}W e_{L} \\
\left(\times 10^{5}\right)\end{array}$ & $W e_{G}$ & $W e_{d}$ & $\begin{array}{c}\text { Oh } \\
\left(\times 10^{-3}\right)\end{array}$ \\
\hline $\begin{array}{l}\text { Low Flow, Low } \\
\text { Press. Water Jet }\end{array}$ & 1.45 & 4.20 & 42.5 & 1.20 & 0.690 & 0.363 & 43 & 1.49 & 2.76 \\
\hline $\begin{array}{c}\text { Low Flow, Low } \\
\text { Press. } 6 \% \text { AFFF Jet }\end{array}$ & 1.45 & 4.20 & 42.5 & 1.20 & 0.617 & 1.51 & 178 & 6.16 & 6.30 \\
\hline $\begin{array}{l}\text { Low Flow, High } \\
\text { Press. Water Jet }\end{array}$ & 0.81 & 4.20 & 135 & 10.7 & 1.23 & 2.05 & 243 & 15.0 & 3.69 \\
\hline $\begin{array}{c}\text { Low Flow, High } \\
\text { Press. } 6 \% \text { AFFF Jet }\end{array}$ & 0.81 & 4.20 & 135 & 10.7 & 1.10 & 8.53 & 1007 & 62.0 & 8.41 \\
\hline $\begin{array}{l}\text { Med. Flow, Med. } \\
\text { Press. Water Jet }\end{array}$ & 1.75 & 12.9 & 88.9 & 5.85 & 1.75 & 1.92 & 228 & 6.50 & 2.51 \\
\hline $\begin{array}{l}\text { Med. Flow, Med. } \\
\text { Press. 3\% AFFF Jet }\end{array}$ & 1.75 & 12.9 & 88.9 & 5.85 & 1.62 & 8.25 & 976 & 27.9 & 5.62 \\
\hline $\begin{array}{l}\text { Med. Flow, Med. } \\
\text { Press. 6\% AFFF Jet }\end{array}$ & 1.75 & 12.9 & 88.9 & 5.85 & 1.56 & 7.98 & 943 & 26.9 & 5.73 \\
\hline $\begin{array}{l}\text { Med. Flow, Med. } \\
\text { Press. 9\% AFFF Jet }\end{array}$ & 1.75 & 12.9 & 88.9 & 5.85 & 1.55 & 7.65 & 901 & 25.7 & 5.63 \\
\hline $\begin{array}{c}\text { Med. Flow, Med. } \\
\text { Press. 12\% AFFF Jet }\end{array}$ & 1.75 & 12.9 & 88.9 & 5.85 & 1.50 & 7.42 & 873 & 24.9 & 5.73 \\
\hline $\begin{array}{l}\text { High Flow, Low } \\
\text { Press. Water Jet }\end{array}$ & 3.68 & 23.6 & 36.8 & 1.16 & 1.52 & 0.692 & 82 & 1.12 & 1.73 \\
\hline $\begin{array}{c}\text { High Flow, Low } \\
\text { Press. 6\% AFFF Jet }\end{array}$ & 3.68 & 23.6 & 36.8 & 1.16 & 1.36 & 2.88 & 340 & 4.62 & 3.95 \\
\hline $\begin{array}{l}\text { High Flow, High } \\
\text { Press. Water Jet }\end{array}$ & 1.91 & 19.8 & 116 & 10.6 & 2.47 & 3.52 & 418 & 11.0 & 2.41 \\
\hline $\begin{array}{l}\text { High Flow, High } \\
\text { Press. } 6 \% \text { AFFF Jet }\end{array}$ & 1.91 & 19.8 & 116 & 10.6 & 2.20 & 14.7 & 1730 & 45.4 & 5.49 \\
\hline
\end{tabular}




\section{B.1 Density}

Density $(\rho)$ measurements of various AFFF solution concentrations were conducted by AFRL personnel at Tyndall Air Force Base, FL on MIL-SPEC C301MS 3-percent AFFF manufactured by Chemguard and Williams ${ }^{\circledR}$, a subsidiary of Tyco International. Table B.1 lists the density of each AFFF solution, and Figure B.1 illustrates the relationship between the AFFF solution and its density. All measurements were taken at approximately $298 \mathrm{~K}$. Density values were extrapolated by measuring the specific gravity $\left(\gamma_{S G}\right)$ of each solution using a Mettler Toledo Densito 30PX with an accuracy of $\pm 1 \mathrm{~kg} \cdot \mathrm{m}^{-3}$. Although the use of this instrument involved drawing a sample of solution through a thin capillary tube, foam or bubbles did not appear to occur. AFFF density was calculated using the following equation where $\rho_{\mathrm{H} 2 \mathrm{O}}$ is the density of water equal to $997.0 \mathrm{~kg} \cdot \mathrm{m}^{3}$ at $298 \mathrm{~K}$ :

$$
\rho=\gamma_{S G} \rho_{H 2 O}
$$

Because AFFF concentrate has a density similar to water in conjunction with also being heavily diluted in water for standard firefighting application purposes, AFFF solution density is a nearly negligible distinguishing property in comparison to water.

Table B.1: Density of Various AFFF Solution Concentrations

\begin{tabular}{|c|c|}
\hline Solution Description & $\begin{array}{c}\text { Density - } \rho \\
\left(\mathrm{kg} \cdot \mathrm{m}^{-3}\right)\end{array}$ \\
\hline $100 \%$ AFFF (pure concentrate) & 1069.7 \\
\hline $12 \%$ AFFF & 1007.0 \\
\hline $9 \%$ AFFF & 1004.4 \\
\hline $6 \%$ AFFF & 1002.4 \\
\hline $3 \%$ AFFF & 999.9 \\
\hline $0.3 \% \mathrm{AFFF}$ & 996.7 \\
\hline $0.03 \%$ AFFF & 996.6 \\
\hline Water & 997.0 \\
\hline
\end{tabular}




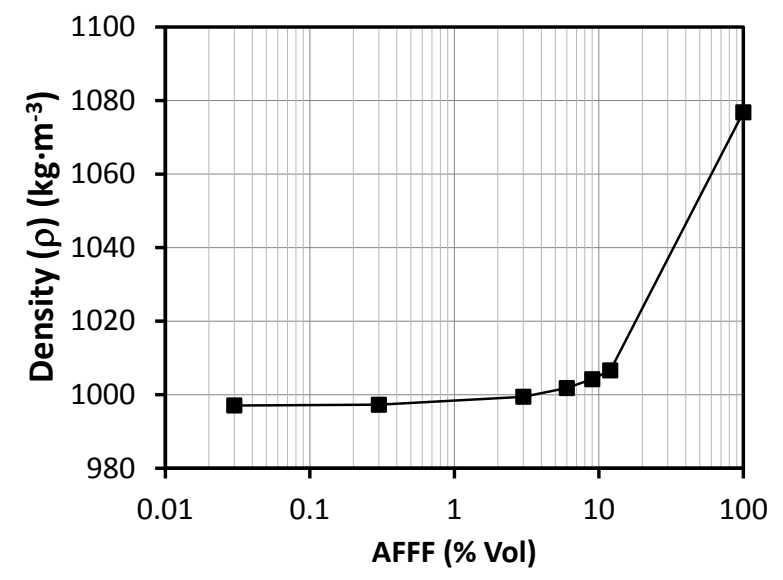

Figure B.1: AFFF Solution Density vs. AFFF Solution Concentration

\section{B.2 Viscosity}

Dynamic viscosity $(\mu)$ at a constant shear rate was derived for various AFFF solution concentrations by AFRL personnel at Tyndall Air Force Base, FL on MIL-SPEC C301MS 3-percent AFFF. Kinematic viscosity $(v)$ was recorded using a size 50 Canon-Fenske Routine viscometer with an effective range of 0.8 to $4 \mathrm{cSt}$. All AFFF measurements fell within this range and were carried out using ASTM standard D445-11a with the exception that measurements were conducted at ambient solution temperatures rather than in a temperature bath [74]. Dynamic viscosity was calculated from the measured kinematic viscosity and the density measurement from Section B.1:

$$
\mu=\rho v
$$

Use of this instrument involved allowing the solution to flow through a capillary tube of varying diameter. It was noted that for concentrations above 0.3-percent AFFF, varying amounts of bubbles were generated above the liquid surface. It is not known what degree of error this introduced. All measurements were taken at approximately $298 \mathrm{~K}$.

Supplemental viscosity data was later provided by Brookfield Engineering Laboratories, Inc. to determine if any AFFF solutions tested by AFRL exhibited non-Newtonian characteristics by measuring their viscous dependence on shear rate. Similar batches of AFFF solution were tested using an LVDV-III+ Ultra Brookfield Rheometer with an accuracy of \pm 1 percent of the current 
test data range. A CPE-40 cone spindle with a CPE-44PY sample cup with an integral temperature probe was used to analyze each batch of AFFF solution. All measurements were taken at $298 \mathrm{~K}$ in the absence of air to prevent foaming. Each sample was equilibrated for at least $10 \mathrm{~min}$ before the start of each run at the lowest spindle RPM, incrementally ramped up to the maximum RPM, and then returned to the lowest spindle RPM to verify repeatability at each previous increment. Table B.2 and Figure B.2a summarize and illustrate the relationship between the AFFF solution concentration and the dynamic viscosity, respectively. Figure B.2b depicts the relationship of AFFF solution dynamic viscosity as a function of shear rate.

Table B.2: Derived Dynamic Viscosity of Various AFFF Solution Concentrations

\begin{tabular}{|c|c|}
\hline Solution & $\begin{array}{c}\text { Dynamic Viscosity }-\mu \\
\left(\mathrm{mPa} \cdot \mathbf{s}^{-1} \text { ) }\right.\end{array}$ \\
\hline $100 \%$ AFFF (pure concentrate) & 2.616 \\
\hline $12 \% \mathrm{AFFF}$ & 1.043 \\
\hline $9 \% \mathrm{AFFF}$ & 1.008 \\
\hline $6 \% \mathrm{AFFF}$ & 1.001 \\
\hline $3 \% \mathrm{AFFF}$ & 0.9639 \\
\hline $0.3 \% \mathrm{AFFF}$ & 0.9394 \\
\hline $0.03 \% \mathrm{AFFF}$ & 0.9500 \\
\hline Water & 0.9224 \\
\hline
\end{tabular}

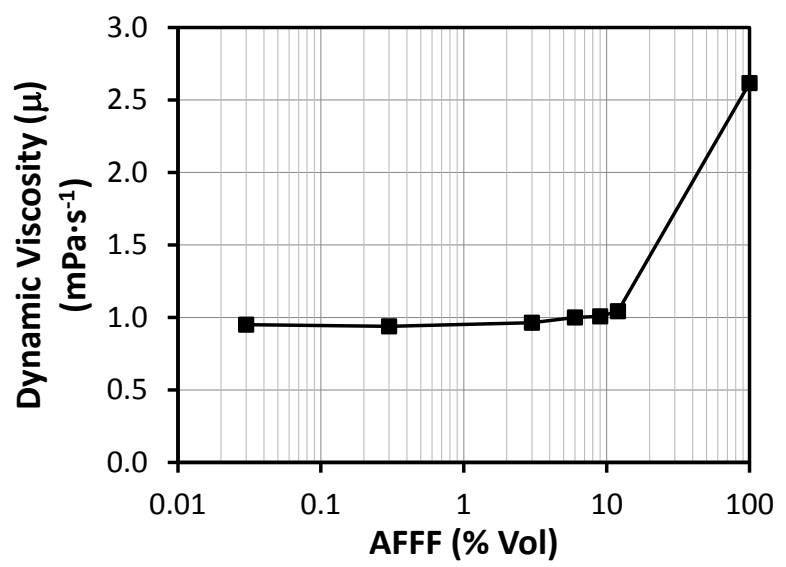

(a)

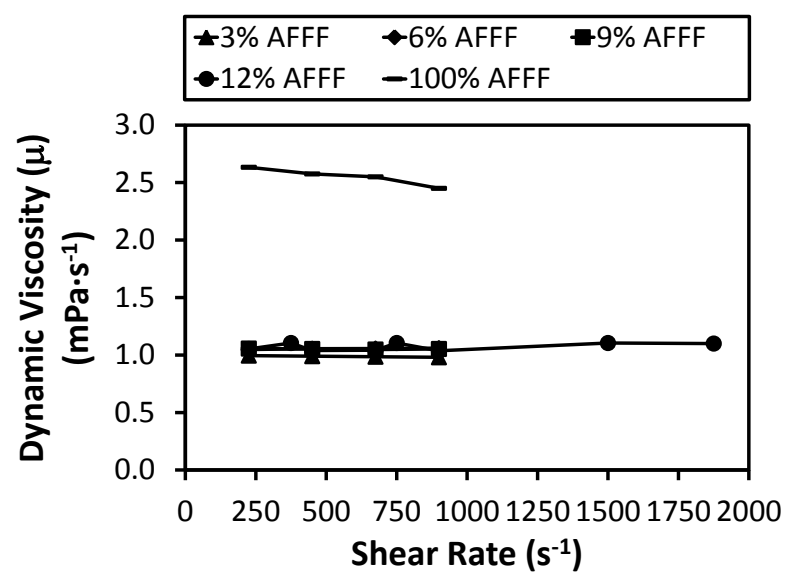

(b)

Figure B.2: (a) AFFF Solution Dynamic Viscosity vs. AFFF Solution Concentration (b) AFFF Solution Dynamic Viscosity vs. Shear Rate for Various AFFF Solution Concentrations 
Both AFRL and Brookfield confirmed that AFFF solutions at practical proportions with water maintain viscosities very similar to that of water. The data collected by Brookfield also confirmed that all AFFF solutions diluted for practical ARFF implementation exhibited primarily Newtonian characteristics with a nearly constant viscosity with respect to shear rate. The 100 -percent AFFF concentrate solution exhibited a very slight dependence on shear rate. The 12-percent AFFF solution, a composition still within an order of magnitude of the manufacturer's recommended proportion with water, was tested up to a shear of $2000 \mathrm{~s}^{-1}$ to ensure no significant dependencies existed at higher thresholds.

This data suggests that AFFF's non-Newtonian nature in aqueous foam form is primarily governed by the multiphase relationship that exists between the AFFF solution and the air bubbles for which it has entrained, rather than the pure liquid state of the AFFF solution.

\section{B.3 Surface Tension}

Equilibrium surface tension and dynamic surface tension measurements were conducted on various AFFF solution concentrations by AFRL personnel at Tyndall Air Force Base, FL on MIL-SPEC C301MS 3-percent AFFF. Additional AFFF dilutions were added for relative comparison. All measurements were taken at approximately $298 \mathrm{~K}$. All equilibrium surface tension data was collected using a Kruss model K10ST digital tensiometer with an accuracy of \pm $0.1 \mathrm{mN} \cdot \mathrm{m}^{-1}$. Equilibrium surface tension measurements were made using a platinum Wilhlemy plate at ambient temperature. Table A.3 lists the equilibrium surface tension data measured for each corresponding solution concentration. Figure B.3a illustrates the equilibrium surface tension as a function of AFFF solution concentration, and Figure A.3b depicts dynamic surface tension as a function of surface age for each respective AFFF solution concentration. Dynamic surface tension measurements were conducted using a Kruss model DVT30 drop volume tensiometer using conventional operating procedures. 
Table B.3: Equilibrium Surface Tension of Various AFFF Solution Concentrations

\begin{tabular}{|c|c|}
\hline Solution & $\begin{array}{c}\text { Equilibrium Surface Tension } \\
\left(\mathbf{m N} \cdot \mathbf{m}^{-1}\right)\end{array}$ \\
\hline $100 \%$ AFFF (pure concentrate) & 23.6 \\
\hline $12 \%$ AFFF & 18.8 \\
\hline $9 \%$ AFFF & 18.2 \\
\hline $6 \%$ AFFF & 17.4 \\
\hline $3 \%$ AFFF & 16.8 \\
\hline $0.3 \%$ AFFF & 17.4 \\
\hline $0.03 \%$ AFFF & 37.3 \\
\hline Water & 72.14 \\
\hline
\end{tabular}

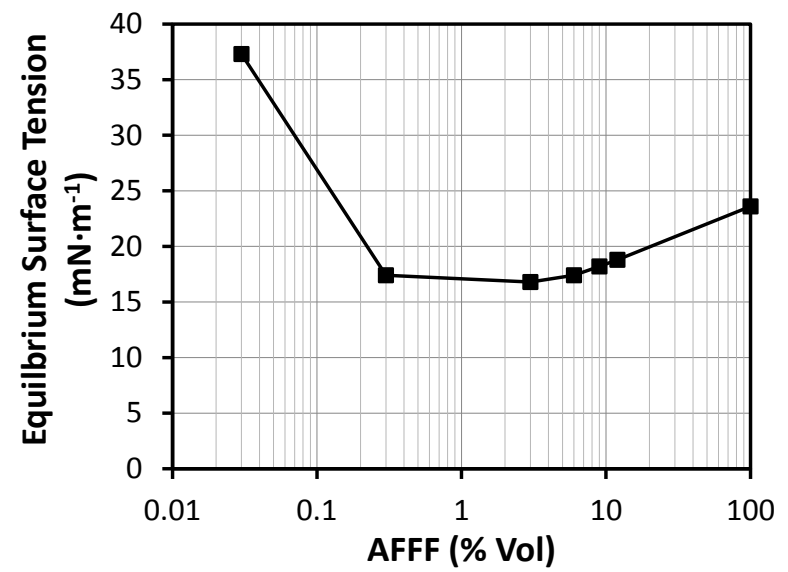

(a)

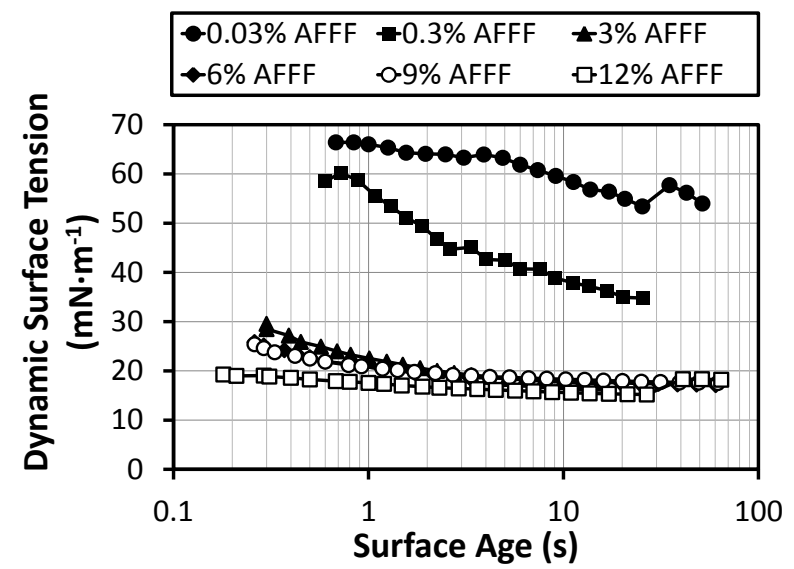

(b)

Figure B.3: (a) Equilibrium Surface Tension vs. AFFF Solution Concentration (b) Dynamic Surface Tension vs. Surface Age for Various AFFF Solution Concentrations

Both the equilibrium and dynamic surface tension of AFFF are important material properties that have exhibited the most divergence compared to water results due to the surfactant nature of AFFF's constituents. Surface tension can significantly affect firefighting jet break-up rate, mean droplet size distribution, and potentially other flow phenomena not characterized in the present study. For AFFF solution dilutions within the same order of magnitude of the manufactured recommended dilution with water, differences in equilibrium and dynamic surface tension are nearly negligible. This indicates AFFF ingredients are selectively proportioned to carefully optimize properties like surface tension, indirectly supporting evidence of past work conducted by Hyland and Williams [22]. 


\section{B.4 Index of Refraction}

Refractive measurements were executed on various AFFF solution concentrations by WVU personnel in the aqueous firefighting agent application laboratory on MIL-SPEC C301MS 3percent AFFF. All measurements were conducted at approximately $298 \mathrm{~K}$. An Atago Palette PR-32 alpha digital refractometer was used to measure the degrees Brix with \pm 0.1 -percent accuracy of each AFFF solution. The instrument is temperature compensated and linearly calibrated using distilled water and a series of small AFFF solution samples representing various dilution levels. Ten measurements were measured and averaged for each solution. Brix measurements are commonly used to measure the sucrose content of liquids for the food and agriculture industry. Brix values were converted to an index of refraction based on available sucrose conversion tables [75]. Results are depicted in Figure A.4a for select AFFF solution concentrations mixed with tap water as well as distilled water. Index of refraction measurements with respect to AFFF solution concentration are illustrated in Figure B.4b. Table B.4 summarizes the information shown in Figures B.4a and b.

Table B.4: Measured Degrees Brix and the Corresponding Index of Various AFFF Solution Dilutions

\begin{tabular}{|c|c|c|}
\hline Solution & $\begin{array}{c}\text { Degrees Brix } \\
(\%)\end{array}$ & $\begin{array}{c}\text { Index of } \\
\text { Refraction } \\
(-)\end{array}$ \\
\hline $100 \%$ AFFF (pure concentrate) & 20.9 & 1.3653 \\
\hline $12 \%$ AFFF & 2.5 & 1.3366 \\
\hline $9 \%$ AFFF & 1.9 & 1.3357 \\
\hline $6 \%$ AFFF & 1.3 & 1.3349 \\
\hline $3 \%$ AFFF & 0.6 & 1.3339 \\
\hline $0.3 \%$ AFFF & 0.1 & 1.3334 \\
\hline $0.03 \%$ AFFF & 0.0 & 1.3330 \\
\hline Water & 0.0 & 1.3330 \\
\hline
\end{tabular}




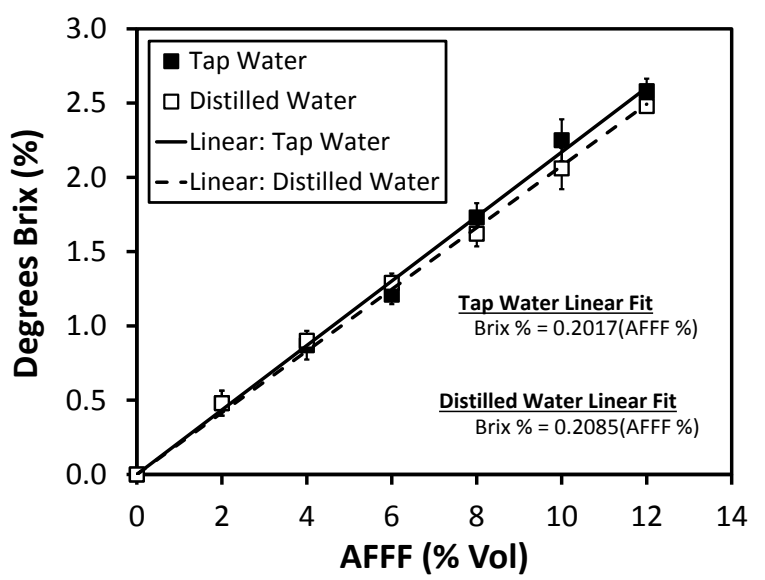

(a)

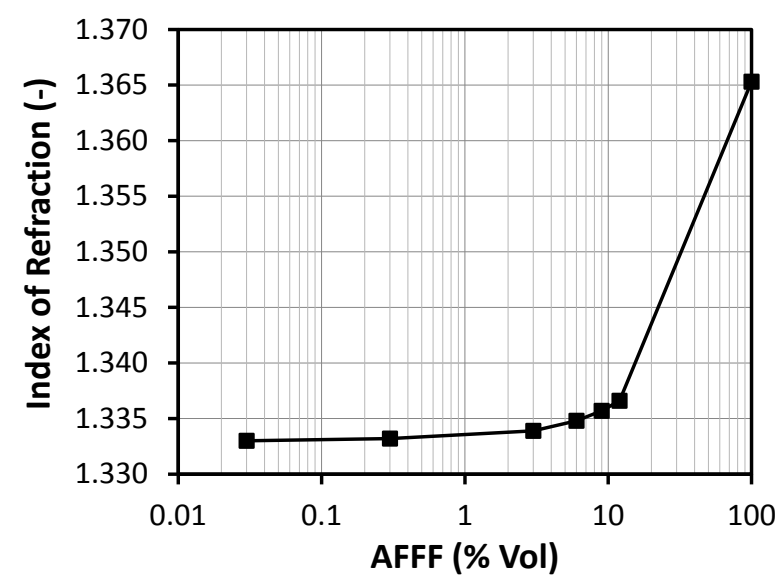

(b)

Figure B.4: (a) Degrees Brix vs. AFFF Solution Concentration (b) Index of Refraction vs. AFFF Solution Concentration

The index of refraction is a key material property that must be defined before PDPA can occur as run-time algorithms used to calculate droplet size by measuring scattered light are dependent upon the amount of refraction generated by the liquid in the droplet. Figures B.4a and $b$ as well as Table B.4 show that the index of refraction varies negligibly for AFFF solution concentrations of practical interest to the ARFF community. 


\section{APPENDIX C: PHASE DOPPLER FIREFIGHTING JET DATA}

Phase Doppler data are presented for all five firefighting jet pressure-flow rate combinations examined in the laboratory. A case summary of data collected is illustrated in Figure 4.2 and listed in Table 4.1 in Section 4.1. The experimental approach is discussed in Section 4.4. All profile data in this appendix compares water and 6-percent AFFF jets. Results collected in profile form are mean axial droplet velocity, mean vertical droplet velocity, mean droplet diameter, RMS droplet diameter, and Sauter mean droplet diameter. Each of these flow parameters are defined in Section 6.3.2. Plot layout and features such as \pm RMS velocity magnitude bars and references to error estimation are also identical to those shown in Figure 6.43 in Section 6.3.2.

Vertical profile measurements spanning the vertical height of each firefighting jet were recorded. A select number of horizontal profiles were also recorded for medium flow, medium pressure jets to measure the full jet width in areas near the nozzle, and approximate half jet widths further downstream where the PDPA traverse was limited in its reach. Select axial and vertical gaseous (air) velocity estimates are also provided for this jet configuration. Droplet size distribution measurements presented similar to those in Figure 6.40 in Section 6.3.1 are also presented at the end of each section highlighting phase Doppler sampling locations where the maximum profile, mean axial droplet velocity was measured. 


\section{C.1 Low Flow, Low Pressure Firefighting Jet Data}

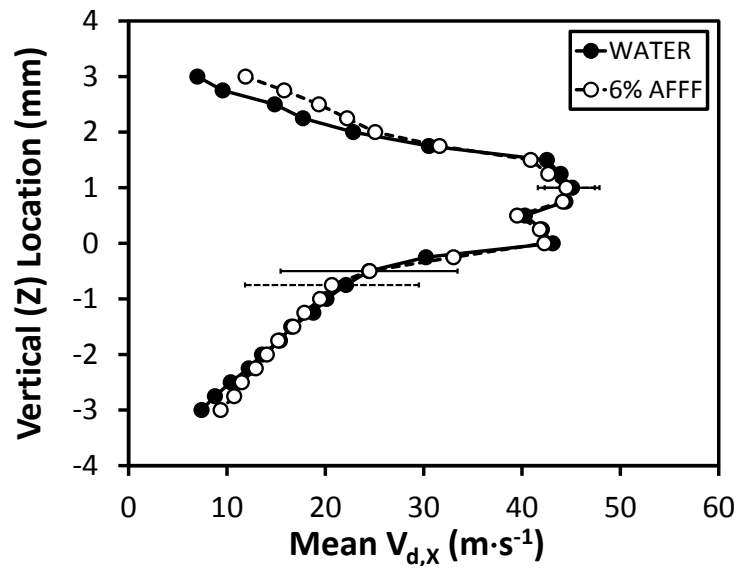

(a) $x=6.4 \mathrm{~mm}$ (0.25 in)

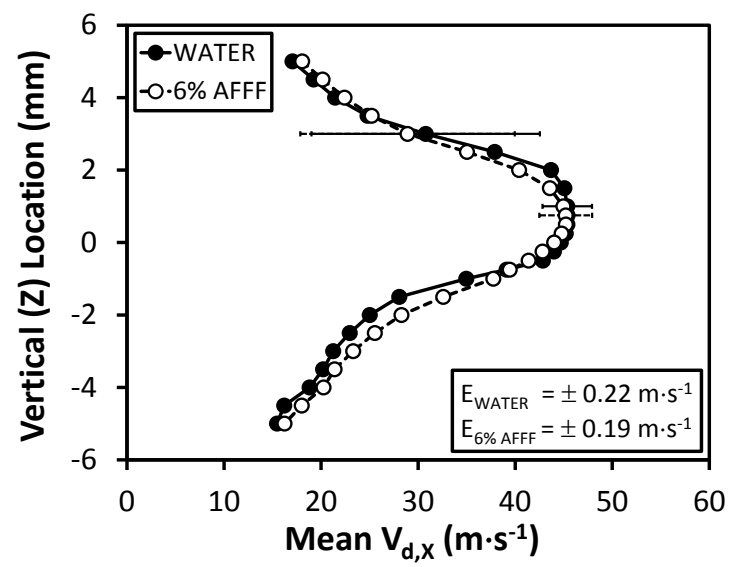

(c) $x=0.152 \mathrm{~m}$ (6 in)

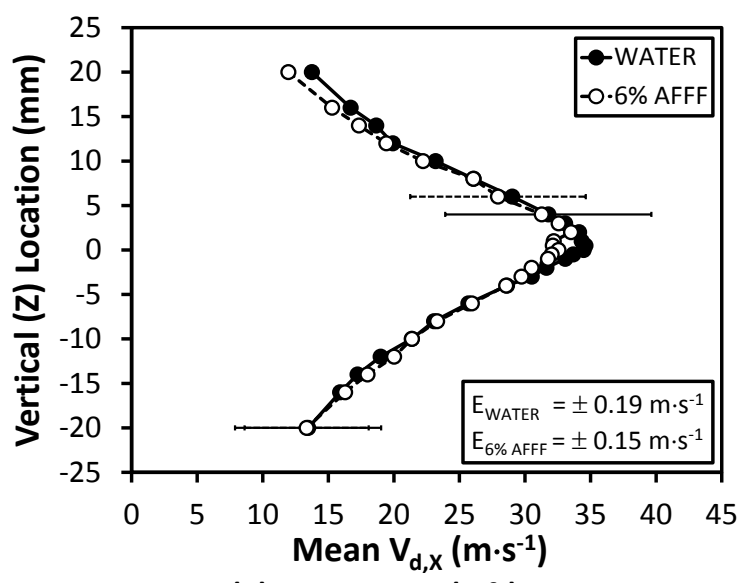

(e) $x=0.914 \mathrm{~m} \mathrm{(3 \textrm {ft } )}$

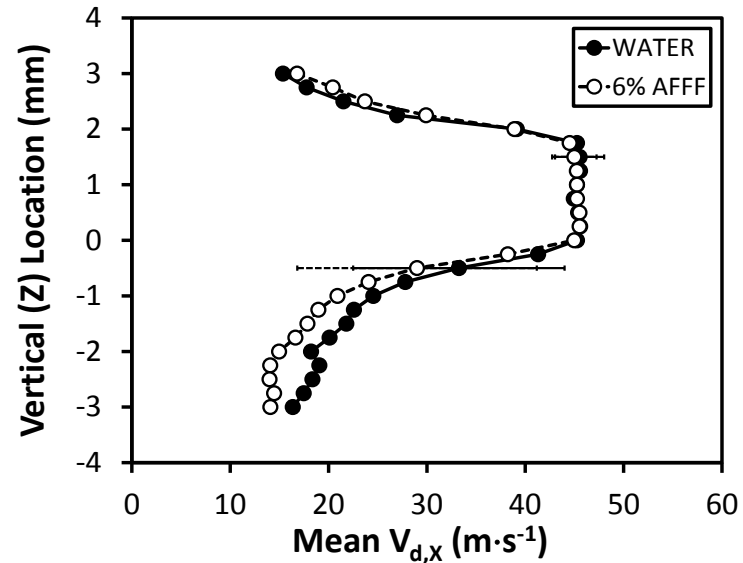

(b) $x=25.4 \mathrm{~mm}$ (1 in)

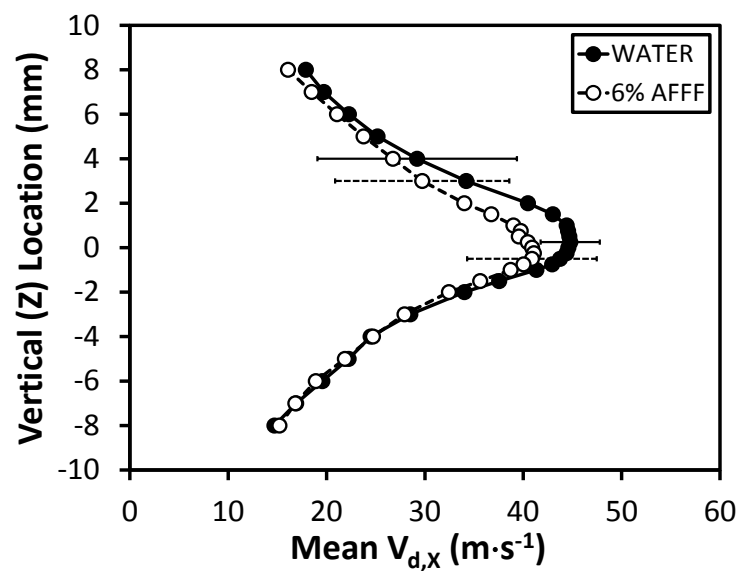

(d) $x=0.305 \mathrm{~m}(1 \mathrm{ft})$

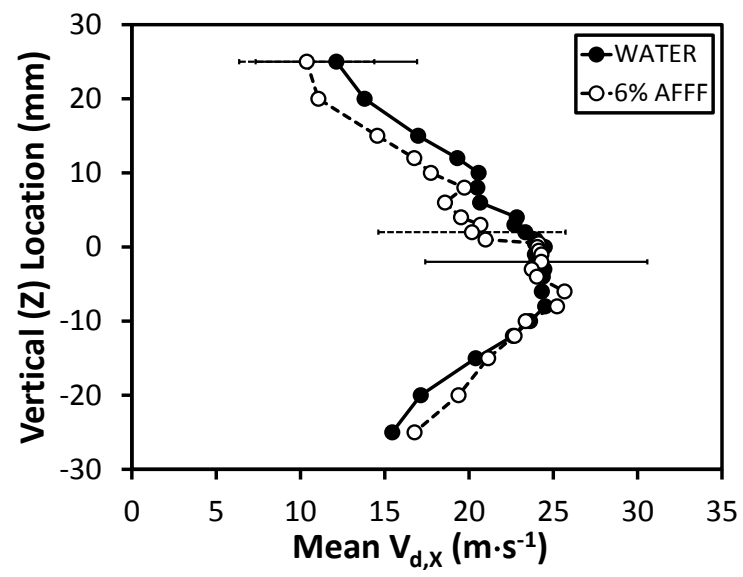

(f) $x=1.52 \mathrm{~m} \mathrm{(5} \mathrm{ft)}$

Figure C.1: Near Field Low Flow, Low Press. Jet Vertical Profiles of Mean Axial Droplet Velocity 


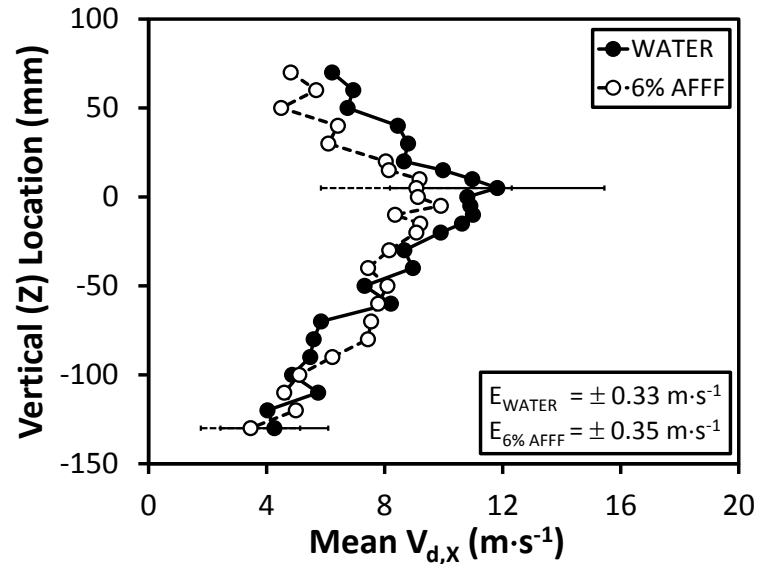

(a) $\mathrm{x}=3.05 \mathrm{~m}(10 \mathrm{ft})$

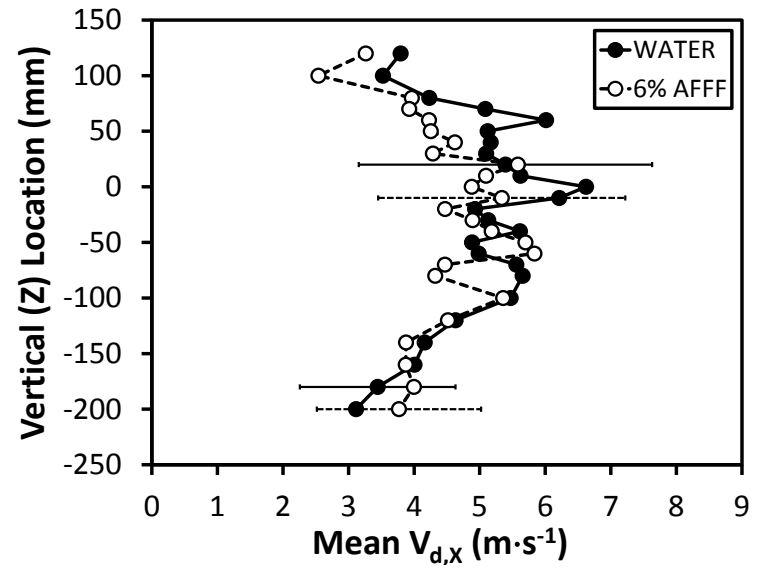

(b) $x=4.57 \mathrm{~m}(15 \mathrm{ft})$

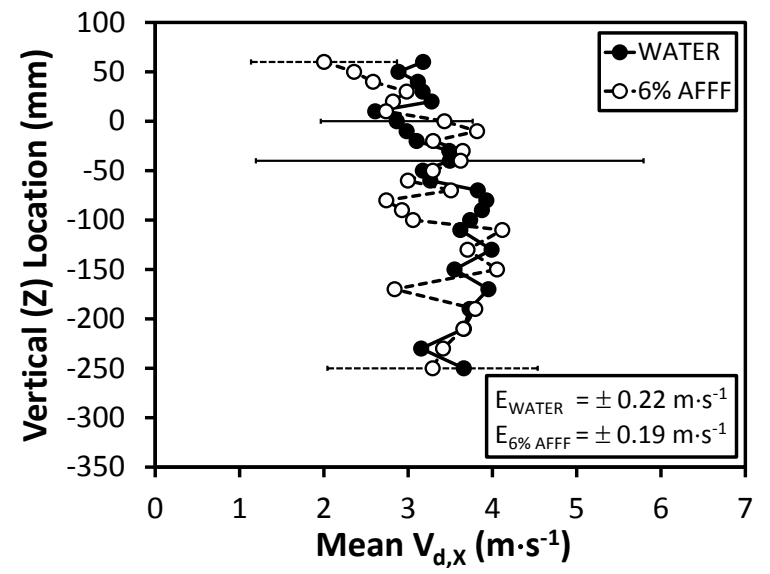

(c) $x=6.10 \mathrm{~m}(20 \mathrm{ft})$

Figure C.2: Far Field Low Flow, Low Press. Jet Vertical Profiles of Mean Axial Droplet Velocity 


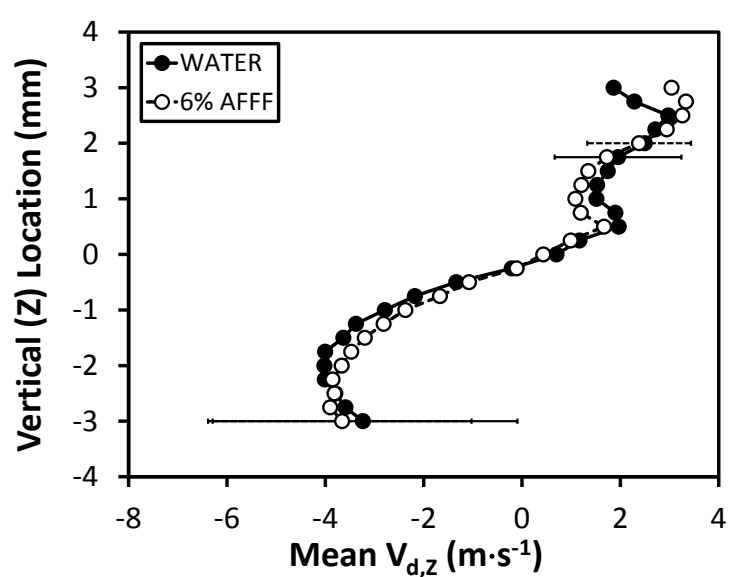

(a) $x=6.4 \mathrm{~mm}(0.25 \mathrm{in})$

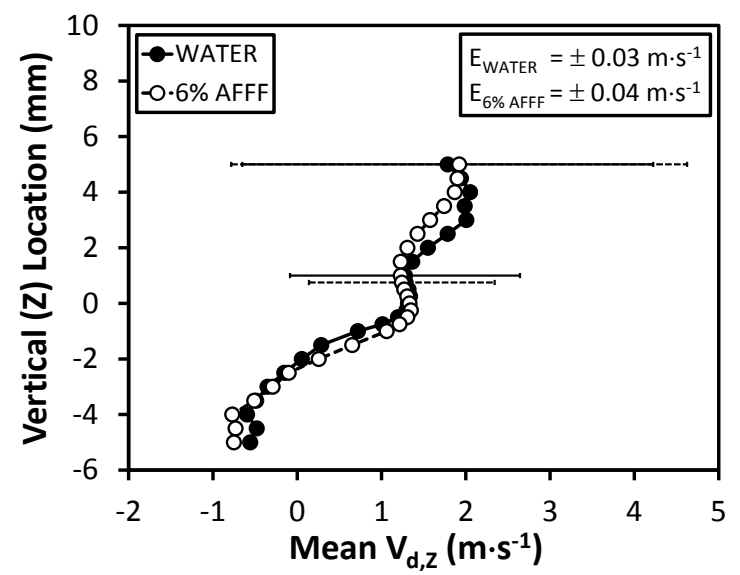

(c) $x=0.152 \mathrm{~m}(6 \mathrm{in})$

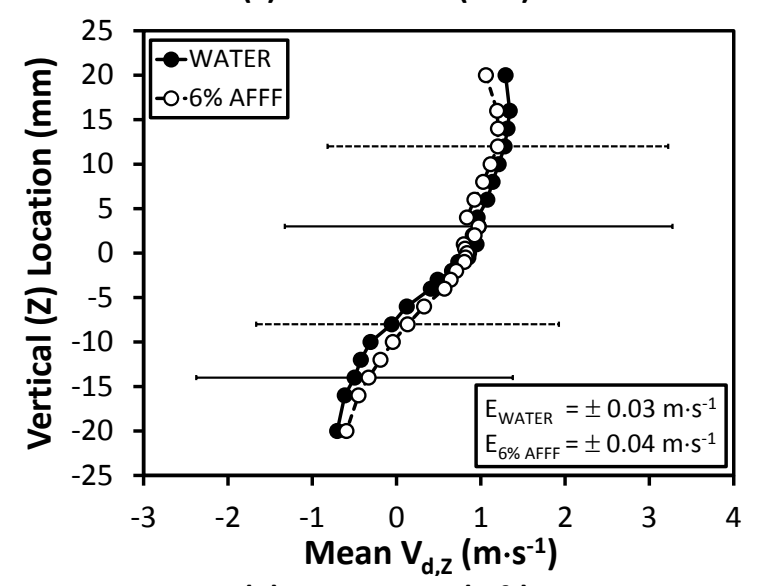

(e) $\mathrm{x}=0.914 \mathrm{~m}(3 \mathrm{ft})$

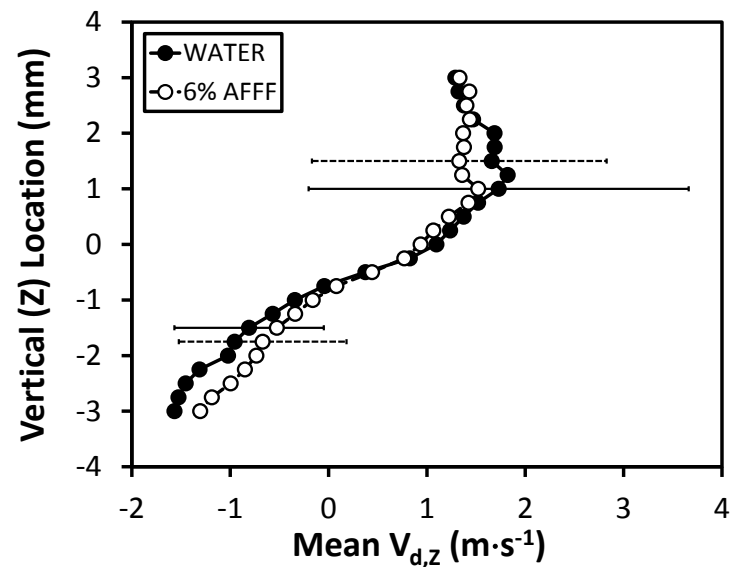

(b) $x=25.4 \mathrm{~mm}(1 \mathrm{in})$

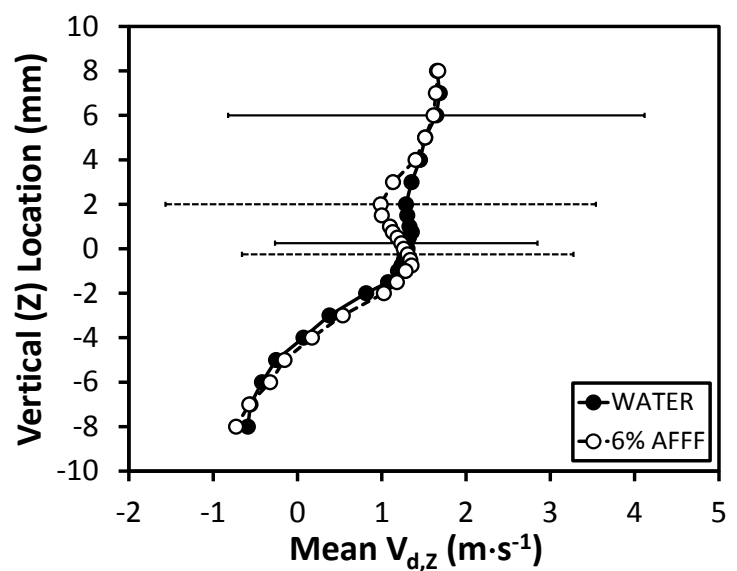

(d) $\mathrm{x}=0.305 \mathrm{~m}(\mathbf{1 f t})$

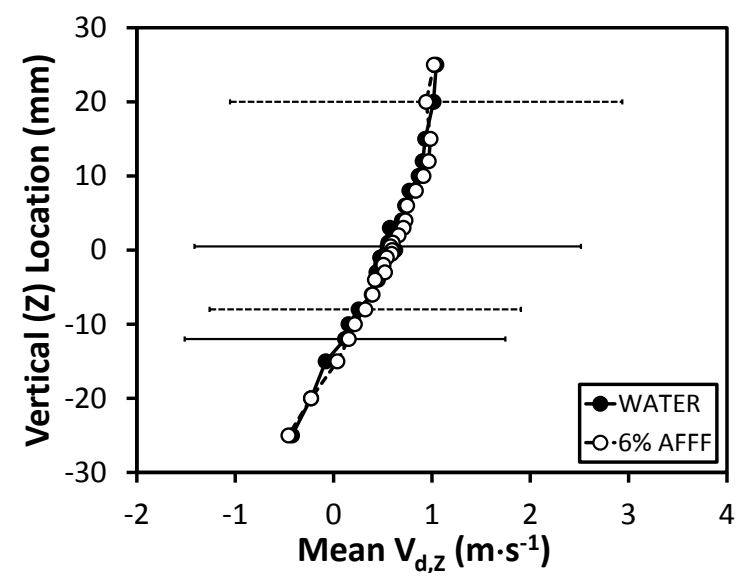

(f) $x=1.52 \mathrm{~m} \mathrm{(5 \textrm {ft } )}$

Figure C.3: Near Field Low Flow, Low Press. Jet Vertical Profiles of Mean Vertical Droplet Velocity 


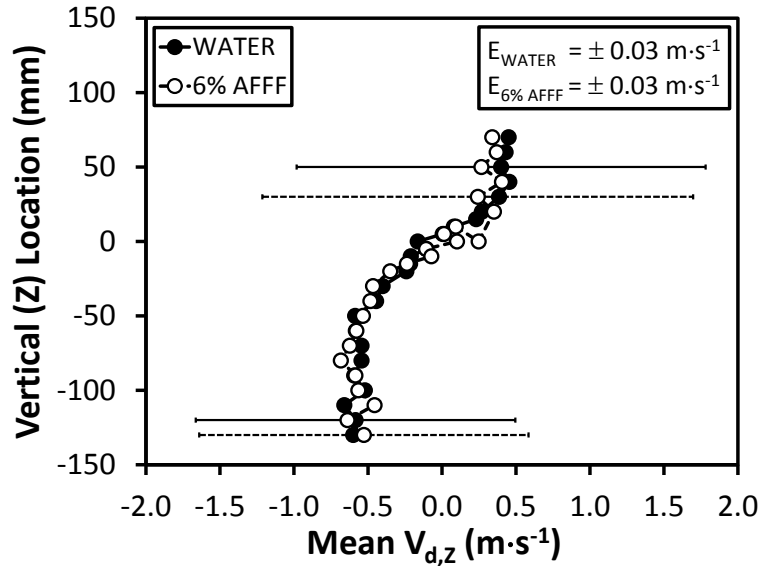

(a) $\mathrm{x}=3.05 \mathrm{~m}(10 \mathrm{ft})$

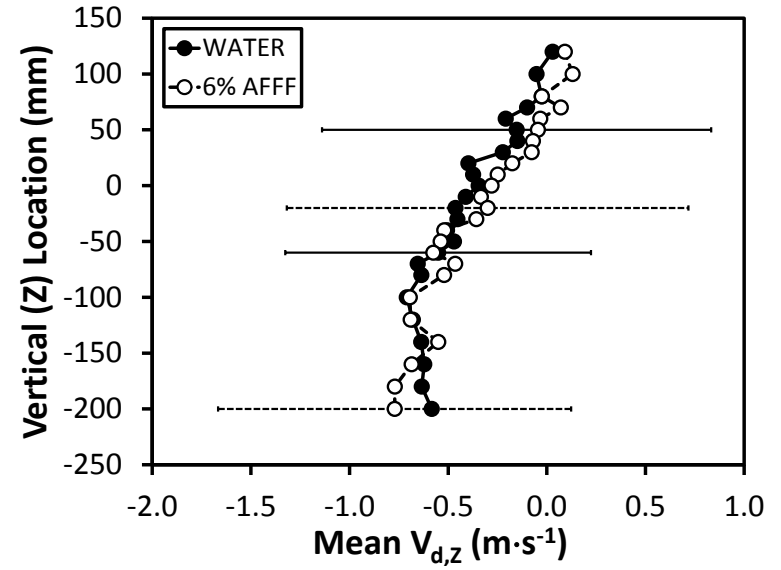

(b) $x=4.57 \mathrm{~m}(15 \mathrm{ft})$

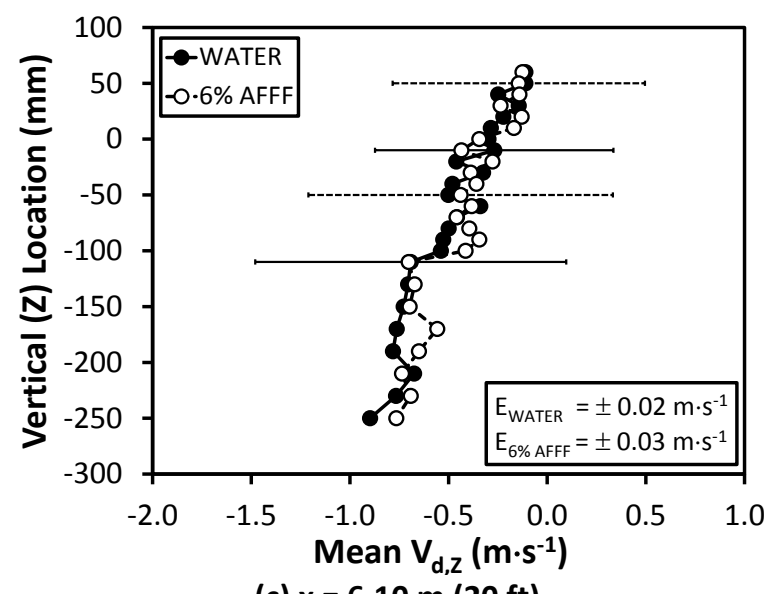

(c) $x=6.10 \mathrm{~m}(20 \mathrm{ft})$

Figure C.4: Far Field Low Flow, Low Press. Jet Vertical Profiles of Mean Vertical Droplet Velocity 


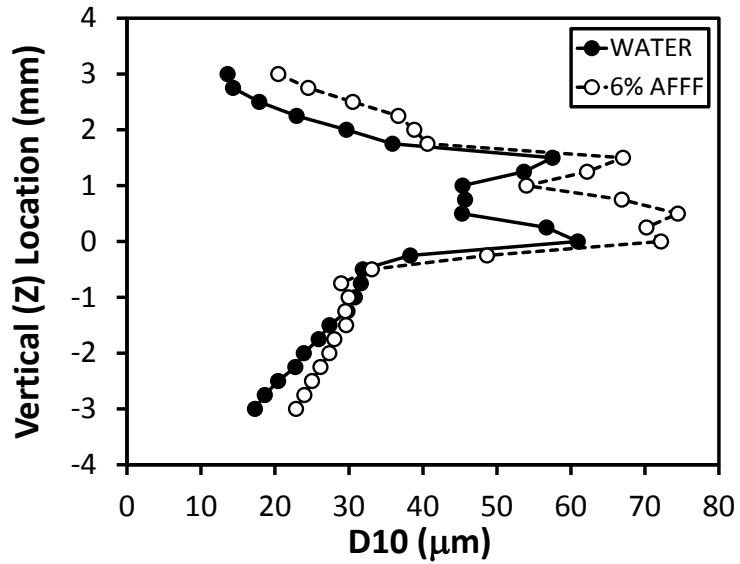

(a) $x=6.4 \mathrm{~mm}(0.25 \mathrm{in})$

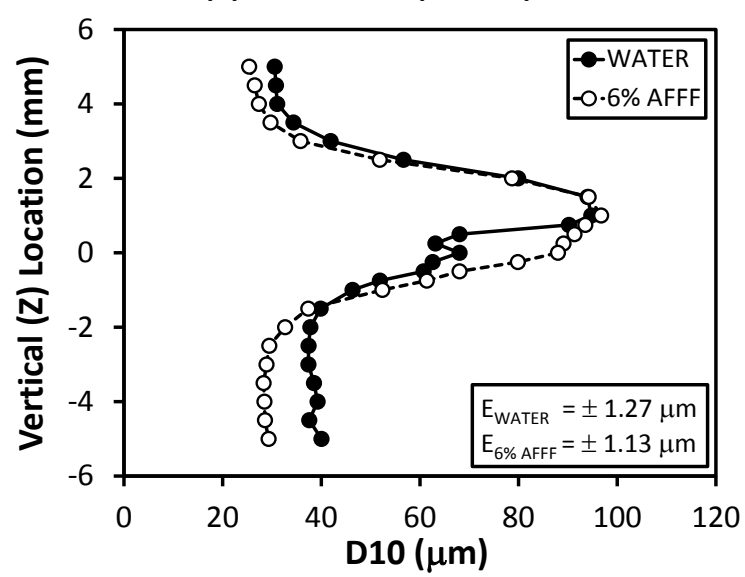

(c) $x=0.152 \mathrm{~m} \mathrm{(6} \mathrm{in)}$

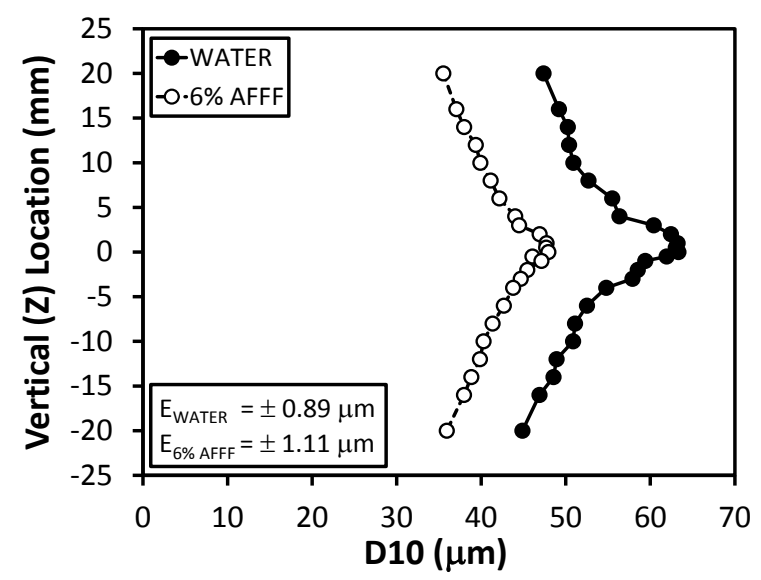

(e) $x=0.914 \mathrm{~m} \mathrm{(3 \textrm {ft } )}$

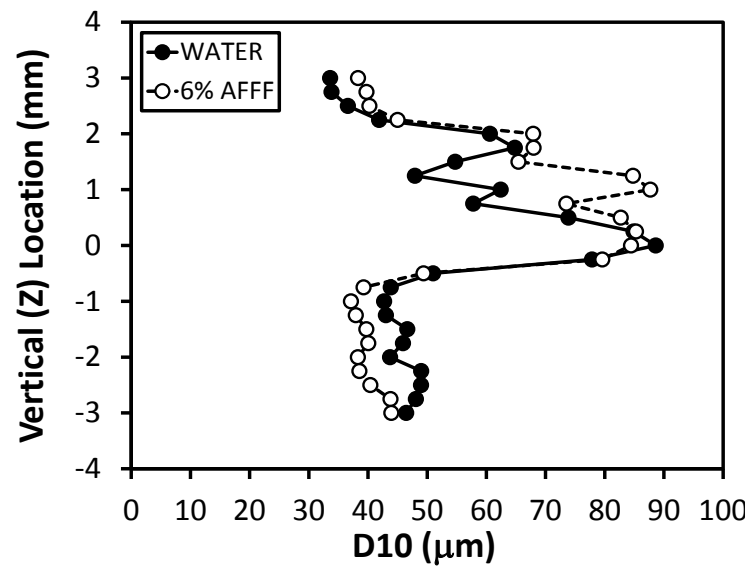

(b) $x=25.4 \mathrm{~mm}(1 \mathrm{in})$

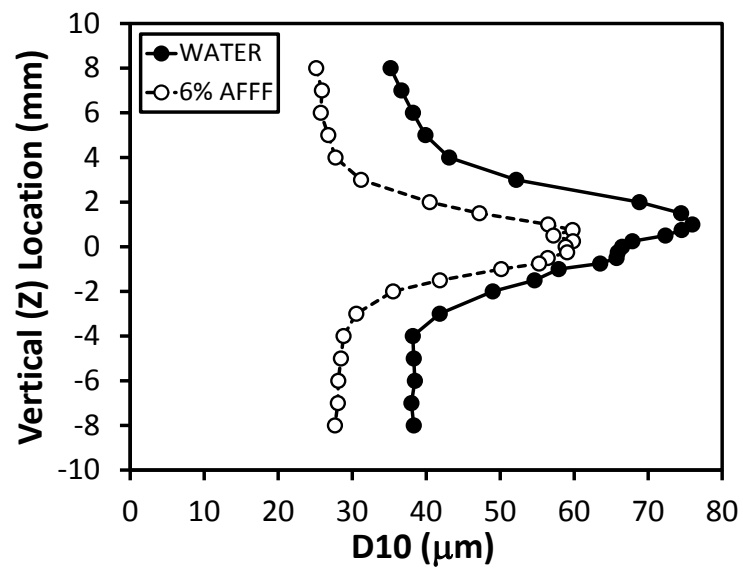

(d) $x=0.305 \mathrm{~m}(1 \mathrm{ft})$

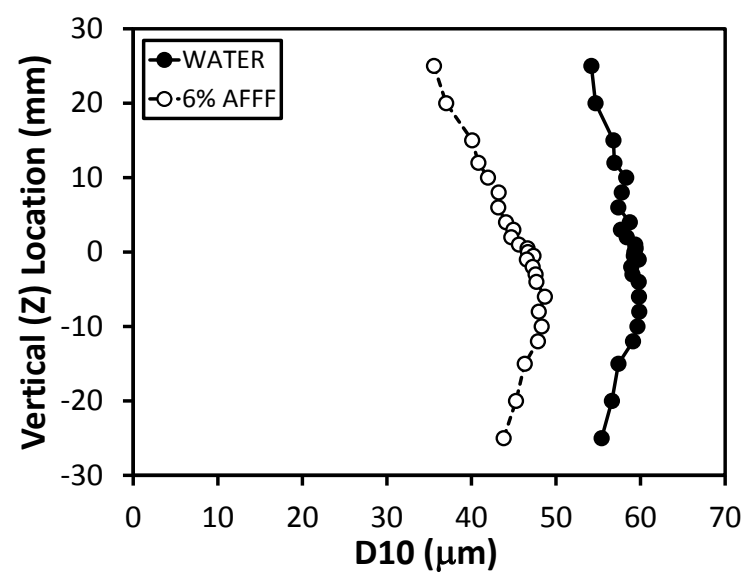

(f) $x=1.52 \mathrm{~m}(5 \mathrm{ft})$

Figure C.5: Near Field Low Flow, Low Press. Jet Vertical Profiles of Mean Droplet Diameter 


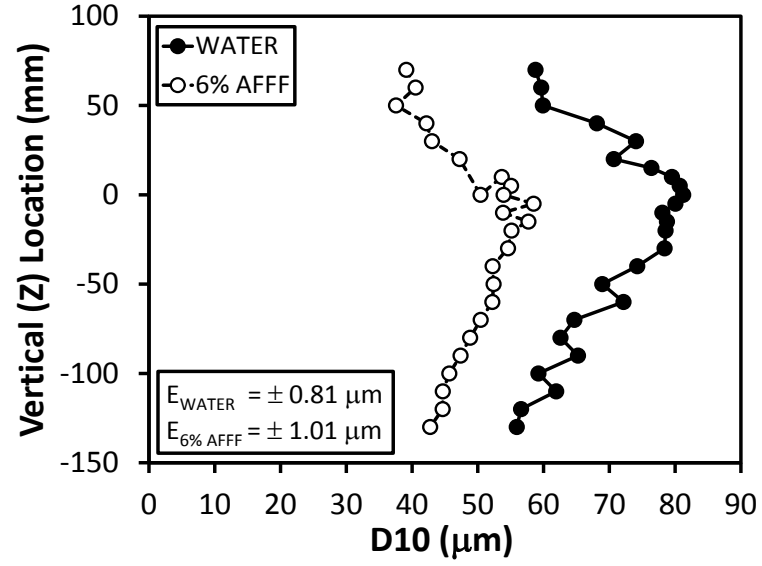

(a) $x=3.05 \mathrm{~m}(10 \mathrm{ft})$

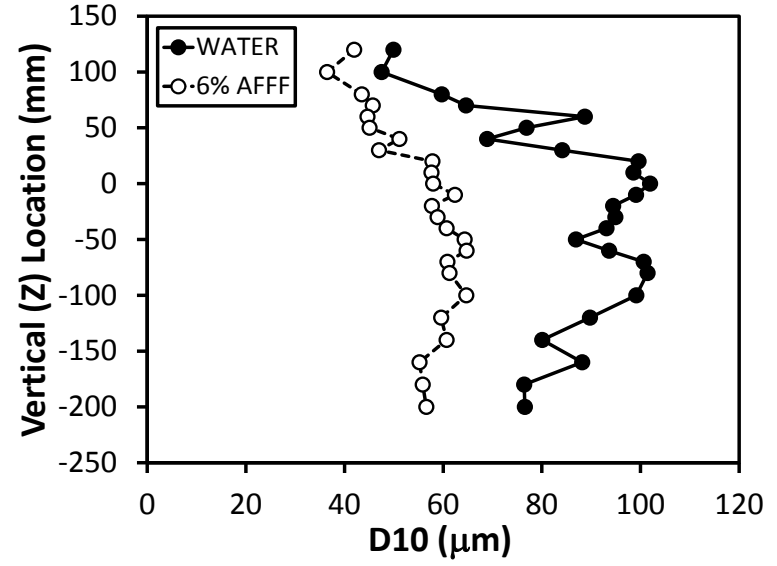

(b) $x=4.57 \mathrm{~m} \mathrm{(15 \textrm {ft } )}$

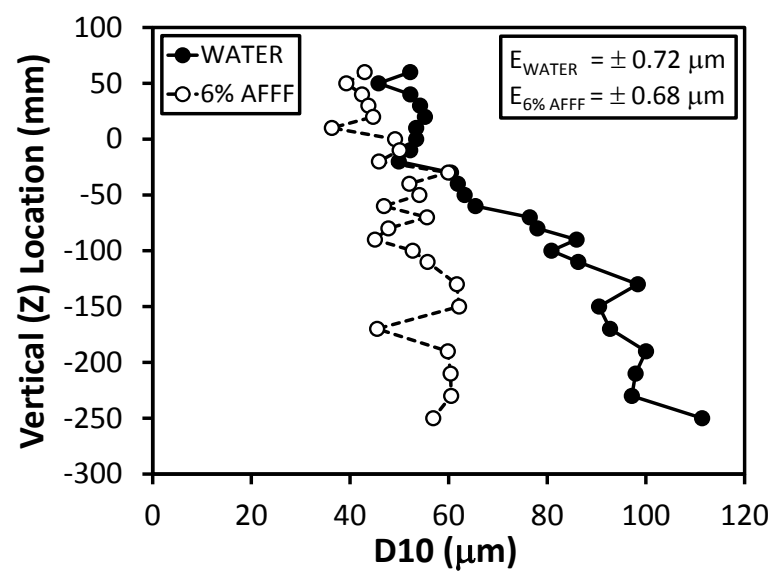

(c) $\mathrm{x}=6.10 \mathrm{~m}(20 \mathrm{ft})$

Figure C.6: Far Field Low Flow, Low Press. Jet Vertical Profiles of Mean Droplet Diameter 


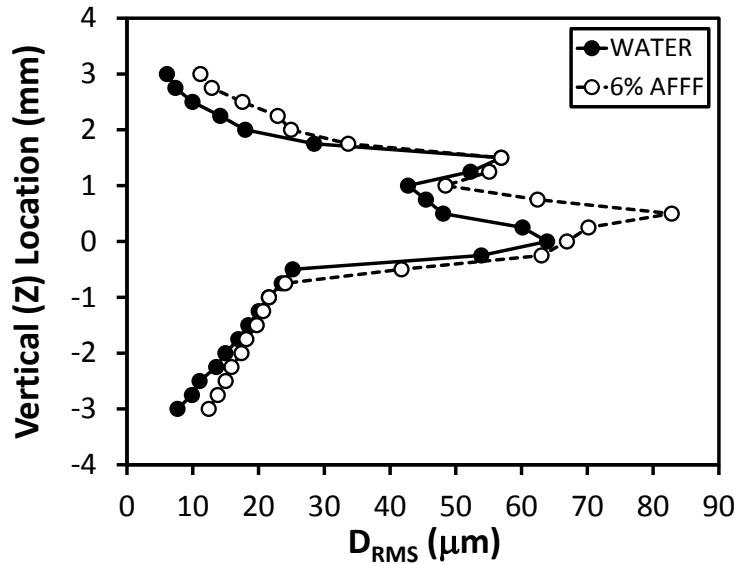

(a) $x=6.4 \mathrm{~mm}(0.25 \mathrm{in})$

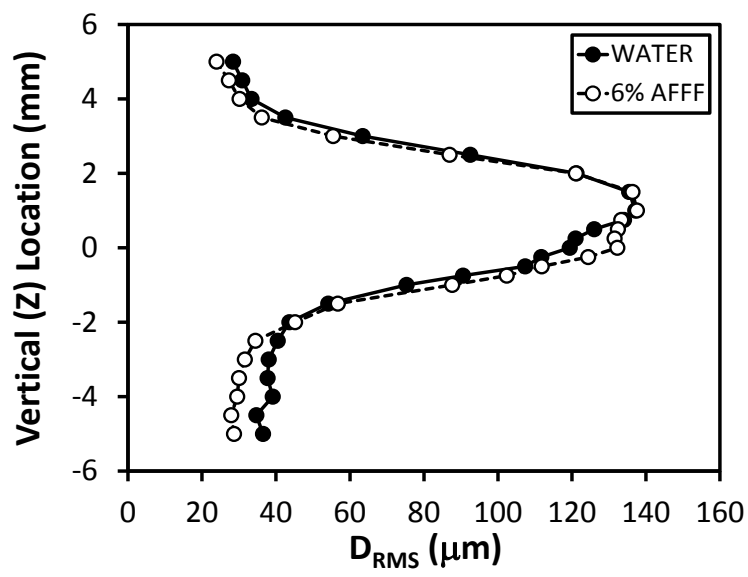

(c) $x=0.152 \mathrm{~m}(6 \mathrm{in})$

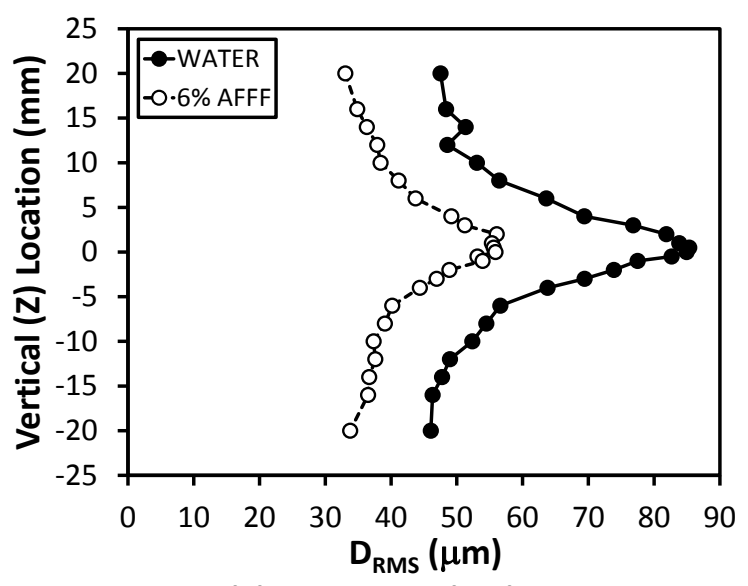

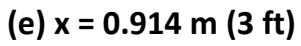

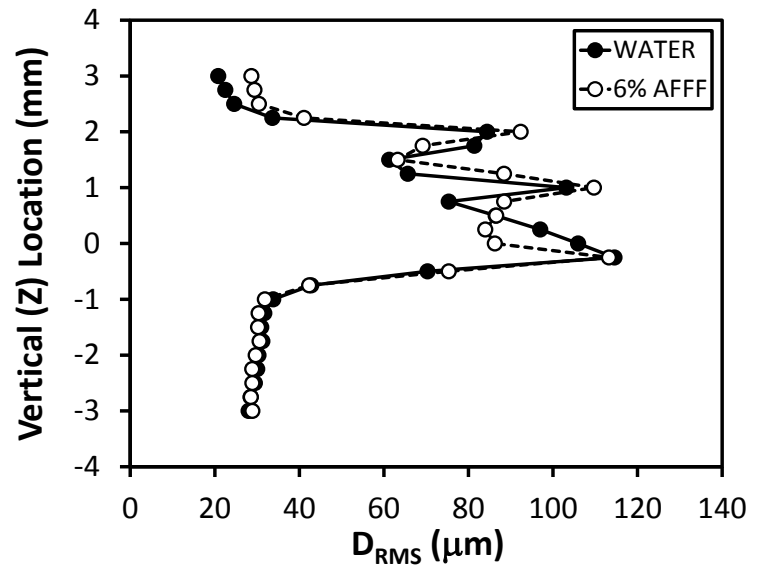

(b) $x=25.4 \mathrm{~mm}(1 \mathrm{in})$

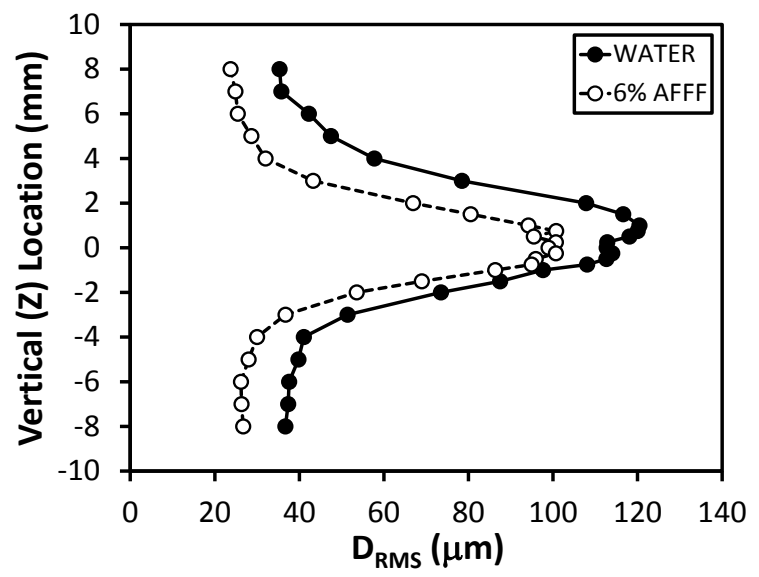

(d) $x=0.305 \mathrm{~m}(1 \mathrm{ft})$

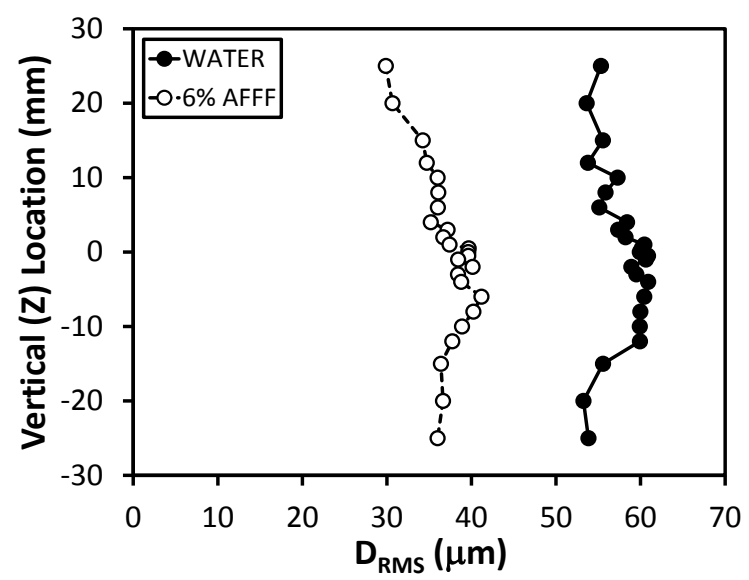

(f) $x=1.52 \mathrm{~m}(5 \mathrm{ft})$

Figure C.7: Near Field Low Flow, Low Press. Jet Vertical Profiles of RMS Droplet Diameter 


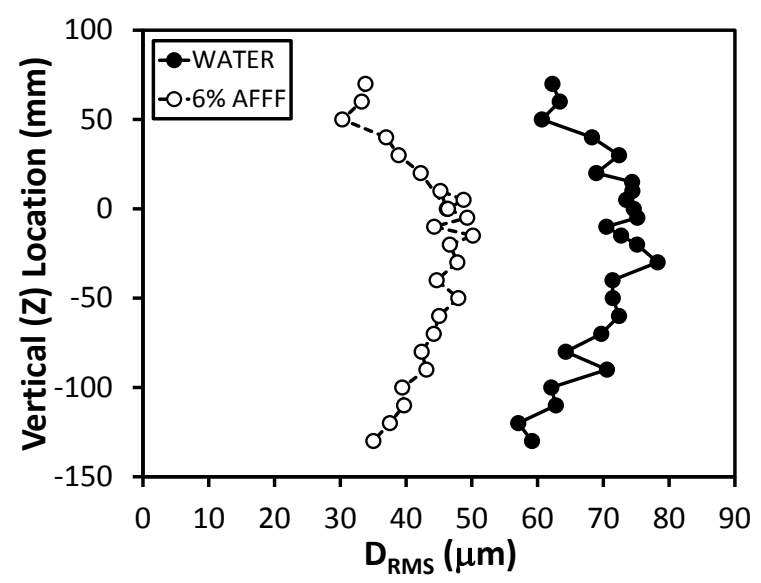

(a) $x=3.05 \mathrm{~m}(10 \mathrm{ft})$

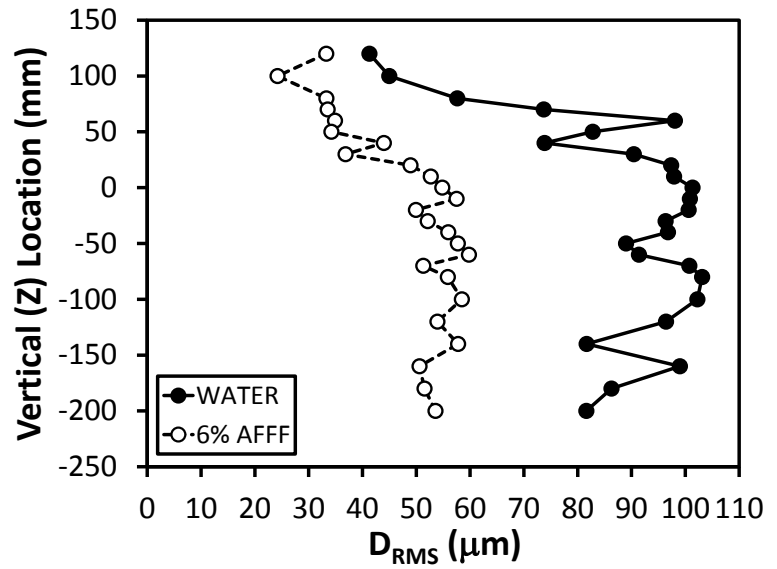

(b) $x=4.57 \mathrm{~m} \mathrm{(15 \textrm {ft } )}$

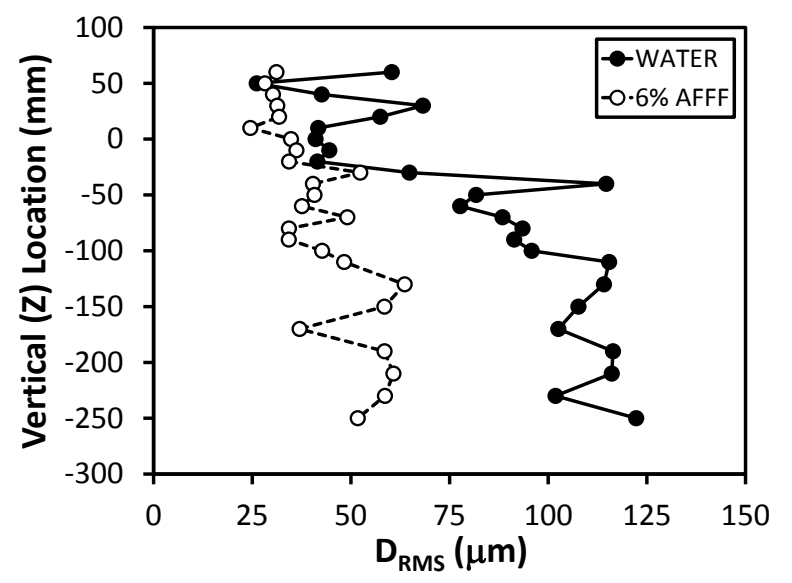

(c) $x=6.10 \mathrm{~m}(20 \mathrm{ft})$

Figure C.8: Far Field Low Flow, Low Press. Jet Vertical Profiles of RMS Droplet Diameter 


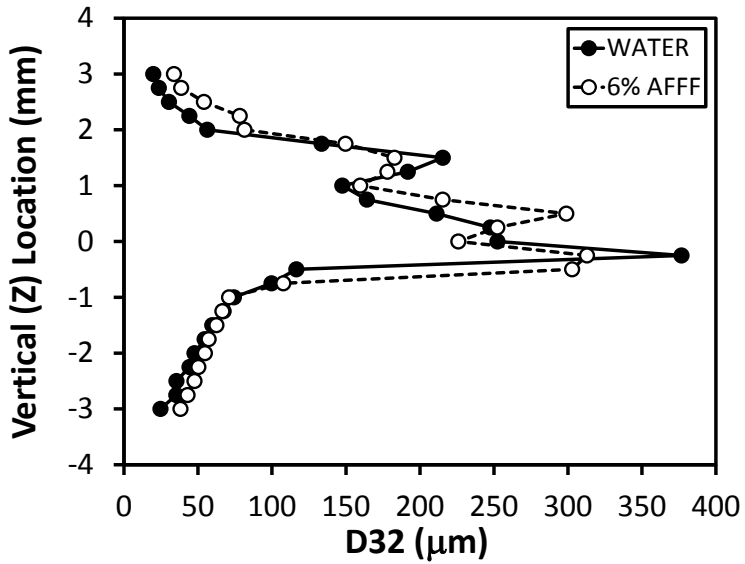

(a) $x=6.4 \mathrm{~mm}(0.25 \mathrm{in})$

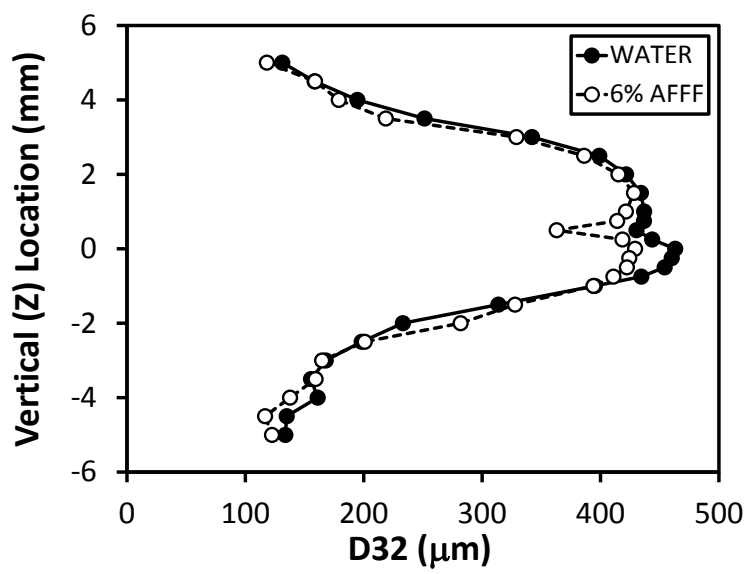

(c) $x=0.152 \mathrm{~m}(6 \mathrm{in})$

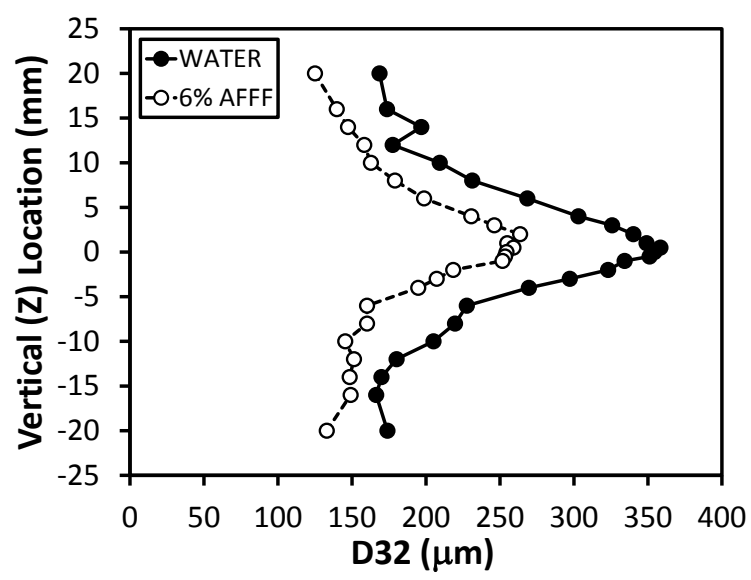

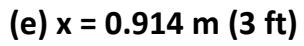

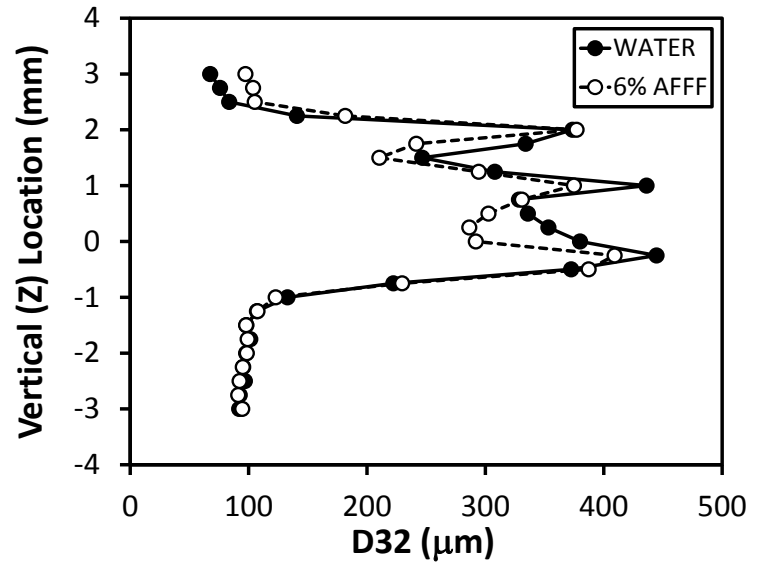

(b) $x=25.4 \mathrm{~mm}(1 \mathrm{in})$

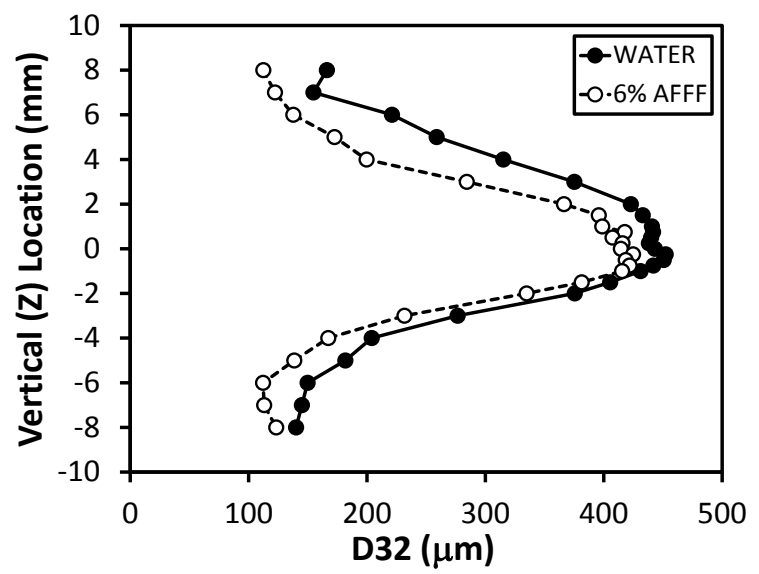

(d) $x=0.305 \mathrm{~m}(1 \mathrm{ft})$

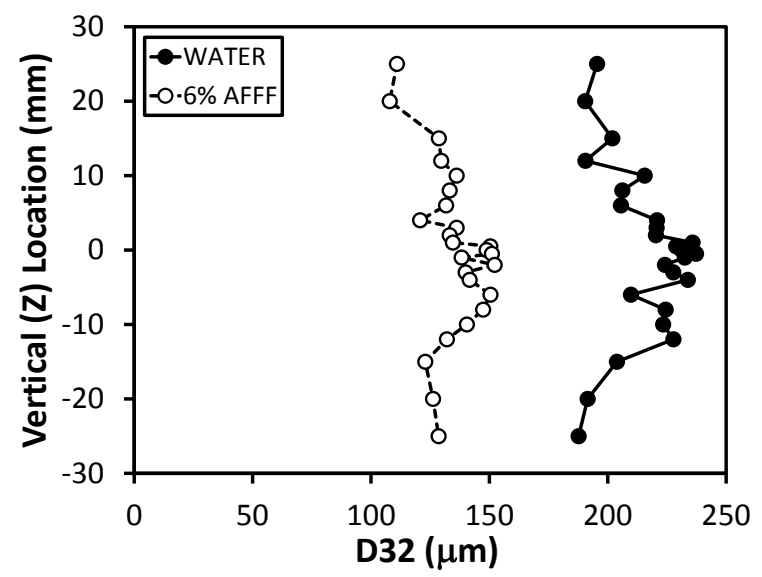

(f) $x=1.52 \mathrm{~m}(5 \mathrm{ft})$

Figure C.9: Near Field Low Flow, Low Press. Jet Vertical Profiles of Sauter Mean Droplet Diameter 


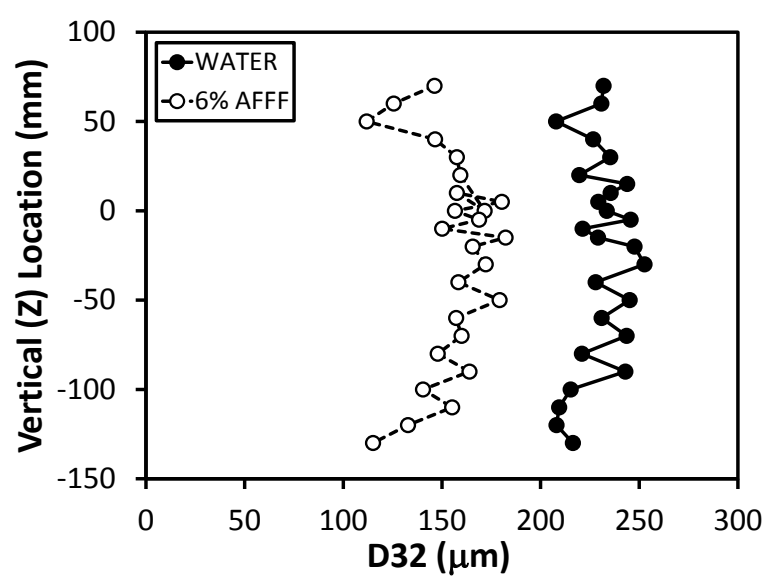

(a) $x=3.05 \mathrm{~m}(10 \mathrm{ft})$

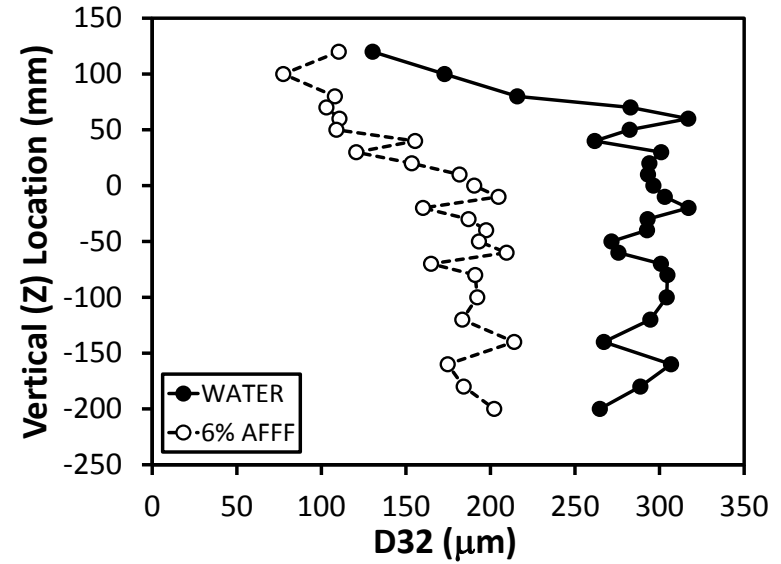

(b) $x=4.57 \mathrm{~m} \mathrm{(15 \textrm {ft } )}$

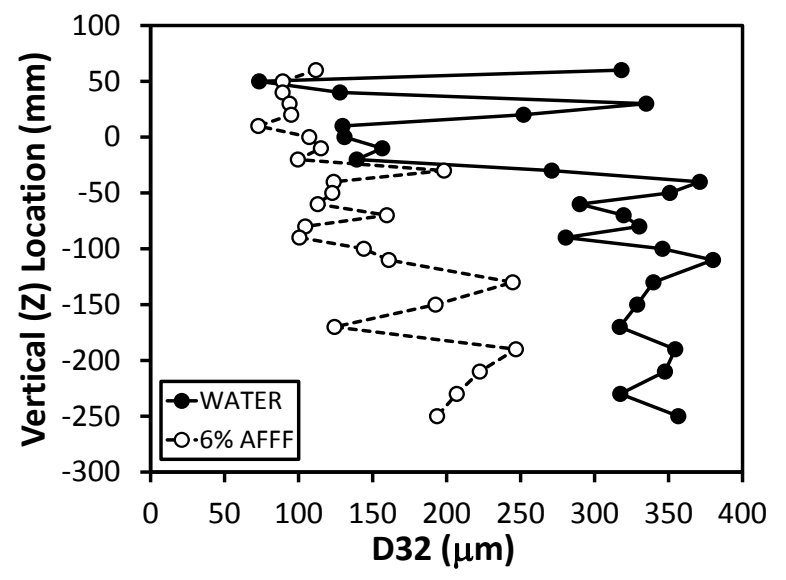

(c) $x=6.10 \mathrm{~m}(20 \mathrm{ft})$

Figure C.10: Far Field Low Flow, Low Press. Jet Vertical Profiles of Sauter Mean Droplet Diameter 


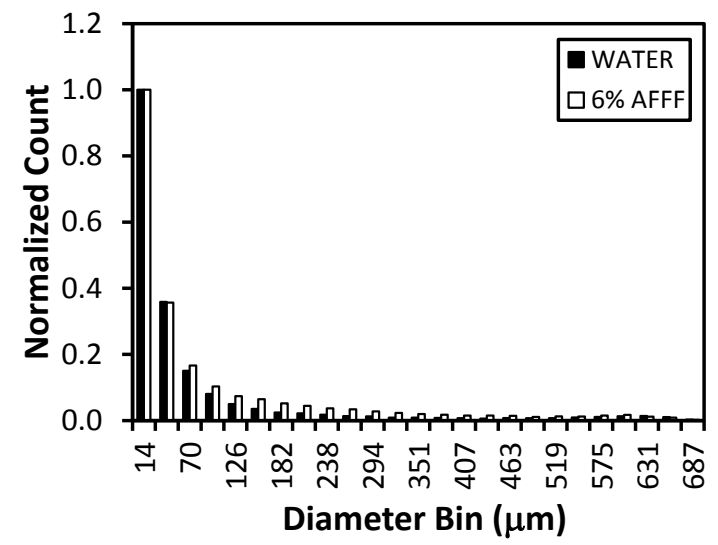

(a) $x=0.152 \mathrm{~m}(6 \mathrm{in})$

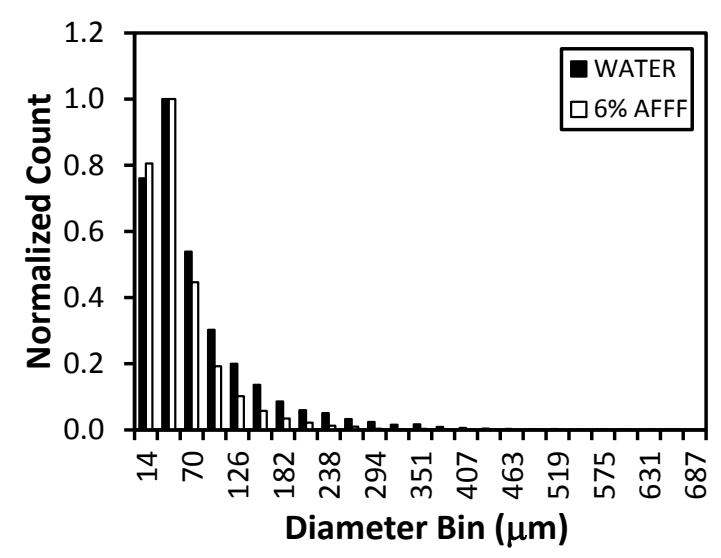

(c) $\mathrm{x}=3.05 \mathrm{~m}(10 \mathrm{ft})$

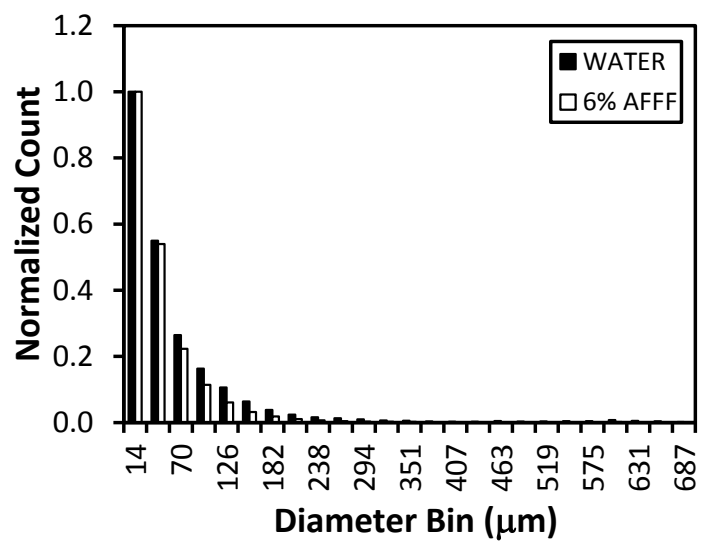

(b) $x=0.914 \mathrm{~m} \mathrm{(3 \textrm {ft } )}$

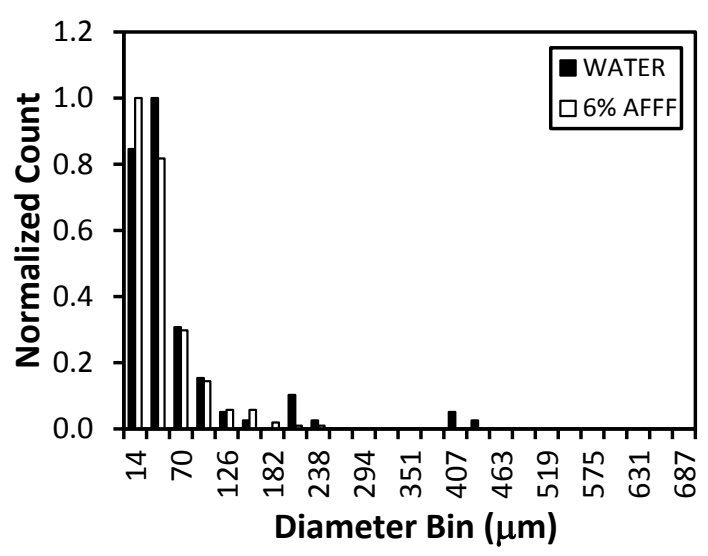

(d) $x=6.10 \mathrm{~m}(20 \mathrm{ft})$

Figure C.11: Low Flow, Low Press. Jet Droplet Size Distribution at the Max. Profile, Mean Axial Droplet Velocity for Select Vertical Profiles 


\section{C.2 Low Flow, High Pressure Firefighting Jet Data}

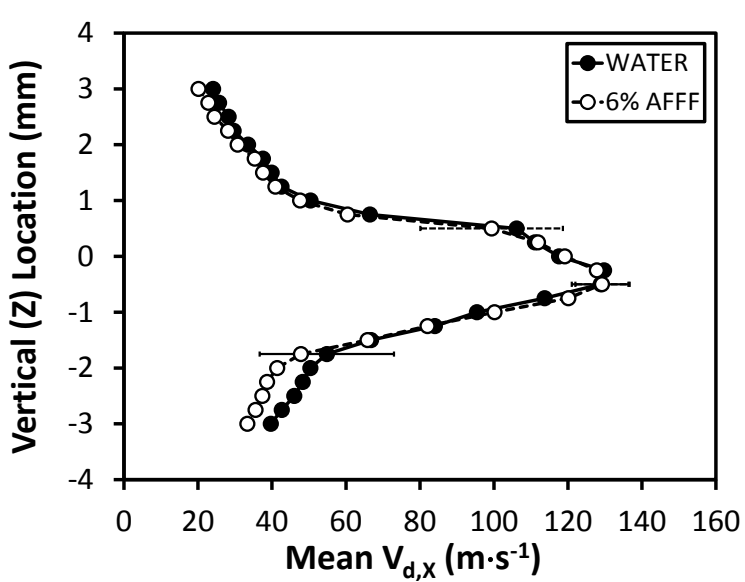

(a) $x=6.4 \mathrm{~mm}(0.25 \mathrm{in})$

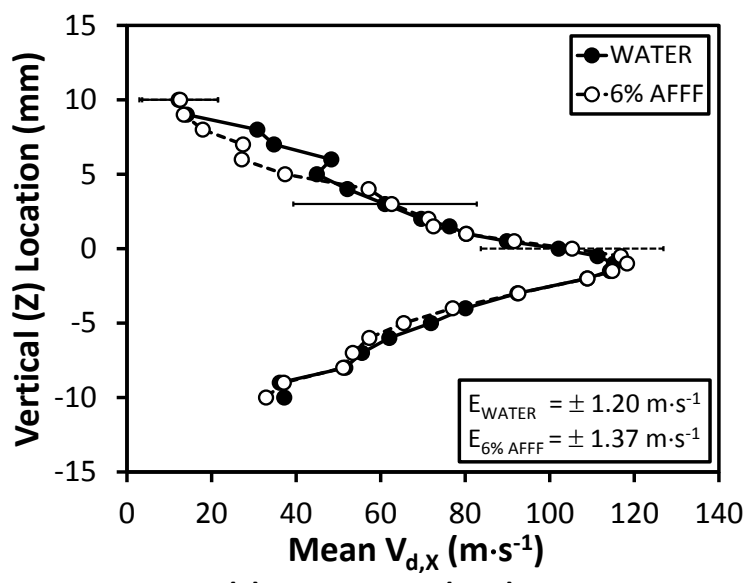

(c) $x=0.152 \mathrm{~m}(6 \mathrm{in})$

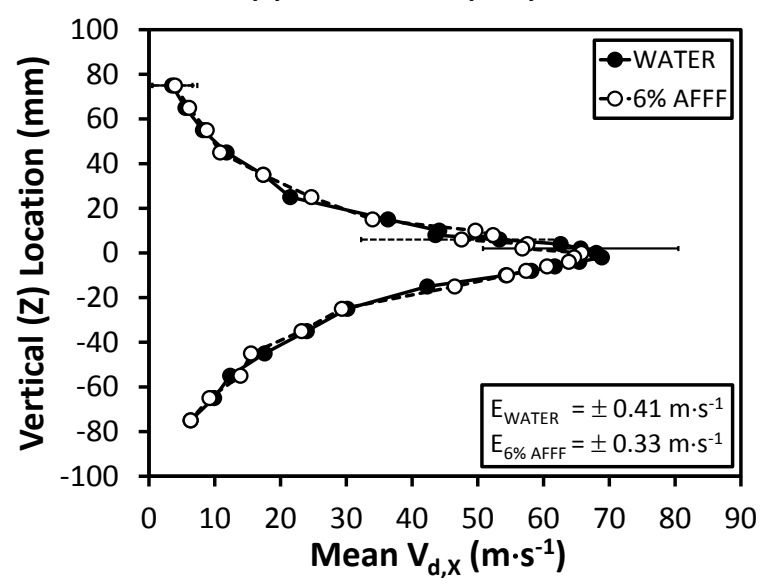

(e) $x=0.914 \mathrm{~m}(3 \mathrm{ft})$

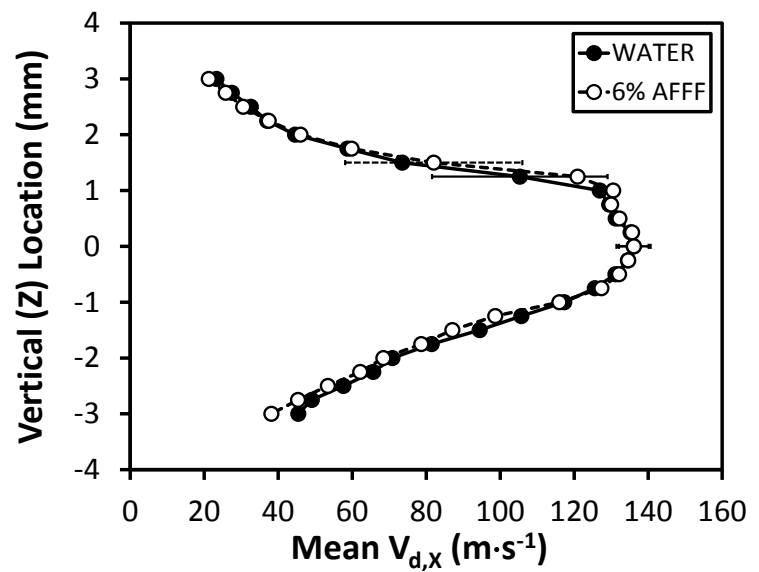

(b) $x=25.4 \mathrm{~mm}$ (1 in)

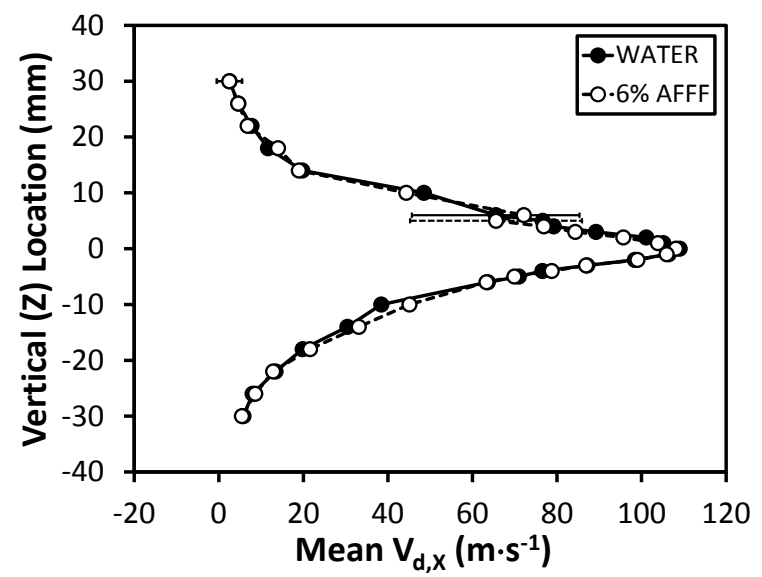

(d) $\mathrm{x}=0.305 \mathrm{~m}(\mathbf{1 f t})$

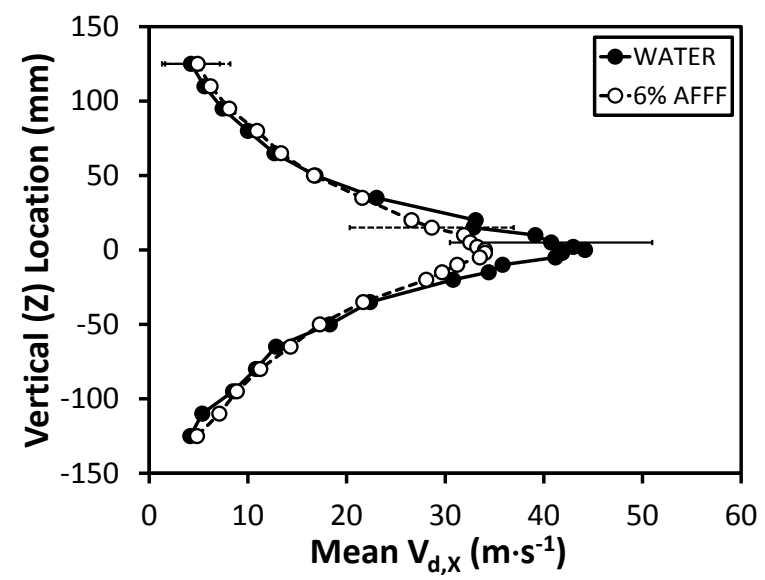

(f) $x=1.52 \mathrm{~m}(5 \mathrm{ft})$

Figure C.12: Near Field Low Flow, High Press. Jet Vertical Profiles of Mean Axial Droplet Velocity 

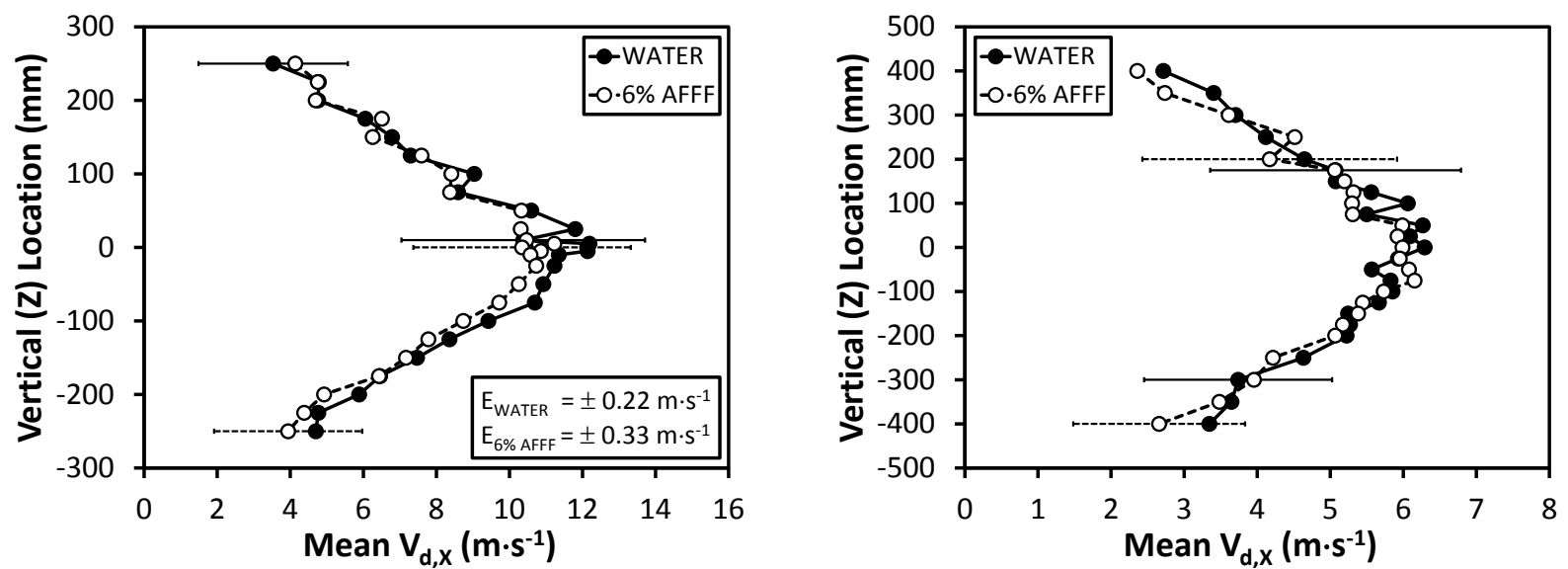

(a) $x=3.05 \mathrm{~m}(10 \mathrm{ft})$

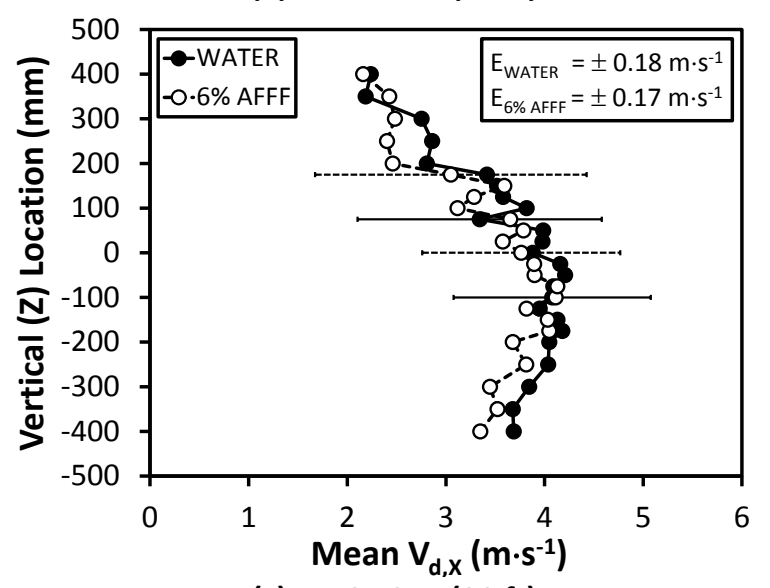

(c) $x=6.10 \mathrm{~m}(20 \mathrm{ft})$

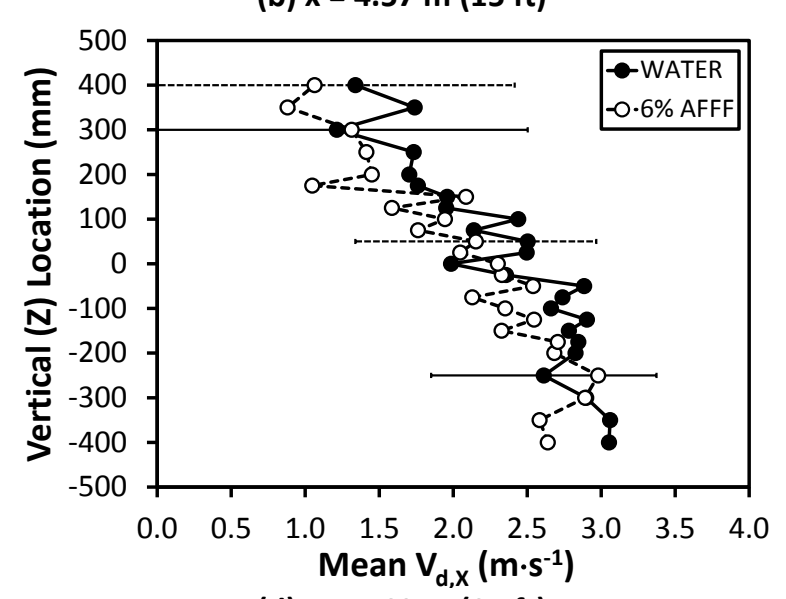

(d) $x=7.62 \mathrm{~m}(25 \mathrm{ft})$

Figure C.13: Far Field Low Flow, High Press. Jet Vertical Profiles of Mean Axial Droplet Velocity 


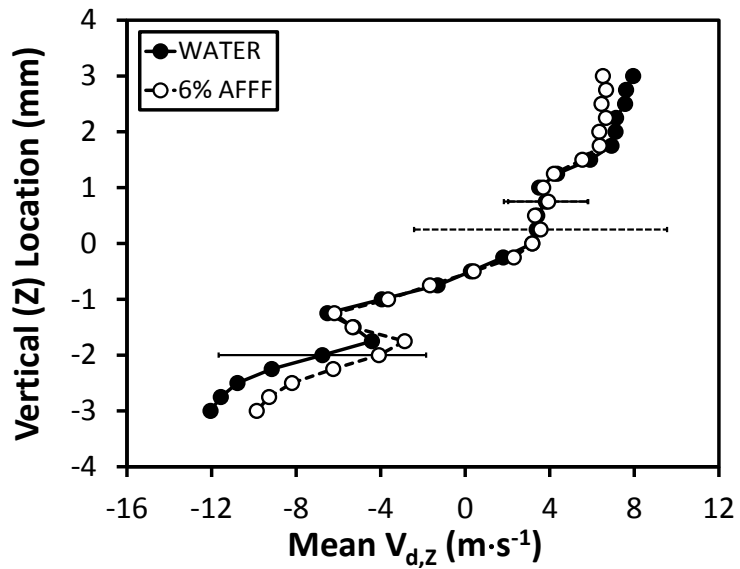

(a) $x=6.4 \mathrm{~mm}$ (0.25 in)

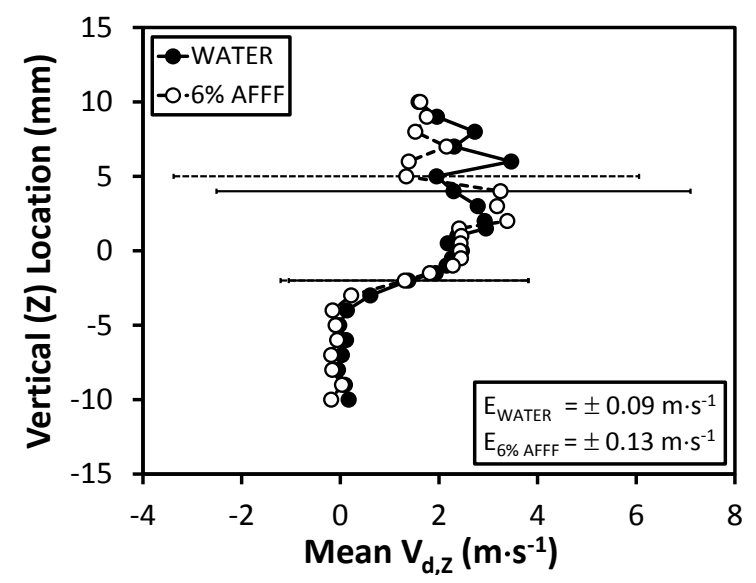

(c) $x=0.152 \mathrm{~m}(6 \mathrm{in})$

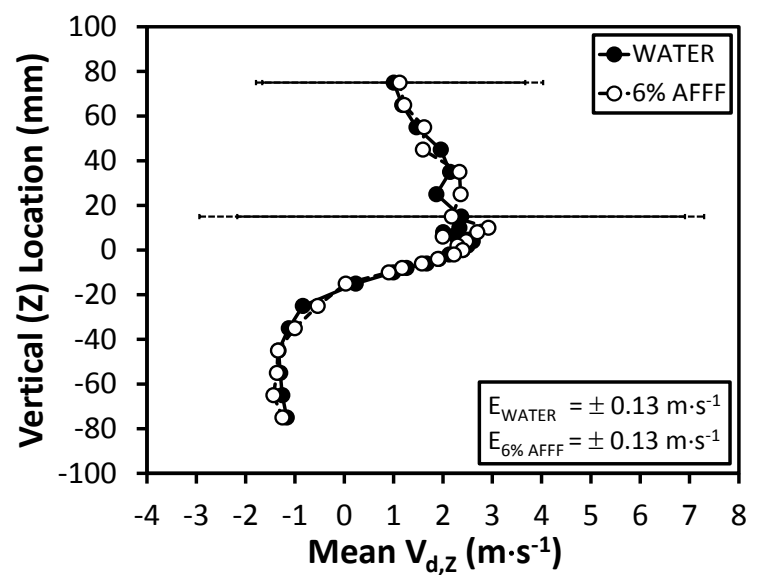

(e) $x=0.914 \mathrm{~m} \mathrm{(3 \textrm {ft } )}$

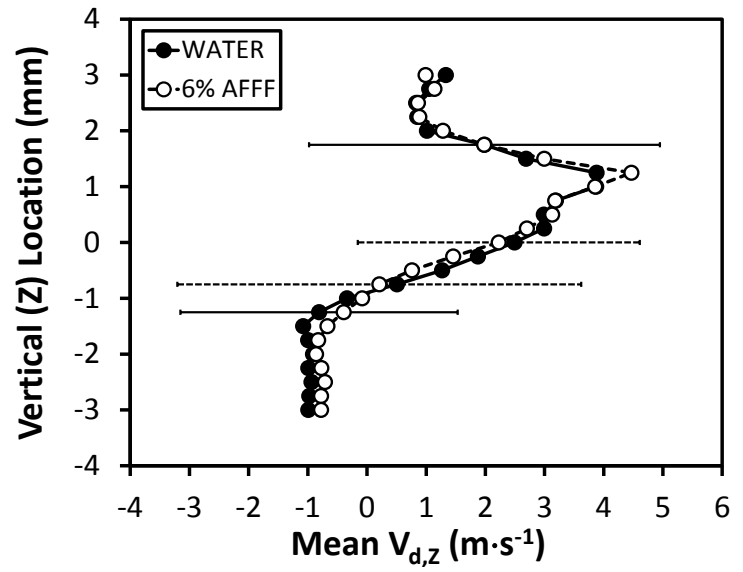

(b) $x=25.4 \mathrm{~mm}(1 \mathrm{in})$

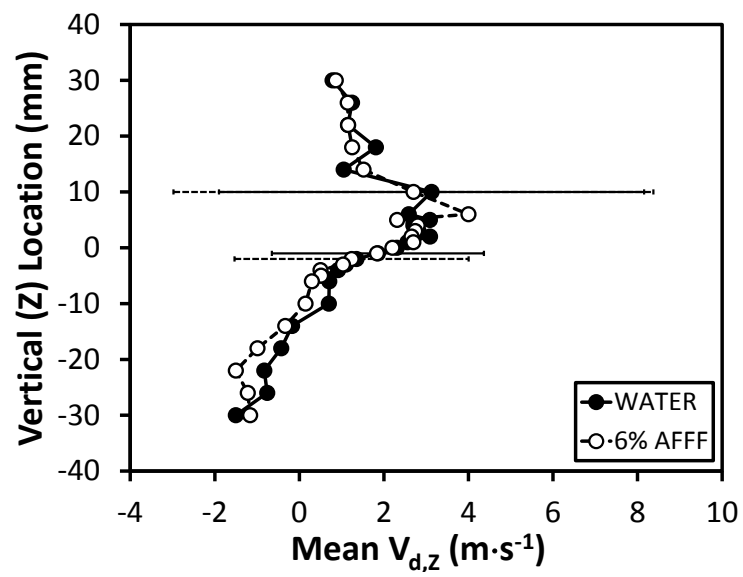

(d) $x=0.305 \mathrm{~m}(1 \mathrm{ft})$

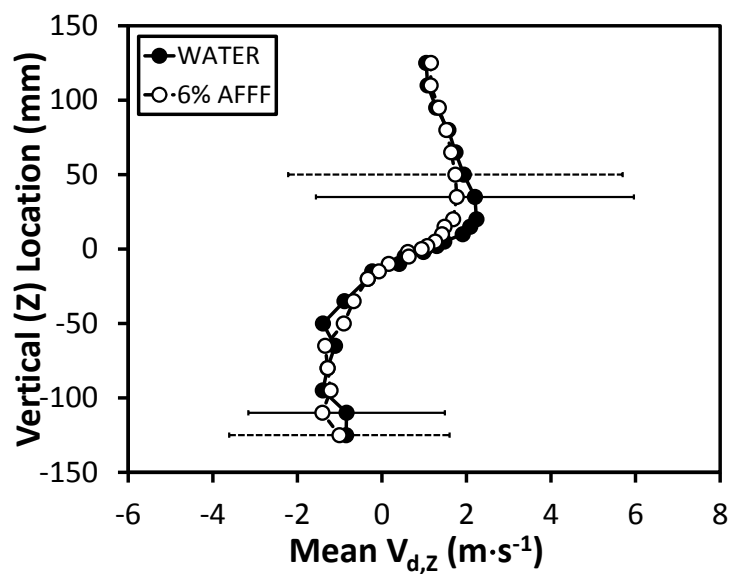

(f) $x=1.52 \mathrm{~m}(5 \mathrm{ft})$

Figure C.14: Near Field Low Flow, High Press. Jet Vertical Profiles of Mean Vertical Droplet Velocity 


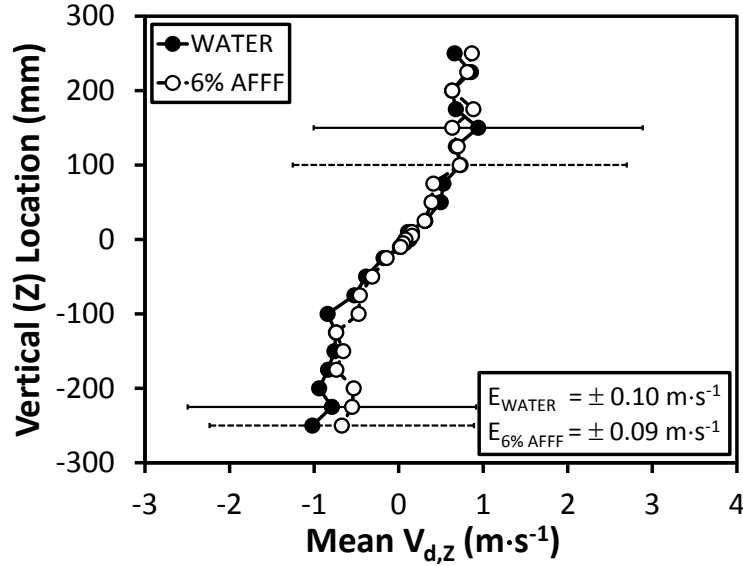

(a) $x=3.05 \mathrm{~m}(10 \mathrm{ft})$

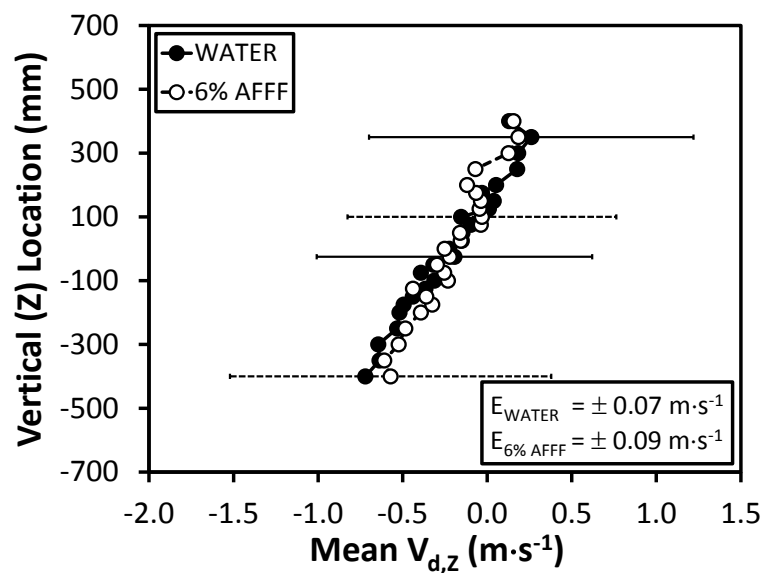

(c) $x=6.10 \mathrm{~m}(20 \mathrm{ft})$

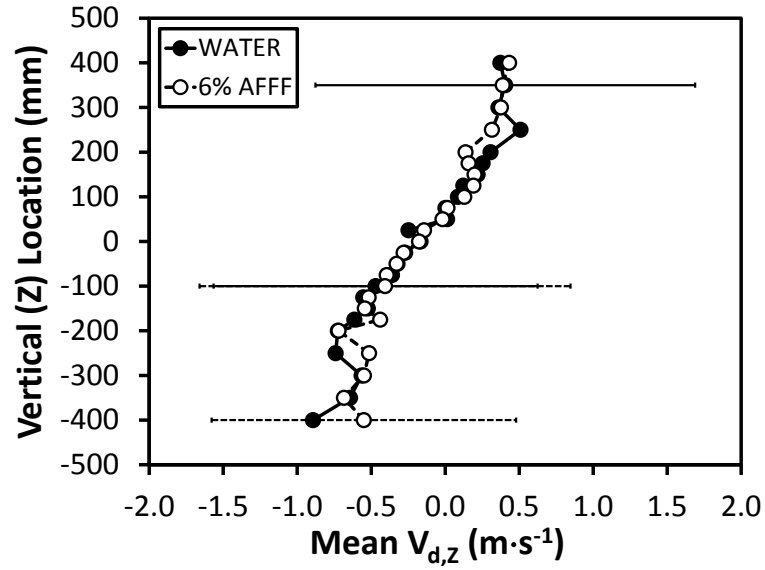

(b) $x=4.57 \mathrm{~m}(15 \mathrm{ft})$

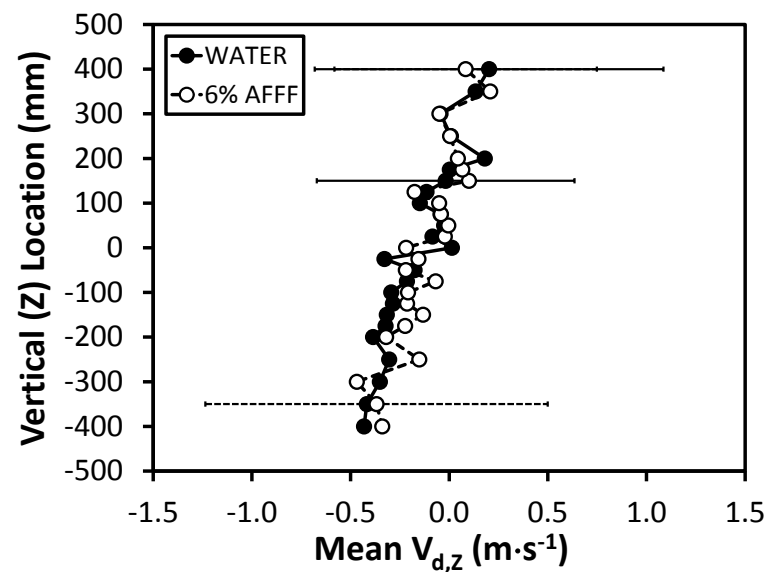

(d) $x=7.62 \mathrm{~m}(25 \mathrm{ft})$

Figure C.15: Far Field Low Flow, High Press. Jet Vertical Profiles of Mean Vertical Droplet Velocity 


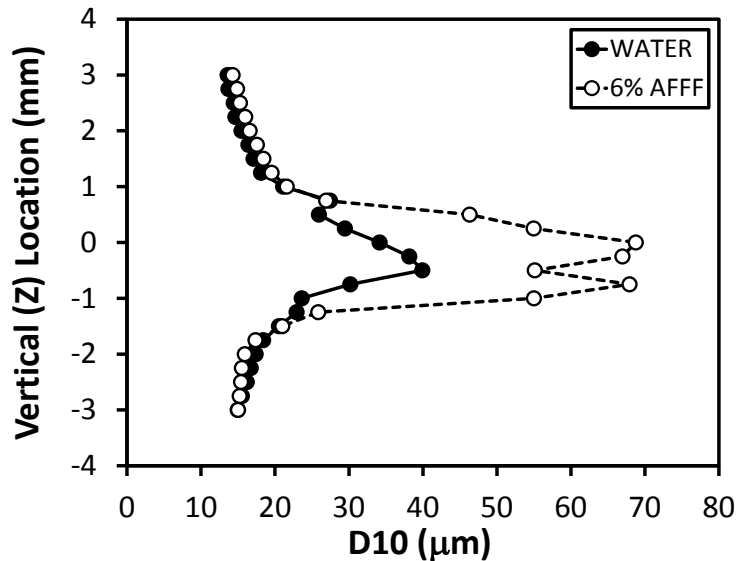

(a) $x=6.4 \mathrm{~mm}(0.25 \mathrm{in})$

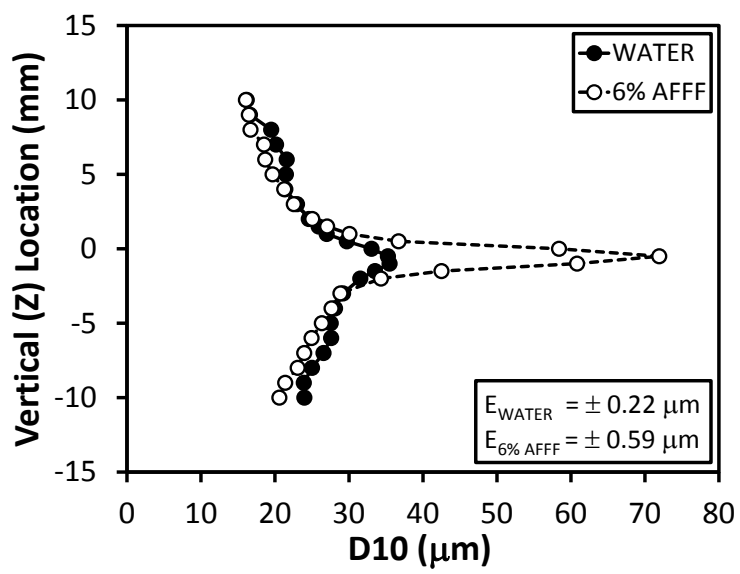

(c) $x=0.152 \mathrm{~m}(6$ in)

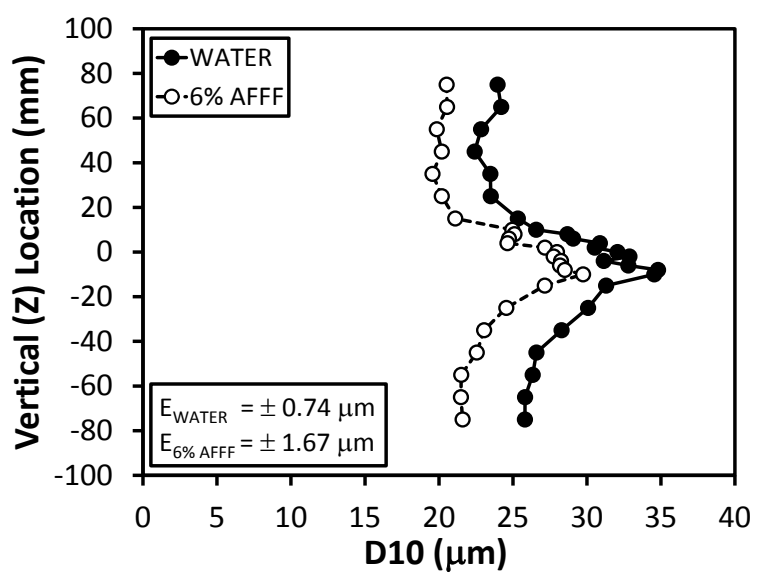

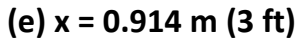

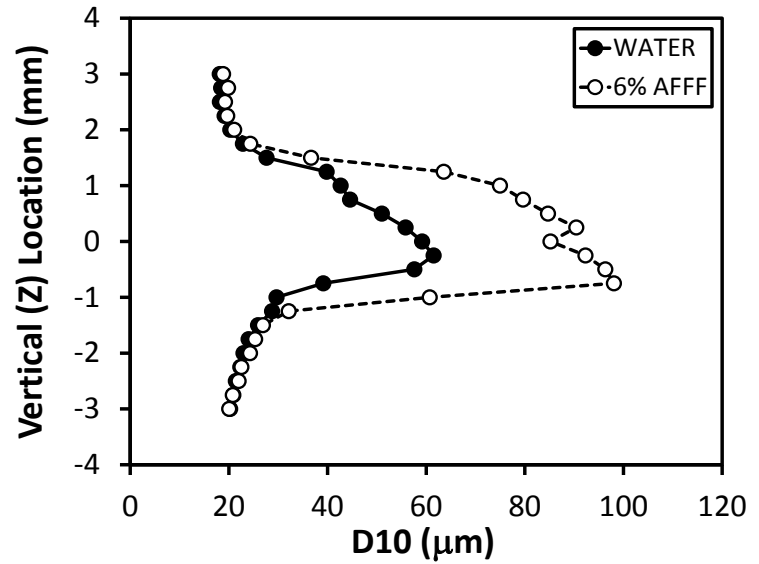

(b) $x=25.4 \mathrm{~mm}(1 \mathrm{in})$

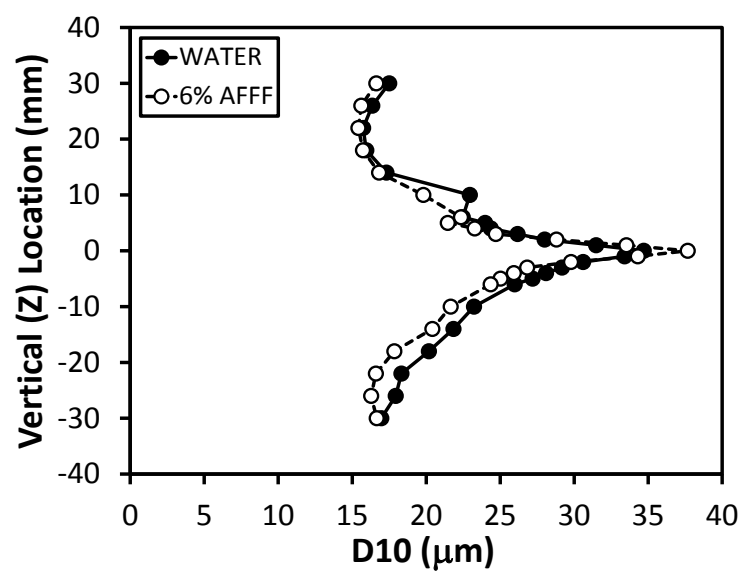

(d) $x=0.305 \mathrm{~m}(1 \mathrm{ft})$

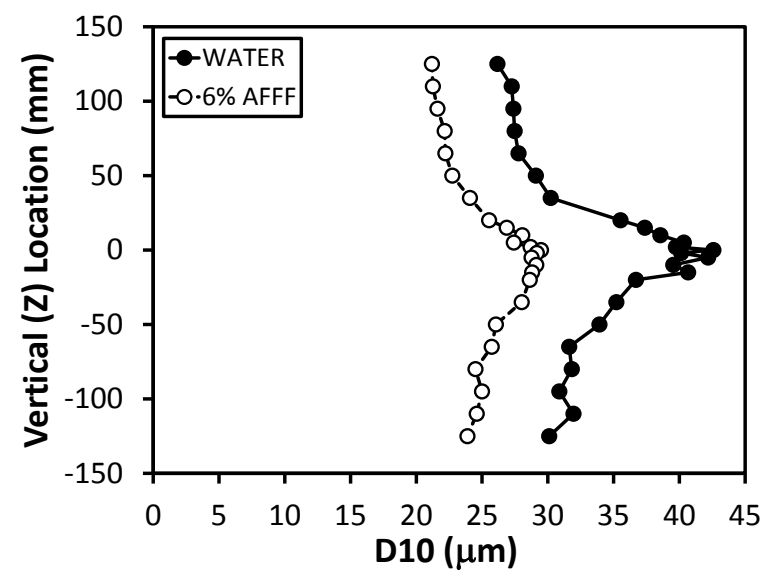

(f) $x=1.52 \mathrm{~m}(5 \mathrm{ft})$

Figure C.16: Near Field Low Flow, High Press. Jet Vertical Profiles of Mean Droplet Diameter 

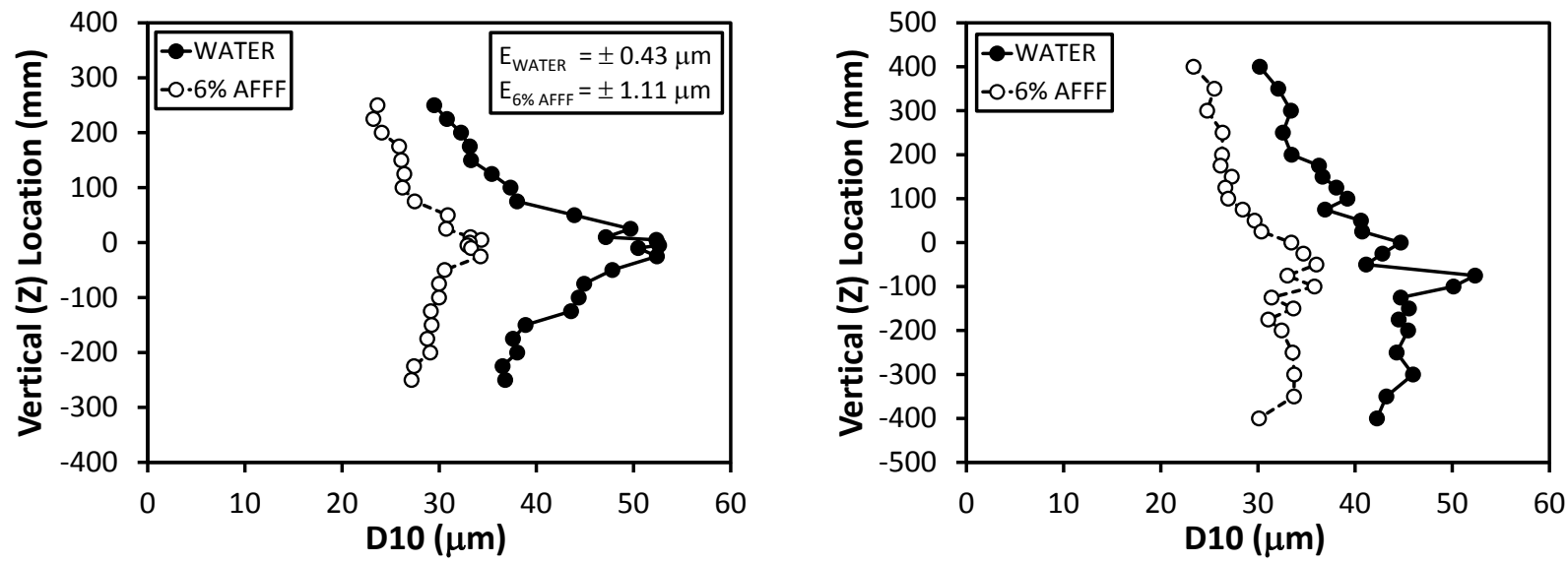

(a) $\mathrm{x}=3.05 \mathrm{~m}(10 \mathrm{ft})$

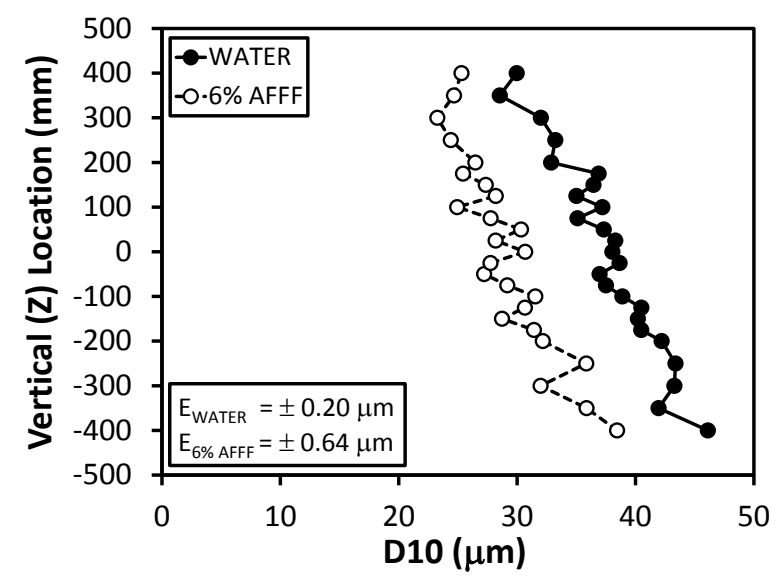

(c) $x=6.10 \mathrm{~m}(20 \mathrm{ft})$

(b) $x=4.57 \mathrm{~m} \mathrm{(15 \textrm {ft } )}$

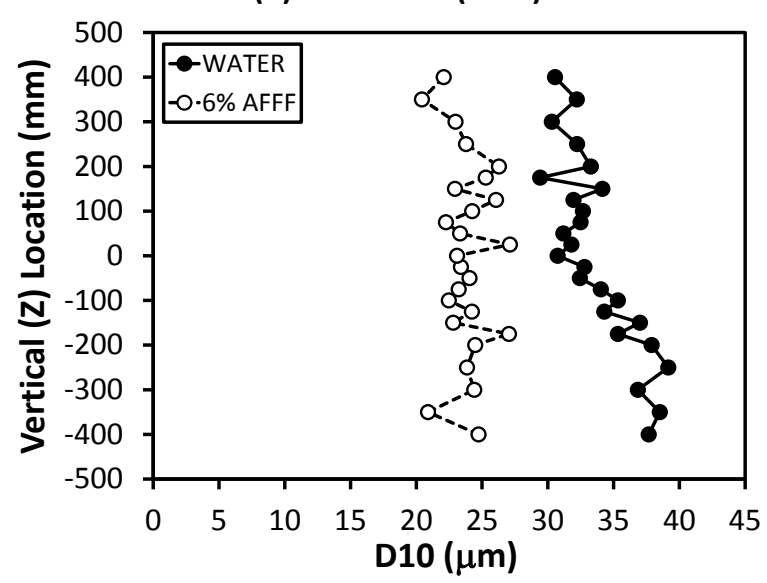

(d) $x=7.62 \mathrm{~m} \mathrm{(25 \textrm {ft } )}$

Figure C.17: Far Field Low Flow, High Press. Jet Vertical Profiles of Mean Droplet Diameter 


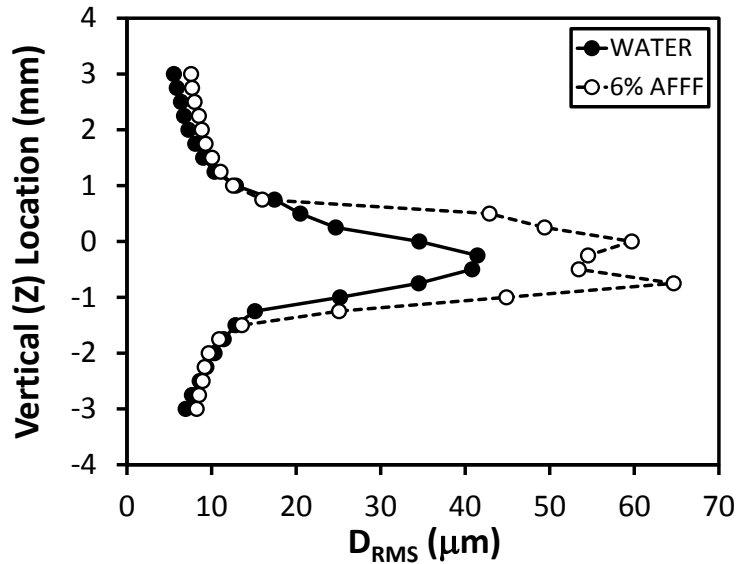

(a) $x=6.4 \mathrm{~mm}(0.25 \mathrm{in})$

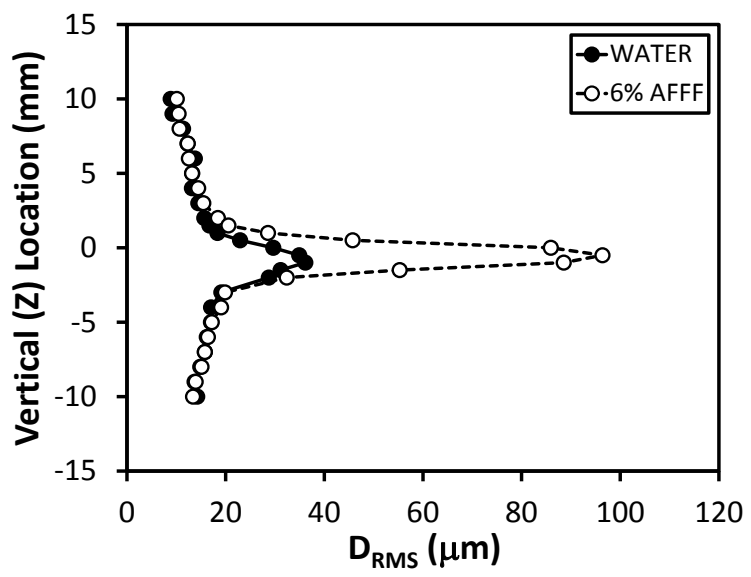

(c) $x=0.152 \mathrm{~m}(6 \mathrm{in})$

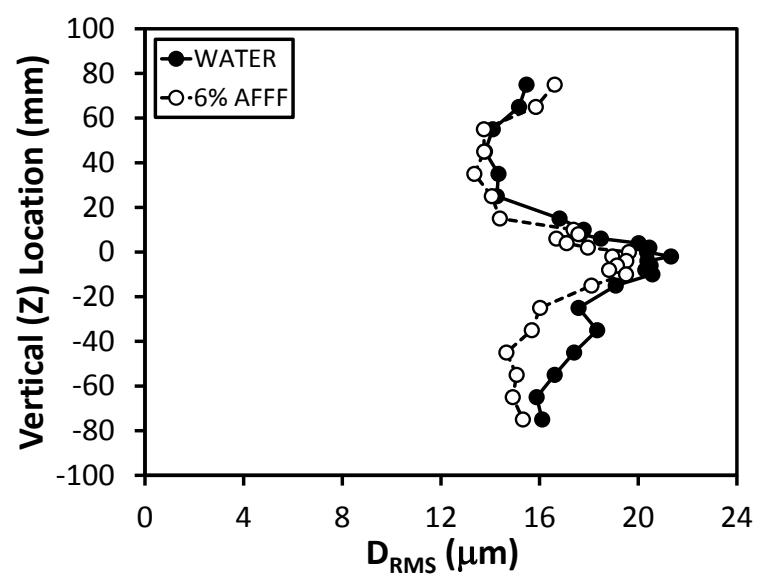

(e) $\mathrm{x}=0.914 \mathrm{~m}(3 \mathrm{ft})$

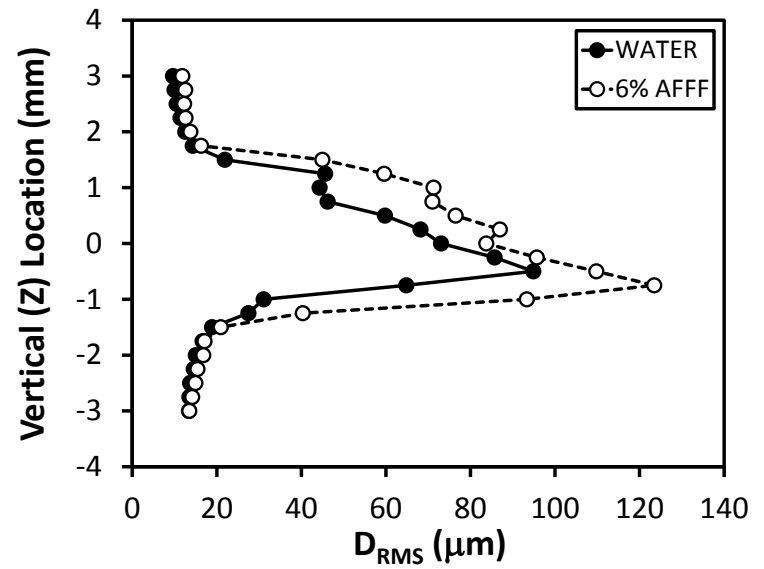

(b) $x=25.4 \mathrm{~mm}(1 \mathrm{in})$

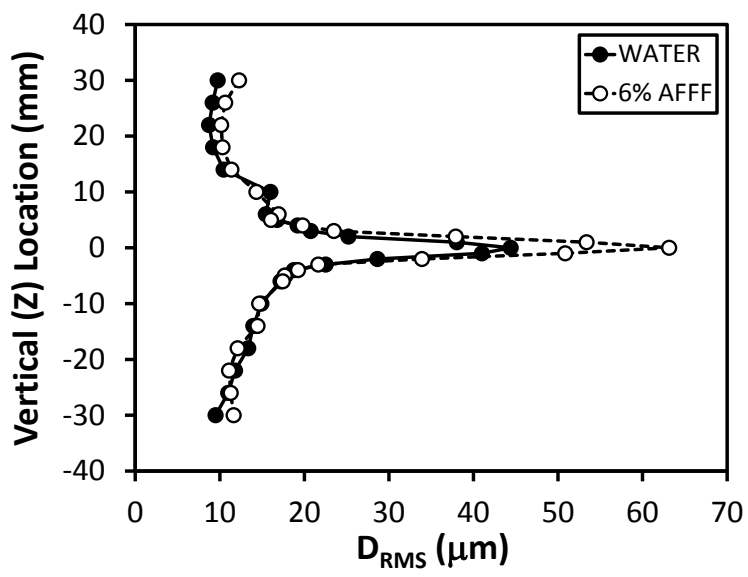

(d) $x=0.305 \mathrm{~m}(1 \mathrm{ft})$

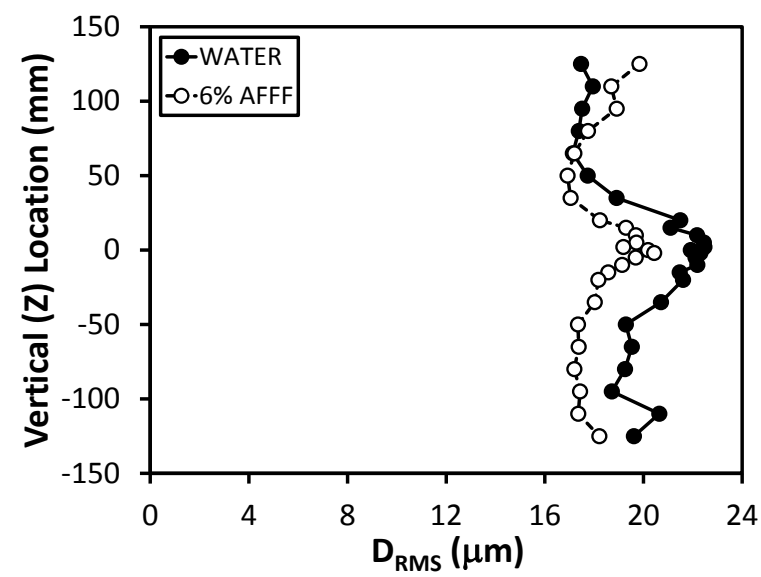

(f) $x=1.52 \mathrm{~m} \mathrm{(5 \textrm {ft } )}$

Figure C.18: Near Field Low Flow, High Press. Jet Vertical Profiles of RMS Droplet Diameter 

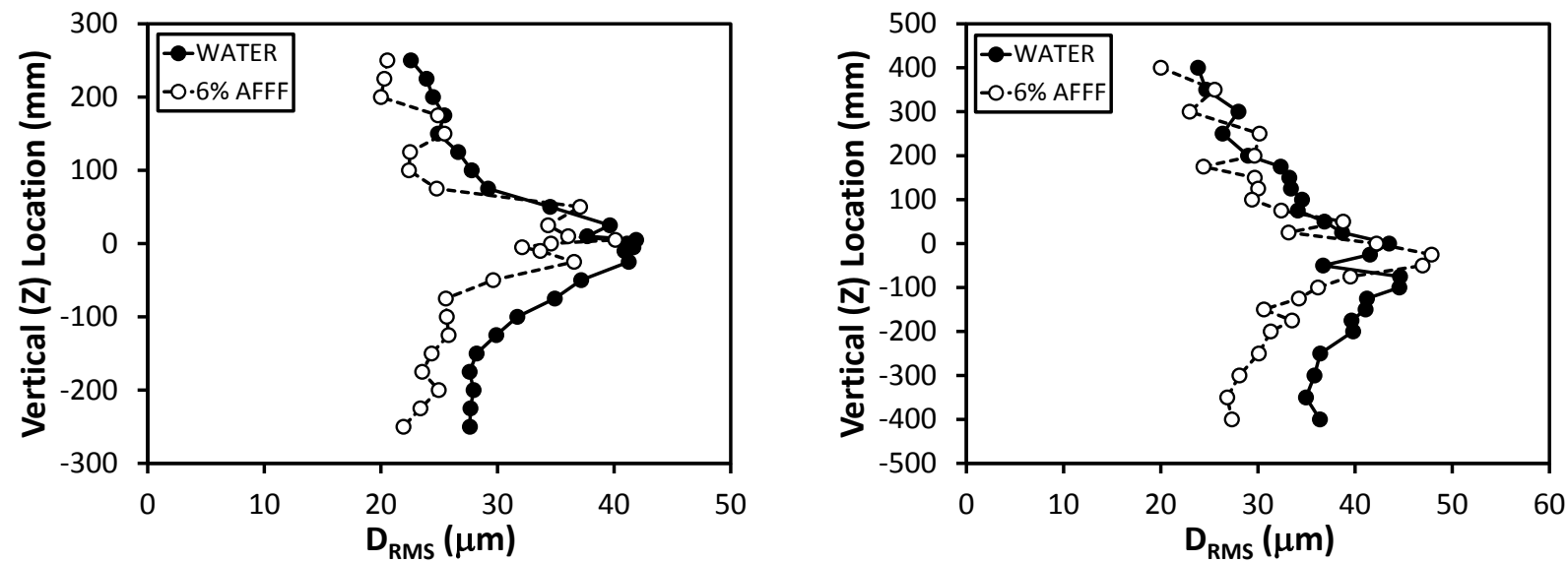

(a) $x=3.05 \mathrm{~m}(10 \mathrm{ft})$

(b) $x=4.57 \mathrm{~m} \mathrm{(15 \textrm {ft } )}$

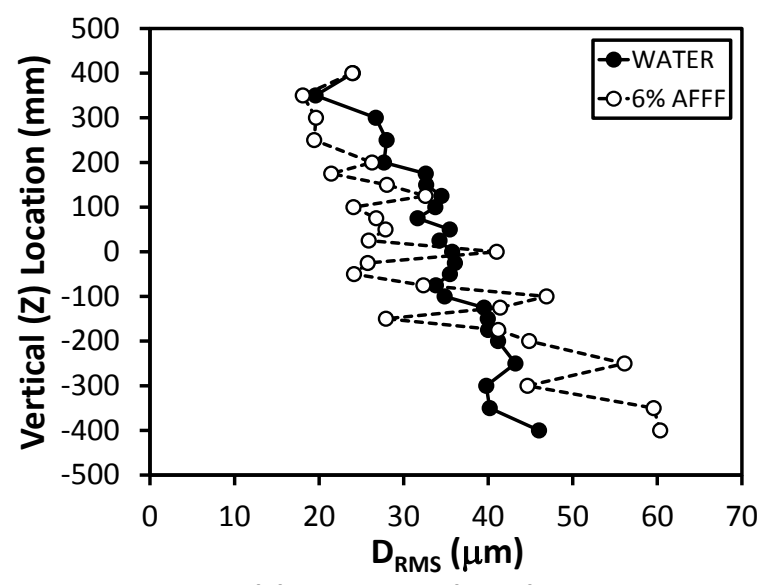

(c) $x=6.10 \mathrm{~m}(20 \mathrm{ft})$

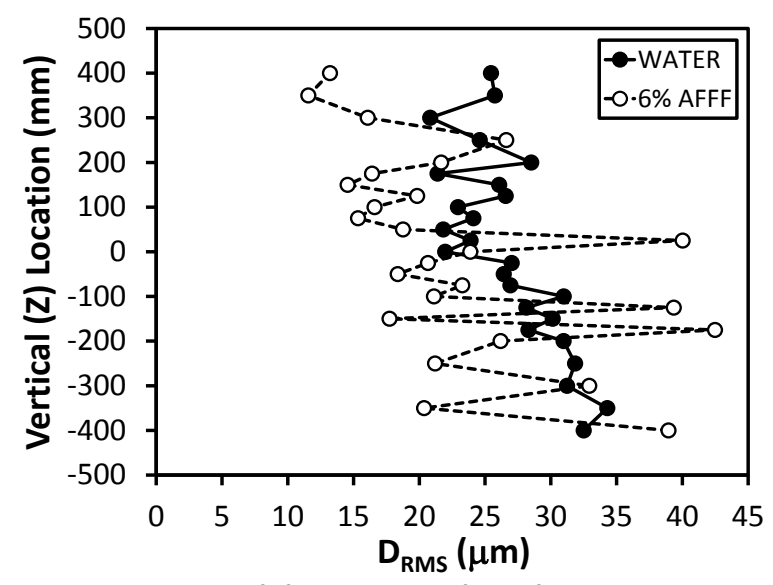

(d) $x=7.62 \mathrm{~m}(25 \mathrm{ft})$

Figure C.19: Far Field Low Flow, High Press. Jet Vertical Profiles of RMS Droplet Diameter 


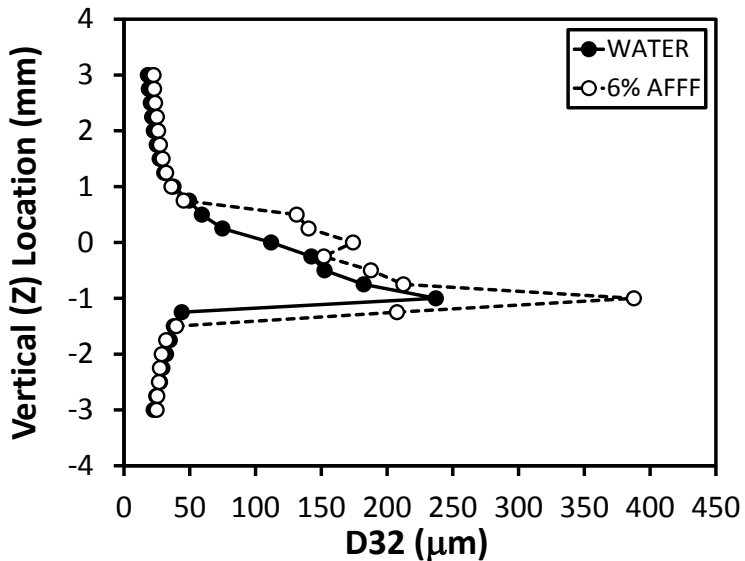

(a) $x=6.4 \mathrm{~mm}(0.25 \mathrm{in})$

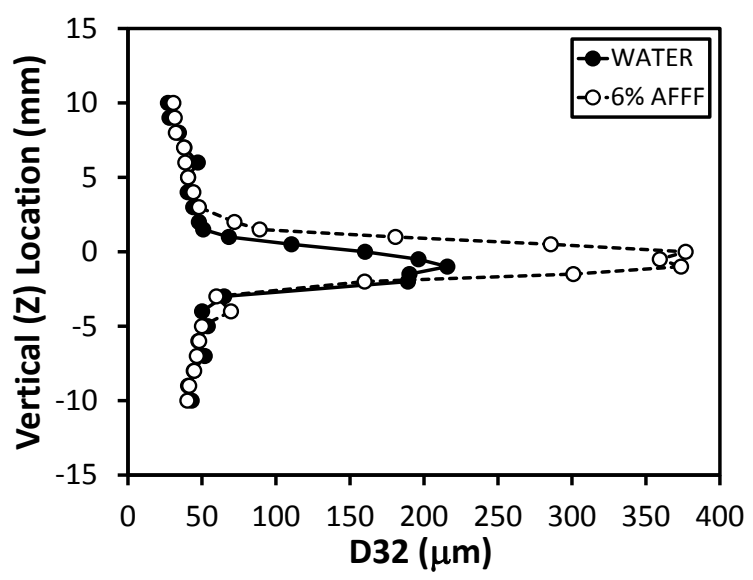

(c) $x=0.152 \mathrm{~m}(6 \mathrm{in})$

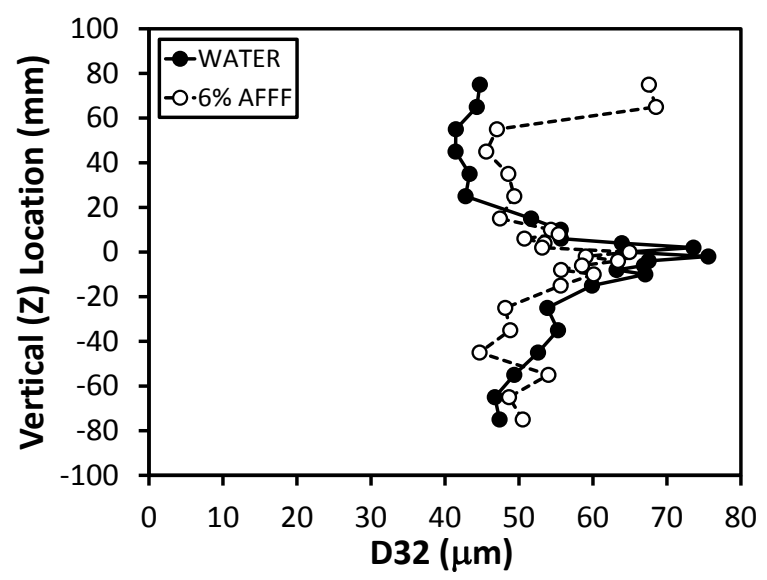

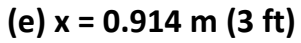

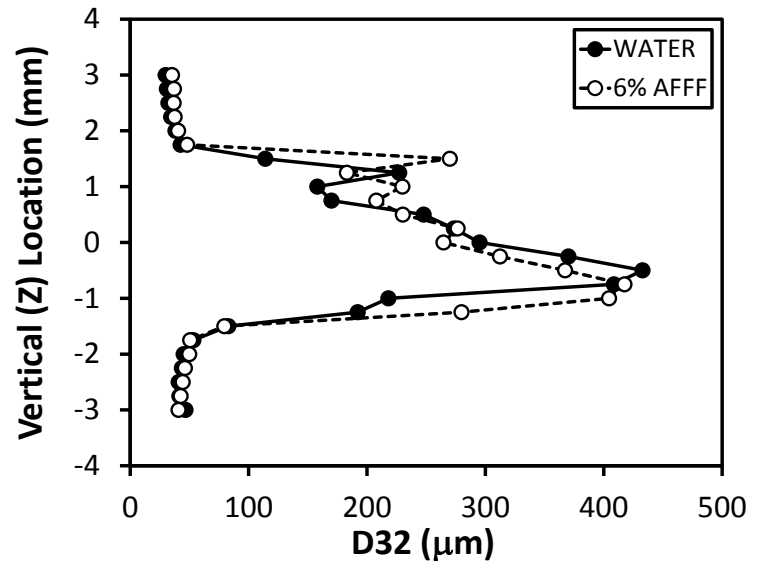

(b) $x=25.4 \mathrm{~mm}(1 \mathrm{in})$

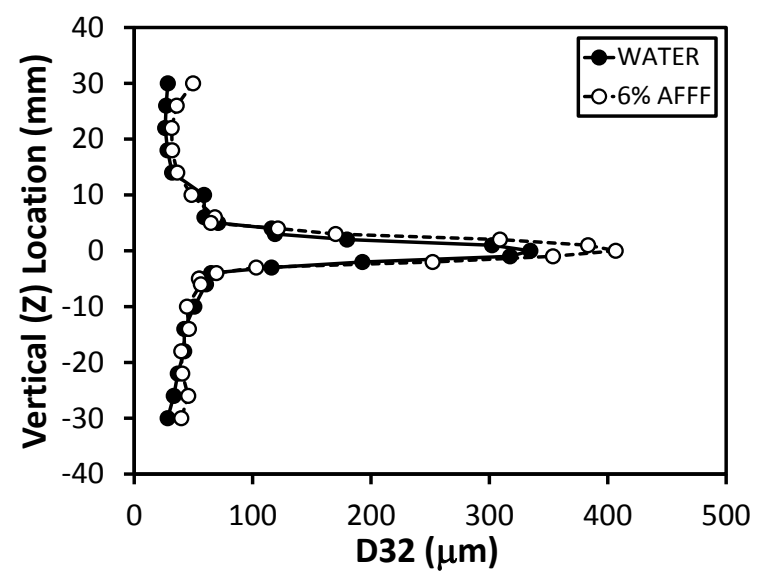

(d) $x=0.305 \mathrm{~m}(1 \mathrm{ft})$

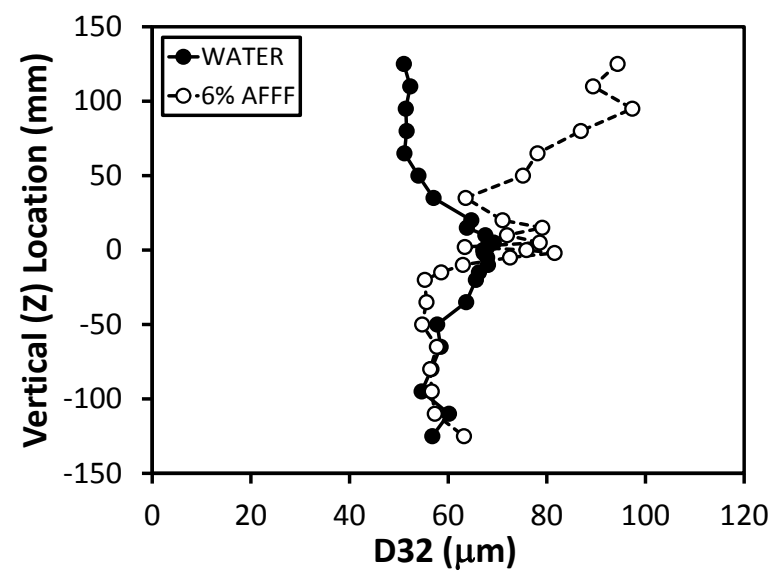

(f) $x=1.52 \mathrm{~m}(5 \mathrm{ft})$

Figure C.20: Near Field Low Flow, High Press. Jet Vertical Profiles of Sauter Mean Droplet Diameter 

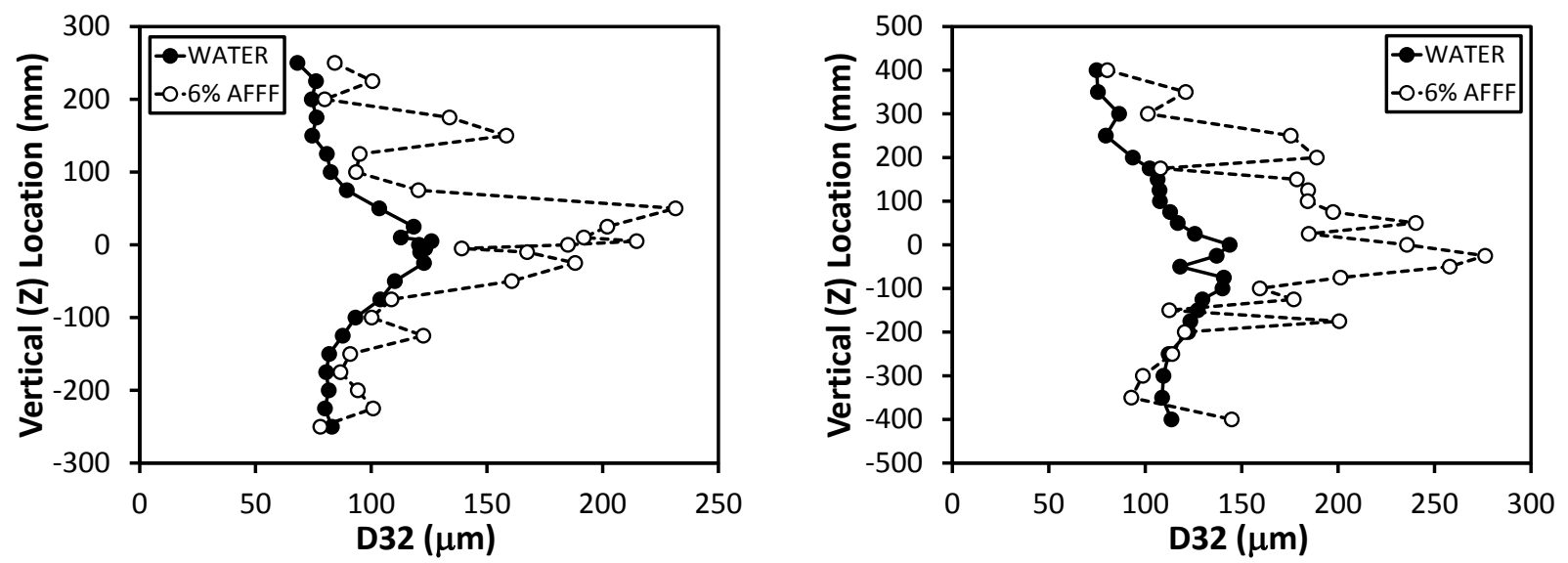

(a) $x=3.05 \mathrm{~m}(10 \mathrm{ft})$

(b) $x=4.57 \mathrm{~m} \mathrm{(15 \textrm {ft } )}$

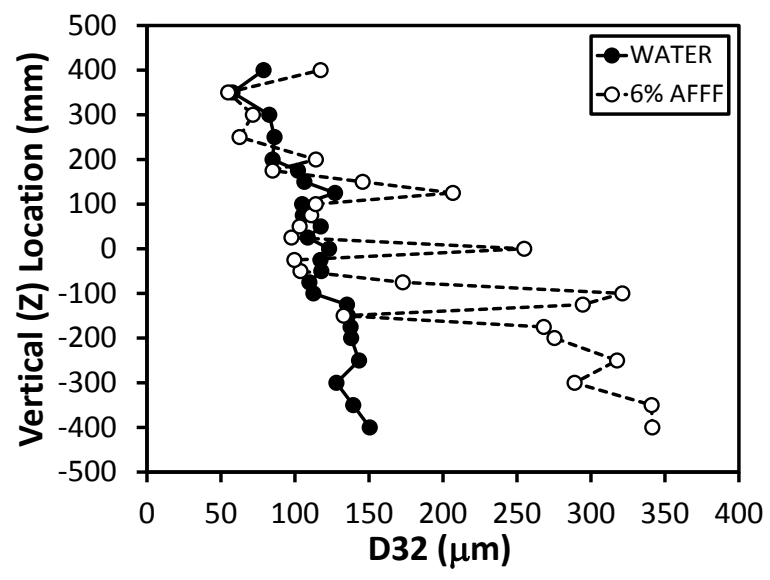

(c) $x=6.10 \mathrm{~m}(20 \mathrm{ft})$

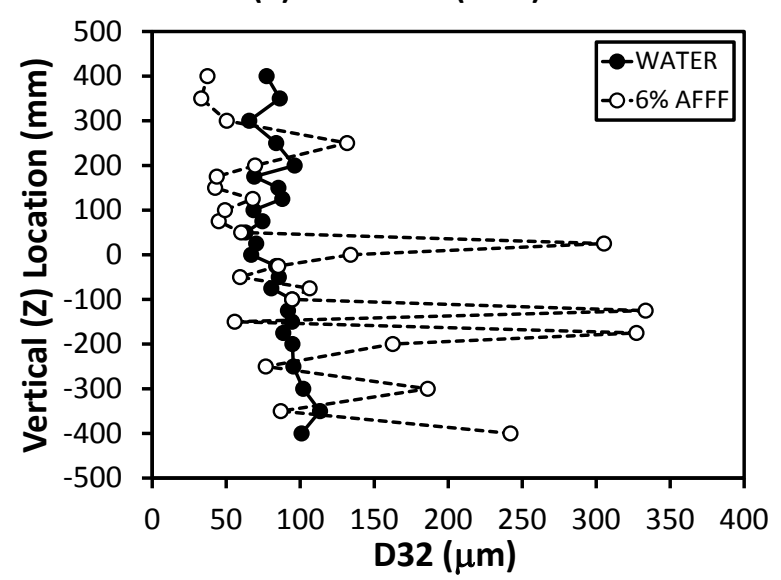

(d) $x=7.62 \mathrm{~m}(25 \mathrm{ft})$

Figure C.21: Far Field Low Flow, High Press. Jet Vertical Profiles of Sauter Mean Droplet Diameter 


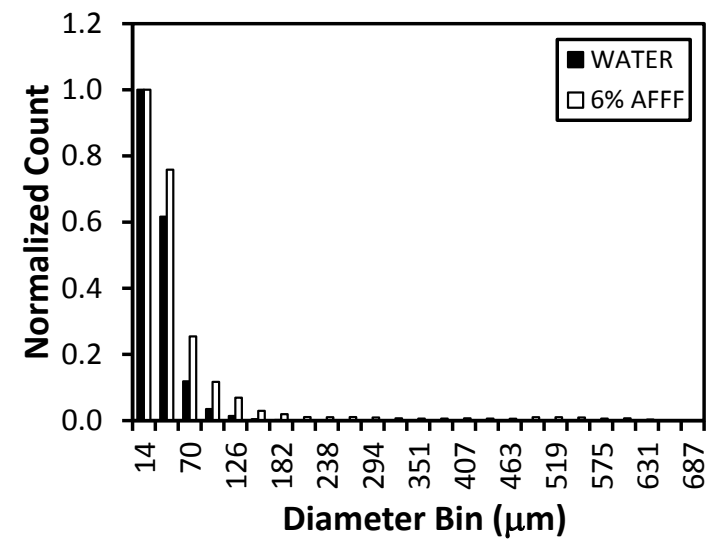

(a) $x=0.152 \mathrm{~m}(6 \mathrm{in})$

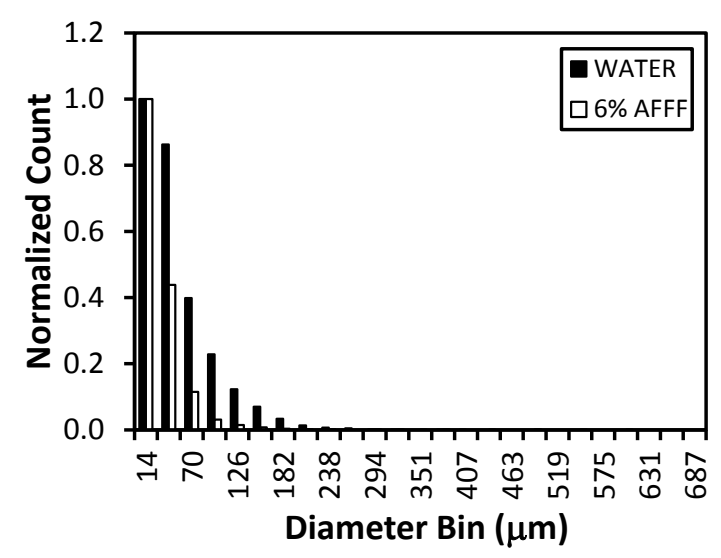

(c) $\mathrm{x}=3.05 \mathrm{~m}(10 \mathrm{ft})$

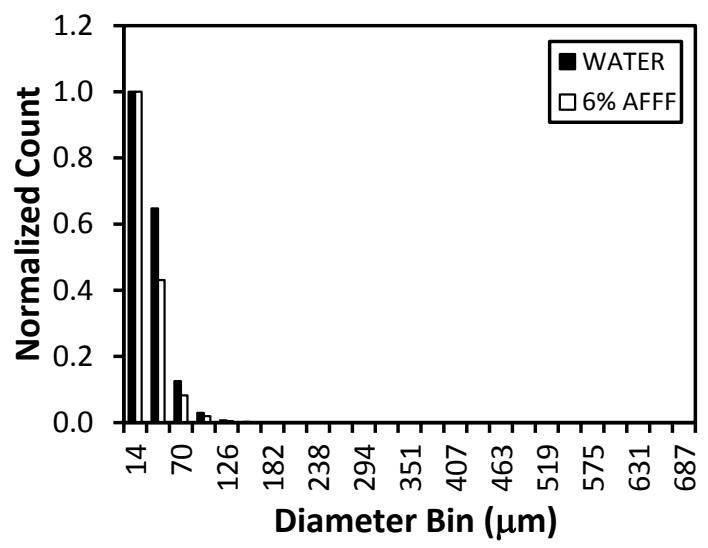

(b) $x=0.914 \mathrm{~m} \mathrm{(3 \textrm {ft } )}$

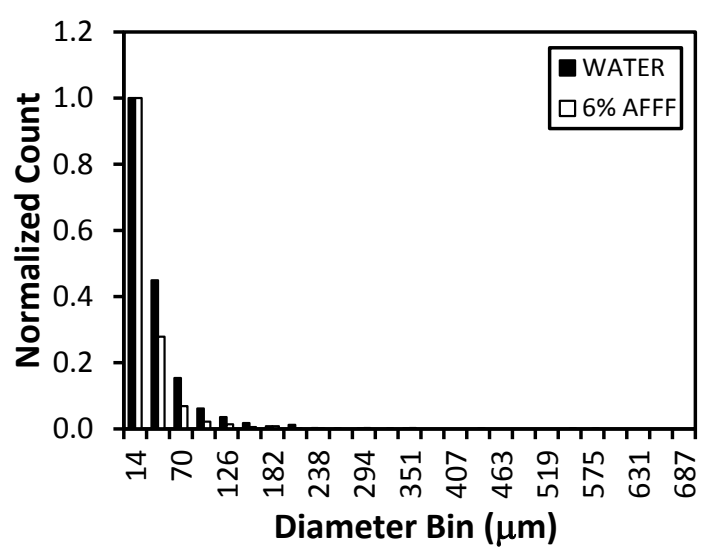

(d) $x=6.10 \mathrm{~m}(20 \mathrm{ft})$

Figure C.22: Low Flow, High Press. Jet Droplet Size Distribution at the Maximum Profile, Mean Axial Droplet Velocity for Select Vertical Profiles 


\section{C.3 Medium Flow, Medium Pressure Firefighting Jet Data}

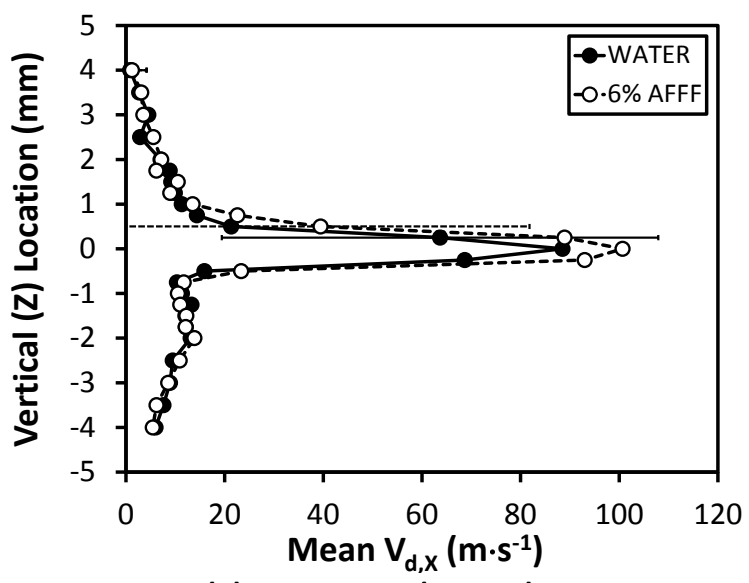

(a) $x=6.4 \mathrm{~mm}$ (0.25 in)

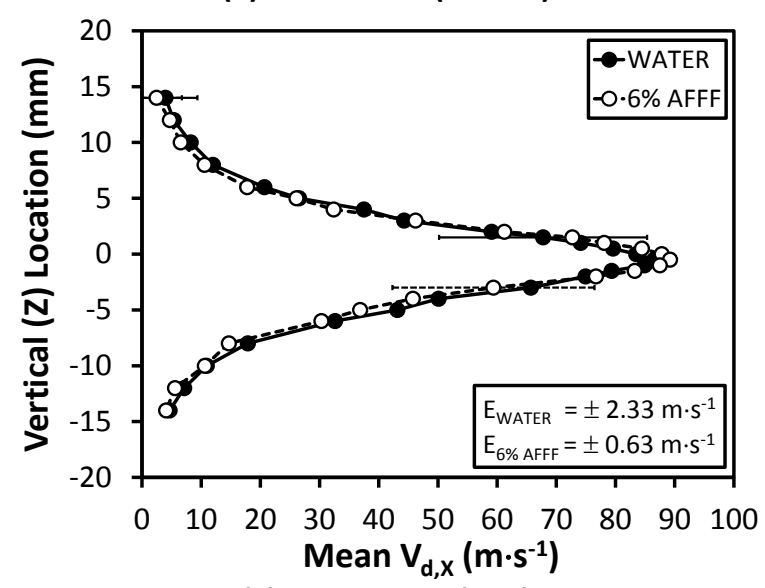

(c) $x=0.152 \mathrm{~m}$ (6 in)

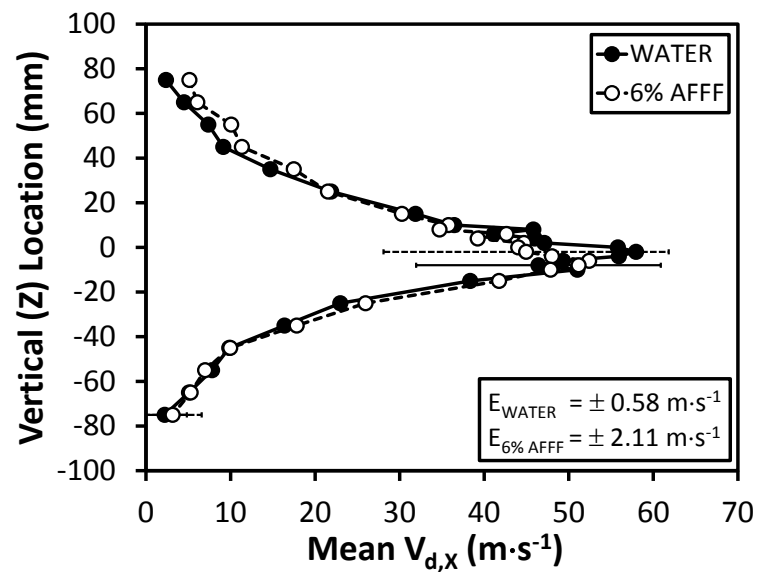

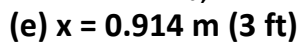

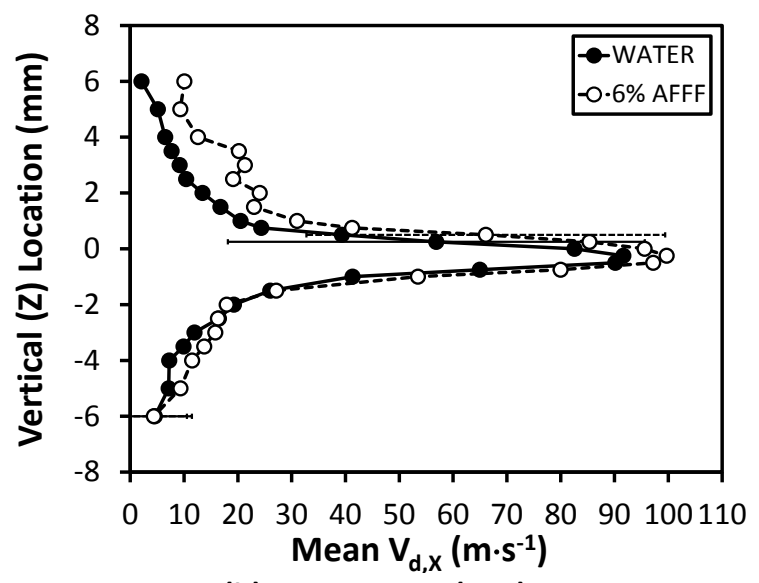

(b) $x=25.4 \mathrm{~mm}$ (1 in)

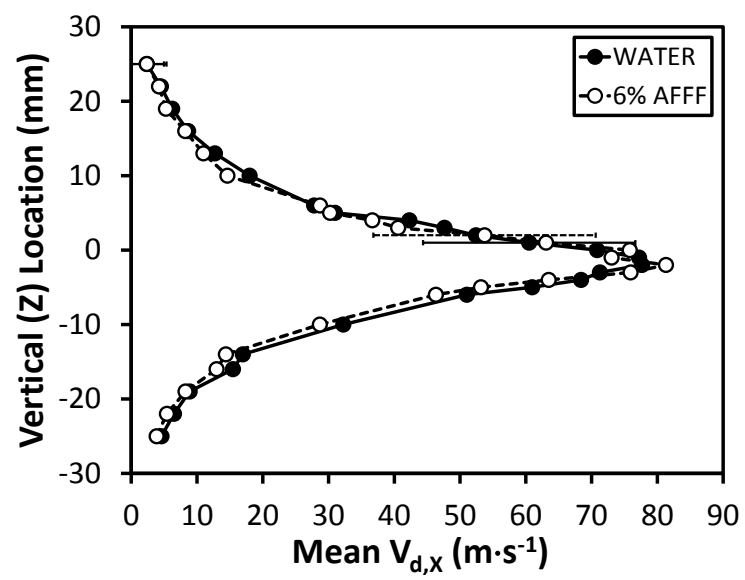

(d) $x=0.305 \mathrm{~m}$ (1ft)

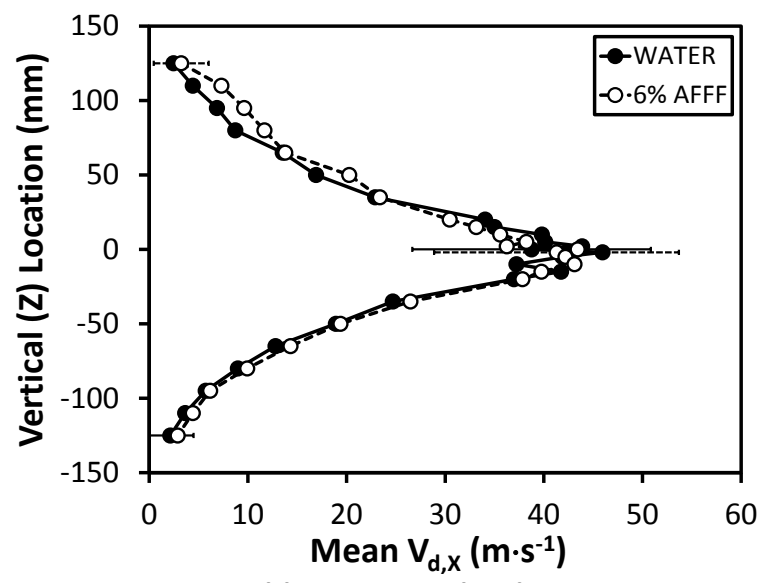

(f) $x=1.52 \mathrm{~m} \mathrm{(5 \textrm {ft } )}$

Figure C.23: Near Field Med. Flow, Med. Press. Jet Vertical Profiles of Mean Axial Droplet Velocity 

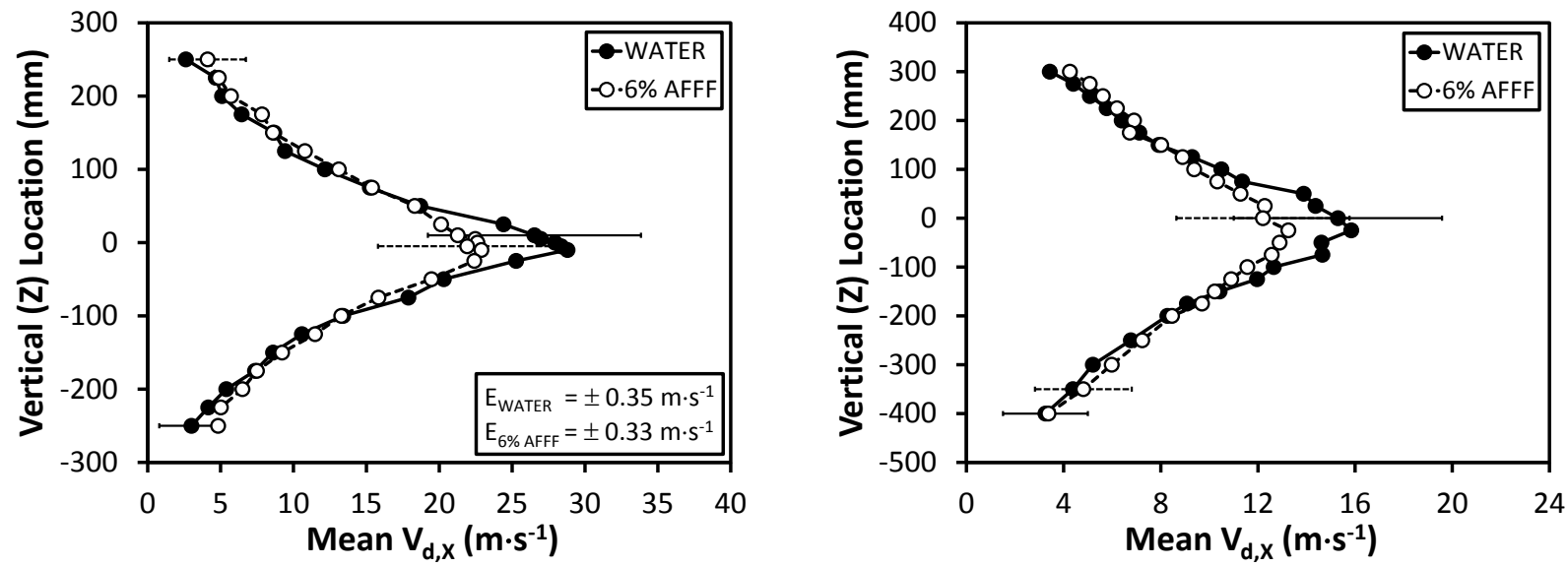

(a) $x=3.05 \mathrm{~m}(10 \mathrm{ft})$

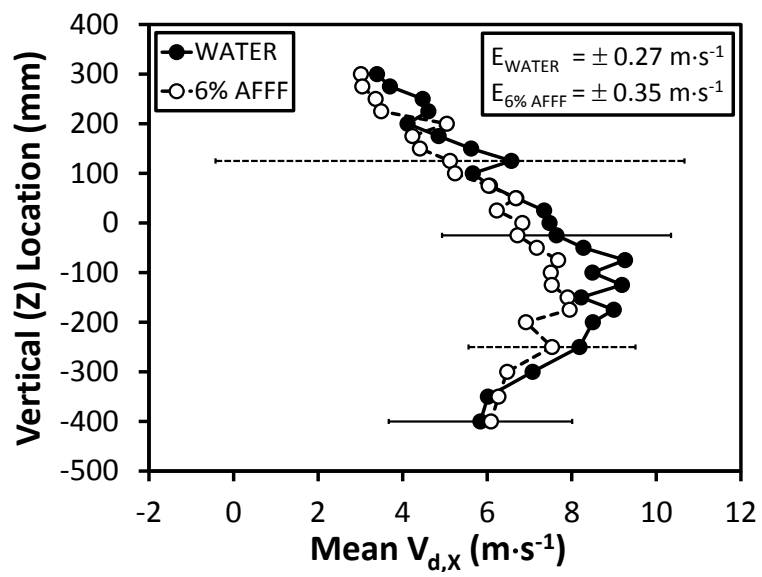

(c) $\mathrm{x}=6.10 \mathrm{~m}(20 \mathrm{ft})$

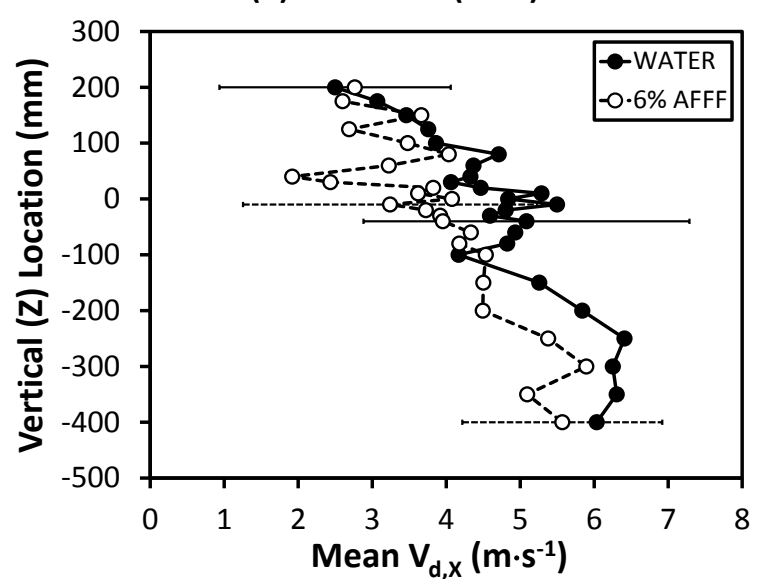

(d) $x=7.62 \mathrm{~m}(25 \mathrm{ft})$

Figure C.24: Far Field Med. Flow, Med. Press. Jet Vertical Profiles of Mean Axial Droplet Velocity 


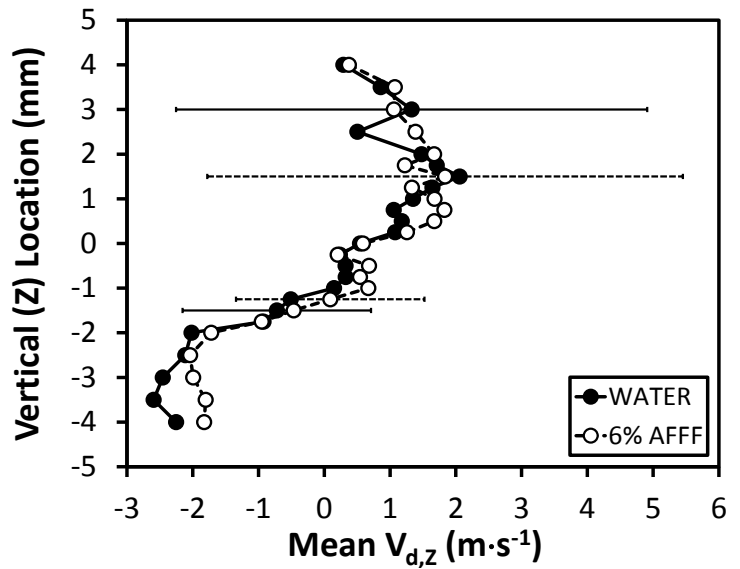

(a) $x=6.4 \mathrm{~mm}(0.25 \mathrm{in})$

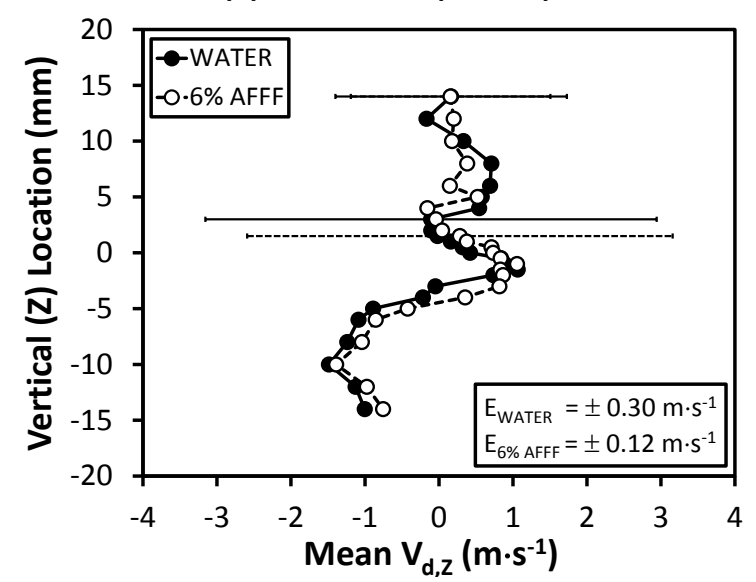

(c) $x=0.152 \mathrm{~m}(6 \mathrm{in})$

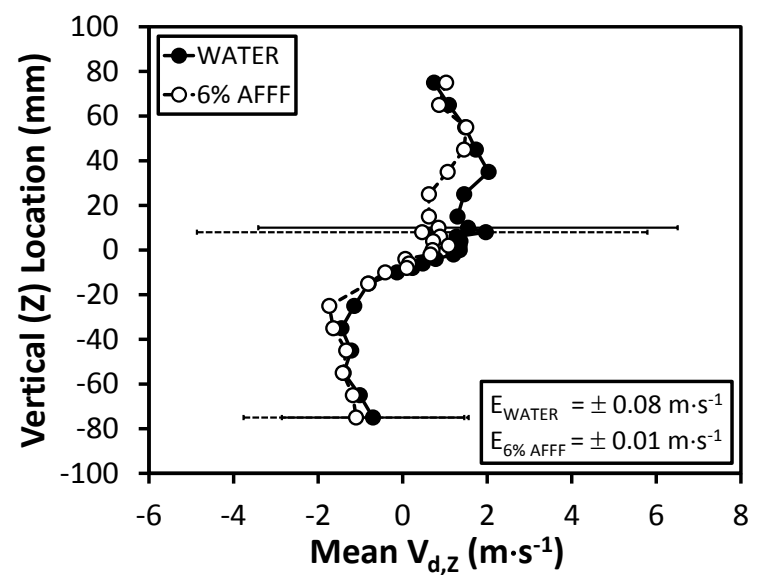

(e) $x=0.914 \mathrm{~m} \mathrm{(3 \textrm {ft } )}$

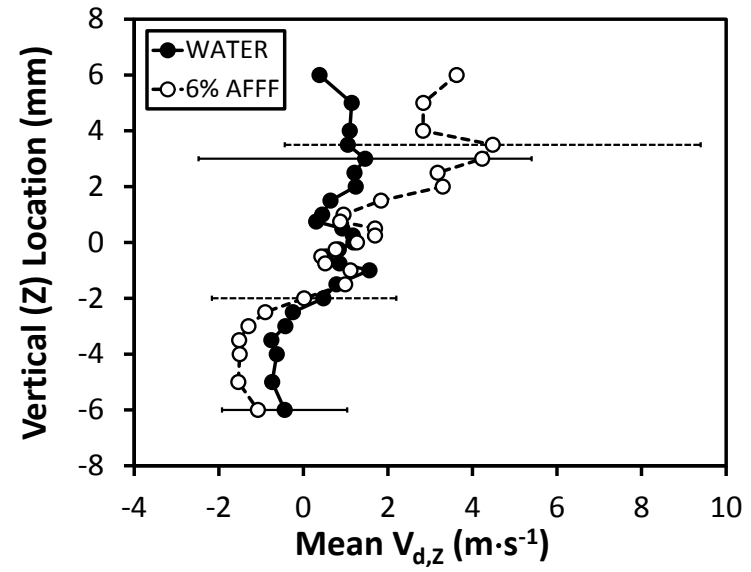

(b) $x=25.4 \mathrm{~mm}$ (1 in)

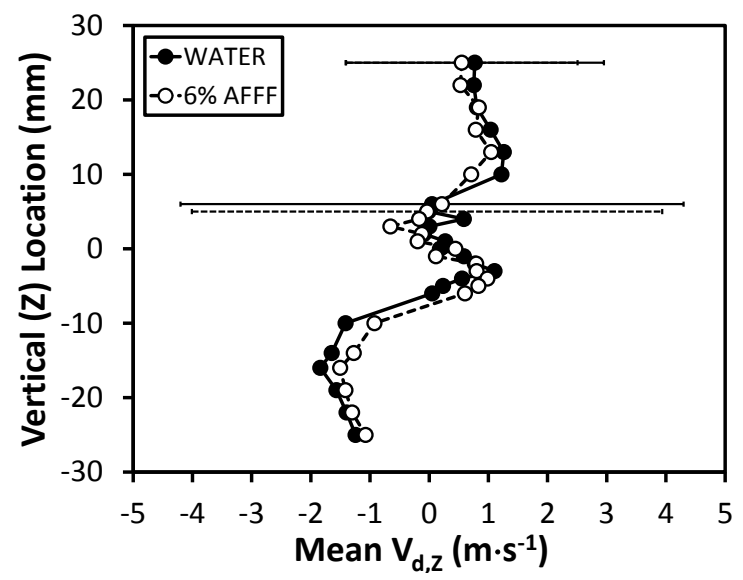

(d) $\mathrm{x}=0.305 \mathrm{~m}(\mathbf{1 f t})$

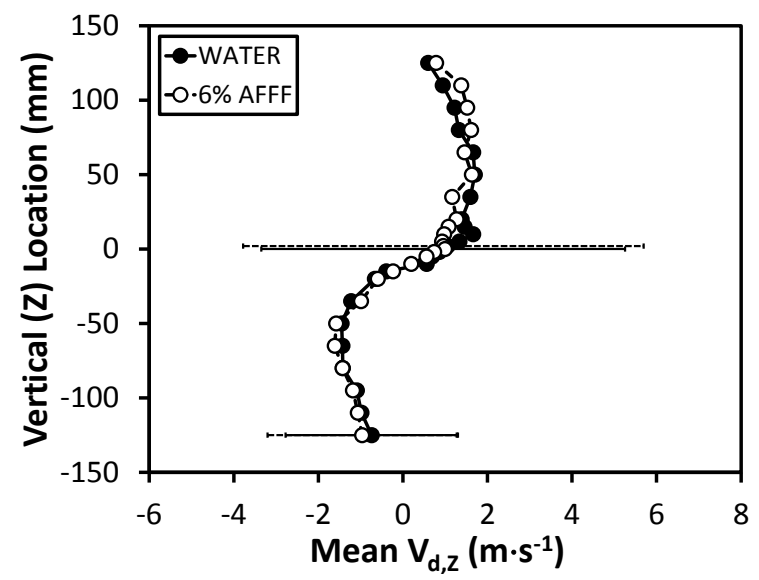

(f) $x=1.52 \mathrm{~m}(5 \mathrm{ft})$

Figure C.25: Near Field Med. Flow, Med. Press. Jet Vertical Profiles of Mean Vertical Droplet Velocity 

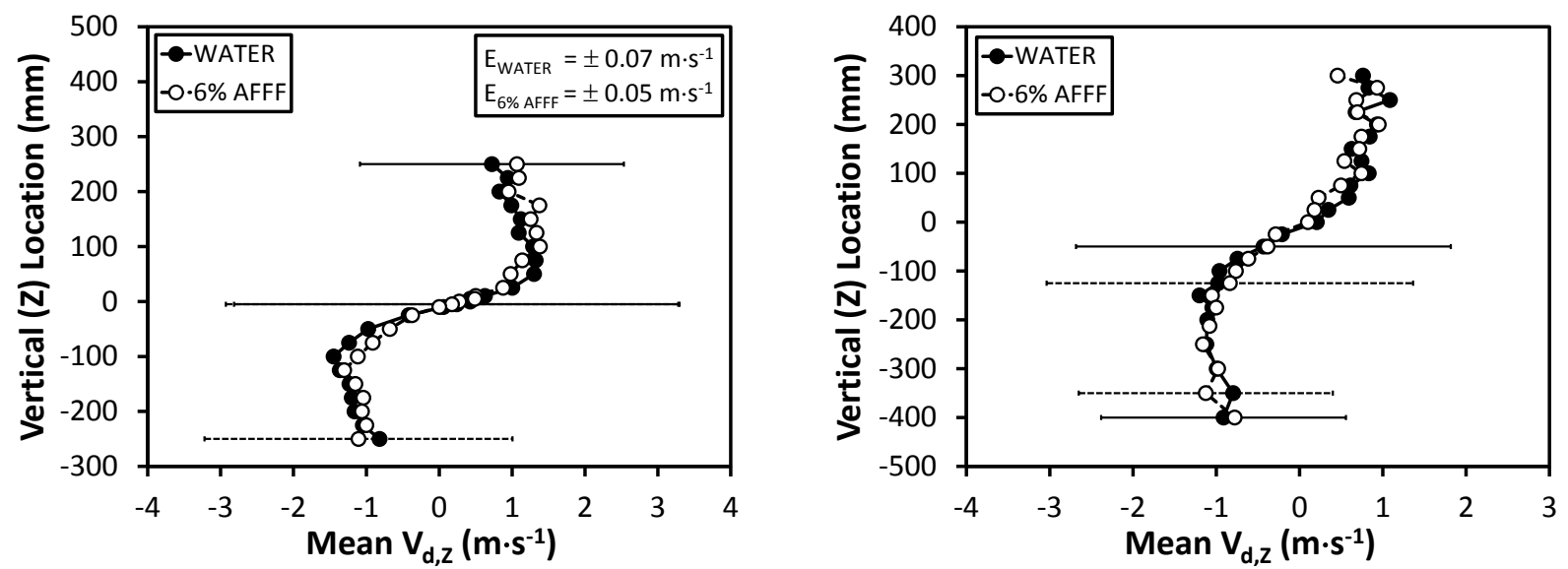

(a) $x=3.05 \mathrm{~m}(10 \mathrm{ft})$

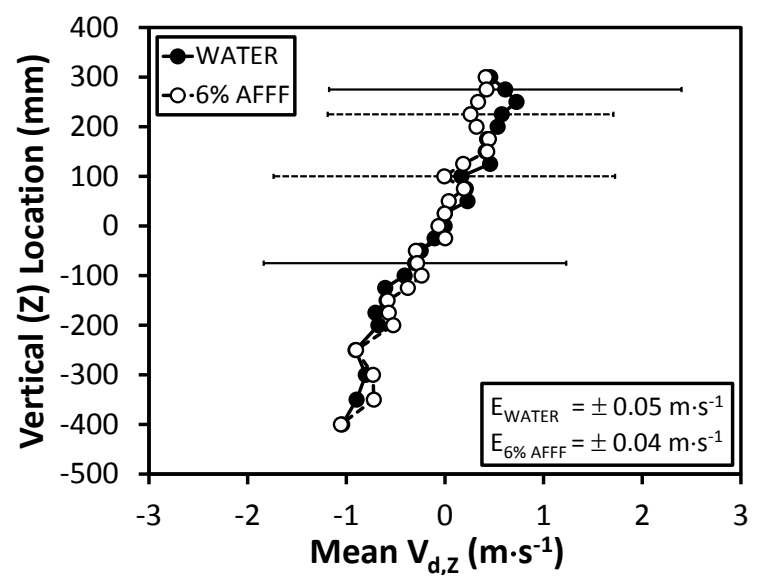

(c) $x=6.10 \mathrm{~m}(20 \mathrm{ft})$

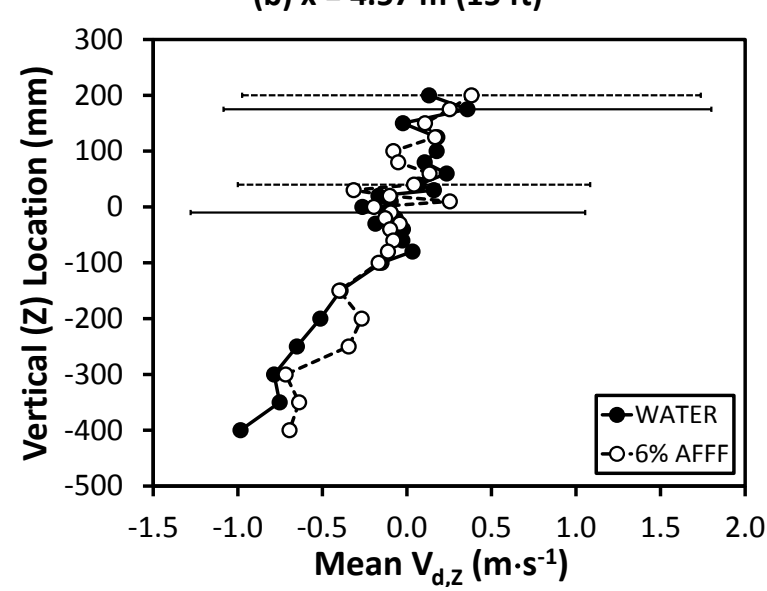

(d) $x=7.62 \mathrm{~m}(25 \mathrm{ft})$

Figure C.26: Far Field Med. Flow, Med. Press. Jet Vertical Profiles of Mean Vertical Droplet Velocity 

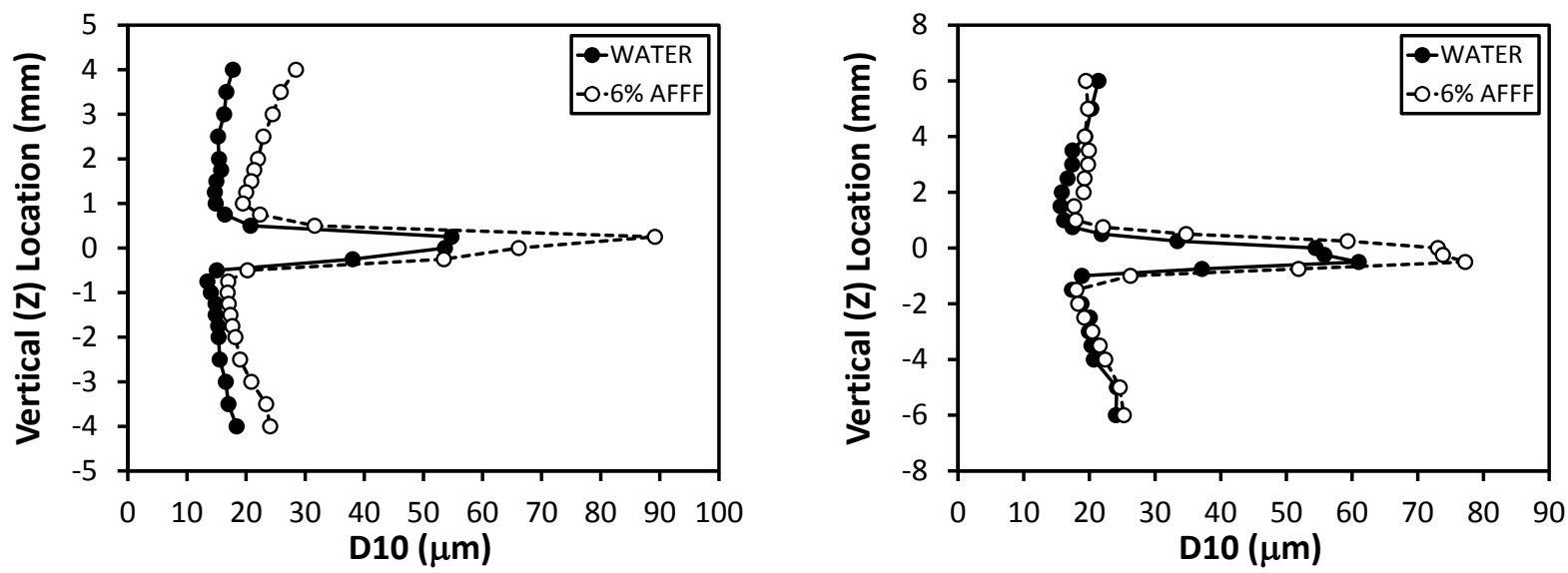

(a) $x=6.4 \mathrm{~mm}(0.25 \mathrm{in})$

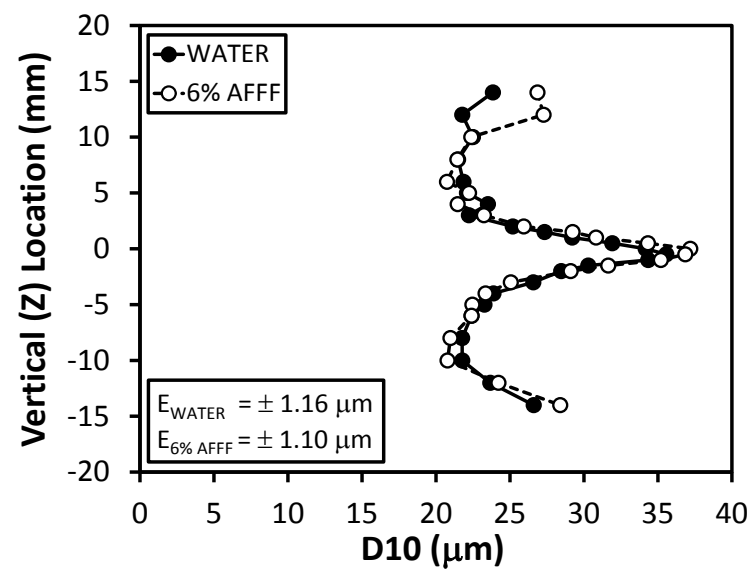

(b) $x=25.4 \mathrm{~mm}(1 \mathrm{in})$

(c) $x=0.152 \mathrm{~m}(6 \mathrm{in})$

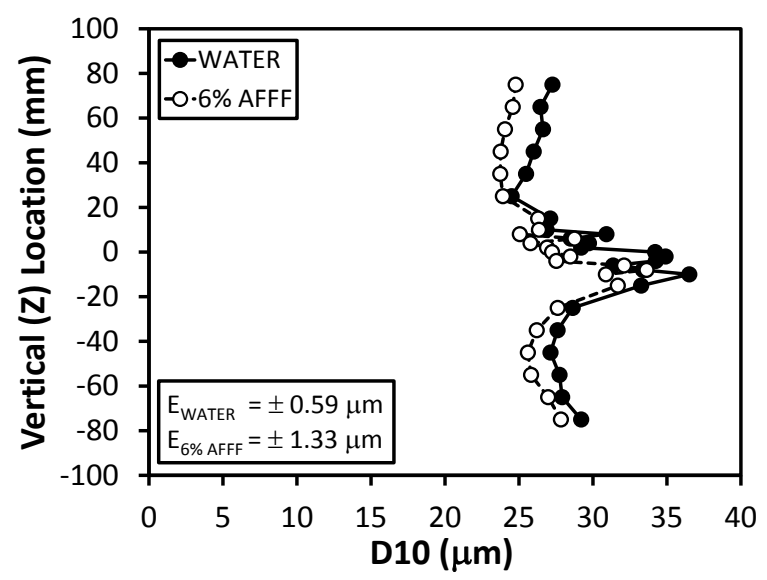

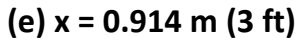

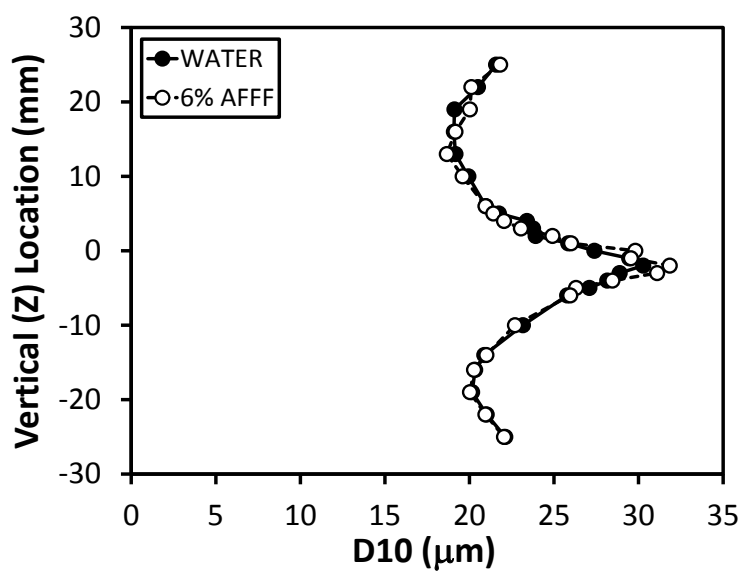

(d) $x=0.305 \mathrm{~m}(1 \mathrm{ft})$

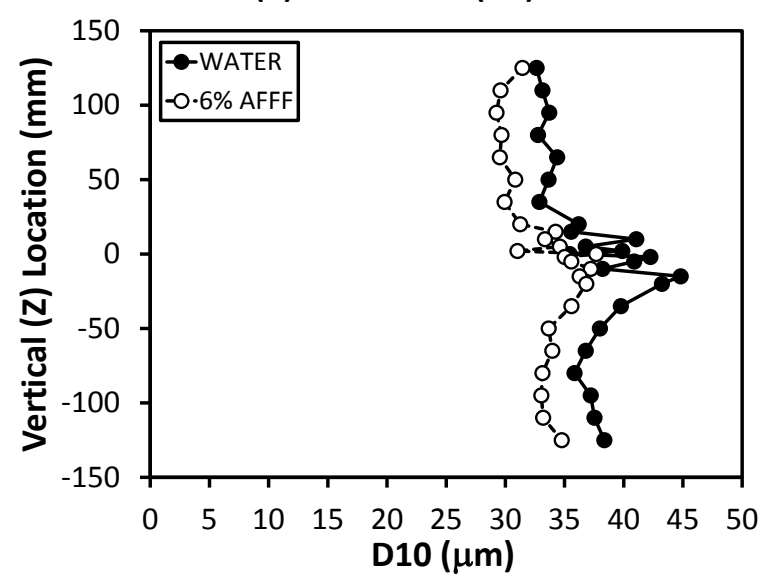

(f) $x=1.52 \mathrm{~m}(5 \mathrm{ft})$

Figure C.27: Near Field Med. Flow, Med. Press. Jet Vertical Profiles of Mean Droplet Diameter 

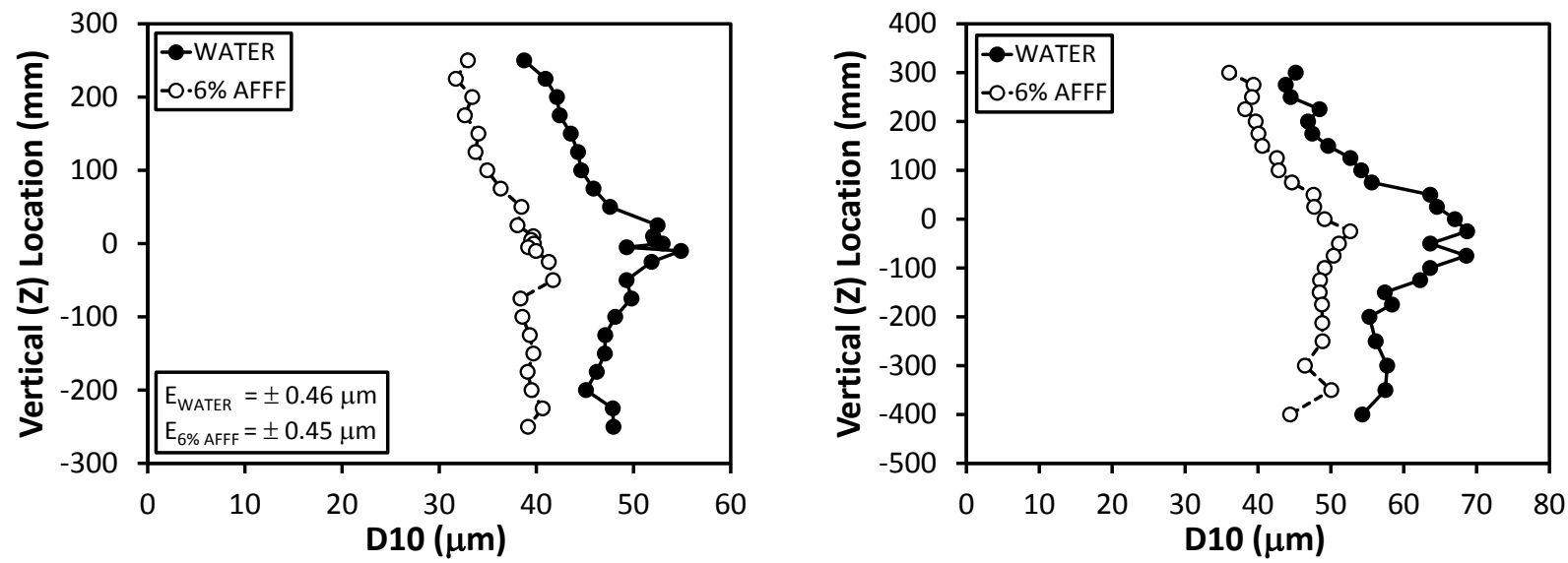

(a) $\mathrm{x}=3.05 \mathrm{~m}(10 \mathrm{ft})$

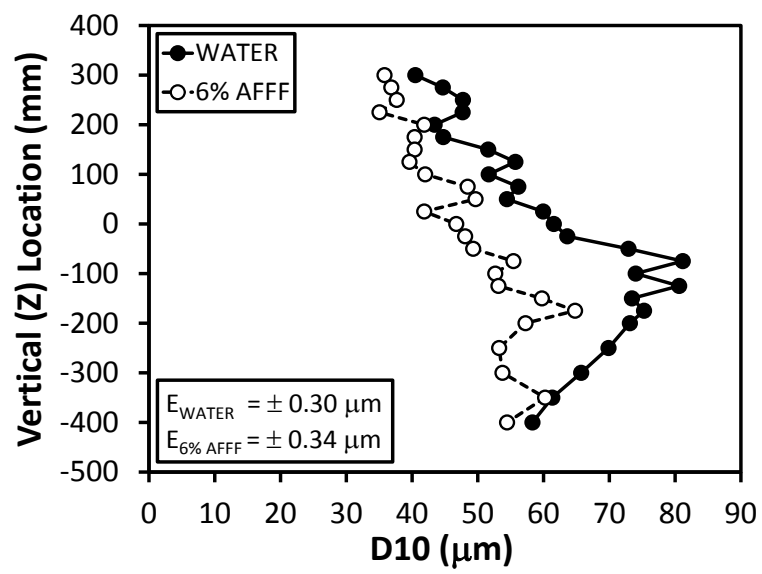

(b) $x=4.57 \mathrm{~m} \mathrm{(15 \textrm {ft } )}$

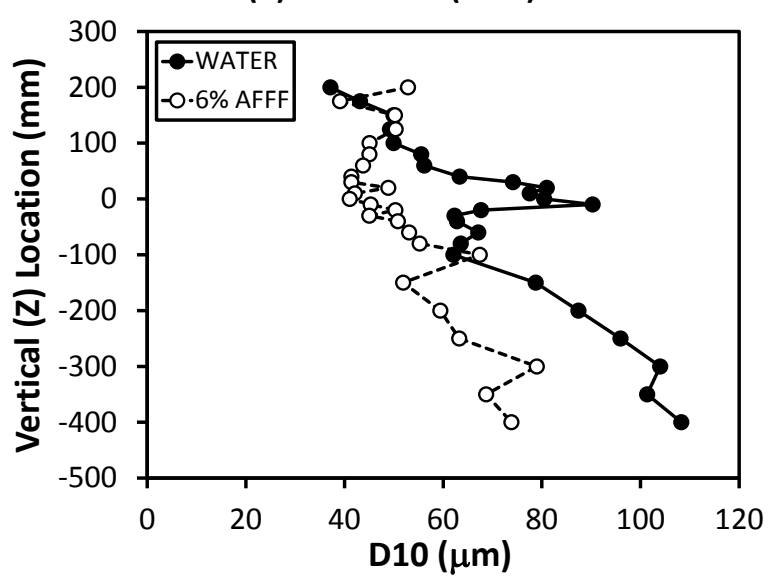

(c) $x=6.10 \mathrm{~m}(20 \mathrm{ft})$

(d) $x=7.62 \mathrm{~m}(25 \mathrm{ft})$

Figure C.28: Far Field Med. Flow, Med. Press. Jet Vertical Profiles of Mean Droplet Diameter 

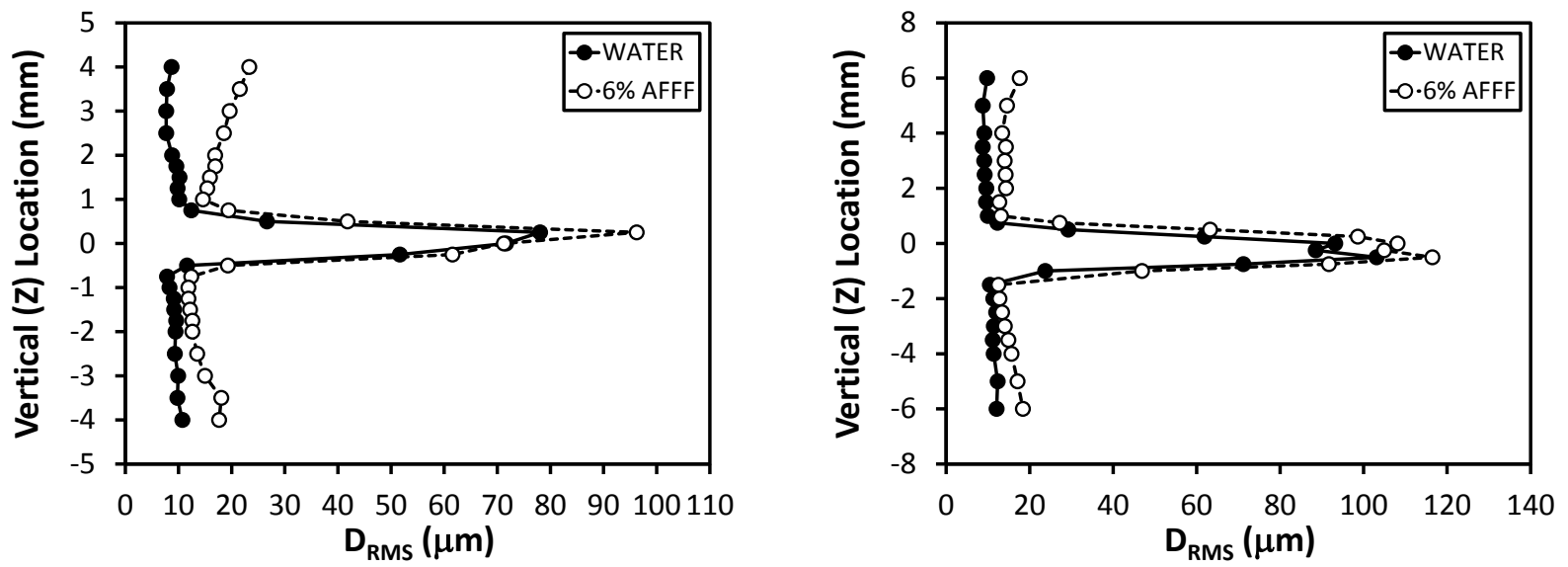

(a) $x=6.4 \mathrm{~mm}(0.25 \mathrm{in})$

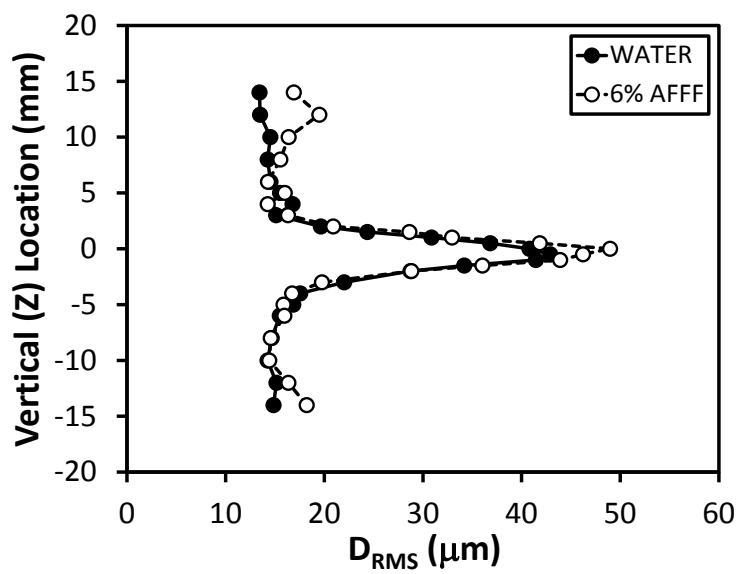

(b) $x=25.4 \mathrm{~mm}(1 \mathrm{in})$

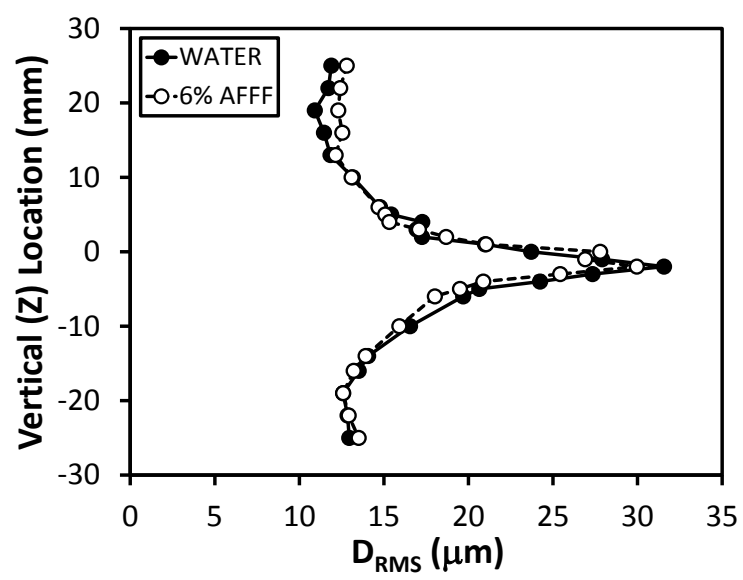

(c) $x=0.152 \mathrm{~m}(6 \mathrm{in})$

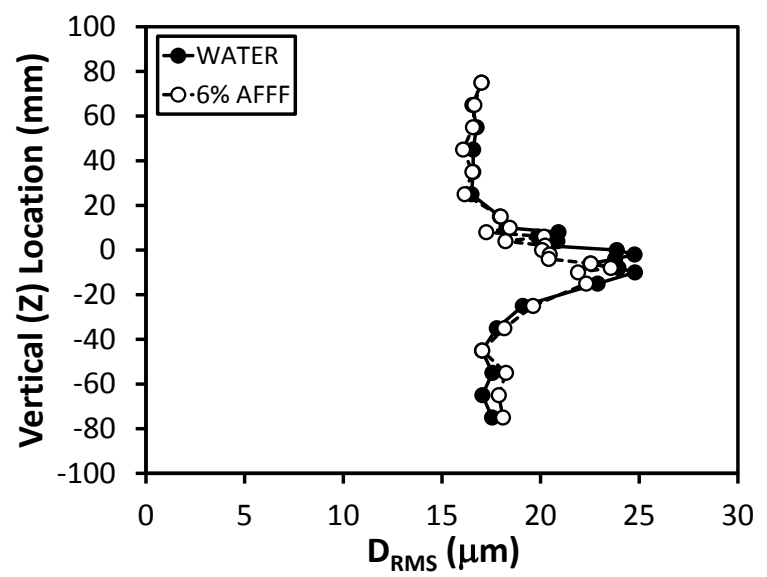

(e) $x=0.914 \mathrm{~m} \mathrm{(3 \textrm {ft } )}$

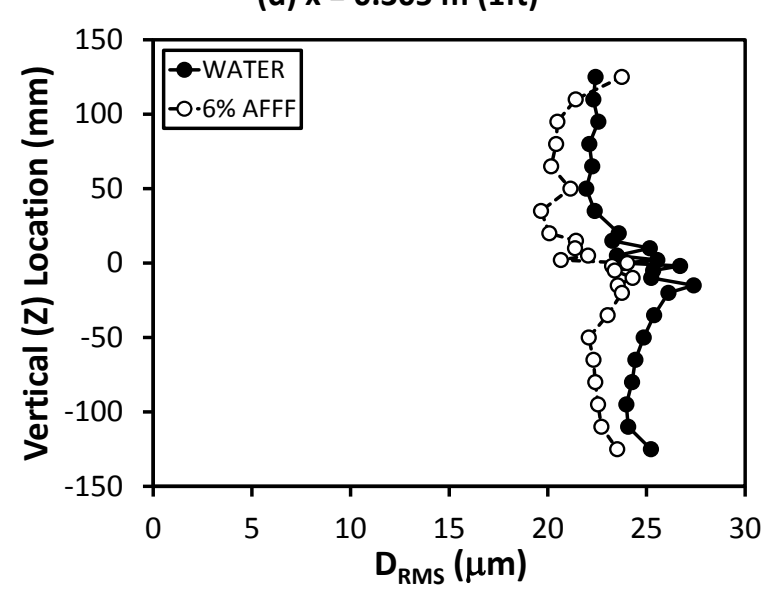

(f) $x=1.52 \mathrm{~m} \mathrm{(5 \textrm {ft } )}$

Figure C.29: Near Field Med. Flow, Med. Press. Jet Vertical Profiles of RMS Droplet Diameter 

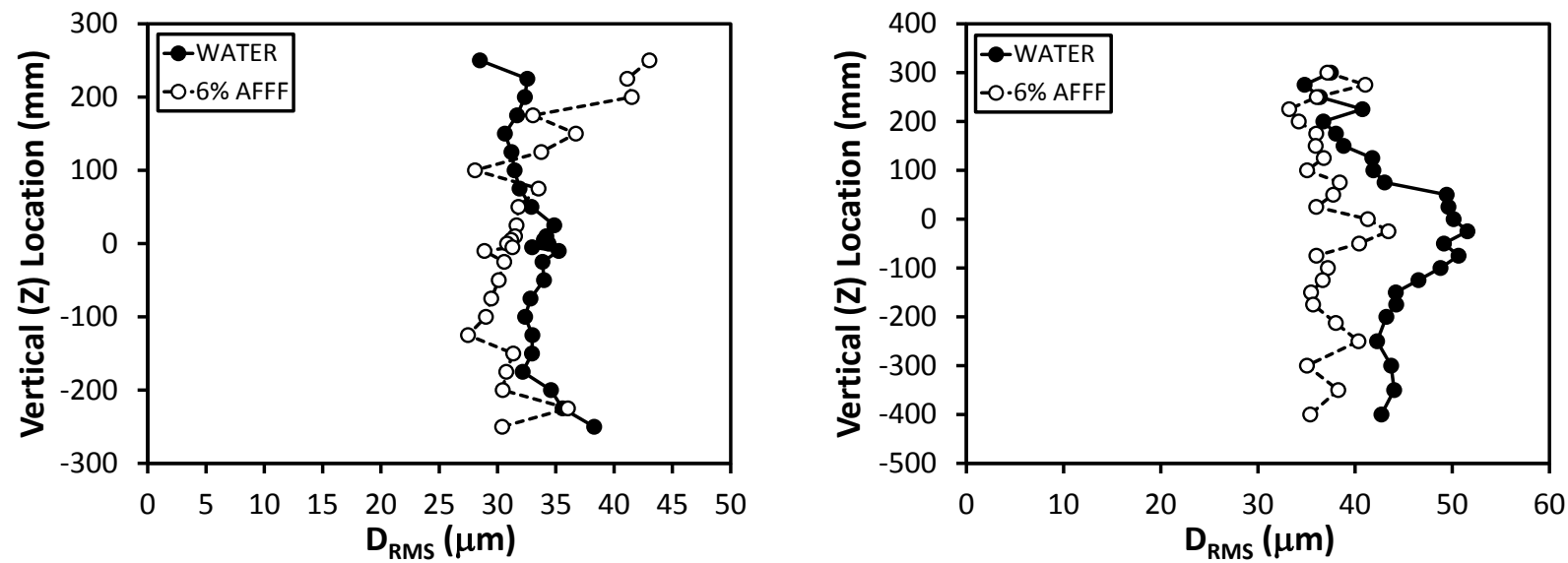

(a) $x=3.05 \mathrm{~m}(10 \mathrm{ft})$

(b) $x=4.57 \mathrm{~m} \mathrm{(15 \textrm {ft } )}$
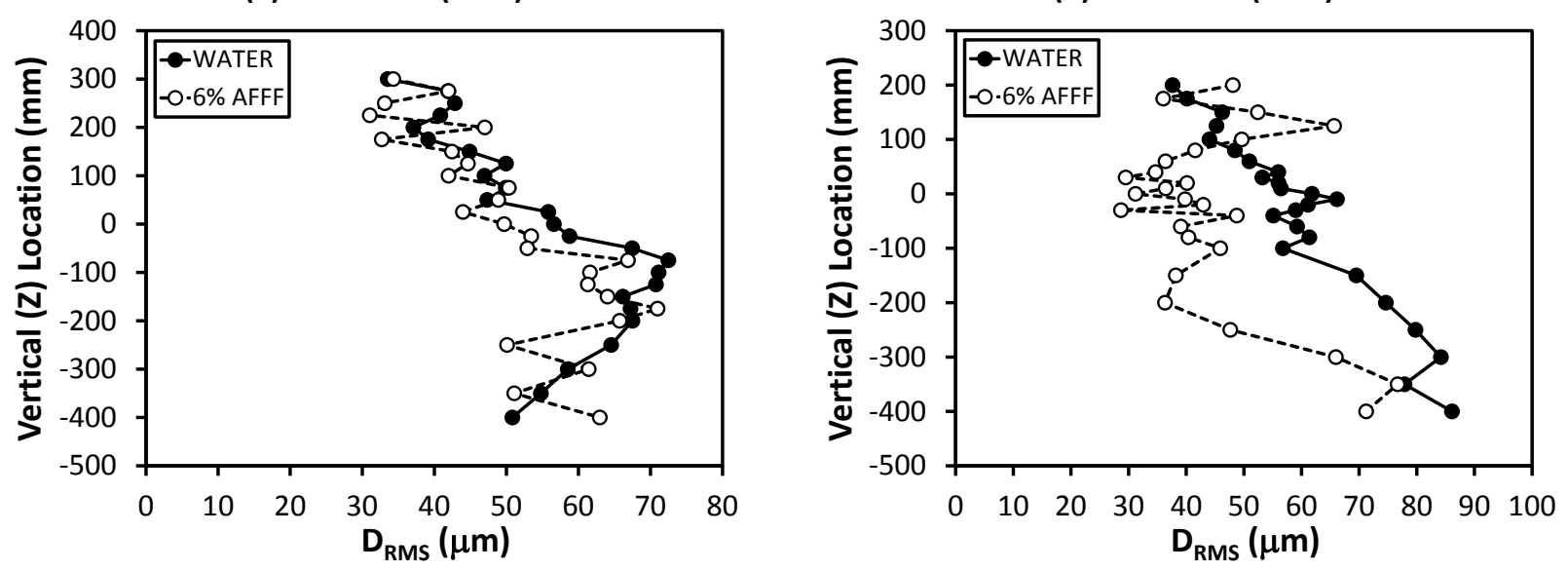

(c) $x=6.10 \mathrm{~m}(20 \mathrm{ft})$

(d) $x=7.62 \mathrm{~m}(25 \mathrm{ft})$

Figure C.30: Far Field Med. Flow, Med. Press. Jet Vertical Profiles of RMS Droplet Diameter 


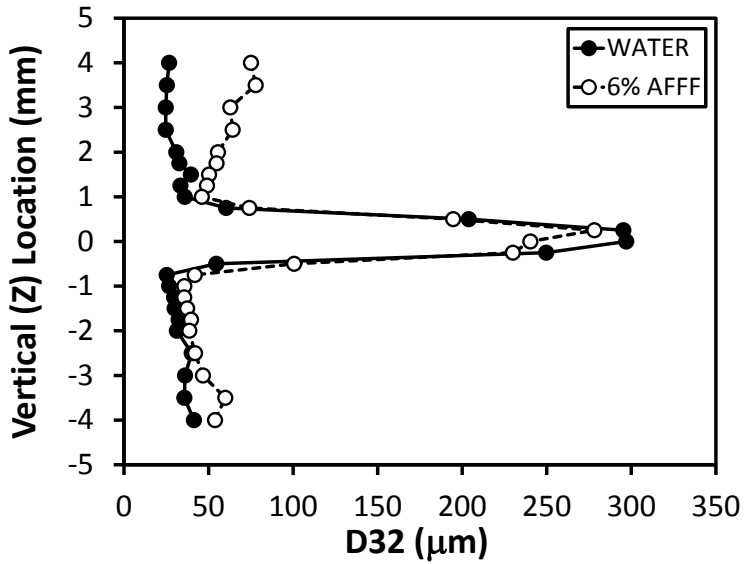

(a) $x=6.4 \mathrm{~mm}(0.25 \mathrm{in})$

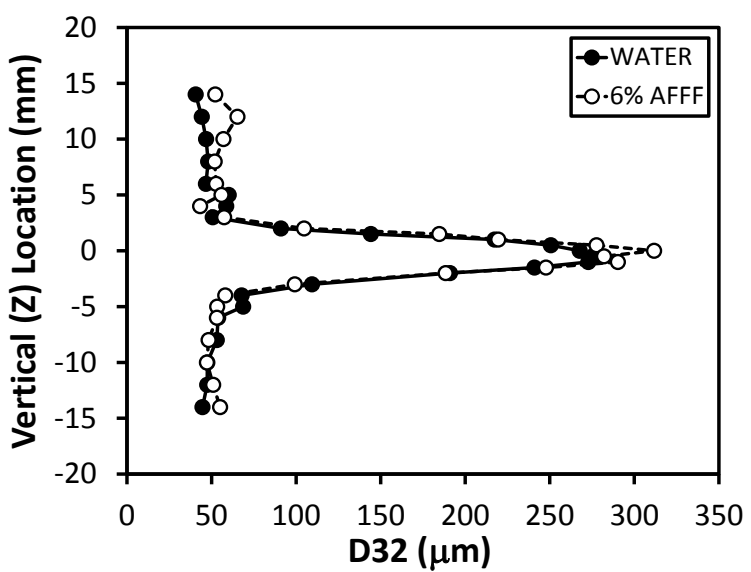

(c) $x=0.152 \mathrm{~m}(6 \mathrm{in})$

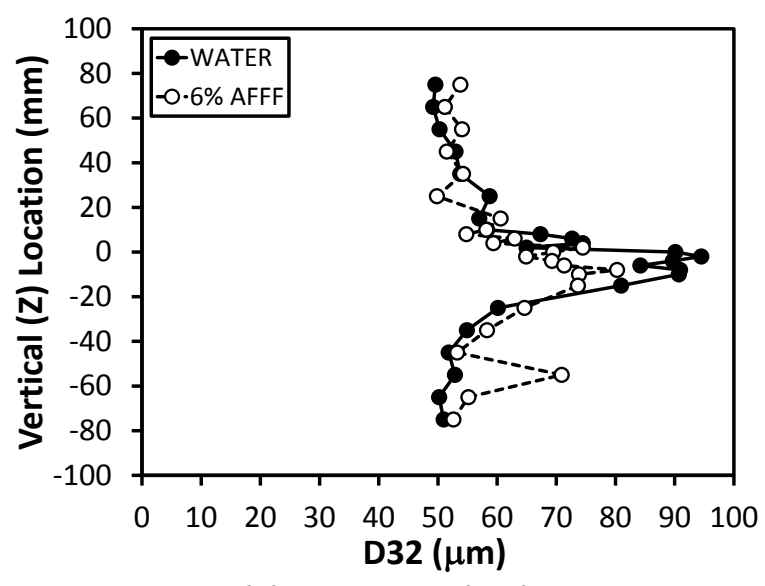

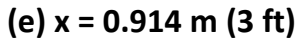

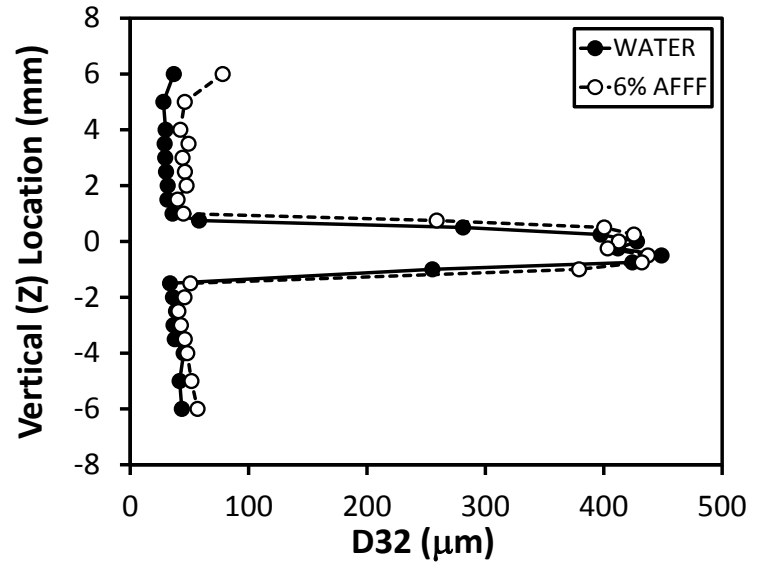

(b) $x=25.4 \mathrm{~mm}(1 \mathrm{in})$

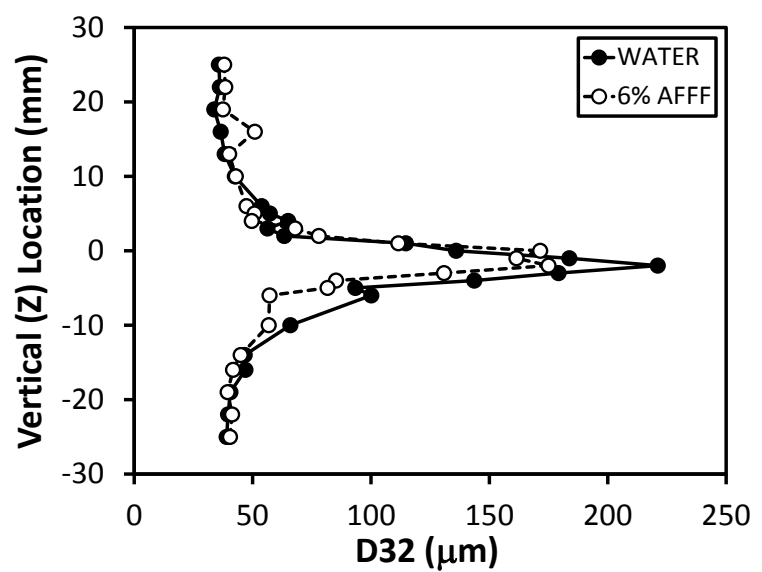

(d) $x=0.305 \mathrm{~m}(\mathbf{1 f t})$

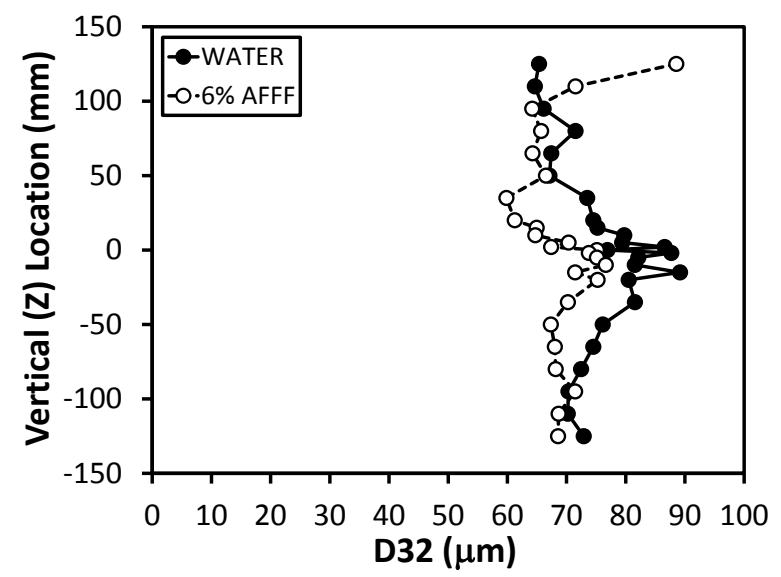

(f) $x=1.52 \mathrm{~m}(5 \mathrm{ft})$

Figure C.31: Near Field Med. Flow, Med. Press. Jet Vertical Profiles of Sauter Mean Droplet Diameter 

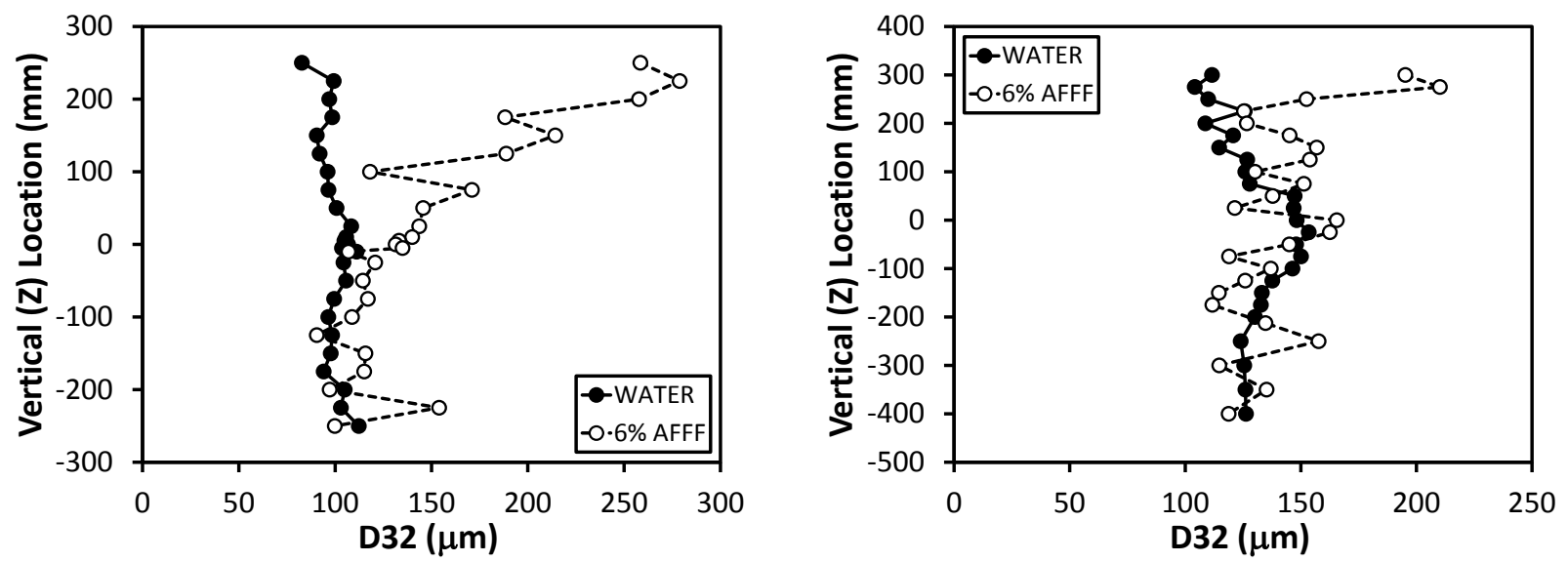

(a) $\mathrm{x}=3.05 \mathrm{~m}(10 \mathrm{ft})$

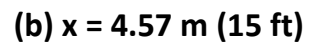

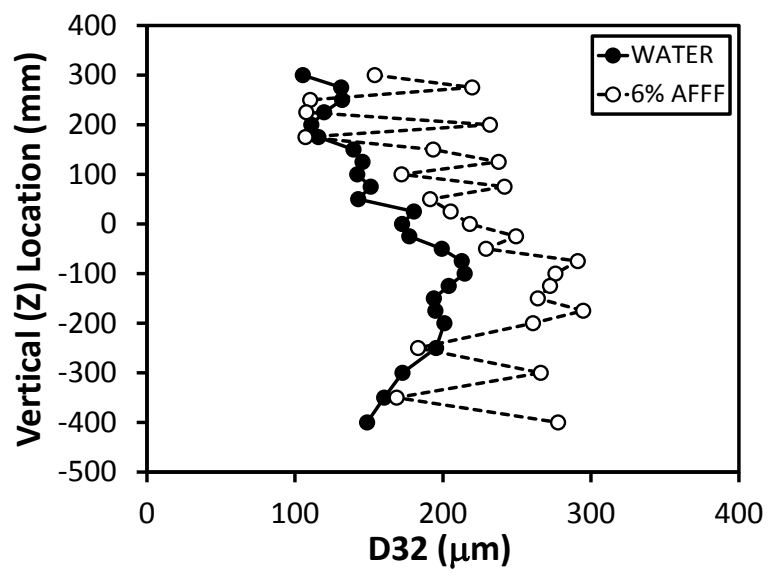

(c) $x=6.10 \mathrm{~m}(20 \mathrm{ft})$

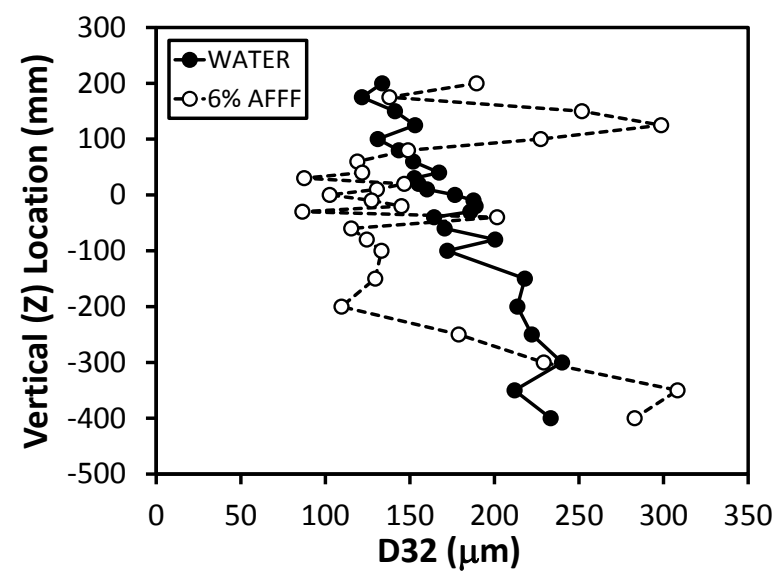

(d) $x=7.62 \mathrm{~m} \mathrm{(25 \textrm {ft } )}$

Figure C.32: Far Field Med. Flow, Med. Press. Jet Vertical Profiles of Sauter Mean Droplet Diameter 


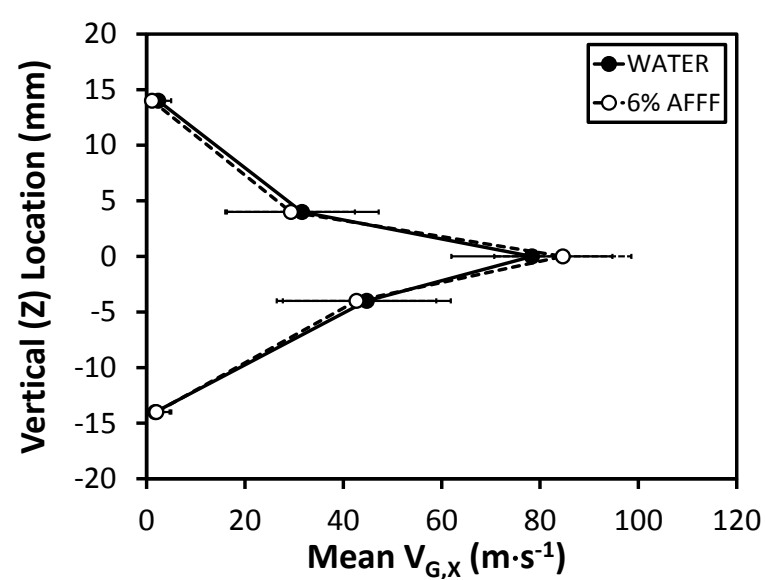

(a) $x=0.152 \mathrm{~m}(6 \mathrm{in})$

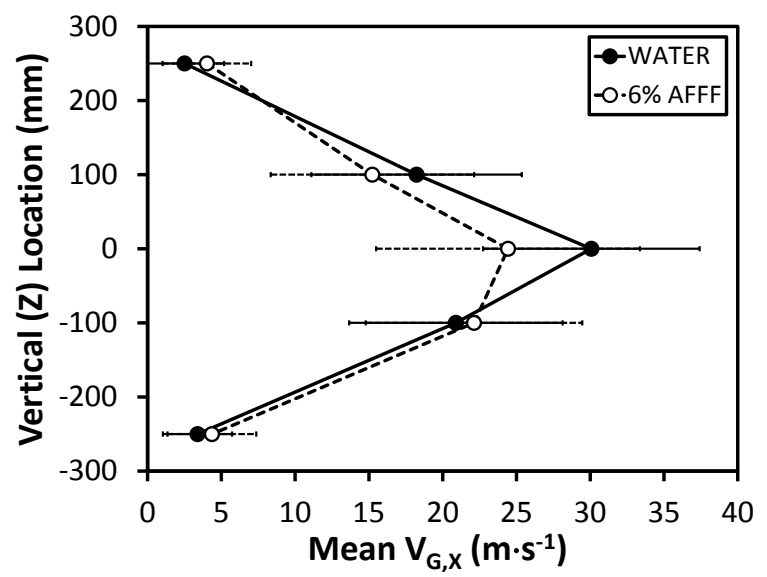

(c) $x=3.05 \mathrm{~m}(10 \mathrm{ft})$

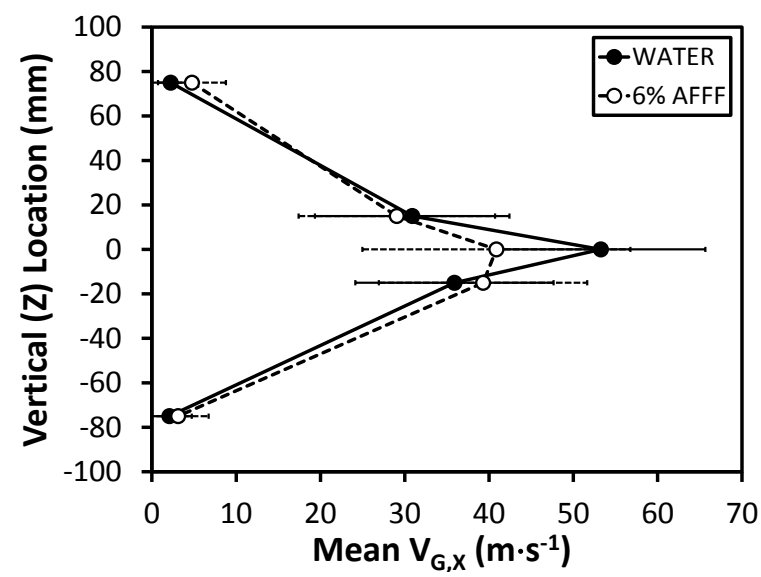

(b) $x=0.914 \mathrm{~m}(3 \mathrm{ft})$

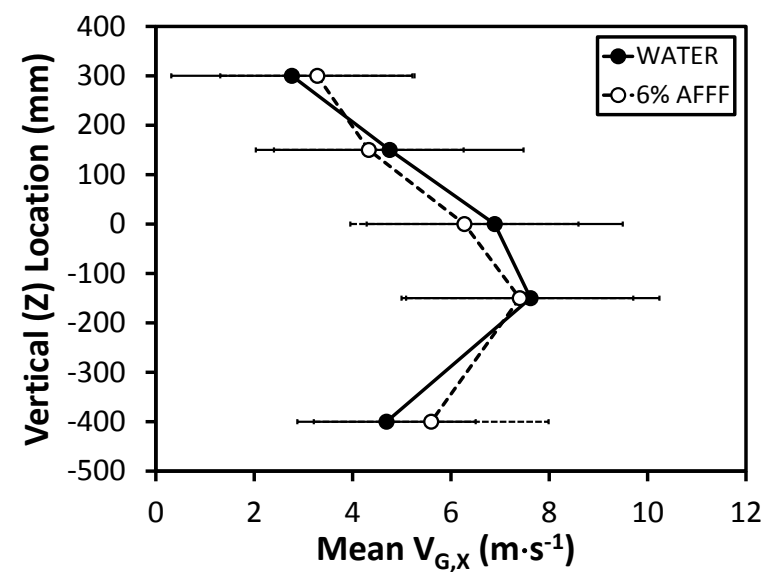

(d) $x=6.10 \mathrm{~m}(20 \mathrm{ft})$

Figure C.33: Select Med. Flow, Med. Press. Jet Vertical Profiles of Estimated Axial Gaseous (Air) Velocity 


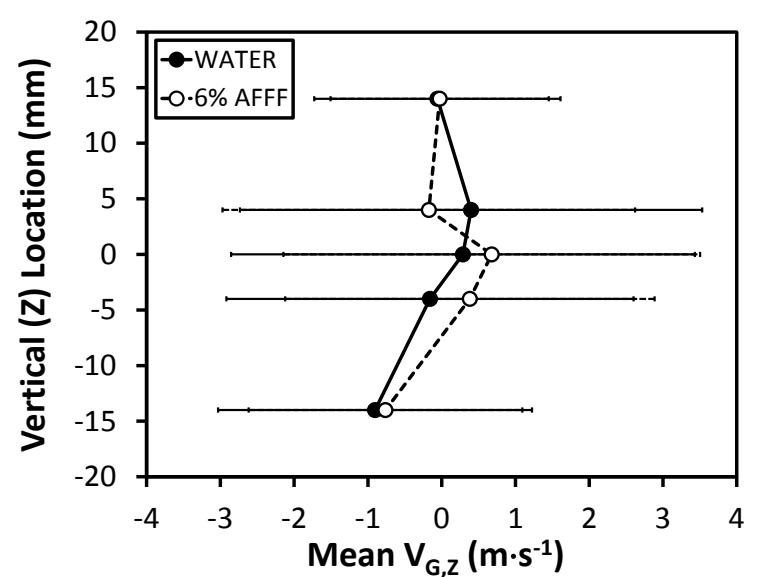

(a) $x=0.152 \mathrm{~m}(6 \mathrm{in})$

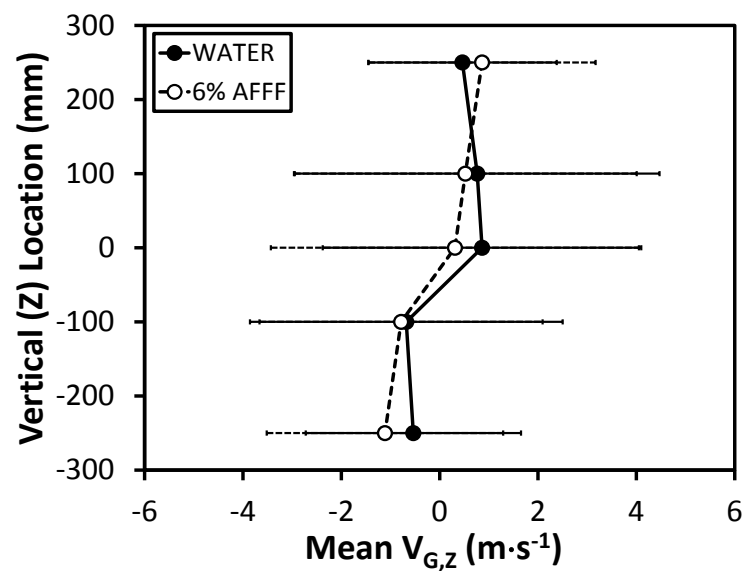

(c) $x=3.05 \mathrm{~m}(10 \mathrm{ft})$

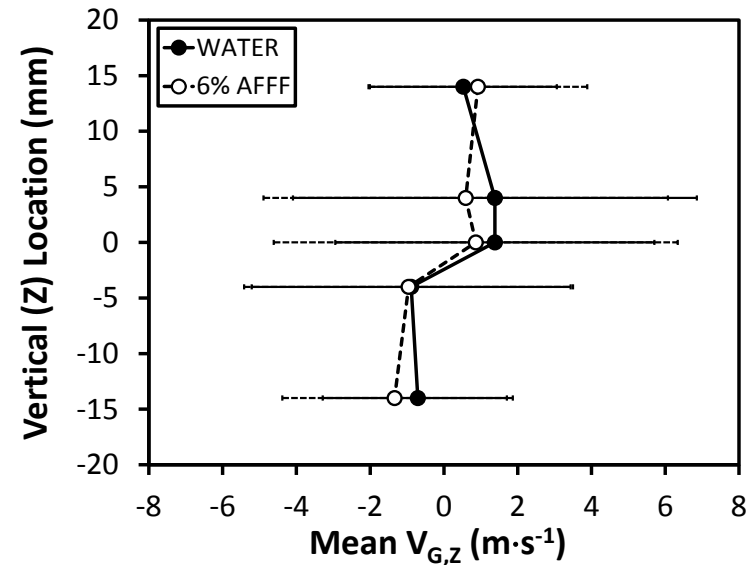

(b) $x=0.914 \mathrm{~m}(3 \mathrm{ft})$

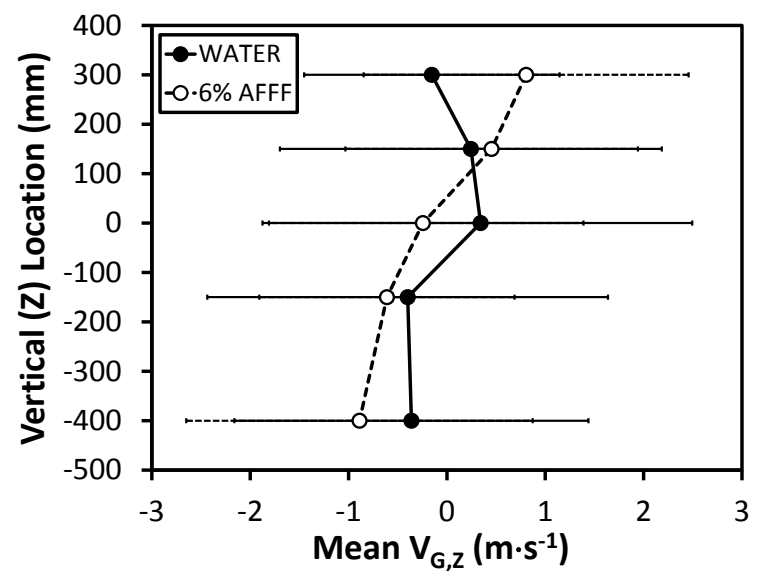

(d) $x=6.10 \mathrm{~m}(20 \mathrm{ft})$

Figure C.34: Select Med. Flow, Med. Press. Jet Vertical Profiles of Estimated Vertical Gaseous (Air) Velocity 

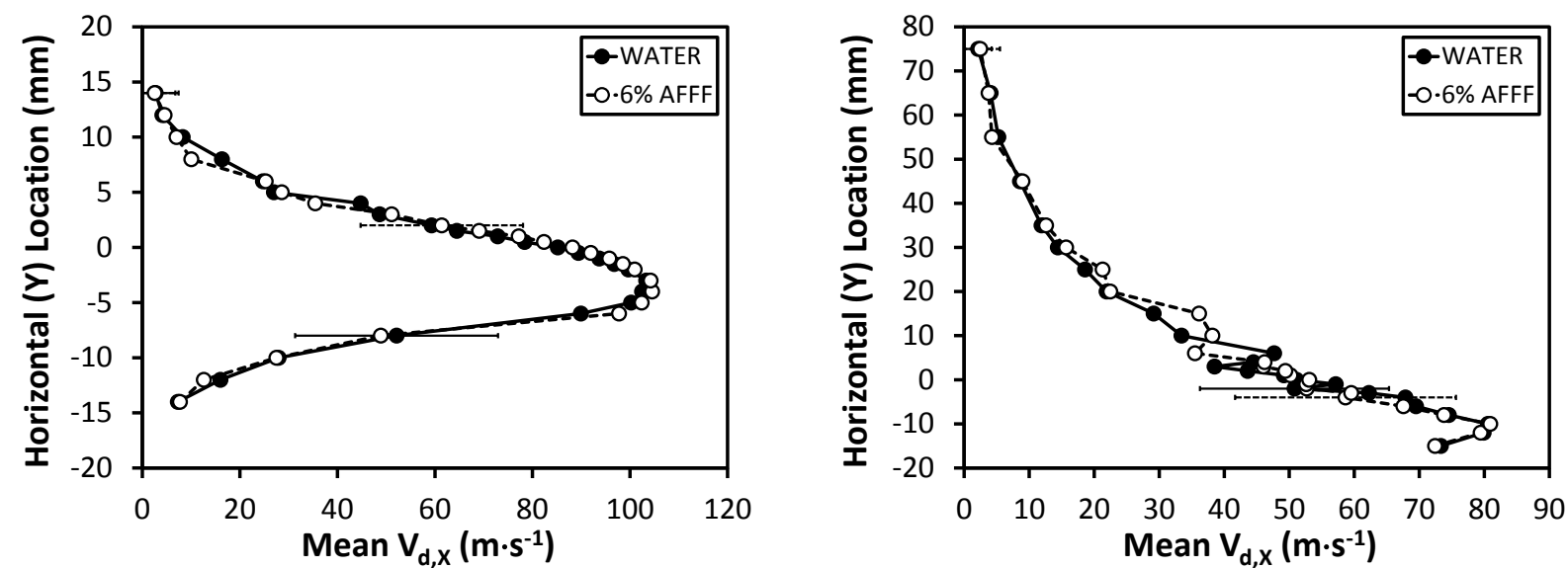

(a) $x=0.152 \mathrm{~m}(6 \mathrm{in})$

(b) $x=0.914 \mathrm{~m}(3 \mathrm{ft})$

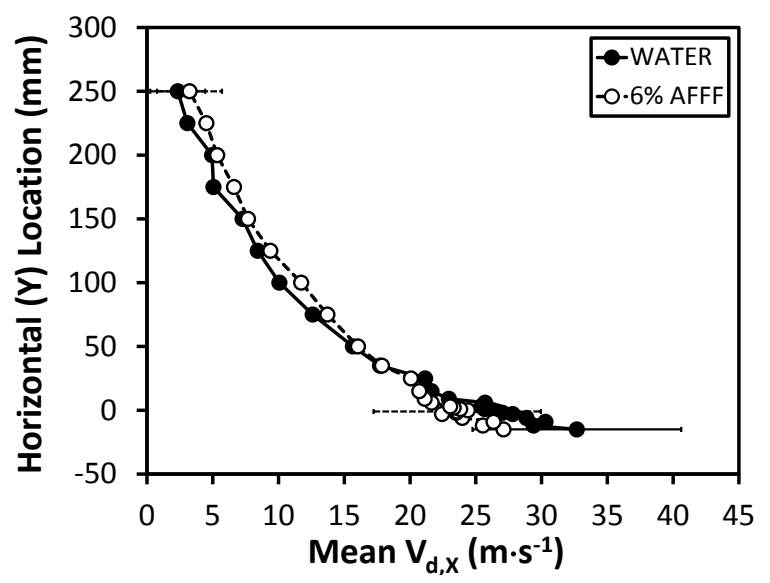

(c) $x=3.05 \mathrm{~m}(10 \mathrm{ft})$

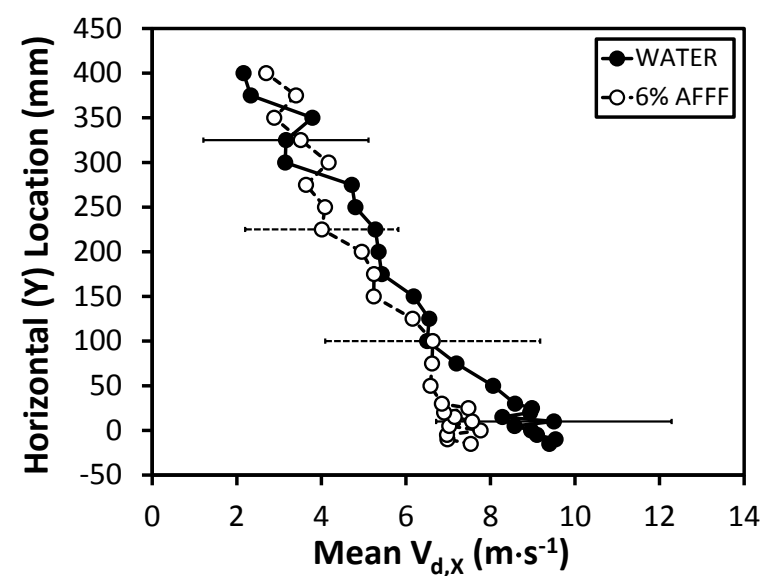

(d) $x=6.10 \mathrm{~m}(20 \mathrm{ft})$

Figure C.35: Select Med. Flow, Med. Pressure Jet Horizontal Profiles of Mean Axial Droplet Velocity 


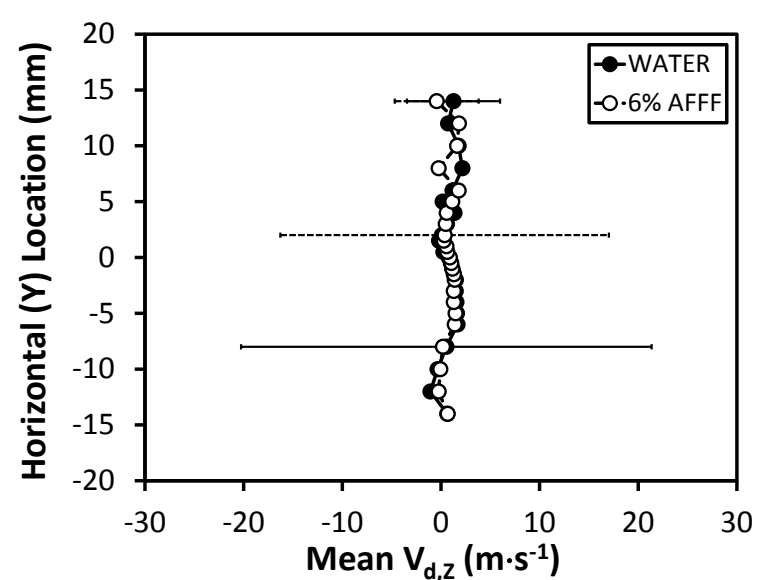

(a) $x=0.152 \mathrm{~m}(6 \mathrm{in})$

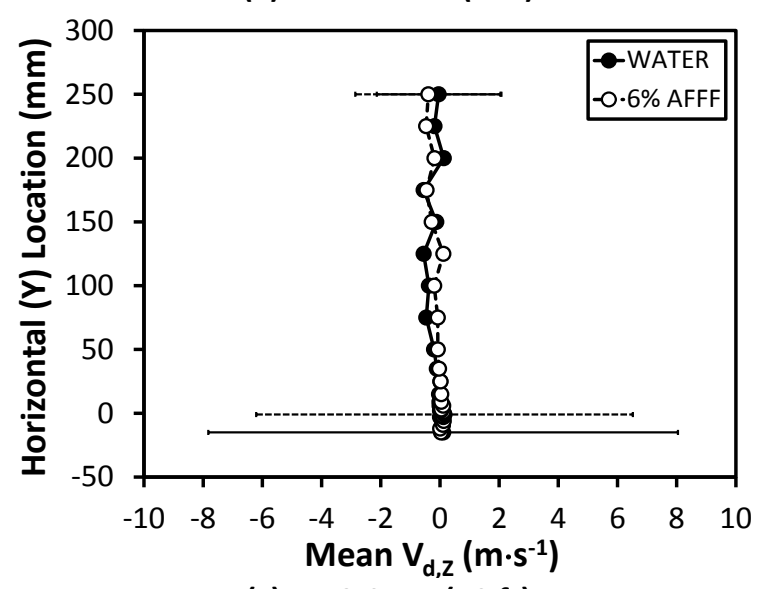

(c) $x=3.05 \mathrm{~m}(10 \mathrm{ft})$

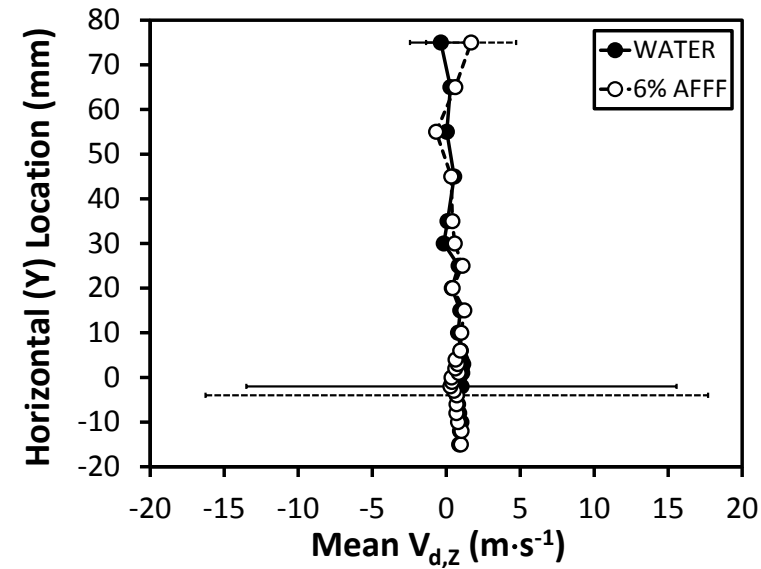

(b) $x=0.914 \mathrm{~m}(3 \mathrm{ft})$

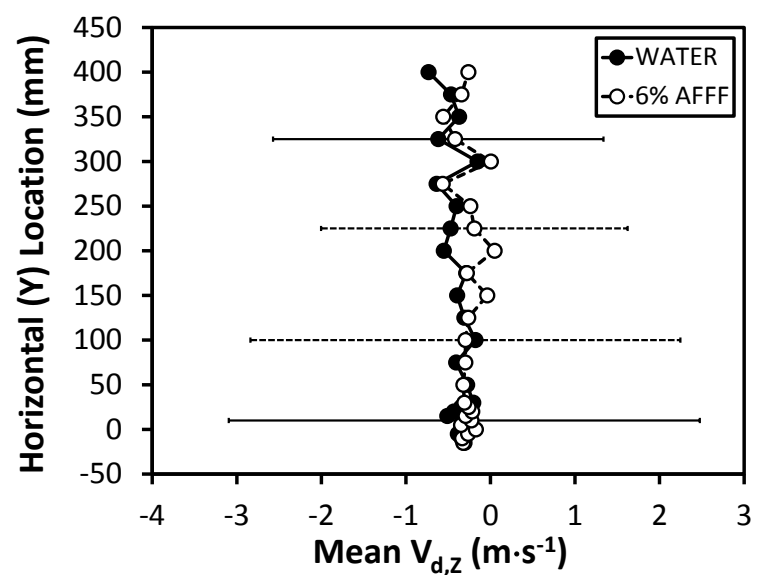

(d) $x=6.10 \mathrm{~m}(20 \mathrm{ft})$

Figure C.36: Select Med. Flow, Med. Press. Jet Horizontal Profiles of Mean Vertical Droplet Velocity 

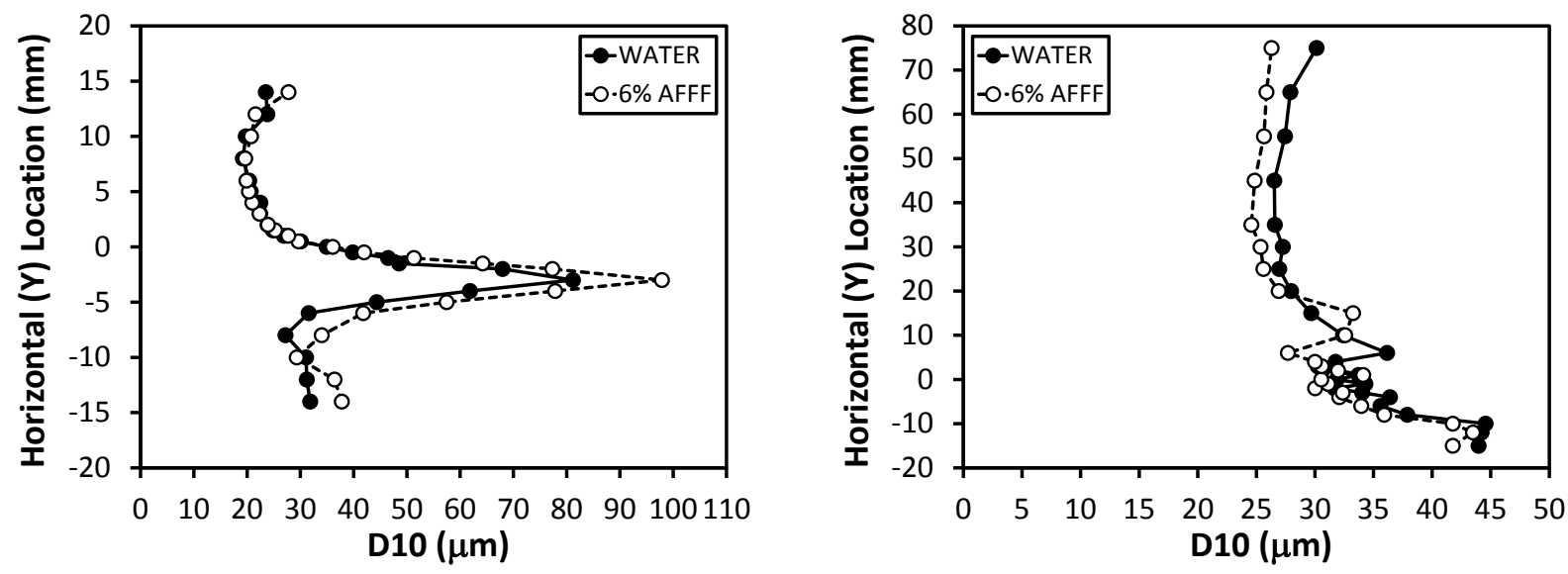

(a) $x=0.152 \mathrm{~m}(6 \mathrm{in})$

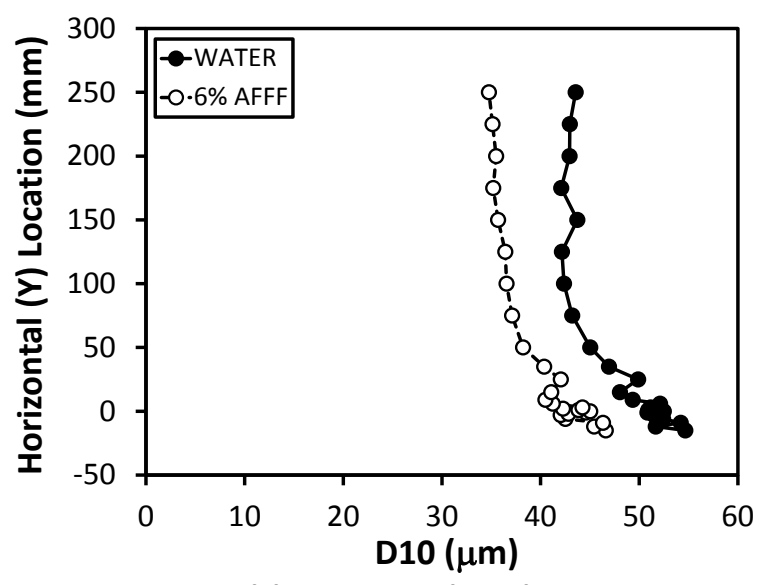

(c) $\mathrm{x}=3.05 \mathrm{~m}(10 \mathrm{ft})$

(b) $x=0.914 \mathrm{~m} \mathrm{(3 \textrm {ft } )}$

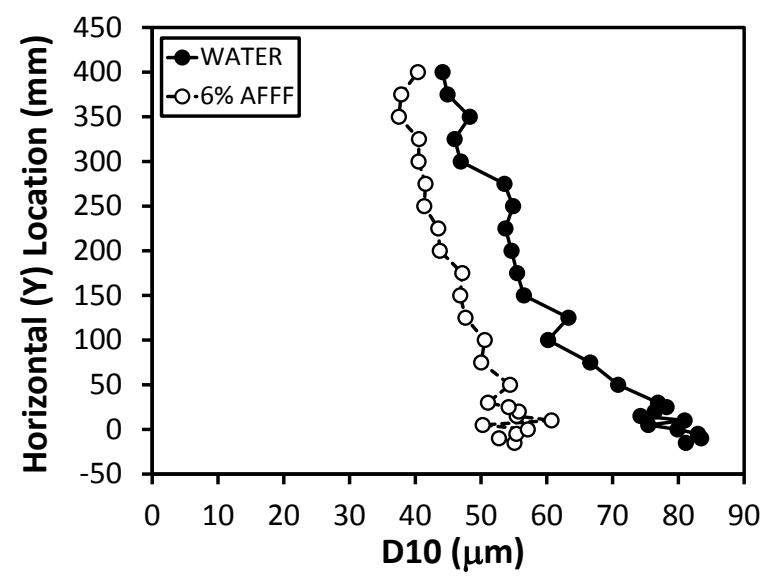

(d) $x=6.10 \mathrm{~m}(20 \mathrm{ft})$

Figure C.37: Select Med. Flow, Med. Press. Jet Horizontal Profiles of Mean Droplet Diameter 

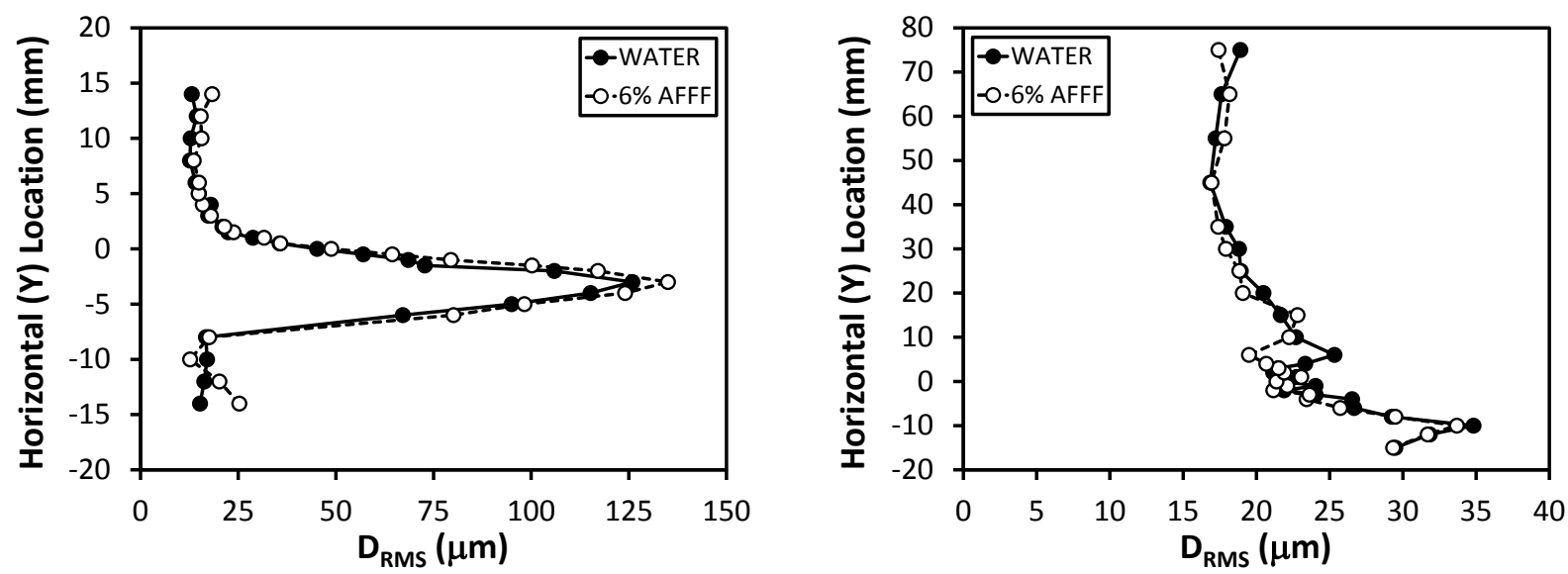

(a) $x=0.152 \mathrm{~m}(6 \mathrm{in})$

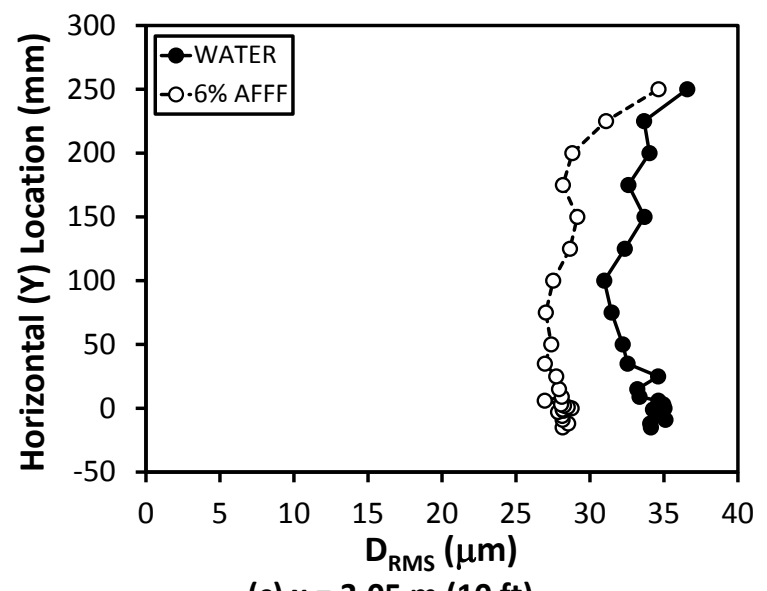

(b) $x=0.914 \mathrm{~m} \mathrm{(3 \textrm {ft } )}$

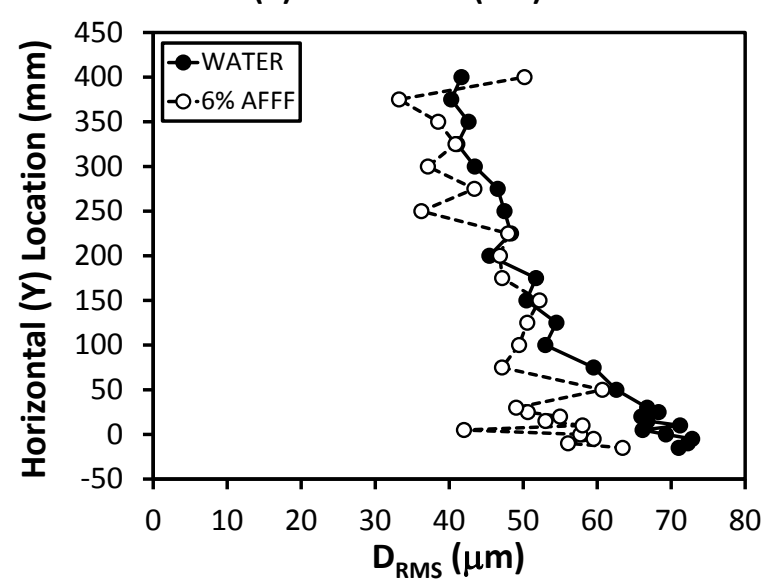

(d) $x=6.10 \mathrm{~m}(20 \mathrm{ft})$

Figure C.38: Select Med. Flow, Med. Press. Jet Horizontal Profiles of RMS Droplet Diameter 

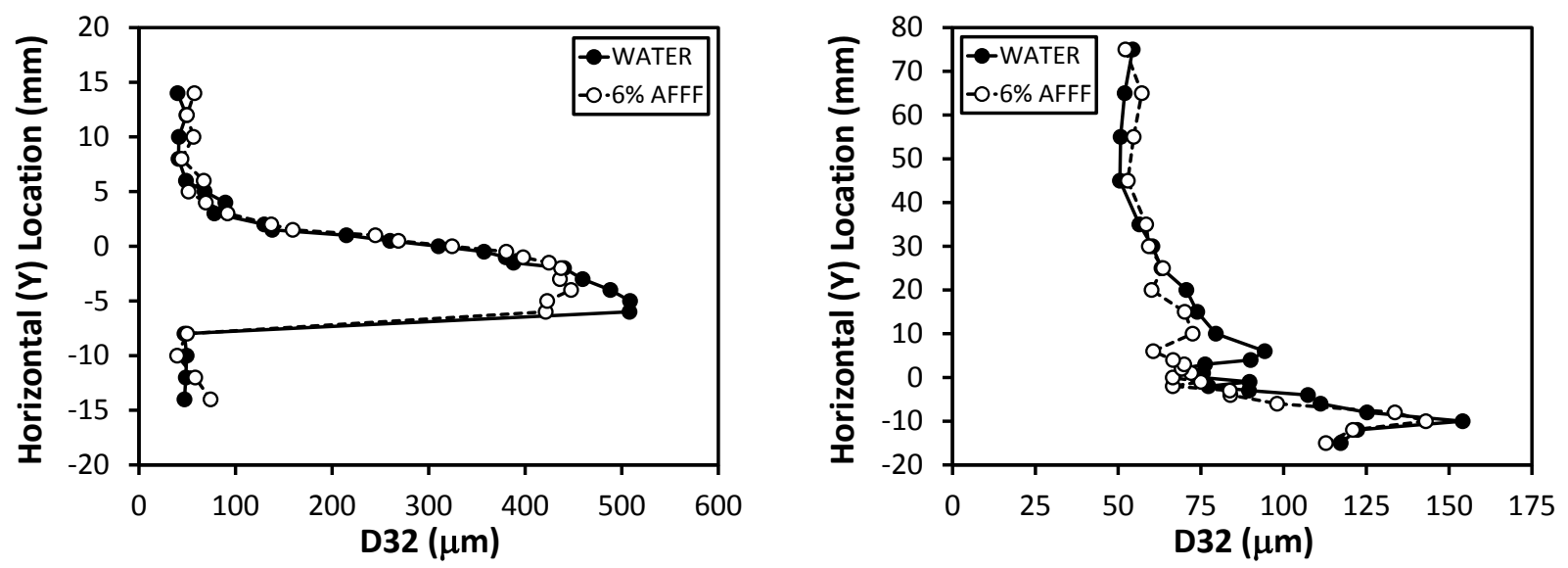

(a) $x=0.152 \mathrm{~m}(6 \mathrm{in})$

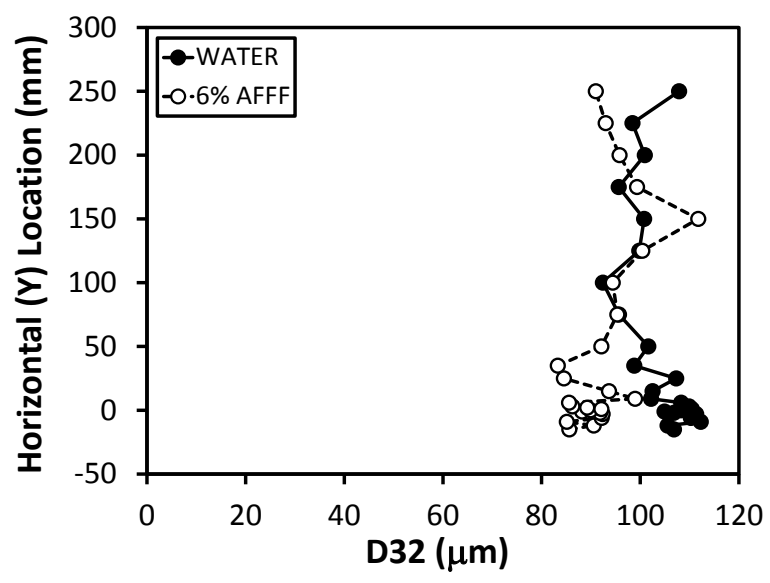

(c) $\mathrm{x}=3.05 \mathrm{~m}(10 \mathrm{ft})$

(b) $x=0.914 \mathrm{~m} \mathrm{(3 \textrm {ft } )}$

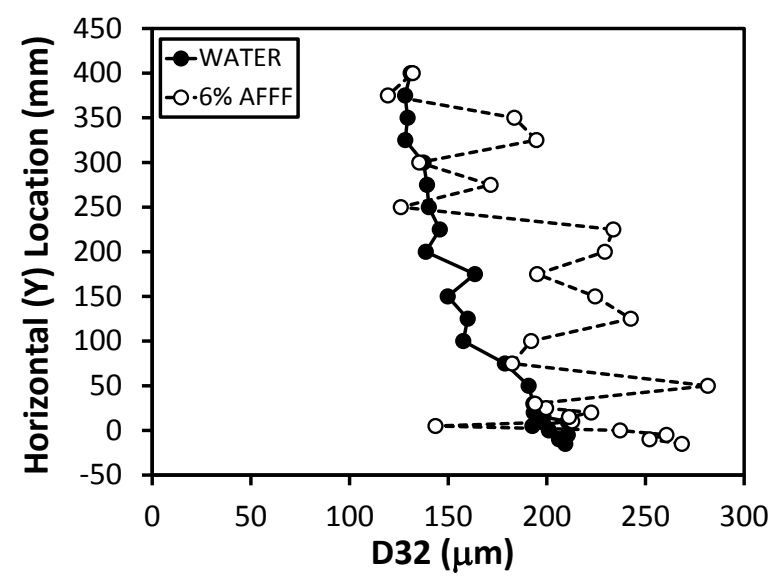

(d) $x=6.10 \mathrm{~m}(20 \mathrm{ft})$

Figure C.39: Select Med. Flow, Med. Press. Jet Horizontal Profiles of Sauter Mean Droplet Diameter 


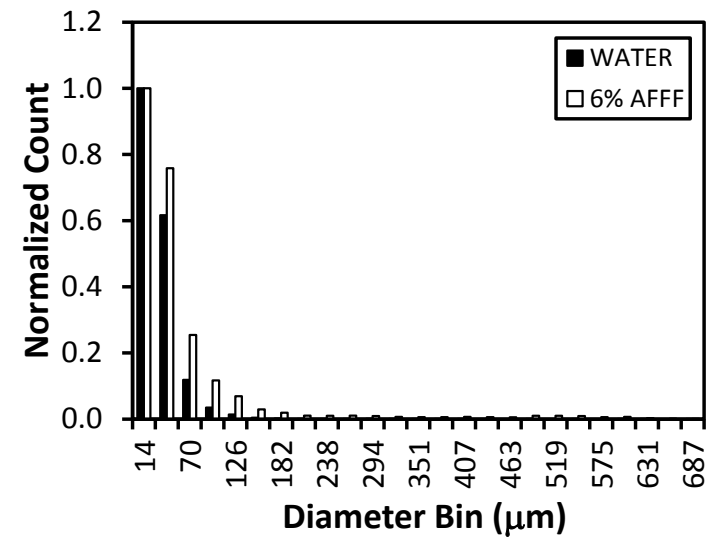

(a) $x=0.152 \mathrm{~m}(6 \mathrm{in})$

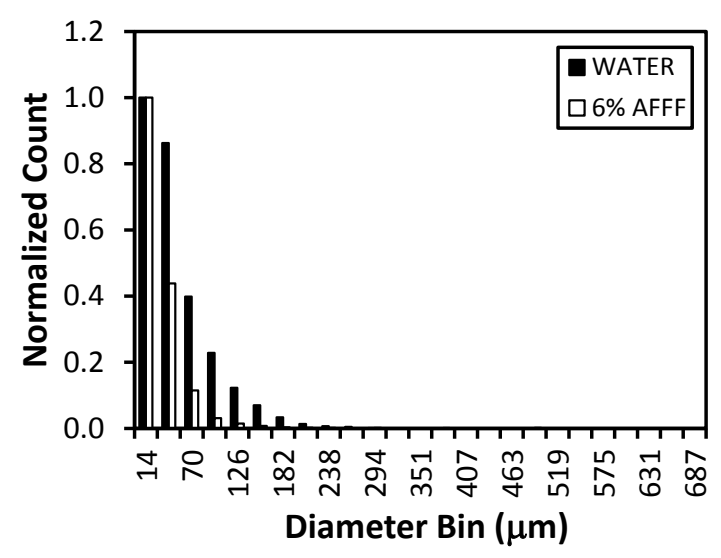

(c) $x=3.05 \mathrm{~m}(10 \mathrm{ft})$

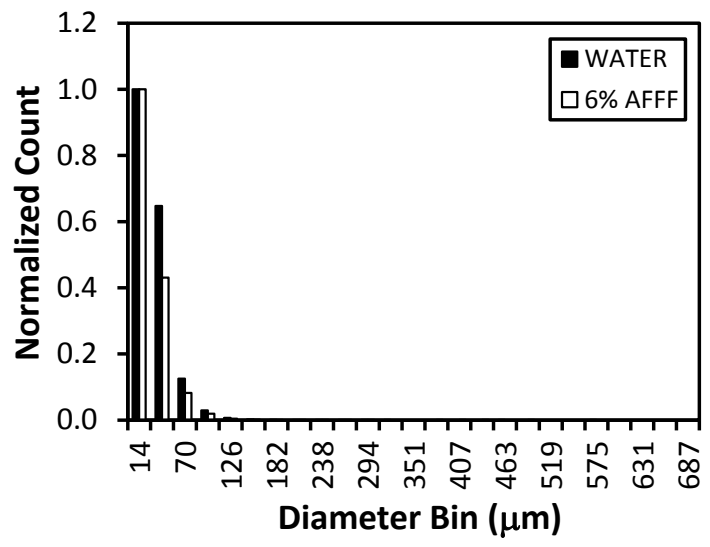

(b) $x=0.914 \mathrm{~m} \mathrm{(3 \textrm {ft } )}$

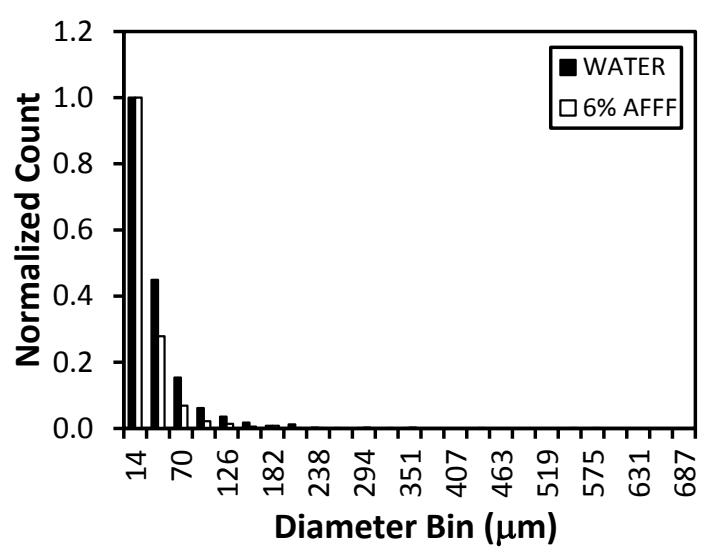

(d) $x=6.10 \mathrm{~m}(20 \mathrm{ft})$

Figure C.40: Med. Flow, Med. Press. Jet Droplet Size Distribution at the Max. Profile, Mean Axial Droplet Velocity for Select Vertical Profiles 


\section{C.4 High Flow, Low Pressure Firefighting Jet Data}

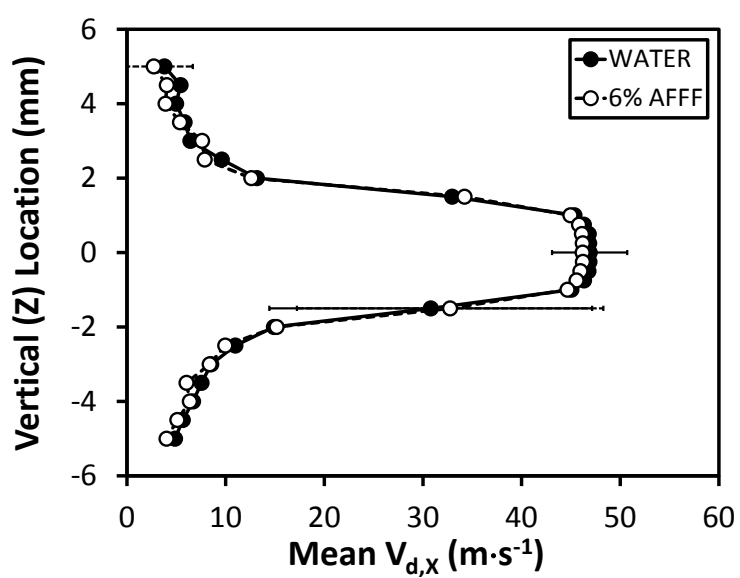

(a) $x=6.4 \mathrm{~mm}$ (0.25 in)

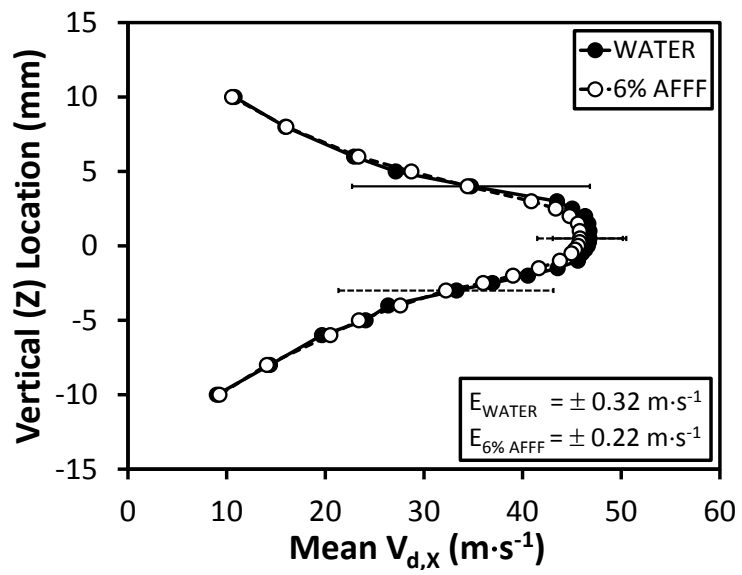

(c) $x=0.152 \mathrm{~m}(6 \mathrm{in})$

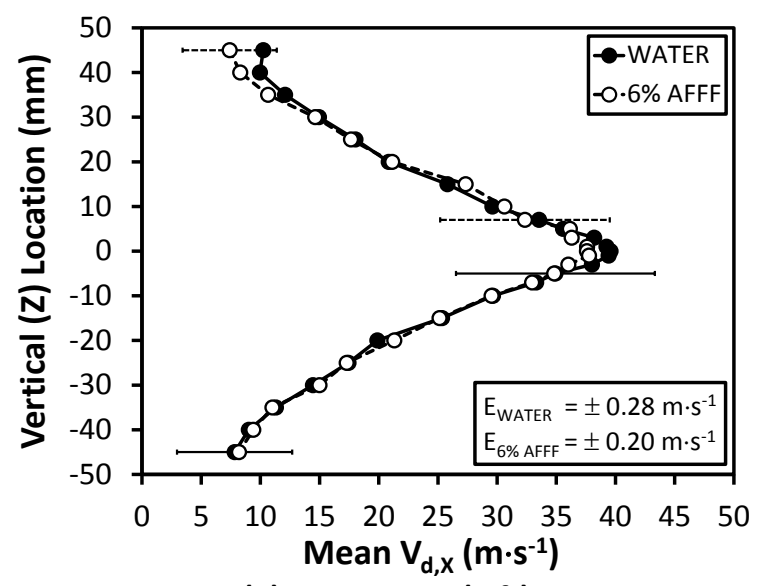

(e) $x=0.914 \mathrm{~m}(3 \mathrm{ft})$

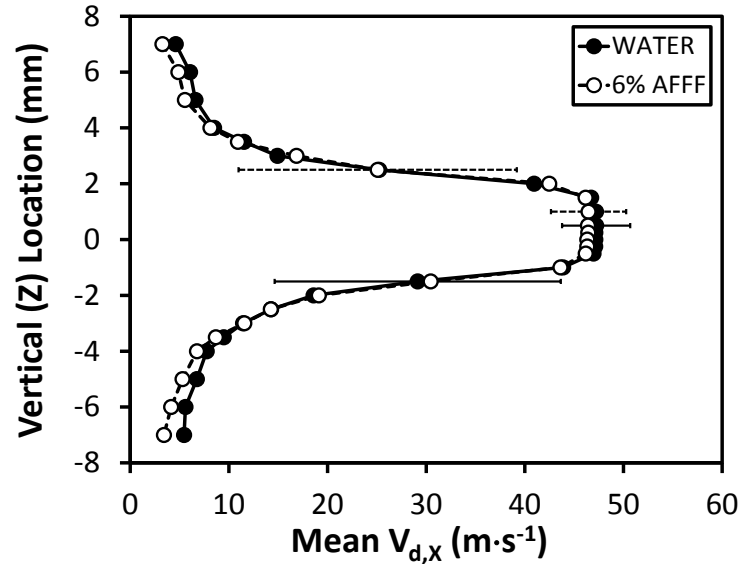

(b) $x=25.4 \mathrm{~mm}$ (1 in)

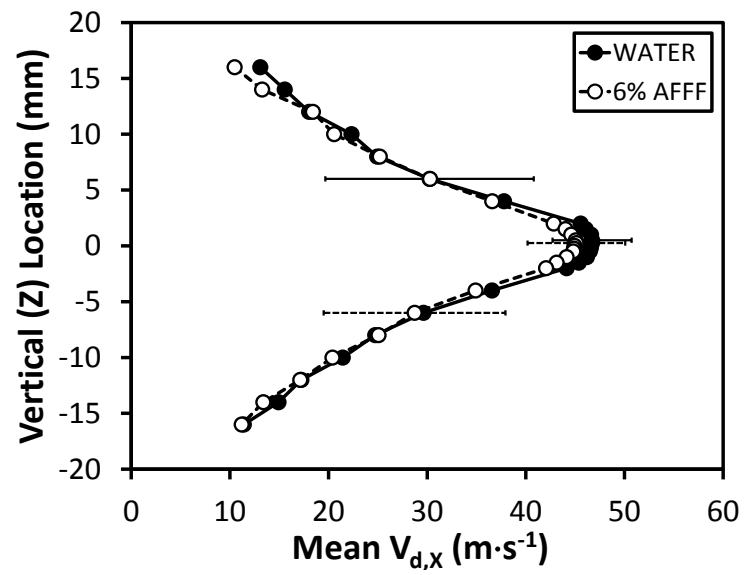

(d) $x=0.305 \mathrm{~m}(1 \mathrm{ft})$

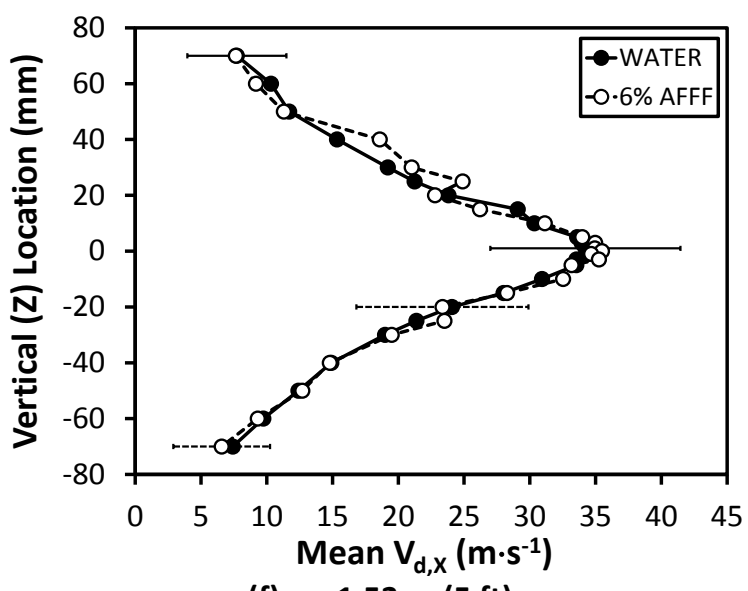

(f) $x=1.52 \mathrm{~m}(5 \mathrm{ft})$

Figure C.41: Near Field High Flow, Low Press. Jet Vertical Profiles of Mean Axial Droplet Velocity 


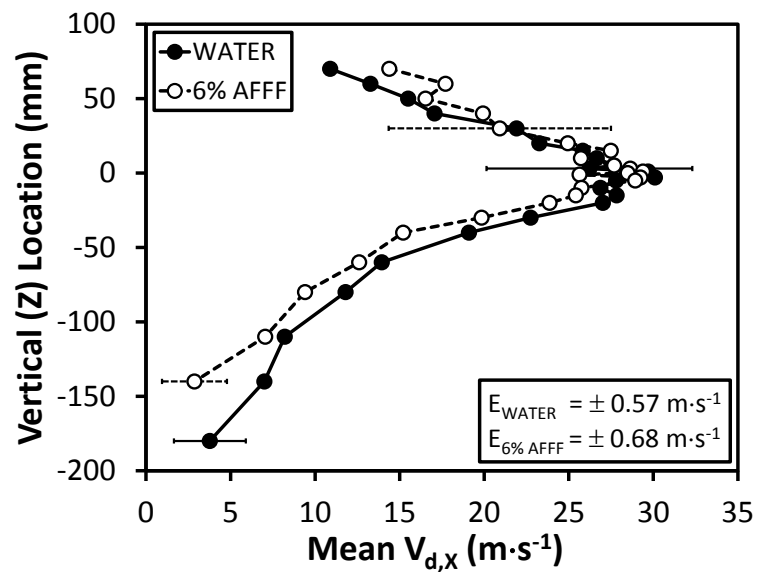

(a) $x=3.05 \mathrm{~m}(10 \mathrm{ft})$

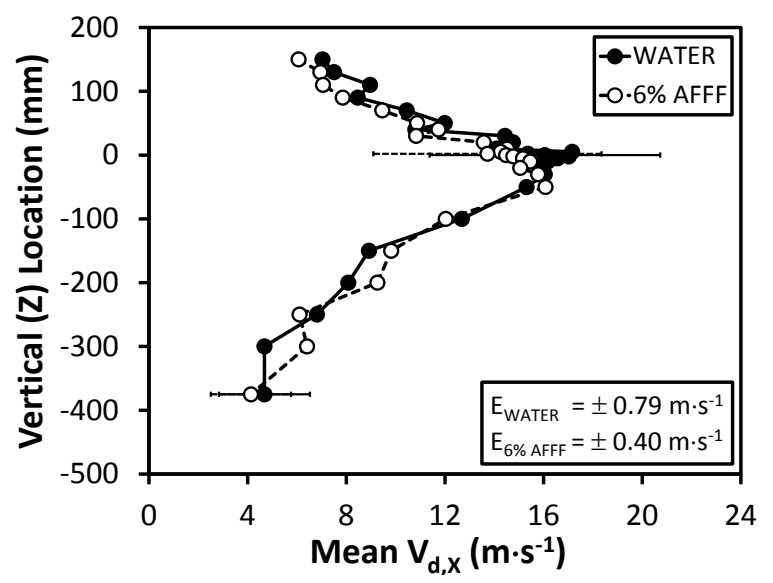

(c) $x=6.10 \mathrm{~m}(20 \mathrm{ft})$

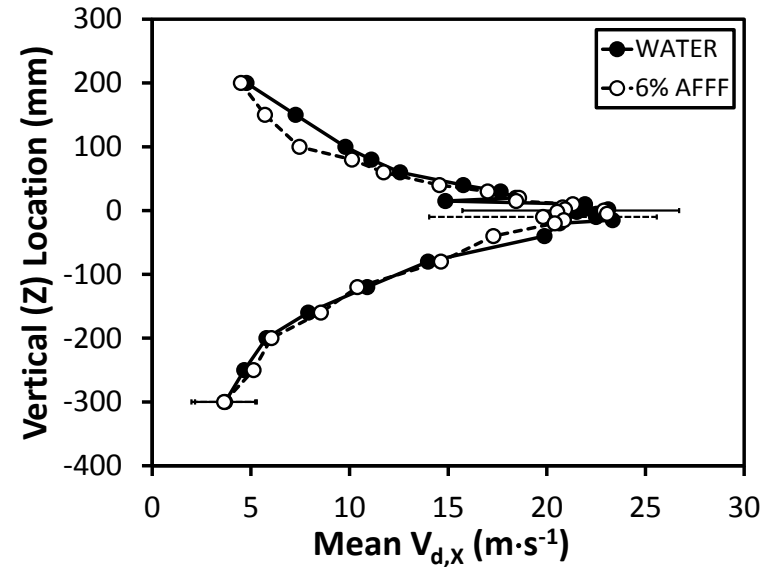

(b) $x=4.57 \mathrm{~m}(15 \mathrm{ft})$

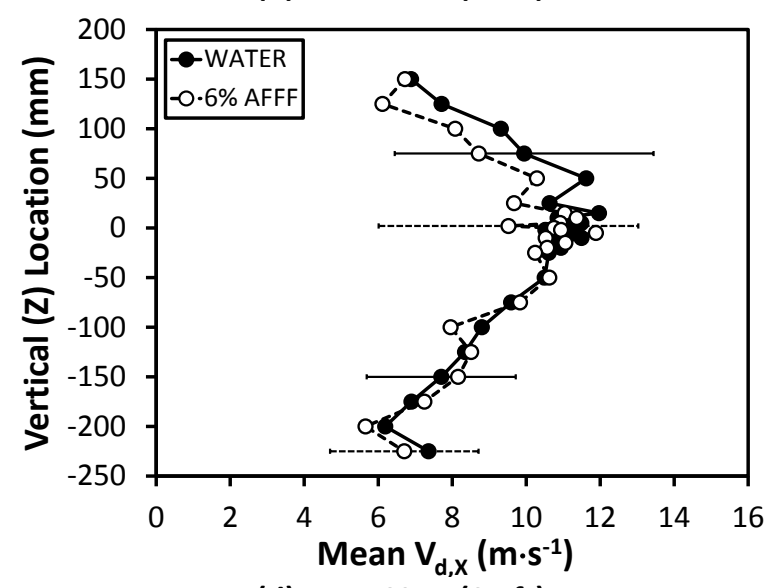

(d) $x=7.62 \mathrm{~m}(25 \mathrm{ft})$

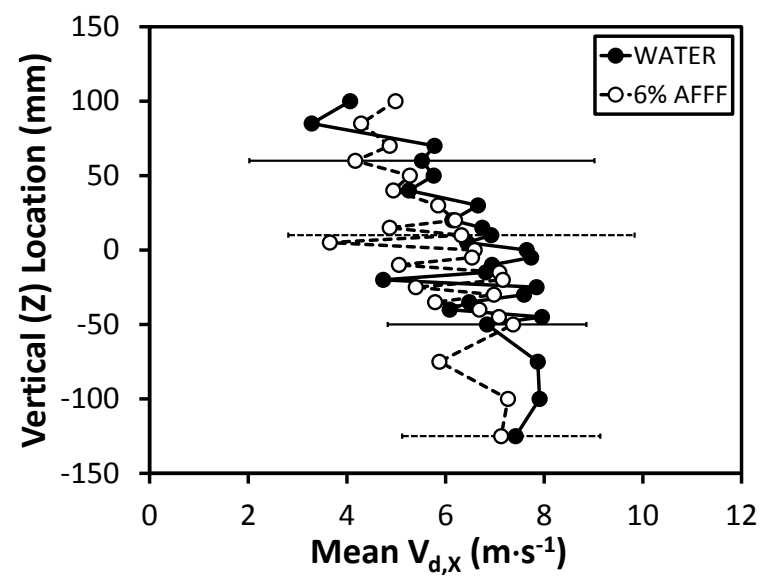

(e) $\mathrm{x}=9.14 \mathrm{~m}(30 \mathrm{ft})$

Figure C.42: Far Field High Flow, Low Press. Jet Vertical Profiles of Mean Axial Droplet Velocity 


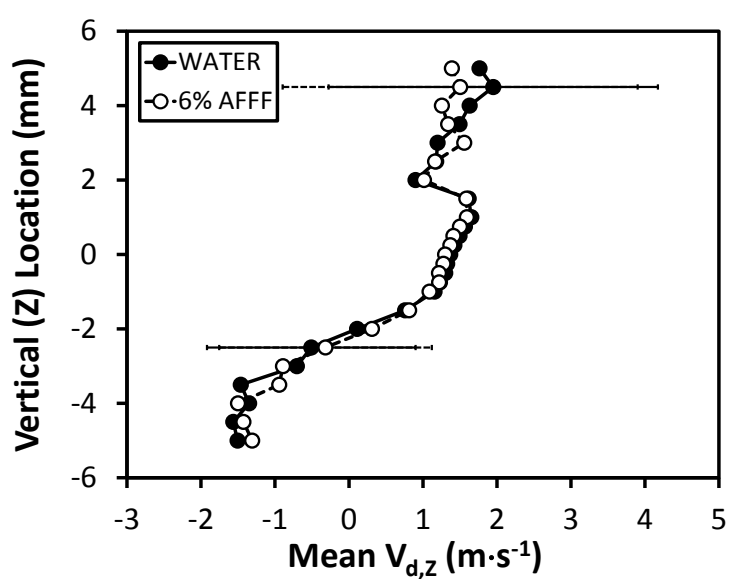

(a) $x=6.4 \mathrm{~mm}(0.25 \mathrm{in})$

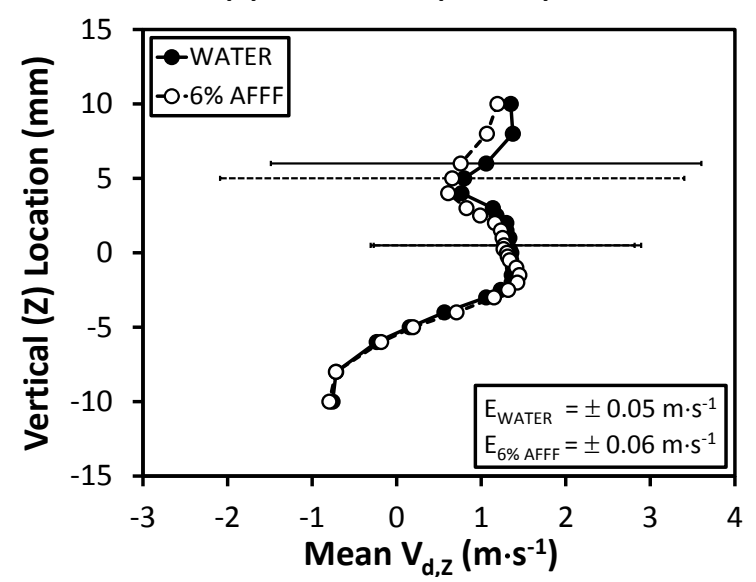

(c) $x=0.152 \mathrm{~m}(6 \mathrm{in})$

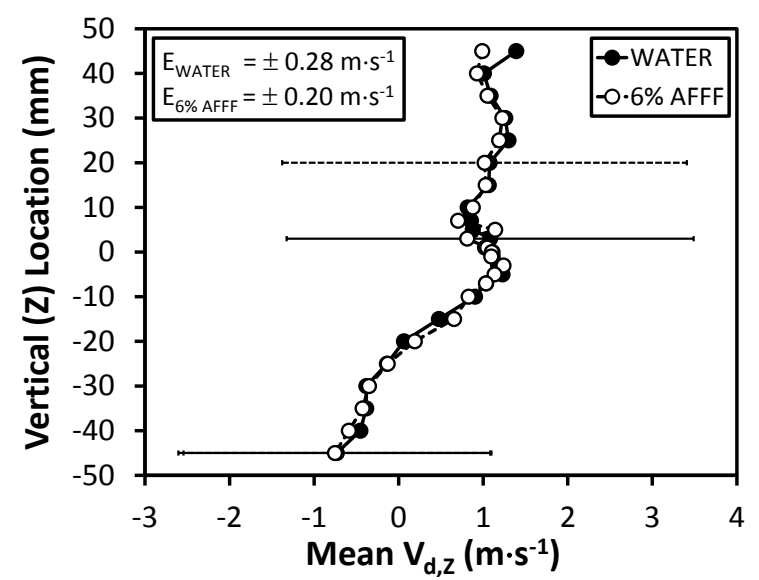

(e) $x=0.914 \mathrm{~m} \mathrm{(3 \textrm {ft } )}$

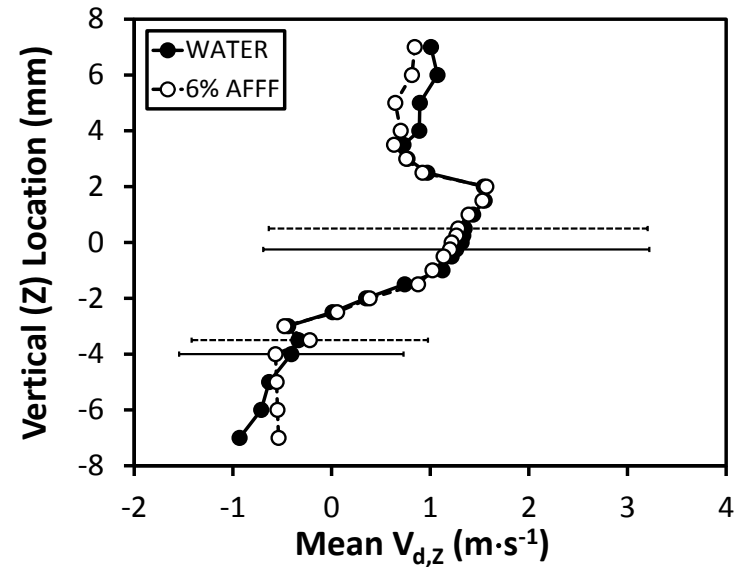

(b) $x=25.4 \mathrm{~mm}(1 \mathrm{in})$

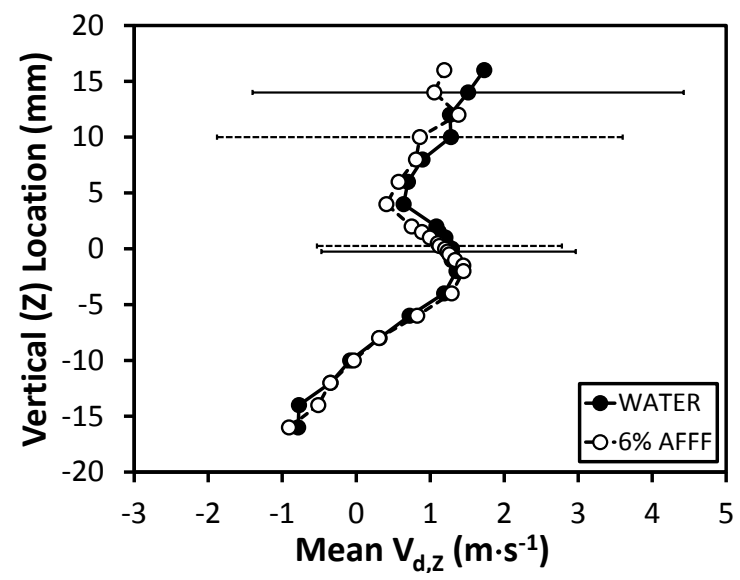

(d) $\mathrm{x}=0.305 \mathrm{~m}(\mathbf{1 f t})$

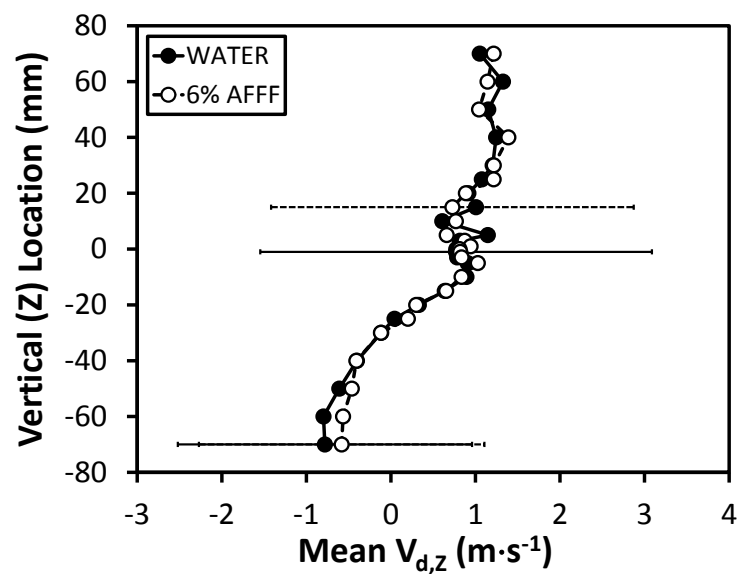

(f) $x=1.52 \mathrm{~m}(5 \mathrm{ft})$

Figure C.43: Near Field High Flow, Low Press. Jet Vertical Profiles of Mean Vertical Droplet Velocity 


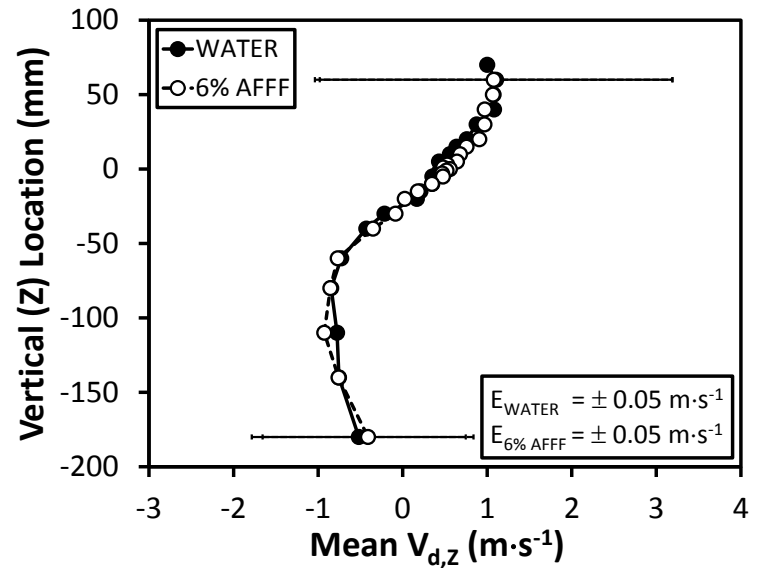

(a) $\mathrm{x}=3.05 \mathrm{~m}(10 \mathrm{ft})$

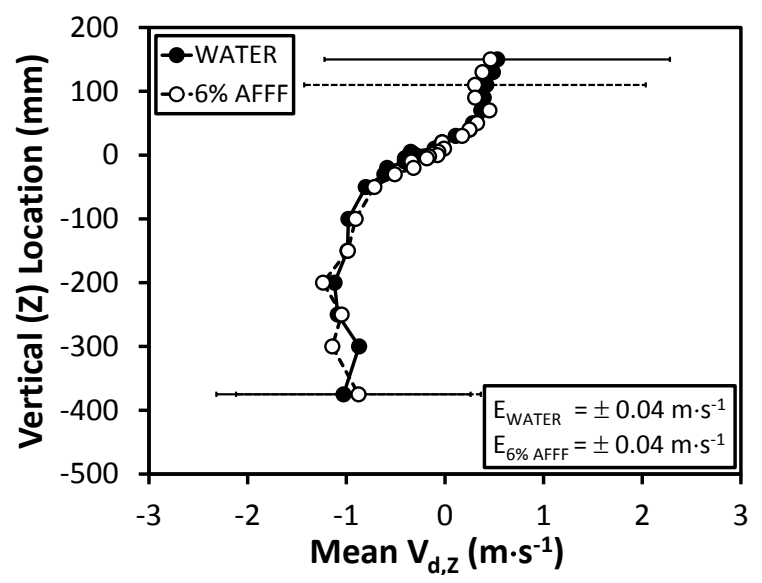

(c) $x=6.10 \mathrm{~m}(20 \mathrm{ft})$

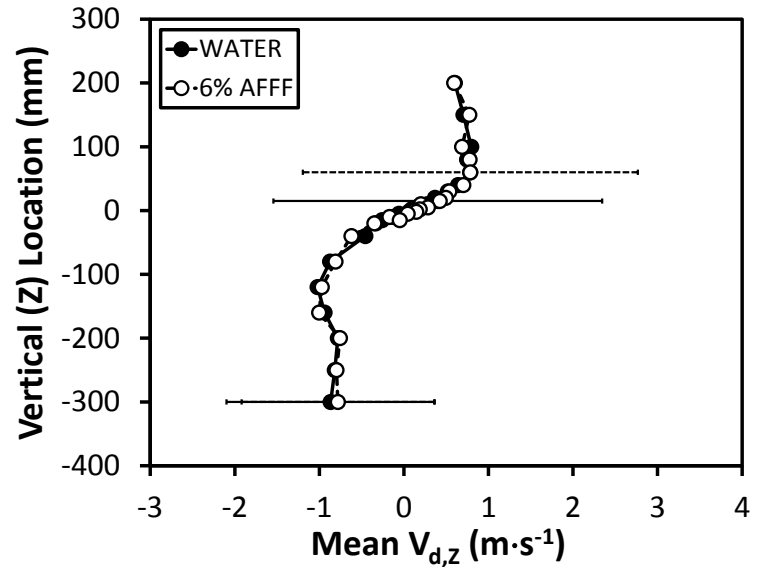

(b) $x=4.57 \mathrm{~m}(15 \mathrm{ft})$

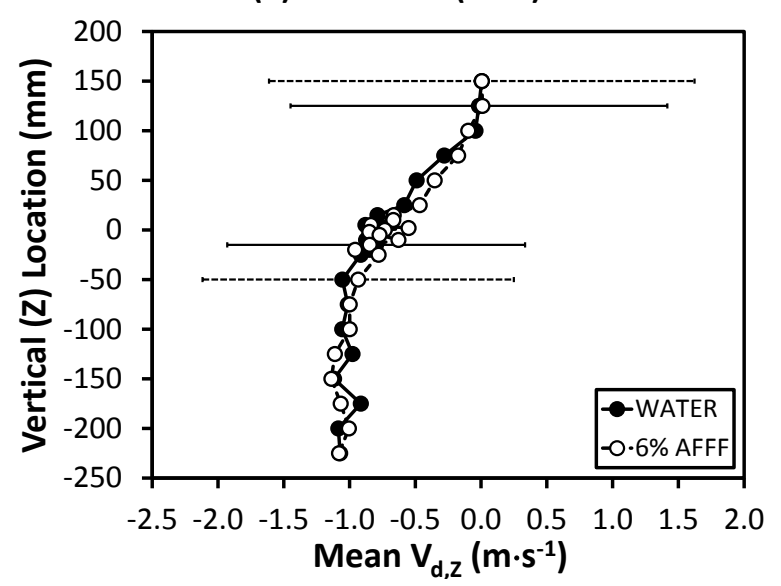

(d) $x=7.62 \mathrm{~m}(25 \mathrm{ft})$

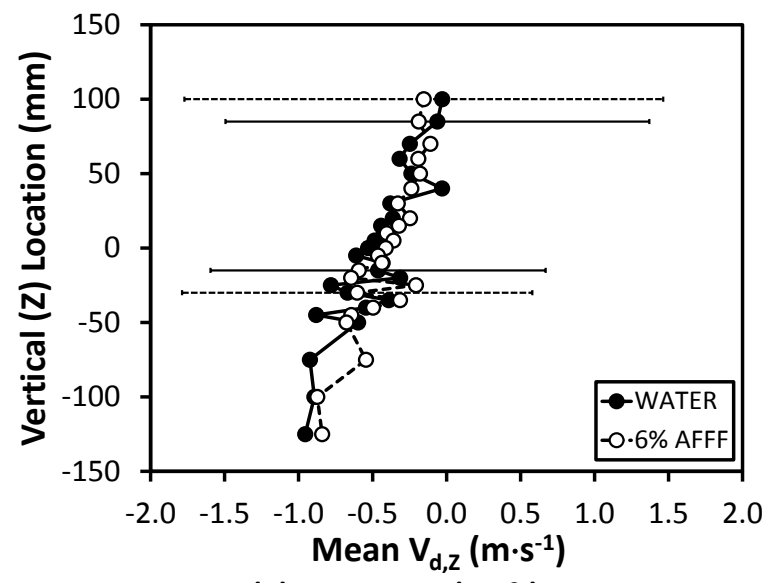

(e) $x=9.14 \mathrm{~m}(30 \mathrm{ft})$

Figure C.44: Far Field High Flow, Low Press. Jet Vertical Profiles of Mean Vertical Droplet Velocity 


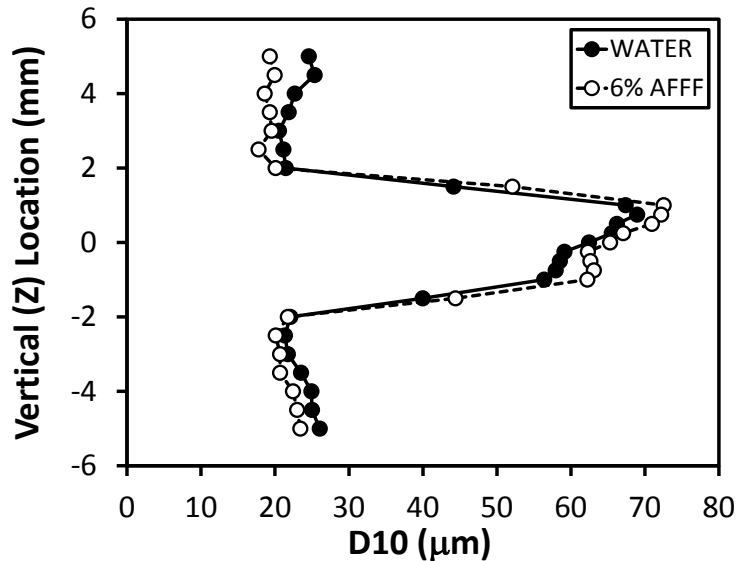

(a) $x=6.4 \mathrm{~mm}(0.25 \mathrm{in})$

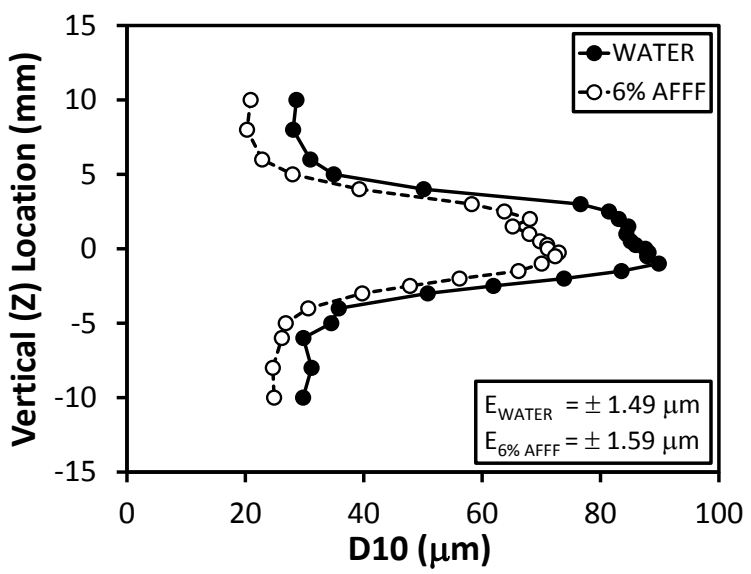

(c) $x=0.152 \mathrm{~m}(6 \mathrm{in})$

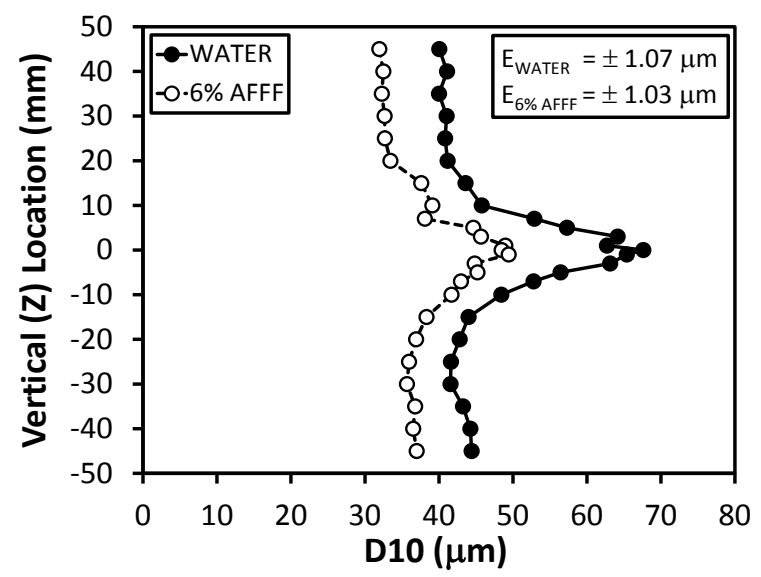

(e) $x=0.914 \mathrm{~m}(3 \mathrm{ft})$

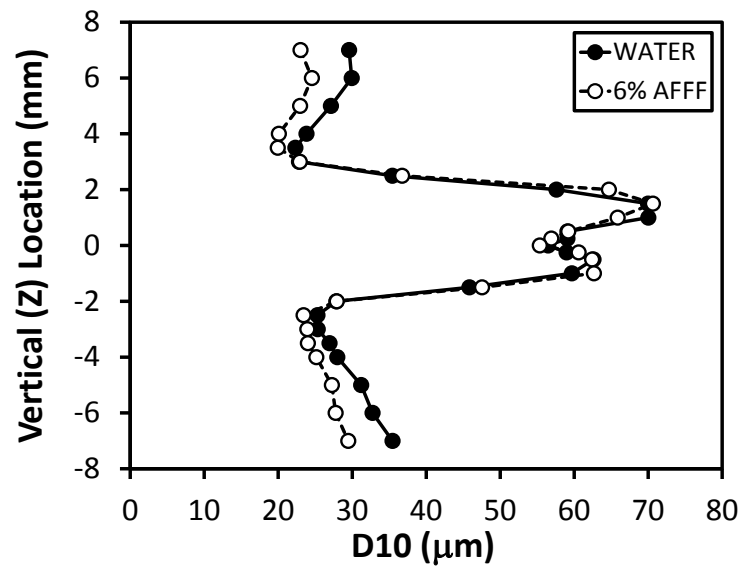

(b) $x=25.4 \mathrm{~mm}(1 \mathrm{in})$

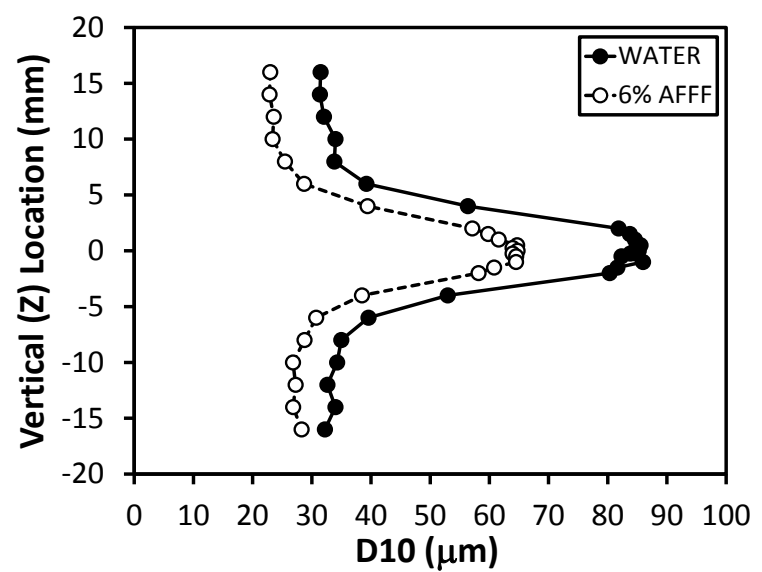

(d) $x=0.305 \mathrm{~m}(1 \mathrm{ft})$

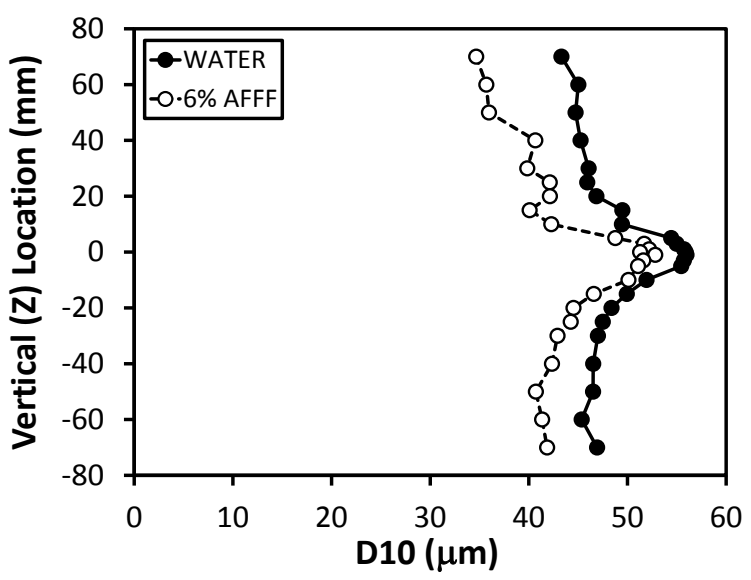

(f) $x=1.52 \mathrm{~m}(5 \mathrm{ft})$

Figure C.45: Near Field High Flow, Low Press. Jet Vertical Profiles of Mean Droplet Diameter 

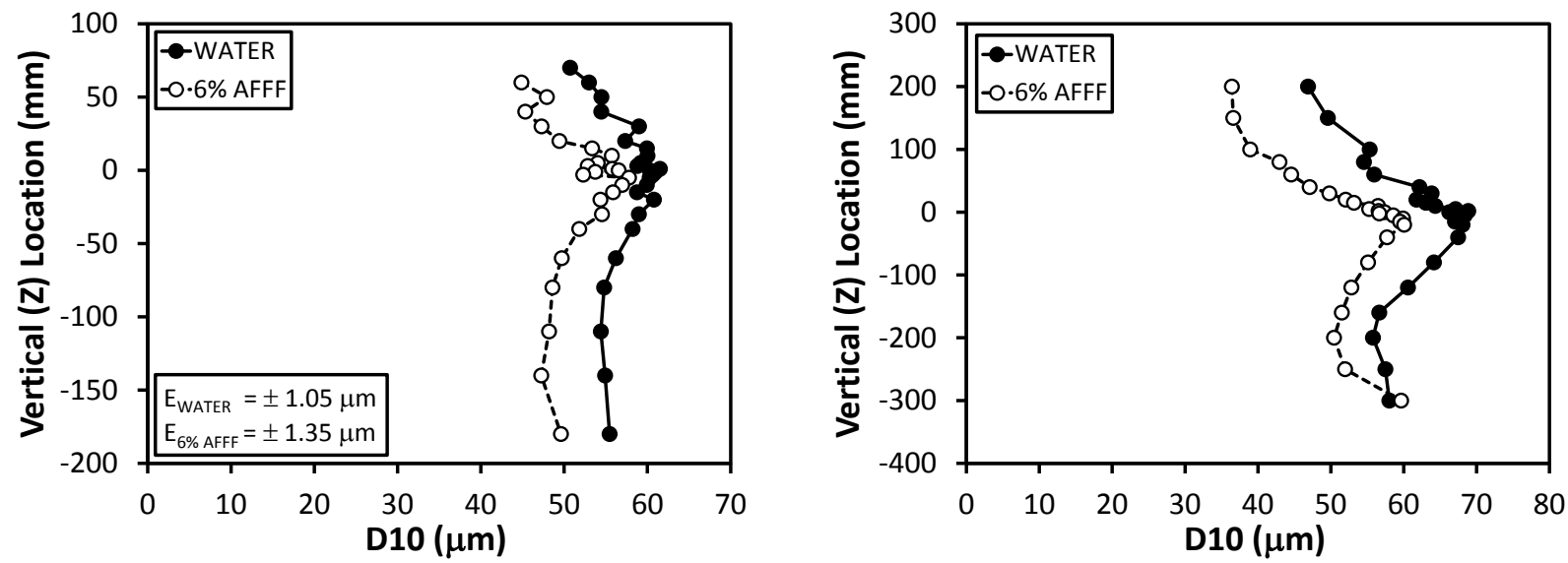

(a) $\mathrm{x}=3.05 \mathrm{~m}(10 \mathrm{ft})$

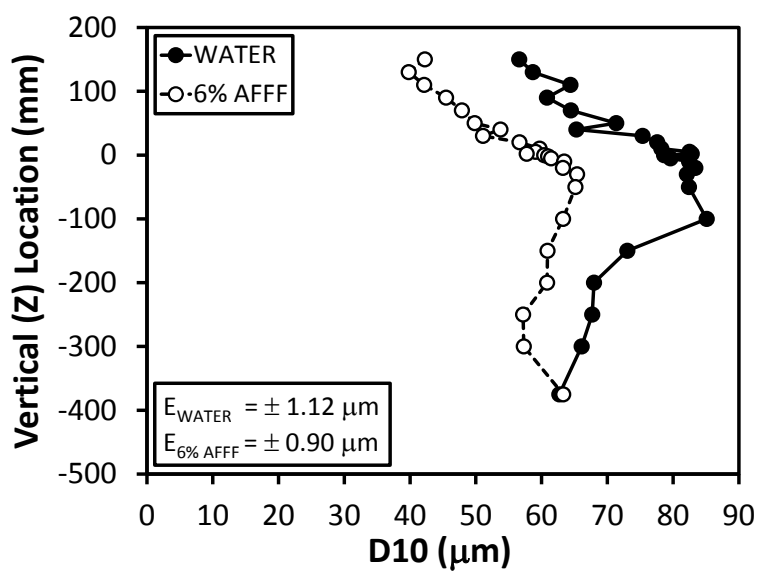

(b) $x=4.57 \mathrm{~m} \mathrm{(15} \mathrm{ft)}$

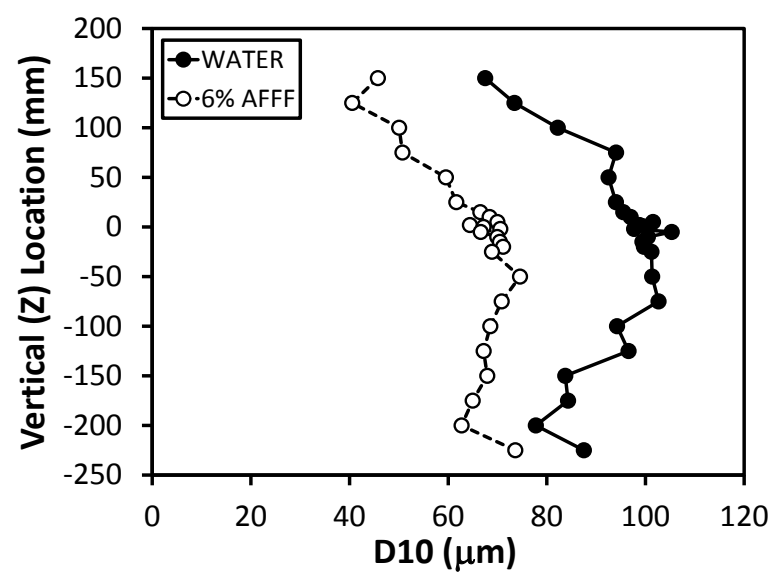

(c) $x=6.10 \mathrm{~m}(20 \mathrm{ft})$

(d) $x=7.62 \mathrm{~m} \mathrm{(25 \textrm {ft } )}$

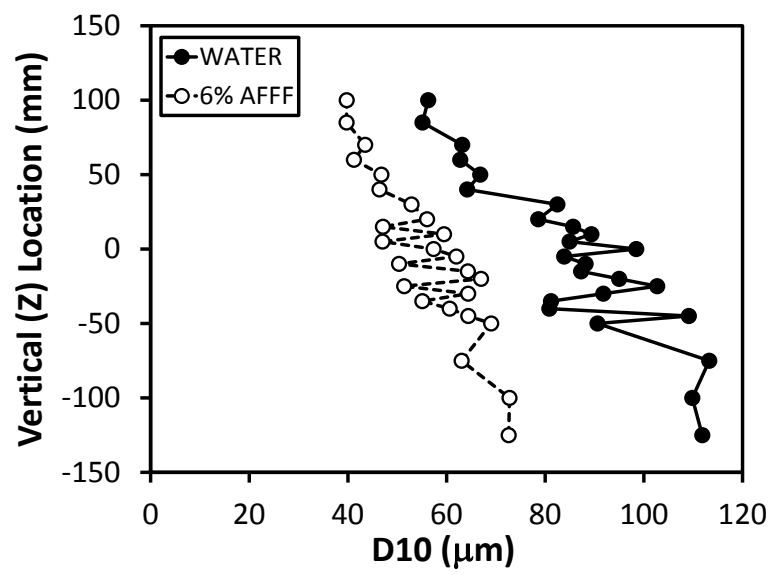

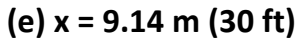

Figure C.46: Far Field High Flow, Low Press. Jet Vertical Profiles of Mean Droplet Diameter 


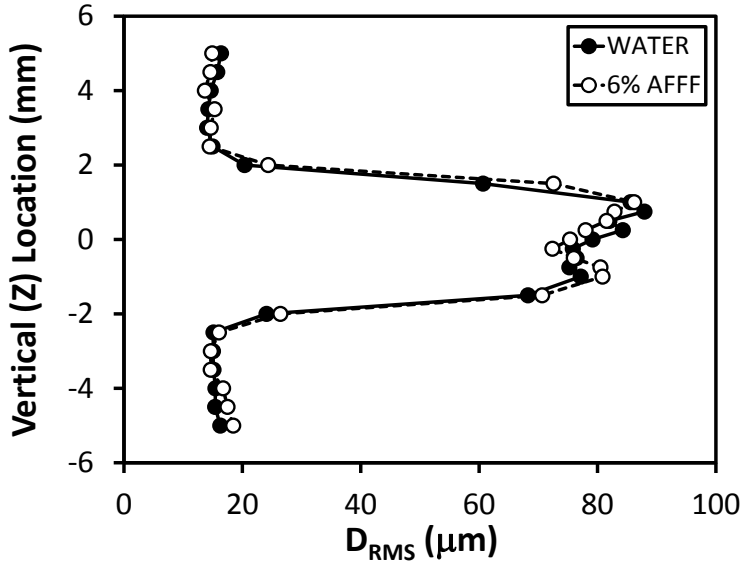

(a) $x=6.4 \mathrm{~mm}(0.25 \mathrm{in})$

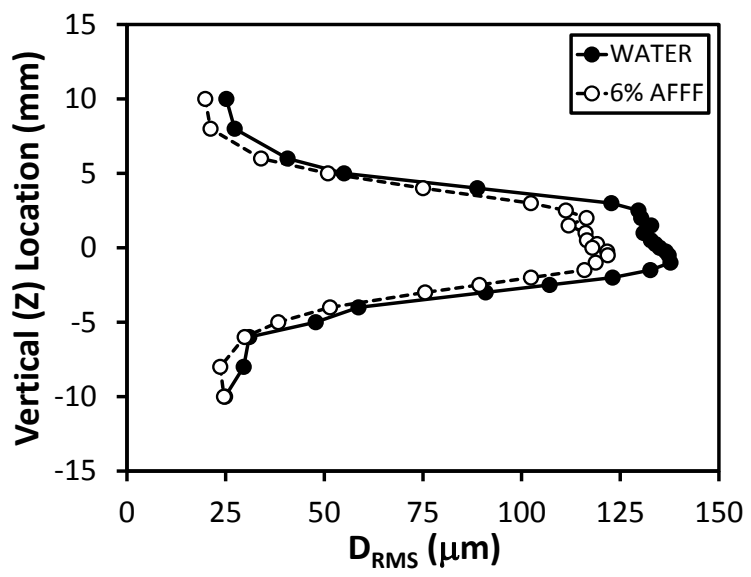

(c) $x=0.152 \mathrm{~m}(6 \mathrm{in})$

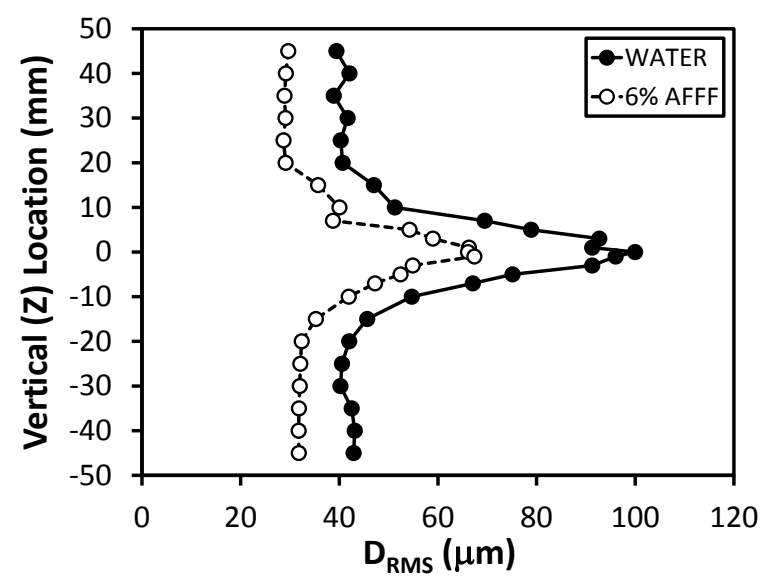

(e) $x=0.914 \mathrm{~m}(3 \mathrm{ft})$

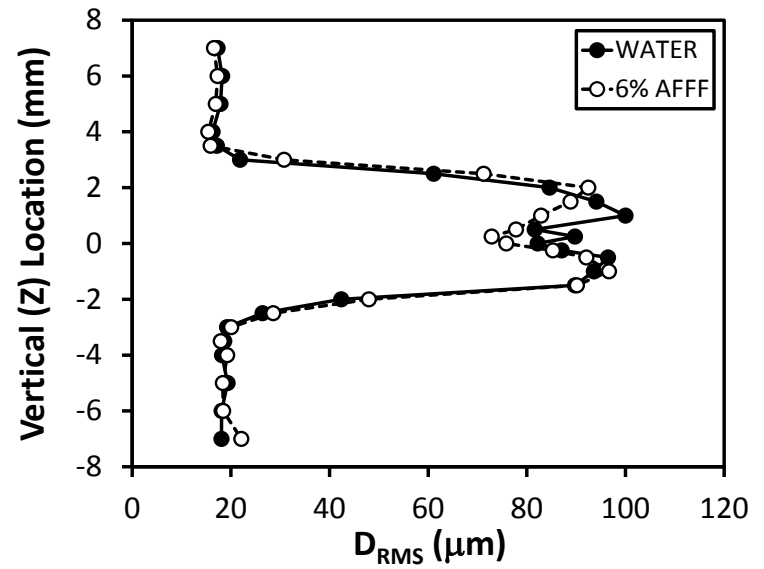

(b) $x=25.4 \mathrm{~mm}(1 \mathrm{in})$

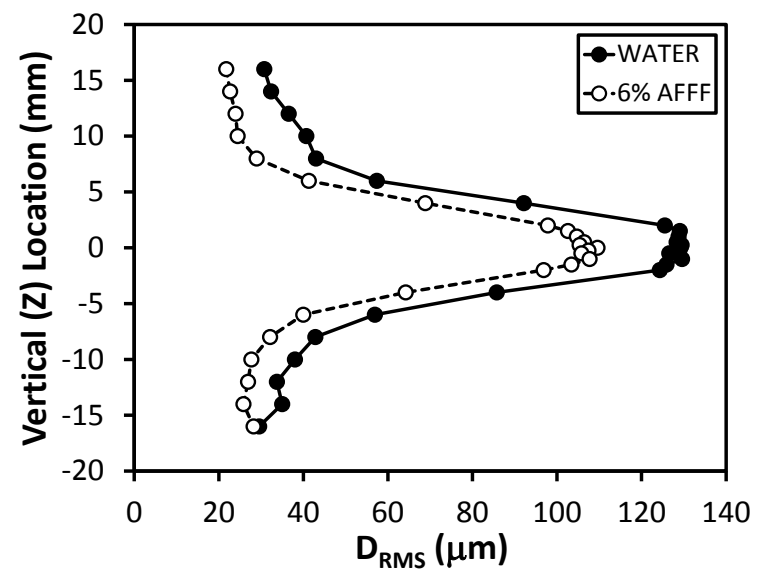

(d) $x=0.305 \mathrm{~m}(1 \mathrm{ft})$

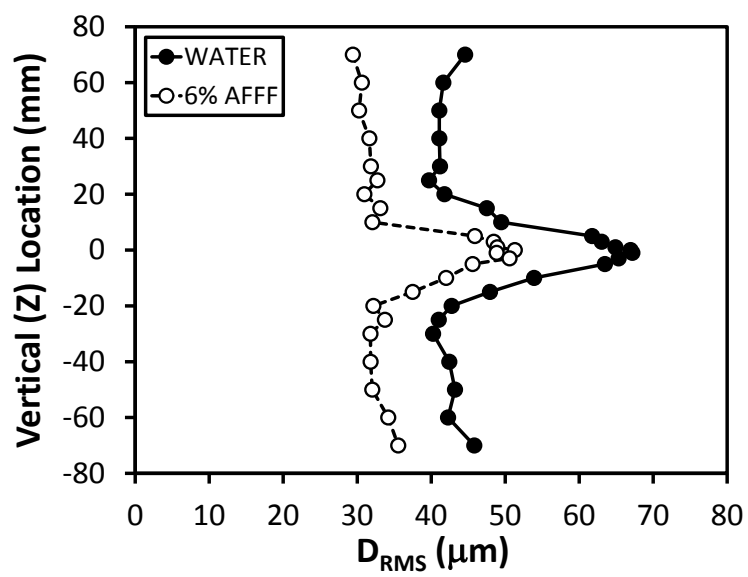

(f) $x=1.52 \mathrm{~m}(5 \mathrm{ft})$

Figure C.47: Near Field High Flow, Low Press. Jet Vertical Profiles of RMS Droplet Diameter 

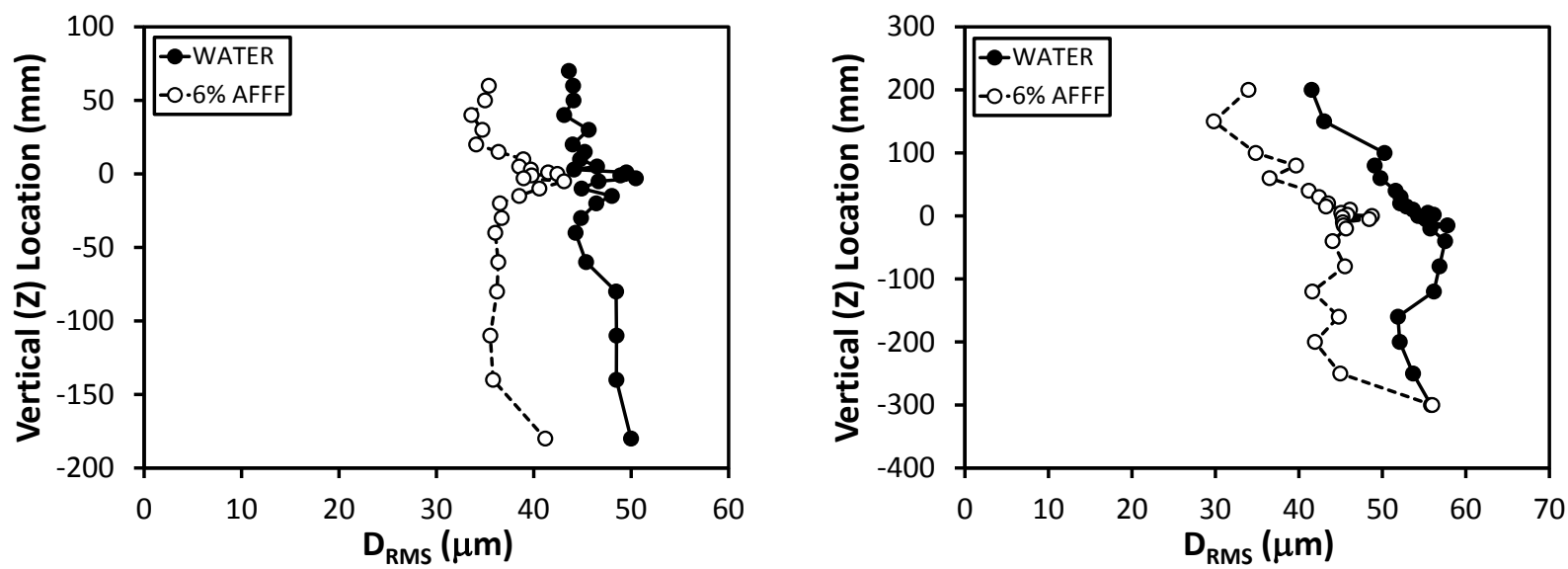

(a) $x=3.05 \mathrm{~m}(10 \mathrm{ft})$

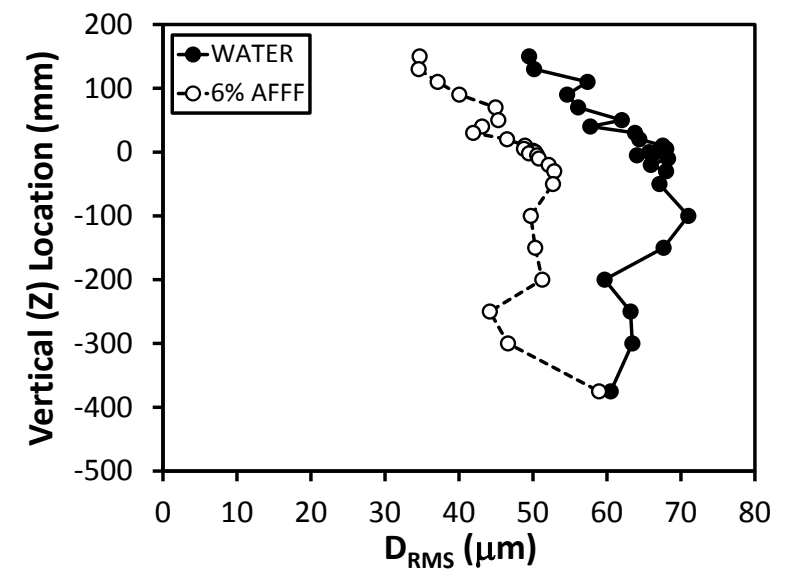

(b) $x=4.57 \mathrm{~m} \mathrm{(15 \textrm {ft } )}$

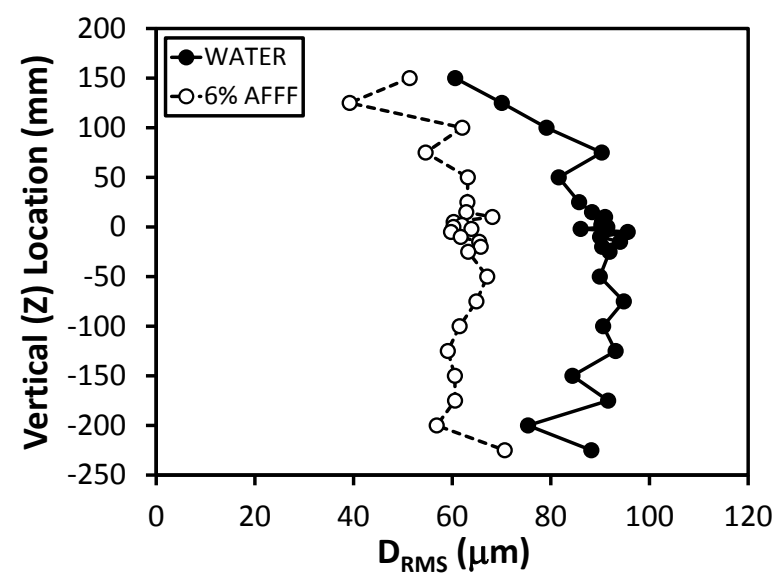

(c) $x=6.10 \mathrm{~m}(20 \mathrm{ft})$

(d) $x=7.62 \mathrm{~m}(25 \mathrm{ft})$

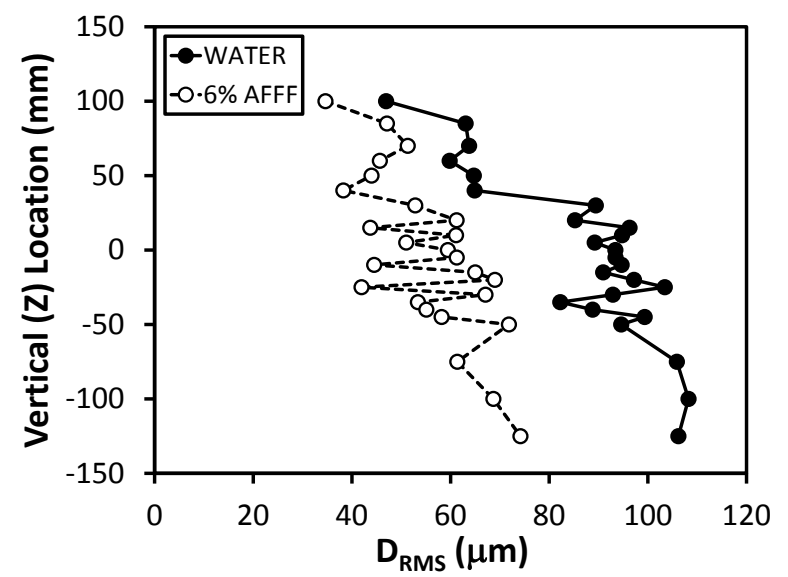

(e) $\mathrm{x}=9.14 \mathrm{~m}(30 \mathrm{ft})$

Figure C.48: Far Field High Flow, Low Press. Jet Vertical Profiles of RMS Droplet Diameter 


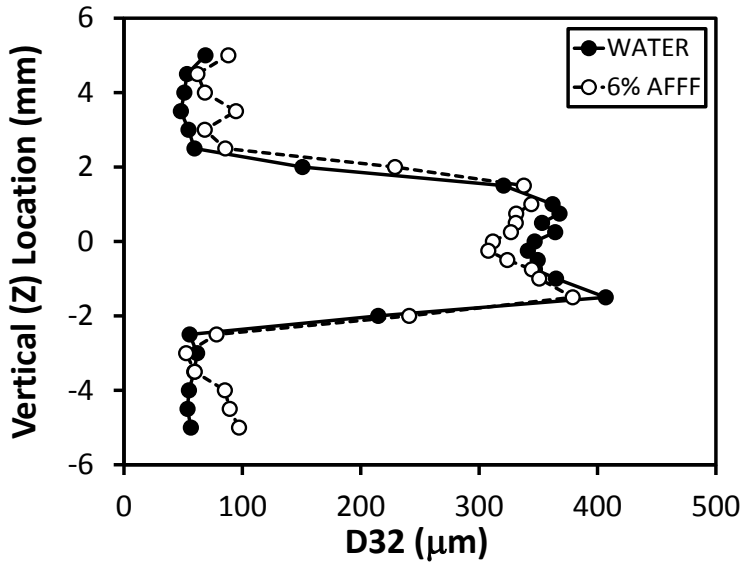

(a) $x=6.4 \mathrm{~mm}(0.25 \mathrm{in})$

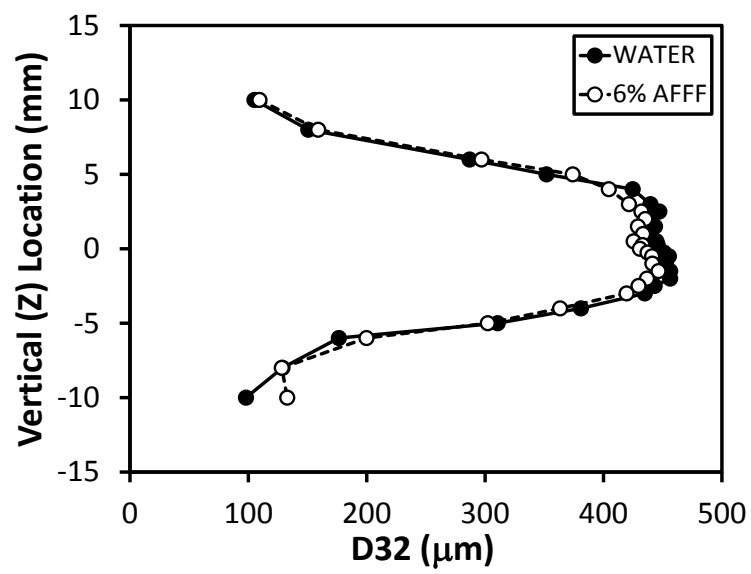

(c) $x=0.152 \mathrm{~m}$ (6 in)

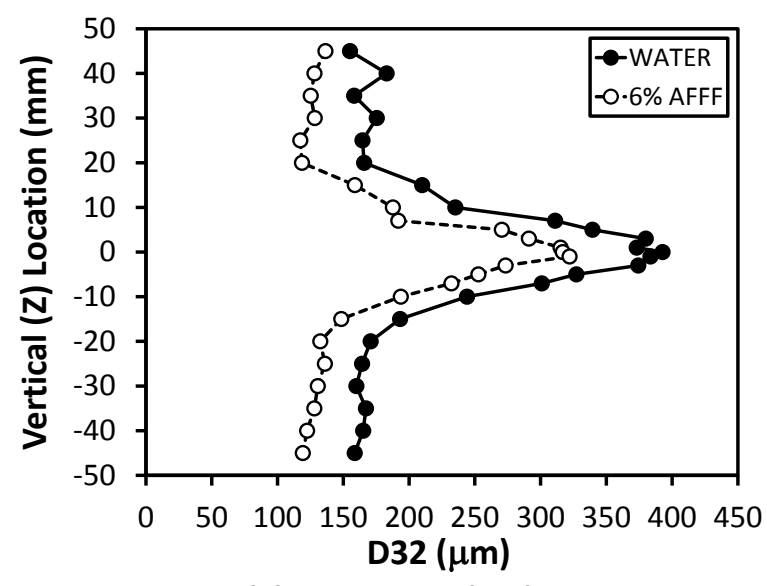

(e) $x=0.914 \mathrm{~m} \mathrm{(3ft)}$

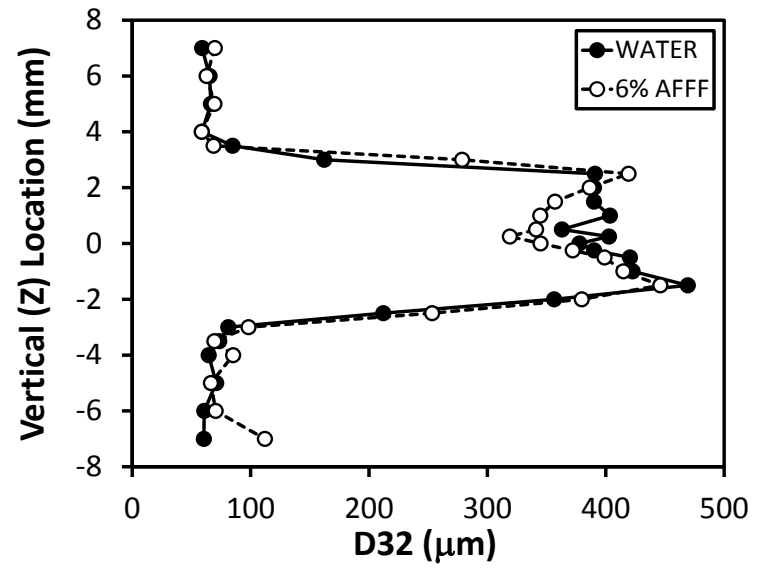

(b) $x=25.4 \mathrm{~mm}$ (1 in)

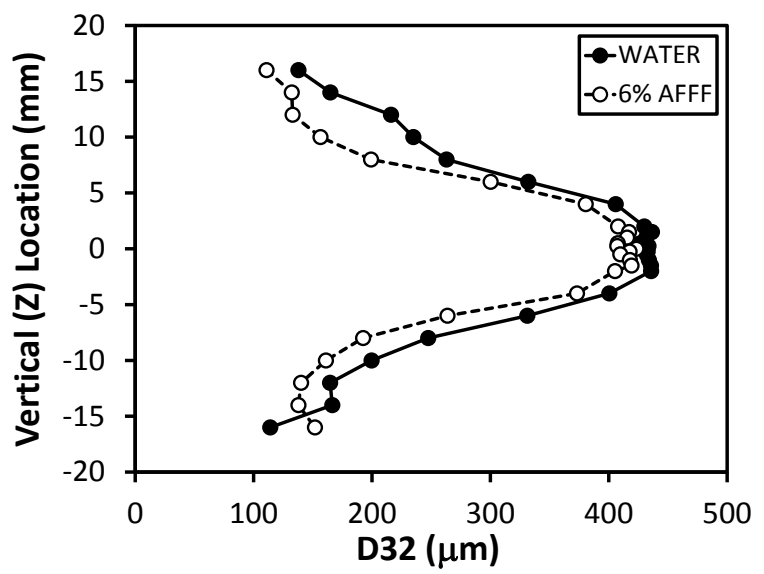

(d) $x=0.305 \mathrm{~m}$ (1ft)

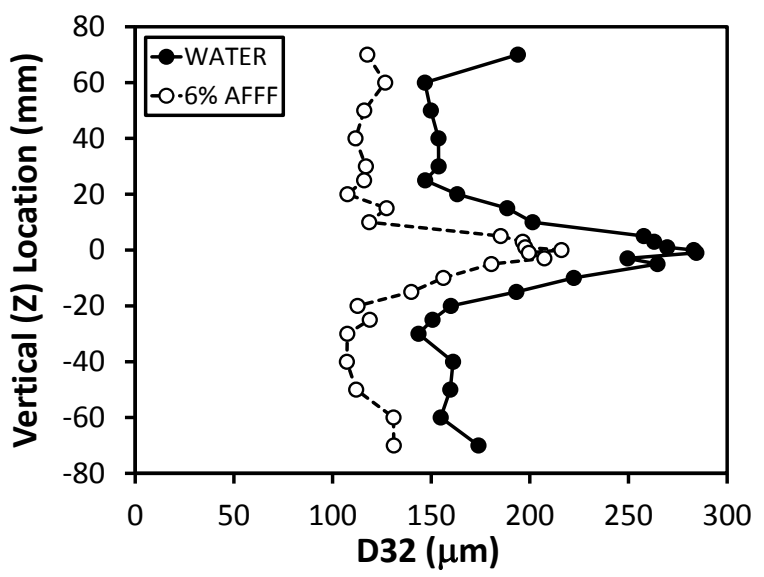

(f) $x=1.52 \mathrm{~m} \mathrm{(5 \textrm {ft } )}$

Figure C.49: Near Field High Flow, Low Press. Jet Vertical Profiles of Sauter Mean Droplet Diameter 

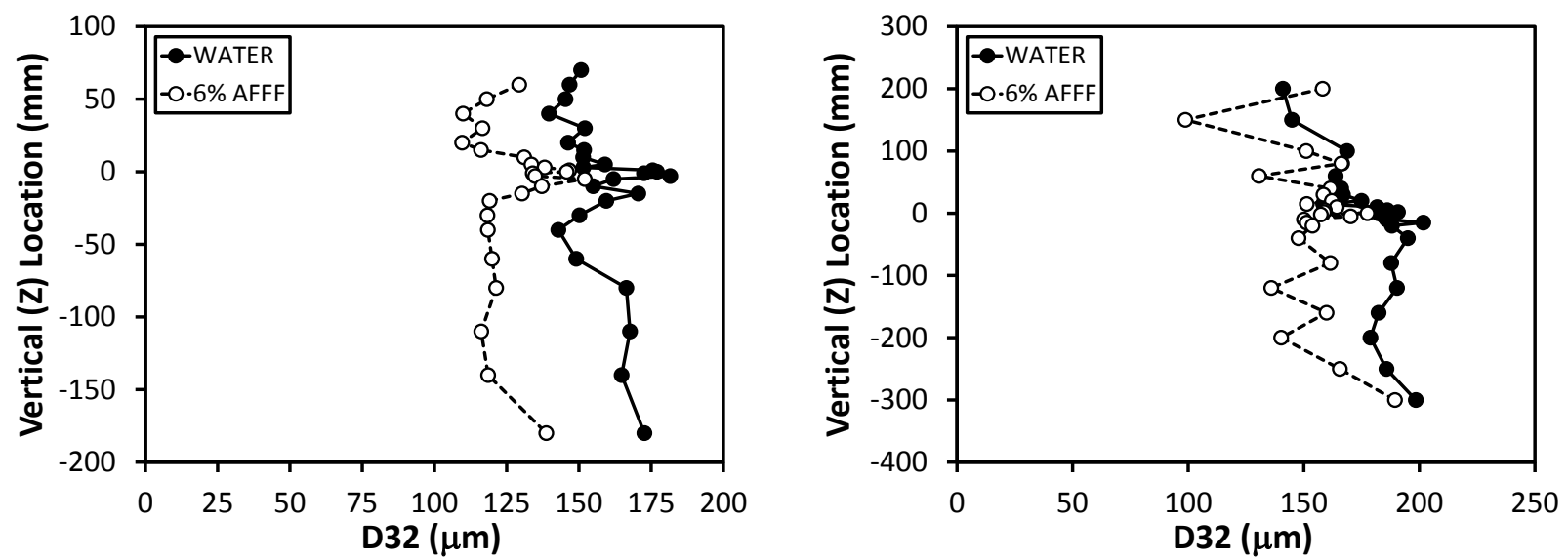

(a) $x=3.05 \mathrm{~m}(10 \mathrm{ft})$

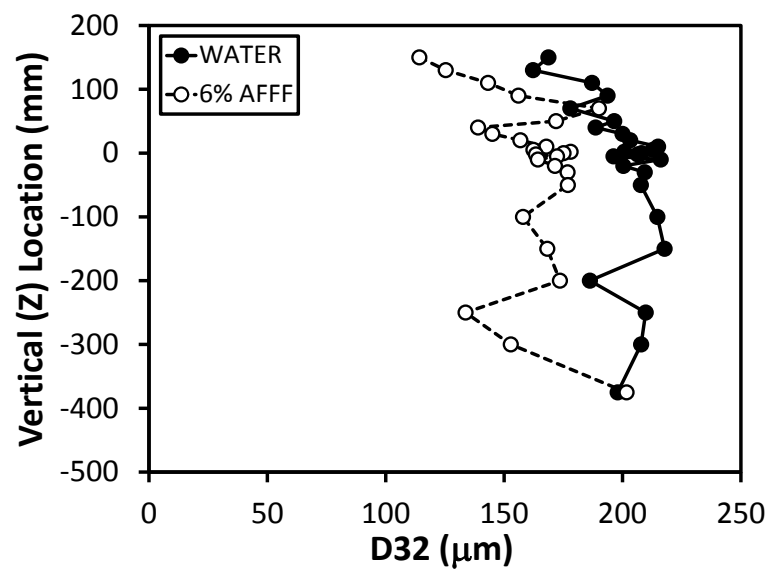

(b) $x=4.57 \mathrm{~m} \mathrm{(15 \textrm {ft } )}$

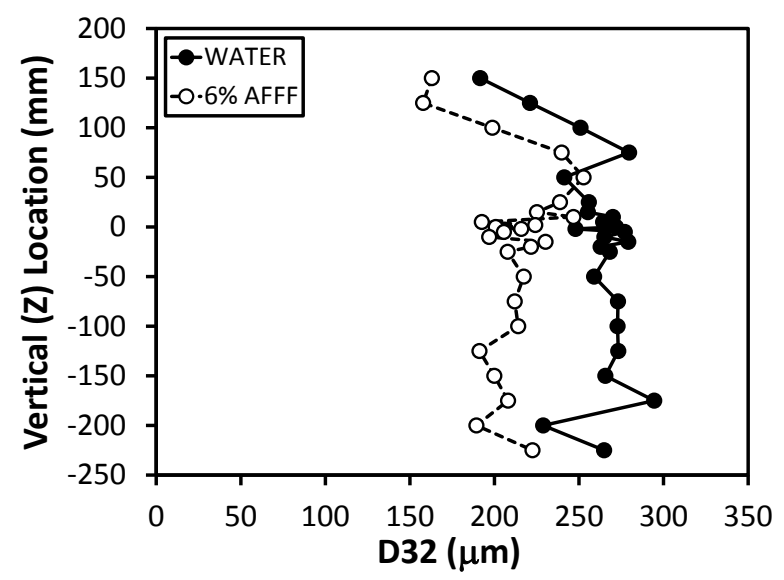

(c) $x=6.10 \mathrm{~m}(20 \mathrm{ft})$

(d) $x=7.62 \mathrm{~m}(25 \mathrm{ft})$

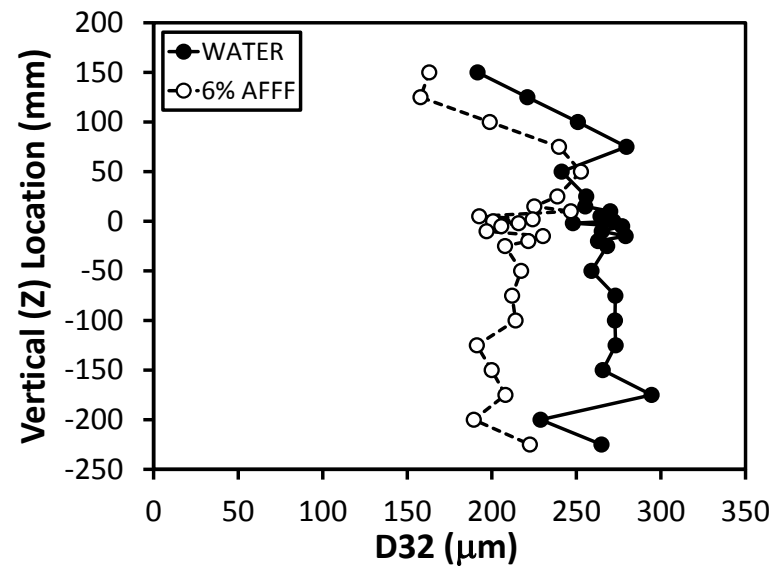

(e) $x=9.14 \mathrm{~m}(30 \mathrm{ft})$

Figure C.50: Far Field High Flow, Low Press. Jet Vertical Profiles of Sauter Mean Droplet Diameter 


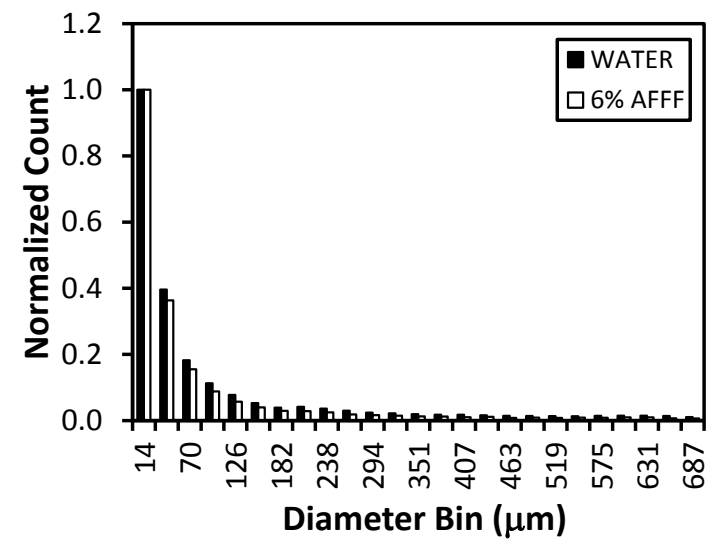

(a) $x=0.152 \mathrm{~m}(6 \mathrm{in})$

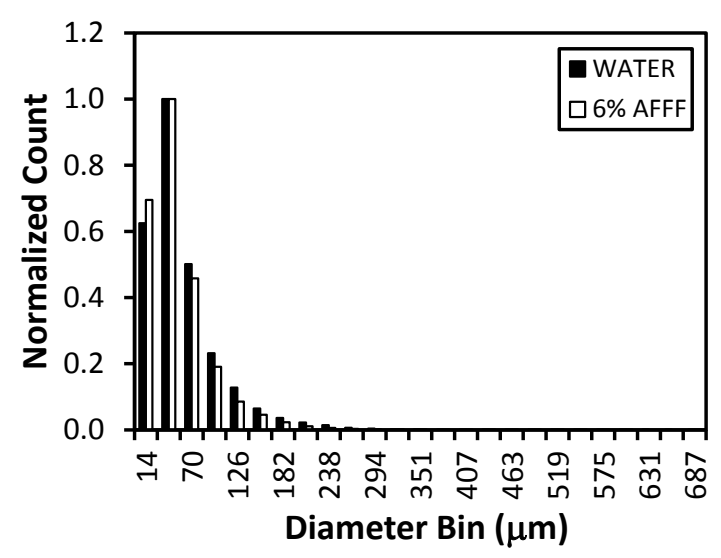

(c) $\mathrm{x}=3.05 \mathrm{~m}(10 \mathrm{ft})$

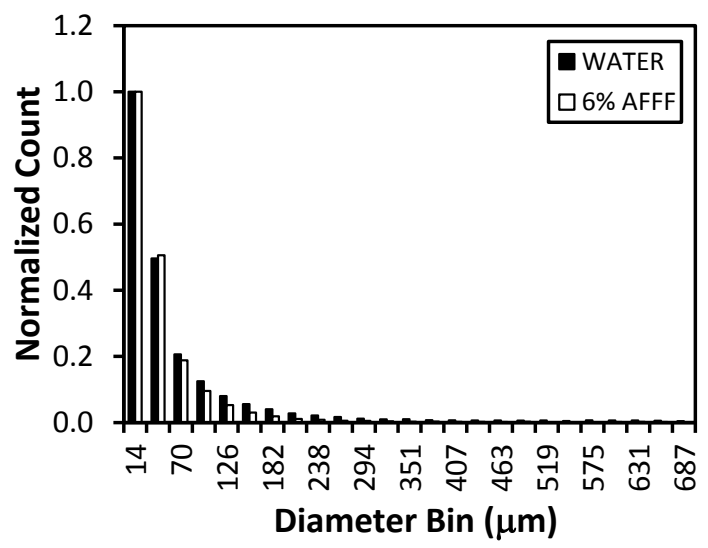

(b) $x=0.914 \mathrm{~m} \mathrm{(3 \textrm {ft } )}$

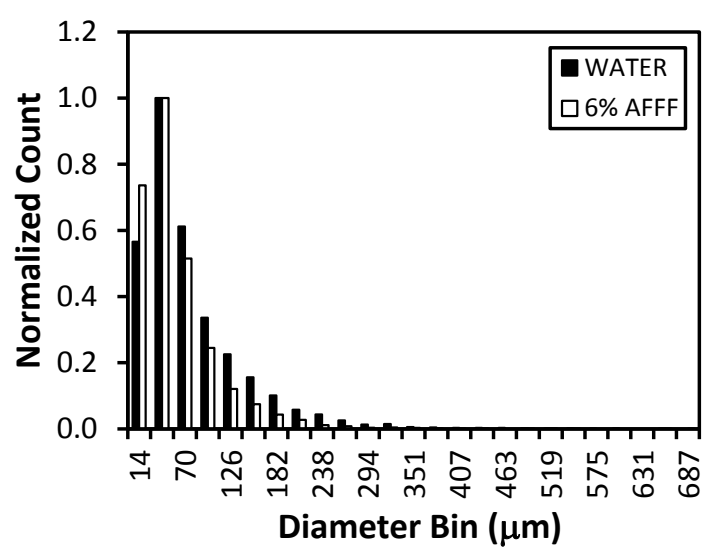

(d) $x=6.10 \mathrm{~m}(20 \mathrm{ft})$

Figure C.51: High Flow, Low Press. Jet Droplet Size Distribution at the Max. Profile, Mean Axial Droplet Velocity for Select Vertical Profiles 


\section{C.5 High Flow, High Pressure Firefighting Jet Data}

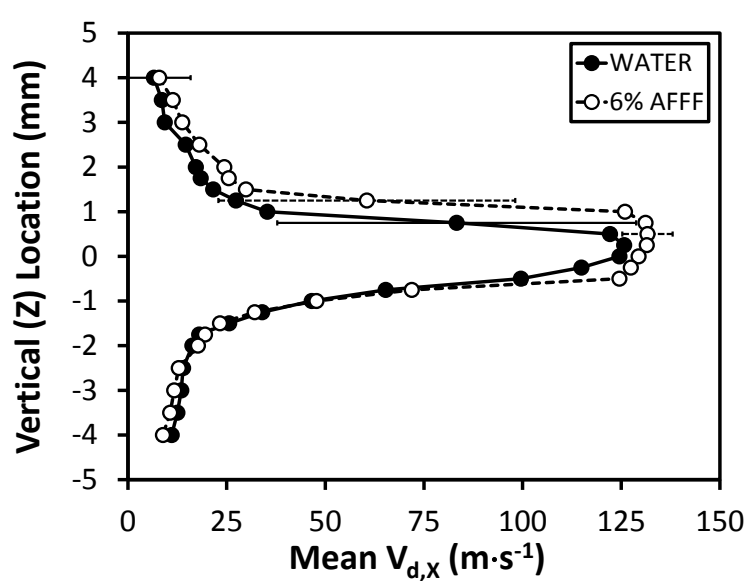

(a) $x=6.4 \mathrm{~mm}(0.25 \mathrm{in})$

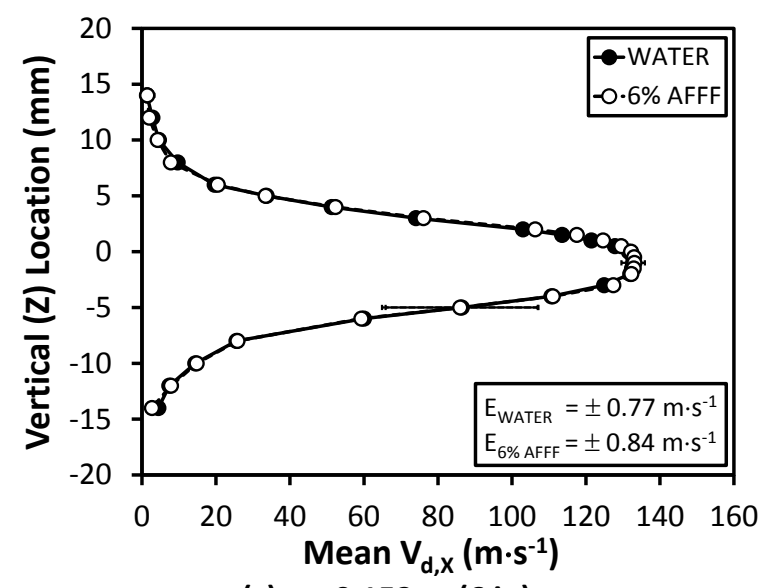

(c) $x=0.152 \mathrm{~m}(6 \mathrm{in})$

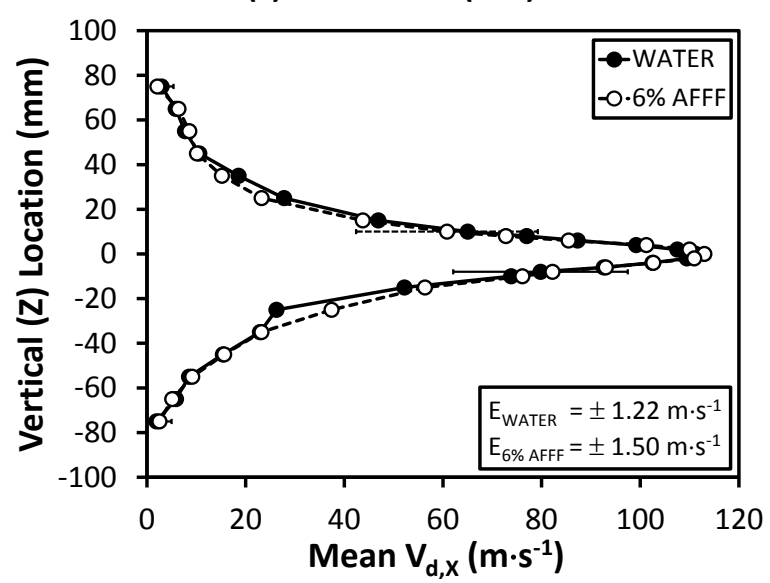

(e) $x=0.914 \mathrm{~m}(3 \mathrm{ft})$

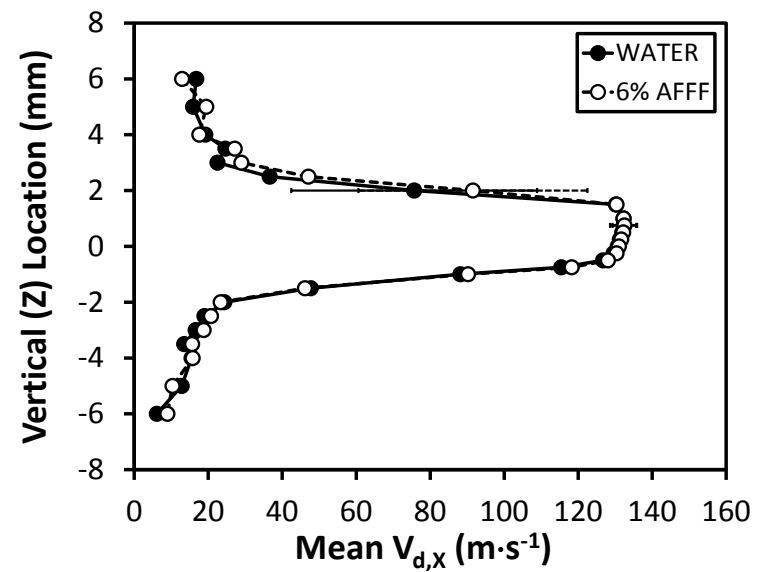

(b) $x=25.4 \mathrm{~mm}$ (1 in)

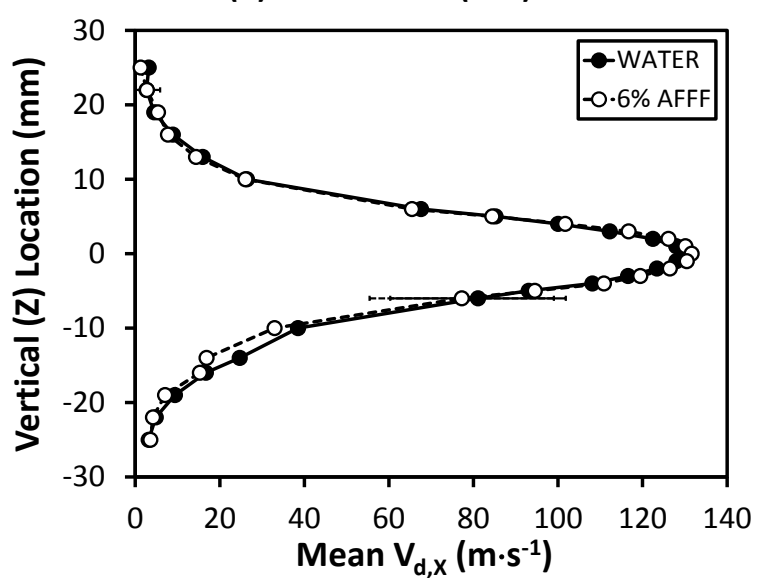

(d) $x=0.305 \mathrm{~m}(\mathbf{1 f t})$

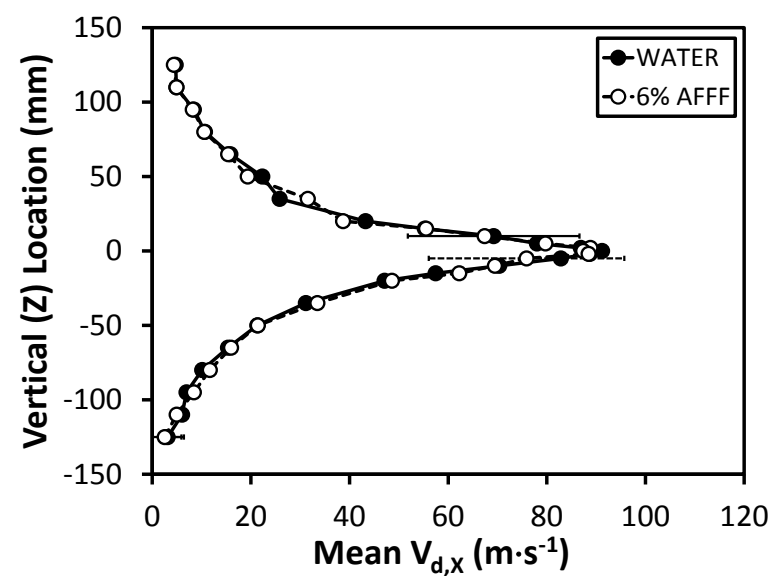

(f) $x=1.52 \mathrm{~m}(5 \mathrm{ft})$

Figure C.52: Near Field High Flow, High Press. Jet Vertical Profiles of Mean Axial Droplet Velocity 

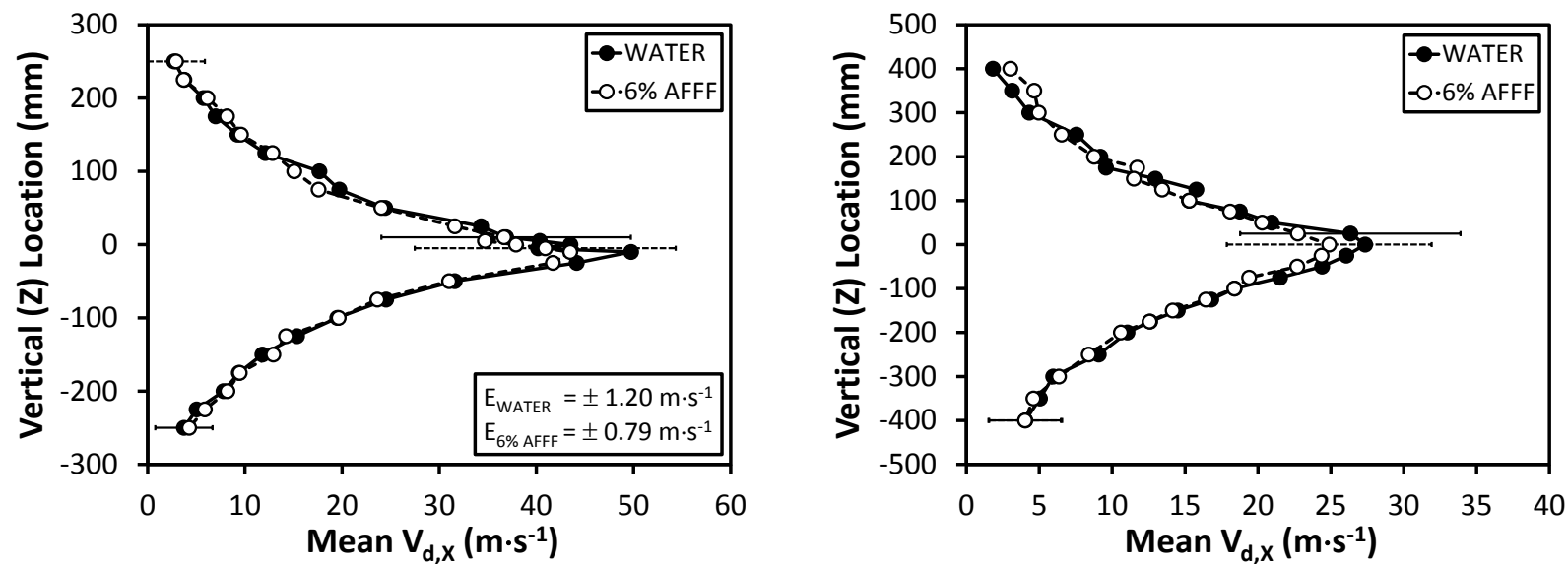

(a) $x=3.05 \mathrm{~m}(10 \mathrm{ft})$

(b) $x=4.57 \mathrm{~m}(15 \mathrm{ft})$

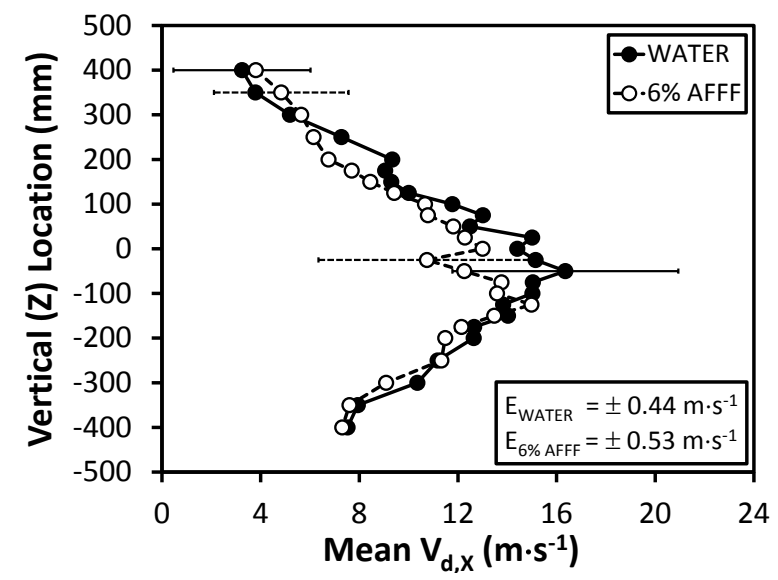

(c) $x=6.10 \mathrm{~m}(20 \mathrm{ft})$

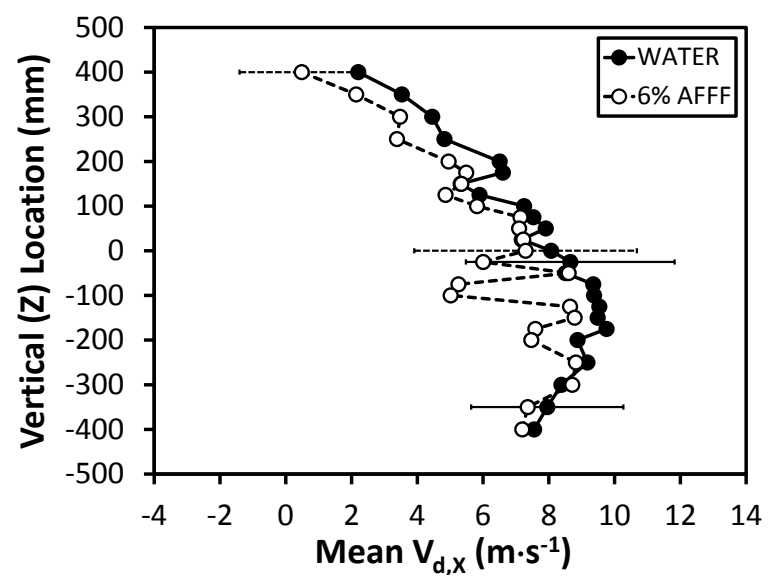

(d) $x=7.62 \mathrm{~m}(25 \mathrm{ft})$

Figure C.53: Far Field High Flow, High Press. Jet Vertical Profiles of Mean Axial Droplet Velocity 


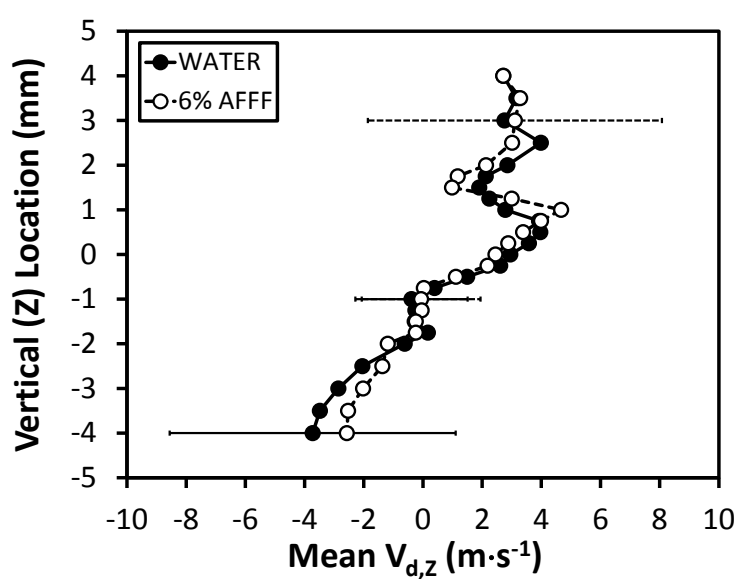

(a) $x=6.4 \mathrm{~mm}(0.25 \mathrm{in})$

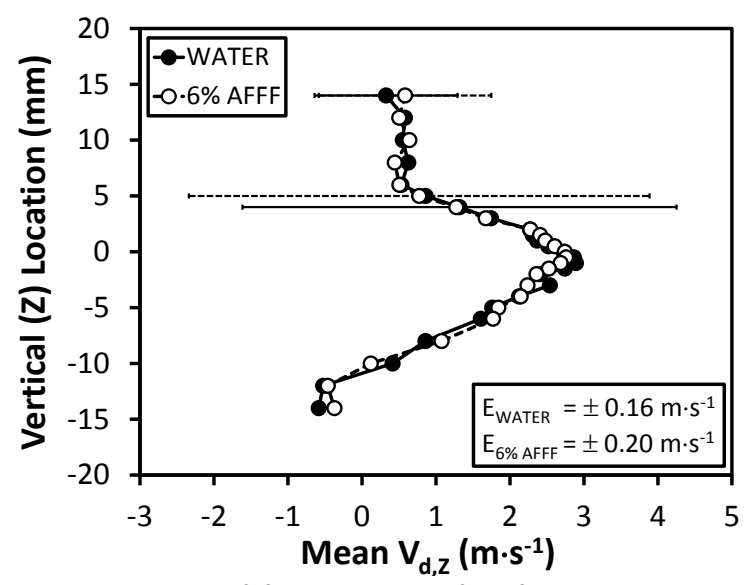

(c) $x=0.152 \mathrm{~m}(6 \mathrm{in})$

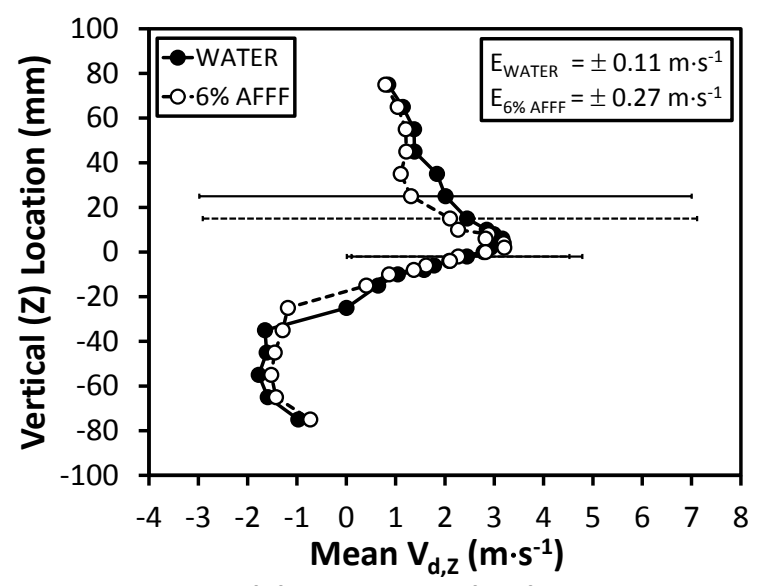

(e) $x=0.914 \mathrm{~m} \mathrm{(3 \textrm {ft } )}$

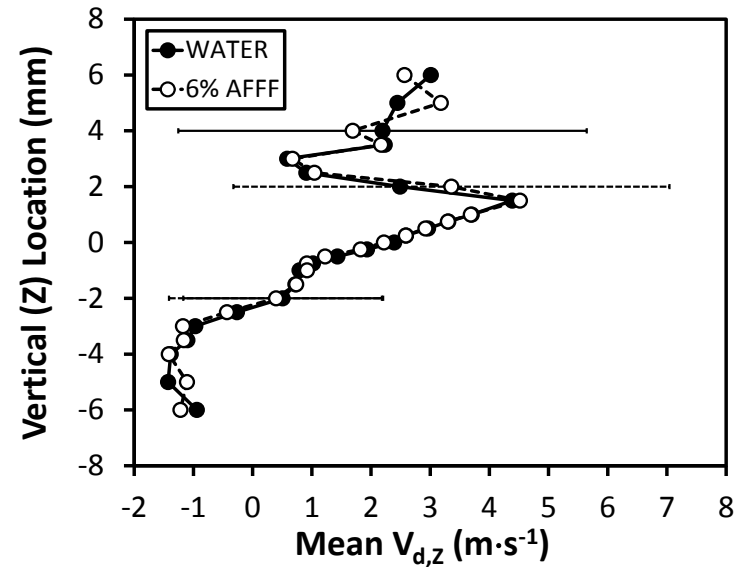

(b) $x=25.4 \mathrm{~mm}(1 \mathrm{in})$

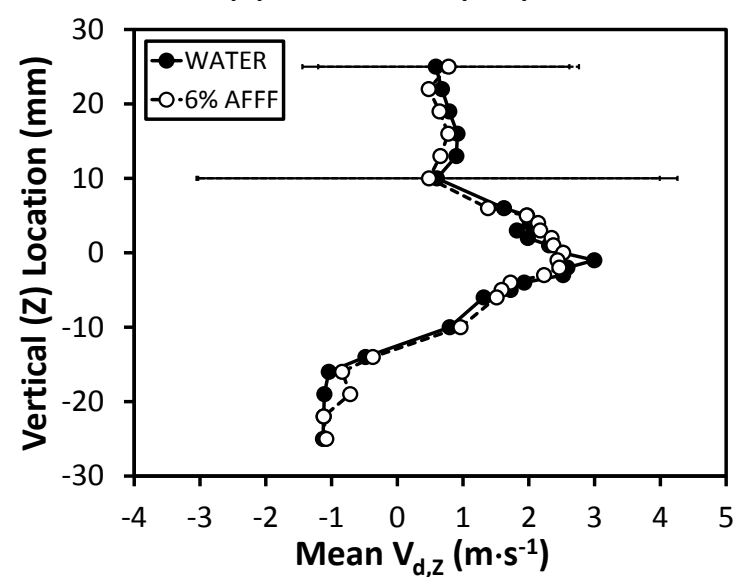

(d) $\mathrm{x}=0.305 \mathrm{~m}(\mathbf{1 f t})$

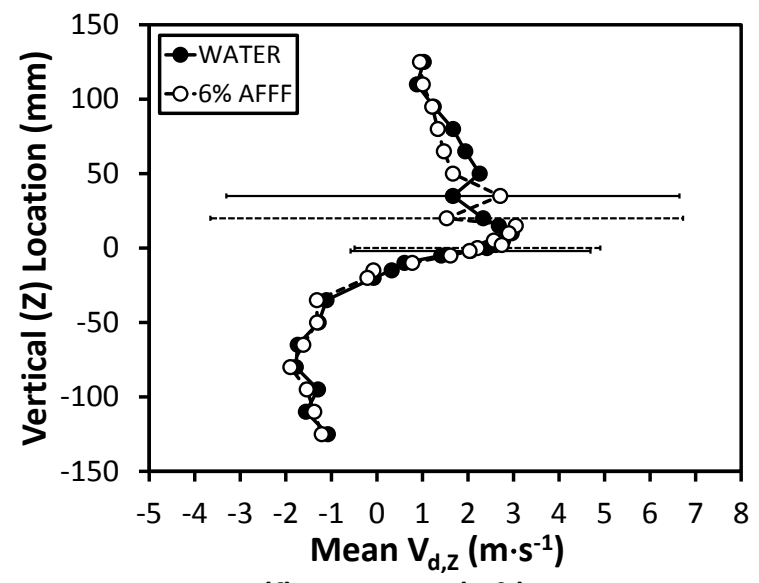

(f) $x=1.52 \mathrm{~m}(5 \mathrm{ft})$

Figure C.54: Near Field High Flow, High Press. Jet Vertical Profiles of Mean Vertical Droplet Velocity 

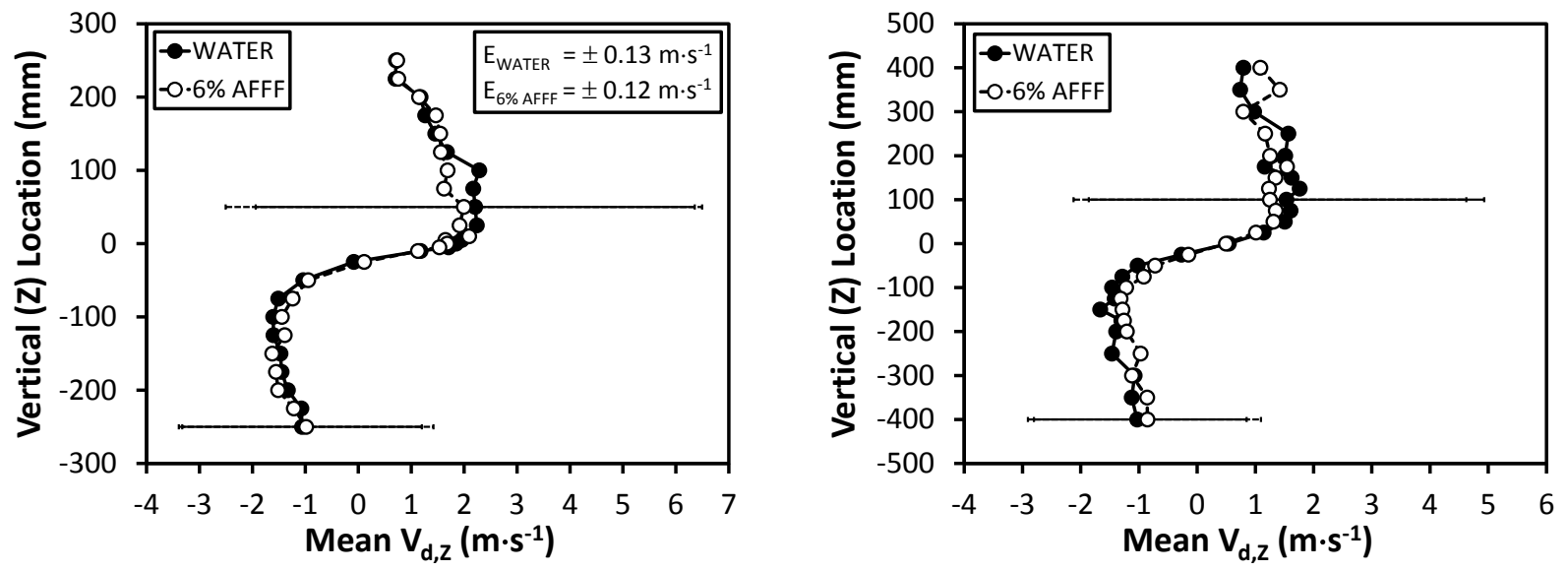

(a) $x=3.05 \mathrm{~m}(10 \mathrm{ft})$

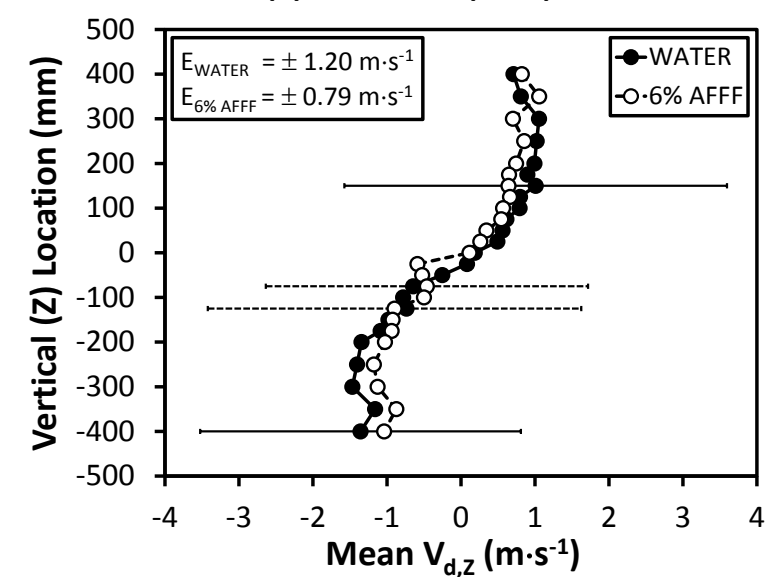

(c) $x=6.10 \mathrm{~m}(20 \mathrm{ft})$

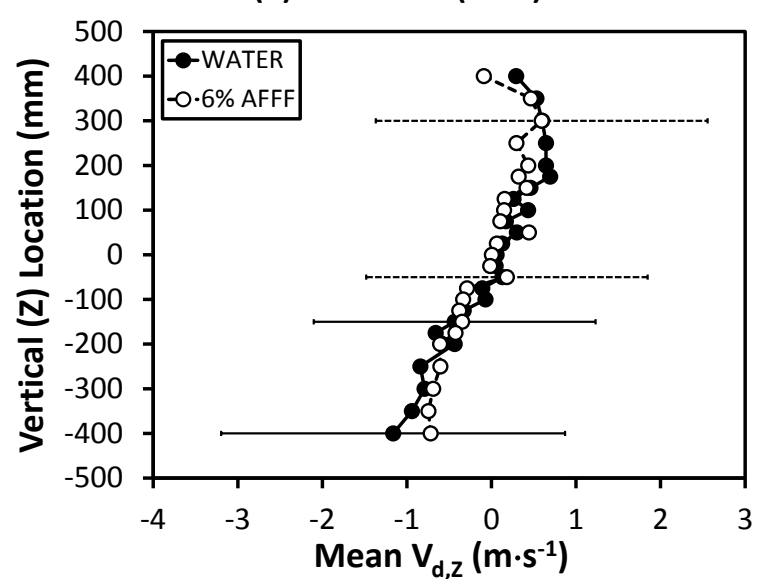

(d) $x=7.62 \mathrm{~m}(25 \mathrm{ft})$

Figure C.55: Far Field High Flow, High Press. Jet Vertical Profiles of Mean Vertical Droplet Velocity 

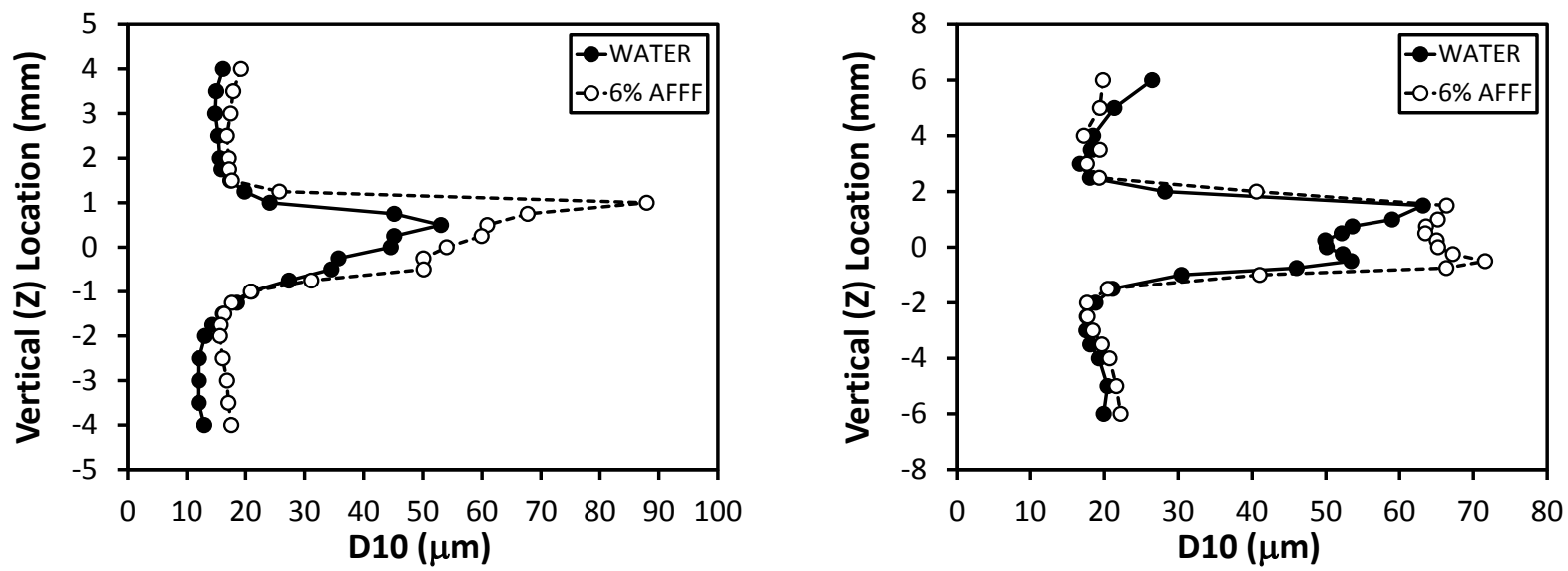

(a) $x=6.4 \mathrm{~mm}(0.25 \mathrm{in})$

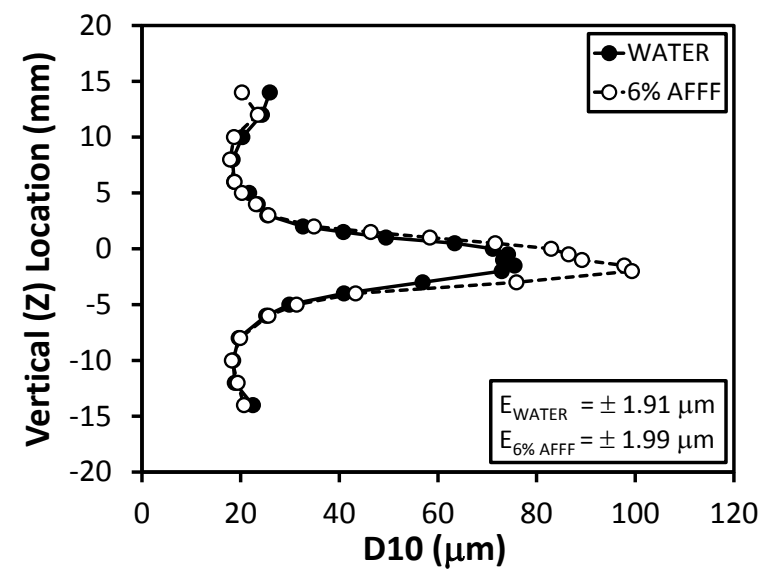

(b) $x=25.4 \mathrm{~mm}(1 \mathrm{in})$

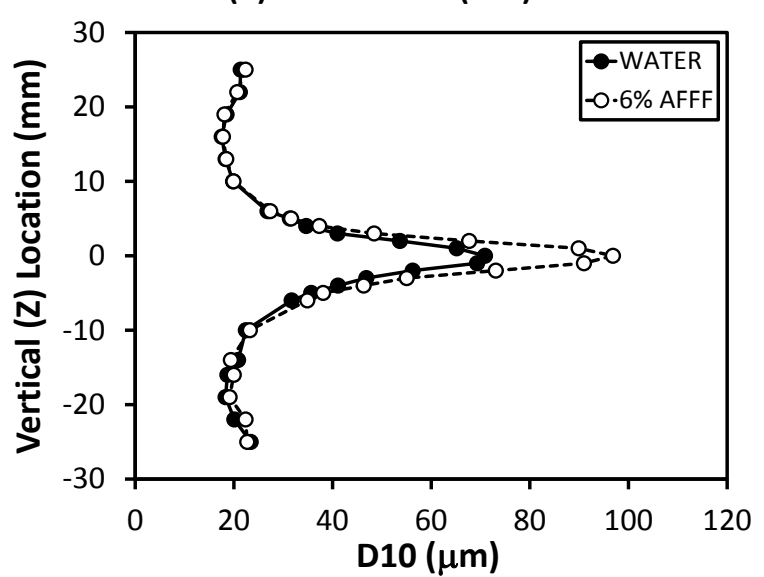

(c) $x=0.152 \mathrm{~m}(6 \mathrm{in})$

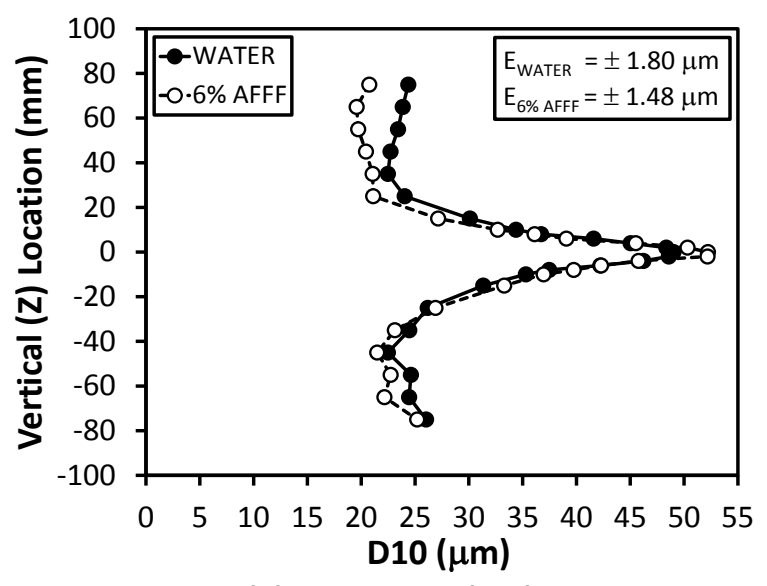

(d) $x=0.305 \mathrm{~m}(\mathbf{1 f t})$

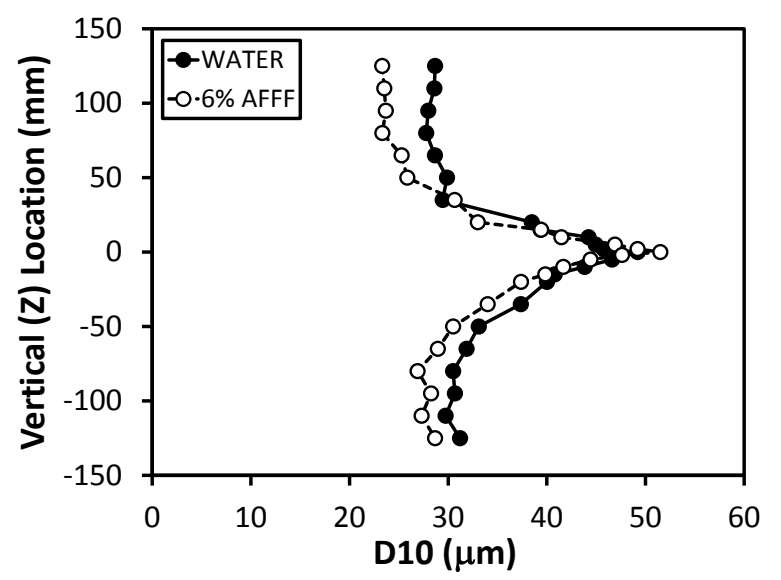

(f) $x=1.52 \mathrm{~m} \mathrm{(5 \textrm {ft } )}$

Figure C.56: Near Field High Flow, High Press. Jet Vertical Profiles of Mean Droplet Diameter 

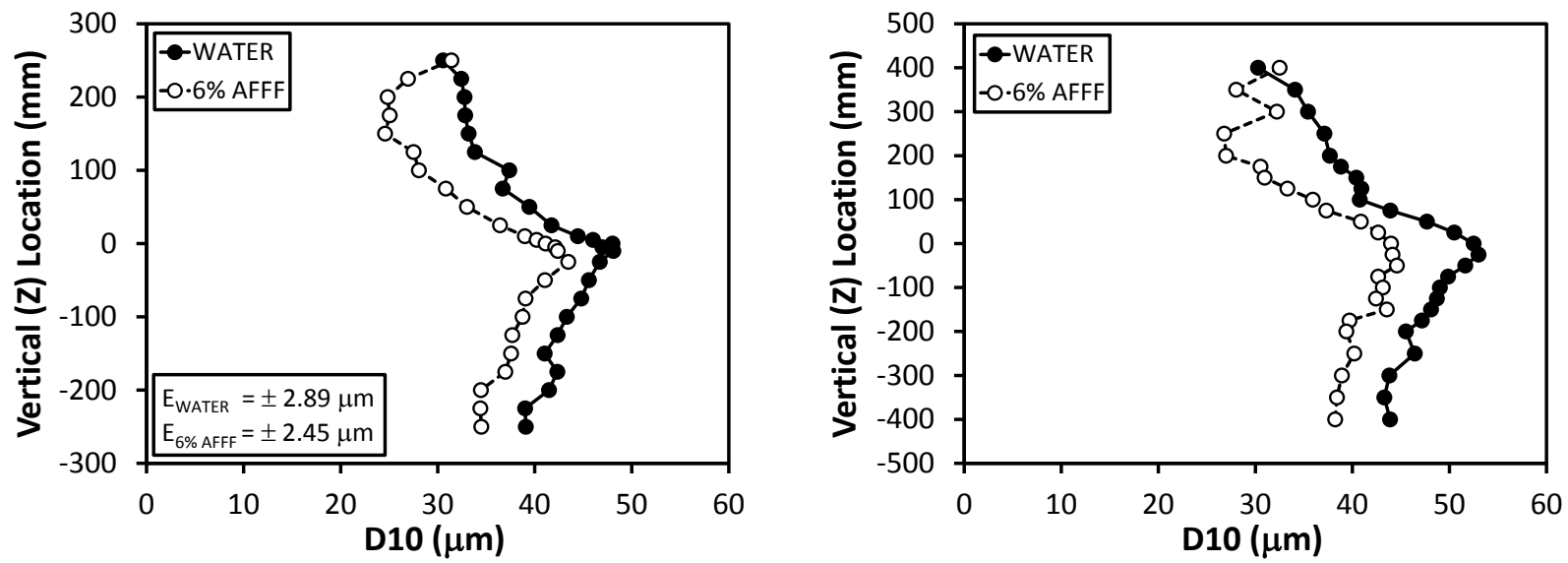

(a) $x=3.05 \mathrm{~m}(10 \mathrm{ft})$

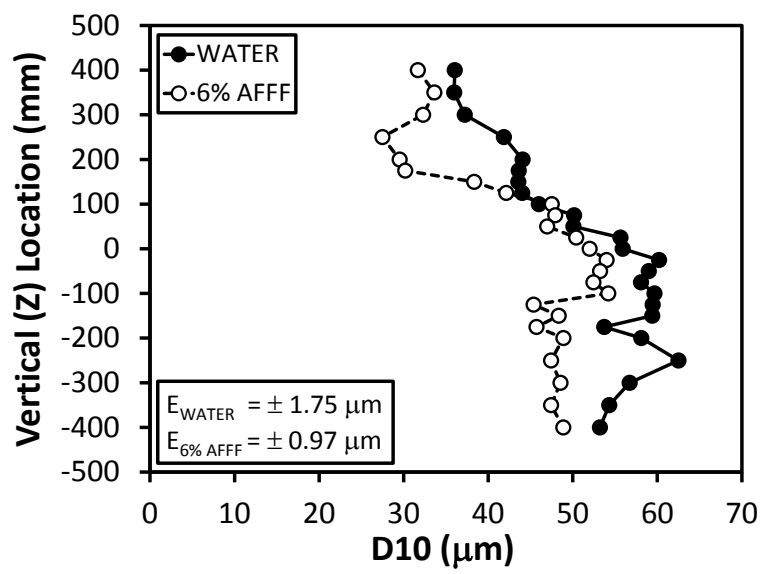

(c) $x=6.10 \mathrm{~m}(20 \mathrm{ft})$

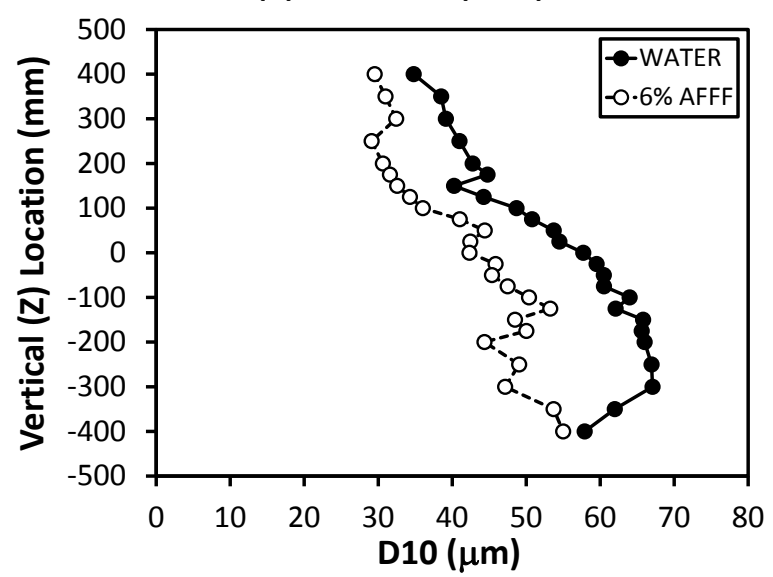

(d) $x=7.62 \mathrm{~m}(25 \mathrm{ft})$

Figure C.57: Far Field High Flow, High Press. Jet Vertical Profiles of Mean Droplet Diameter 

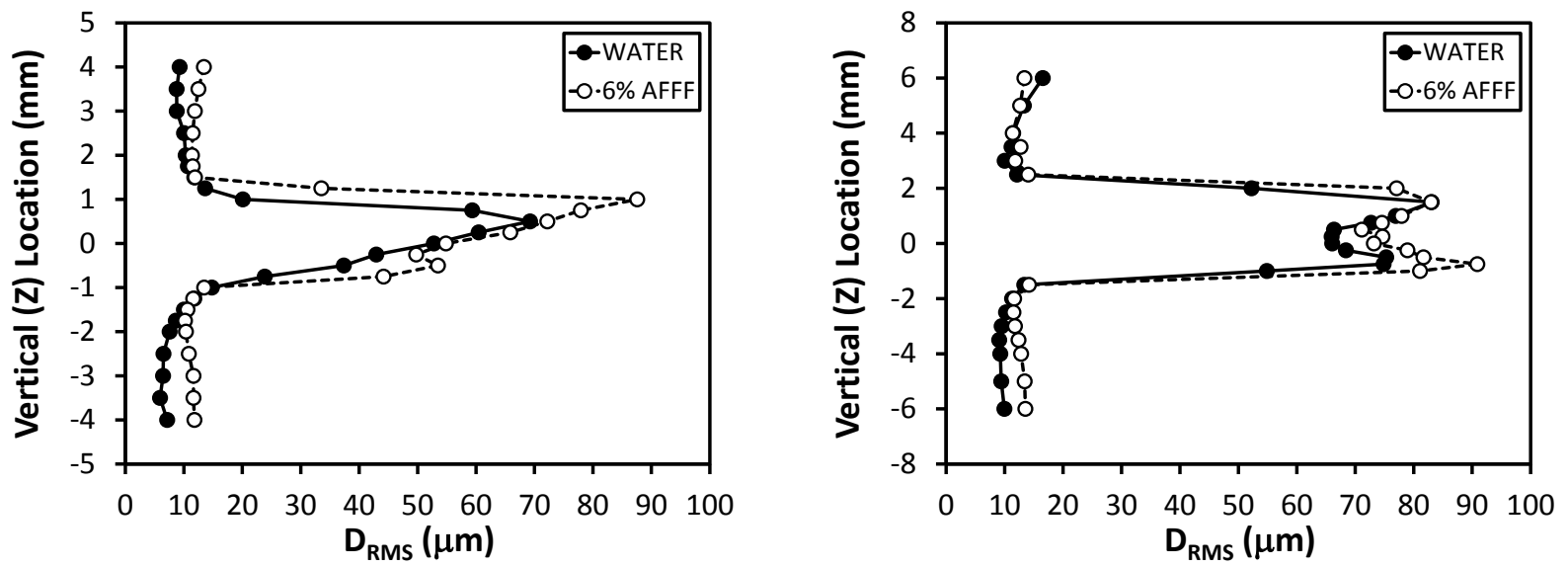

(a) $x=6.4 \mathrm{~mm}(0.25 \mathrm{in})$

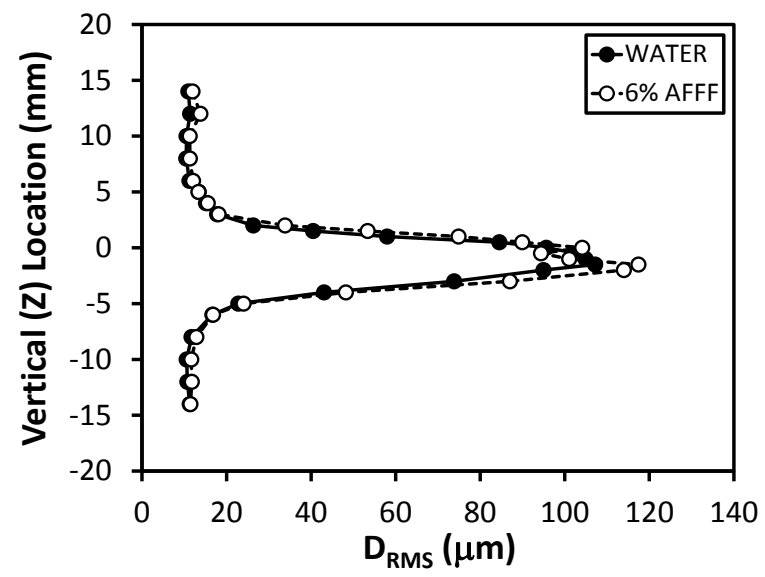

(b) $x=25.4 \mathrm{~mm}(1 \mathrm{in})$

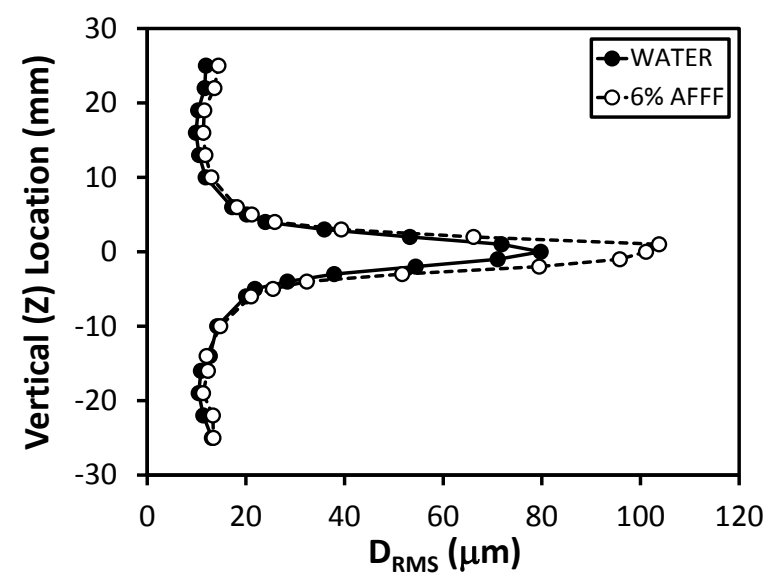

(c) $x=0.152 \mathrm{~m}$ (6 in)

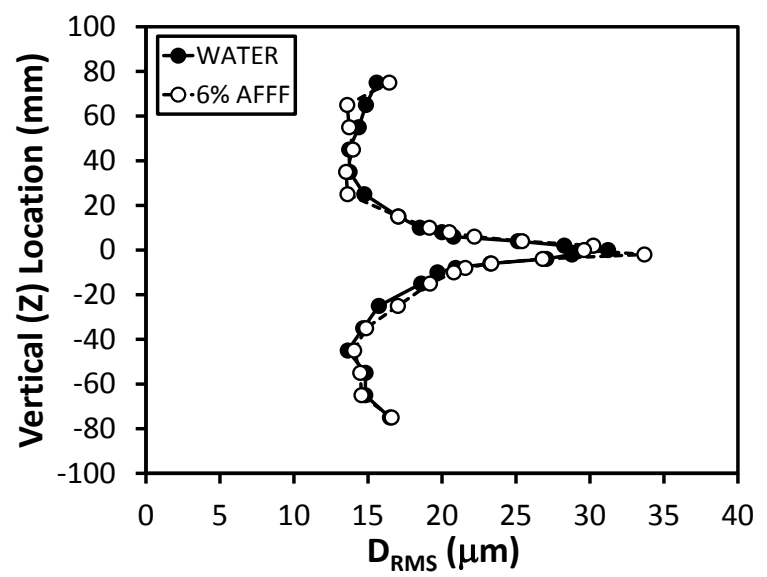

(e) $x=0.914 \mathrm{~m} \mathrm{(3 \textrm {ft } )}$

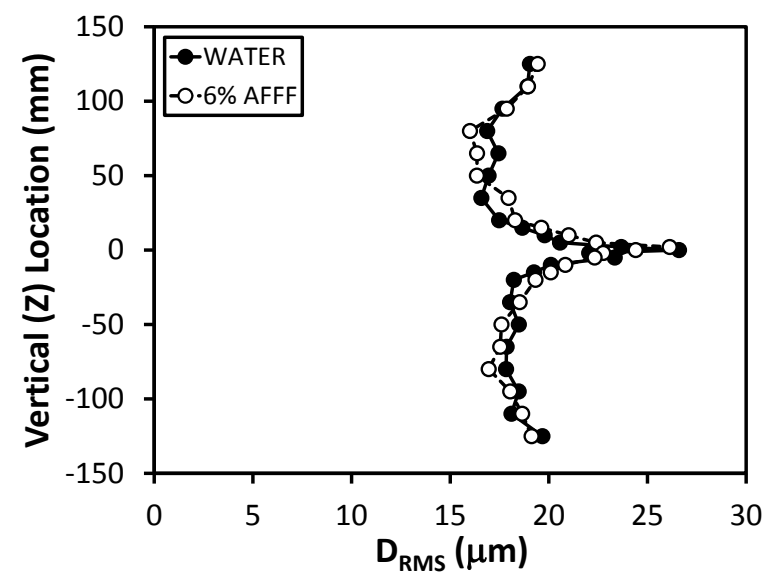

(f) $x=1.52 \mathrm{~m} \mathrm{(5} \mathrm{ft)}$

Figure C.58: Near Field High Flow, High Press. Jet Vertical Profiles of RMS Droplet Diameter 

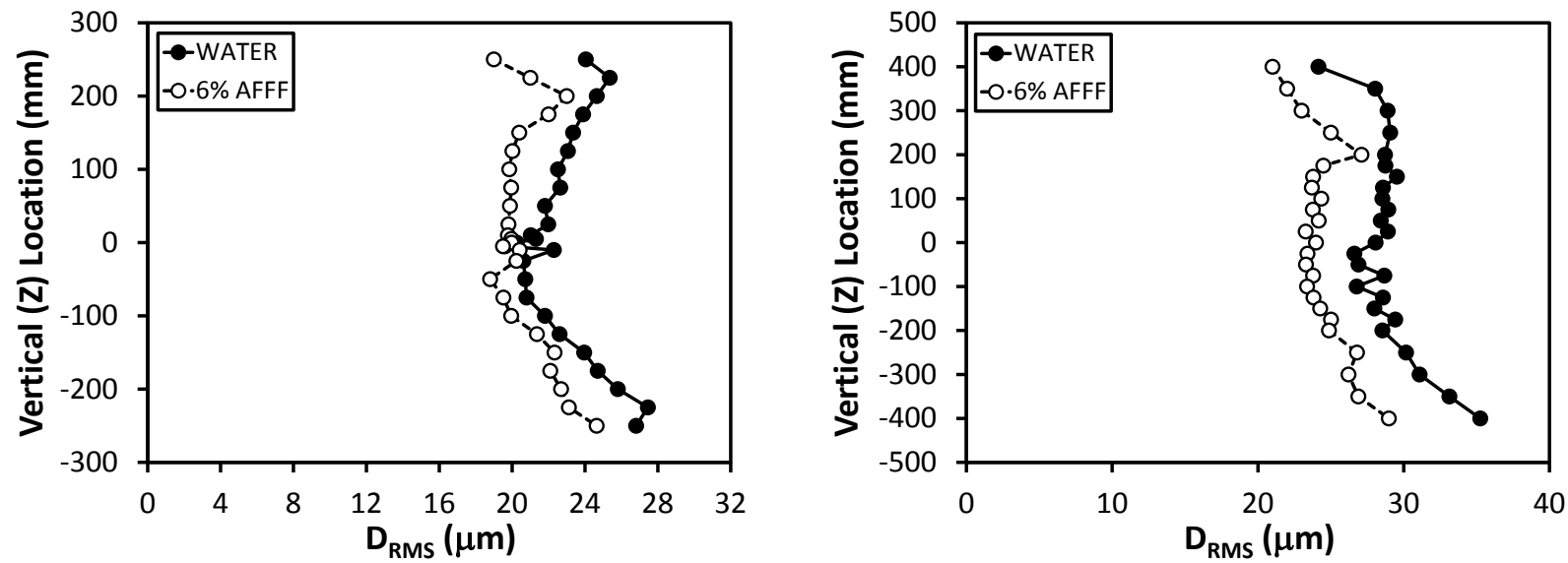

(a) $x=3.05 \mathrm{~m}(10 \mathrm{ft})$

(b) $x=4.57 \mathrm{~m} \mathrm{(15 \textrm {ft } )}$

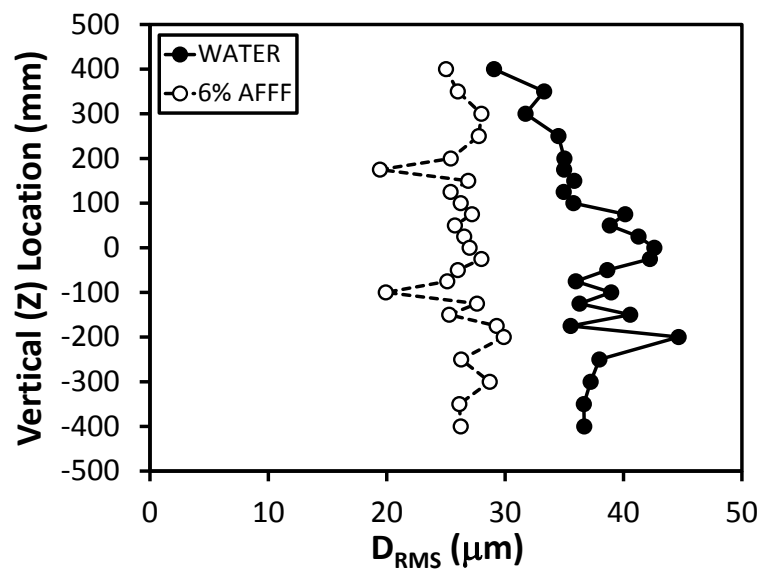

(c) $x=6.10 \mathrm{~m}(20 \mathrm{ft})$

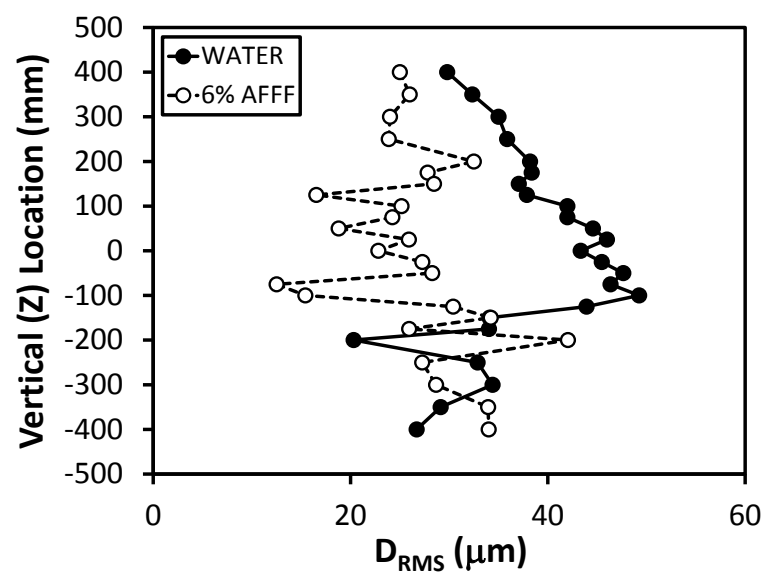

(d) $x=7.62 \mathrm{~m}(25 \mathrm{ft})$

Figure C.59: Far Field High Flow, High Press. Jet Vertical Profiles of RMS Droplet Diameter 


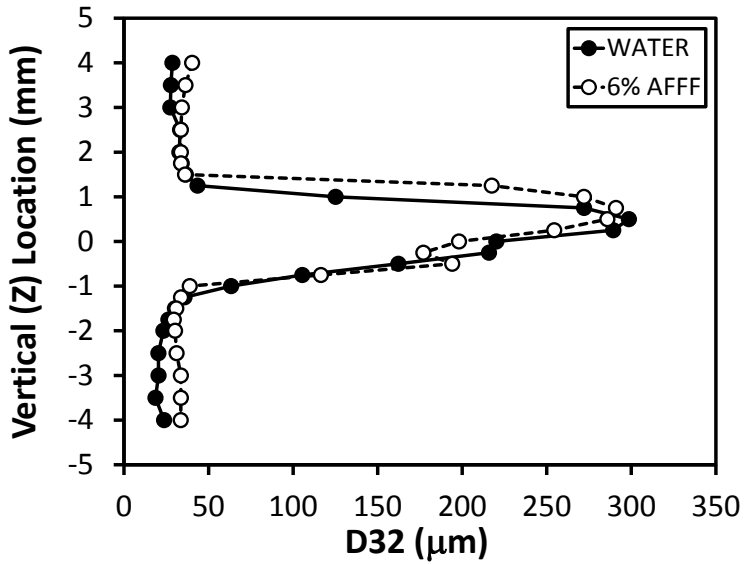

(a) $x=6.4 \mathrm{~mm}(0.25 \mathrm{in})$

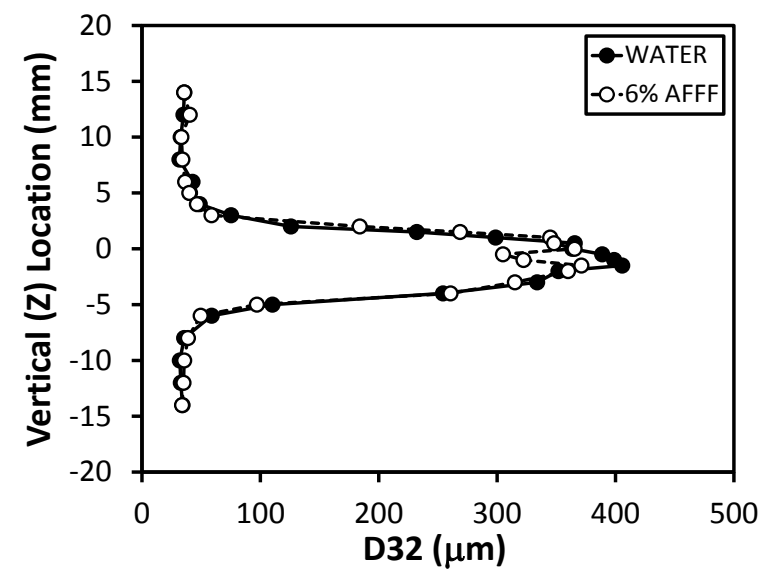

(c) $x=0.152 \mathrm{~m}(6 \mathrm{in})$

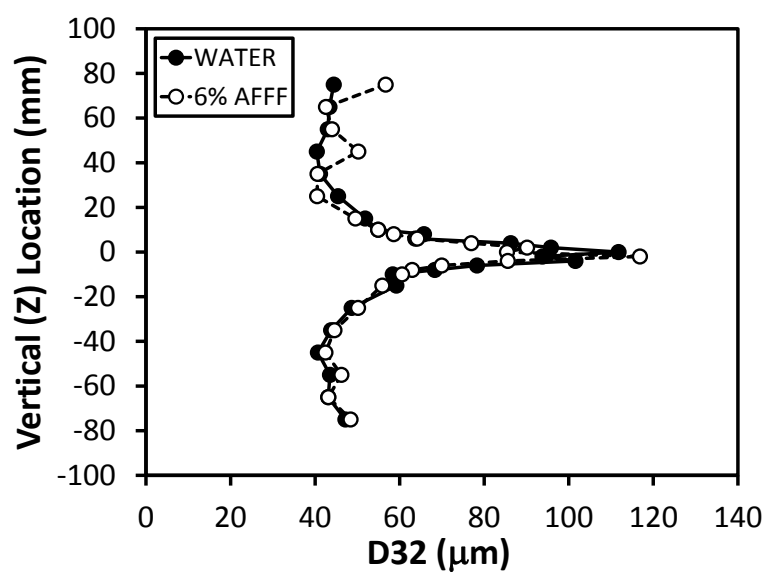

(e) $x=0.914 \mathrm{~m} \mathrm{(3 \textrm {ft } )}$

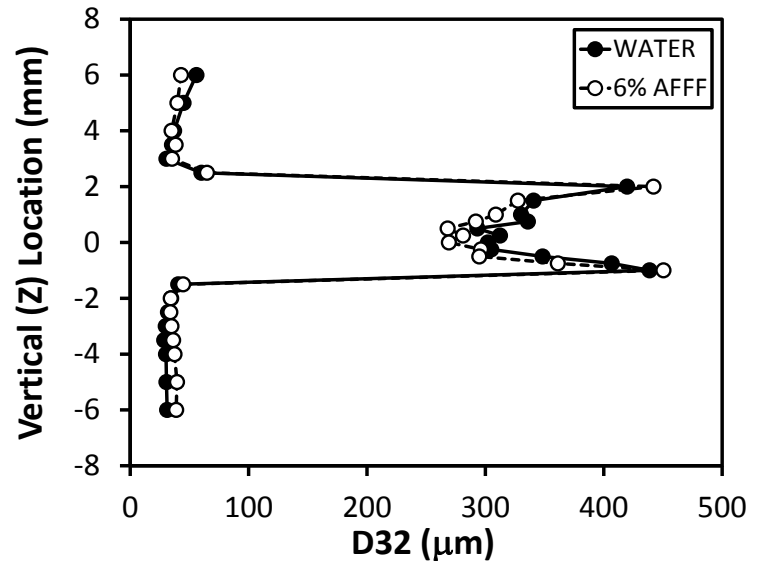

(b) $x=25.4 \mathrm{~mm}(1 \mathrm{in})$

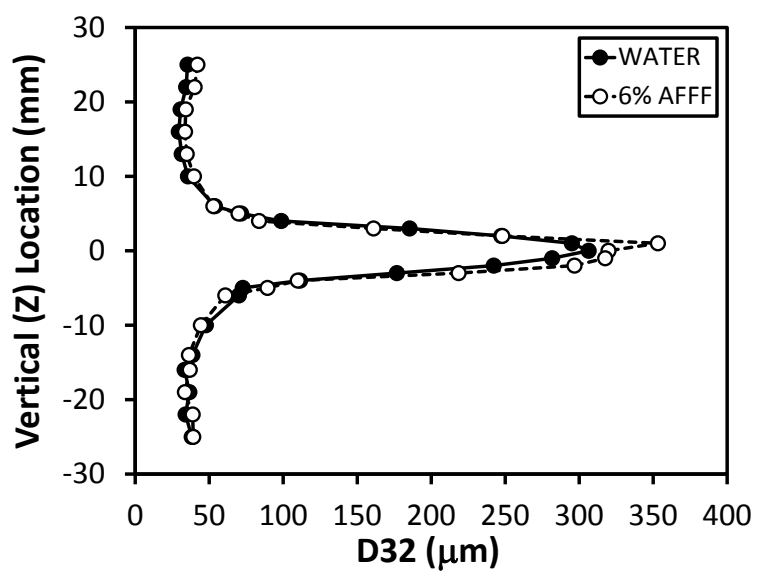

(d) $x=0.305 \mathrm{~m}(1 \mathrm{ft})$

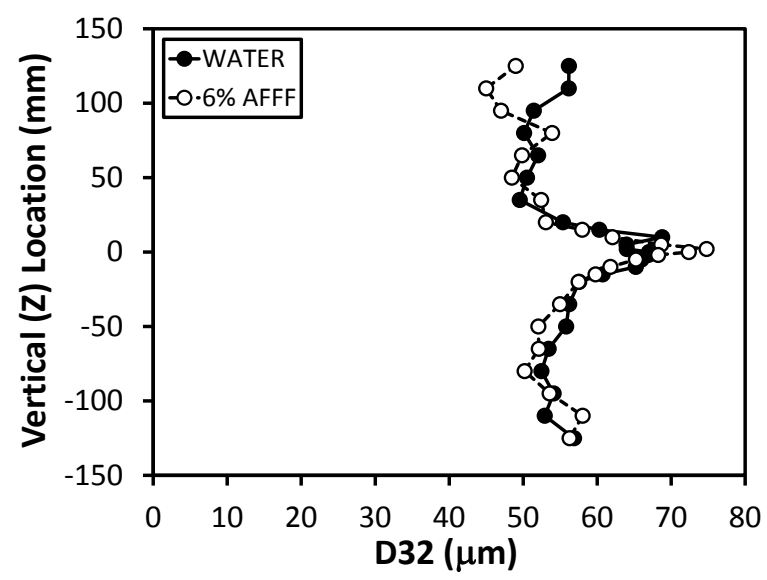

(f) $x=1.52 \mathrm{~m}(5 \mathrm{ft})$

Figure C.60: Near Field High Flow, High Press. Jet Vertical Profiles of Sauter Mean Droplet Diameter 

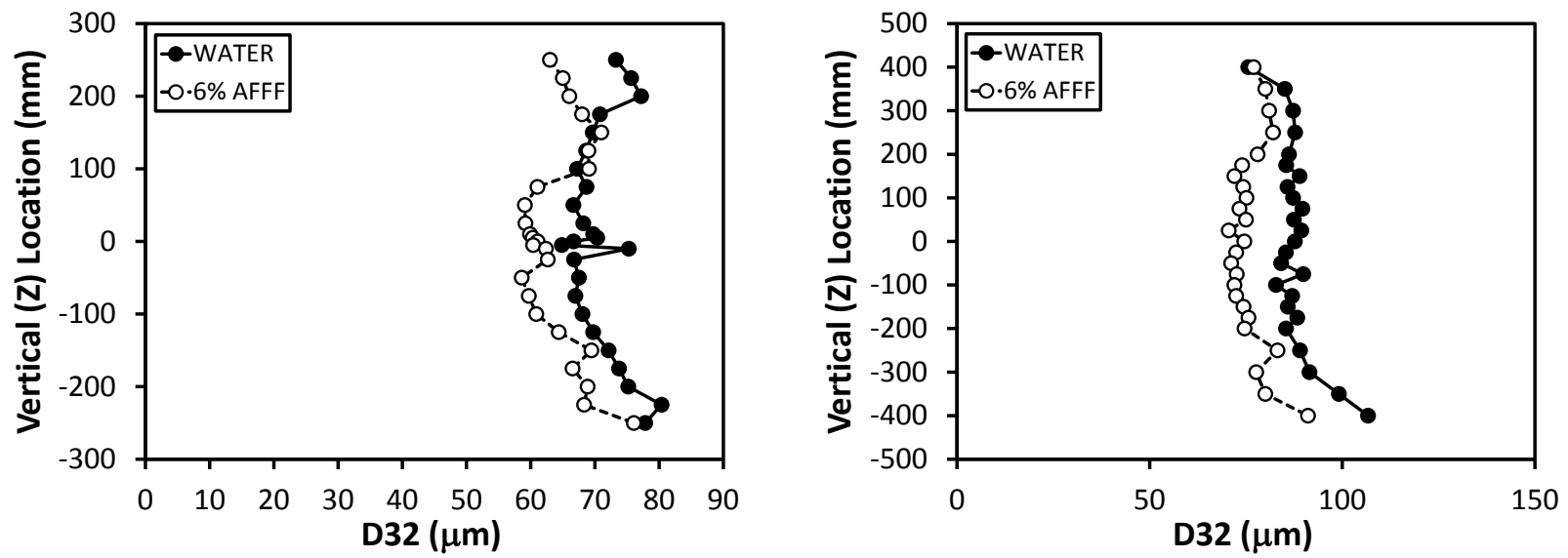

(a) $x=3.05 \mathrm{~m}(10 \mathrm{ft})$

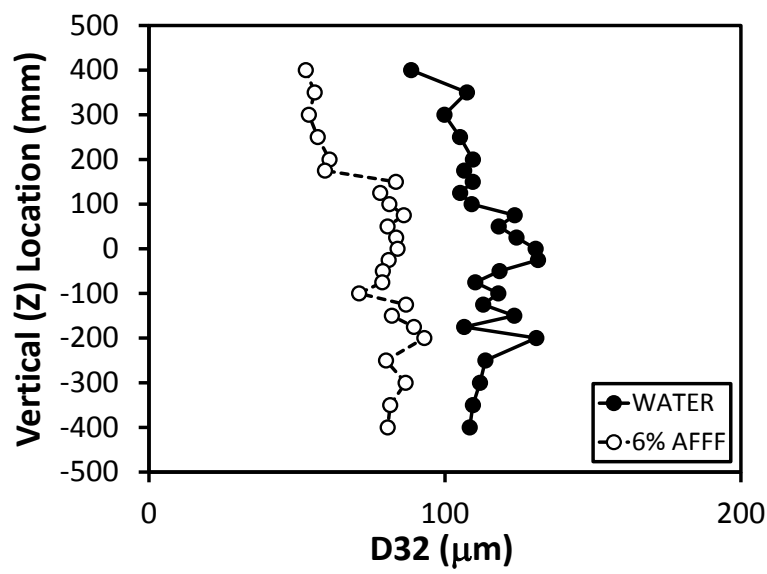

(c) $x=6.10 \mathrm{~m}(20 \mathrm{ft})$

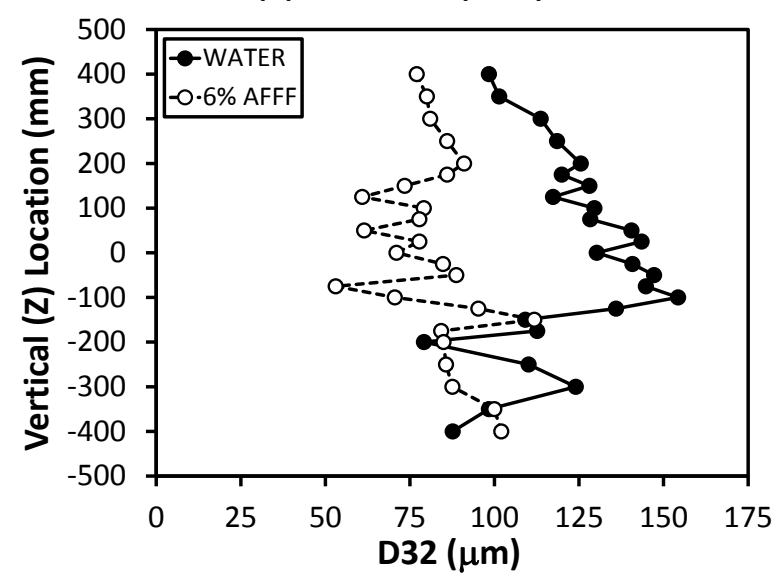

(d) $x=7.62 \mathrm{~m} \mathrm{(25 \textrm {ft } )}$

Figure C.61: Far Field High Flow, High Press. Jet Vertical Profiles of Sauter Mean Droplet Diameter 


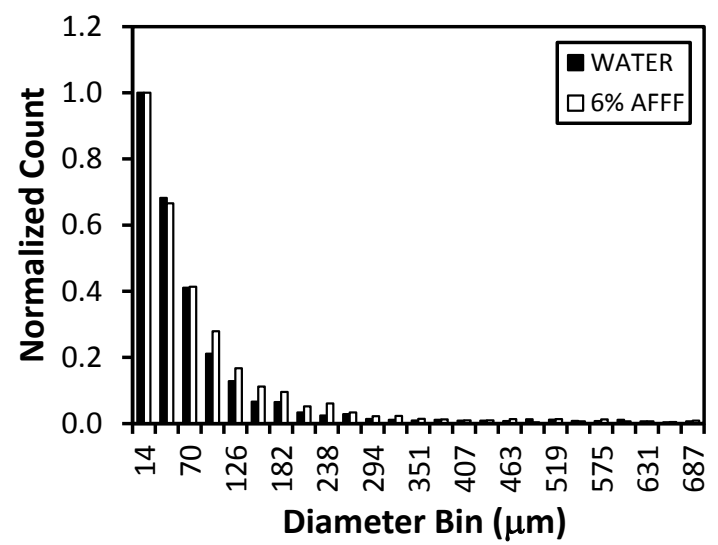

(a) $x=0.152 \mathrm{~m}(6 \mathrm{in})$

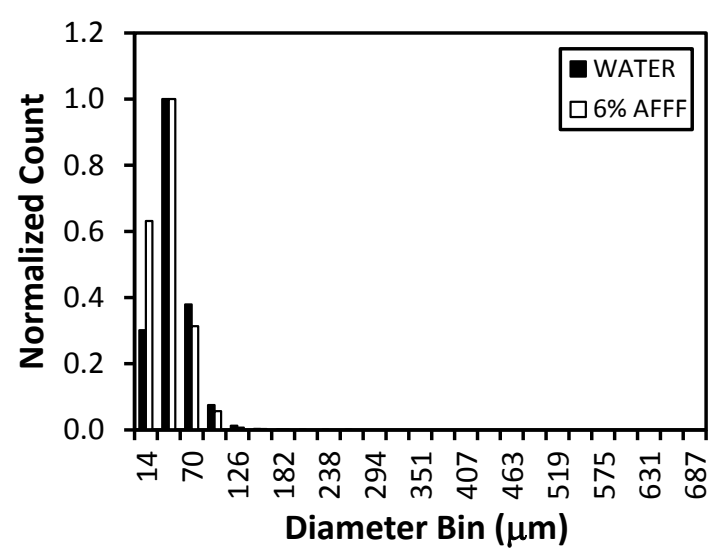

(c) $\mathrm{x}=3.05 \mathrm{~m}(10 \mathrm{ft})$

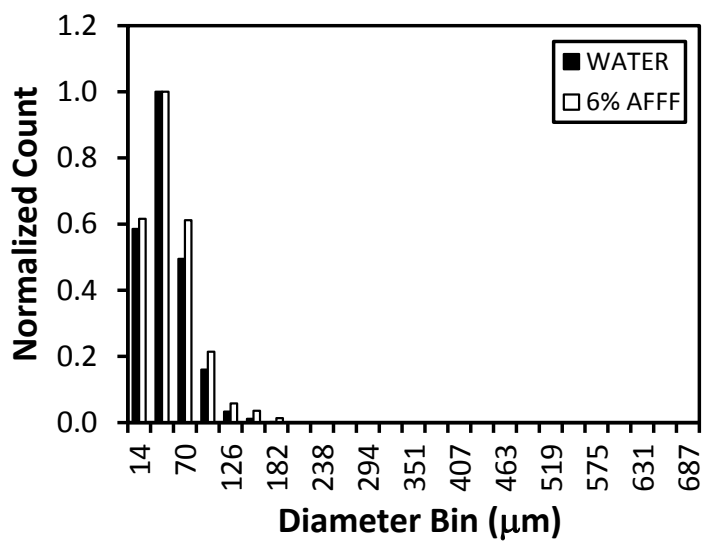

(b) $x=0.914 \mathrm{~m} \mathrm{(3 \textrm {ft } )}$

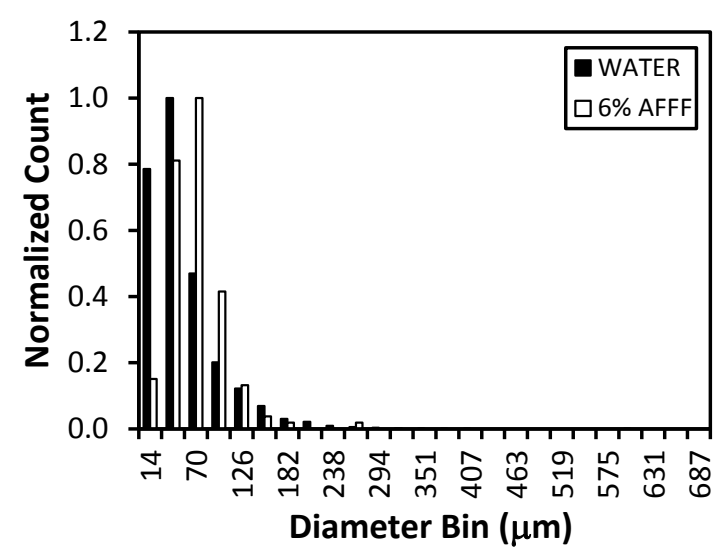

(d) $x=6.10 \mathrm{~m}(20 \mathrm{ft})$

Figure C.62: High Flow, High Press. Jet Droplet Size Distribution at the Max. Profile, Mean Axial Droplet Velocity for Select Vertical Profiles 


\section{APPENDIX D: PDPA CALIBRATION}

An auxiliary method to quantify Phase Doppler droplet size measurement uncertainty was determined via manufacturer calibration using PDPA components specifically used in the present study. A monosize droplet generator (MDG-100 Model KD Scientific 100) was used to produce a stream of uniform, reference droplet sizes made up of distilled water in the range of approximately 100 to $175 \mu \mathrm{m}$ with an uncertainty estimate of \pm 0.5 percent. The droplet generator relies on the principle of applying a constant periodic excitation to a laminar liquid jet, which causes surface disturbance waves to form and grow as the jet slows down. Jet break-up into a single droplet per surface wave period thus occurs. This is an established technique commonly used for fundamental droplet studies and is useful for verification and calibration checks for various instruments. The laminar liquid jet was generated by a constant flow rate pump that delivered fluid through a vibrating orifice. The orifice and column was made to vibrate by a frequency generator and piezo crystal generating one droplet per vibration cycle. The vibration rate and flow rate were controlled by the frequency generator and pump, respectively. Phase Doppler measurements were recorded using reflection light-scattering measurement techniques with the optics positioned in forward-scatter mode. Phase calibration was performed to maintain a relatively constant intensity of approximately $300 \mathrm{mV}$. Verification of the droplet generator stability was obtained by ensuring droplet frequency generation and the phase Doppler data rates were identical. An oscilloscope was used to confirm the Doppler bursts were well formed and repeatable. Measurements were recorded for 12 droplet generation conditions. Phase Doppler measurements were found to vary between 0.01 and 0.76 percent with respect to the expected droplet generator diameter. Table D.1 summarizes the measurement data, and Figure D.1 illustrates the droplet generator vibration frequency as a function of droplet diameter comparing the MGD-100 and mean droplet diameter reported by the PDPA. 
Table D.1: PDPA Droplet Size Calibration Certification Check Points

\begin{tabular}{|c|c|c|c|}
\hline $\begin{array}{c}\text { MDG-100 Vibration } \\
\text { Frequency } \\
(\mathbf{k H z})\end{array}$ & $\begin{array}{c}\text { MDG-100 } \\
\text { Diameter } \\
(\mu \mathrm{m})\end{array}$ & $\begin{array}{l}\text { PDPA Mean } \\
\text { D10 } \\
(\mu \mathrm{m})\end{array}$ & $\begin{array}{c}\text { Absolute } \\
\text { Relative Error } \\
\text { (\%) }\end{array}$ \\
\hline 37.3 & 98.2 & 97.52 & 0.68 \\
\hline 28.2 & 107.8 & 107.44 & 0.36 \\
\hline 23.7 & 114.23 & 114.10 & 0.13 \\
\hline 19.52 & 121.87 & 121.75 & 0.12 \\
\hline 16.54 & 128.78 & 128.96 & 0.18 \\
\hline 14.41 & 134.84 & 134.64 & 0.20 \\
\hline 12.43 & 141.65 & 141.66 & 0.01 \\
\hline 10.67 & 149.05 & 148.85 & 0.20 \\
\hline 9.34 & 155.81 & 155.05 & 0.76 \\
\hline 8.56 & 160.41 & 159.81 & 0.60 \\
\hline 7.42 & 168.23 & 168.17 & 0.06 \\
\hline 6.53 & 175.55 & 176.19 & 0.64 \\
\hline
\end{tabular}

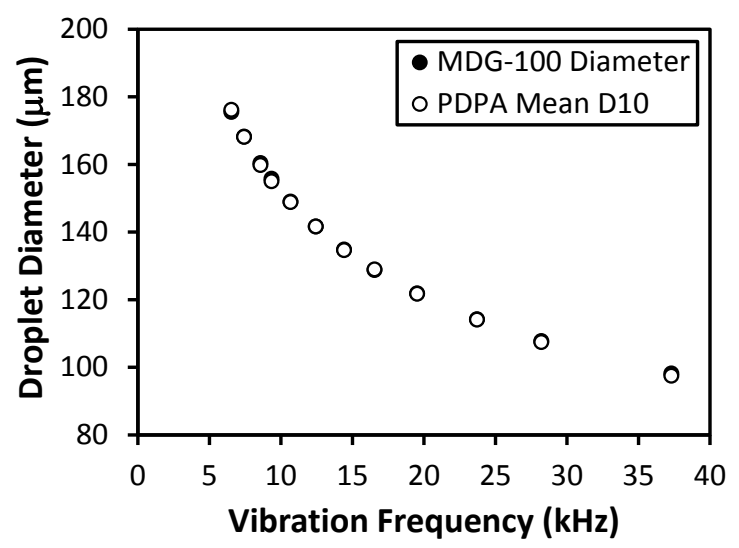

Figure D.1: PDPA Droplet Size Calibration Certification Comparing MDG-100 Diameter and PDPA Mean D10 